
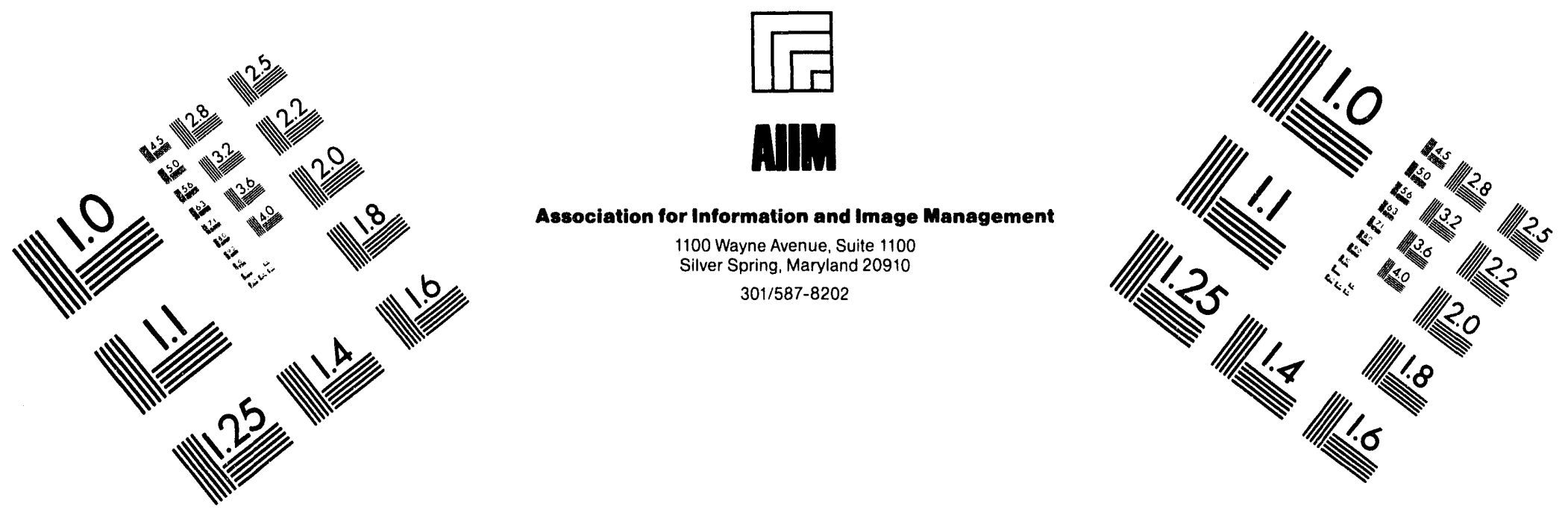

\title{
Centimeter
}

$\begin{array}{llllllllllllllll}1 & 2 & 3 & 4 & 5 & 6 & 7 & 8 & 9 & 10 & 11 & 12 & 13 & 14 & 15 & \mathrm{~mm}\end{array}$ | Inches
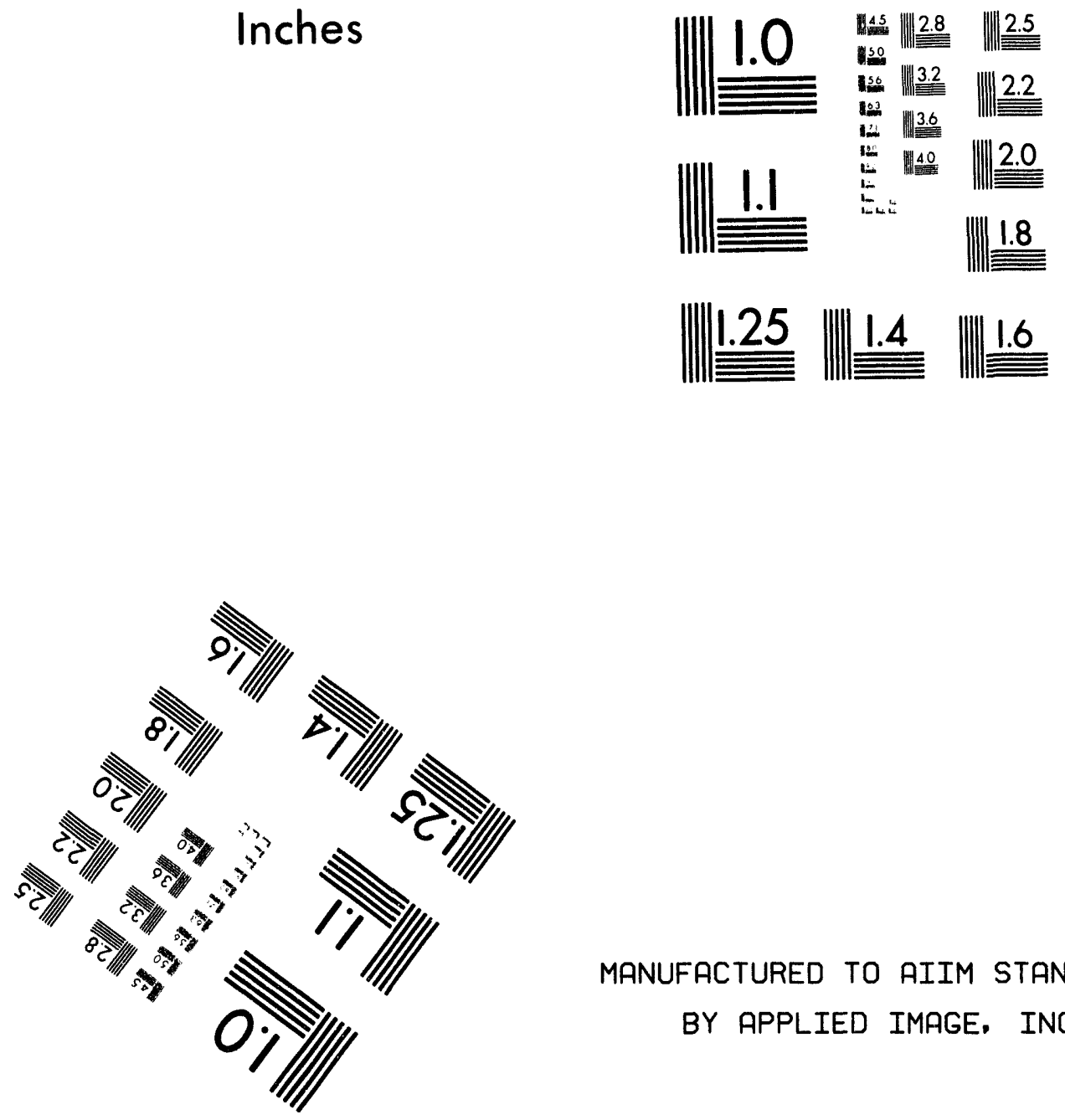

MANUFACTURED TO AIIM STANDARDS BY APPLIED IMAGE, INC.

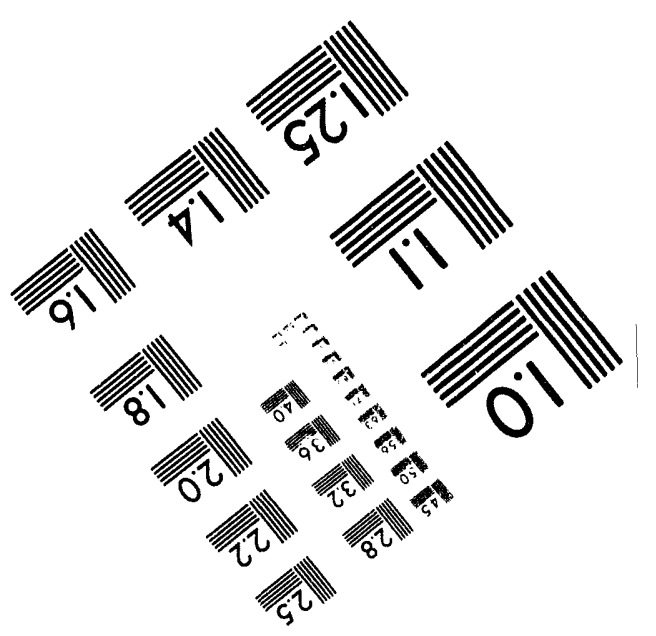



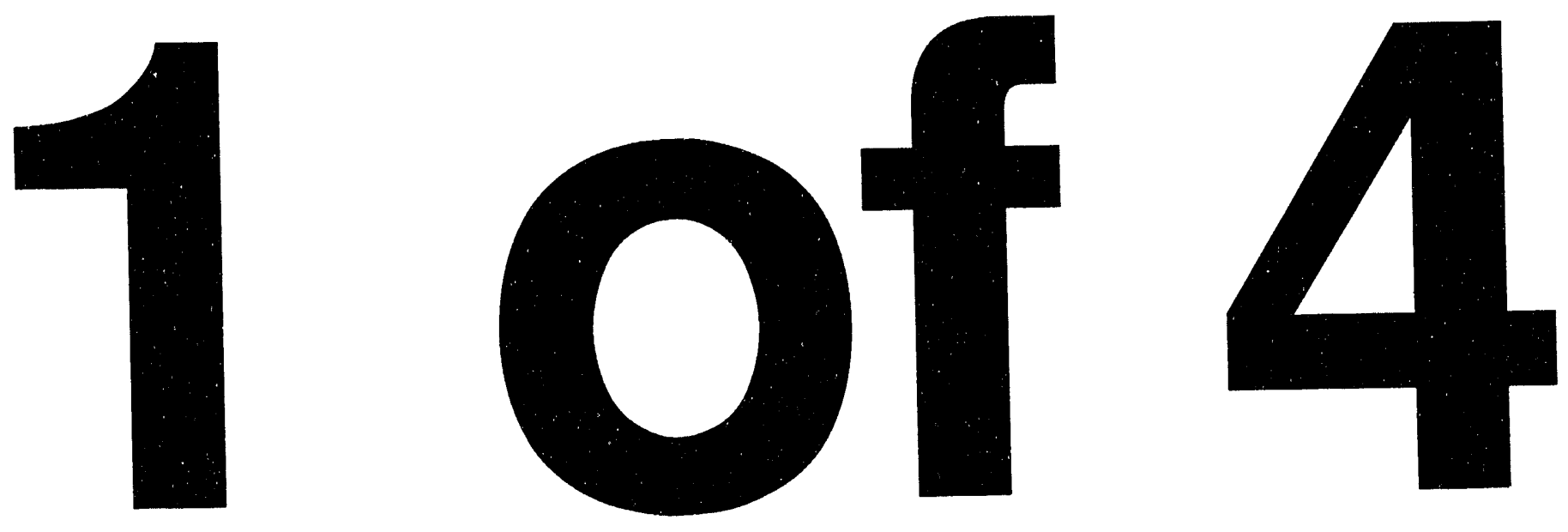


\section{Savannah River Site Environmental Data for 1993}

Environmental Monitoring Section

Environmental Protection Department

Westinghouse Savannah River Company Savannah River Site

Aiken, SC 29808 


\section{Savannah River Site Environmental Data for 1993}

Editor

Margaret W. Arnett

Prepared for the U.S. Department of Energy under contract No. DE-ACO9-89SR18035 Westinghouse Savannah River Company

Savannah River Site, Aiken, SC 29808 


\section{DISCLAIMER}

This report was prepared as an account of work sponsored by an agency of the United States Government. Neither the United States Government nor any agency thereof, nor any of their employees, makes any warranty, express or implied, or assumes any legal liability or responsibility for the accuracy, completeness, or usefulness of any information, apparatus, product, or process disclosed, or represents that its use would not infringe privately owned rights. Reference herein to any specific commercial product, process, or service by trade name, trademark, manufacturér, or otherwise does not necessarily constitute or imply its endorsement, recommendation, or favoring by the United States Government or any agency thereof. The views and opinions of authors expressed herein do not necessarily state or reflect those of the United States Government or any agency thereof.

This report has been reproduced directly from the best available copy.

Available to DOE and DOE contractors from the Office of Scientific and Technical Information, P. O. Box 62, Oak Ridge, TN 37831: prices available from (615) 576-8401.

Available to the public from the National Technical Information Service, U. $S$. Department of Commerce, 5285 Pon Royal Rd., Springfield, VA 22161 


\section{Acknowledgments}

The editor acknowledges with appreciation the efforts of individuals and groups who provided technical expertise, review, and oversight during the preparation of this book.

Environmental Monitoring Section Environmental Protection Department

Sandra Boynton

Jason Chappell

Brian Crandall

Ora Bell Daniel

Ted DeHart

Larry Eldridge

David Filler

Pete Fledderman

Nate Gauerke

Lynn Geary

Manley Grove

Bob Henderson

Craig Hetrick

Timothy Jannik

Linda Karapatakis

Moheb Khalil

Al Mamatey

Priscilla Patterson

Neil Suttles

Dan Vaaler

Roger Wong

Robin Young

Chris Wickham. John Ellinger, Tom Robelen and Tracey Humphrey (for computer support)

Chris Arenz (for distribution database)

Stuart Stinson (for administrative and technical assistance with production of this book)

Judi Phillips and Brenda Walker (for clerical support)

\section{Environmental Advisory Committee}

Dr. Edgar Berkey

Dr. Keros Cartwright

Dr. Stanley N. Davis

Dr. Bernd Kahn

Dr. Ruth Patrick

Dr. Milton Russell

\section{Westinghouse Savannah River Company}

Bill Carlton and Dave Hamby (for dose estimates)

WSRC Technical Information Management (for illustrating, classification, and printing support-Jeff Hammond, manager; Janet Salaymeh, Site Environmental Report project coordinator; Dennis Hendrix, illustrator; Stephanie Doetsch, printing, and Joanne Hay, off-site printing)

\section{Department of Energy-Savannah River}

Chuck Borup (for coordinating the DOE-SR/DOE-HQ review and approval process) 


\section{Introduction}

The figures and tables in this report represent a capsule view of the routine environmental monitoring and surveillance programs at the Savannah River Site. An attempt has been made to include all available data from environmental research programs.

The first section of the book is a collection of maps of radiological and nonradiological sampling locations. Also included are general radiological and nonradiological sampling and analysis schedules; a list of the media sampled, along with sample sizes and representative aliquots; a list of the lower limits of detection for radiological detection instruments; the minimum detectable concentrations for gamma analysis of water and air samples; and the minimum detectable concentrations for gamma analysis of soil, food, fish and wildlife, and vegetation samples.

Following the first section are data tables containing radiological and nonradiological effluent monitoring results, radiological and nonradiological environmental surveillance results, dose estimates, quality assurance activities, and results of nonroutine occurrences and special surveys.

Data tables sometimes present fewer results than would be expected according to the frequency described in the sampling and analysis schedules. There are several reasons for this. Problems may have arisen with sample collection, such as loss of power to the sampling site or inaccessibility to the sampling site (locked gates, flooding, etc.). Results for collected samples can be rejected for such reasons as insufficient sample volume, low chemical yield, or equipment failure. The "number of samples" columns in the tables refer to the number of results used to determine maximum, minimum, and average concentrations.

The following should aid the reader in interpreting the data:

- The uncertainty term is reported with up to-but no more than-three significant figures. In most cases, the result is reported with up to three significant figures, with the last significant figure determined by the quantification of the uncertainty term.

- The reported uncertainty reflects only the counting error-not other components of random and systematic error present in the measurement process. For this reason, some results may imply a greater confidence than the determination would suggest.

- Uncertainties quoted with means represent the deviation of measurements about the mean value. This number is calculated from the results themselves and does not account for the uncertainties of the individual results.

- Averages were calculated using both positive and negative results, except for gammaemitting radionuclides, whose less-than-detectable results were not considered in the averages.

- In tables containing arithmetic mean, maximum, and minimum columns, when only one sample was collected, the single result is reported in the arithmetic mean column. 
Information in this book is summarized in the Savannah River Site Environmental Report for 1993 (WSRC-TR-94-075). Information about the environmental monitoring program, including a complete description of the Environmental Protection Department/Environmental Monitoring Section sampling and analytical procedures, can be found in the "SRS Environmental Monitoring Plan" (WSRC-3Q1-2-1000).

To obtain copies of environmental reports and documents, contact

Manager, Environmental Publications

Westinghouse Savannah River Company

Building 735-16A

Aiken, SC 29808

Telephone: $803-725-3556$ 


\section{Radionuclide and Chemical Nomenclature}

\section{Nomenclature and Half-life for Radionuclides}

\section{Radionuclide}

Americium-241

Americium-243

Antimony-125

Argon-41

Berylium-7

Carbon-14

Cerium-141

Cerium-144

Cesium-134

Cesium-137

Cobalt-58

Cobalt-60

Curium-242

Curium-244

lodine-129

lodine-131

Krypton-85

Krypton-88

Manganese-54

Niobium-95

Osmium-185

Phosphorus-32

Polonium-210

Plutonium-238

Plutonium-239

Potassium -40

Promethium-147

Ruthenium-103

Ruthenium-106

Selenium-75

Stronilum-89

Strontiurn-90

Tritium

Uranium-235

Uranium-238

Xenon-133

Xenon-135

Yttrium-90

Zirconium-95

\section{Symbol}

Am-241

Am-243

Sb-125

Ar- 41

$\mathrm{Be}-7$

C-14

Ce-141

Ce-144

Cs-134

Cs-137

Co-58

Co-60

$\mathrm{Cm}-242$

$\mathrm{Cm}-244$

I-129

|-131

$\mathrm{Kr}-85$

$\mathrm{Kr}-88$

$\mathrm{Mn}-54$

$\mathrm{Nb}-95$

Os- 185

P-32

Po-210

Pu-238

Pu-239

K-40

$\mathrm{Pm}-147$

Ru-103

Ru-106

Se-75

Sr-89

Sr-90

$\mathrm{H}-3$

U-235

U-238

$x e-133$

$X e-135$

Y-90

Zr-95
Half-life ${ }^{a, b}$

$432.7 y$

7.37E3 y

$2.7 y$

$1.83 \mathrm{~h}$

$53 \mathrm{~d}$

$5,730 \mathrm{y}$

$33 \mathrm{~d}$

$284 d$

$2.05 \mathrm{y}$

$30 \mathrm{y}$

$71.3 \mathrm{~d}$

$5.26 \mathrm{y}$

$163 d$

$17.6 \mathrm{y}$

1.7E7 y

$8.05 \mathrm{~d}$

$10.76 \mathrm{y}$

$2.8 \mathrm{~h}$

$312 d$

$35 d$

$94 d$

$14.3 \mathrm{~d}$

$138.4 \mathrm{~d}$

$87.4 \mathrm{y}$

2.4E4 y

$1.26 E 9 y$

$2.62 \mathrm{y}$

$39.6 \mathrm{~d}$

$367 d$

$120.4 d$

$52 d$

$28.1 y$

$12.3 y$

7.1E8 y

4.5E9 y

$5.27 \mathrm{~d}$

$9.16 \mathrm{~h}$

$64 \mathrm{~h}$

$65 d$

a $h=$ hour; $d=$ day; $y=$ year

b Reference: U.S. Department of Health, Education, and Welfare, 1970, Radiological Health Handbook, Food and Drug Administration, Bureau of Radiological Health, Rockville, Md. 
Nomenclature for Elements and Chemical Constituents

Constituent

Symbol

Aluminum

Ammonia

Antimony

Arsenic

Barium

Beryllium

Cadmium

Calcium

Calcium Carbonate

Carbon

Chlorine

Chromium

Cobalt

Copper

Fluorine

Iron

Lead

Lithium

Magnesium

Manganese

Mercury

Nickel

Nitrogen

Nitrate

Nitrite

Oxygen

Ozone

Phosphorus

Phosphate

Potassium

Radium

Rhenium

Selenium

Silver

Sodium

Sulfate

Sulfur Dioxide

Thallium

Uranium

Vanadium

Zinc 


\section{List of Figures}

Figure $1 \quad$ SRS Streams and Savannah River Water Quality Sampling Locations $\ldots \ldots \ldots \ldots \ldots$

Figure 2 SRS Streams and Savannah River Water and Sediment Sampling Locations .......... 2

Figure $3 \quad$ Air Surveillance Stations Located Within 25 Miles of SRS $\ldots \ldots \ldots \ldots \ldots$

Figure $4 \quad$ Off-Site TLD Surveillance Locations $\ldots \ldots \ldots \ldots \ldots \ldots \ldots \ldots \ldots \ldots \ldots$

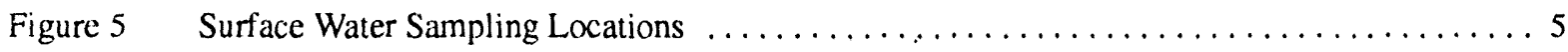

Figure $6 \quad$ Drinking Water Sample Locations $\ldots \ldots \ldots \ldots \ldots \ldots \ldots \ldots \ldots \ldots \ldots \ldots \ldots \ldots \ldots \ldots \ldots \ldots \ldots \ldots$

Figure $7 \quad$ SRS Sediment Sampling Locations $\ldots \ldots \ldots \ldots \ldots \ldots \ldots \ldots \ldots \ldots \ldots \ldots \ldots$

Figure $8 \quad$ Quarterly On-site Vegetation Sampling Locations at SRS $\ldots \ldots \ldots \ldots \ldots \ldots$

Figure $9 \quad$ SWDF Interior Vegetation Sampling Locations $\ldots \ldots \ldots \ldots \ldots \ldots \ldots \ldots$

Figure $10 \quad$ Food/Milk Sampling Locations $\ldots \ldots \ldots \ldots \ldots \ldots \ldots \ldots \ldots \ldots \ldots \ldots \ldots \ldots \ldots$

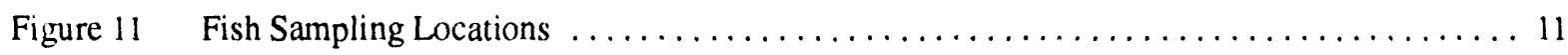




\section{Sampling}

Table 1 General Radiological Sampling and Analysis Schedule $\ldots \ldots \ldots \ldots \ldots \ldots \ldots \ldots \ldots \ldots \ldots \ldots \ldots \ldots \ldots$

Table 2 General Nonradiological Sampling and Analysis Schedule $\ldots \ldots \ldots \ldots \ldots \ldots \ldots \ldots \ldots \ldots \ldots$

Table 3

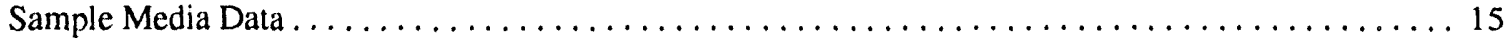

Table 4 Minimum Detertablc Concentrations for Gamma Analysis of Water and Air Samples $\ldots \ldots \ldots \ldots 16$

Table 5 Minimum Deiectable Concer:trations for Gamma Analysis of Soil, Food, Fish and Wildlife,

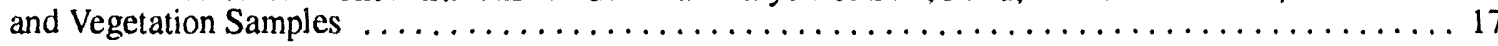

Table 6 Lower Limits of Detection for Radiological Detection Instruments $\ldots \ldots \ldots \ldots \ldots \ldots \ldots \ldots \ldots$

\section{Radiological Effluerit Monitoring}

Table 7

Calculated Migration of Radioactivity from Seepage Basins . . . . . . . . . . . . . . . . . 19

Table 8

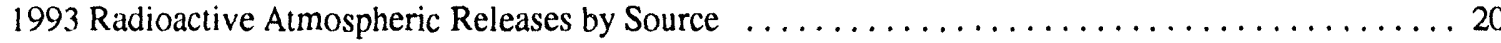

Table 9

1993 Radioactive Atmospheric Releases by Stack/Facility and Comparison of Annual Average Concentrations to DOE Derived Concentration Guides (DCGs) $\ldots \ldots \ldots \ldots \ldots \ldots \ldots \ldots \ldots \ldots \ldots$

Table 10

1993 Radioactive Liquid Releases by Source (Including Direct and Seepage Basin Migration Releases)

Table 11

1993 Liquid Radioactive Releases by Facility and Comparison of Annual Average Radionuclide Concentrations to DOE Derived Concentration Guides (DCGs)

Table 12

Estimated Tritium Releases in SRS Streams and the Savannah River $\ldots \ldots \ldots \ldots \ldots \ldots \ldots \ldots \ldots$

Table 13

Minimum Detectable Activities (MDAs) for Radionuclides in Settleable Solids . . . . . . . . . . . 34

Table 14

Settleable Solids Results for the SRS Radionuclides in Settleable Solids Program $\ldots \ldots \ldots \ldots \ldots . \ldots 35$

\section{Radiological Environmental Surveillance}

Table 15

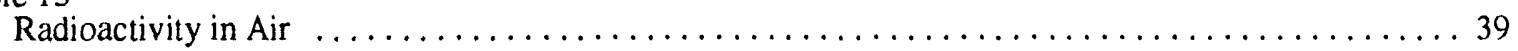

Table 16

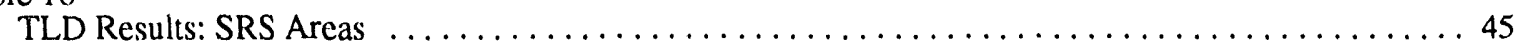

Table 17

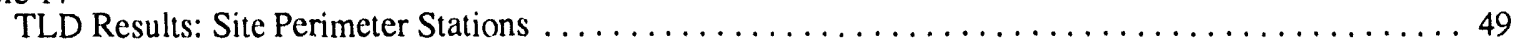

Table 18

TLD Results: Environmental Surveillance (Air Monitoring) Stations $\ldots \ldots \ldots \ldots \ldots \ldots \ldots \ldots$

3 le 19

TLD Results: Population Centers

58 
Table 20

TLD Results: Vogtle Electric Generating Plant Vicinity $\ldots \ldots \ldots \ldots \ldots \ldots \ldots \ldots \ldots \ldots \ldots$

Table 21

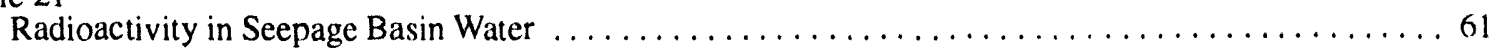

Table 22

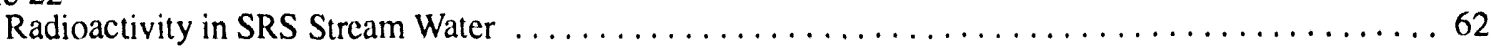

Table 23

Radioactivity in Transport at Sample Points on Four Mile Creek $\ldots \ldots \ldots \ldots \ldots \ldots \ldots \ldots$

Table 24

Radioactivity in Savannah River Water $\ldots \ldots \ldots \ldots \ldots \ldots \ldots \ldots \ldots \ldots \ldots \ldots \ldots$

Table 25

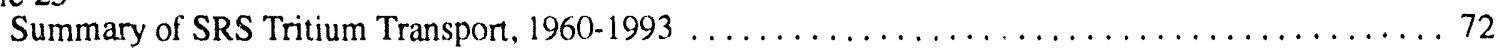

Table 26

Radioactivity in Drinking Water $\ldots \ldots \ldots \ldots \ldots \ldots \ldots \ldots \ldots \ldots \ldots \ldots \ldots \ldots \ldots \ldots \ldots$

Table 27

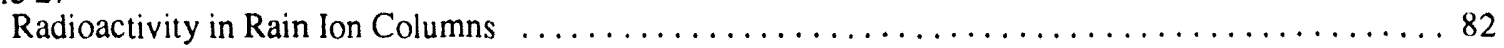

Table 28

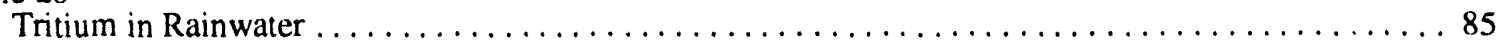

Table 29

Kadioactivity in River and Stream Sediment $\ldots \ldots \ldots \ldots \ldots \ldots \ldots \ldots \ldots \ldots \ldots \ldots \ldots$

Table 30

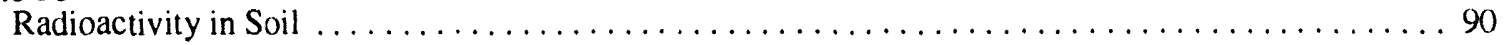

Table 31

Radioactivity in Vegetation (Quarterly Samples) $\ldots \ldots \ldots \ldots \ldots \ldots \ldots \ldots \ldots \ldots \ldots \ldots$

Table 32

Radioactivity in Vegetation (Composites from Seepage and Retention Basins) . . . . . . . . . . . 97

Table 33

Radioactivity in Vegetation (Inside Solid Waste Disposal Facility Fences) . . . . . . . . . . . 98

Table 34

Radioactivity in Vegetation (Outside the Solid Waste Disposal Facility Fences) . . . . . . . . . . 100

Table 35

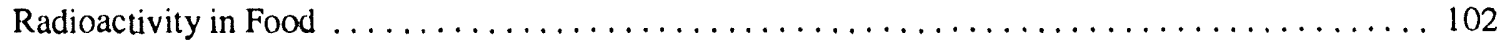

Table 36

Radioactivity in Eggs $\ldots \ldots \ldots \ldots \ldots \ldots \ldots \ldots \ldots \ldots \ldots \ldots \ldots \ldots \ldots \ldots$

Table 37

Radioactivity in Milk $\ldots \ldots \ldots \ldots \ldots \ldots \ldots \ldots \ldots \ldots \ldots \ldots \ldots \ldots \ldots \ldots \ldots \ldots \ldots \ldots$

Table 38

Radioactivity in Fish $\ldots \ldots \ldots \ldots \ldots \ldots \ldots \ldots \ldots \ldots \ldots \ldots \ldots \ldots \ldots \ldots \ldots \ldots \ldots \ldots$

Table 39

Cesium-1 37 in Off-Site Deer $\ldots \ldots \ldots \ldots \ldots \ldots \ldots \ldots \ldots \ldots \ldots \ldots \ldots \ldots \ldots \ldots \ldots \ldots$

Table 40

Comparison of Field and Laboratory Cesium-137 Measurements in Deer and Hogs . . . . . . . . . 126

Table 41

Summary of Cesium -137 in Deer $\ldots \ldots \ldots \ldots \ldots \ldots \ldots \ldots \ldots \ldots \ldots \ldots \ldots \ldots$

Table 42

Strontium in Deer and $\operatorname{Hog}$ Muscle $\ldots \ldots \ldots \ldots \ldots \ldots \ldots \ldots \ldots \ldots \ldots \ldots \ldots \ldots$

Table 43

Strontium in Deer Bone 


\section{Dose}

Table 44

Parameters Used for Adult Consumption Rates and for Atmospheric Dose Calculations

Table 45

Release Locations for 1993 Maximum and Average Individual Doses $\ldots \ldots \ldots \ldots \ldots \ldots \ldots \ldots$

Table 46

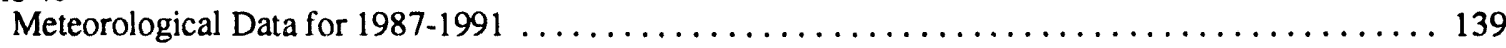

Table 47

50-Mile-Radius ( $80 \mathrm{~km}$ ) Population Distribution Around SRS (1990 Census) $\ldots \ldots \ldots \ldots \ldots \ldots \ldots$

Table 48

50-Mile-Radius ( $80 \mathrm{~km}$ ) Milk, Meat, and Vegetation Production Around SRS $\ldots \ldots \ldots \ldots \ldots \ldots \ldots$

Table 49

Site-Specific Parameters Used with CAP88 Code Used for NESHAPS Calculations . . . . . . . . . . 149

Table 50

1993 Total Site Releases and Maximum Individual Effective Dose Equivalent by Radionuclide

(CAP88 Dose Calculations for 1993 NESHAP Report to EPA)

Table 51

1993 NESHAP Report Data-CAP88 Compared with MAXIGASP . .................. 151

Table 52

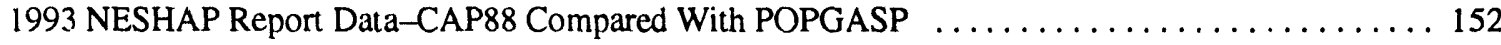

Table 53

Parameters Used for Adult Consumption Rates and for Liquid Dose Calculations . . . . . . . . . 153

Table 54

Site-Specific Parameters Used in Liquid Dose Calculations $\ldots \ldots \ldots \ldots \ldots \ldots \ldots \ldots \ldots \ldots \ldots \ldots \ldots$

Table 55

Maximum Individual Committed Dose at Site Perimeter from Atmospheric Releases

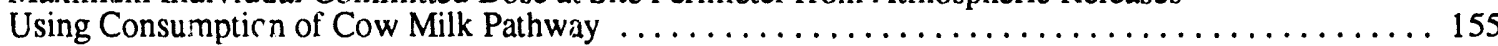

Table 56

Average Individual Committed Dose at Site Perimeter from Atmospheric Releases

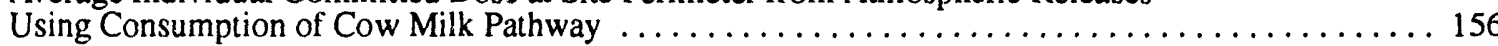

Table 57

Maximum Individual Committed Dose at Site Perimeter from Atmospheric Releases

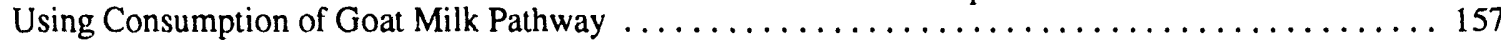

Table 58

50-Mile Collective Dose from Atmospheric Releases $\ldots \ldots \ldots \ldots \ldots \ldots \ldots \ldots \ldots \ldots \ldots \ldots \ldots$

Table 59

Maximum Individual Committed Dose from Liquid Releases $\ldots \ldots \ldots \ldots \ldots \ldots \ldots \ldots \ldots \ldots$

Table 60

Individual Committed Dose from Public Water Supplies at Beaufort-Jasper $\ldots \ldots \ldots \ldots \ldots \ldots \ldots$. 160

Table 61

Individual Committed Dose from Public Water Supplies at Port Wentworth $\ldots \ldots \ldots \ldots \ldots \ldots \ldots 1$

Table 62

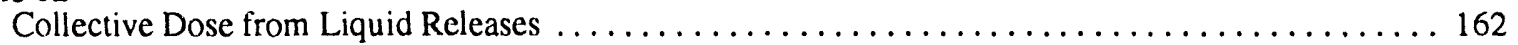

Table 63

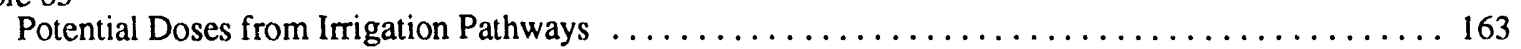

Table 64

Dose from Consumption of Fish from Creek Mouths and Highway $301 \quad \ldots \ldots \ldots \ldots \ldots \ldots \ldots$

Table 65

Calculated Doses to Aquatic Biota from SRS Releases $\ldots \ldots \ldots \ldots \ldots \ldots \ldots \ldots \ldots \ldots \ldots$ 


\section{Nonradiological Effluent Monitoring}

Table 66

National Pollutant Discharge Elimination System Monitoring Data $\ldots \ldots \ldots \ldots \ldots \ldots \ldots \ldots$

Table 67

National Pollutant Discharge Elimination System Stormwater Monitoring Data $\ldots \ldots \ldots \ldots \ldots \ldots$

Nonradiological Environmental Surveillance

Table 68

Savannah River Water Quality ...................................... 199

Table 69

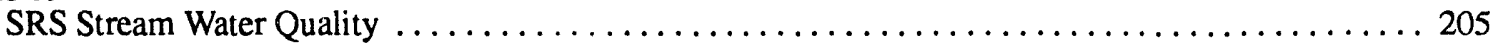

Table 70

Pesticides and Herbicides in Stream and River Water and Sediment $\ldots \ldots \ldots \ldots \ldots \ldots \ldots \ldots \ldots$

Table 71

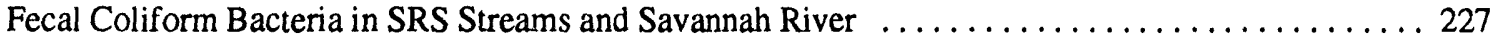

Table 72

Mercury in Fish Flesh $\ldots \ldots \ldots \ldots \ldots \ldots \ldots \ldots \ldots \ldots \ldots \ldots \ldots \ldots \ldots \ldots \ldots, 233$

Table 73

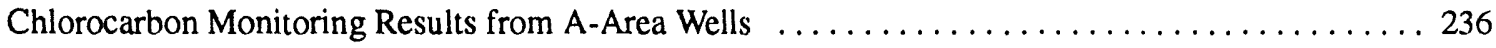

Table 74

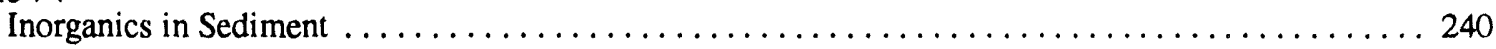

\section{Quality Assurance}

Table 75

Blind Sample Results for Conductivity Field Measurements ..................... 242

Table 76

Blind Sample Results for $\mathrm{pH}$ Field Measurements $\ldots \ldots \ldots \ldots \ldots \ldots \ldots \ldots \ldots \ldots \ldots \ldots \ldots \ldots$

Table 77

EMS Blind Sample Results for Tritium and Strontium $\ldots \ldots \ldots \ldots \ldots \ldots \ldots \ldots \ldots \ldots \ldots . \ldots \ldots$

Table 78

EMS Blind Sample Results for Gross Alpha and Beta and Gamma-Emitting Radionuclides ....... 245

Table 79

QAD Interlaboratory Comparison of Analytical Results ......................... 246

Table 80

QAP Interlaboratory Comparison of Analytical Results $\ldots \ldots \ldots \ldots \ldots \ldots \ldots \ldots \ldots \ldots \ldots \ldots \ldots$

Table 81

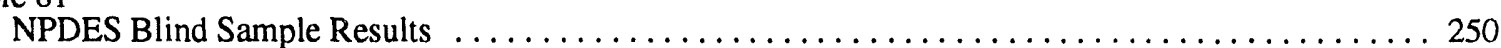

Table 82

NPDES Duplicate Sample Results ..................................... 251

\section{Nonroutine Occurrences}

Table 83

Release and Historical Data from the $195-$ Foot F-Area Stack . . . . . . . . . . . . . . . . 257

Table 84

Comparison of Alpha Concentrations Before and During the Period of the Nonroutine Release

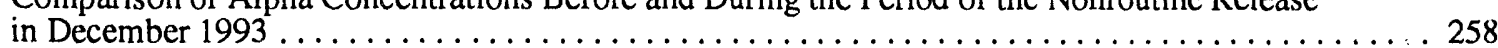




\section{Special Surveys}

Table 85

Savannah River Swamp TLD Exposure Measurements-1993

Table 86

Creek Plantation Survey, Swamp Soil Results ............................... 261

Table 87

Creek Plantation Survey, Swamp Vegetation Results ........................... 262

Table 88

Creek Plantation Survey, Swamp Results 1990, 1991, 1993 .......................... 263

Table 89

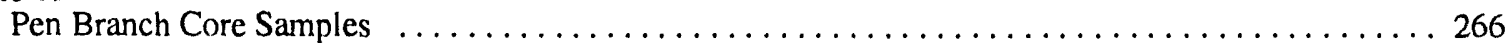

Table 90

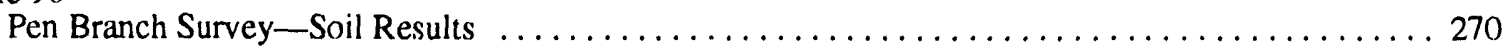

Table 91

Pen Branch Survey, Vegetation Results .................................... 272 


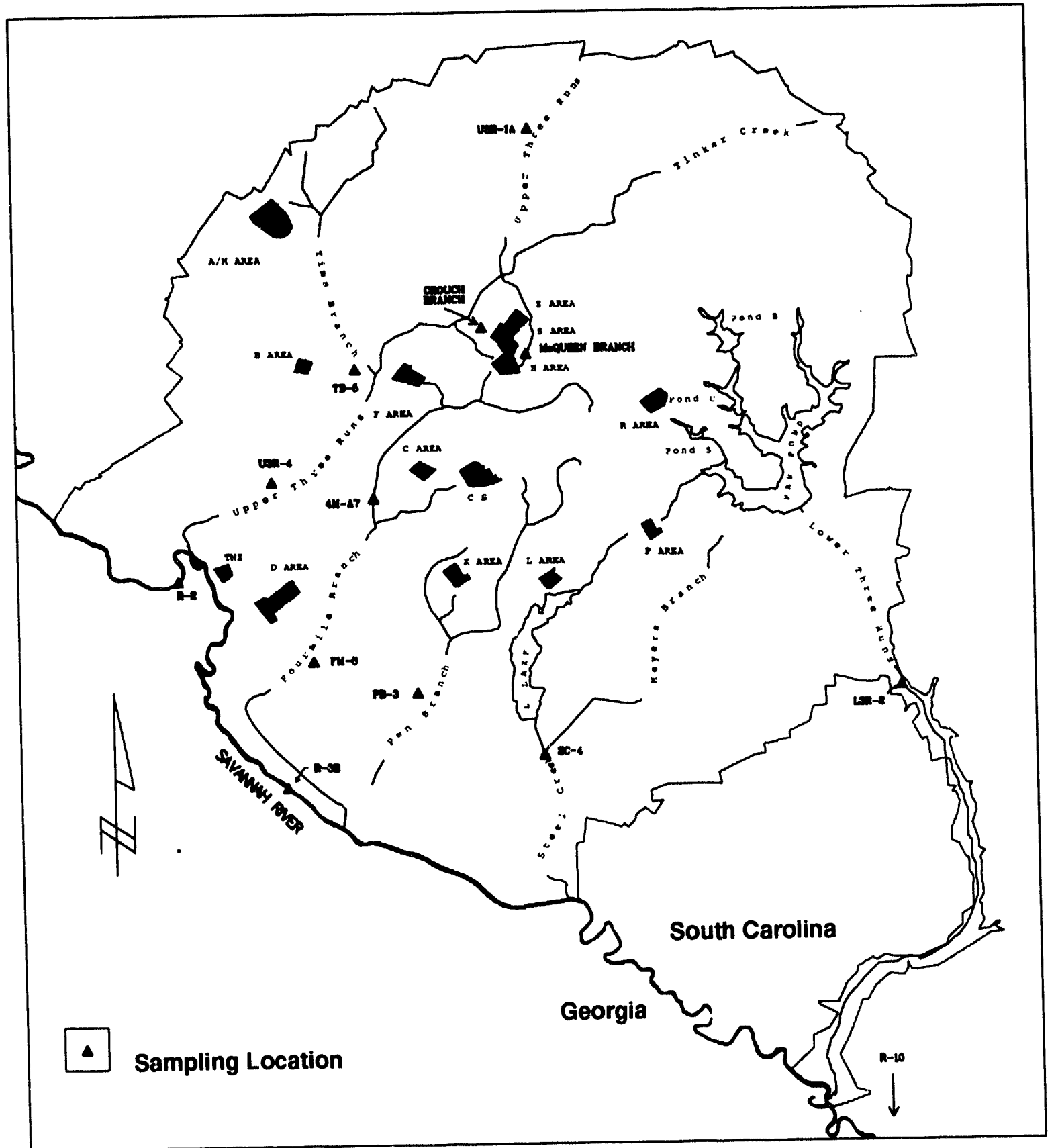

GIS Map

Figure 1 SRS Streams and Savannah River Water Quality Sampling Locations Note: Fourmile Branch is also known as Four Mile Creek.) 


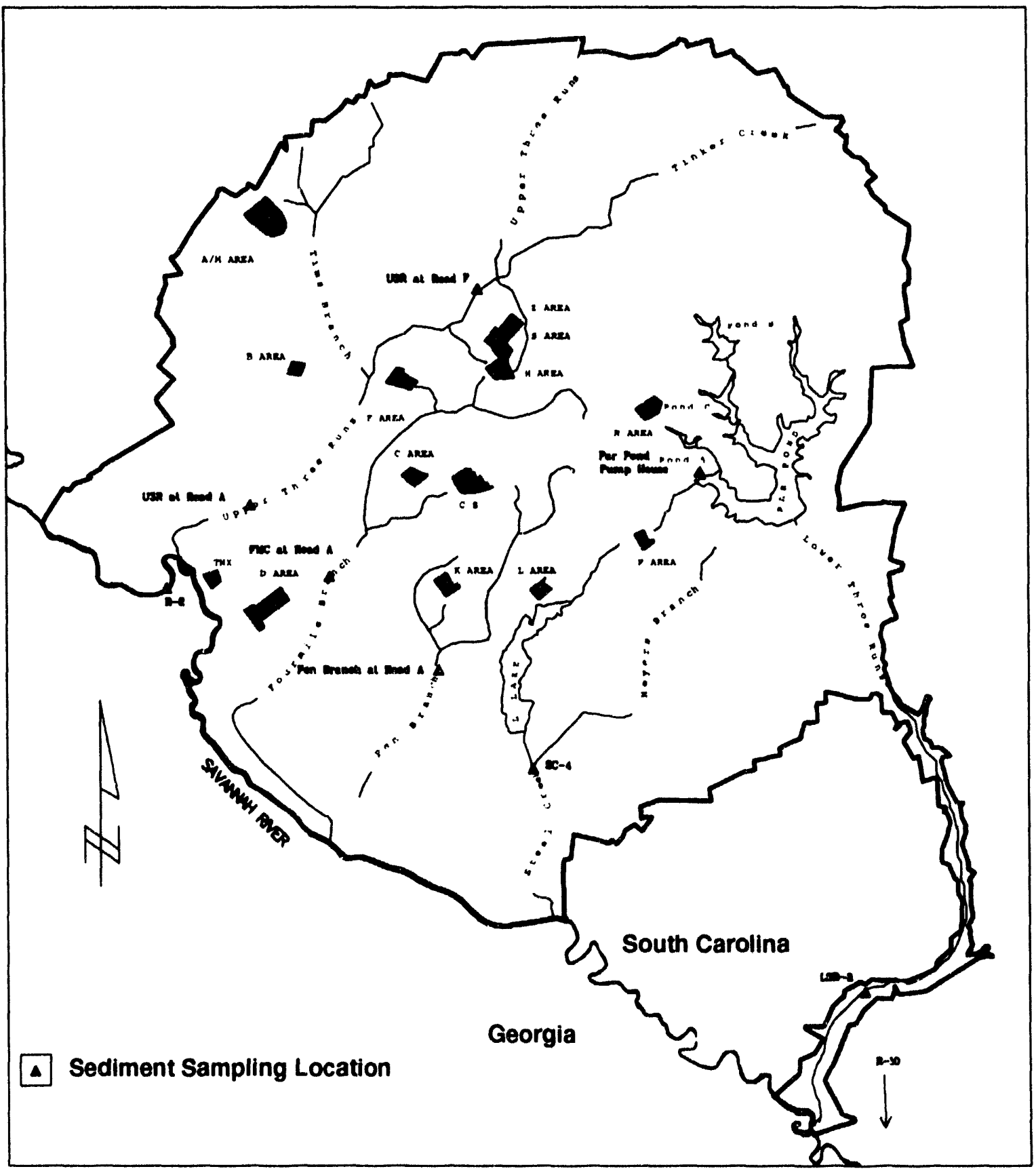

Figure 2 SRS Streams and Savannah River Water and Sediment Sampling Locations Note: Fourmile Branch is also known as Four Mile Creek. 


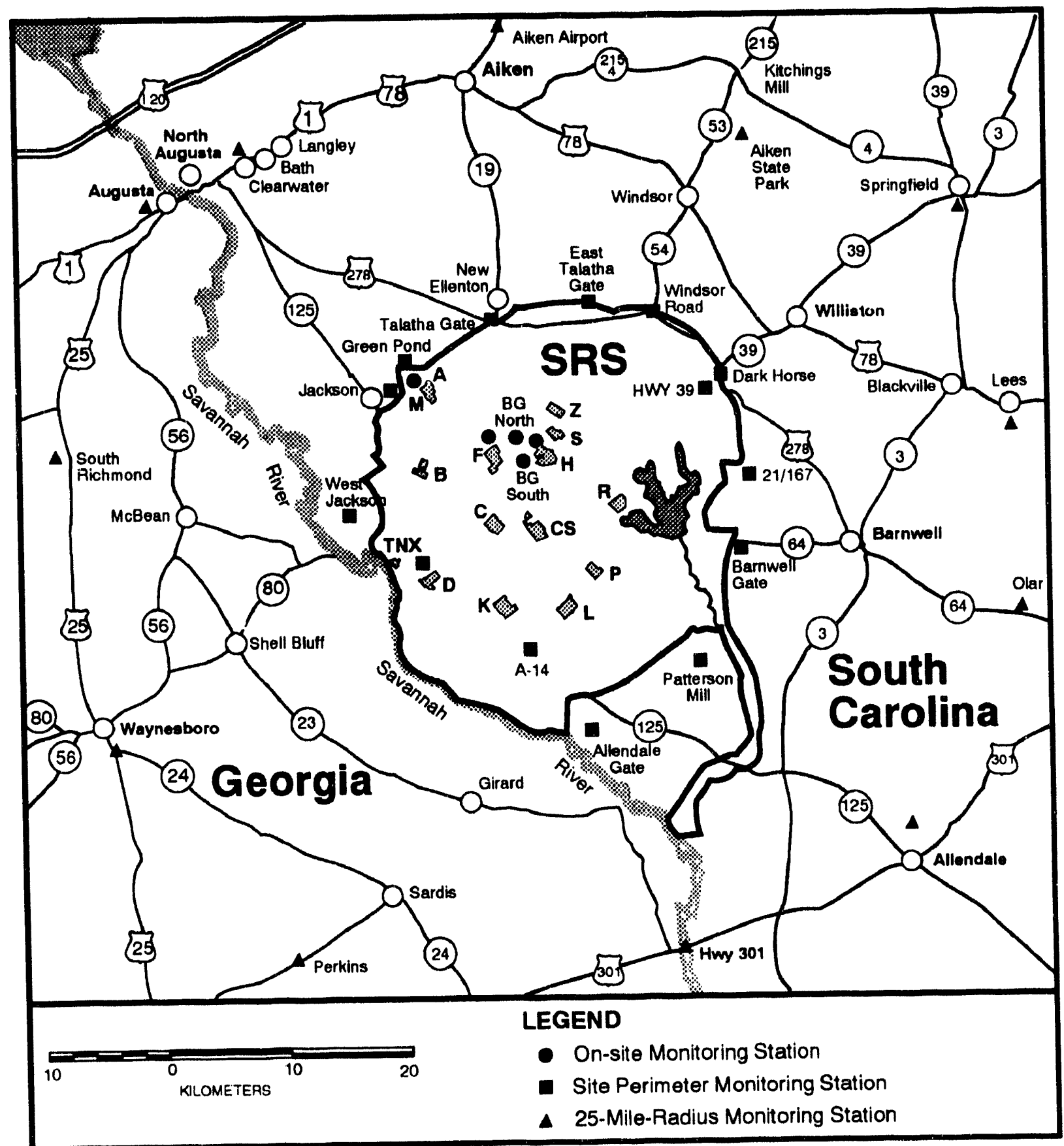

M9300508.01

Figure 3 Air Surveillance Stations Located Within 25 Miles of SRS

Not shown are four stations positioned approximately 100 miles from the site. 


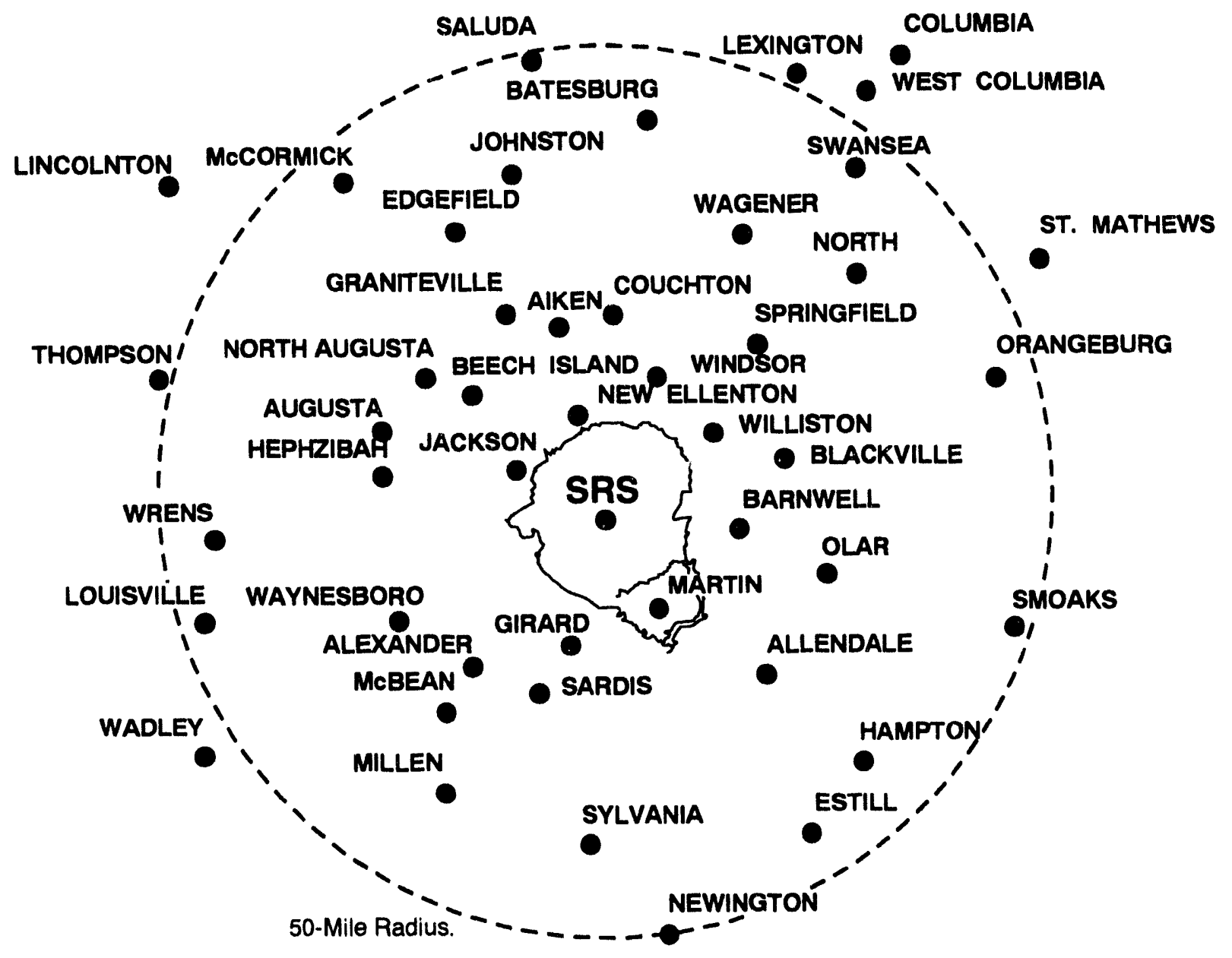

STATESBORO

-

Figure 4 Off-Site TLD Surveillance Locations 


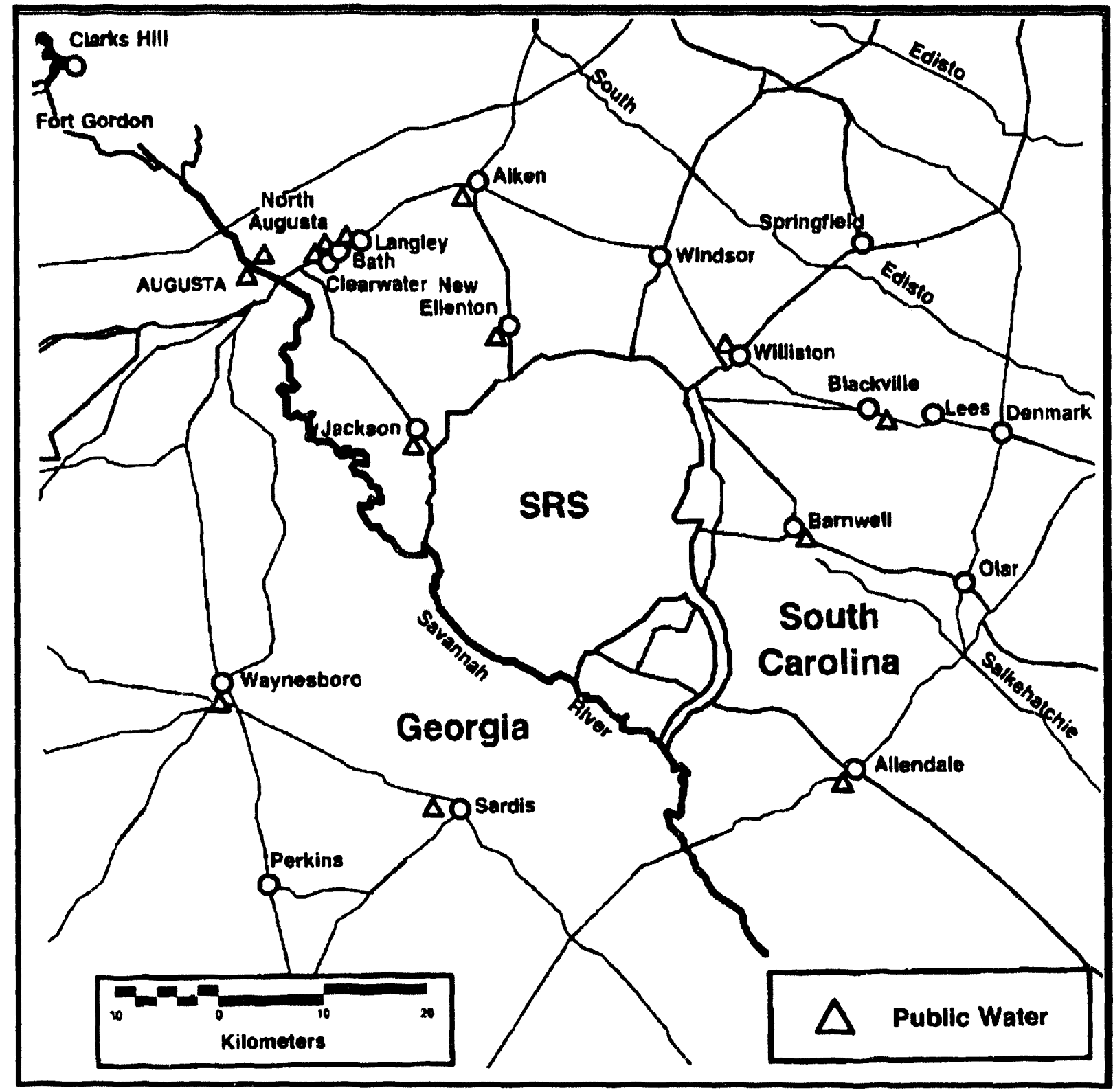

Figure 6 Drinking Water Sample Locations

SRS collects drinking water samples from 14 surrounding towns and cities within a 30-mile radius and from two water treatment plants (not shown on map) that supply treated Savannah River water to Beaufort-Jasper and Port Wentworth consumers. 


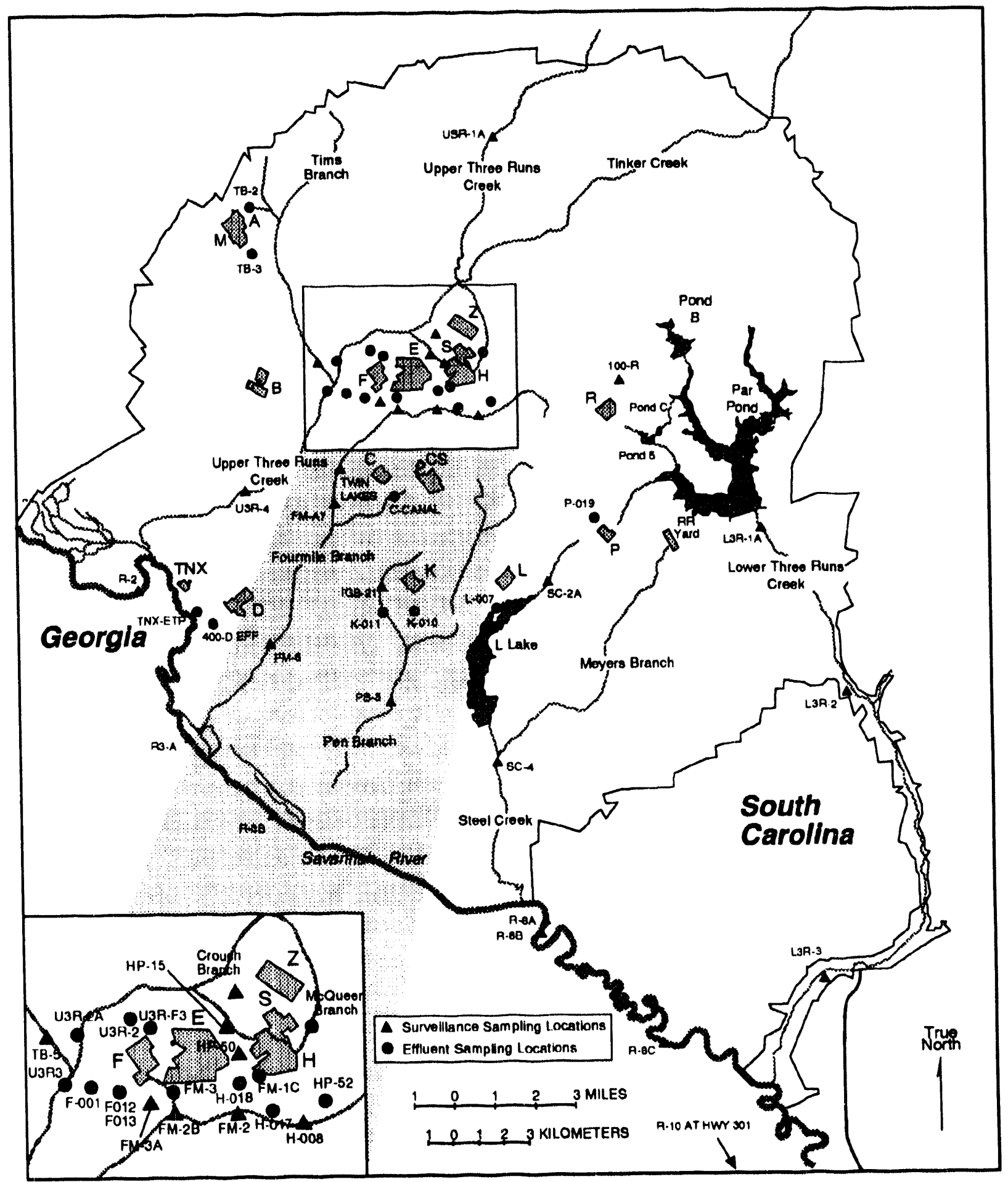

M9300557.01

Figure 5 Surface Water Sampling Locations

Surveillance and sampling points are located on SRS streams and seepage basins and on the Savannah River. 


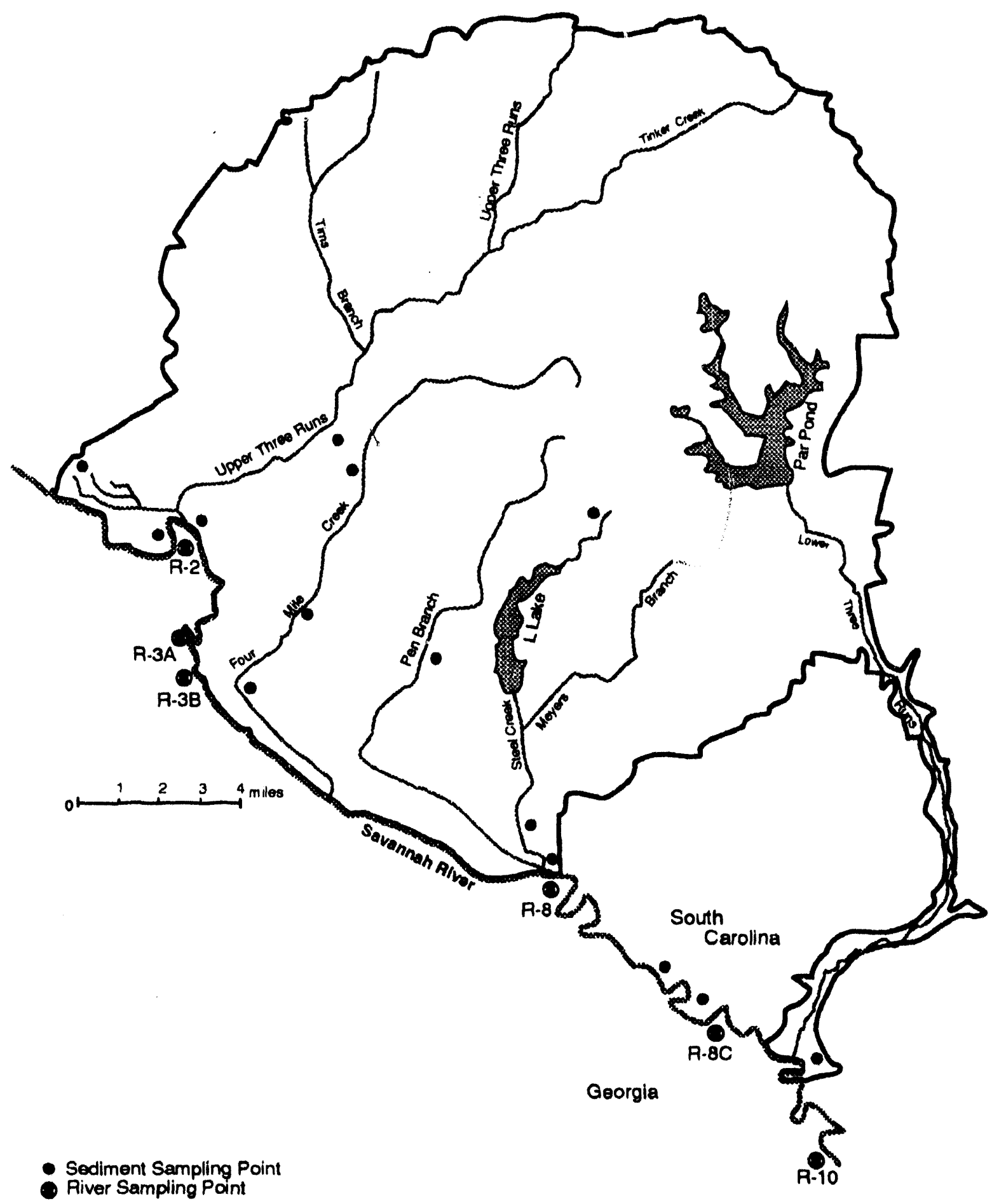

Unnumbered

Figure 7 SRS Sediment Sampling Locations

Sediment samples are collected annually at six locations on the Savannah River-upriver from, adjacent to, and downriver from SRS-and at nine site stream locations. 


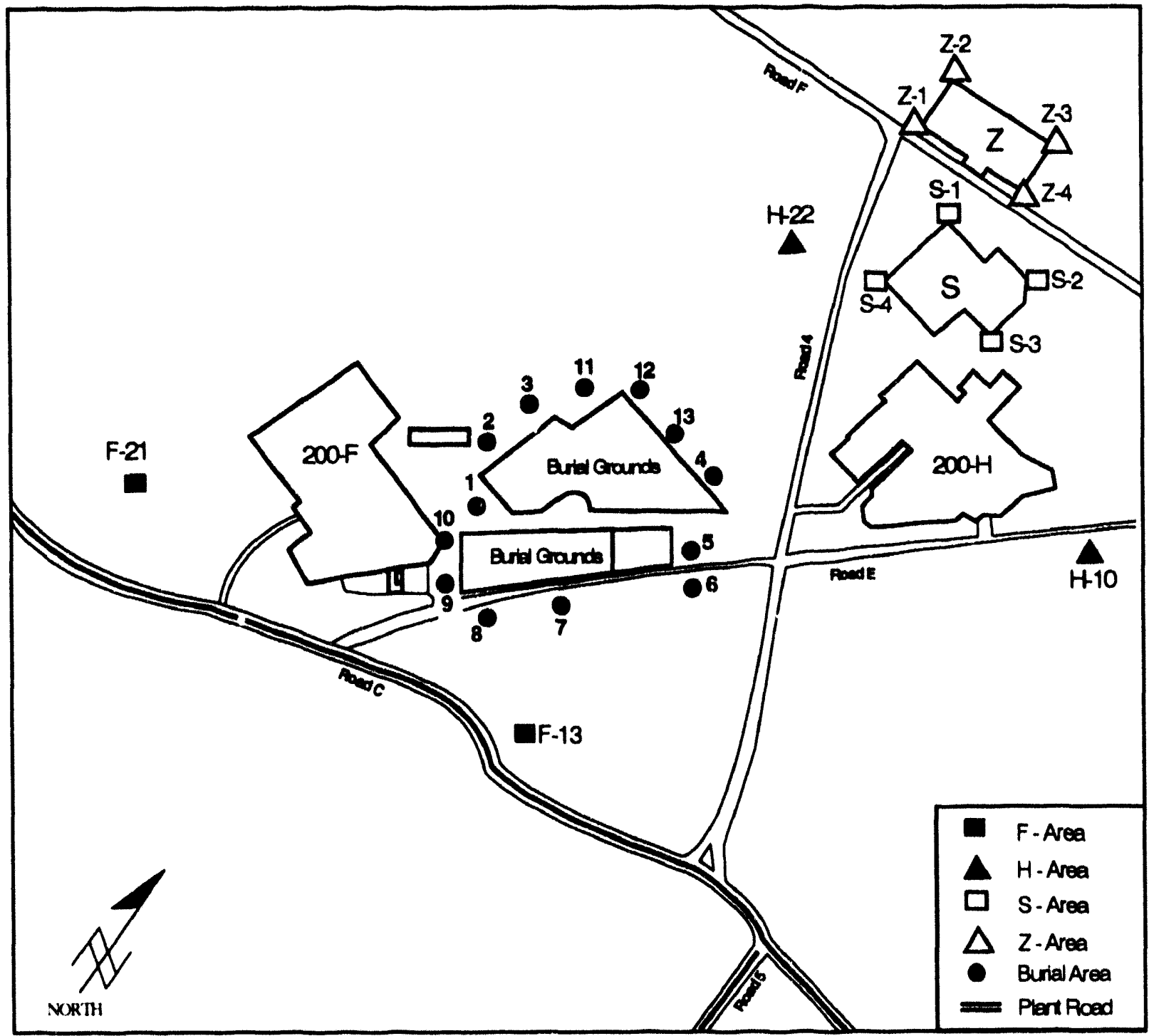

Figure 8 Quarterly On-site Vegetation Sampling Locations at SRS 


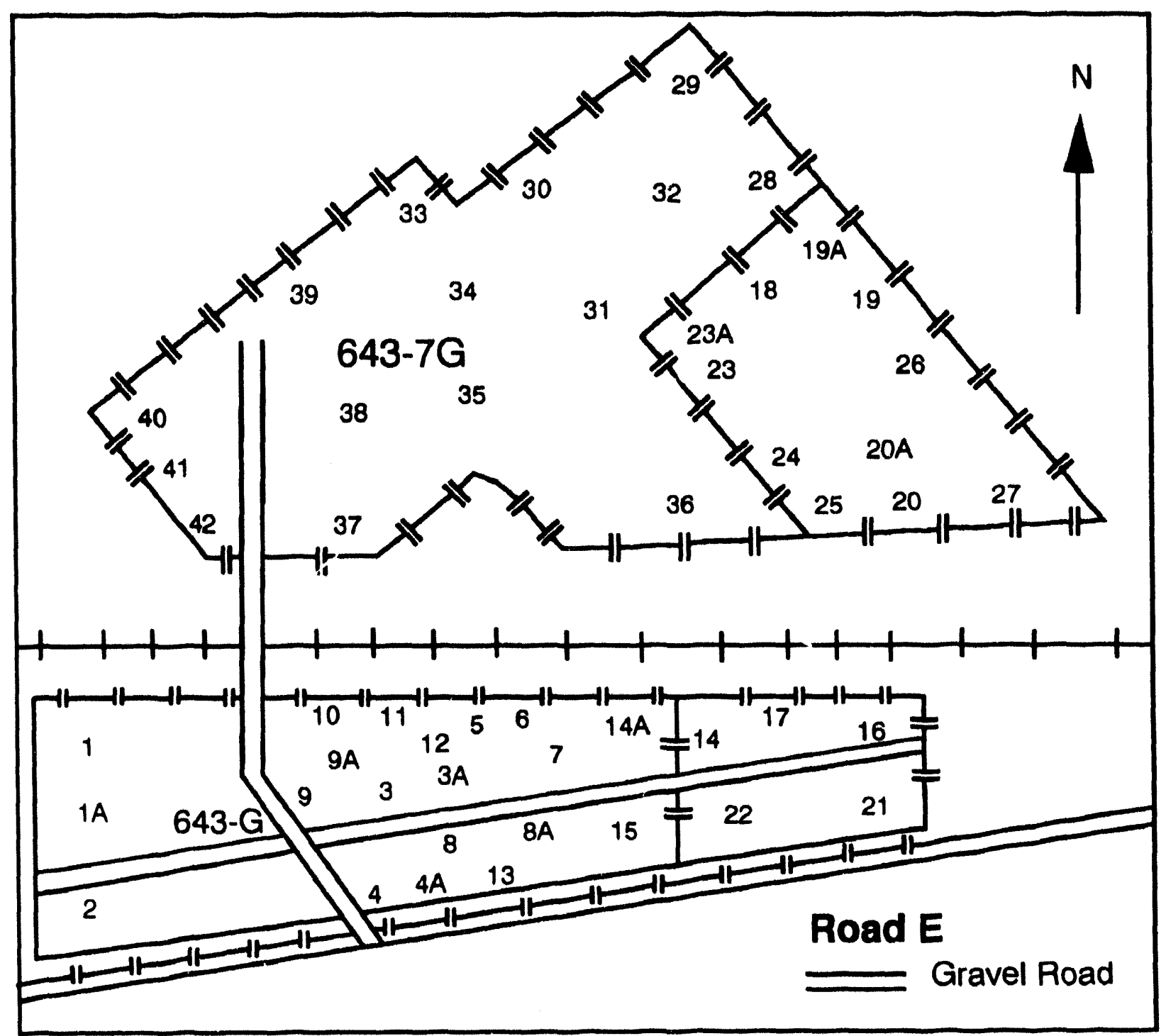

Figure 9 SWDF Interior Vegetation Sampling Locations

Vegetation samples are collected inside the Solid Waste Disposal Facility to determine whether uptake of radioactivity has occurred by vegetation from buried waste.

M92E004.01 


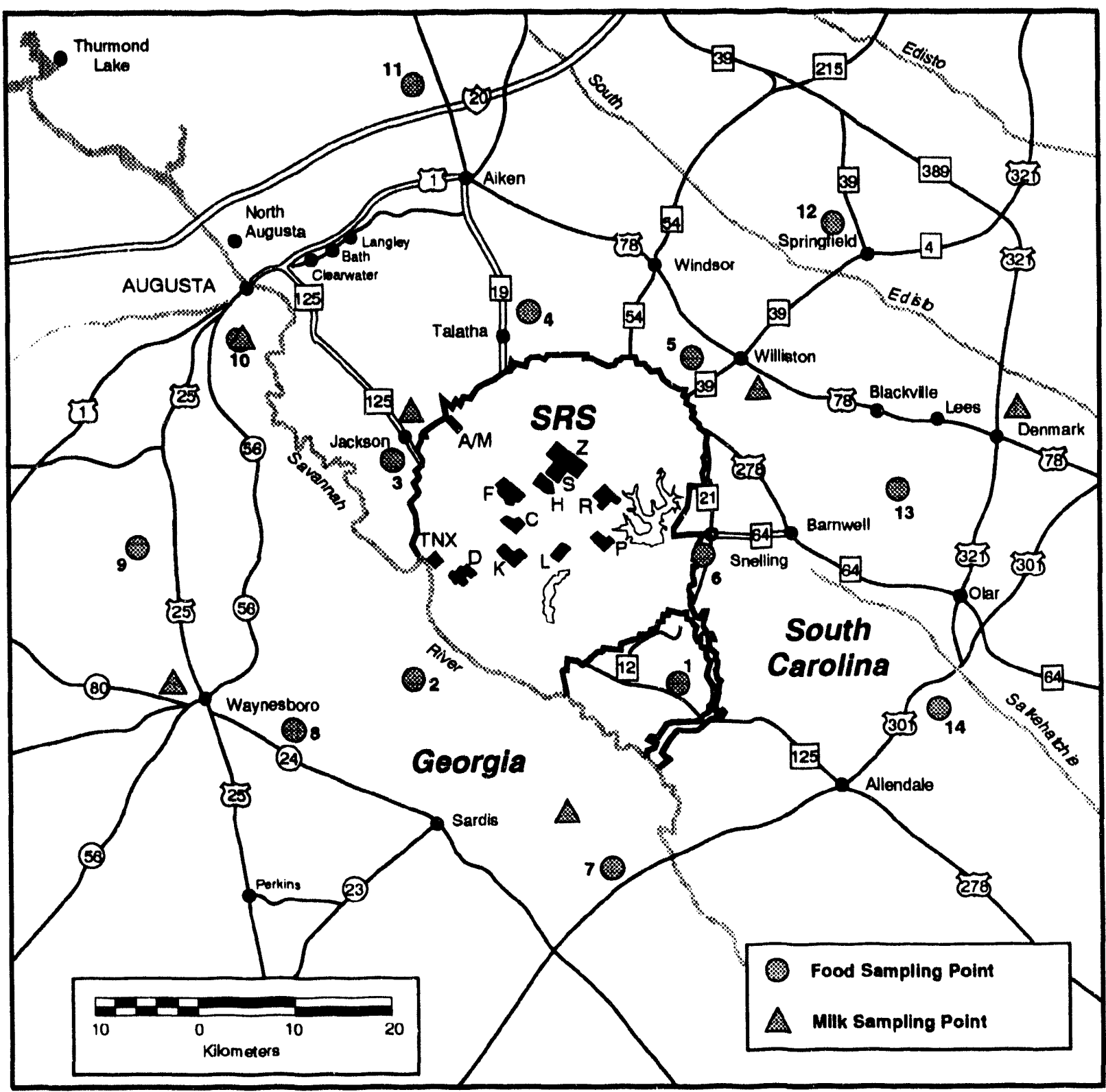

M92F003.09

Figure 10 Food/Milk Sampling Locations

Milk was collected and analyzed in 1992 from five local dairies within a 25-mile radius of SRS. A sixth dairy was sampled early in the year, before going out of business. Milk samples are collected every two weeks. Food samples are collected from 14 locations, also within a 25-mile radius of the site. The collection points on the map correspond to general geographic areas because their exact locations vary from year to year and crop to crop. Food samples are collected annually, except for eggs, which are collected quarterly. 


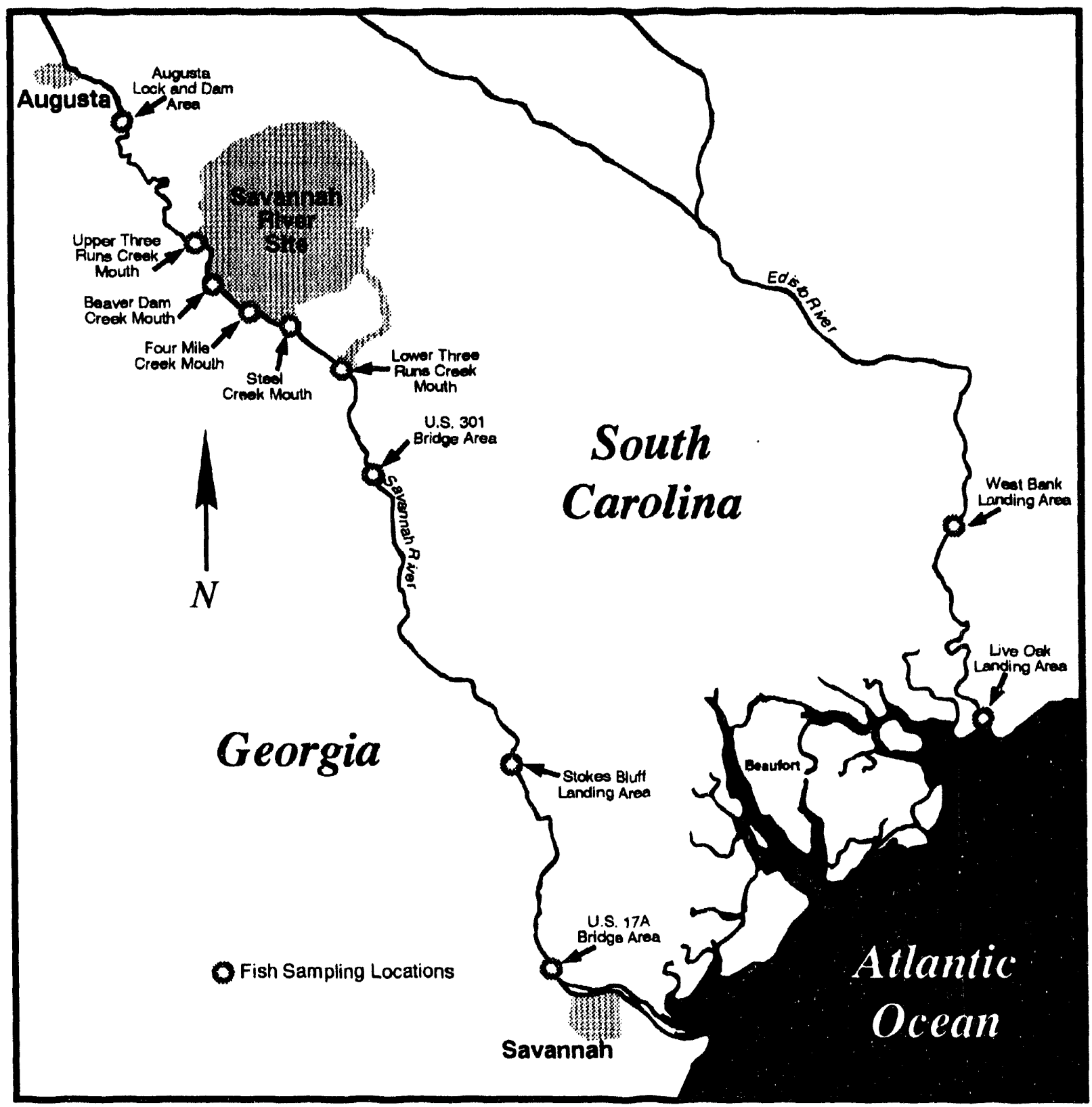

M9300535

Figure 11 Fish Sampling Locations

SRS collects fish on the Savannah River above the site, adjacent to the site, and near the river mouth, as well as on the Edisto River at two control locations 


\section{Table 1}

\section{General Radiological Sampling and Analysis Schedule}

\begin{tabular}{|c|c|c|}
\hline Sample Type & $\begin{array}{l}\text { Sampling } \\
\text { Frequency }\end{array}$ & Frequency of Analysis \\
\hline \multicolumn{3}{|l|}{ Air } \\
\hline Filters & Continuous & $\begin{array}{l}\text { Weekly } \\
\text { Alpha, beta, } \\
\text { Monthly } \\
\text { Gamma emitters, Pu-238, Pu-239, and Sr-89,90 }\end{array}$ \\
\hline Charcoal & Continuous & $\begin{array}{l}\text { Weekly } \\
\quad \text { Gamma emitters (including |-131) }\end{array}$ \\
\hline Silica gel & Continuous & $\begin{array}{l}\text { Biweekly } \\
\text { Tritium }\end{array}$ \\
\hline $\begin{array}{l}\text { Thermoluminescent } \\
\text { dosimeters }\end{array}$ & Continuous & $\begin{array}{l}\text { Quarterly } \\
\text { Gamma emitters }\end{array}$ \\
\hline \multirow[t]{2}{*}{ Streams } & Continuous & $\begin{array}{l}\text { Weekly } \\
\text { Alpha, beta, tritium, and U/Pu }\end{array}$ \\
\hline & & $\begin{array}{l}\text { Monthly } \\
\text { Sr-89,90, Sr-90, and gamma emitters }\end{array}$ \\
\hline \multirow[t]{2}{*}{ Savannah River } & Continuous & $\begin{array}{l}\text { Weekly } \\
\text { Alpha, beta, tritium, and gamma emitters }\end{array}$ \\
\hline & & $\begin{array}{l}\text { Annually } \\
\qquad \mathrm{Sr}-89,90, \mathrm{Sr}-90, \mathrm{Pu}-238 \text {, and } \mathrm{Pu}-239\end{array}$ \\
\hline Seepage basins & Quarterly & Alpha, beta, tritium, gamma emitters, $\mathrm{Sr}-89,90$, and U/Pu \\
\hline Groundwaterb & $\begin{array}{l}\text { Requirements } \\
\text { vary with } \\
\text { location }\end{array}$ & \\
\hline \multicolumn{3}{|l|}{ Food } \\
\hline \multirow[t]{2}{*}{ Milk } & Monthly & Gamma emitters and tritium \\
\hline & Quarterly & Sr-90 \\
\hline Eggs & Quarterly & Tritium, gamma emitters, Sr-90, U/Pu, \\
\hline Other foodstuffs & Annually & $\begin{array}{l}\text { Tritium, gamma emitters, Sr-90, U/Pu, Pu-238, and } \\
\text { Pu-239 }\end{array}$ \\
\hline \multicolumn{3}{|l|}{ Drinking water } \\
\hline On site & $\begin{array}{l}\text { Monthly or } \\
\text { quarterly }\end{array}$ & Alpha, beta, and tritium \\
\hline Off site & Semiannually & Alpha, beta, and tritium \\
\hline Water treatment plants & Daily & $\begin{array}{l}\text { Monthly } \\
\text { Alpha, beta, and tritium }\end{array}$ \\
\hline All locations & Annually & Sr-90 \\
\hline
\end{tabular}

a Analysis frequencies not listed in this column follow the sampling trequency for that medium.

b Sample collection and analytical procedures for groundwater monitoring are discussed in the quarterly groundwater reports. 


\section{Table 1}

\section{General Radiological Sampling and Analysis Schedule}

Page 2 of 2

\begin{tabular}{|c|c|c|}
\hline Semple Type & $\begin{array}{l}\text { Sampling } \\
\text { Frequency }\end{array}$ & Frequency of Analysise \\
\hline Rainwater & Continuous & $\begin{array}{l}\text { Monthly, quarterly, biweekly, } \\
\text { Alpha, beta, tritium, gamma emitters, Pu-238, Pu-239, } \\
\text { Sr-89,90, and Sr-90 }\end{array}$ \\
\hline Soll and sediments & Annually & Gamma emitters, Sr-90, Pu-238, and Pu-239 \\
\hline Vegetation & & Alpha, beta, tritium, $\mathrm{Sr}-89,90$, and gamma emitters \\
\hline $\begin{array}{l}\text { F-Area, H-Area, S-Area, } \\
\text { and Z-Area }\end{array}$ & Quarterly & \\
\hline $\begin{array}{l}\text { Outside burial ground } \\
\text { fence }\end{array}$ & Quarterly & \\
\hline Inside burial ground fence & Annually & \\
\hline $\begin{array}{l}\text { Seepage/retention } \\
\text { basins }\end{array}$ & Annually & \\
\hline Site perimeter & Quarterly & \\
\hline Off site & Quarterly & \\
\hline
\end{tabular}

a Analysis frequencies not listed in this column follow the sampling frequency for that medium. 
Table 2

General Nonradiological Sampling and Analysis Schedule

Page 1 of 1

\begin{tabular}{|c|c|c|}
\hline Environmental Medium & Sampling Frequency & Frequency of Analysis \\
\hline Liquid effluents (NPDES) & Varies with permit requirements & \\
\hline \multirow[t]{3}{*}{ Savannah River } & Continuously & $\begin{array}{l}\text { Quarterly } \\
\text { Metals (monthly composites) }\end{array}$ \\
\hline & Monthly & $\begin{array}{l}\text { Water quality parameters, } \\
\text { temperature, } \mathrm{pH} \text {, dissolved oxygen, } \\
\text { conductivity and fecal coliform }\end{array}$ \\
\hline & Annually & Herbicides \\
\hline \multirow[t]{2}{*}{ Streams } & Weekly & $\begin{array}{l}\text { Quarterly } \\
\text { Metals (weekly composites) }\end{array}$ \\
\hline & Monthly & $\begin{array}{l}\text { Water quality parameters, organics, } \\
\text { pH, dissolved oxygen, temperature, } \\
\text { conductivity, and fecal coliform }\end{array}$ \\
\hline \multicolumn{3}{|l|}{ Drinking water } \\
\hline Primary systems & Annually & Chemicals, metals, and orgarics \\
\hline Smaller systems & Varies with use & Chemicals, metals, and organics \\
\hline All systems & Varies with use & Total coliform and residual chlorine \\
\hline Fish & Monthly & Mercury \\
\hline Sediments & Annually & Herbicides and Inorganics \\
\hline
\end{tabular}




\section{Table 3}

Sample Medla Data

Page 1 of 1

\begin{tabular}{|c|c|c|}
\hline Sample Matrix or Medla & Sample S1ze & Representatlve Aliquot \\
\hline \multicolumn{3}{|l|}{ Gross alpha } \\
\hline Water & $1 \mathrm{~L}$ & $1 \mathrm{~L}$ \\
\hline Vegetation & $1-2 \mathrm{~kg}$ & $0.5 \mathrm{gm}$ \\
\hline Rain (collection pan) & $0.37 \mathrm{~m}^{2}$ & $0.093 \mathrm{~m}^{2}$ (1/4 total sample) \\
\hline Air & whole filter & $800 \mathrm{~m}^{3}$ \\
\hline \multicolumn{3}{|l|}{ Nonvolatlle beta } \\
\hline Water & $1 \mathrm{~L}$ & $1 \mathrm{~L}$ \\
\hline Vegetation & $1-2 \mathrm{~kg}$ & $0.5 \mathrm{gm}$ \\
\hline Air & whole filter & $800 \mathrm{~m}^{3}$ \\
\hline \multicolumn{3}{|l|}{ Strontium-89,90 } \\
\hline River water & $7 L$ & $1 L$ \\
\hline Rain & $0.37 \mathrm{~m}^{2}$ & $0.031 \mathrm{~m}^{2}$ (1/12 total sample) \\
\hline Streams & $1 \mathrm{~L}$ & $1 \mathrm{~L}$ \\
\hline \multicolumn{3}{|l|}{ Air composites } \\
\hline Site perimeter & $20,000 \mathrm{~m}^{3}$ & $4,200 \mathrm{~m}^{3}$ \\
\hline 25-mile radius & $18,000 \mathrm{~m}^{3}$ & $3,600 \mathrm{~m}^{3}$ \\
\hline 100 -mile radius & $6,000 \mathrm{~m}^{3}$ & $1,200 \mathrm{~m}^{3}$ \\
\hline \multicolumn{3}{|l|}{ Strontium-90 } \\
\hline River water & $7 L$ & $7 \mathrm{~L}$ \\
\hline Streams & $6 \mathrm{~L}$ & $3 L$ (duplicates) \\
\hline Milk & $0.5 \mathrm{~L}$ & $0.5 \mathrm{~L}$ \\
\hline Food & $20 \mathrm{~g}$ & $20 \mathrm{~g}$ \\
\hline Rain & $0.37 \mathrm{~m}^{2}$ & $0.031 \mathrm{~m}^{2}$ (1/12 total sample) \\
\hline
\end{tabular}


Table 4

Minimum Detectable Concentrations for Gamma Analysis of Soll, Food, Fish and Wildife, and Vegetation Samples

Page 1 of 1

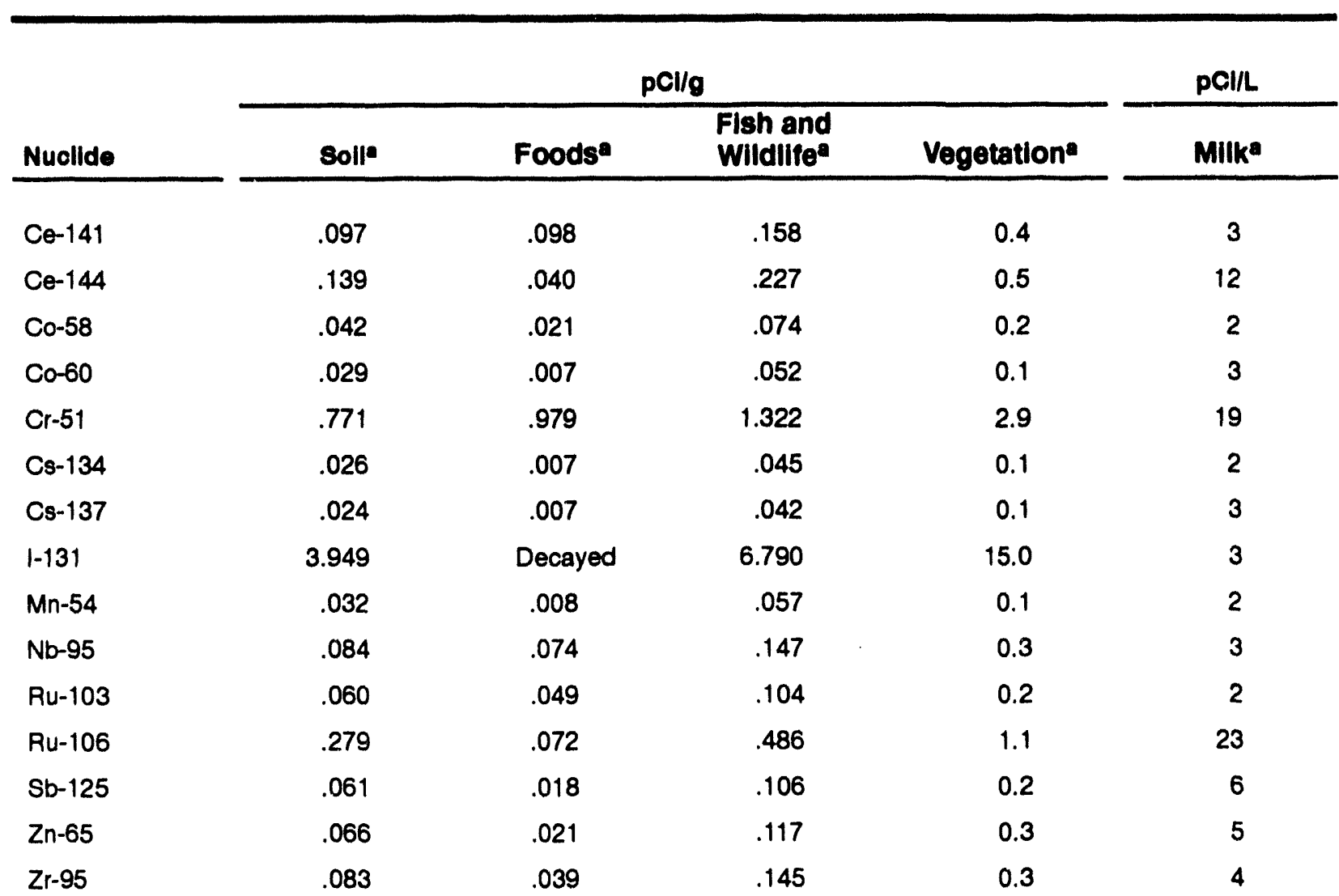

a The Minimum Detectable Concentration (MDC) values are based on a background measurement using a high purity germanium detector with a relative efficiency of $32 \%$. Typical sample volume, decay time, and counting intervals were used in the calculations for each sample type. Some special samples may use different parameters, resulting in different MDC values. Chemical recoveries are assumed to be $100 \%$. The MDC values are calculated at the $95 \%$ confidence level using the

Canberra/Nuclear Data VAXVMS Gamma Spectroscopy Applications and Nuclide Identification software packages. 


\section{Table 5}

Minimum Detectable Concentrations for Gamma Analysis

of Water and Alr Samples

Page 1 of 1

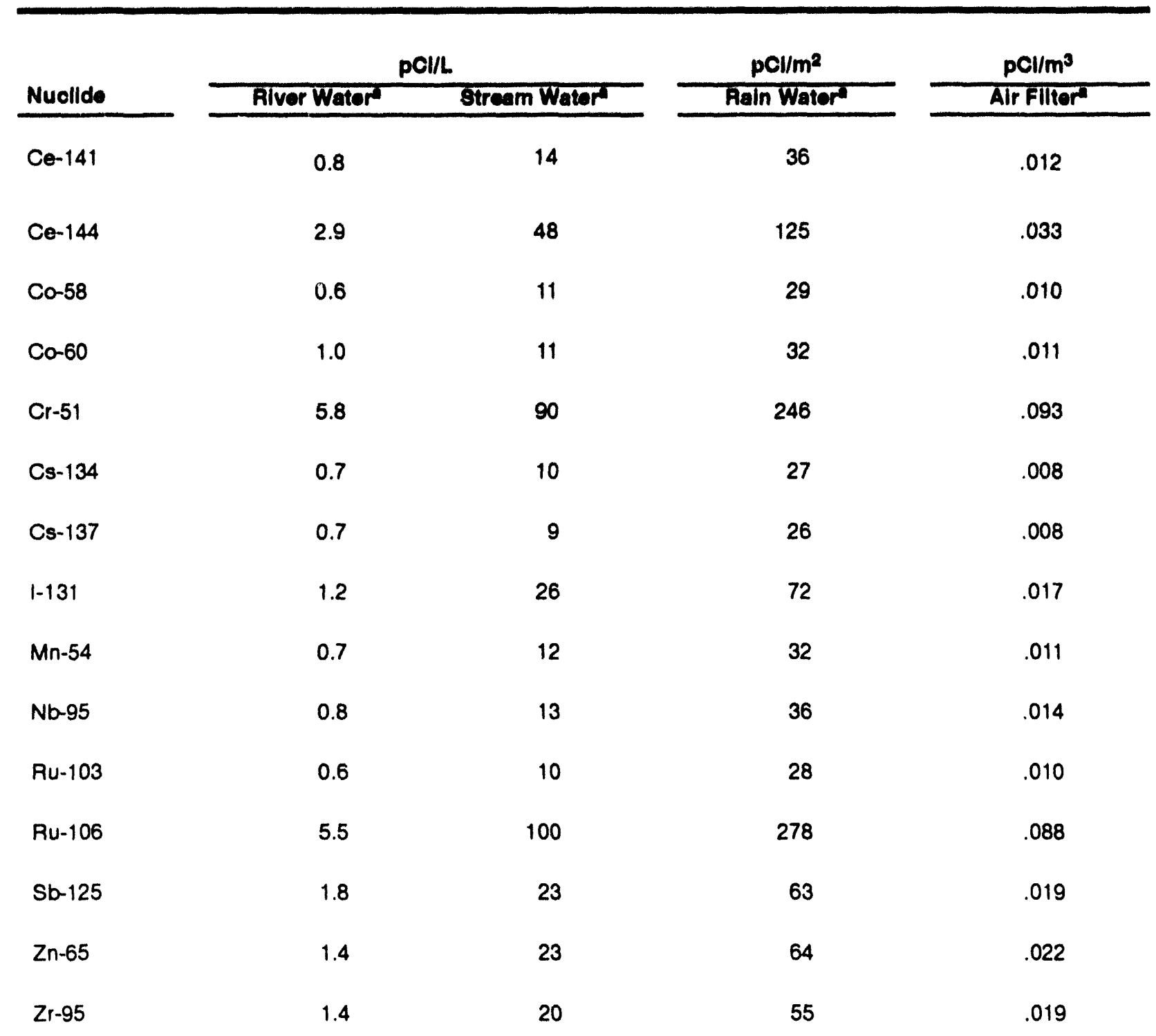

a The Minimum Detectable Concentration (MDC) values are based on a background measurement using a high purity germanium detector with a relative efficiency of $32 \%$. Typical sample volume, decay time, and counting intervals were used

in the calculations for each sample type. Some special samples may use different parameters resulting in different MDC

values. Chemical recoveries are assumed to be $100 \%$. The MDC values are calculated at $95 \%$ confidence level using the

Canberra/Nuclear Data VAXIVMS Gamma Spectroscopy Applications and Nuclide Identification software packages. 
Table 6

Lower Limits of Detection for Radiological Detection Instruments

Pege 1 of 1

\section{Alpha Spectrometer Analysis of Plutonium}

\begin{tabular}{|c|c|}
\hline Sample: & Lower Limlt of Dotection: \\
\hline Air filter & $2.4 E-11 \mu \mathrm{Ci} / \mathrm{m}^{3}$ \\
\hline Rain ion columns & $2.8 E-07 \mu \mathrm{Ci} / \mathrm{m}^{2}$ \\
\hline River water & 4.3E-12 $\mu \mathrm{Ci} / \mathrm{mL}$ \\
\hline Soil and sediment & 8.6E-10 $\mu \mathrm{Ci} / \mathrm{g}$ \\
\hline Foodstuff & 8.6E-11 $\mu \mathrm{Ci} / \mathrm{g}$ \\
\hline
\end{tabular}

\section{Gas-FIow Proportional Counters}

\begin{tabular}{ll}
\hline Nuollde: & Lower LImlt of Deteotion: \\
Gross alpha & $6.23 \mathrm{E}-07 \mu \mathrm{C}$ /sample \\
Gross beta & $1.55 \mathrm{E}-06 \mu \mathrm{C} / \mathrm{sample}$ \\
$\mathrm{Sr}-90$ & $1.89 \mathrm{E}-06 \mu \mathrm{Cl} / \mathrm{sample}$
\end{tabular}

NOTE: The instrument LLD values for the gas-flow proportional counter were calculated at the $95 \%$ confidence level [EMP, 1993]. The counting efficiencies were $28 \%$ for alpha, and $43 \%$ for beta, and Sr-90. The LLD for the actual samples is variable because of the detector used and the effects of sample aliquot size, sample preparation, chemical recovery, counting efficiency, and radioactive decay. The sample counting time was 20 minutes.

\section{Liquid Scintillation Analyses for Weak Beta Emitters}

\begin{tabular}{ll}
\hline Sample: & Lower Limit of Dotoction: \\
Tritium $^{\mathrm{a}}$ & $1.3 \mathrm{E}-06 \mu \mathrm{Cl} / \mathrm{mL}$ \\
Tritium $^{\mathrm{b}}$ & $4.5 \mathrm{E}-07 \mu \mathrm{Ci} / \mathrm{mL}$ \\
Promethium-147 & $7.9 \mathrm{E}-09 \mu \mathrm{Ci} / \mathrm{mL}$ \\
Sulfur-35 & $1.5 \mathrm{E}-09 \mu \mathrm{Ci} / \mathrm{mL}$
\end{tabular}

NOTE: Instrumental LLD values for the liquid scintillation counter were calculatedat the $95 \%$ confidencelevel [EMP, 1993]. The average counting efficiencies were $37 \%$ for tritium, $88 \%$ for Pm-147, and $88 \%$ for S-35. The LLD for the actual samples is variable because of the detector used and the effects of sample preparation, sample aliquot size, chemical recovery, counting efficiency, counting time, and radioactive decay.

a Routine environmental samples (e.g. stream samples and silica gels) are analyzed for tritium using a 20-minute count. b Environmental samples such as drinking water, foodstuffs, and rainwater are analyzed using a 150-minute count. 


\section{Table 7}

\section{Calculated Migration of Radloactivity from Seepage Baains}

Page 1 of 1

\begin{tabular}{|c|c|c|c|c|}
\hline \multirow[b]{2}{*}{ Looation } & \multirow[b]{2}{*}{ Souroe Desoription } & \multicolumn{3}{|c|}{ Tritlum (Curles) } \\
\hline & & 1091 & 1992 & 1903 \\
\hline$F M A 7(F M 3 A+F M 2 B)$ & $\begin{array}{l}\text { 200-F seepage basins to Four Mile } \\
\text { Creek }\end{array}$ & 5,750 & 4,260 & 2,180 \\
\hline FM2B-FM1C & $\begin{array}{l}\text { 200-H seepage basins to Four Mile } \\
\text { Creek }\end{array}$ & 1,810 & 1,470 & 1,020 \\
\hline FM3A-FM3 & $\begin{array}{l}\text { 200-H seepage basin } 4 \text { and Solid } \\
\text { Waste Disposal Facility to Four } \\
\text { Mile Creek }\end{array}$ & 6,420 & 4,090 & 5,330 \\
\hline IGB21 & $\begin{array}{l}\text { K-Area retention basin to Indian } \\
\text { Grave Branch }\end{array}$ & 2,160 & 1,530 & 1,100 \\
\hline \multirow[t]{2}{*}{ SC2A } & $\begin{array}{l}\text { 100-P seepage basin to Steel } \\
\text { Creeka }\end{array}$ & 364 & 232 & 382 \\
\hline & & \multicolumn{3}{|c|}{ Total Strontlum (Milllourles) } \\
\hline Location & Bource Desoription & 1991 & 1992 & 1903 \\
\hline$F M A 7(F M 3 A+F M 2 B)$ & $\begin{array}{l}\text { 200-F seepage basins to Four Mile } \\
\text { Creek }\end{array}$ & 460 & 194 & 150 \\
\hline FM2B-FM1C & $\begin{array}{l}\text { 200-H seepage basins to Four } \\
\text { Mile Creek }\end{array}$ & 72 & 78 & 65 \\
\hline
\end{tabular}

a Includes some secondary effluent releases from P Area when water is diverted from Par Pond. 
Table 8

1993 Radioactive Atmospheric Releases by Source

Page 1 of 1

\begin{tabular}{|c|c|c|c|c|c|c|c|c|}
\hline \multirow[b]{2}{*}{$\begin{array}{l}\text { Radio- } \\
\text { nuollde }\end{array}$} & \multirow[b]{2}{*}{ Half-llfo } & \multicolumn{7}{|c|}{ Curlesa,b } \\
\hline & & Reactors & $\begin{array}{c}\text { Sopare- } \\
\text { tlons }\end{array}$ & $\begin{array}{l}\text { Reaotor } \\
\text { Materiale }\end{array}$ & $\begin{array}{l}\text { Hoavy } \\
\text { Water }\end{array}$ & SATO & $\begin{array}{l}\text { Diffues } \\
\text { and } \\
\text { Fugltivoo }\end{array}$ & Total \\
\hline & \multicolumn{2}{|c|}{ GASES AND VAPORS } & & & & & & \\
\hline$H-3$ (oxide) & $12.3 y$ & $3.85 E+04$ & $9.39 E+04$ & & $4.48 E+02$ & & $4.31 E+01$ & $1.33 E+05$ \\
\hline $\mathrm{H}-3$ (elem) & $12.3 y$ & & $5.82 E+04$ & & & & & $5.82 E+04$ \\
\hline H-3 Total & $12.3 y$ & $3.85 E+04$ & $1.52 E+05$ & & $4.48 E+02$ & & $4.31 E+01$ & $1.91 E+05$ \\
\hline C-14 & $5.7 E 3 y$ & & $1.69 E-02$ & & & & $4.00 E-06$ & $1.69 E-02$ \\
\hline$x \in-135$ & $9.1 \mathrm{~h}$ & & & & & $3.19 E-02$ & & $3.19 E-02$ \\
\hline $1-129$ & $1.6 E 7 y$ & & $4.96 E-03$ & & & & $6.88 E-07$ & $4.96 E-03$ \\
\hline $\mid-131$ & $8 d$ & & $8.89 E-05$ & & & $5.92 E-05$ & & $1.48 E-04$ \\
\hline \multirow[t]{2}{*}{$1-133$} & $20.8 h$ & & & & & $1.96 \mathrm{E}-03$ & & $1.96 E-03$ \\
\hline & AAmTL & LATES & & & & & & \\
\hline S-35 & $87.2 d$ & & & & & & $2.00 E-06$ & $2.00 E-06$ \\
\hline $00-60$ & $5.3 y$ & & $5.89 E-09$ & & & & 3.34E-17 & $5.89 E-09$ \\
\hline $\mathrm{Ni}-63$ & $100 y$ & & & & & & $2.00 E-07$ & $2.00 E-07$ \\
\hline Sr $-89,90^{d}$ & $29.1 y$ & $1.81 E-04$ & $1.88 E-03$ & $8.32 E-05$ & $7.19 E-06$ & $1.19 E-05$ & $1.11 E-04$ & 2.27E-03 \\
\hline Zr-95 & $64 d$ & & & & & & 2.39E-14 & $2.39 E-14$ \\
\hline Ru-106 & $1.0 y$ & $3.99 E-06$ & $5.76 E-09$ & & & & $4.96 E-12$ & $4.00 E-06$ \\
\hline$S b-125$ & $2.8 y$ & & & & & & $7.27 E-15$ & $7.27 E-15$ \\
\hline Cs-134 & $2.1 y$ & & $1.49 \mathrm{E}-06$ & & & & $1.40 E-17$ & $1.49 E-06$ \\
\hline Cs-137 & $30.2 y$ & $1.04 E-04$ & $5.28 E-04$ & & & $1.51 E-06$ & $4.33 E-11$ & $6.34 E-04$ \\
\hline Ce-144 & $285 d$ & & & & & & $1.13 E-13$ & $1.13 E-13$ \\
\hline Eu-154 & $8.6 y$ & & & & & & $3.44 E-13$ & $3.44 E-13$ \\
\hline Eu-155 & $4.7 y$ & & & & & & $1.63 E-13$ & $1.63 E-13$ \\
\hline$U-235,238$ & 4.5E9 y & & $1.86 \mathrm{E}-03$ & $1.55 E-05$ & & $2.89 E-08$ & $4.74 E-05$ & $1.92 E-03$ \\
\hline Pu-238 & $87.7 y$ & & $1.21 E-03$ & & & $1.00 E-08$ & 4.63E-12 & $1.21 E-03$ \\
\hline Pu-239d & $2.4 E 4 y$ & $4.11 E-06$ & $1.06 \mathrm{E}-03$ & $3.50 E-06$ & $8.42 E-07$ & $9.41 E-06$ & $4.70 E-07$ & $1.08 E-03$ \\
\hline Am-241,243 & 7.4E3 y & & $1.42 E-04$ & & & $1.34 E-06$ & $8.86 E-13$ & $1.43 E-04$ \\
\hline $\mathrm{Cm}-242,244$ & $18.1 \mathrm{y}$ & & 4.96E-05 & & & $6.83 E-06$ & $7.33 E-12$ & $5.64 E-05$ \\
\hline
\end{tabular}

One curie equals $3.7 E+10$ Becquerels.

Blank space indicates no quantifiable activity.

Estimated releases from minor unmonitored diffuse and lugitive sources

Includes unidentified beta-gamma emissions

Includes unidentified alpha emissions 


\section{Table 9}

1993 Radloactive Atmospheric Releases by Stack/Facility and Comparison of Annual Average Concentratlons to DOE Derived Concentration Quides (DCQ8)

Pag 1 of 8

\begin{tabular}{|c|c|c|c|c|c|}
\hline stwok or F sollity & Redlonuollde & $\begin{array}{l}\text { Quanilly } \\
\text { Rolosecd During } \\
1005(\mu \mathrm{Cl})\end{array}$ & $\begin{array}{l}\text { Aimosphorlo } \\
\text { Rolease } \\
\text { Volume (mL) }\end{array}$ & $\begin{array}{c}\text { Average Emuent } \\
\text { Conoentrutton } \\
\text { During } 1903 \\
\text { ( } \mu \text { CU/mL) }\end{array}$ & $\begin{array}{c}\text { DOE DCON } \\
(\mu \mathrm{Cl} / \mathrm{mL})\end{array}$ \\
\hline \multicolumn{6}{|l|}{ A-Area (Orro) } \\
\hline \multirow[t]{4}{*}{$738-A$} & $U-235,238$ & $2,89 E-02$ & $4.65 E+14$ & $6.21 E-17$ & $1.0 E-13$ \\
\hline & Pu-238 & $1.00 E-02$ & $4.65 E+14$ & $2.15 E-17$ & $3.0 E-14$ \\
\hline & Am-241,243 & $1.71 E-02$ & $4.65 E+14$ & $3.68 E-17$ & $2.0 E-14$ \\
\hline & $\mathrm{Cm}-242,244$ & $9.30 E-02$ & $4.65 E+14$ & $2.00 E-16$ & $4.0 E-14$ \\
\hline \multirow[t]{3}{*}{ 773-A, B wing } & Cs-137 & $1.17 E+00$ & $1.10 E+15$ & $1.06 E-15$ & $4.0 E-10$ \\
\hline & Am-241,243 & $1.32 E+00$ & $1.10 E+15$ & $1.20 E-15$ & $2.0 E-14$ \\
\hline & $\mathrm{Cm}-242.244$ & $6.74 E+00$ & $1.10 E+15$ & $6.13 E-15$ & $4.0 E-14$ \\
\hline 775-A, C wing & 1.131 & $2.23 E-01$ & $1.10 E+15$ & $2.66 E-16$ & $4.0 E-10$ \\
\hline \multirow{4}{*}{$\begin{array}{l}\text { 773-A, } \\
\text { sendiliter }\end{array}$} & $\mid-131$ & $5.88 E+01$ & $1.10 E+15$ & $5.35 E-14$ & $4.0 E-10$ \\
\hline & 1.133 & $1.96 E+03$ & $1.10 E+15$ & $1.78 E-12$ & $2.0 E-09$ \\
\hline & $x \in-135$ & $3.19 E+04$ & $1.10 E+15$ & $2.90 E-11$ & none listed \\
\hline & Cs-137 & $3.36 \mathrm{E}-01$ & $1.10 E+15$ & $3.05 E-16$ & $4.0 E-10$ \\
\hline \multirow[t]{2}{*}{$778-A$} & $|-13|$ & $6.51 E-02$ & $1.37 E+14$ & $4.75 E-16$ & $4.0 E-10$ \\
\hline & $1-133$ & $1.38 E-01$ & $1.37 E+14$ & $1.01 E-15$ & $2.0 E-09$ \\
\hline \multicolumn{6}{|c|}{ O-Area (O-fiesotor) } \\
\hline C, Main Staok & $H-3$ (oxide) & $1.46 E+08$ & $1.78 E+15$ & $8.20 E-08$ & $1.0 E-07$ \\
\hline \multicolumn{6}{|c|}{ D-Area (Howy Wator Rewark) } \\
\hline 420-D & H-3 (oxide) & $4.08 E+08$ & $2.23 E+14$ & $1.83 E-06$ & $1.0 E-07$ \\
\hline $421-20$ & $H-3$ (oxide) & $1.33 E+07$ & $1.52 E+14$ & $8.75 E-08$ & $1.0 E-07$ \\
\hline $772-D$ & H-3 (oxide) & $2.62 E+07$ & $2.60 E+14$ & $1.01 E-07$ & $1.0 E-07$ \\
\hline \multicolumn{6}{|c|}{ FuAru (eeparatione and Wasto Manegement) } \\
\hline \multirow[t]{3}{*}{ 235-F, sandfilitor } & $P u-238$ & $7.16 E-03$ & $5.26 E+14$ & $1.36 E-17$ & $3.0 E-14$ \\
\hline & Pu-239 & $1.71 E-02$ & $5.26 E+14$ & $3.25 E-17$ & $2.0 E-14$ \\
\hline & $U-235,238$ & $7.49 E-02$ & $5.26 E+14$ & $1.42 E-16$ & 1.0E--13 \\
\hline
\end{tabular}

a Source: DOE Order 5400.5. In cases where different chemical forms have different DCGs, the loweet DCG lor the radionuclide is given. These DCGs are delined as the air concentration of that radionuollde that will give a 50 -year commilted elfective dose equivalent of 100 mrem under conditions of continuous exposure for one year. DCGs are relerence values only and are not considered release limits or standards. 
Table 9

1993 Radloactive Atmospheric Releases by Stack/Facillty and Comparison of Annual Average Concentrations to DOE Derived Concentration Quides (DCQ8)

Poge 2 of 5

\begin{tabular}{|c|c|c|c|c|c|}
\hline steok or Feollity & Radlonuollde & $\begin{array}{l}\text { Quentity } \\
\text { Reloceed During } \\
1903(\mu \mathrm{Cl})\end{array}$ & $\begin{array}{l}\text { Atmoepherto } \\
\text { Releses } \\
\text { Volumo }(\mathrm{mL})\end{array}$ & $\begin{array}{c}\text { Avorege Ermuent } \\
\text { Conoentratton } \\
\text { During } 1093 \\
(\mu \mathrm{CV} / \mathrm{mL})\end{array}$ & $\begin{array}{c}\text { DOE DCQQ" } \\
(\mu \mathrm{C} / \mathrm{mL})\end{array}$ \\
\hline \multicolumn{6}{|c|}{ 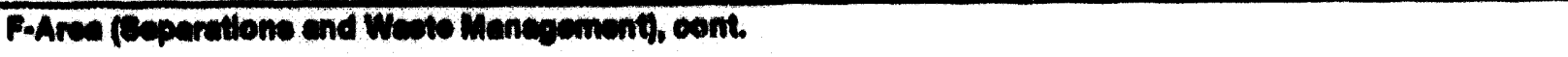 } \\
\hline & Am-241,243 & 5.07E-02 & $5.26 E+14$ & $9.64 E-17$ & $2.0 E-14$ \\
\hline & $\mathrm{Cm}-242,244$ & 2.22E-03 & $5.26 E+14$ & $4.22 E-18$ & $4.0 E-14$ \\
\hline 241f, Miseo. & Co-137 & $1.41 E-01$ & $8.66 \mathrm{E}+13$ & $1.63 E-15$ & $4.0 E-10$ \\
\hline \multirow[t]{2}{*}{ 241-F, Purge } & C9-137 & $1.86 E+00$ & $7.72 E+13$ & $2.41 E-14$ & 4.0E-10 \\
\hline & $A m-241,243$ & $1.84 E-03$ & $7.72 E+13$ & $2.38 E-17$ & $2.0 E-14$ \\
\hline \multirow[t]{3}{*}{ 241-F, Annulus } & Co-60 & $5.89 E-03$ & $2.30 E+14$ & $2.56 \mathrm{E}-17$ & 8.0E-11 \\
\hline & Cs-137 & $1.83 E+00$ & $2.30 E+14$ & $7.96 E-15$ & 4.0E-10 \\
\hline & Am-241,243 & $1.03 E-01$ & $2.30 E+14$ & 4.48E-16 & $2.0 E-14$ \\
\hline \multirow[t]{3}{*}{ 247-F, Maln } & $U-235,238$ & $6.92 E-01$ & $1.50 E+15$ & $4.61 E-16$ & $1.0 E-13$ \\
\hline & Pu-238 & $6.15 E-03$ & $1.50 E+15$ & 4.10E-18 & $3.0 E-14$ \\
\hline & Pu-239 & $1.68 E-02$ & $1.50 E+15$ & $1.12 E-17$ & $2.0 E-14$ \\
\hline 6.1-D, Diesolver & Co-137 & $9.30 E-04$ & $3.09 E+12$ & $3.01 E-16$ & 4.0E-10 \\
\hline 6.4-D, Diseolver & Cs- 137 & $2.95 E-02$ & $2.97 E+12$ & $9.93 E-15$ & 4.0E-10 \\
\hline $800 \mathrm{Coll}$ & Cs-137 & $3.70 E-02$ & $8.90 E+13$ & $4.16 E-16$ & $4.0 E-10$ \\
\hline \multirow[t]{3}{*}{ 772-f, Tomp. } & U-235,238 & $1.02 E+00$ & $5.88 E+14$ & $1.73 E-15$ & $1.0 E-13$ \\
\hline & Pu-238 & $2.53 E-01$ & $5.88 E+14$ & $4.30 E-16$ & $3.0 E-14$ \\
\hline & Pu-239 & $9.55 E-02$ & $5.88 E+14$ & $1.62 E-16$ & $2.0 E-14$ \\
\hline \multirow[t]{3}{*}{$772-4 F$ (Lab) } & U-235,238 & $1.12 E+00$ & $8.13 E+14$ & $1.38 E-15$ & $1.0 E-13$ \\
\hline & Pu-238 & $6.52 E-02$ & $8.13 E+14$ & $8.02 E-17$ & $3.0 E-14$ \\
\hline & Pu-239 & $1.61 E-01$ & $8.13 E+14$ & $1.98 E-16$ & $2.0 E-14$ \\
\hline \multirow[t]{3}{*}{221 f, A Line } & $U-235,238$ & $2.55 E-01$ & $3.53 E+13$ & $7.22 E-15$ & $1.0 E-13$ \\
\hline & Pu-238 & $1.81 E-02$ & $3.53 E+13$ & $5.13 E-16$ & $3.0 E-14$ \\
\hline & Pu-239 & $1.63 E-02$ & $3.53 E+13$ & $4.82 E-16$ & $2.0 E-14$ \\
\hline \multirow[t]{2}{*}{ F, Main Btack } & $C-14$ & $1.48 E+04$ & $4.20 E+15$ & $3.52 E-12$ & $4.0 E-06$ \\
\hline & Sr-89,90 & $3.86 E+02$ & $4.20 E+15$ & $9.19 E-14$ & $9.0 E-12$ \\
\hline
\end{tabular}


Table $\theta$

\section{Radloactive Atmospheric Releases by Stack/Facllity and Comparison of Annual Average Concentrations to DOE Derived Concentration Quldes (DCQ8)}

Pages of 5

\begin{tabular}{|c|c|c|c|c|c|}
\hline steok or Fseollity & Radlonuollde & $\begin{array}{l}\text { Quanitity } \\
\text { Roloeeced During } \\
\text { Poes ( } \mu \mathrm{Cl})\end{array}$ & $\begin{array}{l}\text { Atmoespherlo } \\
\text { Roleateo } \\
\text { Volume }(\mathrm{mL})\end{array}$ & $\begin{array}{l}\text { Average Emuent } \\
\text { Concontration } \\
\text { During } 1993 \\
(\mu \mathrm{CU} / \mathrm{mL})\end{array}$ & $\begin{array}{c}\text { DOE DCQA" } \\
(\mu \mathrm{Cl} / \mathrm{mL})\end{array}$ \\
\hline \multicolumn{6}{|c|}{ 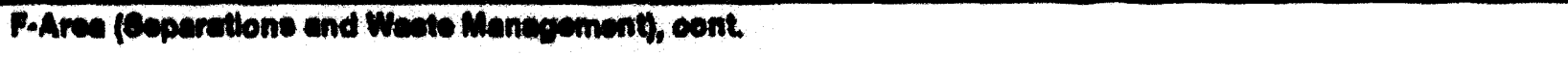 } \\
\hline & $1-129$ & $2.53 E+03$ & $4.20 E+15$ & $6.02 E-13$ & $7.0 E-11$ \\
\hline & $\mid-131$ & $2.94 E+00$ & $4.20 E+15$ & $7.00 E-16$ & $4.0 E-10$ \\
\hline & Ca-134 & $1.38 E+00$ & $4.20 E+15$ & $3.29 E-16$ & $2.0 E-10$ \\
\hline & Co-137 & $4.62 \mathrm{E}+02$ & $4.20 E+15$ & $1.10 E-13$ & $4.0 E-10$ \\
\hline & $U-235,238$ & $1.76 E+03$ & $4.20 E+15$ & 4.19E-13 & $1.0 E-13$ \\
\hline & Pu-238 & $3.26 E+02$ & $4.20 E+15$ & $7.76 E-14$ & $3.0 E-14$ \\
\hline & Pu-239 & $5.75 E+02$ & $4.20 E+15$ & $1.37 E-14$ & $2.0 E-14$ \\
\hline & Am-241,243 & $6.08 E+01$ & $4.20 E+15$ & $1.45 E-14$ & $2.0 E-14$ \\
\hline & $\mathrm{Cm}-242,244$ & 4.31E+01 & $4.20 E+15$ & $1.03 E-14$ & $4.0 E-14$ \\
\hline \multicolumn{6}{|c|}{ 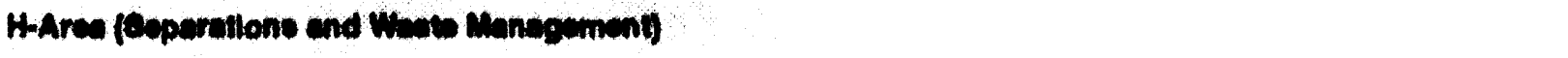 } \\
\hline 230-H, Proceses & None & N/A & N/A & N/A & N/A \\
\hline $241-84 H$, ETF & None & N/A & N/A & N/A & N/A \\
\hline \multirow[t]{2}{*}{$241-H$, Mlec. } & Cs-134 & $1.48 E-02$ & $1.03 E+14$ & $1.44 E-16$ & $2.0 E-10$ \\
\hline & Cs-137 & $2.86 E+00$ & $1.03 E+14$ & $2.78 E-14$ & 4.0E-10 \\
\hline \multirow[t]{3}{*}{$241+$ H, Purge } & Ru-106 & $5.76 E-03$ & $1.47 E+14$ & $3.92 E-17$ & $3.0 E-11$ \\
\hline & Cs-134 & $1.63 E-02$ & $1.47 E+14$ & $1.11 E-16$ & $2.0 E-10$ \\
\hline & C3-137 & $3.96 E+00$ & $1.47 E+14$ & $2.69 E-14$ & 4.0E-10 \\
\hline \multirow[t]{2}{*}{ 241-H, Annulue } & Cs-134 & $8.20 E-02$ & $8.70 E+14$ & $9.43 E-17$ & $2.0 E-10$ \\
\hline & Cs-137 & $1.50 E+01$ & $8.70 E+14$ & $1.72 E-14$ & $4.0 E-10$ \\
\hline $244-H, R B O F b$ & Co-137 & $1.57 E-01$ & $3.53 E+14$ & $4.45 E-16$ & $4.0 E-10$ \\
\hline $\begin{array}{l}244+H_{1} \\
\text { RBOF VVo }\end{array}$ & C8-137 & $4.92 E-02$ & $5.46 E+12$ & $9.01 E-15$ & $4.0 E-10$ \\
\hline 253-H, Waste & $H-3$ (oxide) & $1.67 E+06$ & $5.21 E+13$ & $3.21 E-08$ & $1.0 \mathrm{E}-07$ \\
\hline 299-H, Bullding & Cs-137 & $6.31 E-02$ & $2.76 E+14$ & $2.29 E-16$ & 4.0E-10 \\
\hline
\end{tabular}

a Source: DOE Order 5400.5. In cases where different chemical forms have different DCGa, the lowest DCG for the radionuclide is given. These DCGs are defined as the air concentration of that radionuclide that will give a 50-year committed dose of 100 mrem under conditions of continuous exposure for one year. DCGs are relerence values only and are not considered release limits or standards.

b Receiving Basin lor OHatte Fuel

c Recelving Basin for Offiste Fuel Vessel Vent 


\section{Table 9}

1993 Radloactive Atmospheric Releases by Stack/Facility and Comparison of Annual Average Concentrations to DOE Derived Concentration Guldes (DCGs)

Page 4 of 5

\begin{tabular}{|c|c|c|c|c|c|}
\hline Stack or Faollity & Radlonuolide & $\begin{array}{c}\text { Quantity } \\
\text { Released During } \\
1993(\mu \mathrm{Cl})\end{array}$ & $\begin{array}{l}\text { Atmospheric } \\
\text { Rolease } \\
\text { Volume }(\mathrm{mL})\end{array}$ & $\begin{array}{c}\text { Average Effluent } \\
\text { Concentratlon } \\
\text { During } 1993 \\
(\mu \mathrm{Cl} / \mathrm{mL})\end{array}$ & $\begin{array}{c}\text { DOE DCGsa } \\
(\mu \mathrm{C} / \mathrm{mL})\end{array}$ \\
\hline \multicolumn{6}{|c|}{ 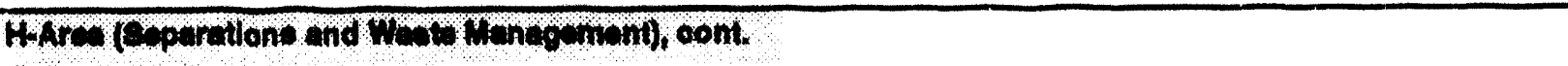 } \\
\hline \multirow[t]{2}{*}{ Tritlum Faollitiea } & $H-3$ (oxide) & $9.39 E+10$ & $1.69 E+15$ & $5.56 E-05$ & $1.0 E-07$ \\
\hline & $\mathrm{H}-3$ (elemental) & $5.82 E+10$ & $1.69 \mathrm{E}+15$ & $3.44 E-05$ & 2.0E-02 \\
\hline \multirow[t]{10}{*}{$H$, Main Stack } & $c-14$ & $2.13 E+03$ & $3.97 E+15$ & $5.37 E-13$ & $4.0 E-06$ \\
\hline & Sr-89,90 & $5.95 E+01$ & $3.97 E+15$ & $1.50 E-14$ & $9.0 E-12$ \\
\hline & $1-129$ & $2.43 E+03$ & $3.97 E+15$ & $6.12 E-13$ & $7.0 E-11$ \\
\hline & $|-13|$ & $8.60 E+01$ & $3.97 E+15$ & $2.17 E-14$ & $4.0 E-10$ \\
\hline & Cs-137 & $4.00 E+01$ & $3.97 E+15$ & $1.01 E-14$ & $4.0 E-10$ \\
\hline & $U-235,238$ & $9.46 \mathrm{E}+01$ & $3.97 E+15$ & $2.38 E-14$ & $1.0 E-13$ \\
\hline & Pu-238 & $8.85 E+02$ & $3.97 E+15$ & $2.23 E-13$ & $3.0 E-14$ \\
\hline & Pu-239 & $1.38 E+02$ & $3.97 E+15$ & $3.48 E-14$ & $2.0 E-14$ \\
\hline & Am-241,243 & $8.07 E+01$ & $3.97 E+15$ & $2.03 E-14$ & $2.0 E-14$ \\
\hline & $\mathrm{Cm}-242,244$ & $6.52 E+00$ & $3.97 E+15$ & $1.64 E-15$ & $4.0 E-14$ \\
\hline \multicolumn{6}{|c|}{ K-Aren (K-Reaton) } \\
\hline K, Main Staok & $H-3$ (oxide) & $3.40 E+10$ & $2.74 E+15$ & $1.24 \mathrm{E}-05$ & $1.0 \mathrm{E}-07$ \\
\hline \multirow[t]{2}{*}{ K, Disessembly } & $H-3$ (oxide) & $1.13 E+09$ & $1.16 E+15$ & $9.74 E-07$ & $1.0 \mathrm{E}-07$ \\
\hline & Cs-137 & $1.12 E+00$ & $1.16 \mathrm{E}+15$ & $9.66 E-16$ & $4.0 E-10$ \\
\hline \multicolumn{6}{|c|}{ L-Ares (L-Resctor) } \\
\hline L, Main Stack & $H-3$ (oxide) & $1.60 E+09$ & $2.62 E+15$ & $6.11 \mathrm{E}-07$ & $1.0 E-07$ \\
\hline \multirow[t]{3}{*}{ L, Dlsaseombly } & $H-3$ (oxide) & $2.61 E+08$ & $1.19 E+15$ & $2.19 E-07$ & $1.0 E-07$ \\
\hline & $R u-106$ & $3.99 E+00$ & $1.19 E+15$ & $3.35 E-15$ & $3.0 \mathrm{E}-11$ \\
\hline & Cs-137 & $1.03 E+02$ & $1.19 E+15$ & $8.66 E-14$ & $4.0 E-10$ \\
\hline \multicolumn{6}{|c|}{ M-Area (Reactor Materiale) } \\
\hline $313-M$ & $U-235,238$ & $4.57 E+00$ & $9.45 E+13$ & $4.84 E-14$ & $1.0 E-13$ \\
\hline $320-M$ & $U-235,238$ & $2.75 \mathrm{E}-02$ & $2.65 E+13$ & $1.04 E-15$ & $1.0 E-13$ \\
\hline $321-M$ & $U-235,238$ & $3.41 E-01$ & $5.91 E+14$ & $5.77 E-16$ & $1.0 E-13$ \\
\hline $\begin{array}{l}\text { Source: DOE O } \\
\text { is given. These } \\
100 \text { mrem unde } \\
\text { release limits or }\end{array}$ & $\begin{array}{l}\text { rder } 5400.5 \text {. In case } \\
\text { DCGs are defined } \\
\text { conditions of conti } \\
\text { standards. }\end{array}$ & $\begin{array}{l}8 \text { where different chem } \\
8 \text { the air concentration } \\
\text { uous exposure for one }\end{array}$ & $\begin{array}{l}\text { forms have differe } \\
\text { nat radionuclide th } \\
\text { ar. DCGs are refer }\end{array}$ & $\begin{array}{l}\text { DCGs, the lowest DCG } \\
\text { will give a } 50 \text {-year com } \\
\text { toe values only and are }\end{array}$ & $\begin{array}{l}\text { the radionuclide } \\
\text { od dose of } \\
\text { t considered }\end{array}$ \\
\hline
\end{tabular}




\section{Table 9}

1993 Radloactive Atmospheric Releases by Stack/Facllity and Comparison of Annual Average Concentrations to DOE Derlved Concentration Guides (DCGs)

Page 5 of 5

\begin{tabular}{|c|c|c|c|c|c|}
\hline Stack or Facillty & Radlonuclide & $\begin{array}{c}\text { Quantity } \\
\text { Released During } \\
1993(\mu \mathrm{Cl})\end{array}$ & $\begin{array}{c}\text { Atmoapherlc } \\
\text { Rolease } \\
\text { Volume (mL) }\end{array}$ & $\begin{array}{c}\text { Average Effluent } \\
\text { Concentration } \\
\text { During } 1993 \\
(\mu \mathrm{Cl} / \mathrm{mL})\end{array}$ & $\begin{array}{c}\text { DOE DCCOA } \\
(\mu \mathrm{C} / \mathrm{mL})\end{array}$ \\
\hline \multicolumn{6}{|c|}{ 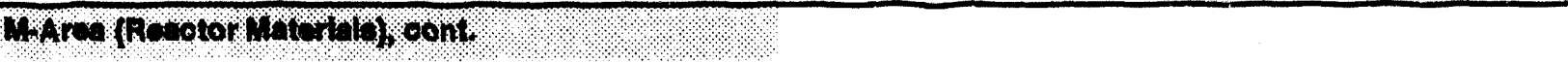 } \\
\hline 321-M (Mach.) & $U-235,238$ & $5.29 E+00$ & $8.68 E+13$ & $6.09 E-14$ & $1.0 E-13$ \\
\hline $322-M$ & $U-235,238$ & $5.31 E+00$ & $6.27 E+14$ & $8.47 E-15$ & $1.0 \mathrm{E}-13$ \\
\hline \multicolumn{6}{|c|}{ 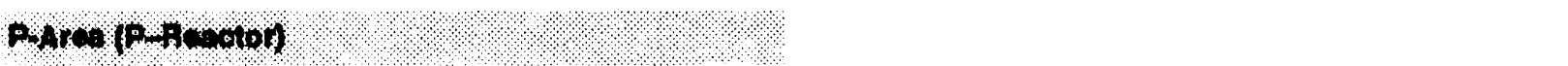 } \\
\hline P, Main Stack & $H-3$ (oxide) & $1.19 E+09$ & $1.22 E+15$ & $9.75 E-07$ & $1.0 E-07$ \\
\hline P, Disassembly & $H-3$ (oxide) & $1.22 E+08$ & $2.86 E+14$ & $4.27 E-07$ & $1.0 E-07$ \\
\hline
\end{tabular}

a Source: DOE Order 5400.5. In cases where different chemical forms have different DCGs, the lowest DCG for the radionuclide is given. These DCGs are defined as the air concentration of that radionuclide that will give a 50-year committed dose of 100 mrem under conditions of continuous exposure for one year. DCGs are reference values only and are not considered release limits or standards. 


\section{Table 10}

1993 Radioactive Liquid Releases by Source

(Including Direct and Seepage Basin Migration Releases)

Page 1 of 1

\begin{tabular}{|c|c|c|c|c|c|c|c|}
\hline \multirow[b]{2}{*}{$\begin{array}{l}\text { Radio- } \\
\text { nuclide }\end{array}$} & \multirow[b]{2}{*}{ Halfillfe } & \multicolumn{6}{|c|}{ Curiesa,b } \\
\hline & & Reactors & $\begin{array}{l}\text { Sopara- } \\
\text { tlons }\end{array}$ & $\begin{array}{l}\text { Reactor } \\
\text { Materials }\end{array}$ & $\begin{array}{l}\text { Heavy } \\
\text { Water }\end{array}$ & SRTC/TNX & Total \\
\hline H-3 (oxide) & $12.3 y$ & $2.29 E+03$ & $9.88 E+03$ & & $4.99 E+02$ & $1.29 E-01$ & $1.27 E+04$ \\
\hline Sr-89,90c & $29.1 y$ & $1.87 \mathrm{E}-01$ & $2.41 E-01$ & & 4.65E-02 & $2.02 E-03$ & 4.77E-01 \\
\hline $1-129$ & $1.6 \mathrm{E} 7 \mathrm{y}$ & & $2.20 E-02$ & & & & $2.20 E-02$ \\
\hline Cs-137 & $30.2 y$ & $1.29 E-02$ & 2.33E-01 & & & & $2.46 E-01$ \\
\hline$P m-147$ & $2.6 y$ & & $7.03 E-03$ & & & & $7.03 E-03$ \\
\hline$U-235,238$ & 4.5E9 y & & $1.14 E-05$ & & & & $1.14 E-05$ \\
\hline Pu-239d & $2.4 E 4 y$ & 5.97E-04 & $8.65 E-03$ & $7.64 E-05$ & & $2.66 \mathrm{E}-04$ & $9.59 E-03$ \\
\hline
\end{tabular}




\section{Table 11}

\section{Liquid Radioactive Releases by Facility and Comparison of Annual Average Radionuclide Concentrations to DOE Derived Concentration Guides (DCGs)}

Page 1 of 4

\begin{tabular}{|c|c|c|c|c|c|}
\hline Stack or Facility & Radionucllde & $\begin{array}{c}\text { Quantity of } \\
\text { Radlonucllides } \\
\text { Released During } \\
1993 \text { (C) }\end{array}$ & $\begin{array}{c}\text { Average Effluent } \\
\text { Concentration } \\
\text { During } 1993 \\
(\mu \mathrm{Cl} / \mathrm{mL})\end{array}$ & $\begin{array}{c}\text { DOE DCGs } \\
(\mu \mathrm{cl} / \mathrm{mL})\end{array}$ & $\begin{array}{c}\text { Fraction of DOE } \\
\text { DCG }\end{array}$ \\
\hline \multicolumn{6}{|l|}{$1+8(x)$} \\
\hline \multirow{3}{*}{$\begin{array}{l}\text { TB-2 A-1 } \\
\text { Outfall }\end{array}$} & $H-3$ & $9.32 E-02$ & $2.02 E-07$ & $2.0 E-03$ & $1.01 E-04$ \\
\hline & $\mathrm{U} / \mathrm{Pu} \quad(\mathrm{Pu}-239)$ & Below MDL & 8.60E-11 & $3.0 E-08$ & $2.87 E-03$ \\
\hline & & & & Sum of Fractions - & $2.97 E-03$ \\
\hline \multicolumn{6}{|c|}{ 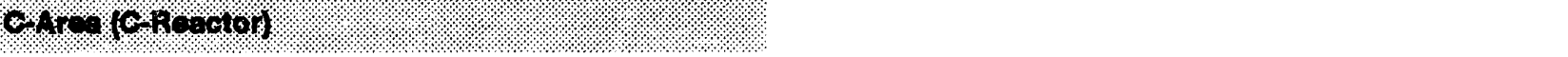 } \\
\hline \multirow[t]{3}{*}{ C-Canal } & $\mathrm{H}-3$ & $1.20 E+01$ & $6.77 E-07$ & $2.0 E-03$ & $3.39 E-04$ \\
\hline & Sr-89,90 & Below MDL & $1.99 E-10$ & $1.0 E-06$ & $1.99 E-04$ \\
\hline & & & & Sum of Fractions - & $5.38 E-04$ \\
\hline \multicolumn{6}{|c|}{ 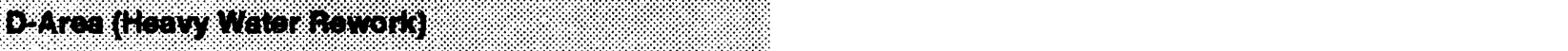 } \\
\hline \multirow[t]{3}{*}{ D-Area Effiuent } & $\mathrm{H}-3$ & $4.99 E+02$ & $7.99 E-06$ & $2.0 E-03$ & $3.99 E-03$ \\
\hline & Sr-89,90 & Below MDL & $2.49 \mathrm{E}-10$ & $1.0 E-06$ & $2.49 E-04$ \\
\hline & & & & Sum of Fractions - & $4.24 E-03$ \\
\hline \multicolumn{6}{|c|}{ 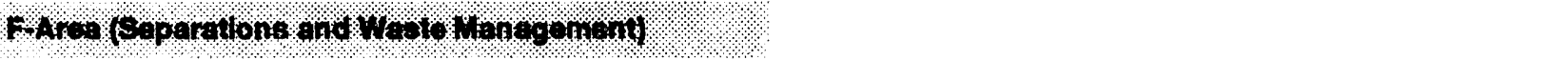 } \\
\hline \multirow[t]{3}{*}{$F-001$} & $\mathrm{H}-3$ & $3.67 E-01$ & $8.95 E-07$ & $2.0 E-03$ & $4.48 E-04$ \\
\hline & Sr-89,90 & Below MDL & $6.91 E-11$ & $1.0 E-06$ & $6.91 E-05$ \\
\hline & & & & Sum of Fractions - & $5.17 E-04$ \\
\hline \multirow[t]{4}{*}{$F-012(281-8 F)$} & $\mathrm{H}-3$ & $7.59 E-02$ & $2.25 E-07$ & $2.0 E-03$ & $1.12 E-04$ \\
\hline & Sr-89,90 & $2.22 E-05$ & $3.62 E-09$ & $1.0 E-06$ & $3.62 E-03$ \\
\hline & Cs-137 & $1.22 E-03$ & $2.51 E-08$ & $3.0 E-06$ & 8.37E-03 \\
\hline & & & & Sum of Fractions - & $1.21 E-02$ \\
\hline \multirow[t]{4}{*}{$F-013(200-F)$} & $\mathrm{H}-3$ & $8.02 E-02$ & $1.58 \mathrm{E}-06$ & $2.0 E-03$ & $7.90 E-04$ \\
\hline & Sr-89,90 & $1.17 \mathrm{E}-05$ & $2.60 E-09$ & $1.0 E-06$ & $2.60 E-03$ \\
\hline & Cs-137 & $2.03 E-03$ & $5.99 \mathrm{E}-09$ & $3.0 E-06$ & $2.00 E-03$ \\
\hline & & & & Sum of Fractions - & $5.39 E-03$ \\
\hline
\end{tabular}

a Source: DOE Order 5400.5. In cases where different chemical forms have different DCGs, the lowest DCG for the radionuclide is given. DCGs are defined as the concentration of that radionuclide that will give a 50-year committed effective dose equivalent of 100 mrem under conditions of continuous exposure for one year. DCGs are reference values only and are not considered release limits or standarck. 
Table 11

1993 Liquid Radioactive Releases by Facility and Comparison of Annual Average Radionuclide Concentrations to DOE Derived Concentration Guides (DCGs)

Page 2 of 4

\begin{tabular}{|c|c|c|c|c|c|}
\hline Stack or Facllity & Radionucllde & $\begin{array}{c}\text { Quantity of } \\
\text { Radionuclides } \\
\text { Released During } \\
1993 \text { (CI) }\end{array}$ & $\begin{array}{c}\text { Average Effluent } \\
\text { Concentration } \\
\text { During } 1993 \\
(\mu \mathrm{Cl} / \mathrm{mL})\end{array}$ & $\begin{array}{c}\text { DOE DCGsa } \\
(\mu \mathrm{Cl} / \mathrm{mL})\end{array}$ & $\begin{array}{c}\text { Fraction of DOE } \\
\text { DCG }\end{array}$ \\
\hline \multicolumn{6}{|c|}{ 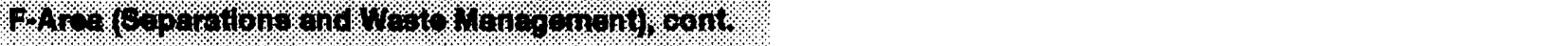 } \\
\hline \multirow[t]{5}{*}{ FM-3 (F-Area) } & $\mathrm{H}-3$ & $3.69 E+00$ & $1.65 E-06$ & $2.0 E-03$ & $8.26 \mathrm{E}-04$ \\
\hline & Sr-89,90 & 3.77E-04 & $6.54 E-10$ & $1.0 E-06$ & $6.54 E-04$ \\
\hline & Cs-137 & 3.67E-04 & $5.85 E-09$ & $3.0 E-06$ & $1.95 E-03$ \\
\hline & Pm-147 & $4.24 \mathrm{E}-03$ & 8.31E-10 & $1.0 E-04$ & 8.31E-06 \\
\hline & & & & Sum of Fractions - & $3.44 E-03$ \\
\hline \multirow{6}{*}{$\begin{array}{l}\text { U3R-2 } \\
\text { (F-Storm Sewer) }\end{array}$} & $H-3$ & $6.42 E-01$ & $1.95 E-06$ & $2.0 E-03$ & 9.77E-.04 \\
\hline & $\mathrm{Sr}-89,90$ & Below MDL & $2.08 E-10$ & $1.0 \mathrm{E}-06$ & $2.08 E-04$ \\
\hline & Cs-137 & $4.48 E-05$ & $7.08 E-09$ & $3.0 E-06$ & $2.36 \mathrm{E}-03$ \\
\hline & $P m-147$ & $4.59 E-04$ & $1.56 E-09$ & $1.0 E-04$ & $1.56 \mathrm{E}-05$ \\
\hline & U/Pu (Pu-239) & $3.30 E-04$ & $8.58 E-10$ & $3.0 E-08$ & $2.86 \mathrm{E}-02$ \\
\hline & & & & Sum of Fractions - & $3.22 E-02$ \\
\hline \multirow[t]{5}{*}{ U3RF-3 (N.F.) } & $\mathrm{H}-3$ & $1.33 E-01$ & $3.61 \mathrm{E}-06$ & $2.0 E-03$ & $1.80 E-03$ \\
\hline & Sr-89,90 & Below MDL & $-4.68 E-11$ & $1.0 E-06$ & $0.00 E+00$ \\
\hline & $P m-147$ & $1.26 \mathrm{E}-04$ & $1.78 E-09$ & $1.0 E-04$ & $1.78 E-05$ \\
\hline & $U-235,238$ & $1.14 \mathrm{E}-05$ & $5.15 E-10$ & $6.0 E-07$ & $8.58 E-04$ \\
\hline & & & & Sum of Fractions - & $2.68 E-03$ \\
\hline \multicolumn{6}{|c|}{ 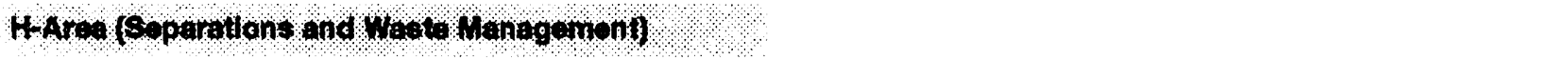 } \\
\hline \multirow[t]{5}{*}{ FM-1C (H-Area) } & $\mathrm{H}-3$ & $6.17 E+00$ & $1.35 \mathrm{E}-05$ & $2.0 E-03$ & $6.76 E-03$ \\
\hline & Sr-89,90 & $1.56 \mathrm{E}-04$ & $9.12 \mathrm{E}-10$ & $1.0 E-06$ & $9.12 E-04$ \\
\hline & Cs-137 & $5.44 E-03$ & $2.55 E-08$ & $3.0 E-06$ & $8.50 E-03$ \\
\hline & Pm-147 & $1.34 E-03$ & 2.17E-09 & $1.0 E-04$ & 2.17E-05 \\
\hline & & & & Sum of Fractions - & $1.62 E-02$ \\
\hline $\begin{array}{l}\text { Source: DOE O } \\
\text { radionuclide is } \mathrm{g} \\
\text { equivalent of } 10 \\
\text { considered relee }\end{array}$ & $\begin{array}{l}\text { rder } 5400.5 \text {. In ca } \\
\text { iven. DCGs are def } \\
0 \text { mrem under conc } \\
\text { ase limits or standa }\end{array}$ & $\begin{array}{l}\text { s where different chemi } \\
\text { od as the concentration } \\
\text { ons of continuous expo } \\
\text { s. }\end{array}$ & $\begin{array}{l}\text { ical forms have differen } \\
\text { of that radionuclide that } \\
\text { sure for one year. DCC }\end{array}$ & $\begin{array}{l}\text { DCGs, the lowest DCG } \\
\text { will give a } 50 \text {-year commi } \\
\text { as are reference values }\end{array}$ & $\begin{array}{l}\text { for the } \\
\text { tted effective dose } \\
\text { only and are not }\end{array}$ \\
\hline
\end{tabular}




\section{Table 11}

\section{Liquid Radioactive Releases by Facility and Comparison of Annual Average Radionuclide Concentrations to DOE Derived Concentration Guides (DCGs)}

Page 3 of 4

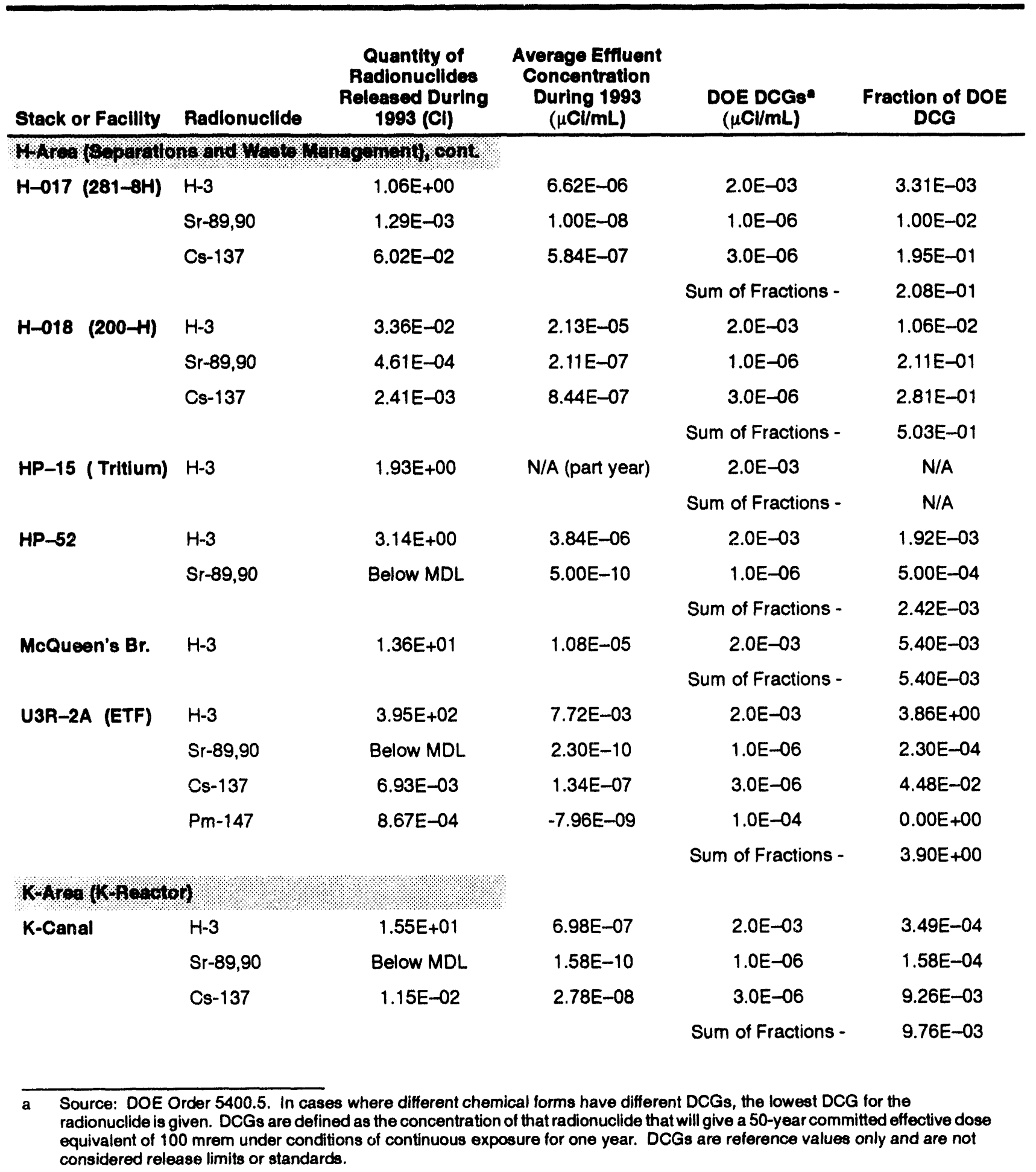




\section{Table 11}

\section{Liquid Radioactive Releases by Facility and Comparison of Annual Average Radionuclide Concentrations to DOE Derived Concentration Guides (DCGs)}

Page 4 of 4

\begin{tabular}{|c|c|c|c|c|c|}
\hline Stack or Facillty & Radlonuclide & $\begin{array}{c}\text { Quantity of } \\
\text { Radionuclldes } \\
\text { Released During } \\
1993 \text { (Cl) }\end{array}$ & $\begin{array}{c}\text { Average Effluent } \\
\text { Concentration } \\
\text { During } 1993 \\
(\mu \mathrm{Cl} / \mathrm{mL})\end{array}$ & $\begin{array}{c}\text { DOE DCGea } \\
(\mu \mathrm{Ci} / \mathrm{mL})\end{array}$ & $\begin{array}{c}\text { Fraction of DOE } \\
\text { DCG }\end{array}$ \\
\hline \multicolumn{6}{|c|}{ 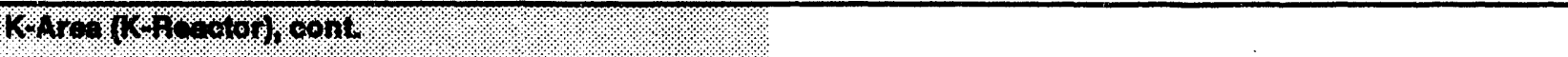 } \\
\hline \multirow[t]{2}{*}{ PB-1 (Sec. Etf.) } & $\mathrm{H}-3$ & $1.96 E-01$ & $7.79 E-06$ & $2.0 E-03$ & $3.90 E-03$ \\
\hline & & & & Sum of Fractions - & $3.90 E-03$ \\
\hline \multirow[t]{2}{*}{ K-Area Purge } & $\mathrm{H}-3$ & $8.05 E+01$ & $2.69 \mathrm{E}-01$ & $2.0 E-03$ & $1.35 E+02$ \\
\hline & & & & Sum of Fractions - & $1.35 E+02$ \\
\hline \multicolumn{6}{|c|}{$1+1+8,+10,10)$} \\
\hline \multirow[t]{3}{*}{$L-007$} & $H-3$ & $9.31 E+00$ & $2.13 E-07$ & $2.0 E-03$ & $1.07 E-04$ \\
\hline & $\mathrm{Sr}-89,90$ & Below MDL & $1.29 E-10$ & $1.0 E-06$ & $1.29 E-04$ \\
\hline & & & & Sum of Fractions - & $2.35 E-04$ \\
\hline \multicolumn{6}{|c|}{ 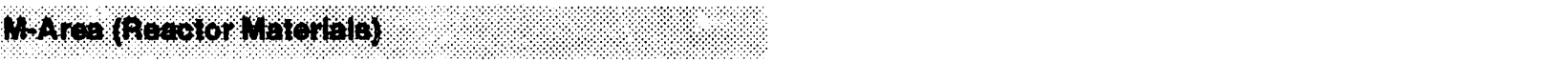 } \\
\hline \multirow[t]{2}{*}{ TB-3 (M-Area) } & $U-235,238$ & Below MDL & $1.47 \mathrm{E}-09$ & $6.0 \mathrm{E}-07$ & 2.45E-03 \\
\hline & & & & Sum of Fractions - & $2.45 E-03$ \\
\hline \multicolumn{6}{|c|}{ P.Area (prisector) } \\
\hline \multirow[t]{3}{*}{ P-019 } & $\mathrm{H}-3$ & $2.86 \mathrm{E}+00$ & $9.61 \mathrm{E}-07$ & $2.0 E-03$ & $4.81 E-04$ \\
\hline & Sr-89,90 & Below MDL & $1.47 E-10$ & $1.0 \mathrm{E}-06$ & $1.47 E-04$ \\
\hline & & & & Sum of Fractions - & $6.27 E-04$ \\
\hline \multicolumn{6}{|l|}{ TNX Ares } \\
\hline \multirow[t]{3}{*}{ TNX-1 } & $\mathrm{H}-3$ & $3.56 \mathrm{E}-02$ & $-1.83 E-08$ & $2.0 E-03$ & $0.00 E+00$ \\
\hline & U/Pu (Pu-239) & Below MDL & $5.97 E-11$ & $3.0 E-08$ & $1.99 E-03$ \\
\hline & & & & Sum of Fractions - & $1.99 E-03$ \\
\hline
\end{tabular}

a Source: DOE Order 5400.5. In cases where different chemical forms have different DCGs, the lowest DCG for the radionuclide is given. DCGs are defined as the concentration of that radionuclide that will give a 50-year committed effective dose equivalent of $100 \mathrm{mrem}$ under conditions of continuous exposure for one year. DCGs are relerence values only and are not considered release limits or standards. 


\section{Table 12}

\section{Estimated Tritium Releases In SRS Streams and the Savannah Rlver}

Page 1 of 3

\begin{tabular}{|c|c|c|c|c|c|c|c|}
\hline & & Direc & $088 c 8$ (C) & & & & \\
\hline Area & Release Point & 1988 & 1989 & 1890 & 1991 & 1992 & 1993 \\
\hline Reactor & & & & & & & \\
\hline $100-P$ & $\begin{array}{l}\text { Par Pond overflow to Lower- } \\
\text { Three Runs Creek }\end{array}$ & 327 & $(321)^{a}$ & $(207)^{a}$ & 221 & 100 & 64 \\
\hline & "Process sewer to Par Pond & & 164 & 67 & $(43)^{a}$ & & \\
\hline & $\begin{array}{l}\text { "Reactor heat exchanger cool- } \\
\text { ing water to Par Pond }\end{array}$ & & 464 & 125 & $(67)^{a}$ & & \\
\hline & "Combined in 1992 (P019) & & & & & $(8)^{a}$ & $(3)^{a}$ \\
\hline $100-L$ & $\begin{array}{l}\text { L-Lake overflow to Steel } \\
\text { Creek }\end{array}$ & 502 & $(556)^{a}$ & $(358)^{a}$ & 723 & 515 & 650 \\
\hline & "Process sewer to L Lake & & 24 & 27 & $(11)^{a}$ & & \\
\hline & $\begin{array}{l}\text { "Reactor heat exchanger cool- } \\
\text { ingwater to L Lake }\end{array}$ & & 98 & 72 & $(112)^{a}$ & & \\
\hline & "Combined in 1992 (LOOT) & & & & & $(58)^{a}$ & $(9)^{a}$ \\
\hline $100-K$ & -Process sewer to Pen Branch & 264 & 100 & 169 & 74 & & \\
\hline & $\begin{array}{l}\text { "Reactor heat exchanger cool- } \\
\text { ingwater to Pen Branch }\end{array}$ & 2,470 & 112 & 249 & $6,470^{b}$ & & \\
\hline & $\begin{array}{l}\text { "Combined in } 1992 \\
\text { (K-Canal) }\end{array}$ & & & & & 126 & 16 \\
\hline & (K008) & & & & & 3 & \\
\hline & K-Area secondary effluent & & & & 6 & 3 & \\
\hline $100-C$ & $\begin{array}{l}\text { Process Sewer to Four Mile } \\
\text { Creek (C-Canal) }\end{array}$ & 11 & 16 & 1 & 13 & 28 & 12 \\
\hline & Subtotal & 3,570 & 978 & 710 & 7,510 & 775 & 742 \\
\hline Separa & lons & & & & & & \\
\hline $200-F$ & Effluent to Four Mile Creek & 14 & 8 & 327 & 6 & 5 & 4 \\
\hline & Effluent to Upper Three Runs & & 2 & 0 & 0 & 1 & 1 \\
\hline $200-H$ & Effluent to Four Mile Creek & 12 & 20 & 22 & 14 & 13 & 12 \\
\hline & Effluent to Upper Three Runs & & 1 & 4 & 5 & 15 & 17 \\
\hline & Effluent Treatment Facility & 101 & 2,070 & 1,200 & 3,070 & 1,010 & 395 \\
\hline & Subtotal & 127 & 3,100 & 1,550 & 3,090 & 1,040 & 426 \\
\hline $400-D$ & $\begin{array}{l}\text { Process sewer to Beaver Dam } \\
\text { Creek }\end{array}$ & 1,740 & 562 & 358 & 681 & 576 & 499 \\
\hline & Subtotal & 1,740 & 562 & 358 & 681 & 576 & 499 \\
\hline & Total Direct Releases ${ }^{\circ}$ & 5,440 & 3,640 & 2,620 & 11,300 & 2,390 & 1,670 \\
\hline
\end{tabular}

Not used in totals because release was counted elsewhere

b Includes heat exchanger leak of December 22-25, 1991

c Because of rounding, sums of individual columns might not equal totals. 
Table 12

Estimated Tritlum Releases in SRS Streams and the Savannah RIver

Page 2 of 3

\begin{tabular}{|c|c|c|c|c|c|c|c|}
\hline \multicolumn{8}{|c|}{ Migration (Curles) } \\
\hline Area & Roloase Point & 1988 & 1989 & 1990 & 1991 & 1992 & 1993 \\
\hline \multirow[t]{3}{*}{$\begin{array}{l}200- \\
F \& H\end{array}$} & $\begin{array}{l}\text { Solid Waste Disposal Facility } \\
\text { and H-Area seepage basin to } \\
\text { Four Mile Creek }\end{array}$ & 3,670 & 3,600 & 4,280 & 6,420 & 4,090 & 5.330 \\
\hline & $\begin{array}{l}\text { 200-F seepage basin to Four } \\
\text { Mile Creek }\end{array}$ & 3,330 & 4,440 & 3,570 & 5,750 & 4,260 & 2,180 \\
\hline & $\begin{array}{l}\text { 200-H seepage basin to Four } \\
\text { Mile Creek }\end{array}$ & 3,980 & 3,310 & 1,900 & 1,810 & 1,470 & 1,020 \\
\hline $100-C$ & $\begin{array}{l}\text { Seepage basin to Four Mile } \\
\text { Creek }\end{array}$ & & & 7 & & & \\
\hline $100-K$ & $\begin{array}{l}904-88 G \text { to Indian Grave } \\
\text { Branch }\end{array}$ & 2,780 & 2,220 & 3,560 & 2,160 & 1,530 & 1,100 \\
\hline \multirow[t]{3}{*}{$100-P$} & Seepage basin to Steel Creek & 133 & 137 & 224 & $(364)^{a}$ & $(232)^{a}$ & $(382)^{a}$ \\
\hline & Subtotal & 13,900 & 13,700 & 13,500 & 16,100 & 11,400 & 9,630 \\
\hline & $\begin{array}{l}\text { Total Direct Releases } \\
\text { and Migrationb }\end{array}$ & 19,300 & 17,300 & 16,100 & 27,400 & 13,800 & 11,300 \\
\hline \multicolumn{8}{|c|}{ stream Transport (Curles) } \\
\hline Area & Release Point & 1988 & 1989 & 1990 & 1991 & 1992 & 1993 \\
\hline $400-D$ & Beaver Dam Creek at swamp & 2,510 & 879 & 756 & 801 & 576 & 499 \\
\hline $\begin{array}{l}20- \\
F \& H\end{array}$ & Four Mile Creek at Road A13 & 11,200 & 11,200 & 9,370 & 13,300 & 8,710 & 9,000 \\
\hline $100-K$ & Pen Branch at Road A & 3,220 & 2,700 & 2,510 & 7,100 & 1,850 & 1,580 \\
\hline $100-L$ & Steel Creek at Road A & 502 & 556 & 358 & 723 & 515 & 650 \\
\hline $100-P$ & Lower Three Runs at Road B & 327 & 321 & 207 & 221 & 100 & 64 \\
\hline \multirow[t]{2}{*}{ ETF } & Upper Three Runs at Road A & 535 & 2,160 & 2,380 & 4,410 & 1,300 & 879 \\
\hline & Totalb & 18,300 & 17,800 & 15,600 & 26,600 & 13,100 & 12,700 \\
\hline
\end{tabular}

a Not used in totals because release was counted elsewhere

b Because of rounding, sums of individual columns might not equal totals. 


\section{Table 12}

Estimated Tritlum Releases in SRS Streams and the Savannah RIver

Page 3 of 3

\begin{tabular}{|c|c|c|c|c|c|c|}
\hline \multicolumn{7}{|c|}{ River Traneport (Curies) } \\
\hline & 1988 & 1989 & 1990 & 1901 & 1992 & 1093 \\
\hline $\begin{array}{l}\text { Tritium measured in the Savannah River } \\
\text { below SRS }\end{array}$ & & 17,110 & 16,570 & 28,700 & 16,000 & 12,600 \\
\hline $\begin{array}{l}\text { Tritium measured in the Savannah Fiver } \\
\text { above SAS }\end{array}$ & & 1,480 & 2,080 & 2,420 & 2,210 & 433 \\
\hline Tritlum measured in the savannah & 14,600 & 15,600 & 14,490 & 26,300 & 13,800 & 12,200 \\
\hline
\end{tabular}

Aiver below 8 As (downrlver minus upriver)"

a Because of rounding, differences in individual columns might not equal totals. 
Table 13

Minimum Detectable Activities (MDAs) for Radionuclides

In Settleable Sollds

Page 1 of 1

\begin{tabular}{|c|c|c|c|}
\hline Nuollde & MDA (pOVeamplo) & Nuollde & MDA (pCl/eamplo) \\
\hline$B e-7$ & $3.32 E+01$ & $\mathrm{Na}-24$ & $1.21 E+01$ \\
\hline$K-40$ & $1.09 E+02$ & $\mathrm{Cr}-51$ & $4.02 E+01$ \\
\hline$M n-54$ & $7.83 E+00$ & $M n-56$ & $8.04 E+00$ \\
\hline Co-57 & $3.31 E+00$ & Co-58 & $6.43 E+00$ \\
\hline$F_{\theta-59}$ & $1.19 E+01$ & $\mathrm{Co}-60$ & $8.22 E+00$ \\
\hline $2 n-65$ & $1.59 E+01$ & $Y-88$ & $1.12 E+01$ \\
\hline $\mathrm{Nb}-95$ & $6.88 E+00$ & Nb-95M & $1.34 E+01$ \\
\hline Zr-95 & $1.18 E+01$ & Mo-99 & $4.40 E+01$ \\
\hline Ru-103 & $5.29 E+00$ & $R u-106$ & $6.63 E+01$ \\
\hline$C d-109$ & $6.74 E+01$ & $S n \cdot 113$ & $5.69 E+00$ \\
\hline$S b-124$ & $6.41 E+00$ & Sb-125 & $1.48 E+01$ \\
\hline$S b-127$ & $1.82 E+01$ & $|-13|$ & $5.03 E+00$ \\
\hline $1-132$ & $9.26 E+00$ & Te-132 & $3.65 E+00$ \\
\hline Ba-133 & $7.69 E+00$ & $1-133$ & $6.82 E+00$ \\
\hline Cs-134 & $6.54 E+00$ & $1-135$ & $2.49 E+01$ \\
\hline$x e-135$ & $4.66 E+00$ & Cs-136 & $6.6 E+0 C$ \\
\hline Cs-137 & $6.47 E+00$ & $C e-139$ & $3.50 E+00$ \\
\hline$B a-140$ & $2.30 E+01$ & La-140 & $9.98 E+00$ \\
\hline $\mathrm{Ce}-141$ & $5.68 E+00$ & $\mathrm{Ce}-143$ & $5.59 E+00$ \\
\hline $\mathrm{Ce}-144$ & $2.57 E+01$ & No-147 & $1.19 E+01$ \\
\hline Eu-154 & $7.01 E+00$ & TI-208 & $9.15 E+00$ \\
\hline TI-210 & $6.15 E+00$ & $\mathrm{~Pb}-211$ & $1.34 E+02$ \\
\hline $\mathrm{Bi}-212$ & $6.17 E+01$ & $\mathrm{~Pb}-212$ & $1.25 E+01$ \\
\hline$B i-214$ & $1.69 E+01$ & $\mathrm{~Pb}-214$ & $1.29 E+01$ \\
\hline Rn-219 & $3.78 E+01$ & Ra-223 & $1.35 \mathrm{E}+01$ \\
\hline Ra-224 & $1.16 E+02$ & Ra-226 & $1.27 E+02$ \\
\hline Th-227 & $3.00 E+01$ & $A c-228$ & $3.22 E+01$ \\
\hline Th-228 & $2.89 E+02$ & Th-231 & $5.41 E+01$ \\
\hline Th-234 & $6.87 E+01$ & U-235 & $7.65 E+00$ \\
\hline \multirow[t]{2}{*}{$A m-241$} & $1.25 E+01$ & Alpha & $6.80 E-01$ \\
\hline & & Beta & $1.53 E-00$ \\
\hline
\end{tabular}




\section{Table 14}

\section{Settleable Sollds Results}

for the SRs Radionuclides in Settleable Sollds Program

Page 1 of 4

\begin{tabular}{|c|c|c|c|c|}
\hline \multirow[b]{2}{*}{ Looation } & \multirow[b]{2}{*}{$\begin{array}{l}\text { Prooseso/Baok- } \\
\text { ground }\end{array}$} & \multicolumn{3}{|c|}{ Hirof cluartor } \\
\hline & & $\begin{array}{l}\text { Tes } \\
\text { mgll }\end{array}$ & $\begin{array}{c}\text { Nonsooflleablo } \\
\text { sollde } \\
\text { mo/l }\end{array}$ & $\begin{array}{l}\text { Sottleable } \\
\text { sollde } \\
\text { moll }\end{array}$ \\
\hline TB-2 & Procese & 1.00 & $<1.00$ & 1.00 \\
\hline U3R-1A & Background & 3.00 & 1.00 & 2.00 \\
\hline$c-004$ & Procese & 3.00 & $<1.00$ & 2.00 \\
\hline FMC RD F & Background & 4.00 & 1.00 & 3.00 \\
\hline FMC-3 & Process & 2.00 & 1.00 & 1.00 \\
\hline FMC RD F & Background & 4.00 & 1.00 & 3.00 \\
\hline FMC-1C & Process & 10.00 & 2.00 & 9.00 \\
\hline FMC RD F & Background & 4.00 & 1.00 & 3.00 \\
\hline HP-52 & Process & 2.00 & 1.00 & 1.00 \\
\hline FMC RD F & Background & 4.00 & 1.00 & 3.00 \\
\hline K CAN.HEAD W'ALL & Process & 8.00 & 2.00 & 6.00 \\
\hline PB RD B & Background & 2.00 & $<1.00$ & 2.00 \\
\hline$L-007$ & Process & 3.00 & 1.00 & 2.00 \\
\hline PB RD B & Background & 2.00 & $<1,00$ & 2.00 \\
\hline TB-3 & Process & $<1.00$ & $<1.00$ & $<1.00$ \\
\hline U3R-1A & Background & 3.00 & 1.00 & 2.00 \\
\hline P-19 & Process & 1.00 & $<1.00$ & 1.00 \\
\hline U3A-1A & Background & 3.00 & 1.00 & 2.00 \\
\hline$B D C$ & Process & 10.00 & 2.00 & 8.00 \\
\hline U3R-1A & Background & 3.00 & 1.00 & 2.00 \\
\hline$T N X-1$ & Process & 1.00 & $<1.00$ & 1.00 \\
\hline PB RD B & Background & 2.00 & $<1.00$ & 2.00 \\
\hline
\end{tabular}


Table 14

Sottleable Sollds Results

for the SRS Radionuclides in Sottleable Sollds Program

Poge 2 of 4

\begin{tabular}{|c|c|c|c|c|}
\hline \multirow[b]{2}{*}{ Looation } & \multirow[b]{2}{*}{$\begin{array}{l}\text { ProcesolBeok- } \\
\text { ground } \\
\end{array}$} & \multicolumn{3}{|c|}{ Eoond curritor } \\
\hline & & $\begin{array}{l}\text { Tes } \\
\text { mg/L }\end{array}$ & $\begin{array}{l}\text { Nonsotileable } \\
\text { Eollde } \\
\text { moll }\end{array}$ & $\begin{array}{l}\text { otiloable } \\
\text { eollde } \\
\text { mall }\end{array}$ \\
\hline TB-2 & Proceses & $<1.00$ & $<1.00$ & $<1.00$ \\
\hline U3R-1A & Background & $<1.00$ & $<1.00$ & $<1.00$ \\
\hline$C-004$ & Process & 4.00 & 3.00 & $<1.00$ \\
\hline FMC RD F & Background & 76.00 & 41.00 & 35.00 \\
\hline FMC-3 & Process & 8.00 & 1.00 & 6.00 \\
\hline FMC RD F & Background & 76.00 & 41.00 & 35.00 \\
\hline FMC-1C & Process & 1.00 & $<1.00$ & 1.00 \\
\hline FMC RD F & Background & 76.00 & 41.00 & 35.00 \\
\hline HP-52 & Process & 12.00 & 4.00 & 8.00 \\
\hline FMC RD F & Background & 76.00 & 41.00 & 35.00 \\
\hline K CAN.HEAD WALL & Process & 12.00 & 4.00 & 9.00 \\
\hline PB RD B & Background & 12.00 & 1.00 & 11.00 \\
\hline$L-007$ & Process & 2.00 & $<1.00$ & 2.00 \\
\hline PB RD B & Background & 12.00 & 1.00 & 11.00 \\
\hline TB-3 & Process & 1.00 & $<1.00$ & 1.00 \\
\hline U3R-1A & Background & $<1.00$ & $<1.00$ & $<1.00$ \\
\hline$P-19$ & Process & 1.00 & $<1.00$ & 1.00 \\
\hline U3R-1A & Background & $<1.00$ & $<1.00$ & $<1.00$ \\
\hline BDC & Process & 8.00 & 2.00 & 6.00 \\
\hline U3R-1A & Background & $<1.00$ & $<1.00$ & $<1.00$ \\
\hline$T N X-1$ & Process & 9.00 & 1.00 & 8.00 \\
\hline PB RD B & Background & 12.00 & 1.00 & 11.00 \\
\hline
\end{tabular}


Table 14

Settleable Sollds Results

for the 8RS Radionuclides in Sottleable Sollds Program

Pago 3 of 4

\begin{tabular}{|c|c|c|c|c|}
\hline \multirow[b]{2}{*}{ Looation } & \multirow[b]{2}{*}{$\begin{array}{l}\text { Procece/Baok- } \\
\text { ground }\end{array}$} & \multicolumn{3}{|c|}{ Third Quartor } \\
\hline & & $\begin{array}{l}\mathrm{Tas} \\
\mathrm{mg} / \mathrm{L}\end{array}$ & $\begin{array}{l}\text { Nonsettleable } \\
\text { Solldo } \\
\text { mg/l }\end{array}$ & $\begin{array}{l}\text { Eottleable } \\
\text { Sollds } \\
\text { mg/L }\end{array}$ \\
\hline TB-2 & Process & $<1.00$ & $<1.00$ & $<1.00$ \\
\hline U3R-1A & Background & 3.00 & $<1.00$ & 3.00 \\
\hline$c-004$ & Process & $<1.00$ & $<1.00$ & $<1.00$ \\
\hline FMC RD F & Background & 15.00 & 6.00 & 10.00 \\
\hline FMC-3 & Proceses & 12.00 & $<1.00$ & 12.00 \\
\hline FMC RD F & Background & 15.00 & 6.00 & 10.00 \\
\hline FMC-1C & Process & 1.00 & $<1.00$ & $<1.00$ \\
\hline FMC RD F & Background & 15.00 & 6.00 & 10.00 \\
\hline HP-52 & Process & 2.00 & 1.00 & 1.00 \\
\hline FMC RD F & Background & 15.00 & 6.00 & 10.00 \\
\hline K CAN.HEAD WALL & Process & 2.00 & $<1.00$ & 2.00 \\
\hline PB RD B & Background & 2.00 & 1.00 & 1.00 \\
\hline$L-007$ & Process & 3.00 & 1.00 & 2.00 \\
\hline PB RD B & Background & 2.00 & 1.00 & 1.00 \\
\hline TB-3 & Process & $<1.00$ & $<1.00$ & $<1.00$ \\
\hline U3R-1A & Background & 3.00 & $<1.00$ & 3.00 \\
\hline P-19 & Process & 1.00 & 1.00 & $<1.00$ \\
\hline U3R-1A & Background & 3.00 & $<1.00$ & 3.00 \\
\hline $\mathrm{BDC}$ & Process & 8.00 & 2.00 & 6.00 \\
\hline U3R-1A & Background & 3.00 & $<1.00$ & 3.00 \\
\hline TNX-1 & Process & 1.00 & $<1.00$ & 1.00 \\
\hline PB RD B & Background & 2.00 & 1.00 & 1.00 \\
\hline
\end{tabular}


Table 14

Settleable Sollds Results

for the SRS Radionuclldes in Settleable Sollds Program

Page 4 of 4

\begin{tabular}{|c|c|c|c|c|}
\hline \multirow[b]{2}{*}{ Location } & \multirow[b]{2}{*}{$\begin{array}{l}\text { Proceso/Baok- } \\
\text { ground }\end{array}$} & \multicolumn{3}{|c|}{ Fourin cuartor } \\
\hline & & $\begin{array}{l}\text { Ts8 } \\
\text { mg/L }\end{array}$ & $\begin{array}{c}\text { Nonsettloable } \\
\text { sollds } \\
\text { moll }\end{array}$ & $\begin{array}{l}\text { Sottleable } \\
\text { Sollde } \\
\text { mgll }\end{array}$ \\
\hline TB-2 & Process & 5.00 & 2.00 & 3.00 \\
\hline U3R-1A & Background & $<1.00$ & $<1.00$ & $<1.00$ \\
\hline$C-004$ & Process & $<1.00$ & $<1.00$ & $<1.00$ \\
\hline FMC RD F & Background & 15.00 & 6.00 & 9.00 \\
\hline FMC-3 & Process & 80.00 & 37.00 & 43.00 \\
\hline FMC RD F & Background & 15.00 & 6.00 & 9.00 \\
\hline FMC-1C & Process & 103.00 & 28.00 & 75.00 \\
\hline FMC RD F & Background & 15.00 & 6.00 & 9.00 \\
\hline HP-52 & Process & 30.00 & 6.00 & 24.00 \\
\hline FMC RD F & Background & 15.00 & 6.00 & 9.00 \\
\hline K CAN.HEAD WALL & Process & $<1.00$ & $<1.00$ & $<1.00$ \\
\hline PB RD B & Background & 6.00 & 1.00 & 5.00 \\
\hline$L-007$ & Process & $<1.00$ & $<1.00$ & $<1.00$ \\
\hline PB RD B & Background & 6.00 & 1.00 & 5.00 \\
\hline TB-3 & Process & $<1.00$ & $<1.00$ & $<1.00$ \\
\hline U3R-1A & Background & $<1.00$ & $<1.00$ & $<1.00$ \\
\hline$P-19$ & Process & $<1.00$ & $<1.00$ & $<1.00$ \\
\hline U3R-1A & Background & $<1.00$ & $<1.00$ & $<1.00$ \\
\hline$B D C$ & Process & 2.00 & $<1.00$ & 2.00 \\
\hline U3R-1A & Background & $<1.00$ & $<1.00$ & $<1.00$ \\
\hline$T N X-1$ & Process & $<1.00$ & $<1.00$ & $<1.00$ \\
\hline PB RD B & Background & 6.00 & 1.00 & 5.00 \\
\hline
\end{tabular}




\section{Table 15}

\section{Radloactivity In Alr}

Page 1 of 6

\begin{tabular}{|c|c|c|c|c|}
\hline Location & $\begin{array}{l}\text { No. of } \\
\text { Samples }\end{array}$ & Arithmotio Mean $\pm \sigma$ & Maximum $\pm \sigma$ & Minimum \pm 0 \\
\hline \multicolumn{5}{|l|}{$\mathrm{H}-3(\mu \mathrm{Cl} / 00)$} \\
\hline \multicolumn{5}{|l|}{ On site } \\
\hline A-Area & 41 & $(3.49 \pm 4.29) E-11$ & $(2.50 \pm 0.17) E-10$ & $(-1.21 \pm 7.02) E-12$ \\
\hline Burial Ground North & 34 & $(2.92 \pm 2.52) E-10$ & $(1.31 \pm 0.04) E-09$ & $(5.87 \pm 0.48) E-11$ \\
\hline Burial Ground South & 43 & (3.64 \pm 3.77$) E-10$ & $(1.94 \pm 0.05) E-09$ & $(2.90 \pm 0.27) E-11$ \\
\hline F-Area & 40 & $(1.09 \pm 0.93) E-10$ & $(5.47 \pm 0.36) E-10$ & $(1.84 \pm 0.24) E-11$ \\
\hline H-Area & 42 & $(1.06 \pm 1.13) E-09$ & $(5.26 \pm 0.08) E-09$ & $(2.99 \pm 0.37) E-11$ \\
\hline \multicolumn{5}{|l|}{ Slto Porimoter } \\
\hline Allendale Gate & 32 & $(9.08 \pm 6.65) E-12$ & $(2.33 \pm 1.32) E-11$ & $(-5.72 \pm 2.23) E-12$ \\
\hline Bamwell Gate & 33 & $(2.58 \pm 4.09) E-11$ & $(2.33 \pm 0.20) E-10$ & $(-0.69 \pm 3.37) E-12$ \\
\hline D-Area & 33 & $(6.03 \pm 4.53) E-11$ & $(1.61 \pm 0.13) E-10$ & $(1.92 \pm 1.95) E-12$ \\
\hline Darkhorse@ Williston Gate & 41 & $(3.04 \pm 2.95) E-11$ & $(1.55 \pm 0.13) E-10$ & $(-1.61 \pm 4.33) E-12$ \\
\hline East Talatha & 42 & $(2.94 \pm 3.22) E-11$ & $(1.75 \pm 0.13) E-10$ & $(-0.58 \pm 2.43) E-12$ \\
\hline Green Pond & 32 & $(3.16 \pm 3.45) E-11$ & $(1.36 \pm 0.14) E-10$ & $(2.74 \pm 3.26) E-12$ \\
\hline Highway 125 @ Road A-14 & 30 & $(3.79 \pm 3.51) E-11$ & $(1.46 \pm 0.18) E-10$ & $(7.34 \pm 9.72) E-12$ \\
\hline Highway $21 / 167$ & 36 & $(2.74 \pm 3.01) E-11$ & $(1.35 \pm 0.16) E-10$ & $(1.94 \pm 3.52) E-12$ \\
\hline $\begin{array}{l}\text { Highway39@ Williston } \\
\text { Gate }\end{array}$ & 42 & $(2.82 \pm 2.66) E-11$ & $(1.23 \pm 0.17) E-10$ & $(-0.43 \pm 1.39) E-11$ \\
\hline Jackson & 32 & $(3.55 \pm 4.88) E-11$ & $(1.86 \pm 0.06) E-10$ & $(-0.13 \pm 2.44) E-12$ \\
\hline Patterson Mill Road & 31 & $(1.38 \pm 1.49) E-11$ & $(6.25 \pm 1.45) E-11$ & $(-1.67 \pm 3.93) E-12$ \\
\hline Talatha Gate & 41 & $(3.63 \pm 3.82) E-11$ & $(1.64 \pm 0.13) E-10$ & $(0.32 \pm 2.02) E-12$ \\
\hline West Jackson & 29 & $(2.67 \pm 2.38) E-11$ & $(1.06 \pm 0.15) E-10$ & $(2.78 \pm 2.54) E-12$ \\
\hline Windsor Road & 41 & $(2.61 \pm 2.21) E-11$ & $(1.19 \pm 0.18) E-10$ & $(0.41 \pm 3.23) E-12$ \\
\hline \multicolumn{5}{|l|}{ 25-Mlle-Radius } \\
\hline Aiken Airport & 28 & $(7.39 \pm 8.28) E-12$ & $(3.07 \pm 0.90) E-11$ & $(-5.14 \pm 3.24) E-12$ \\
\hline Aiken State Park & 32 & $(0.84 \pm 1.02) E-11$ & $(3.10 \pm 0.63) E-11$ & $(-1.09 \pm 1.34) E-11$ \\
\hline Allendale, SC & 34 & $(5.23 \pm 9.16) E-12$ & $(4.19 \pm 0.58) E-11$ & $(-5.02 \pm 4.35) E-12$ \\
\hline Augusta, GA & 43 & $(0.85 \pm 1.23) E-11$ & $(5.06 \pm 0.86) E-11$ & $(-6.47 \pm 9.57) E-12$ \\
\hline Highway $301 @$ State Line & 26 & $(7.41 \pm 7.52) E-12$ & $(2.55 \pm 1.50) E-11$ & $(-4.76 \pm 8.82) E-12$ \\
\hline Langley, SC & 40 & $(9.00 \pm 8.53) E-12$ & $(3.53 \pm 0.84) E-11$ & $(-0.04 \pm 6.65) E-12$ \\
\hline Lees, SC & 39 & $(0.98 \pm 1.31) E-11$ & $(5.28 \pm 0.69) E-11$ & $(-6.66 \pm 3.75) E-12$ \\
\hline Olar, SC & 39 & $(0.86 \pm 1.16) E-11$ & $(4.35 \pm 0.65) E-11$ & $(-8.32 \pm 6.28) E-12$ \\
\hline
\end{tabular}




\section{Table 15}

Radioactivity in Air

Page 2 of 6

\begin{tabular}{|c|c|c|c|c|}
\hline Location & $\begin{array}{l}\text { No. of } \\
\text { Samples }\end{array}$ & Arithmetic Mean $\pm \sigma$ & Maximum $\pm \sigma$ & Minimum $\pm \sigma$ \\
\hline Perkins, GA & 42 & $(1.02 \pm 1.37) \mathrm{E}-11$ & $(7.46 \pm 1.66) \mathrm{E}-11$ & $(-0.27 \pm 1.56) E-11$ \\
\hline South Richmond, GA & 43 & $(1.11 \pm 1.62) E-11$ & $(7.60 \pm 1.61) E-11$ & $(-0.79 \pm 1.79) E-11$ \\
\hline Springfield, SC & 34 & $(1.00 \pm 1.21) E-11$ & $(5.04 \pm 1.29) E-11$ & $(-4.65 \pm 7.23) E-12$ \\
\hline Waynesboro, GA & 42 & $(1.44 \pm 1.79) E-11$ & $(8.92 \pm 0.66) E-11$ & $(-3.89 \pm 4.42) E-12$ \\
\hline \multicolumn{5}{|l|}{ 100-Mile-Radius } \\
\hline Columbia, SC & 4 & $(6.95 \pm 8.42) E-12$ & $(1.95 \pm 0.78) E-11$ & $(1.80 \pm 4.28) E-12$ \\
\hline Greenville, SC & 4 & $(-1.84 \pm 9.31) E-12$ & $(7.95 \pm 8.20) E-12$ & $(-1.33 \pm 1.36) E-11$ \\
\hline Macon, GA & 4 & $(3.26 \pm 4.96) \mathrm{E}-12$ & $(1.02 \pm 0.53) E-11$ & $(1.53 \pm 5.61) E-12$ \\
\hline Savannah, GA & 3 & $(1.05 \pm 1.18) E-11$ & $(2.40 \pm 1.59) \mathrm{E}-11$ & $(1.81 \pm 6.24) E-12$ \\
\hline \multicolumn{5}{|l|}{$\mathrm{Mn}-54(\mu \mathrm{Cl} / \mathrm{cc})$} \\
\hline \multicolumn{5}{|l|}{ Site Perimeter } \\
\hline Barnwell Gate & 2 & $(9.54 \pm 2.24) E-15$ & $(1.11 \pm 0.10) E-14$ & $(7.96 \pm 0.79) E-15$ \\
\hline Site Perimeter & 1 & $(9.32 \pm 1.55) E-16$ & $(9.32 \pm 1.55) E-16$ & $(9.32 \pm 1.55) E-16$ \\
\hline \multicolumn{5}{|l|}{ Co-60 ( $\mu \mathrm{Cl} / \mathrm{cc})$} \\
\hline \multicolumn{5}{|l|}{ Site Perimeter } \\
\hline Barnwell Gate & 2 & $(4.09 \pm 0.35) E-14$ & $(4.33 \pm 0.11) E-14$ & $(3.84 \pm 0.10) E-14$ \\
\hline Site Perimeter & 2 & $(2.99 \pm 0.89) E-15$ & $(3.62 \pm 0.25) E-15$ & $(2.36 \pm 0.24) E-15$ \\
\hline \multicolumn{5}{|l|}{ Pu-238 ( $\mu \mathrm{Cl} / \mathrm{cc})$} \\
\hline \multicolumn{5}{|l|}{ On Site } \\
\hline A-Area & 11 & $(0.61 \pm 1.71) E-18$ & $(3.69 \pm 1.95) E-18$ & $(-2.55 \pm 1.90) E-18$ \\
\hline Burial Ground North & 12 & $(1.32 \pm 4.32) \mathrm{E}-17$ & $(1.49 \pm 0.19) E-16$ & $(-1.72 \pm 0.86) E-17$ \\
\hline Burial Ground South & 10 & $(3.41 \pm 3.51) E-18$ & $(9.60 \pm 1.82) E-18$ & $(-1.18 \pm 1.18) E-18$ \\
\hline F-Area & 11 & $(2.21 \pm 1.80) E-17$ & $(5.88 \pm 0.49) E-17$ & $(0.74 \pm 1.64) E-18$ \\
\hline H-Area & 11 & $(3.59 \pm 5.59) \mathrm{E}-17$ & $(1.93 \pm 0.12) E-16$ & $(1.69 \pm 0.64) E-18$ \\
\hline \multicolumn{5}{|l|}{ Site Perimeter } \\
\hline Site Perimeter & 45 & $(5.19 \pm 9.86) E-19$ & $(4.81 \pm 1.29) \mathrm{E}-18$ & $(-1.72 \pm 0.99) E-18$ \\
\hline \multicolumn{5}{|l|}{ 25-Mile-Radius } \\
\hline 25-Mile-Radius & 49 & $(3.86 \pm 8.98) E-19$ & $(3.65 \pm 2.58) E-18$ & $(-1.35 \pm 1.35) E-18$ \\
\hline \multicolumn{5}{|l|}{ 100-Mile-Radius } \\
\hline 100-Mile-Radius & 45 & $(0.70 \pm 1.59) E-18$ & $(5.86 \pm 3.10) E-18$ & $(-2.37 \pm 1.68) E-18$ \\
\hline
\end{tabular}


Table 15

Radioactivity in Air

Page 3 of 6

\begin{tabular}{|c|c|c|c|c|}
\hline Location & $\begin{array}{c}\text { No. of } \\
\text { Samples }\end{array}$ & Arithmetic Mean $\pm \sigma$ & Maximum $\pm \sigma$ & Minimum $\pm \sigma$ \\
\hline \multicolumn{5}{|l|}{ Pu-239 ( $\mu \mathrm{Cl} / \mathrm{cc})$} \\
\hline \multicolumn{5}{|l|}{ On Site } \\
\hline A-Area & 11 & $(1.33 \pm 1.39) E-18$ & $(4.60 \pm 2.66) E-18$ & $(00.00 \pm 2.04) E-18$ \\
\hline Burial Ground North & 12 & $(5.23 \pm 3.77) E-18$ & $(1.31 \pm 0.62) E-17$ & $(8.58 \pm 6.79) E-19$ \\
\hline Burial Ground South & 9 & $(2.02 \pm 1.85) E-18$ & $(3.44 \pm 1.27) E-18$ & $(-1.95 \pm 3.39) E-18$ \\
\hline F-Area & 11 & $(2.27 \pm 3.29) E-17$ & $(1.19 \pm 7.02) E-16$ & $(4.40 \pm 1.80) E-18$ \\
\hline H-Area & 11 & $(1.15 \pm 1.65) E-17$ & $(5.77 \pm 0.63) E-17$ & $(0.85 \pm 2.24) E-18$ \\
\hline \multicolumn{5}{|l|}{ Slte Perimeter } \\
\hline Site Perimeter & 47 & $(2.57 \pm 7.15) E-19$ & $(2.06 \pm 0.97) E-18$ & $(-2.76 \pm 1.60) E-18$ \\
\hline \multicolumn{5}{|l|}{ 25-Mile-Radius } \\
\hline 25-Mile-Radius & 44 & $(3.02 \pm 9.88) E-19$ & $(3.64 \pm 3.64) E-18$ & $(-3.06 \pm 2.28) E-18$ \\
\hline \multicolumn{5}{|l|}{ 100-Mile-Radius } \\
\hline 100-Mile-Radius & 45 & $(-0.04 \pm 2.05) E-18$ & $(4.44 \pm 1.90) E-18$ & $(-1.04 \pm 0.77) E-17$ \\
\hline \multicolumn{5}{|l|}{$\mathrm{Sr}-89,90(\mu \mathrm{Cl} / \mathrm{cc})$} \\
\hline \multicolumn{5}{|l|}{ On Slte } \\
\hline A-Area & 12 & $(0.33 \pm 4.41) E-16$ & $(1.10 \pm 0.54) E-15$ & $(-5.05 \pm 3.12) E-16$ \\
\hline Burial Ground North & 12 & $(1.39 \pm 4.55) E-16$ & $(1.19 \pm 0.53) E-15$ & $(-5.91 \pm 3.22) E-16$ \\
\hline Burial Ground South & 12 & $(2.42 \pm 4.36) E-16$ & $(1.27 \pm 0.54) E-15$ & $(-3.69 \pm 3.34) E-16$ \\
\hline F-Area & 12 & $(0.32 \pm 3.19) E-16$ & $(8.29 \pm 5.12) E-16$ & $(-4.59 \pm 2.31) E-16$ \\
\hline H-Area & 12 & $(0.50 \pm 3.10) E-16$ & $(5.45 \pm 3.27) E-16$ & $(-4.53 \pm 3.26) E-16$ \\
\hline \multicolumn{5}{|l|}{ Site Perimeter } \\
\hline Site Perimeter & 52 & $(-0.06 \pm 8.89) E-17$ & $(2.67 \pm 1.18) E-16$ & $(-1.82 \pm 1.01) E-16$ \\
\hline \multicolumn{5}{|l|}{ 25-Mile-Radius } \\
\hline 25-Mile-Radius & 51 & $(0.69 \pm 9.67) E-17$ & $(3.27 \pm 1.46) E-16$ & $(-1.86 \pm 1.03) E-16$ \\
\hline \multicolumn{5}{|l|}{ 100-Mile-Radius } \\
\hline 100-Mile-Radius & 52 & $(-0.32 \pm 3.00) E-16$ & $(5.79 \pm 4.65) E-16$ & $(-6.92 \pm 2.99) E-16$ \\
\hline \multicolumn{5}{|l|}{ Gross A ( $\mu \mathrm{Cl} / \mathrm{cc})$} \\
\hline \multicolumn{5}{|l|}{ On Site } \\
\hline A-Area & 51 & $(1.89 \pm 0.89) E-15$ & $(4.01 \pm 0.77) E-15$ & $(3.44 \pm 2.65) E-16$ \\
\hline Burial Ground North & 45 & $(2.12 \pm 0.87) E-15$ & $(4.06 \pm 0.77) E-15$ & $(4.96 \pm 2.06) E-16$ \\
\hline Burial Ground South & 51 & $(1.74 \pm 0.72) E-15$ & $(3.56 \pm 0.75) E-15$ & $(3.90 \pm 1.89) E-16$ \\
\hline F-Area & 51 & $(1.89 \pm 0.71) E-15$ & $(3.63 \pm 0.45) E-15$ & $(4.49 \pm 2.87) E-16$ \\
\hline
\end{tabular}


Tâble 15

Radioactivity in Air

Page 4 of 6

\begin{tabular}{|c|c|c|c|c|}
\hline Location & $\begin{array}{c}\text { No. of } \\
\text { Samples }\end{array}$ & Arithmetic Mean $\pm \sigma$ & Maximum $\pm \sigma$ & Minimum $\pm \sigma$ \\
\hline H-Area & 50 & $(1.96 \pm 0.84) E-15$ & $(3.75 \pm 0.45) E-15$ & $(3.69 \pm 1.90) E-16$ \\
\hline $\begin{array}{l}\text { PP-2 Par Pond } \\
\text { Pumphouse }^{\mathrm{a}}\end{array}$ & 3 & $(1.59 \pm 0.84) E-15$ & $(2.52 \pm 0.39) E-15$ & $(9.05 \pm 2.55) E-16$ \\
\hline Par Pond Bubble-Upa & 3 & $(9.74 \pm 8.16) E-16$ & $(1.88 \pm 0.34) E-15$ & $(3.08 \pm 1.79) E-16$ \\
\hline \multicolumn{5}{|l|}{ Site Perimeter } \\
\hline Allendale Gate & 50 & $(1.74 \pm 0.73) E-15$ & $(3.61 \pm 0.73) E-15$ & $(4.56 \pm 1.99) E-16$ \\
\hline Barnwell Gate & 51 & $(1.78 \pm 0.82) E-15$ & $(3.99 \pm 0.74) E-15$ & $(4.78 \pm 3.68) E-16$ \\
\hline D-Area & 51 & $(1.67 \pm 0.70) E-15$ & $(3.97 \pm 0.47) E-15$ & $(4.19 \pm 2.85) E-16$ \\
\hline Darkhorse @ Williston Gate & 50 & $(2.09 \pm 0.94) E-15$ & $(4.35 \pm 0.78) E-15$ & $(4.56 \pm 3.10) E-16$ \\
\hline East Talatha & 51 & $(2.00 \pm 0.85) E-15$ & $(4.35 \pm 0.79) E-15$ & $(5.35 \pm 2.96) E-16$ \\
\hline Green Pond & 51 & $(1.75 \pm 0.85) E-15$ & $(4.74 \pm 0.81) E-15$ & $(3.32 \pm 2.55) E-16$ \\
\hline Highway $125 @$ Road A-14 & 49 & $(1.71 \pm 0.90) E-15$ & $(3.65 \pm 0.67) E-15$ & $(1.31 \pm 1.47) E-16$ \\
\hline Highway $21 / 167$ & 51 & $(2.05 \pm 0.88) E-15$ & $(4.33 \pm 0.79) E-15$ & $(5.64 \pm 2.15) E-16$ \\
\hline $\begin{array}{l}\text { Highway } 39 @ \text { Williston } \\
\text { Gate }\end{array}$ & 52 & $(2.00 \pm 0.93) E-15$ & $(4.72 \pm 0.83) E-15$ & $(5.44 \pm 3.01) E-16$ \\
\hline Jackson & 51 & $(1.48 \pm 0.76) E-15$ & $(3.28 \pm 0.71) E-15$ & $(3.55 \pm 2.74) E-16$ \\
\hline Patterson Mill Road & 51 & $(1.95 \pm 0.97) E-15$ & $(5.02 \pm 0.83) E-15$ & $(4.35 \pm 2.79) E-16$ \\
\hline Talatha Gate & 50 & $(1.60 \pm 0.76) E-15$ & $(3.99 \pm 0.77) E-15$ & $(3.24 \pm 2.34) E-16$ \\
\hline West Jackson & 49 & $(1.58 \pm 0.71) E-15$ & $(3.26 \pm 0.62) E-15$ & $(0.98 \pm 1.99) E-16$ \\
\hline Windsor Road & 51 & $(2.05 \pm 0.81) E-15$ & $(4.54 \pm 0.51) E-15$ & $(4.33 \pm 2.77) E-16$ \\
\hline \multicolumn{5}{|l|}{ 25-Mile-Radius } \\
\hline Aiken Airport & 47 & $(2.00 \pm 0.81) E-15$ & $(4.01 \pm 0.75) E-15$ & $(3.99 \pm 2.71) E-16$ \\
\hline Aiken State Park & 51 & $(1.71 \pm 0.74) E-15$ & $(3.51 \pm 0.65) E-15$ & $(3.30 \pm 2.54) E-16$ \\
\hline Allendale, SC & 45 & $(1.55 \pm 0.87) E-15$ & $(4.38 \pm 0.79) E-15$ & $(4.26 \pm 2.84) E-16$ \\
\hline Augusta, GA & 49 & $(1.96 \pm 0.83) E-15$ & $(4.52 \pm 0.82) E-15$ & $(3.09 \pm 2.56) E-16$ \\
\hline Highway 301 @ State Line & 34 & $(1.84 \pm 0.93) E-15$ & $(4.48 \pm 0.81) E-15$ & $(6.57 \pm 3.24) E-16$ \\
\hline Langley, SC & 48 & $(1.79 \pm 0.96) \mathrm{E}-15$ & $(4.36 \pm 0.87) E-15$ & $(3.97 \pm 2.82) E-16$ \\
\hline Lees, SC & 51 & $(1.64 \pm 0.82) E-15$ & $(4.04 \pm 0.75) E-15$ & $(3.78 \pm 2.95) E-16$ \\
\hline Olar, SC & 49 & $(1.73 \pm 1.01) \mathrm{E}-15$ & $(5.11 \pm 0.84) \mathrm{E}-15$ & $(2.99 \pm 2.50) E-16$ \\
\hline Perkins, GA & 51 & $(1.85 \pm 0.89) E-15$ & $(4.09 \pm 0.78) E-15$ & $(1.19 \pm 1.46) E-16$ \\
\hline South Richmond, GA & 49 & $(1.92 \pm 0.98) E-15$ & $(3.99 \pm 0.74) E-15$ & $(4.41 \pm 2.82) E-16$ \\
\hline
\end{tabular}

a Temporary sampling location installed and operated to support the 1991 drawdown of Par Pond; removed from service in February 1993 


\section{Table 15}

\section{Radioactivity in Air}

Page 5 of 6

\begin{tabular}{|c|c|c|c|c|}
\hline Location & $\begin{array}{l}\text { No. of } \\
\text { Samples }\end{array}$ & Arithmetic Mean $\pm \sigma$ & Maximum $\pm \sigma$ & Minimum $\pm \sigma$ \\
\hline Springfield, SC & 50 & $(1.68 \pm 0.76) E-15$ & $(3.99 \pm 0.74) E-15$ & $(5.11 \pm 2.94) E-16$ \\
\hline Waynesboro, GA & 47 & $(1.82 \pm 0.77) E-15$ & $(3.44 \pm 0.45) E-15$ & $(3.30 \pm 2.57) E-16$ \\
\hline \multicolumn{5}{|l|}{ 100-Mile-Radius } \\
\hline Columbia, SC & 51 & $(2.26 \pm 1.06) E-15$ & $(4.92 \pm 0.86) E-15$ & $(7.45 \pm 3.42) E-16$ \\
\hline Greenville, SC & 41 & $(1.99 \pm 0.90) E-15$ & $(3.86 \pm 0.63) E-15$ & $(5.46 \pm 3.14) E-16$ \\
\hline Macon, GA & 42 & $(1.93 \pm 0.98) E-15$ & $(4.15 \pm 0.51) E-15$ & $(4.41 \pm 1.98) E-16$ \\
\hline Savannah, GA & 51 & $(1.75 \pm 0.82) E-15$ & $(3.69 \pm 0.73) E-15$ & $(0.95 \pm 1.92) E-16$ \\
\hline \multicolumn{5}{|l|}{ Gross B ( $\mu \mathrm{Cl} / \mathrm{cc})$} \\
\hline \multicolumn{5}{|l|}{ On Site } \\
\hline A-Area & 51 & $(1.74 \pm 0.57) E-14$ & $(3.67 \pm 0.11) E-14$ & $(5.84 \pm 0.55) E-15$ \\
\hline Burial Ground North & 45 & $(1.80 \pm 0.59) E-14$ & $(3.10 \pm 0.15) E-14$ & $(5.11 \pm 0.55) E-15$ \\
\hline Burial Ground South & 51 & $(1.58 \pm 0.45) E-14$ & $(3.61 \pm 0.11) E-14$ & $(6.34 \pm 0.58) E-15$ \\
\hline F-Area & 51 & $(1.92 \pm 0.57) E-14$ & $(4.11 \pm 0.12) E-14$ & $(5.84 \pm 0.57) E-15$ \\
\hline H-Area & 50 & $(1.75 \pm 0.52) E-14$ & $(3.63 \pm 0.11) E-14$ & $(4.63 \pm 0.54) E-15$ \\
\hline $\begin{array}{l}\text { PP-2 Par Pond } \\
\text { Pumphouse }^{\mathrm{a}}\end{array}$ & 3 & $(1.73 \pm 1.09) E-14$ & $(2.99 \pm 0.11) E-14$ & $(1.08 \pm 0.09) E-14$ \\
\hline Par Pond Bubble-Upa & 3 & $(1.62 \pm 1.10) E-14$ & $(2.88 \pm 0.11) E-14$ & $(8.89 \pm 0.88) E-15$ \\
\hline \multicolumn{5}{|l|}{ Site Perimeter } \\
\hline Allendale Gate & 50 & $(1.83 \pm 0.53) E-14$ & $(3.51 \pm 0.11) E-14$ & $(4.50 \pm 0.53) E-15$ \\
\hline Barnwell Gate & 51 & $(1.98 \pm 1.01) E-14$ & $(6.30 \pm 0.15) E-14$ & $(6.92 \pm 0.60) E-15$ \\
\hline D-Area & 51 & $(1.70 \pm 0.46) E-14$ & $(3.43 \pm 0.11) \mathrm{E}-14$ & $(5.59 \pm 0.56) E-15$ \\
\hline Darkhorse @ Williston Gate & 50 & $(2.08 \pm 0.62) E-14$ & $(4.11 \pm 0.12) E-14$ & $(5.99 \pm 0.58) E-15$ \\
\hline East Talatha & 51 & $(2.04 \pm 0.70) E-14$ & $(4.42 \pm 0.12) E-14$ & $(2.94 \pm 0.70) E-15$ \\
\hline Green Pond & 51 & $(1.78 \pm 0.51) E-14$ & $(3.63 \pm 0.11) E-14$ & $(6.75 \pm 0.60) E-15$ \\
\hline Highway $125 @$ Road A-14 & 49 & $(1.74 \pm 0.49) E-14$ & $(3.29 \pm 0.11) E-14$ & $(5.26 \pm 0.56) E-15$ \\
\hline Highway 21/167 & 51 & $(2.08 \pm 0.60) E-14$ & $(4.09 \pm 0.12) E-14$ & $(5.86 \pm 0.57) E-15$ \\
\hline $\begin{array}{l}\text { Highway39@ Williston } \\
\text { Gate }\end{array}$ & 52 & $(1.99 \pm 0.60) E-14$ & $(3.81 \pm 0.12) E-14$ & $(6.75 \pm 0.60) E-15$ \\
\hline Jackson & 51 & $(1.56 \pm 0.50) E-14$ & $(3.36 \pm 0.11) E-14$ & $(5.62 \pm 0.57) E-15$ \\
\hline Patterson Mill Road & 51 & $(1.93 \pm 0.53) E-14$ & $(2.99 \pm 0.15) E-14$ & $(5.29 \pm 0.56) \mathrm{E}-15$ \\
\hline Talatha Gate & 50 & $(1.67 \pm 0.51) E-14$ & $(3.40 \pm 0.11) E-14$ & $(3.87 \pm 0.68) E-15$ \\
\hline
\end{tabular}

a Temporary sampling location installed and operated to support the 1991 drawdown of Par Pond; removed from service in February 1993 
Table 15

Radloactivity in Air

Page 6 of 6

\begin{tabular}{|c|c|c|c|c|}
\hline Location & $\begin{array}{l}\text { No. of } \\
\text { Samples }\end{array}$ & Arithmetic Mean $\pm \sigma$ & Maximum $\pm \sigma$ & Minimum $\pm \sigma$ \\
\hline West Jackson & 49 & $(1.69 \pm 0.48) E-14$ & $(3.83 \pm 0.11) E-14$ & $(5.74 \pm 0.57) E-15$ \\
\hline Windsor Road & 51 & $(2.03 \pm 0.60) E-14$ & $(4.55 \pm 0.12) E-14$ & $(6.79 \pm 0.60) E-15$ \\
\hline \multicolumn{5}{|l|}{ 25-Mile-Radius } \\
\hline Aiken Airport & 47 & $(1.83 \pm 0.51) E-14$ & $(3.63 \pm 0.12) E-14$ & $(5.80 \pm 0.81) E-15$ \\
\hline Aiken State Park & 51 & $(1.74 \pm 0.46) E-14$ & $(3.52 \pm 0.11) E-14$ & $(6.63 \pm 0.60) E-15$ \\
\hline Allendale, SC & 45 & $(1.62 \pm 0.54) E-14$ & $(3.31 \pm 0.11) E-14$ & $(5.81 \pm 0.81) E-15$ \\
\hline Augusta, GA & 49 & $(1.76 \pm 0.56) E-14$ & $(3.40 \pm 0.19) E-14$ & $(5.79 \pm 0.57) E-15$ \\
\hline Highway 301 @ State Line & 34 & $(1.94 \pm 0.50) E-14$ & $(3.05 \pm 0.19) E-14$ & $(1.16 \pm 0.10) E-14$ \\
\hline Langley, SC & 48 & $(1.91 \pm 0.65) E-14$ & $(3.41 \pm 0.18) E-14$ & $(6.44 \pm 0.59) E-15$ \\
\hline Lees, SC & 51 & $(1.75 \pm 0.57) E-14$ & $(2.93 \pm 0.10) E-14$ & $(5.75 \pm 0.58) E-15$ \\
\hline Olar, SC & 49 & $(1.85 \pm 0.61) E-14$ & $(3.35 \pm 0.11) E-14$ & $(7.82 \pm 0.63) E-15$ \\
\hline Perkins, GA & 51 & $(1.86 \pm 0.54) E-14$ & $(2.98 \pm 0.14) E-14$ & $(5.59 \pm 0.56) E-15$ \\
\hline South Richmond, GA & 49 & $(1.87 \pm 0.58) E-14$ & $(3.17 \pm 0.11) E-14$ & $(7.25 \pm 0.61) E-15$ \\
\hline Springfield, SC & 50 & $(1.81 \pm 0.51) E-14$ & $(3.46 \pm 0.11) E-14$ & $(2.49 \pm 0.47) E-15$ \\
\hline Waynesboro, GA & 47 & $(1.85 \pm 0.47) E-14$ & $(3.41 \pm 0.11) E-14$ & $(4.32 \pm 0.53) E-15$ \\
\hline \multicolumn{5}{|l|}{ 100-Mile-Radlus } \\
\hline Columbia, SC & 51 & $(1.99 \pm 0.50) E-14$ & $(3.47 \pm 0.12) E-14$ & $(1.10 \pm 0.07) E-14$ \\
\hline Greenville, SC & 41 & $(2.21 \pm 0.74) E-14$ & $(4.29 \pm 0.29) E-14$ & $(1.01 \pm 0.10) E-14$ \\
\hline Macon, GA & 42 & $(1.92 \pm 0.62) E-14$ & $(3.96 \pm 0.12) E-14$ & $(9.00 \pm 0.95) E-15$ \\
\hline Savannah, GA & 51 & $(1.81 \pm 0.53) E-14$ & $(3.28 \pm 0.10) E-14$ & $(6.17 \pm 0.53) E-15$ \\
\hline
\end{tabular}


Table 16

TLD Results: SRS Areas

Page 1 of 4

\begin{tabular}{|c|c|c|c|c|c|}
\hline Location & Quarter $\mathrm{I}^{\mathrm{b}}$ & Quarter $2^{b}$ & Quarter $3^{b}$ & Quarter $4^{b}$ & mR/Yearb \\
\hline 643-7G BUILDING TLD 1 & 0.24 & 0.24 & 0.23 & 0.26 & 88.4 \\
\hline 643-7G BUILDING TLD 2 & 0.25 & 0.24 & 0.25 & 0.26 & 90.5 \\
\hline 643-7G BUILDING TLD 3 & 0.36 & 0.32 & 0.33 & 0.35 & 124.5 \\
\hline 643-7G BUILDING TLD 4 & 0.78 & 0.73 & 0.75 & 0.71 & 270.7 \\
\hline 643-G BUILDING TLD 1 & 0.22 & 0.21 & 0.22 & 0.23 & 80.1 \\
\hline 643-G BUILDING TLD 2 & 0.27 & 0.26 & 0.27 & 0.29 & 98.8 \\
\hline 643-G BUILDING TLD 3 & 0.94 & 0.95 & 0.94 & 0.95 & 344.7 \\
\hline $643-G$ BUILDING TLD 4 & 0.29 & 0.29 & 0.29 & 0.31 & 106.7 \\
\hline B-AREA TLD 1 & 0.65 & 0.69 & 0.65 & 0.73 & 248.6 \\
\hline B-AREA TLD 2 & 0.20 & 0.18 & 0.20 & 0.22 & 72.4 \\
\hline B-AREA TLD 3 & 0.20 & 0.18 & 0.20 & 0.20 & 71.5 \\
\hline B-AREA TLD 4 & 0.20 & 0.18 & 0.20 & 0.20 & 71.1 \\
\hline C AREA TLD 1 & 0.17 & 0.18 & 0.20 & 0.18 & 67.3 \\
\hline C AREA TLD 2 & 0.17 & 0.18 & 0.20 & 0.19 & 67.5 \\
\hline C AREA TLD 3 & 0.18 & 0.19 & 0.20 & 0.20 & 70.5 \\
\hline C AREA TLD 4 & 0.17 & 0.18 & 0.20 & 0.20 & 68.6 \\
\hline CLASSIFICATION YARD TLD 1 & 0.17 & 0.18 & 0.20 & 0.19 & 67.1 \\
\hline CLASSIFICATION YARD TLD 2 & 0.17 & 0.19 & 0.21 & 0.19 & 69.6 \\
\hline CLASSIFICATION YARD TLD 3 & 0.20 & 0.21 & 0.23 & 0.22 & 78.2 \\
\hline CLASSIFICATION YARD TLD 4 & 0.16 & 0.16 & 0.17 & 0.17 & 60.1 \\
\hline D AREA TLD 1 & 0.17 & 0.18 & 0.20 & 0.20 & 68.1 \\
\hline D AREA TLD 2 & 0.15 & 0.16 & 0.17 & 0.16 & 58.9 \\
\hline D AREA TLD 3 & 0.20 & 0.19 & 0.22 & 0.21 & 74.9 \\
\hline D AREA TLD 4 & 0.20 & 0.20 & 0.23 & 0.21 & 76.8 \\
\hline D AREA TLD 5 & 0.16 & 0.16 & 0.19 & 0.18 & 62.8 \\
\hline D AREA TLD 6 & 0.16 & 0.15 & 0.15 & 0.16 & 55.9 \\
\hline F AREA TLD 1 & 0.22 & 0.19 & 0.20 & 0.21 & 74.7 \\
\hline F AREA TLD 2 & 0.23 & 0.20 & 0.22 & 0.24 & 80.4 \\
\hline
\end{tabular}

a $m R=$ milliRoentgen, exposure unit for gamma radiation. One $m R$ is approximately equal to $1 \mathrm{mrem}$.

b An 18\% uncertainty is associated with each result. 
Table 16

TLD Results: SRS Areas

Pago 2 of 4

Quarterly Exposure in mR/Day

Yearly Exposure in mR/Yeara

Location

Quarter

Quarter $\mathbf{2}^{\mathbf{b}}$

Quarter $3^{b}$

Quarter $4^{b}$

mR/Yoarb

F AREA TLD 3

0.24

0.22

0.23

0.24

85.0

F AREA TLD 4

0.24

0.22

0.23

0.25

86.1

F AREA TLD 5

0.36

0.33

0.35

0.34

125.7

F AREA TLD 6

0.27

0.24

0.26

0.28

95.7

H AREA TLD 1

0.24

0.21

0.23

0.23

84.2

H AREA TLD 2

0.36

H AREA TLD 3

0.23

c

0.24

0.26

103.9

H AREA TLD 4

0.39

0.30

0.22

0.23

89.1

H AREA TLD 5

0.34

0.39

0.40

0.41

145.9

H AREA TLD 6

0.25

0.32

0.36

0.42

132.7

H AREA TLD 7

0.25

0.23

0.25

0.26

89.9

H AREA TLD 8

0.25

0.24

0.23

0.25

88.2

K AREA TLD 1

0.21

0.22

0.25

0.25

88.3

K AREA TLD 2

0.17

0.2

0.24

0.24

82.5

K AREA TLD 3

0.24

0.17

0.19

0.18

64.6

K AREA TLD 4

0.20

0.25

0.27

0.26

93.0

LAREA TLD 1

0.18

0.21

0.22

0.21

76.0

LAREA TLD 2

0.18

0.19

0.20

0.20

70.9

L AREA TLD 3

0.18

0.18

0.21

0.20

69.6

L AREA TLD 4

0.20

0.19

0.20

0.21

70.1

M AREA TLD 1

0.26

0.20

0.22

0.22

76.8

M AREA TLD 2

0.19

0.25

0.26

0.27

95.1

M AREA TLD 3

0.29

0.18

0.19

0.19

69.2

$M$ AREA TLD 4

0.21

0.26

0.29

0.29

102.6

$M$ AREA TLD 5

0.22

0.20

0.20

0.21

74.4

M AREA TLD 6

0.20

0.21

0.22

0.23

80.0

$M$ AREA TLD 7

0.26

0.18

0.19

0.20

70.9

$M$ AREA TLD 8

0.28

0.23

0.25

0.25

90.4

0.27

0.28

0.31

103.5

a $\mathrm{mR}=$ milliRoentgen, exposure unit for gamma radiation. One $\mathrm{mR}$ is approximately equal to $1 \mathrm{mrem}$.

b An $18 \%$ uncertainty is associated with each result.

c Blank spaces indicate missing TLD results. 


\section{Table 16}

TLD Results: SRS Areas

Page 3 of 4

Quarterly Exposure in mR/Day Yearly Exposure in mR/Year"

\begin{tabular}{|c|c|c|c|c|c|}
\hline Location & Quarter $1^{b}$ & Quarter $2^{b}$ & Quartor $3^{b}$ & Quarter $4^{b}$ & mP/Yearb \\
\hline N-AREA TLD 1 & 0.24 & 0.25 & 0.28 & 0.27 & 95.4 \\
\hline N-AREA TLD 2 & 0.23 & 0.24 & 0.27 & 0.26 & 91.5 \\
\hline N-AREA TLD 3 & 0.31 & 0.32 & 0.35 & 0.34 & 120.8 \\
\hline N-AREA TLD 4 & 0.31 & 0.33 & 0.35 & 0.35 & 122.2 \\
\hline N-AREA TLD 5 & 1.52 & 1.49 & 1.14 & 0.89 & 460.4 \\
\hline P AREA TLD 1 & 0.19 & 0.21 & 0.22 & 0.22 & 77.0 \\
\hline P AREA TLD 2 & 0.17 & 0.18 & 0.20 & 0.19 & 67.7 \\
\hline P AREA TLD 3 & 0.19 & 0.18 & 0.21 & 0.20 & 70.4 \\
\hline P AREA TLD 4 & 0.19 & 0.19 & 0.22 & 0.21 & 74.1 \\
\hline R AREA TLD 1 & 0.18 & 0.18 & 0.20 & 0.19 & 68.5 \\
\hline R AREA TLD 2 & 0.19 & 0.19 & 0.22 & 0.21 & 74.3 \\
\hline R AREA TLD 3 & 0.16 & c & 0.18 & 0.18 & 62.9 \\
\hline R AREA TLD 4 & 0.19 & 0.19 & 0.22 & 0.22 & 74.8 \\
\hline R AREA TLD 5 & 0.18 & 0.19 & 0.22 & 0.21 & 73.3 \\
\hline R AREA TLD 6 & 0.18 & 0.17 & 0.20 & 0.19 & 67.2 \\
\hline R AREA TLD 7 & 0.18 & 0.19 & 0.21 & 0.20 & 71.6 \\
\hline RAILROAD AT ROADS E \& F 1 & 0.23 & 0.22 & 0.23 & 0.24 & 83.6 \\
\hline RAILROAD AT ROADS E \& F 2 & 0.24 & 0.22 & 0.24 & 0.24 & 85.8 \\
\hline S AREA TLD 1 & 0.23 & 0.21 & 0.23 & 0.25 & 84.1 \\
\hline S AREA TLD 2 & 0.32 & 0.31 & 0.32 & 0.34 & 116.8 \\
\hline S AREA TLD 3 & 0.30 & 0.28 & 0.28 & 0.31 & 106.7 \\
\hline S AREA TLD 4 & 0.28 & 0.25 & 0.25 & 0.27 & 95.4 \\
\hline TECHNICAL AREA TLD 1 & 0.23 & 0.20 & 0.22 & & 78.6 \\
\hline TECHNICAL AREA TLD 2 & 0.30 & 0.27 & 0.29 & 0.29 & 104.7 \\
\hline TECHNICAL AREA ILLD 3 & 0.23 & 0.23 & 0.23 & 0.24 & 84.5 \\
\hline TECHNICAL AREA TLD 4 & 0.25 & 0.29 & 0.31 & 0.25 & 99.8 \\
\hline TECHNICAL AREA TLD 5 & 0.23 & 0.22 & 0.22 & 0.23 & 82.1 \\
\hline TECHNICAL AREA TLD 6 & 0.23 & 0.22 & 0.23 & 0.24 & 83.9 \\
\hline
\end{tabular}

$m R=$ milliRoentgen, exposure unit for gamma radiation. One $m R$ is approximately equal to $1 \mathrm{mrem}$. An $18 \%$ uncertainty is associated with each result.

Blank spaces indicate missing TLD results. 


\section{Table 16}

\section{TLD Rosults: SRS Areas}

Page 4 of 4

\begin{tabular}{|c|c|c|c|c|c|}
\hline Location & Quartor $1^{b}$ & Quartor $2^{b}$ & Quartor $3^{b}$ & Quartor $4^{b}$ & mR/Yoart \\
\hline TECHNICAL AREA TLD 7 & 0.26 & 0.24 & 0.24 & 0.23 & 88.4 \\
\hline TECHNICAL AREA TLD 8 & 0.25 & 0.22 & 0.21 & 0.25 & 84.6 \\
\hline T AREA TLD 1 & 0.16 & 0.16 & 0.18 & 0.17 & 61.8 \\
\hline T AREA TLD 2 & 0.21 & 0.22 & 0.26 & 0.23 & 83.5 \\
\hline T AREA TLD 3 & 0.20 & 0.21 & 0.21 & 0.19 & 74.3 \\
\hline T AREA TLD 4 & 0.26 & 0.26 & 0.29 & 0.28 & 100.5 \\
\hline$Z$ AREA TLD 1 & 0.20 & 0.18 & 0.20 & 0.19 & 70.3 \\
\hline Z AREA TLD 2 & 0.19 & 0.18 & 0.19 & 0.20 & 69.6 \\
\hline Z AREA TLD 3 & 0.19 & 0.18 & 0.18 & 0.21 & 69.3 \\
\hline Z AREA TLD 4 & 0.23 & 0.21 & 0.22 & 0.22 & 80.1 \\
\hline
\end{tabular}

a $\mathrm{mR}=$ milliRoentgen, exposure unit for gamma radiation. One $\mathrm{mR}$ is approximately equal to $1 \mathrm{mrem}$.

b An $18 \%$ uncertainty is associated with each result. 
Table 17

TLD Results: Site Perimeter Stations

।

Page 1 of 7

Quartorly Exposure in mR/Day

Yearly Exposure in mA/Yeara

\begin{tabular}{|c|c|c|c|c|c|}
\hline Location & Quartor $1^{b}$ & Quartor $\mathbf{2 b}^{b}$ & Quarter $3^{b}$ & Quarter $4^{b}$ & mR/Yearb \\
\hline SITE PERIMETER TLD 1 & 0.16 & 0.17 & 0.16 & 0.18 & 61.1 \\
\hline SITE PERIMETER TLD 1.25 & 0.17 & 0.18 & 0.17 & 0.18 & 64.0 \\
\hline SITE PERIMETER TLD 1.5 & 0.18 & 0.10 & 0.17 & 0.20 & 66.7 \\
\hline SITE PERIMETER TLD 1.75 & 0.16 & 0.17 & 0.15 & 0.17 & 60.3 \\
\hline SITE PERIMETER TLD 2 & 0.23 & 0.25 & 0.24 & 0.23 & 86.8 \\
\hline SITE PERIMETER TLD 2.25 & 0.19 & 0.19 & 0.19 & 0.21 & 71.0 \\
\hline SITE PERIMETER TLD 2.5 & 0.17 & 0.19 & 0.17 & $c$ & 63.8 \\
\hline SITE PERIMETER TLD 2.75 & 0.16 & 0.18 & 0.16 & 0.18 & 61.5 \\
\hline SITE PERIMETER TLD 3 & 0.16 & 0.17 & 0.17 & 0.18 & 62.7 \\
\hline SITE PERIMETER TLD 3.25 & & 0.18 & 0.15 & & 61.7 \\
\hline SITE PERIMETER TLD 3.5 & 0.17 & 0.17 & 0.16 & 0.18 & 62.6 \\
\hline SITE PERIMETER TLD 3.75 & 0.20 & 0.21 & 0.20 & 0.23 & 77.6 \\
\hline SITE PERIMETER TLD 4 & 0.20 & 0.21 & 0.20 & 0.22 & 75.0 \\
\hline SITE PERIMETER TLD 4.25 & 0.17 & 0.17 & 0.16 & 0.18 & 63.6 \\
\hline SITE PERIMETER TLD 4.5 & 0.17 & 0.20 & 0.17 & 0.19 & 66.9 \\
\hline SITE PERIMETER TLD 4.75 & 0.16 & 0.17 & 0.16 & 0.18 & 62.5 \\
\hline SITE PERIMETER TLD 5 & 0.20 & 0.21 & 0.19 & 0.23 & 75.9 \\
\hline SITE PERIMETER TLD 5.25 & 0.19 & 0.20 & 0.18 & 0.21 & 70.9 \\
\hline SITE PERIMETER TLD 5.5 & 0.20 & 0.22 & 0.21 & 0.21 & 76.3 \\
\hline SITE PERIMETER TLD 5.75 & 0.23 & 0.26 & 0.24 & 0.27 & 91.6 \\
\hline SITE PERIMETER TLD 6 & 0.25 & 0.26 & 0.24 & 0.27 & 92.7 \\
\hline SITE PERIMETER TLO 6.25 & 0.25 & 0.26 & 0.25 & 0.18 & 85.9 \\
\hline SITE PERIMETER TLD 6.5 & 0.20 & 0.21 & 0.20 & 0.23 & 76.6 \\
\hline SITE PERIMETER TLD 6.75 & 0.16 & 0.18 & 0.16 & 0.18 & 62.7 \\
\hline SITE PERIMETER TLD 7 & 0.21 & 0.22 & 0.19 & 0.22 & 77.2 \\
\hline SITE PERIMETER TLD 7.25 & 0.22 & 0.23 & 0.22 & & 81.7 \\
\hline SITE PERIMETER TLD 7.5 & 0.19 & 0.19 & 0.18 & 0.21 & 70.0 \\
\hline SITE PERIMETER TLD 7.75 & 0.19 & 0.31 & 0.19 & 0.21 & 82.6 \\
\hline
\end{tabular}

a $m R=$ milliRoentgen. One $m R$ is approximately equal to $1 \mathrm{mrem}$.

b An $18 \%$ uncertainty is associated with each result.

c Blank spaces indicate missing TLD results. 
Table 17

TLD Results: Site Perimeter Stations

Page 2 of 7

Quartorly Expooure in mR/Day

Yearly Exposure in mA/Ycar"

\begin{tabular}{|c|c|c|c|c|c|}
\hline Looation & Quarter 16 & Quarter $2^{b}$ & Quartor $3 b$ & Quarter $4^{b}$ & mAVYoarb \\
\hline SITE PERIMETER TLD 8 & 0.18 & 0.19 & 0.18 & 0.20 & 68.1 \\
\hline SITE PERIMETER TLD 8.25 & 0.20 & 0.22 & 0.20 & 0.23 & 77.7 \\
\hline SITE PERIMETER TLD 8.5 & 0.20 & 0.22 & 0.19 & 0.22 & 76.6 \\
\hline SITE PERIMETER TLD 8.75 & 0.19 & 0.20 & 0.18 & $\cdot$ & 68.4 \\
\hline SITE PERIMETER TLD 9 & 0.20 & 0.21 & 0.19 & 0.21 & 73.4 \\
\hline SITE PERIMETER TLD 9.25 & 0.17 & 0.18 & 0.16 & 0.19 & 63.1 \\
\hline SITE PERIMETER TLD 9.5 & 0.17 & 0.17 & 0.16 & 0.18 & 62.7 \\
\hline SITE PERIMETER TLD 9.75 & 0.17 & 0.17 & 0.15 & 0.18 & 61.5 \\
\hline SITE PERIMETER TLD 10 & 0.15 & 0.17 & 0.15 & 0.18 & 60.7 \\
\hline SITE PERIMETER TLD 11 & 0.17 & 0.17 & 0.16 & 0.19 & 62.6 \\
\hline SITE PERIMETER TLD 12 & 0.17 & 0.18 & 0.16 & 0.19 & 63.6 \\
\hline SITE PERIMETER TLD 13 & 0.16 & & 0.15 & 0.18 & 59.5 \\
\hline SITE PERIMETER TLD 14 & 0.17 & & 0.17 & 0.19 & 65.6 \\
\hline SITE PERIMETER TLD 15 & 0.17 & & 0.17 & 0.20 & 66.5 \\
\hline SITE PERIMETER TLD 16 & & & 0.20 & 0.24 & 81.1 \\
\hline SITE PERIMETER TLD 17 & 0.21 & & 0.21 & 0.24 & 80.6 \\
\hline SITE PERIMETER TLD 18 & 0.18 & & 0.19 & 0.21 & 70.9 \\
\hline SITE PERIMETER TLD 19 & 0.18 & & 0.19 & 0.21 & 69.9 \\
\hline SITE PERIMETER TLD 20 & 0.27 & 0.26 & 0.31 & 0.27 & 101.9 \\
\hline SITE PERIMETER TLD 21 & 0.20 & 0.18 & 0.22 & 0.18 & 71.2 \\
\hline SITE PERIMETER TLD 22 & 0.20 & 0.20 & 0.22 & 0.19 & 73.7 \\
\hline SITE PERIMETER TLD 23 & 0.22 & 0.21 & 0.25 & 0.20 & 79.7 \\
\hline SITE PERIMETER TLD 24 & 0.19 & 0.19 & 0.22 & 0.18 & 71.9 \\
\hline SITE PERIMETER TLD 25 & 0.18 & 0.19 & 0.22 & 0.18 & 69.7 \\
\hline SITE PERIMETER TLD 26 & 0.19 & 0.18 & 0.21 & 0.17 & 68.8 \\
\hline SITE PERIMETER TLD 27 & 0.21 & 0.20 & 0.22 & 0.19 & 75.1 \\
\hline SITE PERIMETEA TLD 28 & 0.21 & 0.20 & 0.23 & 0.19 & 76.2 \\
\hline SITE PERIMETER TLD 29 & 0.18 & 0.18 & 0.21 & 0.16 & 66.6 \\
\hline
\end{tabular}


Table 17

TLD Results: Site Perimeter Stations

Page 3 of 7

Quartorly Exposure In mR/Day

Yearly Exposurs in mR/Year"

Location

Quarter 1

Quarter $2^{b}$

Quartor $3^{b}$

Quarter $4^{b} \quad$ mA/Yeart

SITE PERIMETER TLD 30

0.10

0.21

0.24

0.20

77.5

SITE PERIMETER TLD 31

0.19

0.19

0.21

0.18

70.0

SITE PERIMETER TLD 32

0.20

0.19

0.23

0.19

73.5

SITE PERIMETER TLD 33

0.21

0.20

0.24

0.19

76.2

SITE PERIMETER TLD 34

0.20

0.19

0.23

0.19

74.2

SITE PERIMETER TLD 35

0.19

0.19

0.21

0.17

70.8

SITE PERIMETER TLD 36

0.22

0.21

0.25

0.20

79.8

SITE PERIMETER TLD 37

0.21

0.21

0.24

0.19

77.4

SITE PERIMETER TLD 38

0.23

0.22

0.28

0.23

87.9

SITE PERIMETER TLD 39

0.18

0.22

0.18

72.0

SITE PERIMETER TLD 40

0.17

0.21

0.16

64.7

SITE PERIMETER TLD 41

0.16

0.20

0.18

65.9

SITE PERIMETER TLD 42

0.19

SITE PERIMETER TLD 43

0.20

0.25

0.23

68.6

SITE PERIMETER TLD 44

0.16

0.21

0.18

82.7

SITE PERIMETER TLD 45

0.17

0.20

0.18

67.4

SITE PERIMETER TLD 46

0.18

0.22

0.19

66.8

SITE PERIMETER TLD 47

0.18

0.21

0.18

71.5

SITE PERIMETER TLD 48

0.18

0.21

0.18

69.3

SITE PERIMETER TLD 49

0.24

0.29

0.26

70.4

SITE PERIMETER TLD 50

0.16

0.20

0.16

97.8

SITE PERIMETER TLD 51

0.17

0.21

0.17

64.4

SITE PERIMETER TLD 52

0.19

0.23

0.19

66.1

SITE PERIMETER TLD 53

0.16

0.19

0.16

73.9

SITE PERIMETER TLD 54

0.15

0.18

0.15

61.9

SITE PERIMETER TLD 55

0.14

0.18

0.14

59.1

SITE PERIMETER TLD 56

0.14

0.15

0.17

0.13

56.7

SITE PERIMETER TLD 57

0.15

0.16

0.19

0.16

54.8

59.6

$m R=m i l l i R$ oentgen. One $m R$ is approximately equal to $1 \mathrm{mrem}$

An $18 \%$ uncertainty is associated with each result.

Blank spaces indicate missing TLD results. 
Table 17

TLD Results: Slte Perimeter Stations

Page 4 of 7

Quartorty Exposure in mR/Day

Yearly Expoeuro in mA/Ycar

\begin{tabular}{|c|c|c|c|c|c|}
\hline Location & Quarter 16 & Quartor $2^{b}$ & Quarter $3^{b}$ & Quartor $4^{b}$ & $m R / Y c a r b$ \\
\hline SITE PERIMETER TLD 57.25 & 0.16 & 0.17 & 0.20 & 0.17 & 63.1 \\
\hline SITE PERIMETER TLD 57.5 & 0.16 & 0.17 & 0.20 & 0.17 & 64.0 \\
\hline SITE PERIMETER TLD 57.75 & 0.17 & 0.17 & 0.20 & 0.18 & 65.9 \\
\hline SITE PERIMETER TLD 58 & 0.20 & 0.21 & 0.23 & 0.21 & 78.0 \\
\hline SITE PERIMETER TLD 58.25 & 0.17 & 0.17 & 0.20 & 0.17 & 64.8 \\
\hline SITE PERIMETER TLD 58.5 & 0.16 & 0.18 & 0.2 & 0.17 & 65.8 \\
\hline SITE PERIMETER TLD 58.75 & 0.17 & 0.19 & 0.21 & c & 69.1 \\
\hline SITE PERIMETER TLD 59 & 0.16 & 0.17 & 0.19 & 0.17 & 62.7 \\
\hline SITE PERIMETER TLD 59.25 & 0.18 & 0.19 & 0.21 & 0.17 & 68.6 \\
\hline SITE PERIMETER TLD 59.5 & 0.20 & 0.22 & 0.24 & 0.21 & 78.6 \\
\hline SITE PERIMETER TLD 59.75 & 0.17 & 0.19 & 0.21 & 0.19 & 69.8 \\
\hline SITE PERIMETER TLD 60 & 0.19 & 0.20 & 0.24 & 0.20 & 76.4 \\
\hline SITE PERIMETER TLD 60.25 & 0.16 & 0.18 & 0.20 & 0.16 & 64.0 \\
\hline SITE PERIMETER TLD 60.5 & 0.15 & 0.17 & 0.19 & 0.17 & 61.7 \\
\hline SITE PERIMETER TLD 60.75 & 0.16 & 0.28 & 0.19 & 0.17 & 72.3 \\
\hline SITE PERIMETER TLD 61 & 0.17 & 0.18 & 0.20 & 0.17 & 65.7 \\
\hline SITE PERIMETER TLD 61.25 & 0.18 & 0.18 & 0.22 & 0.18 & 68.9 \\
\hline SITE PERIMETER TLD 61.5 & 0.16 & 0.17 & 0.19 & 0.16 & 62.5 \\
\hline SITE PERIMETER TLD 61.75 & 0.18 & 0.19 & 0.22 & 0.18 & 71.3 \\
\hline SITE PERIMETER TLD 62 & 0.16 & 0.18 & 0.21 & 0.17 & 66.1 \\
\hline SITE PERIMETER TLD 62.25 & 0.16 & 0.17 & 0.19 & 0.17 & 63.3 \\
\hline SITE PERIMETER TLD 62.5 & 0.18 & 0.19 & 0.22 & 0.19 & 71.4 \\
\hline SITE PERIMETER TLD 62.75 & 0.22 & 0.22 & 0.25 & 0.21 & 83.4 \\
\hline SITE PERIMETER TLD 63 & 0.18 & & 0.19 & 0.17 & 65.8 \\
\hline SITE PERIMETER TLD 63.25 & 0.18 & 0.16 & 0.18 & 0.17 & 63.3 \\
\hline SITE PERIMETER TLD 63.5 & 0.18 & 0.16 & 0.18 & 0.18 & 64.1 \\
\hline SITE PERIMETER TLD 63.75 & 0.17 & 0.15 & 0.17 & 0.15 & 58.2 \\
\hline SITE PERIMETER TLD 64 & 0.17 & 0.15 & 0.17 & 0.16 & 58.6 \\
\hline
\end{tabular}

a $m R=$ milliRoentgen. One $m R$ is approximately equal to $1 \mathrm{mrem}$.

An $18 \%$ uncertainty is associated with each result.

Blank spaces indicate missing TLD results. 
Table 17

TLD Results: Site Perimeter Stations

Page 5 of 7

\begin{tabular}{|c|c|c|c|c|c|}
\hline Loontion & Quartor $1^{b}$ & Quartor $2^{b}$ & Quarter $3^{b}$ & Quarter $4^{b}$ & mR/Yearb \\
\hline SITE PERIMETER TLD 64.25 & 0.16 & 0.14 & 0.16 & 0.15 & 56.8 \\
\hline SITE PERIMETER TLD 64.5 & 0.18 & 0.15 & 0.17 & 0.16 & 60.5 \\
\hline SITE PERIMETER TLD 64.75 & 0.17 & 0.15 & 0.17 & 0.16 & 58.5 \\
\hline SITE PERIMETER TLD 65 & 0.19 & 0.17 & 0.19 & 0.19 & 67.0 \\
\hline SITE PERIMETER TLD 65.25 & c & 0.19 & 0.22 & 0.21 & 76.3 \\
\hline SITE PERIMETER TLD 65.5 & 0.25 & 0.24 & 0.26 & 0.25 & 90.6 \\
\hline SITE PERIMETER TLD 65.75 & 0.22 & 0.19 & 0.22 & 0.21 & 76.4 \\
\hline SITE PERIMETER TLD 66 & 0.23 & 0.22 & 0.25 & 0.24 & 87.2 \\
\hline SITE PERIMETER TLD 66.25 & 0.25 & 0.23 & 0.25 & 0.26 & 90.4 \\
\hline SITE PERIMETER TLD 66.5 & 0.19 & 0.17 & 0.19 & 0.19 & 67.1 \\
\hline SITE PERIMETER TLD 66.75 & 0.21 & 0.18 & 0.20 & 0.20 & 72.9 \\
\hline SITE PERIMETER TLD 67 & 0.19 & 0.16 & 0.19 & 0.17 & 64.8 \\
\hline SITE PERIMETER TLD 67.25 & 0.21 & 0.19 & 0.21 & 0.20 & 72.8 \\
\hline SITE PERIMETER TLD 67.5 & & 0.17 & 0.21 & 0.19 & 69.3 \\
\hline SITE PERIMETER TLD 67.75 & 0.17 & 0.15 & 0.16 & 0.16 & 58.9 \\
\hline SITE PERIMETER TLD 68 & 0.17 & 0.14 & 0.17 & 0.16 & 58.6 \\
\hline SITE PERIMETER TLD 68.25 & 0.17 & 0.15 & 0.17 & 0.16 & 59.3 \\
\hline SITE PERIMETER TLD 68.5 & 0.20 & 0.17 & 0.19 & 0.18 & 67.8 \\
\hline SITE PERIMETER TLD 68.75 & 0.20 & 0.18 & 0.22 & 0.20 & 73.8 \\
\hline SITE PERIMETER TLD 69 & 0.24 & 0.21 & 0.23 & 0.22 & 81.9 \\
\hline SITE PERIMETER TLD 69.25 & 0.17 & 0.15 & 0.17 & 0.16 & 59.0 \\
\hline SITE PERIMETER TLD 69.5 & 0.18 & 0.16 & 0.18 & 0.17 & 62.2 \\
\hline SITE PERIMETER TLD 69.75 & 0.21 & 0.18 & 0.19 & 0.19 & 69.8 \\
\hline SITE PERIMETER TLD 70 & 0.19 & 0.17 & 0.19 & 0.18 & 67.8 \\
\hline SITE PERIMETER TLD 70.25 & 0.17 & 0.15 & 0.16 & 0.15 & 57.9 \\
\hline SITE PERIMETER TLD 70.5 & 0.19 & 0.16 & 0.18 & 0.17 & 63.5 \\
\hline SITE PERIMETER TLD 70.75 & 0.20 & 0.18 & 0.20 & 0.19 & 71.0 \\
\hline SITE PERIMETER TLD 71 & 0.18 & 0.16 & 0.18 & 0.17 & 62.2 \\
\hline
\end{tabular}

a $m R=m i l l i R o e n t g e n$. One $m R$ is approximately equal to $1 \mathrm{mrem}$.

b An 18\% uncertainty is associated with each result.

c Blank spaces indicate misesing TLD results. 


\section{Table 17}

\section{TLD Results: Site Perimeter Stations}

Page 6 of 7

\begin{tabular}{|c|c|c|c|c|c|}
\hline Location & Quarter $1^{b}$ & Quarter 2b & Quarter 3b & Quarter $\mathbf{4}^{\mathbf{b}}$ & mR/Yearb \\
\hline SITE PERIMETER TLD 71.25 & 0.21 & 0.18 & 0.20 & 0.20 & 71.9 \\
\hline SITE PERIMETER TLD 71.5 & 0.20 & 0.18 & 0.20 & 0.19 & 70.2 \\
\hline SITE PERIMETER TLD 71.75 & 0.21 & 0.19 & 0.21 & 0.20 & 73.7 \\
\hline SITE PERIMETER TLD 72 & 0.20 & 0.17 & 0.19 & 0.18 & 68.4 \\
\hline SITE PERIMETER TLD 72.25 & 0.18 & 0.16 & 0.18 & 0.18 & 64.1 \\
\hline SITE PERIMETER TLD 72.5 & 0.19 & 0.17 & 0.18 & 0.18 & 65.6 \\
\hline SITE PERIMETER TLD 72.75 & 0.18 & 0.16 & 0.18 & 0.17 & 63.6 \\
\hline SITE PERIMETER TLD 73 & 0.17 & 0.15 & 0.17 & 0.16 & 59.7 \\
\hline SITE PERIMETER TLD 73.25 & 0.18 & 0.17 & 0.19 & 0.18 & 65.0 \\
\hline SITE PERIMETER TLD 73.5 & 0.19 & 0.16 & 0.18 & 0.17 & 64.1 \\
\hline SITE PERIMETER TLD 73.75 & 0.17 & 0.15 & 0.18 & 0.16 & 60.1 \\
\hline SITE PERIMETER TLD 74 & 0.17 & 0.15 & 0.17 & 0.16 & 59.2 \\
\hline SITE PERIMETER TLD 74.25 & 0.16 & 0.14 & 0.16 & 0.15 & 55.7 \\
\hline SITE PERIMETER TLD 74.5 & 0.16 & 0.13 & 0.16 & 0.15 & 55.8 \\
\hline SITE PERIMETER TLD 74.75 & 0.17 & $c$ & 0.17 & 0.16 & 61.9 \\
\hline SITE PERIMETER TLD 75 & 0.16 & 0.14 & 0.16 & 0.15 & 55.4 \\
\hline SITE PERIMETER TLD 75.25 & 0.17 & 0.15 & 0.17 & 0.16 & 58.8 \\
\hline SITE PERIMETER TLD 75.5 & 0.15 & 0.13 & 0.15 & 0.15 & 52.8 \\
\hline SITE PERIMETER TLD 75.75 & 0.16 & 0.14 & 0.16 & 0.15 & 55.5 \\
\hline SITE PERIMETER TLD 76 & 0.17 & 0.15 & 0.16 & 0.16 & 58.3 \\
\hline SITE PERIMETER TLD 76.25 & 0.18 & 0.16 & 0.18 & 0.17 & 63.0 \\
\hline SITE PERIMETER TLD 76.5 & 0.18 & 0.15 & 0.17 & 0.16 & 60.5 \\
\hline SITE PERIMETER TLD 76.75 & 0.18 & 0.15 & 0.18 & 0.16 & 60.6 \\
\hline SITE PERIMETER TLD 77 & 0.19 & 0.16 & 0.18 & 0.16 & 63.1 \\
\hline SITE PERIMETER TLD 78 & 0.19 & 0.16 & 0.18 & 0.18 & 65.4 \\
\hline SITE PERIMETER TLD 79 & 0.19 & 0.16 & 0.19 & 0.18 & 65.4 \\
\hline SITE PERIMETER TLD 79.25 & 0.0 & 0.17 & 0.18 & 0.18 & 67.0 \\
\hline SITE PERIMETER TLD 79.5 & 0.21 & 0.19 & 0.21 & 0.20 & 73.8 \\
\hline
\end{tabular}


Table 17

TLD Results: Site Perimeter Stations

Page 7 of 7

Quarterly Exposure in mA/Day

Yearly Exposure in mR/Yeara

\begin{tabular}{lccccc} 
Location & Quarter 1b $^{b}$ & Quarter $^{\text {b }}$ & Quarter $3^{b}$ & Quarter 4b & mR/Yearb \\
\hline SITE PERIMETER TLD 79.75 & 0.22 & 0.20 & 0.21 & 0.20 & 76.7 \\
ALLIED GENERAL 1 & 0.31 & 0.20 & 0.25 & 0.20 & 87.1 \\
ALLIED GENERAL 2 & 0.17 & 0.16 & 0.20 & 0.16 & 62.7 \\
ALLIED GENERAL 3 & 0.19 & 0.18 & 0.22 & 0.17 & 68.5 \\
ALLIED GENERAL 4 & 0.17 & 0.17 & 0.20 & 0.16 & 64.8 \\
PUMPHOUSE ROAD 1 & 0.20 & 0.19 & 0.21 & 0.21 & 74.0 \\
PUMPHOUSE ROAD 2 & 0.15 & 0.16 & 0.22 & 0.21 & 69.1 \\
PUMPHOUSE ROAD 3 & 0.20 & 0.14 & 0.16 & 0.15 & 58.4 \\
PUMPHOUSE ROAD 4 & 0.17 & 0.15 & 0.17 & 0.16 & 58.8 \\
PUMPHOUSE ROAD 5 & 0.24 & 0.22 & 0.25 & 0.23 & 85.6 \\
PUMPHOUSE ROAD 6 & 0.20 & 0.18 & 0.21 & 0.20 & 71.8
\end{tabular}

a $m R=$ milliRoentgen. One $m R$ is approximately equal to $1 \mathrm{mrem}$.

b An 18\% uncertainty is associated with each result. 
Table 18

TLD Results: Environmental Surveillance (Alr Monitoring) Stations

Paga 1 of 2

\begin{tabular}{|c|c|c|c|c|c|}
\hline Location & Quarter $1^{b}$ & Quarter $\mathbf{2}^{\text {b }}$ & Quarter $3^{b}$ & Quarter $4^{b}$ & mR/Yearb \\
\hline 400 D BUILDING 614c & 0.16 & 0.22 & 0.17 & 0.20 & 68.0 \\
\hline A AREA BUIL.DING 614 & 0.21 & 0.25 & 0.24 & 0.25 & 87.4 \\
\hline AIKEN AIRPORT BUILDING 614 & 0.18 & 0.22 & 0.18 & 0.20 & 71.4 \\
\hline $\begin{array}{l}\text { AIKEN STATE PARK } \\
\text { BUILDING } 614\end{array}$ & 0.14 & 0.18 & 0.14 & 0.16 & 56.8 \\
\hline ALLENDALE BUILDING 614 & 0.16 & 0.25 & 0.37 & 0.12 & 82.3 \\
\hline $\begin{array}{l}\text { ALLENDALE GATE } \\
\text { BUILDING } 614\end{array}$ & 0.13 & 0.17 & 0.13 & 0.16 & 53.5 \\
\hline AUGUSTA BUILDING 614 & 0.20 & 0.21 & 0.20 & 0.23 & 77.5 \\
\hline $\begin{array}{l}\text { BARNWELL GATE } \\
\text { BUILDING } 614\end{array}$ & 0.17 & 0.23 & 0.17 & 0.21 & 70.8 \\
\hline $\begin{array}{l}\text { BURIAL GROUND-NORTH } \\
\text { BUILDING } 614\end{array}$ & 0.27 & 0.35 & 0.32 & 0.35 & 117.8 \\
\hline $\begin{array}{l}\text { BURIAL GROUND-SOUTH } \\
\text { BUILDING } 614\end{array}$ & 0.19 & 0.24 & 0.22 & 0.24 & 81.2 \\
\hline COLUMBIA BUILDING 614 TLD 2 & 0.23 & 0.23 & 0.23 & 0.24 & 85.5 \\
\hline COLUMBIA BUILDING 614 TLD 1 & 0.23 & 0.23 & 0.26 & 0.21 & 84.7 \\
\hline DARKHORSE BUILDING 614 & 0.17 & 0.22 & 0.18 & 0.19 & 70.0 \\
\hline EAST TALATHA BUILDING 614 & 0.17 & 0.19 & 0.18 & d & 65.6 \\
\hline F-AREA BUILDING 614 & 0.25 & 0.30 & 0.28 & 0.30 & 103.2 \\
\hline GREENPOND BUILDING 614 & 0.15 & 0.21 & 0.16 & 0.20 & 65.7 \\
\hline $\begin{array}{l}\text { GREENVILLE BUILDING } 614 \\
\text { TLD } 2\end{array}$ & 0.30 & 0.29 & 0.37 & 0.34 & 119.3 \\
\hline $\begin{array}{l}\text { GREENVILLE BUILDING } 614 \\
\text { TLD } 1\end{array}$ & 0.28 & 0.30 & 0.34 & 0.38 & 118.3 \\
\hline H-AREA BUILDING 614 & 0.22 & 0.27 & 0.29 & 0.32 & 100.4 \\
\hline HWY 39 BUILDING 614 & 0.17 & 0.21 & 0.19 & 0.20 & 70.8 \\
\hline HWY 301 BUILDING 614 & 0.44 & & & 0.25 & 126.0 \\
\hline $\begin{array}{l}\text { INTERSECTION OF HWYS } 21 \& \\
167\end{array}$ & 0.22 & 0.28 & 0.23 & 0.26 & 89.4 \\
\hline JACKSON BUILDING 614 & 0.17 & 0.24 & 0.18 & 0.21 & 73.2 \\
\hline $\begin{array}{l}\text { a } m R=\text { milliRoentgen, exposure uni } \\
\text { b An } 18 \% \text { uncertainty is associated } \\
\text { c "614" is the number assigned to ai } \\
\text { d Blank spaces indicate missing TL }\end{array}$ & $\begin{array}{l}\text { Jamma radia } \\
\text { gach result. } \\
\text { itoring static } \\
\text { ults. }\end{array}$ & & & & \\
\hline
\end{tabular}




\section{Table 18}

\section{TLD Results: Environmental Survelllance (Air Monitoring) Stations}

Pagu 2 of 2

\begin{tabular}{|c|c|c|c|c|c|}
\hline Location & Quarter 1b & Quarter $2^{b}$ & Quarter $3^{b}$ & Quarter $4^{b}$ & mR/Yearb \\
\hline LANGLEY BUILDING $614^{\circ}$ & 0.21 & 0.28 & 0.23 & 0.26 & 89.2 \\
\hline LEES BUILDING 614 & 0.14 & 0.18 & 0.14 & 0.16 & 57.1 \\
\hline MACON BUILDING 614 TLD 2 & d & 0.28 & 0.28 & & 98.7 \\
\hline MACON BUILDING 614 TLD 1 & & 0.30 & 0.31 & 0.31 & 109.7 \\
\hline OLAR BUILDING 614 & 0.15 & 0.19 & 0.16 & 0.17 & 60.9 \\
\hline $\begin{array}{l}\text { PATTERSON MILL RD. } \\
\text { BUILDING } 614^{\circ}\end{array}$ & 0.14 & 0.20 & 0.15 & 0.18 & 61.2 \\
\hline PERKINS BUILDING 614 & 0.15 & 0.22 & 0.16 & 0.19 & 64.8 \\
\hline RD. A-14 BUILDING 614 & 0.17 & 0.20 & 0.16 & 0.19 & 65.1 \\
\hline $\begin{array}{l}\text { SAVANNAH BUILDING } 614 \\
\text { TLD } 2\end{array}$ & 0.20 & 0.17 & 0.18 & 0.19 & 66.7 \\
\hline $\begin{array}{l}\text { SAVANNAH BUILDING } 614 \\
\text { TLD } 1\end{array}$ & 0.19 & 0.17 & 0.20 & 0.21 & 68.4 \\
\hline $\begin{array}{l}\text { SOUTH RICHMOND } \\
\text { BUILDING } 614\end{array}$ & 0.22 & 0.30 & 0.23 & 0.26 & 92.8 \\
\hline SPRINGFIELD BUILDING 614 & 0.25 & 0.30 & 0.25 & 0.28 & 99.7 \\
\hline TALATHA GATE BUILDING 614 & 0.19 & 0.24 & 0.21 & 0.23 & 80.0 \\
\hline WAYNESBORO BUILDING 614 & 0.21 & 0.24 & 0.21 & 0.24 & 82.4 \\
\hline WEST JACKSON BUILDING 614 & 0.22 & 0.24 & 0.21 & 0.23 & 83.1 \\
\hline WINDSOR ROAD BUILDING 614 & 0.16 & 0.19 & 0.18 & 0.19 & 66.1 \\
\hline
\end{tabular}

a $\mathrm{mR}=$ milliRoentgen, exposure unit for gamma radiation. One $\mathrm{mR}$ is approximately equal to $1 \mathrm{mrem}$

b An $18 \%$ uncertainty is associated with each result.

c " 614 " is the number assigned to air monitoring stations.

d Blank spaces indicate missing TLD results. 
Table 19

TLD Results: Population Centers

Page 1 of 2

\begin{tabular}{|c|c|c|c|c|c|}
\hline Location & Quarter $1^{b}$ & Quarter 2b & Quarter $3^{b}$ & Quarter $4^{b}$ & mR/Yearb \\
\hline AIKEN (LAURENS ST) & 0.23 & 0.25 & 0.27 & 0.27 & 88.9 \\
\hline AIKEN (SILVER BLL:F RD) & 0.26 & 0.27 & 0.27 & 0.28 & 95.0 \\
\hline ALEXANDER & 0.16 & c & 0.18 & 0.21 & 58.3 \\
\hline ALLENDALE & 0.18 & 0.19 & 0.12 & 0.21 & 59.9 \\
\hline AUGUSTA (CENTRAL AVE) & 0.23 & 0.24 & 0.26 & 0.32 & 91.2 \\
\hline AUGUSTA (CENTRAL \& TOUPE) & 0.21 & 0.22 & 0.22 & 0.26 & 79.2 \\
\hline AUGUSTA (EVE \& ELLIS) & 0.20 & 0.20 & 0.21 & 0.24 & 74.6 \\
\hline AUGUSTA (MILLEDGEVILLE RD) & 0.21 & 0.22 & 0.23 & 0.26 & 79.6 \\
\hline AUGUSTA (REYNOLDS ST) & 0.27 & 0.27 & 0.28 & 0.31 & 98.4 \\
\hline AUGUSTA (WALTON WAY) & 0.28 & 0.27 & 0.28 & 0.30 & 98.9 \\
\hline AUGUSTA (WATKINS ST) & 0.24 & 0.25 & 0.25 & 0.28 & 88.6 \\
\hline BARNWELL & 0.25 & 0.24 & 0.33 & 0.26 & 95.3 \\
\hline BATESBURG & 0.28 & 0.28 & 0.33 & 0.30 & 102.6 \\
\hline BEECH ISLAND & 0.27 & 0.26 & 0.35 & 0.25 & 100.3 \\
\hline BLACKVILLE & 0.22 & 0.25 & 0.27 & 0.28 & 88.2 \\
\hline COLUMBIA (ATLAS ST) & 0.26 & 0.26 & 0.27 & 0.25 & 94.8 \\
\hline COLUMBIA (DENTSVILLE) & 0.28 & 0.28 & 0.27 & 0.31 & 103.4 \\
\hline COLUMBIA (EAU CLAIRE) & & & 0.29 & 0.33 & 113.9 \\
\hline COLUMBIA (HARDEN ST) & 0.26 & 0.28 & 0.27 & 0.30 & 102.1 \\
\hline COLUMBIA (HEADQUARTERS) & 0.27 & 0.28 & 0.29 & 0.33 & 106.4 \\
\hline COLUMBIA (INDUSTRIAL PARK) & 0.26 & 0.27 & 0.27 & 0.32 & 101.0 \\
\hline COLUMBIA (NORTH COLUMBIA) & 0.25 & 0.26 & 0.25 & 0.27 & 93.7 \\
\hline COLUMBIA (SHANDON) & 0.23 & 0.25 & 0.25 & 0.27 & 90.7 \\
\hline COLUMBIA (ST ANDREWS) & 0.26 & 0.27 & 0.27 & 0.28 & 99.2 \\
\hline COUCHTON & & 0.27 & 0.30 & 0.28 & 100.5 \\
\hline EDGEFIELD & 0.27 & 0.25 & 0.26 & 0.27 & 92.2 \\
\hline ESTILL & 0.31 & 0.26 & 0.23 & 0.33 & 100.4 \\
\hline GIRARD & 0.26 & 0.28 & 0.30 & 0.32 & 100.9 \\
\hline GRANITEVILLE & 0.24 & 0.22 & 0.29 & & 87.7 \\
\hline HAMPTON & 0.28 & 0.22 & 0.29 & 0.35 & 101.6 \\
\hline HEPHZIBAH & 0.17 & 0.17 & & 0.18 & 61.3 \\
\hline
\end{tabular}

a $m R=$ exposure unit for gamma radiation. One $\mathrm{mR}$ is approximately equal to $1 \mathrm{mrem}$.

b An $18 \%$ uncertainty is associated with each measured value.

c Blank spaces indicate missing TLD results. 


\section{Table 19}

\section{TLD Results: Population Centers}

Page 2 of 2

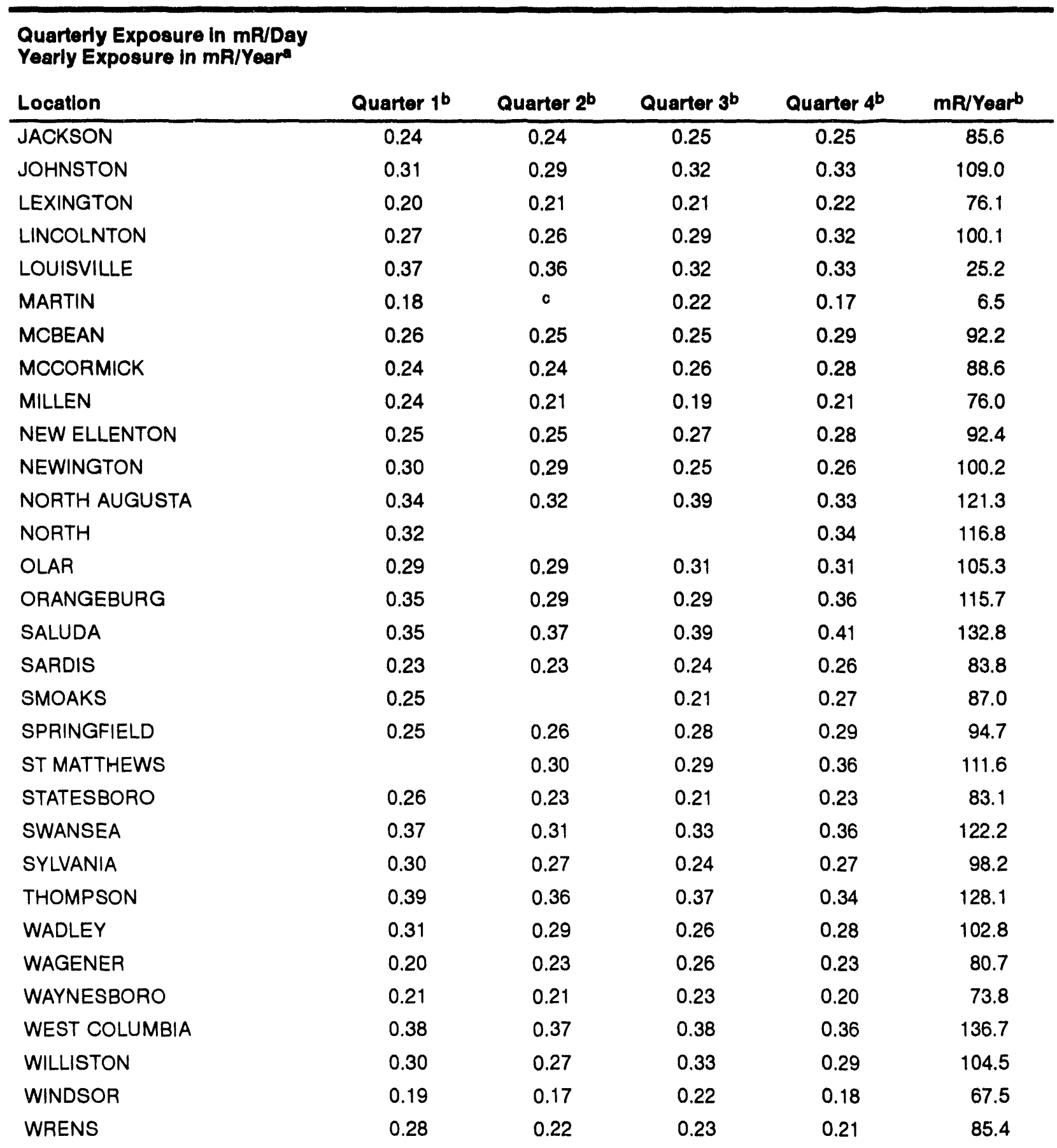

$\mathrm{mR}=$ exposure unit for gamma radiation. One $\mathrm{mR}$ is approximately equal to $1 \mathrm{mrem}$.

b An $18 \%$ uncertainty is associated with each measured value.

c Blank spaces indicate missing TLD results. 
Table 20

TLD Results: Vogtle Electric Generating Plant Vicinity

Page 1 of 1

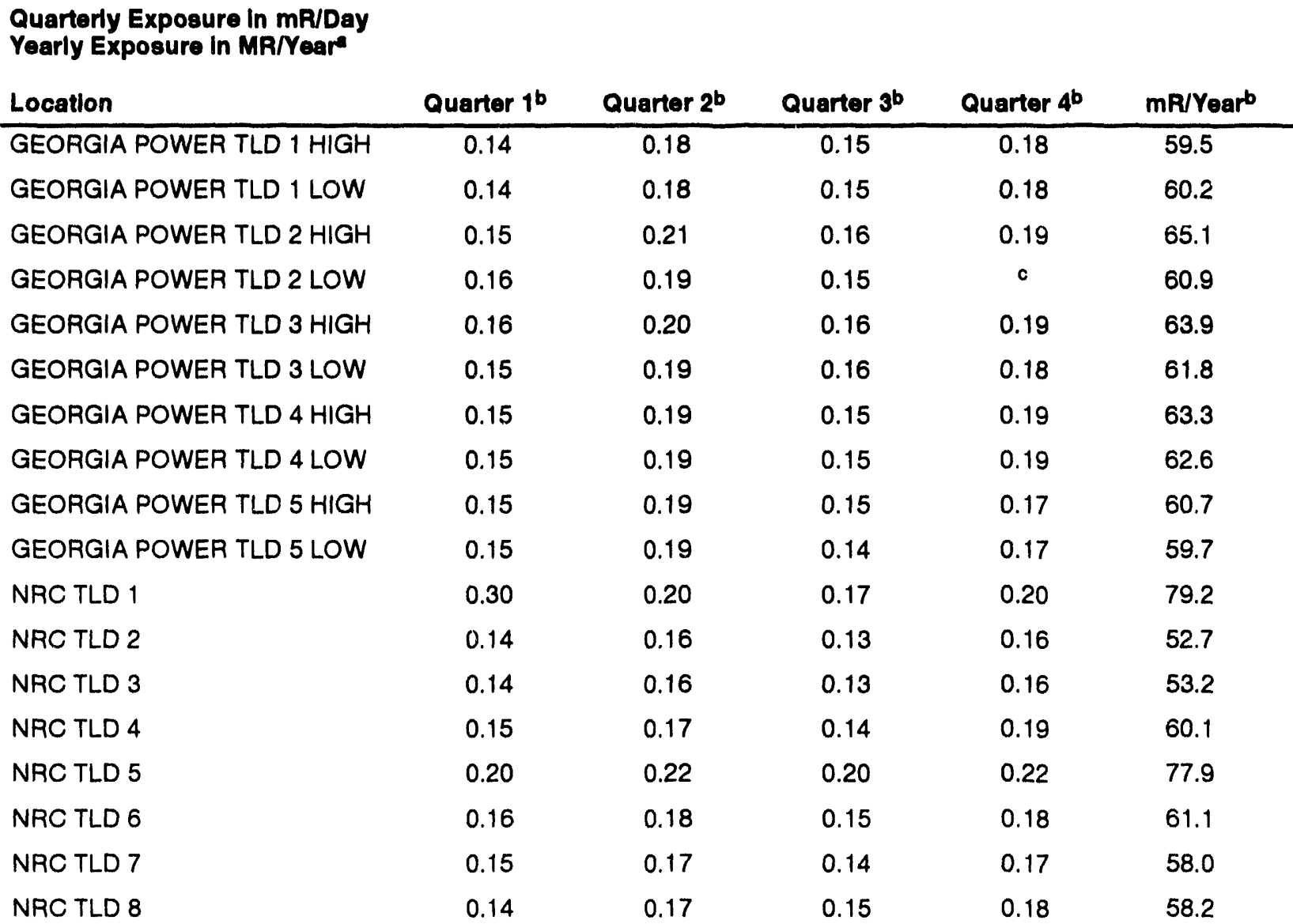

\footnotetext{
$m R=$ milliRoentgen. One $m R$ is approximately equal to $1 \mathrm{mrem}$.

An $18 \%$ uncertainty is associated with each result.

Blank spaces indicate missing TLD results.
} 
Table 21

Radloactivity in Seepage Basin Water

Page 1 of 1

\begin{tabular}{|c|c|c|c|c|}
\hline Location & $\begin{array}{l}\text { No. of } \\
\text { Samples }\end{array}$ & Arlthmetic Mean $\pm \sigma$ & Maximum $\pm \sigma$ & Minimum $\pm \sigma$ \\
\hline \multicolumn{5}{|l|}{$\mathrm{H}-3(\mu \mathrm{Cl} / \mathrm{mL})$} \\
\hline Burial Ground Seepage Basin & 12 & $(4.32 \pm 3.16) E-05$ & $(9.03 \pm 0.19) E-05$ & $(9.01 \pm 0.54) E-06$ \\
\hline CSB-1 C-Area Seepage Basin & 3 & $(2.43 \pm 2.77) E-05$ & $(5.53 \pm 0.42) E-05$ & $(2.13 \pm 3.79) E-06$ \\
\hline E Basin North & 11 & $(1.06 \pm 1.07) E-05$ & $(4.00 \pm 0.10) E-05$ & $(2.39 \pm 0.49) E-06$ \\
\hline E Basin South & 3 & $(3.99 \pm 3.75) E-05$ & $(8.31 \pm 0.19) E-05$ & $(1.50 \pm 0.06) E-05$ \\
\hline LSB-1 L-Area Seepage Basin & 4 & $(2.04 \pm 2.11) E-06$ & $(3.25 \pm 3.22) E-06$ & $(-1.13 \pm 3.74) E-06$ \\
\hline PSB-1 P-Area Seepage Basin & 2 & $(-0.86 \pm 4.04) E-05$ & $(1.99 \pm 6.34) E-05$ & $(-3.72 \pm 7.86) E-05$ \\
\hline TNX-SB Seepage Basin & 12 & $(2.92 \pm 0.73) E-06$ & $(3.97 \pm 0.50) E-06$ & $(1.76 \pm 0.36) E-06$ \\
\hline \multicolumn{5}{|l|}{ C8-137 ( $\mu \mathrm{Cl} / \mathrm{mL})$} \\
\hline CSB-1 C-Area Seepage Basin & 2 & $(1.74 \pm 0.30) E-08$ & $(1.95 \pm 0.73) E-08$ & $(1.52 \pm 0.54) E-08$ \\
\hline PSB-1 P-Area Seepage Basin & 2 & $(2.90 \pm 0.26) E-08$ & $(3.09 \pm 0.54) E-08$ & $(2.71 \pm 0.65) E-08$ \\
\hline \multicolumn{5}{|l|}{$\mathrm{Sr}-89,90(\mu \mathrm{Cl} / \mathrm{mL})$} \\
\hline LSB-1 L-Area Seepage Basin & 4 & $(1.28 \pm 2.06) E-08$ & $(4.22 \pm 1.94) E-08$ & $(-0.32 \pm 1.64) E-08$ \\
\hline \multicolumn{5}{|l|}{$\mathrm{U} / \mathrm{Pu}(\mu \mathrm{Cl} / \mathrm{mL})$} \\
\hline TNX-SB Seepage Basin & 12 & $(2.44 \pm 6.15) E-09$ & $(1.43 \pm 0.88) E-08$ & $(-4.08 \pm 3.51) E-09$ \\
\hline \multicolumn{5}{|l|}{ Gross A $(\mu \mathrm{Cl} / \mathrm{mL})$} \\
\hline Burial Ground Seepage Basin & 11 & $(1.23 \pm 0.95) E-09$ & $(3.89 \pm 2.24) E-09$ & $(4.52 \pm 4.67) E-10$ \\
\hline CSB-1 C-Area Seepage Basin & 3 & $(0.16 \pm 1.01) E-09$ & $(0.76 \pm 1.48) E-09$ & $(-1.00 \pm 0.85) E-09$ \\
\hline E Basin North & 11 & $(2.10 \pm 1.94) E-09$ & $(5.30 \pm 1.44) E-09$ & $(1.52 \pm 4.11) E-10$ \\
\hline E Basin South & 3 & $(1.57 \pm 1.47) E-09$ & $(3.24 \pm 0.97) E-09$ & $(4.98 \pm 5.11) E-10$ \\
\hline LSB-1 L-Area Seepage Basin & 4 & $(0.82 \pm 1.90) E-09$ & $(3.51 \pm 2.11) E-09$ & $(-8.49 \pm 8.14) E-10$ \\
\hline PSB-1 P-Area Seepage Basin & 2 & $(1.14 \pm 0.69) E-09$ & $(1.62 \pm 1.71) E-09$ & $(0.65 \pm 1.48) E-09$ \\
\hline TNX-SB Seepage Basin & 12 & $(-0.19 \pm 2.40) E-09$ & $(4.34 \pm 4.44) E-09$ & $(-3.16 \pm 2.85) E-09$ \\
\hline \multicolumn{5}{|l|}{ Gross B $(\mu \mathrm{Cl} / \mathrm{mL})$} \\
\hline Burial Ground Seepage Basin & 11 & $(1.08 \pm 0.52) E-08$ & $(1.86 \pm 0.18) E-08$ & $(4.81 \pm 1.09) E-09$ \\
\hline CSB-1 C-Area Seepage Basin & 3 & $(6.33 \pm 2.75) E-08$ & $(9.25 \pm 0.81) \mathrm{E}-08$ & $(3.78 \pm 0.58) E-08$ \\
\hline E Basin North & 11 & $(3.54 \pm 1.47) E-09$ & $(6.36 \pm 1.21) E-09$ & $(1.01 \pm 0.87) E-09$ \\
\hline E Basin South & 3 & $(4.43 \pm 3.36) E-09$ & $(8.11 \pm 1.28) E-09$ & $(1.51 \pm 0.95) E-09$ \\
\hline LSB-1 L-Area Seepage Basin & 4 & $(1.58 \pm 0.90) E-08$ & $(2.44 \pm 0.55) E-08$ & $(3.24 \pm 4.01) E-09$ \\
\hline PSB-1 P-Area Seepage Basin & 2 & $(3.51 \pm 0.76) E-08$ & $(4.05 \pm 0.62) E-08$ & $(2.97 \pm 0.54) E-08$ \\
\hline TNX-SB Seepage Basin & 12 & $(4.95 \pm 7.36) E-09$ & $(2.02 \pm 1.02) E-08$ & $(-0.77 \pm 1.06) E-08$ \\
\hline
\end{tabular}




\section{Table 22}

Radloactivity in SRS Stream Water

Page 1 of 5

\begin{tabular}{|c|c|c|c|c|}
\hline Location & $\begin{array}{c}\text { No. of } \\
\text { Samples }\end{array}$ & Arlitimmotlo Mean $\pm \sigma$ & Maximum $\pm \sigma$ & Minimum $\pm \sigma$ \\
\hline \multicolumn{5}{|l|}{$\mathrm{H}-3(\mu \mathrm{Cl} / \mathrm{mL})$} \\
\hline \multicolumn{5}{|l|}{ Tims Branch } \\
\hline TB-5 Near Road C & 26 & $(9.88 \pm 3.15) E-07$ & $(1.56 \pm 0.31) E-06$ & $(3.03 \pm 4.25) E-07$ \\
\hline \multicolumn{5}{|l|}{ Upper Three Runs } \\
\hline Crouch Branch at Road 4 & 26 & $(2.09 \pm 0.44) E-05$ & $(3.23 \pm 0.09) E-05$ & $(1.18 \pm 0.05) E-05$ \\
\hline $\begin{array}{l}\text { U3R-1A Treadway Bridge Rd. } \\
\text { 8-1 }\end{array}$ & 52 & $(5.48 \pm 4.47) E-07$ & $(2.73 \pm 0.42) E-06$ & $(-2.35 \pm 4.09) E-07$ \\
\hline U3R-3 at Road C & 26 & $(3.67 \pm 3.15) E-06$ & $(1.32 \pm 0.05) E-05$ & $(6.07 \pm 3.02) E-07$ \\
\hline U3R-4 at Road $A$ & 26 & $(3.58 \pm 3.55) E-06$ & $(1.79 \pm 0.07) E-05$ & $(-1.40 \pm 4.13) E-07$ \\
\hline \multicolumn{5}{|l|}{ Four Mile Creok } \\
\hline Caster Creek-SE of C-Area & 53 & $(3.90 \pm 0.43) E-06$ & $(4.79 \pm 0.49) E-06$ & $(2.38 \pm 0.39) E-06$ \\
\hline FM-2 at Road 4 & 26 & $(4.33 \pm 1.77) E-05$ & $(6.84 \pm 0.10) E-05$ & $(1.10 \pm 0.06) E-05$ \\
\hline FM-2B Above F-Area Effluent & 26 & $(1.26 \pm 0.22) E-04$ & $(1.64 \pm 0.07) E-04$ & $(7.65 \pm 0.51) E-0.5$ \\
\hline FM-3A Below F-Area Effluent & 26 & $(2.04 \pm 0.47) E-03$ & $(3.39 \pm 0.05) E-03$ & $(1.44 \pm 0.03) E-03$ \\
\hline FM-6 at Road A-12.2 & 26 & $(2.22 \pm 0.55) E-04$ & $(3.09 \pm 0.02) E-04$ & $(6.13 \pm 0.08) E-05$ \\
\hline FM-A7 at Road A-7 & 26 & $(5.17 \pm 1.11) E-04$ & $(6.89 \pm 0.10) E-04$ & $(3.36 \pm 0.09) E-04$ \\
\hline $\mathrm{H}-008$ Outfall & 52 & $(2.79 \pm 1.88) E-06$ & $(9.12 \pm 0.44) E-06$ & $(0.23 \pm 3.79) E-07$ \\
\hline HP-50 Tritium Facility Outfall & 26 & $(2.38 \pm 3.79) E-05$ & $(1.86 \pm 0.02) E-04$ & $(3.61 \pm 0.35) E-06$ \\
\hline Twin Lakes-West of C-Area & 53 & $(1.81 \pm 0.15) E-05$ & $(2.19 \pm 0.08) E-05$ & $(1.52 \pm 0.07) E-05$ \\
\hline \multicolumn{5}{|l|}{ Pen Branch } \\
\hline $\begin{array}{l}\text { IGB-21 800ft South of Rd. } \\
6-1\end{array}$ & 52 & $(1.07 \pm 0.27) E-03$ & $(1.72 \pm 0.03) E-03$ & $(4.21 \pm 0.11) E-04$ \\
\hline $\mathrm{K}-011$ Outfall at B Road & 52 & $(0.95 \pm 1.32) E-04$ & $(7.48 \pm 0.03) E-04$ & $(3.22 \pm 0.07) E-05$ \\
\hline PB-3 at Road A-13.2 & 26 & $(4.02 \pm 1.14) E-05$ & $(6.50 \pm 0.15) E-05$ & $(1.72 \pm 0.07) E-05$ \\
\hline \multicolumn{5}{|l|}{ Steel Creek } \\
\hline SC-2A 1 mile above Road B & 26 & $(1.62 \pm 0.86) E-04$ & $(2.59 \pm 0.04) E-04$ & $(9.46 \pm 0.55) E-06$ \\
\hline SC-4 Steel Creek at Road A & 26 & $(5.15 \pm 0.53) E-06$ & $(6.20 \pm 0.51) E-06$ & $(4.13 \pm 0.46) E-06$ \\
\hline \multicolumn{5}{|l|}{ Lower Three Runs Creek } \\
\hline L3R-1A at Road B & 26 & $(1.93 \pm 0.80) E-06$ & $(3.51 \pm 0.47) E-06$ & $(9.38 \pm 3.04) E-07$ \\
\hline L3R-2 Patterson Mill Rd & 26 & $(9.03 \pm 3.89) E-07$ & $(1.69 \pm 0.32) E-06$ & $(0.91 \pm 4.07) E-07$ \\
\hline L3R-3 at Highway 125 & 12 & $(5.38 \pm 2.37) E-07$ & $(9.07 \pm 4.08) E-07$ & $(1.31 \pm 4.07) E-07$ \\
\hline R-Area Downstream of R-1 & 52 & $(3.69 \pm 3.75) E-06$ & $(2.04 \pm 0.06) E-05$ & $(1.04 \pm 0.41) E-06$ \\
\hline
\end{tabular}




\section{Table 22}

\section{Radioactivity in SRS Stream Water}

Page 2 of 5

\begin{tabular}{|c|c|c|c|c|}
\hline Looation & $\begin{array}{c}\text { No. of } \\
\text { Samplese }\end{array}$ & Arlthmotlo Mean $\pm \sigma$ & Maximum $\pm \sigma$ & Minimum \pm 0 \\
\hline \multicolumn{5}{|l|}{$8-35(\mu \mathrm{Cl} / \mathrm{mL})$} \\
\hline \multicolumn{5}{|l|}{ Four Mile Creek } \\
\hline FM-6 at Road A-12.2 & 12 & $(0.71 \pm 1.14) E-09$ & $(2.78 \pm 0.83) E-09$ & $(-1.69 \pm 0.60) E-09$ \\
\hline \multicolumn{5}{|l|}{ Pen Branch } \\
\hline PB-3 at Road A-13.2 & 12 & $(0.02 \pm 2.18) E-09$ & $(5.46 \pm 0.56) E-09$ & $(-3.36 \pm 0.96) E-09$ \\
\hline \multicolumn{5}{|l|}{ Steel Creok } \\
\hline SC-4 Steel Creek at Road A & 12 & $(-0.34 \pm 1.40) E-09$ & $(2.03 \pm 0.80) E-09$ & $(-3.36 \pm 0.96) E-09$ \\
\hline \multicolumn{5}{|l|}{ Lower Three Runs Creek } \\
\hline L3R-2 Patterson Mill Rd & 12 & $(-0.27 \pm 2.54) E-09$ & $(6.99 \pm 0.54) E-09$ & $(-3.00 \pm 0.99) E-09$ \\
\hline \multicolumn{5}{|l|}{$\mathrm{C} 8-137(\mu \mathrm{C} / / \mathrm{mL})$} \\
\hline \multicolumn{5}{|l|}{ Four Mile Creek } \\
\hline FM-2 at Road 4 & 13 & $(1.91 \pm 0.81) E-08$ & $(3.32 \pm 0.42):-08$ & $(8.42 \pm 1.71) E-09$ \\
\hline FM-2B Above F-Area Effluent & 13 & $(1.72 \pm 0.52) E-08$ & $(2.89 \pm 0.38) E-08$ & $(1.04 \pm 0.38) E-08$ \\
\hline FM-3A Below F-Area Effluent & 2 & $(9.25 \pm 7.93) E-09$ & $(1.49 \pm 0.42) E-08$ & $(3.65 \pm 1.12) E-09$ \\
\hline FM-6 at Road A-12.2 & 2 & $(7.68 \pm 4.39) E-09$ & $(1.08 \pm 0.29) E-08$ & $(4.57 \pm 1.24) E-09$ \\
\hline FM-A7 at Road A-7 & 6 & $(1.16 \pm 0.39) E-08$ & $(1.94 \pm 0.59) E-08$ & $(8.44 \pm 1.24) E-09$ \\
\hline $\mathrm{H}-008$ Outfall & 2 & $(5.58 \pm 3.15) E-09$ & $(7.81 \pm 2.78) E-09$ & $(3.36 \pm 1.25) E-09$ \\
\hline \multicolumn{5}{|l|}{ Steol Creok } \\
\hline SC-2A 1 mile above Road $B$ & 3 & $(1.39 \pm 0.62) E-08$ & $(2.08 \pm 0.41) E-08$ & $(8.81 \pm 2.53) E-09$ \\
\hline \multicolumn{5}{|l|}{ Lower Three Runs Creek } \\
\hline L3R-2 Patterson Mill Rd & 1 & $(3.75 \pm 0.92) E-09$ & $(3.75 \pm 0.92) E-09$ & $(3.75 \pm 0.92) E-09$ \\
\hline R-Area Downstream of R-1 & 2 & $(1.52 \pm 0.16) E-08$ & $(1.64 \pm 0.48) E-08$ & $(1.41 \pm 0.24) E-08$ \\
\hline \multicolumn{5}{|l|}{$P m-147(\mu \mathrm{Cl} / \mathrm{mL})$} \\
\hline \multicolumn{5}{|l|}{ Upper Three Runs } \\
\hline U3R-3 at Road C & 12 & $(2.58 \pm 7.86) E-09$ & $(1.35 \pm 0.23) E-08$ & $(-1.93 \pm 0.44) E-08$ \\
\hline \multicolumn{5}{|l|}{$8 \mathrm{r}-89,90(\mu \mathrm{Cl} / \mathrm{mL})$} \\
\hline \multicolumn{5}{|l|}{ Upper Three Runs } \\
\hline Crouch Branch at Road 4 & 12 & $(4.01 \pm 6.78) E-10$ & $(1.53 \pm 0.52) E-09$ & $(-5.44 \pm 3.97) E-10$ \\
\hline U3R-3 at Road C & 12 & $(0.72 \pm 4.25) E-10$ & $(7.83 \pm 4.73) E-10$ & $(-4.43 \pm 3.79) E-10$ \\
\hline \multicolumn{5}{|l|}{ Four Mlle Creek } \\
\hline FM-2 at Road 4 & 12 & $(3.53 \pm 2.02) E-09$ & $(6.08 \pm 1.10) E-09$ & $(-0.42 \pm 1.30) E-09$ \\
\hline FM-2B Above F-Area Effluent & 12 & $(8.27 \pm 2.18) E-09$ & $(1.24 \pm 0.10) E-08$ & $(5.92 \pm 0.86) E-09$ \\
\hline
\end{tabular}




\section{Table 22}

\section{Radloactlvity in SRS Stroam Water}

Page 3 of 6

\begin{tabular}{|c|c|c|c|c|}
\hline Looatlon & $\begin{array}{l}\text { No. of } \\
\text { Samples }\end{array}$ & Arithmotlo Mean \pm 0 & Maximum \pm 0 & Minimum \pm 0 \\
\hline FM-3A Below F-Area Efluent & 12 & $(3.96 \pm 7.34) E-10$ & $(1.18 \pm 0.66) E-09$ & $(-1.44 \pm 1.08) E-09$ \\
\hline FM-6 at Road A-12.2 & 12 & $(5.22 \pm 1.16) E-09$ & $(6.50 \pm 0.39) E-09$ & $(2.78 \pm 0.28) E-09$ \\
\hline FM-A7 at Road A-7 & 12 & $(1.27 \pm 0.14) E-08$ & $(1.53 \pm 0.14) E-08$ & $(1.03 \pm 0.13) E-08$ \\
\hline H-008 Outfall & 12 & $(3.43 \pm 6.30) E-10$ & $(1.64 \pm 0.51) E-09$ & $(-5.76 \pm 4.33) E-10$ \\
\hline \multicolumn{5}{|l|}{ Pon Branoh } \\
\hline $\begin{array}{l}\text { IGB-21 800tt South of Rd. } \\
6-1\end{array}$ & 4 & $(1.71 \pm 3.81) E-10$ & $(6.72 \pm 4.99) E-10$ & $(-2.47 \pm 4.01) E-10$ \\
\hline $\mathrm{K}-011$ Outfall at B Road & 12 & $(2.70 \pm 4.93) E-10$ & $(1.12 \pm 0.50) E-09$ & $(-6.04 \pm 3.94) E-10$ \\
\hline PB-3 at Road A-13.2 & 12 & $(1.79 \pm 1.52) E-10$ & $(4.90 \pm 1.81) E-10$ & $(-0.84 \pm 1.48) E-10$ \\
\hline \multicolumn{5}{|l|}{ Steol Creok } \\
\hline SC-2A 1 mile above Road $B$ & 12 & $(3.60 \pm 3.14) E-10$ & $(8.35 \pm 5.29) E-10$ & $(-2.88 \pm 4.15) E-10$ \\
\hline SC-4 Steel Creek at Road A & 12 & $(1.82 \pm 1.60) E-10$ & $(4.63 \pm 1.83) E-10$ & $(-0.98 \pm 1.34) E-10$ \\
\hline \multicolumn{5}{|l|}{ Lower Three Runs Creek } \\
\hline L3R-1A at Road B & 12 & $(5.17 \pm 4.56) E-10$ & $(1.33 \pm 0.59) E-09$ & $(-0.77 \pm 4.27) E-10$ \\
\hline L3R-2 Patterson Mill Rd & 12 & $(1.92 \pm 1.69) E-10$ & $(5.79 \pm 1.80) E-10$ & $(-0.64 \pm 1.46) E-10$ \\
\hline L3R-3 at Highway 125 & 12 & $(1.78 \pm 3.52) E-10$ & $(8.18 \pm 3.70) E-10$ & $(-5.15 \pm 2.67) E-10$ \\
\hline \multicolumn{5}{|l|}{$\mathrm{U} / \mathrm{Pu}(\mu \mathrm{Cl} / \mathrm{mL})$} \\
\hline \multicolumn{5}{|l|}{ Upper Three Runs } \\
\hline U3R- -4 at Road $A$ & 25 & $(0.49 \pm 1.44) E-10$ & $(2.98 \pm 1.94) E-10$ & $(-2.66 \pm 2.44) E-10$ \\
\hline \multicolumn{5}{|l|}{ Four Mile Creek } \\
\hline FM-6 at Road A-12.2 & 25 & $(0.57 \pm 1.77) E-10$ & $(4.67 \pm 2.87) E-10$ & $(-1.80 \pm 1.75) E-10$ \\
\hline \multicolumn{5}{|l|}{ Pen Branch } \\
\hline PB-3 at Road A-13.2 & 25 & $(0.22 \pm 1.19) E-10$ & $(2.97 \pm 2.39) E-10$ & $(-1.71 \pm 1.57) E-10$ \\
\hline \multicolumn{5}{|l|}{ Lower Three Runs Creek } \\
\hline L3R-2 (Weekly Grab) & 45 & $(0.44 \pm 1.76) E-10$ & $(7.15 \pm 4.41) E-10$ & $(-2.69 \pm 2.44) E-10$ \\
\hline L3R-2 Patterson Mill Rd & 24 & $(0.68 \pm 1.52) E-10$ & $(3.56 \pm 3.60) E-10$ & $(-2.67 \pm 2.44) E-10$ \\
\hline \multicolumn{5}{|l|}{ Gross A $(\mu \mathrm{Cl} / \mathrm{mL})$} \\
\hline \multicolumn{5}{|l|}{ Tims Branch } \\
\hline TB-5 Near Road C & 26 & $(1.54 \pm 1.01) E-09$ & $(4.99 \pm 1.05) E-09$ & $(3.06 \pm 2.06) E-10$ \\
\hline \multicolumn{5}{|l|}{ Upper Three Runs } \\
\hline Crouch Branch at Road 4 & 26 & $(2.64 \pm 3.56) E-10$ & $(1.67 \pm 0.84) E-09$ & $(-1.81 \pm 1.62) E-10$ \\
\hline $\begin{array}{l}\text { U3R-1A Treadway Bridge RD } \\
8-1\end{array}$ & 51 & $(2.26 \pm 1.27) E-09$ & $(5.92 \pm 0.79) E-09$ & $(5.25 \pm 2.54) E-10$ \\
\hline
\end{tabular}


Table 22

Radloactivity In SRS Stream Water

Page 4 of 5

\begin{tabular}{|c|c|c|c|c|}
\hline Looation & $\begin{array}{c}\text { No. of } \\
\text { samplee }\end{array}$ & Artithmotio Mean \pm 0 & Maximum \pm 0 & Minimum $\pm \sigma$ \\
\hline U3R-3 at Road C & 26 & $(1.77 \pm 0.86) E-09$ & $(3.90 \pm 0.75) E-09$ & $(5.30 \pm 2.92) E-10$ \\
\hline U3R-4 at Road $A$ & 26 & $(1.77 \pm 0.97) E-09$ & $(3.57 \pm 0.57) E-09$ & $(-1.06 \pm 0.98) E-10$ \\
\hline \multicolumn{5}{|l|}{ Four Millo Creek } \\
\hline FM-2 at Road 4 & 26 & $(1.20 \pm 0.80) E-09$ & $(4.18 \pm 0.83) E-09$ & $(4.59 \pm 2.66) E-10$ \\
\hline FM-2B Above F-Area Effluent & 26 & $(4.28 \pm 7.14) E-10$ & $(3.64 \pm 1.40) E-09$ & $(-2.44 \pm 1.66) E-10$ \\
\hline FM-3A Below F-Area Efrluent & 26 & $(1.50 \pm 0.75) E-09$ & $(3.60 \pm 0.76) E-09$ & $(2.49 \pm 2.55) E-10$ \\
\hline FM-6 at Road A-12.2 & 26 & $(3.50 \pm 3.08) E-10$ & $(1.03 \pm 0.45) E-09$ & $(-1.85 \pm 1.46) E-10$ \\
\hline FM-A7 at Road $A-7$ & 26 & $(1.15 \pm 0.65) E-09$ & $(2.68 \pm 0.60) E-09$ & $(0.73 \pm 2.82) E-10$ \\
\hline $\mathrm{H}-008$ Outfall & 52 & $(2.35 \pm 0.98) E-09$ & $(5.14 \pm 0.90) E-09$ & $(3.90 \pm 3.50) E-10$ \\
\hline HP-50 Tritium Facility Outfall & 26 & $(3.16 \pm 1.44) E-09$ & $(6.85 \pm 0.99) E-09$ & $(1.54 \pm 0.42) E-09$ \\
\hline \multicolumn{5}{|l|}{ Pen Branoh } \\
\hline $\begin{array}{l}\text { IGB-21 800tt South of Rd. } \\
6-1\end{array}$ & 4 & $(1.38 \pm 1.51) E-10$ & $(3.50 \pm 2.40) E-10$ & $(-0.06 \pm 2.28) E-10$ \\
\hline $\mathrm{K}-011$ Outfall at B Road & 52 & $(1.11 \pm 2.21) E-10$ & $(9.45 \pm 7.70) E-10$ & $(-2.08 \pm 1.87) E-10$ \\
\hline PB-3 at Road A-13.2 & 26 & $(2.59 \pm 3.45) E-10$ & $(1.27 \pm 0.43) E-09$ & $(-1.53 \pm 1.37) E-10$ \\
\hline \multicolumn{5}{|l|}{ Steel Creek } \\
\hline SC-2A 1 mile above Road B & 26 & $(5.41 \pm 2.92) E-10$ & $(1.47 \pm 0.46) E-09$ & $(-0.25 \pm 1.83) E-10$ \\
\hline $\mathrm{SC}-4$ Steel Creek at Road A & 26 & $(0.46 \pm 3.05) E-10$ & $(1.22 \pm 0.48) E-09$ & $(-1.81 \pm 1.63) E-10$ \\
\hline \multicolumn{5}{|l|}{ Lower Three Runs Creek } \\
\hline L3R-1A at Road $B$ & 26 & $(0.69 \pm 1.86) E-10$ & $(5.54 \pm 2.67) E-10$ & $(-1.65 \pm 1.47) E-10$ \\
\hline L3R-2 Patterson Mill Rd & 26 & $(1.26 \pm 1.89) E-10$ & $(5.99 \pm 3.33) E-10$ & $(-1.49 \pm 1.34) E-10$ \\
\hline L3R-3 at Highway 125 & 12 & $(1.60 \pm 2.65) E-10$ & $(6.92 \pm 3.83) E-10$ & $(-3.40 \pm 3.24) E-10$ \\
\hline R-Area Downstream of R-1 & 52 & $(3.31 \pm 3.90) E-10$ & $(1.75 \pm 0.62) E-09$ & $(-1.90 \pm 1.48) E-10$ \\
\hline \multicolumn{5}{|l|}{ Gross B $(\mu \mathrm{Cl} / \mathrm{mL})$} \\
\hline \multicolumn{5}{|l|}{ Tims Branch } \\
\hline TB-5 Near Road C & 26 & $(2.48 \pm 1.23) E-09$ & $(5.33 \pm 0.80) E-09$ & $(4.08 \pm 4.19) E-10$ \\
\hline \multicolumn{5}{|l|}{ Upper Three Runs } \\
\hline Crouch Branch at Road 4 & 26 & $(1.94 \pm 0.64) E-09$ & $(3.86 \pm 0.62) E-09$ & $(1.13 \pm 0.57) E-09$ \\
\hline $\begin{array}{l}\text { U3R-1A Treadway Bridge RD } \\
8-1\end{array}$ & 51 & $(1.41 \pm 0.89) E-09$ & $(3.54 \pm 0.46) E-09$ & $(-1.10 \pm 4.09) E-10$ \\
\hline U3R-3 at Road C & 26 & $(1.46 \pm 0.82) E-09$ & $(3.94 \pm 0.82) E-09$ & $(4.97 \pm 3.62) E-10$ \\
\hline U3R-4 at Road A & 26 & $(1.50 \pm 1.01) E-09$ & $(3.94 \pm 0.83) E-09$ & $(2.05 \pm 4.36) E-10$ \\
\hline
\end{tabular}


Table 22

Radloactivity in 8 As stream Water

Page 5 of 5

\begin{tabular}{|c|c|c|c|c|}
\hline Location & $\begin{array}{l}\text { No. of } \\
\text { Samplee }\end{array}$ & Artthmetio Mean \pm 0 & Maximum \pm 0 & Minimum $\pm \sigma$ \\
\hline \multicolumn{5}{|l|}{ Four Mile Creak } \\
\hline FM-2 at Road 4 & 26 & $(1.91 \pm 1.07) E-08$ & $(4.24 \pm 0.15) E-08$ & $(6.49 \pm 0.70) E-09$ \\
\hline FM-2B Above F-Area Effluent & 26 & $(2.75 \pm 1.15) E-08$ & $(7.27 \pm 0.21) E-08$ & $(1.61 \pm 0.10) E-08$ \\
\hline FM-3A Below F-Area Effluent & 26 & $(4.76 \pm 1.05) E-09$ & $(6.92 \pm 0.78) E-09$ & $(2.66 \pm 0.46) E-09$ \\
\hline FM-6 at Road A-12.2 & 26 & $(1.41 \pm 0.37) E-08$ & $(2.20 \pm 0.12) E-08$ & $(5.41 \pm 0.68) E-09$ \\
\hline FM-A7 at Road A-7 & 26 & $(3.16 \pm 0.58) E-08$ & $(4.35 \pm 0.17) E-08$ & $(2.05 \pm 0.11) E-08$ \\
\hline H-008 Outfall & 52 & $(5.31 \pm 1.31) E-09$ & $(8.65 \pm 0.81) E-09$ & $(3.09 \pm 0.56) E-09$ \\
\hline HP-50 Tritium Facility Outfall & 26 & $(3.64 \pm 1.65) E-09$ & $(8.12 \pm 0.79) E-09$ & $(1.42 \pm 0.48) E-09$ \\
\hline \multicolumn{5}{|l|}{ Pon Branoh } \\
\hline $\begin{array}{l}\text { IGB-21 800H South of Rd. } \\
6-1\end{array}$ & 4 & $(8.39 \pm 4.21) E-10$ & $(1.41 \pm 0.50) E-09$ & $(4.02 \pm 5.08) E-10$ \\
\hline K-011 Outfall at B Road & 52 & $(1.71 \pm 0.63) E-09$ & $(3.16 \pm 0.63) E-09$ & $(2.32 \pm 4.30) E-10$ \\
\hline PB-3 at Road $A-13.2$ & 26 & $(1.22 \pm 0.54) E-09$ & $(2.86 \pm 0.57) E-09$ & $(3.68 \pm 3.62) E-10$ \\
\hline \multicolumn{5}{|l|}{ Steol Creok } \\
\hline SC-2A 1 mile above Road B & 26 & $(4.27 \pm 1.67) E-09$ & $(9.78 \pm 0.87) E-09$ & $(1.70 \pm 0.50) E-09$ \\
\hline SC-4 Steel Creak at Road A & 26 & $(1.49 \pm 0.55) E-09$ & $(2.79 \pm 0.56) E-09$ & $(6.88 \pm 4.42) E-10$ \\
\hline \multicolumn{5}{|l|}{ Lower Three Runs Creek } \\
\hline L3R-1A at Road $B$ & 26 & $(3.32 \pm 1.05) E-09$ & $(5.46 \pm 0.71) E-09$ & $(1.73 \pm 0.49) E-09$ \\
\hline L3R-2 Patterson Mill Rd & 26 & $(2.74 \pm 1.09) E-09$ & $(5.33 \pm 0.74) E-09$ & $(1.22 \pm 0.49) E-09$ \\
\hline L3R-3 at Highway 125 & 12 & $(2.06 \pm 0.68) E-09$ & $(3.43 \pm 0.80) E-09$ & $(1.16 \pm 0.57) E-09$ \\
\hline R-Area Downstream of R-1 & 52 & $(8.28 \pm 4.11) E-09$ & $(2.42 \pm 0.13) E-08$ & $(3.60 \pm 0.59) E-09$ \\
\hline
\end{tabular}




\section{Table 23}

Radioactivity in Transport at Sample Points on Four Mlle Creek

Page 1 of 1

\begin{tabular}{|c|c|c|c|}
\hline & \multicolumn{3}{|c|}{ Tritum (ourles) } \\
\hline Looation & 7867 & 1002 & 10083 \\
\hline FM3 & 6 & 5 & 4 \\
\hline FM1C & 11 & 5 & 6 \\
\hline $\mathrm{H}-017$ & & & 1 \\
\hline$H-008$ & 5 & 4 & 5 \\
\hline FM2 & 456 & 421 & 322 \\
\hline FM2B & 1820 & 1480 & 1040 \\
\hline FMBA & 6430 & 4100 & 5320 \\
\hline FMA7 & 14000 & 9840 & 8560 \\
\hline \multirow[t]{2}{*}{ FMG } & 13300 & 8710 & 9000 \\
\hline & \multicolumn{3}{|c|}{ Total Strontlum (MIIIlourlee) } \\
\hline Location & 1991 & 1992 & 1993 \\
\hline FM3 & 4 & 1 & a \\
\hline FM1C & 3 & 1 & a \\
\hline $\mathrm{H}-017$ & & 7 & 1 \\
\hline FM2 & 51 & 42 & 26 \\
\hline FM2B & 83 & 86 & 66 \\
\hline FMЗА & 4 & 1 & a \\
\hline FMA7 & 547 & 280 & 216 \\
\hline FM6 & 377 & 219 & 227 \\
\hline
\end{tabular}

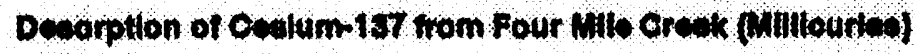

1993

$160^{\mathrm{b}}$

a No activity quantified for year

h Amount of C8-137 estimated in transport at FMA7 minus direct releases. This valve is included in dose calculations and reflects previous releases to Four Mile Creek that were sorbed in the creek bed. 


\section{Table 24}

\section{Radioactivity in Savannah River Water}

Page 1 of 4

\begin{tabular}{|c|c|c|c|c|}
\hline Location & $\begin{array}{l}\text { No. of } \\
\text { Samples }\end{array}$ & Arithmetic Mean $\pm \sigma$ & Maximum $\pm \sigma$ & Minimum $\pm \sigma$ \\
\hline \multicolumn{5}{|l|}{$\mathrm{H}-3(\mu \mathrm{Cl} / \mathrm{mL})$} \\
\hline \multicolumn{5}{|l|}{ Edisto River } \\
\hline $\begin{array}{l}\text { E-1 Edisto River, } \\
\text { Aiken State Park }\end{array}$ & 52 & $(1.66 \pm 1.37) E-07$ & $(4.39 \pm 2.98) E-07$ & $(-1.83 \pm 1.15) E-07$ \\
\hline \multicolumn{5}{|l|}{ Savannah River } \\
\hline River Mile 160 & 2 & $(1.81 \pm 1.19) E-07$ & $(2.65 \pm 1.41) E-07$ & $(0.96 \pm 1.41) E-07$ \\
\hline$R-2(R M-160)$ above SRS & 50 & $(0.70 \pm 1.73) E-07$ & $(7.26 \pm 3.52) E-07$ & $(-2.08 \pm 1.39) E-07$ \\
\hline$R-2$ (monthly grab) & 13 & $(0.79 \pm 1.40) E-07$ & $(2.97 \pm 1.09) E-07$ & $(-1.80 \pm 1.39) E-07$ \\
\hline$R-3 A(R M-151)$ above Vogtle & 52 & $(2.82 \pm 3.36) E-07$ & $(1.48 \pm 0.12) E-06$ & $(-1.85 \pm 1.43) E-07$ \\
\hline Vogtle Discnarge & 19 & $(8.77 \pm 8.63) E-07$ & $(3.84 \pm 0.17) E-06$ & $(-0.86 \pm 1.42) E-07$ \\
\hline River Mile 150 & 1 & $(3.55 \pm 0.17) E-06$ & $(3.55 \pm 0.17) E-06$ & $(3.55 \pm 0.17) E-06$ \\
\hline R-3B (monthly grab) & 13 & $(1.72 \pm 3.95) E-07$ & $(1.36 \pm 0.15) E-06$ & $(-1.35 \pm 1.40) E-07$ \\
\hline R-3B (RM-150) below Vogtle & 52 & $(6.11 \pm 8.97) E-07$ & $(5.69 \pm 0.17) E-06$ & $(-1.47 \pm 1.41) E-07$ \\
\hline $\begin{array}{l}\text { R-8A (RM-141) below Steel } \\
\text { Crk }\end{array}$ & 26 & $(9.37 \pm 5.11) E-07$ & $(1.92 \pm 0.15) E-06$ & $(0.66 \pm 1.41) E-07$ \\
\hline $\begin{array}{l}\text { R-8C (RM-134)below Little } \\
\text { Hell }\end{array}$ & 26 & $(1.22 \pm 0.58) E-06$ & $(2.33 \pm 0.34) E-06$ & $(1.06 \pm 1.42) E-07$ \\
\hline River Mile 140 & 3 & $(2.25 \pm 0.15) E-06$ & $(2.39 \pm 0.16) E-06$ & $(2.08 \pm 0.15) E-06$ \\
\hline River Mile 120 (monthly grab) & 13 & $(1.36 \pm 0.88) E-06$ & $(3.83 \pm 0.13) E-06$ & $(0.58 \pm 1.42) E-07$ \\
\hline River Mile 120 & 53 & $(1.24 \pm 0.46) E-06$ & $(2.32 \pm 0.33) E-06$ & $(4.11 \pm 1.48) E-07$ \\
\hline \multicolumn{5}{|l|}{$\mathrm{s}-35(\mu \mathrm{Cl} / \mathrm{mL})$} \\
\hline \multicolumn{5}{|l|}{ Savannah River } \\
\hline River Mile 160 & 1 & $(-2.57 \pm 4.74) E-10$ & $(-2.57 \pm 4.74) E-10$ & $(-2.57 \pm 4.74) E-10$ \\
\hline$R-2(R M-160)$ above SRS & 12 & $(1.10 \pm 1.90) E-09$ & $(5.41 \pm 0.55) E-09$ & $(-1.06 \pm 0.69) E-09$ \\
\hline Plant Vogtle & 5 & $(6.41 \pm 9.84) E-10$ & $(2.02 \pm 0.45) E-09$ & $(-7.18 \pm 4.95) E-10$ \\
\hline R-3B (RM-150) below Vogtle & 12 & $(4.86 \pm 9.77) E-10$ & $(2.19 \pm 0.45) E-09$ & $(-9.12 \pm 4.95) E-10$ \\
\hline River Mile 120 & 12 & $(1.03 \pm 1.72) E-09$ & $(5.32 \pm 0.55) E-09$ & $(-8.70 \pm 8.21) E-10$ \\
\hline \multicolumn{5}{|l|}{$\mathrm{Sr}-90, \mu \mathrm{Cl} / \mathrm{mL}$} \\
\hline \multicolumn{5}{|l|}{ Savannah River } \\
\hline River Mile 160 & 1 & $(9.06 \pm 5.24) E-11$ & $(9.06 \pm 5.24) E-11$ & $(9.06 \pm 5.24) E-11$ \\
\hline River Mile 160 & 1 & $(1.97 \pm 2.67) E-11$ & $(1.97 \pm 2.67) E-11$ & $(1.97 \pm 2.67) E-11$ \\
\hline$R-2(R M-160)$ above SRS & 12 & $(0.60 \pm 1.13) E-10$ & $(1.74 \pm 0.87) E-10$ & $(-2.51 \pm 1.51) E-10$ \\
\hline R-2 (monthly grab) & 12 & $(7.09 \pm 7.40) E-11$ & $(2.07 \pm 0.90) E-10$ & $(-0.62 \pm 1.10) E-10$ \\
\hline
\end{tabular}




\section{Table 24}

\section{Radioactivity in Savannah River Water}

Page 2 of 4

\begin{tabular}{|c|c|c|c|c|}
\hline Location & $\begin{array}{l}\text { No. of } \\
\text { Samples }\end{array}$ & Arithmetic Mean $\pm \sigma$ & Maximum $\pm \sigma$ & Minimum $\pm \sigma$ \\
\hline R-3A (RM-151) above Vogtle & 12 & $(7.68 \pm 8.06) E-11$ & $(2.41 \pm 1.35) E-10$ & $(-1.42 \pm 8.62) E-11$ \\
\hline Plant Vcgtle & 5 & $(1.35 \pm 1.29) E-10$ & $(3.45 \pm 1.40) E-10$ & $(0.51 \pm 8.82) E-11$ \\
\hline River Mile 150 & 1 & $(0.04 \pm 2.22) E-10$ & $(0.04 \pm 2.22) E-10$ & $(0.04 \pm 2.22) E-10$ \\
\hline R-3B (RM-150) below Vogtle & 12 & $(1.20 \pm 0.73) E-10$ & $(2.44 \pm 0.98) E-10$ & $(0.17 \pm 9.92) E-11$ \\
\hline River Mile 140 & 1 & $(5.43 \pm 8.48) E-11$ & $(5.43 \pm 8.48) E-11$ & $(5.43 \pm 8.48) E-11$ \\
\hline River Mile 120 & 12 & $(1.24 \pm 0.60) E-10$ & $(2.23 \pm 0.95) E-10$ & $(0.09 \pm 1.22) E-10$ \\
\hline \multicolumn{5}{|l|}{$\mathrm{Pu}-238, \mu \approx \mathrm{l} / \mathrm{mL}$} \\
\hline \multicolumn{5}{|l|}{ Savannah River } \\
\hline River Mile 160 & 1 & $(-3.71 \pm 3.71) E-13$ & $(-3.71 \pm 3.71) E-13$ & $(-3.71 \pm 3.71) E-13$ \\
\hline R-2 (RM-160) above SRS & 10 & $(1.27 \pm 6.88) E-13$ & $(0.86 \pm 1.92) E-12$ & $(-1.18 \pm 0.62) E-12$ \\
\hline$R-3 A(R M-151)$ above Vogtle & 12 & $(3.25 \pm 8.76) E-13$ & $(2.21 \pm 1.11) E-12$ & $(-8.63 \pm 4.99) E-13$ \\
\hline Plant Vogtle & 5 & $(0.30 \pm 1.02) E-12$ & $(1.21 \pm 0.86) E-12$ & $(-1.40 \pm 0.66) E-12$ \\
\hline River Mile 150 & 1 & $(6.56 \pm 6.56) E-13$ & $(6.56 \pm 6.56) E-13$ & $(6.56 \pm 6.56) E-13$ \\
\hline$R-3 B(R M-150)$ below Vogtle & 12 & $(1.37 \pm 6.35) E-13$ & $(1.18 \pm 0.84) E-12$ & $(-1.17 \pm 0.83) E-12$ \\
\hline River Mile 140 & 1 & $(1.56 \pm 0.90) E-12$ & $(1.56 \pm 0.90) E-12$ & $(1.56 \pm 0.90) E-12$ \\
\hline River Mile 120 & 12 & $(0.22 \pm 7.45) E-13$ & $(1.74 \pm 1.05) E-12$ & $(-1.08 \pm 0.62) E-12$ \\
\hline \multicolumn{5}{|l|}{$\mathrm{Pu}-239, \mu \mathrm{Cl} / \mathrm{mL}$} \\
\hline \multicolumn{5}{|l|}{ Savannah River } \\
\hline River Mile 160 & 1 & $(-3.70 \pm 8.28) E-13$ & $(-3.70 \pm 8.28) E-13$ & $(-3.70 \pm 8.28) E-13$ \\
\hline$R-2(R M-160)$ above SRS & 12 & $(2.64 \pm 6.14) E-13$ & $(9.85 \pm 5.21) E-13$ & $(-0.83 \pm 2.20) E-12$ \\
\hline R-3A (RM-151) above Vogtle & 11 & $(4.01 \pm 6.10) E-13$ & $(1.31 \pm 0.65) E-\{2$ & $(-2.88 \pm 7.61) E-13$ \\
\hline Plant Vogtle & 3 & $(-1.51 \pm 4.92) E-13$ & $(0.00 \pm 2.48) E-10$ & $(-7.00 \pm 5.22) E-13$ \\
\hline River Mile 150 & 1 & $(-0.65 \pm 1.13) E-12$ & $(-0.65 \pm 1.13) E-12$ & $(-0.65 \pm 1.13) E-12$ \\
\hline R-3B (RM-150) below Vogtle & 9 & $(1.06 \pm 6.52) E-13$ & $(9.07 \pm 5.24) E-13$ & $(-1.18 \pm 0.8+) E-12$ \\
\hline River Mile 140 & 1 & $(0.00 \pm 7.32) E-13$ & $(0.00 \pm 7.32) E-13$ & $(0.00 \pm 7.32) E-13$ \\
\hline River Mile 120 & 12 & $(-0.47 \pm 1.05) E-12$ & $(1.20 \pm 0.69) E-12$ & $(-2.87 \pm 2.14) E-12$ \\
\hline \multicolumn{5}{|l|}{$\mathrm{Sr}-89,90, \mu \mathrm{Cl} / \mathrm{mL}$} \\
\hline \multicolumn{5}{|l|}{ Savannah River } \\
\hline $\begin{array}{l}\text { R-8A (RM-141) } \\
\text { below Steel Crk }\end{array}$ & 12 & $(0.94 \pm 5.09) E-10$ & $(1.10 \pm 0.57) E-09$ & $(-6.85 \pm 5.77) E-10$ \\
\hline R-8B (RM-141) & 12 & $(3.58 \pm 4.10) E-10$ & $(8.92 \pm 6.89) E-10$ & $(-3.40 \pm 5.37) E-10$ \\
\hline
\end{tabular}


Table 24

Radioactivity in Savannah River Water

Page 3 of 4

\begin{tabular}{|c|c|c|c|c|}
\hline Location & $\begin{array}{l}\text { No. of } \\
\text { Samples }\end{array}$ & Arithmetic Mean $\pm \sigma$ & Maximum $\pm \sigma$ & Minimum $\pm \sigma$ \\
\hline $\begin{array}{l}\text { R-8C (RM-134) } \\
\text { below Little Hell }\end{array}$ & 12 & $(4.30 \pm 4.72) E-10$ & $(1.34 \pm 0.72) E-09$ & $(-2.79 \pm 4.53) E-10$ \\
\hline Gross A, $\mu \mathrm{Ci} / \mathrm{mL}$ & & & : & \\
\hline \multicolumn{5}{|l|}{ Edisto River } \\
\hline $\begin{array}{l}\text { E-1 Edisto River, } \\
\text { Aiken State Park }\end{array}$ & 51 & $(1.06 \pm 0.49) E-09$ & $(2.47 \pm 0.60) E-09$ & $(2.72 \pm 2.14) E-10$ \\
\hline \multicolumn{5}{|l|}{ Savannah River } \\
\hline River Mile 160 & 2 & $(3.21 \pm 2.53) E-10$ & $(5.00 \pm 3.95) E-10$ & $(1.42 \pm 3.17) E-10$ \\
\hline R-2 (RM-160) above SRS & 50 & $(0.39 \pm 2.00) E-10$ & $(5.86 \pm 3.61) E-10$ & $(-2.86 \pm 2.56) E-10$ \\
\hline $\mathrm{R}-2$ (monthly grab) & 13 & $(2.51 \pm 3.35) E-10$ & $(1.00 \pm 0.37) E-09$ & $(-1.62 \pm 1.55) E-10$ \\
\hline R-3A (RM-151) above Vogtle & 52 & $(0.73 \pm 2.01) E-10$ & $(6.93 \pm 3.72) E-10$ & $(-2.06 \pm 1.95) E-10$ \\
\hline Vogtle Discharge & 19 & $(1.27 \pm 3.47) E-10$ & $(9.61 \pm 6.30) E-10$ & $(-2.32 \pm 2.06) E-10$ \\
\hline River Mile 150 & 1 & $(5.29 \pm 4.36) E-10$ & $(5.29 \pm 4.36) E-10$ & $(5.29 \pm 4.36) E-10$ \\
\hline R-3B (monthly grab) & 13 & $(2.50 \pm 3.69) E-10$ & $(9.40 \pm 7.07) E-10$ & $(-1.86 \pm 1.76) E-10$ \\
\hline R-3B (RM-150) below Vogtle & 52 & $(1.11 \pm 1.79) E-10$ & $(5.14 \pm 2.96) E-10$ & $(-1.99 \pm 1.80) E-10$ \\
\hline $\begin{array}{l}\text { R-8A (RM-141) below Steel } \\
\text { Crk }\end{array}$ & 26 & $(055 \pm 2.03) E-10$ & $(5.68 \pm 3.08) E-10$ & $(-1.83 \pm 1.63) E-10$ \\
\hline $\begin{array}{l}\text { R-8B (RM-141) below Steel } \\
\text { Crk }\end{array}$ & 26 & $(0.35 \pm 2.25) E-10$ & $(5.94 \pm 4.07) E-10$ & $(-2.70 \pm 2.41) E-10$ \\
\hline $\begin{array}{l}\text { R-8C (RM-134) } \\
\text { below Little Hell } 26\end{array}$ & 26 & $(0.60 \pm 2.05) E-10$ & $(4.05 \pm 2.70) E-10$ & $(-1.83 \pm 1.63) E-10$ \\
\hline River Mile 140 & 3 & $(0.95 \pm 2.23) E-10$ & $(3.53 \pm 3.95) E-10$ & $(-0.35 \pm 2.71) E-10$ \\
\hline River Mile 120 (monthly grab) & 13 & $(1.55 \pm 2.90) E-10$ & $(7.05 \pm 4.36) E-10$ & $(-1.92 \pm 1.59) E-10$ \\
\hline River Mile 120 & 53 & $(0.09 \pm 1.44) E-10$ & $(3.25 \pm 3.42) E-10$ & $(-2.91 \pm 2.61) E-10$ \\
\hline \multicolumn{5}{|l|}{ Gross $B, \mu \mathrm{Ci} / \mathrm{mL}$} \\
\hline \multicolumn{5}{|l|}{ Edisto River } \\
\hline $\begin{array}{l}\text { E-1 Edisto River, } \\
\text { Aiken State Park }\end{array}$ & 51 & $(1.40 \pm 0.49) E-09$ & $(2.68 \pm 0.57) E-09$ & $(0.58 \pm 4.50) E-10$ \\
\hline \multicolumn{5}{|l|}{ Savannah River } \\
\hline River Mile 160 & 2 & $(1.77 \pm 0.66) E-09$ & $(2.24 \pm 0.58) E-09$ & $(1.30 \pm 0.53) E-09$ \\
\hline R-2 (RM-160) above SRS & 50 & $(1.72 \pm 0.63) E-09$ & $(3.17 \pm 0.78) \mathrm{E}-09$ & $(3.93 \pm 4.97) E-10$ \\
\hline R-2 (monthly grab) & 13 & $(1.77 \pm 0.69) E-09$ & $(2.85 \pm 0.69) E-09$ & $(9.91 \pm 5.20) E-10$ \\
\hline $\mathrm{R}-3 \mathrm{~A}(\mathrm{RM}-151)$ above Vogtle & 52 & $(1.78 \pm 0.67) E-09$ & $(3.29 \pm 0.66) E-09$ & $(5.95 \pm 4.36) E-10$ \\
\hline Vogtle Discharge & 19 & $(2.57 \pm 0.85) E-09$ & $(4.36 \pm 0.69) E-09$ & $(1.53 \pm 0.54) E-09$ \\
\hline
\end{tabular}




\section{Table 24}

\section{Radioactivity in Savannah River Water}

Page 4 of 4

\begin{tabular}{lcccc}
\hline & $\begin{array}{c}\text { No. of } \\
\text { Samples }\end{array}$ & Arlthmetic Mean $\pm \sigma$ & Maximum $\pm \sigma$ & Minimum $\pm \sigma$ \\
\hline Location & 1 & $(3.53 \pm 0.64) E-09$ & $(3.53 \pm 0.64) E-09$ & $(3.53 \pm 0.64) E-09$ \\
River Mile 150 & 13 & $(1.88 \pm 0.39) E-09$ & $(2.70 \pm 0.62) E-09$ & $(1.35 \pm 0.45) E-09$ \\
R-3B (RM-150) below Vogtle & 52 & $(1.85 \pm 0.50) E-09$ & $(3.41 \pm 0.61) E-09$ & $(9.01 \pm 5.28) E-10$ \\
$\begin{array}{l}\text { R-8A (RM-141) below Steel } \\
\text { Crk }\end{array}$ & 26 & $(1.77 \pm 0.50) E-09$ & $(2.89 \pm 0.63) E-09$ & $(8.65 \pm 4.52) E-10$ \\
$\begin{array}{l}\text { R-8B (RM-141) below Steel } \\
\text { Crk }\end{array}$ & 26 & $(1.72 \pm 0.59) E-09$ & $(2.69 \pm 0.63) E-09$ & $(6.22 \pm 4.60) E-10$ \\
$\begin{array}{l}\text { R-8C (RM-134) } \\
\text { below Little Hell }\end{array}$ & 26 & $(1.71 \pm 0.63) E-09$ & $(2.97 \pm 0.66) E-09$ & $(7.22 \pm 4.17) E-10$ \\
River Mile 140 & & & & \\
River Mile 120 (monthly grab) & 13 & $(1.92 \pm 0.55) E-09$ & $(2.89 \pm 0.59) E-09$ & $(8.81 \pm 5.42) E-10$ \\
River Mile 120 & 53 & $(1.76 \pm 0.51) E-09$ & $(3.12 \pm 0.63) E-09$ & $(9.59 \pm 5.28) E-10$
\end{tabular}


Table 25

Summary of SRS Tritium Transport, 1960-1993

Page 1 of 1

\begin{tabular}{|c|c|c|c|}
\hline \multirow[b]{2}{*}{ Year } & \multicolumn{3}{|c|}{ Estimated Tritium Transport (Cl) } \\
\hline & $\begin{array}{c}\text { Based on Polnt-of- } \\
\text { Release } \\
\text { Concentrations } \\
\text { and Flow Rates }\end{array}$ & $\begin{array}{c}\text { Based on Stream } \\
\text { Concentrations and } \\
\text { Flow Rates }\end{array}$ & $\begin{array}{c}\text { Based on Savannah River } \\
\text { Concentrations and } \\
\text { Flow Rates }\end{array}$ \\
\hline 1960 & $64,000^{b}$ & 69,600 & 73,700 \\
\hline 1961 & $69,000^{b}$ & 83,000 & 77,000 \\
\hline 1962 & $58,000^{b}$ & 64,000 & 63,000 \\
\hline 1963 & $97,000^{b}$ & 96,900 & 122,800 \\
\hline 1964 & $111,000^{b}$ & 131,600 & 143,000 \\
\hline 1965 & 108,400 & 109,200 & 100,200 \\
\hline 1966 & 84,900 & 97,800 & 78,300 \\
\hline 1967 & 70,600 & 77,000 & 68,500 \\
\hline 1968 & 63,800 & 67,200 & 61,800 \\
\hline 1969 & 64,600 & 64,000 & 58,100 \\
\hline 1970 & 36,900 & 43,200 & 31,800 \\
\hline 1971 & 38,200 & 44,700 & 39,100 \\
\hline 1972 & 16,800 & 47,300 & 45,300 \\
\hline 1973 & 71,100 & 62,800 & 61,100 \\
\hline 1974 & 59,900 & 54,600 & 46,000 \\
\hline 1975 & 55,600 & 50,000 & 49,500 \\
\hline 1976 & 59,600 & 47,400 & 51,100 \\
\hline 1977 & 43,800 & 39,700 & 42,500 \\
\hline 1978 & 37,600 & 35,300 & 36,600 \\
\hline 1979 & 29,400 & 27,100 & 30,600 \\
\hline 1980 & 24,900 & 28,800 & 30,700 \\
\hline 1981 & 23,900 & 22,100 & 25,100 \\
\hline 1982 & 32,200 & 31,300 & 30,600 \\
\hline 1983 & 34,200 & 33,000 & 33,000 \\
\hline 1984 & 32,800 & 32,600 & 33,200 \\
\hline 1985 & 25,000 & 22,300 & 24,100 \\
\hline 1986 & 27,800 & 22,300 & 22,100 \\
\hline 1987 & 22,700 & 20,500 & 26,200 \\
\hline 1988 & 19,300 & 18,300 & 14,600 \\
\hline 1989 & 17,300 & 17,800 & 15,600 \\
\hline 1990 & 16,100 & 15,600 & 14,500 \\
\hline 1991 & 27,400 & 26,600 & 26,300 \\
\hline 1992 & 13,800 & 13,100 & 13,800 \\
\hline 1993 & 11,300 & 12,700 & 12,200 \\
\hline
\end{tabular}

a Includes direct releases to streams and migration from F-Area, $\mathrm{H}$-Area, K-Area, and P-Area seepage basins and the Solid Waste Disposal Facility to streams

b Includes heat exchanger cooling water released from P-Area (of Par Pond origin) to Steel Creek 


\section{Table 26}

\section{Radioactivity in Drinking Water}

Page 1 of 9

\begin{tabular}{|c|c|c|c|c|}
\hline Location & $\begin{array}{l}\text { No. of } \\
\text { Samples }\end{array}$ & Arithmetic Mean $\pm \sigma$ & Maximum $\pm \sigma$ & Minimum $\pm \sigma$ \\
\hline \multicolumn{5}{|l|}{$\mathrm{H}-3, \mu \mathrm{Cl} / \mathrm{mL}$} \\
\hline \multicolumn{5}{|l|}{ On Site } \\
\hline $\begin{array}{l}\text { 105-C C-Area Dom. Water } \\
\text { Faucet }\end{array}$ & 12 & $(-1.24 \pm 1.43) E-07$ & $(0.86 \pm 1.21) E-07$ & $(-3.59 \pm 1.37) E-07$ \\
\hline $\begin{array}{l}105-\text { K K-Area Dom. Water } \\
\text { Faucet }\end{array}$ & 12 & $(-0.51 \pm 2.17) E-07$ & $(2.56 \pm 1.53) E-07$ & $(-3.66 \pm 1.41) E-07$ \\
\hline $\begin{array}{l}\text { 105-L L-Area Dom. Water } \\
\text { Faucet }\end{array}$ & 12 & $(-1.13 \pm 1.28) E-07$ & $(1.20 \pm 1.11) E-07$ & $(-3.40 \pm 1.37) E-07$ \\
\hline $\begin{array}{l}\text { 105-P P-Area Dom. Water } \\
\text { Faucet }\end{array}$ & 12 & $(2.11 \pm 4.17) E-07$ & $(1.39 \pm 0.15) E-06$ & $(-1.85 \pm 1.37) E-07$ \\
\hline $\begin{array}{l}221-F \text { F-Area Dom. Water } \\
\text { Faucet }\end{array}$ & 12 & $(-1.46 \pm 0.89) E-07$ & $(-0.07 \pm 1.43) E-07$ & $(-3.21 \pm 1.40) E-07$ \\
\hline $\begin{array}{l}\text { 221-H H-Area Dom. Water } \\
\text { Faucet }\end{array}$ & 12 & $(-1.80 \pm 1.53) E-07$ & $(0.37 \pm 1.54) E-07$ & $(-4.19 \pm 1.37) E-07$ \\
\hline $\begin{array}{l}241-24 H \text { H-Area Dom. Water } \\
\text { Faucet }\end{array}$ & 4 & $(-1.44 \pm 1.00) E-07$ & $(-0.53 \pm 1.09) E-07$ & $(-2.84 \pm 1.44) E-07$ \\
\hline $\begin{array}{l}\text { 617-G Wackenhut Training } \\
\text { Facility }\end{array}$ & 4 & $(9.74 \pm 2.07) E-07$ & $(1.26 \pm 0.13) E-06$ & $(7.70 \pm 1.44) E-07$ \\
\hline $\begin{array}{l}\text { 618-G Class. Yard } \\
\text { Lunchroom }\end{array}$ & 4 & $(-1.83 \pm 1.12) E-07$ & $(-0.51 \pm 1.21) \mathrm{E}-07$ & $(-2.77 \pm 1.37) E-07$ \\
\hline $\begin{array}{l}661-G \text { Firing Range } \\
\text { (pumphouse) }\end{array}$ & 4 & $(9.38 \pm 1.65) E-07$ & $(1.11 \pm 0.15) E-06$ & $(7.50 \pm 1.42) E-07$ \\
\hline $\begin{array}{l}\text { 679-T TNX Dom. Water } \\
\text { Faucet }\end{array}$ & 4 & $(-2.64 \pm 1.18) E-07$ & $(-1.45 \pm 1.47) E-07$ & $(-4.24 \pm 1.35) E-07$ \\
\hline 681-1G Dom. Water Faucet & 2 & $(-1.65 \pm 0.65) E-07$ & $(-1.19 \pm 1.20) E-07$ & $(-2.11 \pm 1.44) E-07$ \\
\hline 681-3G Dom. Water Faucet & 4 & $(-6.35 \pm 8.34) E-08$ & $(0.29 \pm 1.21) E-07$ & $(-1.57 \pm 1.37) E-07$ \\
\hline 701-12G Patrol Gate 7 & 4 & $(-2.65 \pm 4.46) E-08$ & $(0.40 \pm 1.39) E-07$ & $(-0.53 \pm 1.21) E-07$ \\
\hline 701-13G Patrol Gate 6 & 4 & $(2.53 \pm 0.15) E-06$ & $(2.71 \pm 0.14) E-06$ & $(2.40 \pm 0.16) E-06$ \\
\hline $\begin{array}{l}\text { 701-1F F-Area Dom. Water } \\
\text { Faucet }\end{array}$ & 4 & $(-1.72 \pm 1.11) E-07$ & $(-0.53 \pm 1.21) E-07$ & $(-2.92 \pm 1.36) E-07$ \\
\hline $\begin{array}{l}701-1 \mathrm{H} \text { H-Area Dom. Water } \\
\text { Faucet }\end{array}$ & 4 & $(-2.23 \pm 1.71) E-07$ & $(0.18 \pm 121) E-07$ & $(-3.76 \pm 1.36) E-07$ \\
\hline 701-3G Barnwell Gate & 3 & $(-1.74 \pm 0.69) E-07$ & $(-1.29 \pm 1.38) E-07$ & $(-2.53 \pm 1.19) E-07$ \\
\hline 701-4G Williston Gate & 4 & $(-1.33 \pm 0.44) E-07$ & $(-0.81 \pm 1.37) E-07$ & $(-1.77 \pm 1.38) E-07$ \\
\hline 701-5G Talatha Gate & 4 & $(5.02 \pm 0.82) E-07$ & $(5.96 \pm 1.25) E-07$ & $(4.14 \pm 1.40) E-07$ \\
\hline 701-6G Jackson Gate & 4 & $(-2.03 \pm 1.50) E-07$ & $(0.20 \pm 1.47) E-07$ & $(-3.08 \pm 1.38) E-07$ \\
\hline 701-8G Patroi Gate 8 & 4 & $(2.16 \pm 0.06) E-06$ & $(2.21 \pm 0.16) E-06$ & $(2.10 \pm 0.15) E-06$ \\
\hline 703-A EOC (kitchen) & 4 & $(-1.89 \pm 1.45) E-07$ & $(0.21 \pm 1.47) E-07$ & $(-3.14 \pm 1.37) E-07$ \\
\hline
\end{tabular}




\section{Table 26}

\section{Radloactivity in Drinking Water}

Page 2 of 9

\begin{tabular}{|c|c|c|c|c|}
\hline Location & $\begin{array}{c}\text { No. of } \\
\text { Samples }\end{array}$ & Arithmetic Mean $\pm \sigma$ & Maximum \pm 0 & Minimum $\pm \sigma$ \\
\hline $\begin{array}{l}\text { 704-F F-Area Dom. Water } \\
\text { Faucet }\end{array}$ & 4 & $(0.01 \pm 1.29) E-07$ & $(1.15 \pm 1.52) E-07$ & $(-1.83 \pm 1.39) E-07$ \\
\hline $\begin{array}{l}\text { 704-H H-Area Dom. Water } \\
\text { Faucet }\end{array}$ & 4 & $(-0.16 \pm 1.60) E-07$ & $(1.73 \pm 1.53) E-07$ & $(-2.18 \pm 1.39) E-07$ \\
\hline $\begin{array}{l}\text { 704-S S-Area Dom. Water } \\
\text { Faucet }\end{array}$ & 4 & $(-0.79 \pm 2.06) E-07$ & $(1.94 \pm 1.48) E-07$ & $(-2.46 \pm 1.36) E-07$ \\
\hline 708-A A-Area Cafeteria & 4 & $(-1.71 \pm 1.66) E-07$ & $(0.00 \pm 1.46) E-07$ & $(-3.63 \pm 1.36) E-07$ \\
\hline 735-7G Par Pond Lab & 3 & $(-1.68 \pm 1.16) E-07$ & $(-0.96 \pm 1.46) E-07$ & $(-3.02 \pm 1.39) E-07$ \\
\hline 760-G Forestry Building & 4 & $(8.03 \pm 1.97) E-07$ & $(1.04 \pm 0.15) E-06$ & $(6.38 \pm 1.41) E-07$ \\
\hline $\begin{array}{l}\text { 772-F F-Area Dom. Water } \\
\text { Faucet }\end{array}$ & 4 & $(-1.29 \pm 1.10) E-07$ & $(-0.58 \pm 1.09) E-07$ & $(-2.93 \pm 1.39) E-07$ \\
\hline $\begin{array}{l}\text { Central Shops } 690-G \text { Ford } \\
\text { Building }\end{array}$ & 4 & $(-1.52 \pm 1.25) E-07$ & $(-0.40 \pm 1.37) E-07$ & $(-2.86 \pm 1.36) E-07$ \\
\hline TC-1 B-Area Cafeteria & 4 & $(-1.60 \pm 1.82) E-07$ & $(0.62 \pm 1.48) E-07$ & $(-3.29 \pm 1.36) E-07$ \\
\hline Z-Area Building 704-Z & 4 & $(-1.83 \pm 0.81) E-07$ & $(-0.78 \pm 1.46) E-07$ & $(-2.74 \pm 1.37) E-07$ \\
\hline \multicolumn{5}{|l|}{ Surrounding Towns } \\
\hline Aiken SC, Post Office & 2 & $(1.08 \pm 0.28) E-07$ & $(1.28 \pm 1.48) E-07$ & $(0.88 \pm 1.41) E-07$ \\
\hline Allendale SC, Post Office & 2 & $(-1.84 \pm 0.02) E-07$ & $(-1.82 \pm 1.41) E-07$ & $(-1.85 \pm 1.45) E-07$ \\
\hline $\begin{array}{l}\text { Augusta GA, Post Office (8th } \\
\text { St.) }\end{array}$ & 2 & $(-3.61 \pm 1.36) E-08$ & $(-0.27 \pm 1.46) E-07$ & $(-0.46 \pm 1.40) E-07$ \\
\hline Barnwell SC, Post Office & 2 & $(-2.43 \pm 0.61) E-07$ & $(-2.00 \pm 1.40) E-07$ & $(-2.86 \pm 1.46) E-07$ \\
\hline Bath SC, Post Office & 2 & $(1.05 \pm 0.90) E-07$ & $(1.68 \pm 1.43) E-07$ & $(0.41 \pm 1.47) E-07$ \\
\hline Blackville SC, Post Office & 2 & $(1.16 \pm 2.85) E-08$ & $(0.32 \pm 1.46) E-07$ & $(-0.09 \pm 1.41) E-07$ \\
\hline Clearwater SC, Post Office & 2 & $(2.28 \pm 0.88) E-07$ & $(2.90 \pm 1.44) E-07$ & $(1.66 \pm 1.49) E-07$ \\
\hline Jackson SC, Post Office & 2 & $(4.15 \pm 1.90) E-07$ & $(5.49 \pm 1.51) E-07$ & $(2.81 \pm 1.41) E-07$ \\
\hline Langley SC, Post Office & 2 & $(-1.23 \pm 1.09) E-07$ & $(-0.46 \pm 1.46) E-07$ & $(-2.00 \pm 1.39) E-07$ \\
\hline N. Augusta SC, Post Office & 2 & $(-1.30 \pm 0.99) E-07$ & $(-0.61 \pm 1.39) E-07$ & $(-2.00 \pm 1.47) E \cdot 07$ \\
\hline New Ellenton SC, Post Office & 2 & $(1.80 \pm 2.11) E-07$ & $(3.30 \pm 1.50) E-07$ & $(0.31 \pm 1.42) E-07$ \\
\hline ORA Site, ORA Building & 2 & $(-1.17 \pm 0.69) E-07$ & $(-0.69 \pm 1.45) E-07$ & $(-1.66 \pm 1.39) E-07$ \\
\hline $\begin{array}{l}\text { Sardis GA, Post Office (Farm } \\
\text { St.) }\end{array}$ & 2 & $(-2.69 \pm 3.56) E-08$ & $(-0.02 \pm 1.47) E-07$ & $(-0.52 \pm 1.39) E-07$ \\
\hline $\begin{array}{l}\text { Talatha SC, Tap Near Water } \\
\text { Tank }\end{array}$ & 2 & $(1.64 \pm 1.00) E-07$ & $(2.34 \pm 1.48) E-07$ & $(0.93 \pm 1.41) E-07$ \\
\hline Waynesboro GA, Post Office & 2 & $(-0.13 \pm 2.05) E-07$ & $(1.32 \pm 1.41) E-07$ & $(-1.58 \pm 1.46) E-07$ \\
\hline Williston SC, Post Office & 2 & $(0.43 \pm 1.53) E-07$ & $(1.51 \pm 1.45) E-07$ & $(-0.65 \pm 1.46) E-07$ \\
\hline
\end{tabular}




\section{Table 26}

\section{Radloactlvity In Drinking Water}

Page 3 of 9

\begin{tabular}{|c|c|c|c|c|}
\hline Location & $\begin{array}{l}\text { No. of } \\
\text { Samples }\end{array}$ & Arithmetic Mean $\pm \sigma$ & Maximum $\pm \sigma$ & Minimum \pm 0 \\
\hline \multicolumn{5}{|c|}{ Drinking Water from Water Treatment Plants } \\
\hline Beaufort Finished & 12 & $(7.12 \pm 4.63) E-07$ & $(1.25 \pm 0.12) E-06$ & $(-0.03 \pm 1.20) E-07$ \\
\hline Beaufort Raw & 12 & $(7.24 \pm 4.25) E-07$ & $(1.38 \pm 0.12) E-06$ & $(1.11 \pm 1.21) \mathrm{E}-07$ \\
\hline D-Area Finished & 12 & $(-0.17 \pm 2.18) E-07$ & $(2.95 \pm 1.49) E-07$ & $(-2.98 \pm 1.37) E-07$ \\
\hline D-Area Raw & 12 & $(1.93 \pm 1.35) E-07$ & $(3.81 \pm 1.51) E-07$ & $(-0.87 \pm 1.52) E-07$ \\
\hline N. Augusta Finishud & 12 & $(0.40 \pm 1.03) E-07$ & $(2.20 \pm 1.22) E-07$ & $(-1.17 \pm 1.53) E-07$ \\
\hline N. Augusta Raw & 12 & $(0.32 \pm 1.82) E-07$ & $(1.92 \pm 1.11) E-07$ & $(-4.52 \pm 1.51) E-07$ \\
\hline Savannah Finished & 12 & $(9.67 \pm 2.48) E-07$ & $(1.35 \pm 0.12) E-06$ & $(6.02 \pm 1.51) E-07$ \\
\hline Savannah Raw & 12 & $(9.83 \pm 3.42) E-07$ & $(1.35 \pm 0.15) E-06$ & $(4.19 \pm 1.50) E-07$ \\
\hline \multicolumn{5}{|l|}{ EMS Sampler } \\
\hline Beaufort Raw & 22 & $(7.75 \pm 4.58) E-07$ & $(1.37 \pm 0.15) E-06$ & $(0.51 \pm 1.55) E-07$ \\
\hline \multicolumn{5}{|l|}{$\mathrm{Sr}-90, \mu \mathrm{Cl} / \mathrm{mL}$} \\
\hline \multicolumn{5}{|l|}{ On Sltea } \\
\hline $\begin{array}{l}\text { 105-L L-Area Dom. Water } \\
\text { Faucet }\end{array}$ & 1 & $(-2.43 \pm 3.92) E-10$ & & \\
\hline $\begin{array}{l}\text { 221-F F-Area Dom. Water } \\
\text { Faucet }\end{array}$ & 1 & $(1.28 \pm 4.35) E-10$ & & \\
\hline $\begin{array}{l}\text { 221-H H-Area Dom. Water } \\
\text { Faucet }\end{array}$ & 1 & $(6.40 \pm 4.64) \mathrm{E}-10$ & & \\
\hline $\begin{array}{l}617-G \text { Wackenhut Training } \\
\text { Facility }\end{array}$ & 1 & $(-2.56 \pm 4.49) E-10$ & & \\
\hline 681-1G Dom. Water Faucet & 1 & $(1.11 \pm 4.61) E-10$ & & \\
\hline $\begin{array}{l}701-1 \mathrm{H} \mathrm{H} \text {-Area Dom. Water } \\
\text { Faucet }\end{array}$ & 1 & $(-2.32 \pm 4.52) E-10$ & & \\
\hline 701-5G Taiatha Gate & 1 & $(4.00 \pm 4.21) E-10$ & & \\
\hline 703-A EOC (kitchen) & 1 & $(2.35 \pm 5.26) E-10$ & & \\
\hline $\begin{array}{l}\text { 704-H H-Area Dom. Water } \\
\text { Faucet }\end{array}$ & 1 & $(-1.21 \pm 6.33) E-10$ & & \\
\hline $\begin{array}{l}\text { 704-S S-Area Dom. Water } \\
\text { Faucet }\end{array}$ & 1 & $(-3.40 \pm 4.49) E-10$ & & \\
\hline 735-7G Par Pond Lab & 1 & $(-3.31 \pm 3.99) E-10$ & & \\
\hline $\begin{array}{l}\text { Central Shops } 690-\text { G Ford } \\
\text { Building }\end{array}$ & 1 & $(-2.28 \pm 4.27) E-10$ & & \\
\hline TC-1 B-Area Cafeteria & 1 & $(-0.03 \pm 4.42) E-10$ & & \\
\hline
\end{tabular}

a Annual result reported in arithmetic mean column 


\section{Table 26}

\section{Radioactivity in Drinking Water}

Page 4 of 8

\begin{tabular}{lcr}
\hline Location & $\begin{array}{c}\text { No. of } \\
\text { Samples }\end{array}$ & Arithmetic Mean \pm 0 \\
\hline Surrounding Towns & & \\
$\quad$ Aiken SC, Post Office & 1 & $(8.35 \pm 5.03) E-10$ \\
Allendale SC, Post Office & 1 & $(3.56 \pm 9.76) E-10$ \\
Augusta GA, Post Office (8th & 1 & $(-0.95 \pm 5.52) E-10$ \\
St.) & & $(-0.23 \pm 6.75) E-10$ \\
Barnwell SC, Post Office & 1 & $(9.17 \pm 6.17) E-10$ \\
Bath SC, Post Office & 1 & $(4.16 \pm 5.77) E-10$ \\
Blackville SC, Post Office & 1 & $(1.12 \pm 6.32) E-10$ \\
Clearwater SC, Post Office & 1 & $(-0.75 \pm 2.47) E-09$ \\
Jackson SC, Post Office & 1 & $(-7.28 \pm 7.71) E-10$ \\
Langley SC, Post Office & 1 & $(-2.76 \pm 5.34) E-10$ \\
N. Augusta SC, Post Office & 1 & $(5.84 \pm 5.26) E-10$ \\
New Ellenton SC, Post Office & 1 & $(-2.60 \pm 5.76) E-10$ \\
ORA Site, ORA Building & 1 & $(-4.47 \pm 7.30) E-10$ \\
Sardis GA, Post Office (Farm & 1 & \\
St.) & & \\
Talatha SC, Tap Near Water & 1 & $(-2.06 \pm 5.90) E-10$ \\
Tank & & $(3.67 \pm 8.05) E-10$ \\
Waynesboro GA, Post Office & 1 & $(7.22 \pm 5.19) E-10$ \\
Williston SC, Post Office & 1 & \\
Din & &
\end{tabular}

Drin'sing Water from Water Treatment Plantsa

$\begin{array}{llr}\text { Beaufort Finished } & 1 & (4.44 \pm 5.88) E-10 \\ \text { Beaufort Raw } & 1 & (2.77 \pm 4.34) E-10 \\ \text { D-Area Finished } & 1 & (-3.60 \pm 6.16) E-10 \\ \text { D-Area Raw } & 1 & (2.49 \pm 5.13) E-10 \\ \text { N. Augusta Finished } & 1 & (2.01 \pm 5.20) E-10 \\ \text { N. Augusta Raw } & 1 & (3.88 \pm 5.43) E-10 \\ \text { Savannah Finished } & 1 & (1.41 \pm 4.85) E-10 \\ \text { Savannah Raw } & 1 & (-2.77 \pm 5.28) E-10\end{array}$

Gross A, $\mu \mathrm{Cl} / \mathrm{mL}$

On Slte

105-C C-Area Dom. Water

Faucet

$12 \quad(4.96 \pm 5.18) E-10 \quad(1.92 \pm 0.48) E-09 \quad(-0.05 \pm 1.78) E-$

a Annual result reported in arithmetic mean column 


\section{Table 26}

\section{Radloactlvity in Drinking Water}

Page 5 of 9

\begin{tabular}{|c|c|c|c|c|}
\hline Location & $\begin{array}{l}\text { No. of } \\
\text { Samples }\end{array}$ & Arlthmetlo Mean $\pm \sigma$ & Maximum \pm 0 & Minimum $\pm \sigma$ \\
\hline $\begin{array}{l}\text { 105-K K-Area Dom. Water } \\
\text { Faucet }\end{array}$ & 12 & $(4.38 \pm 4.91) E-10$ & $(1.24 \pm 0.44) E-09$ & $(-1.66 \pm 1.37) E-$ \\
\hline $\begin{array}{l}\text { 105-L L-Area Dom. Water } \\
\text { Faucet }\end{array}$ & 12 & $(4.83 \pm 3.01) E-10$ & $(9.99 \pm 4.51) E-10$ & $(-0.07 \pm 1.89) E-$ \\
\hline $\begin{array}{l}\text { 105-P P-Area Dom. Water } \\
\text { Faucet }\end{array}$ & 12 & $(6.22 \pm 6.62) E-10$ & $(2.11 \pm 0.53) E-09$ & $(-0.07 \pm 1.65)$ E-- \\
\hline $\begin{array}{l}\text { 221-F F-Area Dom. Water } \\
\text { Faucet }\end{array}$ & 12 & $(3.84 \pm 1.35) E-09$ & $(6.12 \pm 1.22) E-09$ & $(1.83 \pm 0.74) E-0$ \\
\hline $\begin{array}{l}221-\mathrm{H} H \text { H-Area Dom. Water } \\
\text { Faucet }\end{array}$ & 12 & $(3.26 \pm 2.05) E-09$ & $(7.44 \pm 1.21) E-09$ & $(6.49 \pm 3.82) E-1$ \\
\hline $\begin{array}{l}241-24 \mathrm{H} \text { H-Area Dom. Water } \\
\text { Faucet }\end{array}$ & 4 & $(4.17 \pm 3.04) E-09$ & $(8.41 \pm 1.14) E-09$ & $(1.48 \pm 0.58) E-09$ \\
\hline $\begin{array}{l}\text { 617-G Wackenhut Training } \\
\text { Facility }\end{array}$ & 4 & $(2.21 \pm 0.60) E-09$ & $(2.86 \pm 0.56) E-09$ & $(1.60 \pm 0.46) E-09$ \\
\hline $\begin{array}{l}\text { 618-G Class. Yard Lunch- } \\
\text { room }\end{array}$ & 4 & $(1.48 \pm 0.45) E-09$ & $(1.73 \pm 0.55) E-09$ & $(8.01 \pm 6.71) E-10$ \\
\hline $\begin{array}{l}\text { 661-G Firing Range } \\
\text { (pumphouse) }\end{array}$ & 4 & $(2.37 \pm 1.50) E-09$ & $(3.78 \pm 0.60) E-09$ & $(2.66 \pm 5.82) E-10$ \\
\hline $\begin{array}{l}\text { 679-T TNX Dom. Water } \\
\text { Faucet }\end{array}$ & 4 & $(2.16 \pm 1.88) E-09$ & $(4.42 \pm 2.10) E-09$ & $(3.95 \pm 5.72) E-10$ \\
\hline 681-1G Dom. Water Faucet & 2 & $(9.07 \pm 2.84) E-10$ & $(1.11 \pm 0.43) E-09$ & $(7.06 \pm 3.83) E-10$ \\
\hline 681-3G Dom. Water Faucet & 4 & $(1.19 \pm 0.18) E-09$ & $(1.36 \pm 0.47) E-09$ & $(9.73 \pm 3.92) E-10$ \\
\hline 701-12G Patrol Gate 7 & 4 & $(5.20 \pm 4.70) E-10$ & $(1.21 \pm 0.40) E-09$ & $(1.54 \pm 2.83) E-10$ \\
\hline 701-13G Patrol Gate 6 & 4 & $(1.58 \pm 0.43) E-09$ & $(1.95 \pm 0.48) E-09$ & $(1.04 \pm 0.38) E-09$ \\
\hline $\begin{array}{l}\text { 701-1F F-Area Dom. Water } \\
\text { Faucet }\end{array}$ & 4 & $(3.43 \pm 1.18) E-09$ & $(5.11 \pm 1.38) E-09$ & $(2.45 \pm 0.41) E-09$ \\
\hline $\begin{array}{l}701-1 \text { H H-Area Dom. Water } \\
\text { Faucet }\end{array}$ & 4 & $(3.90 \pm 2.15) E-09$ & $(5.41 \pm 0.90) E-09$ & $(7.15 \pm 4.43) E-10$ \\
\hline 701-3G Barnwell Gate & 3 & $(1.68 \pm 2.43) E-10$ & $(4.42 \pm 2.85) E-10$ & $(-0.23 \pm 1.85) E-10$ \\
\hline 701-4G Williston Gate & 4 & $(0.39 \pm 1.90) E-10$ & $(3.12 \pm 2.45) E-10$ & $(-1.27 \pm 1.15) E-10$ \\
\hline 701-5G Talatha Gate & 4 & $(1.32 \pm 0.10) E-08$ & $(1.46 \pm 0.12) E-08$ & $(1.23 \pm 0.12) E-08$ \\
\hline 701-6G Jackson Gate & 4 & $(2.82 \pm 0.45) E-09$ & $(3.43 \pm 0.61) E-09$ & $(2.46 \div 0.51) E-09$ \\
\hline 701-8G Patrol Gate 8 & 4 & $(6.68 \pm 5.52) E-10$ & $(1.38 \pm 0.49) E-09$ & $(2.03 \pm 2.06) E-10$ \\
\hline 703-A EOC (kitchen) & 4 & $(9.98 \pm 6.18) E-10$ & $(1.59 \pm 0.54) E-09$ & $(3.70 \pm 3.04) E-10$ \\
\hline $\begin{array}{l}\text { 704-F F-Area Dom. Water } \\
\text { Faucet }\end{array}$ & 4 & $(2.54 \pm 1.27) E-09$ & $(3.77 \pm 0.59) E-09$ & $(1.03 \pm 0.55) E-09$ \\
\hline $\begin{array}{l}\text { 704-H H-Area Dom. Water } \\
\text { Faucet }\end{array}$ & 4 & $(2.78 \pm 1.62) E-09$ & $(4.32 \pm 0.75) E-09$ & $(5.03 \pm 5.62) E-10$ \\
\hline
\end{tabular}


Table 26

Radloactivity in Drinking Water

Page 6 of 9

\begin{tabular}{|c|c|c|c|c|}
\hline Looation & $\begin{array}{l}\text { No. of } \\
\text { Samples }\end{array}$ & Arithmotio Mean $\pm \sigma$ & Maximum \pm 0 & Minimum $\pm \sigma$ \\
\hline $\begin{array}{l}\text { 704-s S-Area Dom. Water } \\
\text { Faucet }\end{array}$ & 4 & $(3.23 \pm 0.30) E-09$ & $(3.51 \pm 0.69) E-09$ & $(2.86 \pm 0.60) E-09$ \\
\hline $\begin{array}{l}\text { 708-A A-Area Cafe } \\
\text { (restroom) }\end{array}$ & 4 & $(1.23 \pm 0.41) E-09$ & $(1.62 \pm 0.80) E-09$ & $(8.07 \pm 5.21) E-10$ \\
\hline 735-7G Par Pond Lab & 3 & $(4.75 \pm 5.43) E-09$ & $(1.09 \pm 0.14) E-08$ & $(5.15 \pm 4.68) E-10$ \\
\hline $760-G$ Forestry Building & 4 & $(2.68 \pm 0.95) E-09$ & $(4.03 \pm 0.73) E-09$ & $(2.00 \pm 0.48) E-09$ \\
\hline $\begin{array}{l}\text { 772-F F-Area Dom. Water } \\
\text { Faucet }\end{array}$ & 4 & $(3.68 \pm 1.46) E-09$ & $(5.82 \pm 1.11) E-09$ & $(2.60 \pm 0.86) E-09$ \\
\hline $\begin{array}{l}\text { Central Shops 690-G Ford } \\
\text { Building }\end{array}$ & 4 & $(0.50 \pm 3.03) E-10$ & $(3.38 \pm 3.58) E-10$ & $(-3.40 \pm 3.28) E-10$ \\
\hline TC-1 B-Area Cafoteria & 4 & $(1.17 \pm 0.50) E-09$ & $(1.68 \pm 0.51) E-09$ & $(5.40 \pm 4.92) E-10$ \\
\hline Z-Area Building 704-Z & 4 & $(2.77 \pm 1.41) E-09$ & $(4.13 \pm 0.78) E-09$ & $(1.07 \pm 0.40) E-09$ \\
\hline \multicolumn{5}{|l|}{ Surrounding Towns } \\
\hline Aiken SC, Post Office & 2 & $(1.40 \pm 0.40) E-09$ & $(1.69 \pm 0.53) E-09$ & $(1.12 \pm 0.36) E-09$ \\
\hline Allendale SC, Post Office & 2 & $(-1.51 \pm 2.39) E-10$ & $(0.17 \pm 4.97) E-10$ & $(-3.20 \pm 2.90) E-10$ \\
\hline $\begin{array}{l}\text { Augusta GA, Post Office (8th } \\
\text { St.) }\end{array}$ & 2 & $(0.10 \pm 1.82) E-10$ & $(1.39 \pm 2.57) E-10$ & $(-1.19 \pm 1.06) E-10$ \\
\hline Barnwell SC, Post Office & 2 & $(0.87 \pm 1.33) E-10$ & $(1.82 \pm 2.04) E-10$ & $(-0.07 \pm 2.58) E-10$ \\
\hline Bath SC, Post Office & 2 & $(1.32 \pm 0.74) E-09$ & $(1.84 \pm 0.55) E-09$ & $(7.95 \pm 2.82) E-10$ \\
\hline Blackville SC, Post Office & 2 & $(1.94 \pm 0.58) E-10$ & $(2.34 \pm 4.97) E-10$ & $(1.53 \pm 3.34) E-10$ \\
\hline Clearwater SC, Post Office & 2 & $(1.16 \pm 0.10) E-09$ & $(1.23 \pm 0.75) E-09$ & $(1.09 \pm 1.22) E-09$ \\
\hline Jackson SC, Post Office & 2 & $(2.88 \pm 1.79) E-09$ & $(4.15 \pm 0.94) E-09$ & $(1.62 \pm 0.49) E-09$ \\
\hline Langley SC, Post Office & 2 & $(1.81 \pm 1.47) E-09$ & $(2.84 \pm 0.61) E-09$ & $(7.68 \pm 2.58) \mathrm{E}-10$ \\
\hline N. Augusta SC, Post Office & 2 & $(-8.31 \pm 8.79) E-11$ & $(-0.21 \pm 1.66) E-10$ & $(-1.45 \pm 1.42) E-10$ \\
\hline New Ellenton SC, Post Office & 2 & $(6.42 \pm 4.34) E-10$ & $(9.48 \pm 3.86) E-10$ & $(3.35 \pm 2.18) E-10$ \\
\hline ORA Site, ORA Building & 2 & $(6.69 \pm 6.47) E-10$ & $(1.13 \pm 0.41) E-09$ & $(2.12 \pm 1.70) E-10$ \\
\hline $\begin{array}{l}\text { Sardis GA, Post Office (Farm } \\
\text { St.) }\end{array}$ & 2 & $(-4.01 \pm 4.11) E-10$ & $(-1.10 \pm 8.79) E-10$ & $(-6.92 \pm 4.89) E-10$ \\
\hline $\begin{array}{l}\text { Talatha SC, Tap Near Water } \\
\text { Tank }\end{array}$ & 2 & $(3.98 \pm 2.33) \mathrm{E}-10$ & $(5.63 \pm 3.51) \mathrm{E}-10$ & $(2.33 \pm 1.88) E-10$ \\
\hline Waynesboro GA, Post Office & 2 & $(6.74 \pm 5.84) E-10$ & $(1.09 \pm 1.22) E-09$ & $(2.61 \pm 4.69) E-10$ \\
\hline Williston SC, Post Office & 2 & $(4.14 \pm 6.06) E-10$ & $(8.42 \pm 4.14) E-10$ & $(-0.14 \pm 3.79) E-10$ \\
\hline \multicolumn{5}{|c|}{ Drinking Water from Water Treatment Plants } \\
\hline Beaufort Finished & 12 & $(0.47 \pm 2.14) E-10$ & $(5.15 \pm 4.23) E-10$ & $(-2.47 \pm 2.01) E-10$ \\
\hline Beaufort Raw & 12 & $(1.88 \pm 1.94) E-10$ & $(5.49 \pm 2.67) E-10$ & $(-0.44 \pm 3.28) E-10$ \\
\hline D-Area Finished & 12 & $(-0.34 \pm 3.05) E-10$ & $(7.48 \pm 5.61) E-10$ & $(-2.91 \pm 2.54) E-10$ \\
\hline
\end{tabular}




\section{Table 26}

\section{Radloactivity in Drinking Water}

Page 7 of 9

\begin{tabular}{|c|c|c|c|c|}
\hline Looation & $\begin{array}{c}\text { No. of } \\
\text { samples }\end{array}$ & Arthmotio Moan $\pm \sigma$ & Maximum $\pm \sigma$ & Minimum $\pm \sigma$ \\
\hline D-Area Raw & 12 & $(0.65 \pm 1.07) E-10$ & $(2.16 \pm 2.43) E-10$ & $(-0.44 \pm 1.98) E-10$ \\
\hline N. Augusta Finished & 12 & $(0.11 \pm 1.11) E-10$ & $(2.21 \pm 2.48) E-10$ & $(-1.69 \pm 1.47) E-10$ \\
\hline N. Augusta Raw & 12 & $(0.25 \pm 1.37) E-10$ & $(1.22 \pm 2.18) E-10$ & $(-1.69 \pm 1.53) E-10$ \\
\hline Savannah Finished & 12 & $(1.04 \pm 2.17) E-10$ & $(5.00 \div 5.68) E-10$ & $(-2.29 \pm 1.93) E-10$ \\
\hline Savannah Raw & 12 & $(1.36 \pm 2.39) E-10$ & $(6.12 \pm 2.99) E-10$ & $(-1.22 \pm 1.18) E-10$ \\
\hline \multicolumn{5}{|l|}{ EMS samplor } \\
\hline Beautort Raw & 22 & $(1.62 \pm 2.16) E-10$ & $(5.66 \pm 3.64) E-10$ & $(-2.29 \pm 1.87) E-10$ \\
\hline \multicolumn{5}{|l|}{ Grose B, $\mu$ ClimL } \\
\hline \multicolumn{5}{|l|}{ On Slte } \\
\hline $\begin{array}{l}\text { 105-C C-Area Dom. Water } \\
\text { Faucet }\end{array}$ & 12 & $(9.17 \pm 3.71) E-10$ & $(1.54 \pm 0.49) E-09$ & $(3.69 \pm 4.12) E-10$ \\
\hline $\begin{array}{l}\text { 105-K K-Area Dom. Water } \\
\text { Faucet }\end{array}$ & 12 & $(3.24 \pm 1.32) E-09$ & $(5.46 \pm 0.64) E-09$ & $(1.74 \pm 0.51) E-09$ \\
\hline $\begin{array}{l}\text { 105-L L-Area Dom. Water } \\
\text { Faucet }\end{array}$ & 12 & $(1.38 \pm 0.36) E-09$ & $(1.93 \pm 0.54) E-09$ & $(6.24 \pm 4.58) E-10$ \\
\hline $\begin{array}{l}\text { 105-P P-Area Dom. Water } \\
\text { Faucet }\end{array}$ & 12 & $(2.05 \pm 0.74) E-09$ & $(4.03 \pm 0.72) E-09$ & $(1.33 \pm 0.43) E-09$ \\
\hline $\begin{array}{l}\text { 221-F F-Area Dom. Water } \\
\text { Faucet }\end{array}$ & 12 & $(3.96 \pm 1.21) E-09$ & $(6.49 \pm 0.74) E-09$ & $(1.71 \pm 0.56) E-09$ \\
\hline $\begin{array}{l}221-H \text { H-Area Dom. Water } \\
\text { Faucet }\end{array}$ & 12 & $(3.98 \pm 1.10) E-09$ & $(5.35 \pm 0.68) E-09$ & $(2.38 \pm 0.58) E-09$ \\
\hline $\begin{array}{l}241-24 \mathrm{H} \text { H-Area Dom, Water } \\
\text { Faucet }\end{array}$ & 4 & $(4.75 \pm 1.75) E-09$ & $(i .14 \pm 0.75) E-09$ & $(3.12 \pm 0.55) E-09$ \\
\hline $\begin{array}{l}617-G \text { Wackenhut Training } \\
\text { Facility }\end{array}$ & 4 & $(1.60 \pm 0.57) E-09$ & $(2.29 \pm 0.53) E-09$ & $(9.36 \pm 4.43) E-10$ \\
\hline $\begin{array}{l}\text { 618-G Class. Yard } \\
\text { Lunchroom }\end{array}$ & 4 & $(1.69 \pm 0.34) E-09$ & $(2.07 \pm 0.62) E-09$ & $(1.25 \pm 0.85) E-09$ \\
\hline $\begin{array}{l}661-G \text { Firing Range } \\
\text { (pumphouse) }\end{array}$ & 4 & $(1.56 \pm 0.80) E-09$ & $(2.23 \pm 0.50) E-09$ & $(4.03 \pm 5.72) E-10$ \\
\hline $\begin{array}{l}679-T \text { TNX Dom. Water } \\
\text { Faucet }\end{array}$ & 4 & $(4.09 \pm 0.46) E-09$ & $(4.59 \pm 0.68) E-09$ & $(3.55 \pm 0.62) E-09$ \\
\hline 681-1G Dom. Water Faucet & 2 & $(3.64 \pm 0.56) E-09$ & $(4.03 \pm 0.72) E-09$ & $(3.24 \pm 0.59) E-09$ \\
\hline 681-3G Dom. Water Faucet & 4 & $(3.45 \pm 0.40) E-09$ & $(3.72 \pm 0.66) E-09$ & $(2.86 \pm 0.57) E-09$ \\
\hline 701-12G Patrol Gate 7 & 4 & $(1.42 \pm 0.54) E-09$ & $(2.17 \pm 0.59) E-09$ & $(9.41 \pm 5.42) E-10$ \\
\hline 701-13G Patrol Gate 6 & 4 & $(1.50 \pm 0.56) E-09$ & $(2.00 \pm 0.58) E-09$ & $(7.28 \pm 4.27) E-10$ \\
\hline 701-1F F-Area Dom. Water & 4 & $(5.21 \pm 1.21) E-09$ & $(6.47 \pm 0.53) E-09$ & $(4.00 \pm 0.68) E-09$ \\
\hline
\end{tabular}


Table 26

Radioactivity in Drinking Water

Page 8 of 9

\begin{tabular}{|c|c|c|c|c|}
\hline Losation & $\begin{array}{c}\text { No. of } \\
\text { samplese }\end{array}$ & Arithmotlo Moen \pm 0 & Maximum $\pm \sigma$ & Minimum $\pm \sigma$ \\
\hline $\begin{array}{l}\text { 701-1H H-Area Dom. Water } \\
\text { Faucel }\end{array}$ & 4 & $(5.02 \pm 2.22) E-09$ & $(6.82 \pm 0.75) E-09$ & $(2.17 \pm 0.60) E-09$ \\
\hline 701-3G Barnwell Mate & 3 & $(3.70 \pm 1.86) E-10$ & $(4.86 \pm 4.27) E-10$ & $(1.56 \pm 4.82) E-10$ \\
\hline 701-4G Williston Gate & 4 & $(1.26 \pm 1.72) E-10$ & $(2.80 \pm 4.70) E-10$ & $(-0.73 \pm 4.01) E-10$ \\
\hline 701-5G Talatha Gate & 4 & $(7.39 \pm 1.89) E-09$ & $(8.92 \pm 0.79) E-09$ & $(4.94 \pm 0.63) E-09$ \\
\hline 701-6G Jackson Gate & 4 & $(2.47 \pm 0.33) E-09$ & $(2.86 \pm 0.60) E-09$ & $(2.16 \pm 0.50) E-09$ \\
\hline 701-8G Patrol Gate 8 & 4 & $(1.40 \pm 1.06) E-09$ & $(2.92 \pm 0.66) E-09$ & $(6.76 \pm 4.36) E-10$ \\
\hline 703-A EOC (kitchen) & 4 & $(1.17 \pm 0.43) E-09$ & $(1.62 \pm 0.52) E-09$ & $(7.03 \pm 4.68) E-10$ \\
\hline $\begin{array}{l}\text { 704-F F-Area Dom. Water } \\
\text { Faucet }\end{array}$ & 4 & $(3.36 \pm 1.67) E-09$ & $(5.69 \pm 0.50) E-09$ & $(2.01 \pm 0.56) E-09$ \\
\hline $\begin{array}{l}\text { 704-H H-Area Dom. Water } \\
\text { Faucet }\end{array}$ & 4 & $(6.77 \pm 3.79) E-09$ & $(1.19 \pm 0.10) E-08$ & $(3.59 \pm 0.51) E-09$ \\
\hline $\begin{array}{l}\text { 704-S S-Area Dom. Water } \\
\text { Faucet }\end{array}$ & 4 & $(3.77 \pm 0.55) E-09$ & $(4.29 \pm 0.68) E-09$ & $(3.12 \pm 0.56) E-09$ \\
\hline $\begin{array}{l}\text { 708-A A-Area Cafeteria } \\
\text { (restroom) }\end{array}$ & 4 & $(1.03 \pm 0.16) E-09$ & $(1.21 \pm 0.59) E-09$ & $(8.81 \pm 4.76) E-10$ \\
\hline 735-7G Par Pond Lab & 3 & $(2.99 \pm 4.19) E-09$ & $(7.72 \pm 0.83) E-09$ & $(-2.62 \pm 4.70) E-10$ \\
\hline $760-G$ Forestry Building & 4 & $(2.32 \pm 0.59) E-09$ & $(3.13 \pm 0.67) E-09$ & $(1.73 \pm 0.50) E-09$ \\
\hline $\begin{array}{l}772-F \text { F-Area Dom. Water } \\
\text { Faucet }\end{array}$ & 4 & $(4.37 \pm 0.79) E-09$ & $(4.99 \pm 0.72) E-09$ & $(3.24 \pm 0.62) E-09$ \\
\hline $\begin{array}{l}\text { Central Shops 690-G Ford } \\
\text { Building }\end{array}$ & 4 & $(7.45 \pm 7.65) E-10$ & $(1.74 \pm 0.53) E-09$ & $(0.50 \pm 7.75) E-10$ \\
\hline TC-1 B-Area Cafeteria & 4 & $(1.24 \pm 0.66) E-09$ & $(2.05 \pm 0.52) E-09$ & $(4.86 \pm 4.44) E-10$ \\
\hline Z-Area Building 704-Z & 4 & $(2.78 \pm 1.82) E-09$ & $(5.41 \pm 0.75) F-09$ & $(1.54 \pm 0.50) E-09$ \\
\hline \multicolumn{5}{|l|}{ Surrounding Towns } \\
\hline Aiken SC, Post Office & 2 & $(1.23 \pm 0.36) E-09$ & $(1.48 \pm 0.55) E-09$ & $(9.69 \pm 3.87) E-10$ \\
\hline Allendale SC, Post Office & 2 & $(1.39 \pm 0.55) E-09$ & $(1.77 \pm 0.62) E-09$ & $(9.99 \pm 4.20) E-10$ \\
\hline $\begin{array}{l}\text { Augusta GA, Post Office (8th } \\
\text { St.) }\end{array}$ & 2 & $(1.73 \pm 0.19) E-09$ & $(1.87 \pm 0.58) E-09$ & $(1.59 \pm 0.42) E-09$ \\
\hline Barnwell SC, Post Office & 2 & $(5.96 \pm 4.56) \mathrm{E}-10$ & $(9.19 \pm 5.38) E-10$ & $(2.74 \pm 3.48) E-10$ \\
\hline Bath SC, Post Office & 2 & $(1.22 \pm 0.14) E-09$ & $(1.32 \pm 0.40) E-09$ & $(1.13 \pm 0.54) E-09$ \\
\hline Blackville SC, Post Office & 2 & $(1.19 \pm 0.83) E-09$ & $(1.78 \pm 0.60) E-09$ & $(6.10 \pm 3.90) E-10$ \\
\hline Clearwater SC, Post Office & 2 & $(5.66 \pm 6.34) E-10$ & $(1.01 \pm 0.58) E-09$ & $(1.17 \pm 3.79) E-10$ \\
\hline Jackson SC, Post Office & 2 & $(1.77 \pm 1.39) E-09$ & $(2.76 \pm 0.64) E-09$ & $(7.84 \pm 3.89) E-10$ \\
\hline Langley SC, Post Office & 2 & $(2.02 \pm 0.79) E-09$ & $(2.58 \pm 0.60) E-09$ & $(1.45 \pm 0.40) E-09$ \\
\hline N. Augusta SC, Post Office & 2 & $(9.07 \pm 5.19) E-10$ & $(1.27 \pm 0.54) E-09$ & $(5.40 \pm 3.69) E-10$ \\
\hline
\end{tabular}




\section{Table 26}

\section{Radloactivity in Drinking Water}

Page 8 of 9

\begin{tabular}{|c|c|c|c|c|}
\hline Looation & $\begin{array}{l}\text { No. of } \\
\text { Samplece }\end{array}$ & Arithmotlo Moan \pm 0 & Maximum \pm 0 & Minimum $\pm \sigma$ \\
\hline New Ellenton SC, Post Office & 2 & $(1.28 \pm 1.38) E-09$ & $(2.25 \pm 0.58) E-09$ & $(3.07 \pm 3.45) E-10$ \\
\hline ORA Site, ORA Bullding & 2 & $(0.06 \pm 2.72) E-10$ & $(1.99 \pm 4.69) E-10$ & $(-1.86 \pm 3.06) E-10$ \\
\hline $\begin{array}{l}\text { Sardis GA, Post Office (Farm } \\
\text { St.) }\end{array}$ & 2 & $(1.07 \pm 0.81) E-09$ & $(1.65 \pm 0.63) E-09$ & $(5.03 \pm 4.03) E-10$ \\
\hline $\begin{array}{l}\text { Talatha SC, Tap Near Water } \\
\text { Tank }\end{array}$ & 2 & $(9.00 \pm 7.81) E-10$ & $(1.45 \pm 0.55) E-09$ & $(3.48 \pm 3.44) E-10$ \\
\hline Waynesboro GA, Post Office & 2 & $(6.40 \pm 6.04) E-10$ & $(1.07 \pm 0.57) E-09$ & $(2.12 \pm 3.85) E-10$ \\
\hline Williston SC, Post Office & 2 & $(7.82 \pm 0.87) E-10$ & $(8.44 \pm 5.50) E-10$ & $(7.21 \pm 3.89) E-10$ \\
\hline \multicolumn{5}{|c|}{ Drinking Water from Wator Troatment Plante } \\
\hline Beaufort Finished & 12 & $(1.81 \pm 0.51) E-09$ & $(2.76 \pm 0.6 .5) E-09$ & $(1.05 \pm 0.51) E-09$ \\
\hline Beaufort Raw & 12 & $(1.65 \pm 0.30) E-09$ & $(2.1 z \pm 0.62) E-09$ & $(1.19 \pm 0.50) E-09$ \\
\hline D-Area Finished & 12 & $(2.01 \pm 0.88) E-09$ & $(4.01 \pm 0.70) E-09$ & $(1.14 \pm 0.50) E-09$ \\
\hline D-Area Raw & 12 & $(1.56 \pm 0.45) E-09$ & $(2.16 \pm 0.61) E-09$ & $(8.45 \pm 3.66) E-10$ \\
\hline N. Augusta Finished & 12 & $(1.22 \pm(.63) E-09$ & $(2.40 \pm 0.60) E-09$ & $(3.44 \pm 5.24) E-10$ \\
\hline N. Augusta Raw & 12 & $(1.31 \pm 0.27) E-09$ & $(1.60 \pm 0.50) E-09$ & $(8.48 \pm 4.43) E-10$ \\
\hline Savannah Finished & 12 & $(1.67 \pm 0.47) E-09$ & $(2.46 \pm 0.55) E-09$ & $(9.43 \pm 4.90) E-10$ \\
\hline Savannah Raw & 12 & $(1.65 \pm 0.65) E-09$ & $(2.50 \pm 0.53) E-09$ & $(8.02 \pm 3.72) E-10$ \\
\hline \multicolumn{5}{|l|}{ EMS Sampler } \\
\hline Beaufort Raw & 22 & $(1.84 \pm 0.45) E-09$ & $(3.18 \pm 0.60) E-09$ & $(1.13 \pm 0.56) E-09$ \\
\hline
\end{tabular}



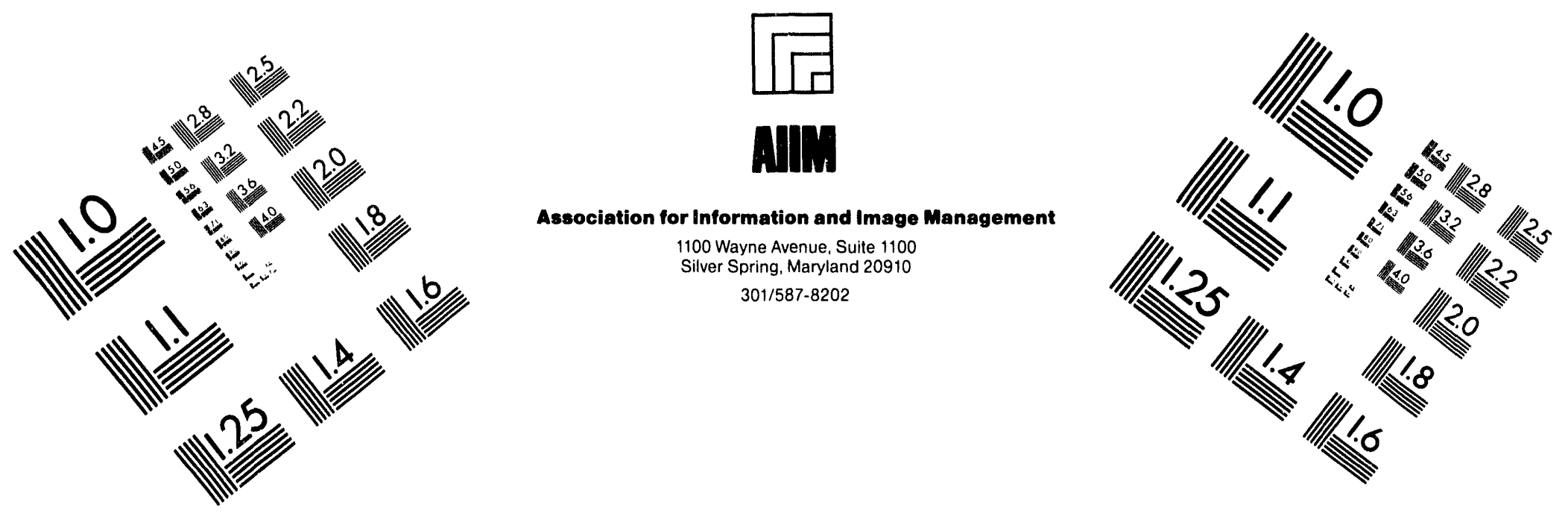

\section{Centimeter}

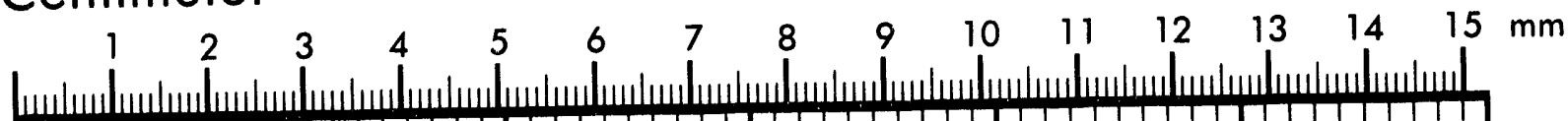

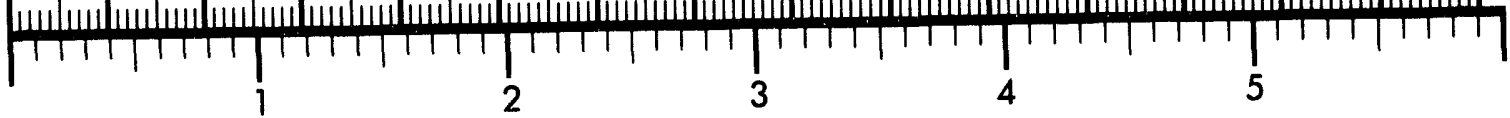

Inches
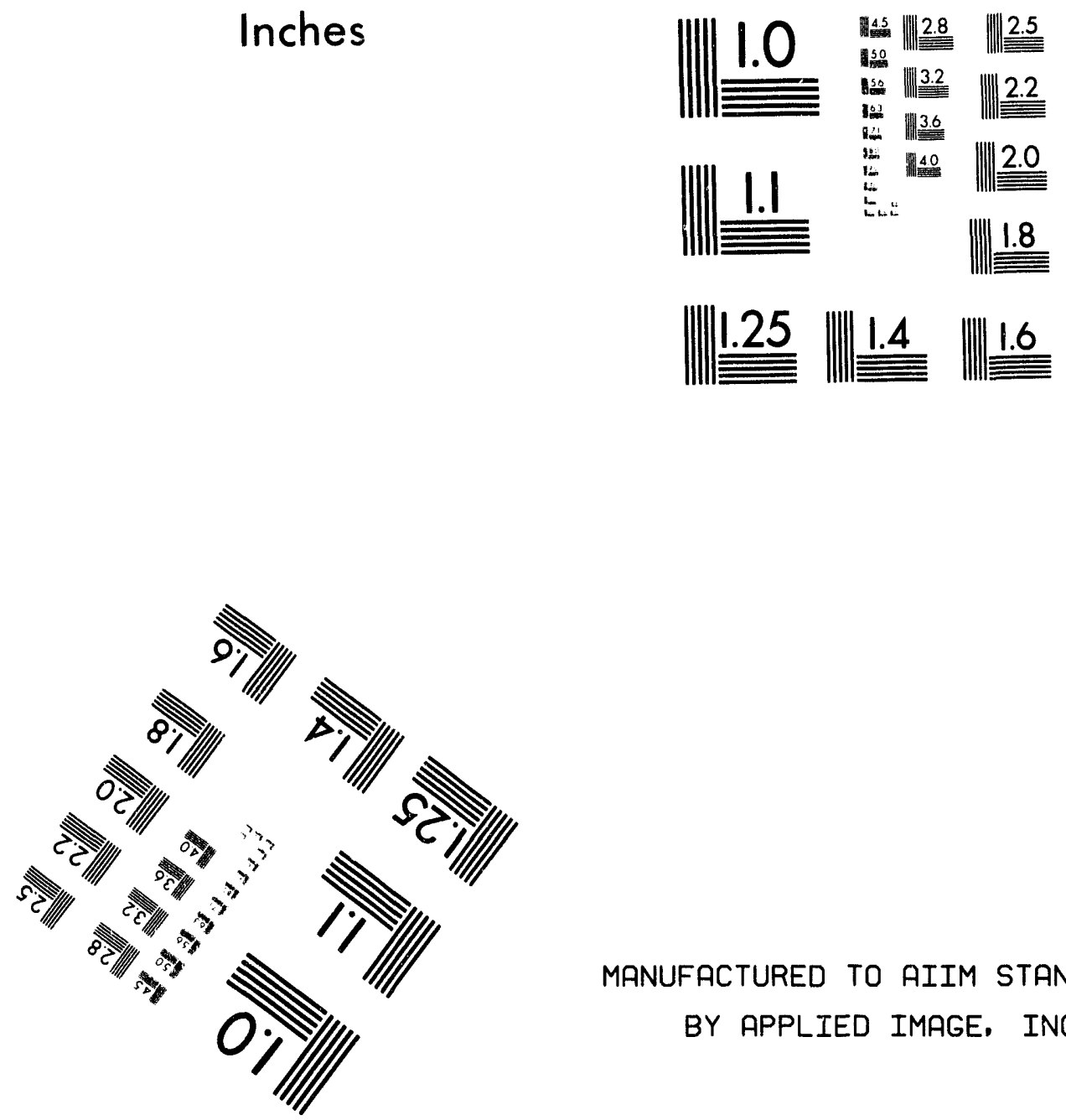

MANUFACTURED TO AIIM STANDARDS

BY APPLIED IMAGE, INC.

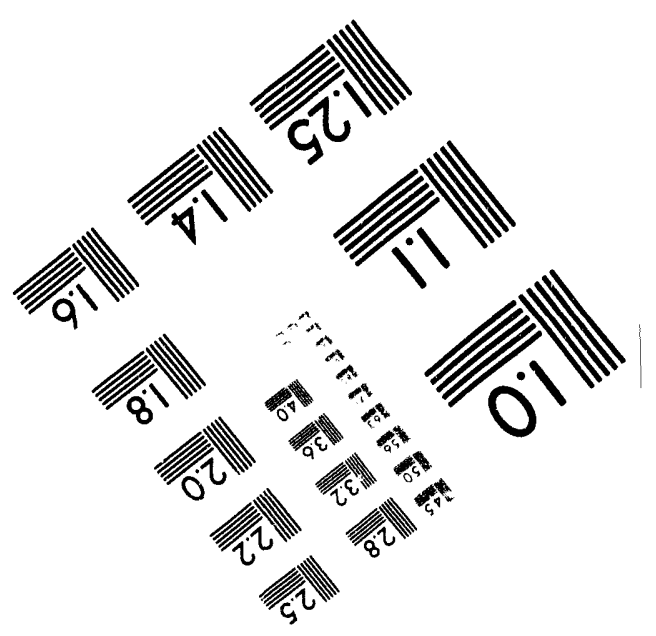



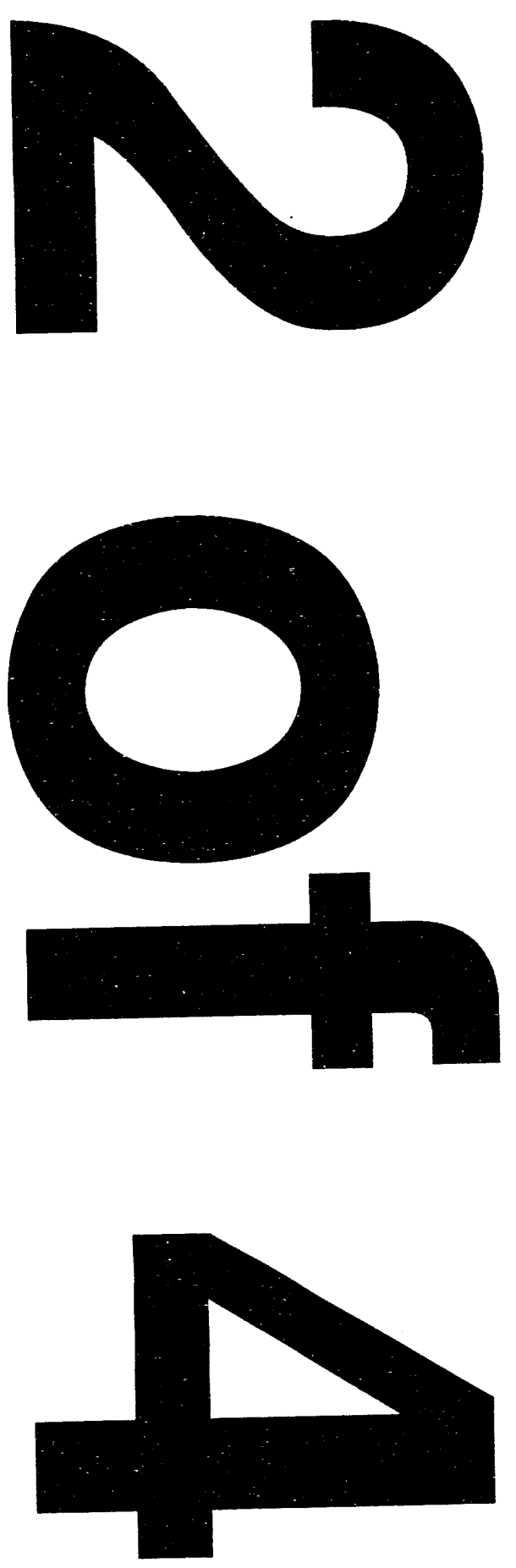
Table 27

Radioactivity in Rain Ion Columns

Page 1 of 3

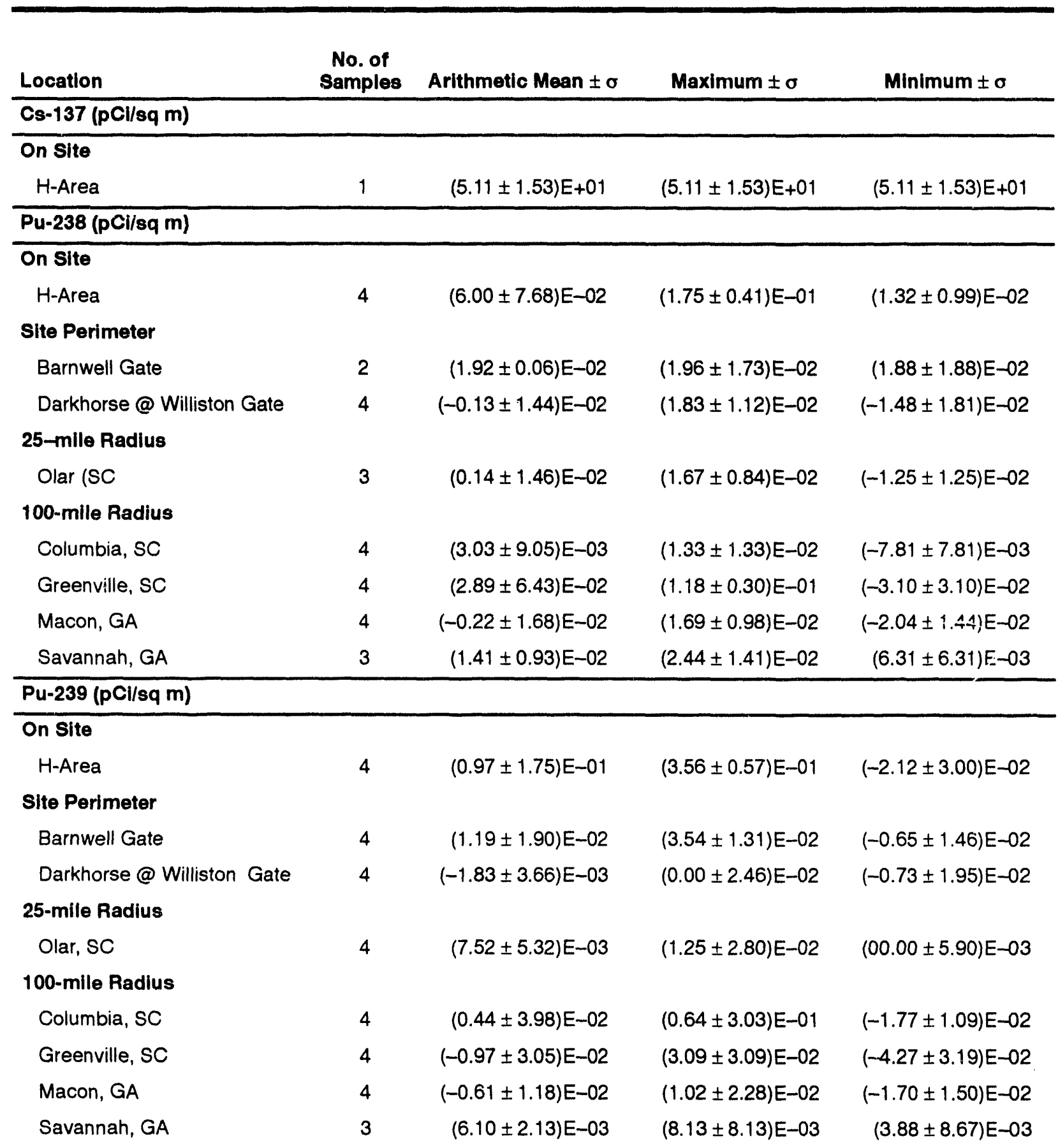




\section{Table 27}

Radioactivity in Rain Ion Columns

Page 2 of 3

\begin{tabular}{|c|c|c|c|c|}
\hline Location & $\begin{array}{c}\text { No. of } \\
\text { Samples }\end{array}$ & Arithmetic Mean $\pm \sigma$ & Maximum $\pm \sigma$ & Minimum $\pm \sigma$ \\
\hline \multicolumn{5}{|l|}{ Sr-89,90 (pCi/sq m) } \\
\hline \multicolumn{5}{|l|}{ On Site } \\
\hline H-Area & 4 & $(1.56 \pm 2.22) E+00$ & $(3.97 \pm 3.97) E+00$ & $(-1.04 \pm 4.44) E+00$ \\
\hline \multicolumn{5}{|l|}{ Site Perimeter } \\
\hline Barnwell Gate & 4 & $(1.72 \pm 2.89) E+00$ & $(5.15 \pm 4.16) E+00$ & $(-1.73 \pm 6.16) E+00$ \\
\hline Darkhorse@ WillistonGate & 4 & $(1.58 \pm 5.11) E+00$ & $(7.34 \pm 7.03) E+00$ & $(-4.88 \pm 4.37) E+00$ \\
\hline \multicolumn{5}{|l|}{ 25-mile Radius } \\
\hline Olar, SC & 4 & $(0.73 \pm 3.87) E+00$ & $(4.75 \pm 6.68) E+00$ & $(-4.00 \pm 4.27) E+00$ \\
\hline \multicolumn{5}{|l|}{ 100-mile Radius } \\
\hline Columbia, SC & 4 & $(0.88 \pm 1.92) E+00$ & $(3.45 \pm 4.64) E+00$ & $(-1.17 \pm 5.53) E+00$ \\
\hline Greenville, SC & 4 & $(-1.91 \pm 5.07) E+00$ & $(4.24 \pm 5.62) E+00$ & $(-8.17 \pm 4.19) E+00$ \\
\hline Macon, GA & 4 & $(4.68 \pm 9.30) E+00$ & $(1.79 \pm 0.82) E+01$ & $(-2.18 \pm 4.72) E+00$ \\
\hline Savannah, GA & 4 & $(1.81 \pm 3.87) E+00$ & $(6.97 \pm 5.40) E+00$ & $(-2.16 \pm 4.70) E+00$ \\
\hline \multicolumn{5}{|l|}{ Gross A (pCl/sq m) } \\
\hline \multicolumn{5}{|l|}{ On Site } \\
\hline H-Area & 13 & $(1.92 \pm 2.57) E+00$ & $(8.86 \pm 4.21) E+00$ & $(-0.29 \pm 2.07) E+00$ \\
\hline \multicolumn{5}{|l|}{ Site Perimeter } \\
\hline Barnwell Gate & 13 & $(-0.05 \pm 1.42) E+00$ & $(2.17 \pm 2.65) E+00$ & $(-2.97 \pm 1.70) E+00$ \\
\hline Darkhorse@ WillistonGate & 13 & $(-0.39 \pm 1.63) E+00$ & $(3.44 \pm 2.92) E+00$ & $(-1.72 \pm 1.43) E+00$ \\
\hline \multicolumn{5}{|l|}{ 25-mile Radius } \\
\hline Olar, SC & 13 & $(0.28 \pm 1.68) E+00$ & $(3.94 \pm 2.59) E+00$ & $(-1.80 \pm 1.47) E+00$ \\
\hline \multicolumn{5}{|l|}{ 100-mile Radius } \\
\hline Columbia, SC & 4 & $(6.53 \pm 4.11) E+00$ & $(1.17 \pm 0.77) E+01$ & $(1.87 \pm 4.39) E+00$ \\
\hline Greenville, SC & 4 & $(3.17 \pm 5.80) E+00$ & $(9.42 \pm 8.53) E+00$ & $(-2.71 \pm 2.40) E+00$ \\
\hline Macon, GA & 4 & $(6.00 \pm 7.71) E+00$ & $(1.47 \pm 1.02) E+01$ & $(-0.67 \pm 4.33) E+00$ \\
\hline Savannah, GA & 4 & $(4.82 \pm 5.06) E+00$ & $(1.01 \pm 0.77) E+01$ & $(-0.47 \pm 3.54) E+00$ \\
\hline \multicolumn{5}{|l|}{ Gross B (pCi/sq m) } \\
\hline \multicolumn{5}{|l|}{ On Site } \\
\hline H-Area & 13 & $(5.96 \pm 5.01) E+01$ & $(1.72 \pm 0.13) E+02$ & $(2.94 \pm 4.46) E+00$ \\
\hline \multicolumn{5}{|l|}{ Site Perimeter } \\
\hline Barnwell Gate & 13 & $(7.27 \pm 7.95) \mathrm{E}+01$ & $(2.50 \pm 0.13) E+02$ & $(4.75 \pm 4.59) E+00$ \\
\hline Darkhorse @ WillistonGate & 13 & $(5.66 \pm 4.28) \mathrm{E}+01$ & $(1.63 \pm 0.12) E+02$ & $(9.71 \pm 4.84) E+00$ \\
\hline
\end{tabular}


Table 27

Radioactivity in Rain Ion Columns

Page 3 of 3

\begin{tabular}{lcccr}
\hline Location & $\begin{array}{c}\text { No. of } \\
\text { Samples }\end{array}$ & Arithmetic Mean $\pm \sigma$ & Maximum $\pm \sigma$ & Minimum $0 \sigma$ \\
\hline $\begin{array}{l}\text { 25-mile Radius } \\
\text { Olar, SC }\end{array}$ & 13 & $(5.81 \pm 4.14) E+01$ & $(1.49 \pm 0.11) E+02$ & $(0.84 \pm 4.21) E+00$ \\
$\begin{array}{l}\text { 100-mile Radius } \\
\text { Columbia, SC }\end{array}$ & 4 & $(3.14 \pm 1.87) E+02$ & $(5.79 \pm 0.32) E+02$ & $(1.39 \pm 0.17) E+02$ \\
$\begin{array}{l}\text { Greenville, SC } \\
\text { Macon, GA }\end{array}$ & 4 & $(3.62 \pm 3.20) E+02$ & $(7.06 \pm 0.31) E+02$ & $(5.07 \pm 1.28) E+01$ \\
Savannah, GA & 4 & $(2.97 \pm 3.56) E+02$ & $(7.98 \pm 0.31) E+02$ & $(-8.14 \pm 7.80) E+00$ \\
& 4 & $(1.47 \pm 1.36) E+02$ & $(2.82 \pm 0.24) E+02$ & $(1.68 \pm 1.16) E+01$
\end{tabular}




\section{Table 28}

\section{Tritium in Rainwater}

Page 1 of 2

\begin{tabular}{|c|c|c|c|c|}
\hline Location & $\begin{array}{c}\text { No. of } \\
\text { Samples }\end{array}$ & Arithmetic Mean $\pm \sigma$ & Maximum $\pm \sigma$ & Minimum $\pm \sigma$ \\
\hline \multicolumn{5}{|l|}{$(\mu \mathrm{Cl} / \mathrm{mL})$} \\
\hline \multicolumn{5}{|l|}{ On Site } \\
\hline A-Area & 26 & $(0.68 \pm 1.20) E-06$ & $(5.36 \pm 0.47) E-06$ & $(-4.20 \pm 3.88) E-07$ \\
\hline Burial Ground North & 26 & $(1.11 \pm 1.82) E-05$ & $(6.87 \pm 0.14) E-05$ & $(3.14 \pm 4.04) E-07$ \\
\hline Burial Ground South & 26 & $(1.51 \pm 1.59) E-05$ & $(5.24 \pm 0.12) E-05$ & $(3.37 \pm 4.01) E-07$ \\
\hline F-Area & 26 & $(5.56 \pm 9.73) E-06$ & $(4.55 \pm 0.11) E-05$ & $(-1.17 \pm 3.83) E-07$ \\
\hline H-Area & 25 & $(4.25 \pm 3.05) E-05$ & $(1.10 \pm 0.02) E-04$ & $(5.77 \pm 3.99) E-07$ \\
\hline \multicolumn{5}{|l|}{ Site Perimeter } \\
\hline Allendale Gate & 25 & $(1.07 \pm 3.58) E-07$ & $(1.15 \pm 0.42) E-06$ & $(-6.32 \pm 3.70) E-07$ \\
\hline Barnwell Gate & 25 & $(0.63 \pm 1.01) E-06$ & $(3.82 \pm 0.46) E-06$ & $(-4.74 \pm 3.88) E-07$ \\
\hline D-Area & 24 & $(3.03 \pm 4.92) E-06$ & $(2.23 \pm 0.07) E-05$ & $(-0.30 \pm 2.83) E-07$ \\
\hline Darkhorse @ Williston Gate & 26 & $(6.00 \pm 7.92) E-07$ & $(2.85 \pm 0.43) E-06$ & $(-3.72 \pm 3.92) E-07$ \\
\hline East Talatha & 25 & $(8.75 \pm 9.10) E-07$ & $(2.90 \pm 0.43) E-06$ & $(-1.79 \pm 3.12) E-07$ \\
\hline Green Pond & 26 & $(6.59 \pm 8.58) E-07$ & $(4.51 \pm 0.46) E-06$ & $(-1.87 \pm 3.94) E-07$ \\
\hline Highway $125 @$ Road A-14 & 25 & $(0.75 \pm 1.29) \mathrm{E}-06$ & $(4.67 \pm 0.47) E-06$ & $(-4.91 \pm 4.02) E-07$ \\
\hline Highway 21/167 & 25 & $(2.81 \pm 5.27) \mathrm{E}-07$ & $(2.43 \pm 0.43) E-06$ & $(-2.74 \pm 3.87) E-07$ \\
\hline Highway39 @ Williston Gate & 26 & $(4.60 \pm 8.13) E-07$ & $(3.10 \pm 0.28) E-06$ & $(-4.77 \pm 3.70) E-07$ \\
\hline Jackson & 26 & $(5.87 \pm 9.88) \mathrm{E}-07$ & $(3.56 \pm 0.45) E-06$ & $(-5.05 \pm 3.72) E-07$ \\
\hline Patterson Mill Road & 26 & $(2.65 \pm 4.10) E-07$ & $(1.24 \pm 0.41) E-06$ & $(-4.26 \pm 3.81) E-07$ \\
\hline Talatha Gate & 26 & $(0.64 \pm 1.34) E-06$ & $(4.36 \pm 0.47) E-06$ & $(-6.05 \pm 3.86) E-07$ \\
\hline West Jackson & 26 & $(0.89 \pm 1.27) E-06$ & $(4.15 \pm 0.47) E-06$ & $(-6.84 \pm 3.85) E-07$ \\
\hline Windsor Road & 26 & $(6.02 \pm 9.71) E-07$ & $(3.14 \pm 0.44) E-06$ & $(-7.46 \pm 3.89) E-07$ \\
\hline \multicolumn{5}{|l|}{ 25-Mile-Radius } \\
\hline Aiken Airport & 26 & $(1.20 \pm 3.34) E-07$ & $(7.77 \pm 4.13) \mathrm{E}-07$ & $(-6.23 \pm 2.77) E-07$ \\
\hline Aiken State Park & 26 & $(3.35 \pm 6.43) E-07$ & $(2.01 \pm 0.42) E-06$ & $(-7.47 \pm 3.82) E-07$ \\
\hline Allendale, SC & 26 & $(1.26 \pm 3.02) \mathrm{E}-07$ & $(8.67 \pm 3.05) \mathrm{E}-07$ & $(-4.13 \pm 4.21) E-07$ \\
\hline Augusta, GA & 23 & $(1.23 \pm 5.24) E-07$ & $(1.22 \pm 0.42) E-06$ & $(-6.01 \pm 3.83) E-07$ \\
\hline Highway 301 @ State Line & 18 & $(-0.39 \pm 4.08) E-07$ & $(1.02 \pm 0.41) E-06$ & $(-9.61 \pm 3.61) E-07$ \\
\hline Langley, SC & 26 & $(1.93 \pm 4.14) E-07$ & $(1.13 \pm 0.41) E-06$ & $(-4.12 \pm 3.80) E-07$ \\
\hline Lees, SC & 26 & $(1.49 \pm 4.14) E-07$ & $(1.02 \pm 0.31) E-06$ & $(-6.32 \pm 3.86) E-07$ \\
\hline Olar, SC & 26 & $(1.55 \pm 4.01) E-07$ & $(1.16 \pm 0.42) E-06$ & $(-6.63 \pm 3.96) E-07$ \\
\hline Perkins, GA & 25 & $(0.54 \pm 2.53) E-07$ & $(6.60 \pm 4.12) E-07$ & $(-4.89 \pm 3.90) E-07$ \\
\hline
\end{tabular}


Table 28

Tritium in Rainwater

Page 2 of 2

\begin{tabular}{|c|c|c|c|c|}
\hline Location & $\begin{array}{l}\text { No. of } \\
\text { Samples }\end{array}$ & Arithmetic Mean $\pm \sigma$ & Maximum $\pm \sigma$ & Minimum $\pm \sigma$ \\
\hline South Richmond, GA & 26 & $(1.31 \pm 4.72) \mathrm{E}-07$ & $(1.41 \pm 0.32) E-06$ & $(-5.92 \pm 3.78) E-07$ \\
\hline Springfield, SC & 25 & $(2.70 \pm 7.52) E-07$ & $(3.15 \pm 0.25) E-06$ & $(-9.97 \pm 3.59) E-07$ \\
\hline Waynesboro, GA & 26 & $(3.18 \pm 7.30) E-07$ & $(2.56 \pm 0.43) E-06$ & $(-6.51 \pm 3.81) E-07$ \\
\hline \multicolumn{5}{|l|}{ 100-Mile-Radius } \\
\hline Columbia, SC & 4 & $(1.46 \pm 4.01) E-07$ & $(7.32 \pm 4.02) E-07$ & $(-1.58 \pm 3.90) E-07$ \\
\hline Greenville, SC & 4 & $(-1.52 \pm 4.12) E-07$ & $(3.30 \pm 4.08) E-07$ & $(-6.77 \pm 3.81) E-07$ \\
\hline Macon, GA & 4 & $(0.94 \pm 1.89) E-07$ & $(2.71 \pm 2.95) E-07$ & $(-1.05 \pm 3.87) E-07$ \\
\hline Savannah, GA & 4 & $(2.03 \pm 2.26) E-07$ & $(5.39 \pm 4.09) E-07$ & $(0.65 \pm 4.08) E-07$ \\
\hline
\end{tabular}


Table 29

Radioactivity in River and Stream Sediment

Page 1 of 3

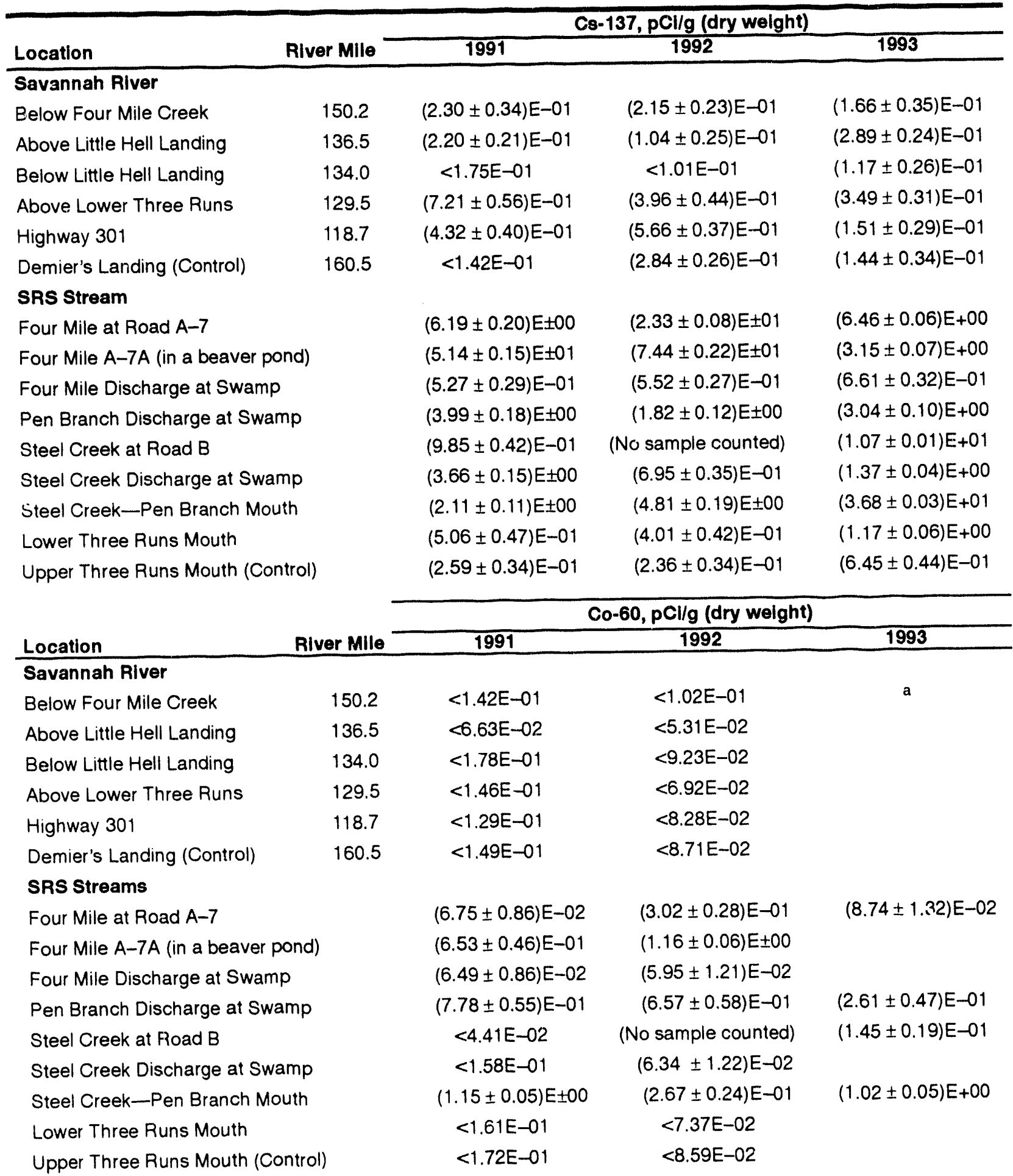

a Blank space indicates activity was below the lower limit of detection. 
Table 29

Radioactivity in River and Stream Sediment

Page 2 of 3

\begin{tabular}{l} 
Location \\
\hline Savannah River \\
Below Four Mile Creek \\
Above Little Hell Landing \\
Below Little Hell Landing \\
Above Lower Three Runs \\
Highway 301 \\
Demier's Landing (Control) \\
SRS Streams \\
Four Mile at Road A-7 \\
Four Mile A-7A (in a beaver pond) \\
Four Mile Discharge at Swamp \\
Pen Branch Discharge at Swamp \\
Steel Creek at Road B \\
Steel Creek Discharge at Swamp \\
Steel Creek-Pen Branch Mouth \\
Lower Three Runs Mouth \\
Upper Three Runs Mouth Control
\end{tabular}

Sr-90, pCI/g (dry weight)

\begin{tabular}{rrrr} 
& \multicolumn{1}{c}{1991} & \multicolumn{1}{c}{1992} & \multicolumn{1}{c}{1993} \\
\cline { 2 - 4 } 150.2 & $(1.62 \pm 1.89) E-02$ & $(1.12 \pm 1.84) E-02$ & $(5.40 \pm 3.17) E-02$ \\
136.5 & $(-0.17 \pm 1.76) E-02$ & $(1.29 \pm 2.13) E-02$ & $(-2.49 \pm 1.41) E-02$ \\
134.0 & $(-1.36 \pm 2.24) E-02$ & $(-0.94 \pm 5.13) E-02$ & $(-0.18 \pm 1.91) E-02$ \\
129.5 & $(3.85 \pm 2.06) E-02$ & $(-1.84 \pm 4.88) E-02$ & $(-2.43 \pm 1.38) E-02$ \\
118.7 & $(2.10 \pm 1.72) E-02$ & $(3.10 \pm 5.44) E-02$ & $(-1.45 \pm 1.47) E-02$ \\
160.5 & $(-2.42 \pm 2.41) E-02$ & $(3.03 \pm 1.65) E-02$ & $(6.68 \pm 3.23) E-02$
\end{tabular}

$(4.12 \pm 0.39) E-01$

(2.34 \pm 0.13$) \mathrm{E} \pm 00$

$(2.47 \pm 0.26) E-01$

$(9.11 \pm 0.53) E-01$

$(1.85 \pm 0.12) \mathrm{E} \pm 00$

$(4.35 \pm 1.71) E-02$

$(5.10 \pm 2.11) E-02$

$(9.63 \pm 5.69) \mathrm{E}-02$

$(1.15 \pm 1.56) E-02$

$(1.24 \pm 0.25) E-01$

$(-3.34 \pm 4.67)$ E-02

(3.48 \pm 2.38$) E-02$

$(5.10 \pm 1.95) E-02$

$(-6.87 \pm 6.54) E-02$

$(-0.16 \pm 2.23) E-02$

$(4.25 \pm 2.17) E-02$

$(4.73 \pm 5.10) E-02$

$(2.71 \pm 1.62) \mathrm{E}-02$

(No sample counted)

$(1.78 \pm 4.95) E-02$

$(8.22 \pm 2.57) E-02$

$(1.29 \pm 0.23) E-01$

$(3.70 \pm 2.05) E-02$

$(-0.96 \pm 2.20) E-02$

$(5.59 \pm 3.01) E-02$

$(1.30 \pm 1.65) E-02$

(3.49 \pm 3.10$) E-02$

\begin{tabular}{lrrrr} 
& & \multicolumn{3}{c}{ Pu-238, pCl/g (dry weight) } \\
\cline { 3 - 5 } Location & River Mile & \multicolumn{1}{c}{1991} & \multicolumn{1}{c}{1992} & \multicolumn{1}{c}{1993} \\
\hline Savannah River & & & & \\
Below Four Mile Creek & 150.2 & $(2.89 \pm 4.20) E-03$ & $(1.14 \pm 0.14) E-02$ & $(-2.15 \pm 2.15) E-04$ \\
Above Little Hell Landing & 136.5 & $(1.52 \pm 0.23) E-03$ & $(1.45 \pm 0.23) E-02$ & $(2.02 \pm 1.78) E-04$ \\
Below Little Hell Landing & 134.0 & $(8.49 \pm 0.33) E-04$ & $(1.17 \pm 0.94) E-03$ & $(3.78 \pm 1.89) E-04$ \\
Above Lower Three Runs & 129.5 & $(2.93 \pm 1.20) E-04$ & $(3.57 \pm 3.14) E-04$ & $(-4.97 \pm 4.97) E-04$ \\
Highway 301 & 118.7 & $(1.42 \pm 1.31) E-04$ & $(2.06 \pm 1.58) E-03$ & $(00.00 \pm 5.65) E-04$ \\
Demier's Landing (Control) & 160.5 & $(-0.80 \pm 1.00) E-04$ & $(3.60 \pm 0.98) E-03$ & $(2.00 \pm 2.00) E-04$ \\
SRS Streams & & & & \\
Four Mile at Road A-7 & & $(7.78 \pm 0.26) E-02$ & $(2.93 \pm 0.08) E-01$ & $(7.50 \pm 0.41) E-02$ \\
Four Mile A-7A (in a beaver pond) & & $(1.18 \pm 0.01) E \pm 00$ & $(1.82 \pm 0.04) E+00$ & $(3.08 \pm 0.26) E-02$ \\
Four Mile Discharge at Swamp & $(1.88 \pm 0.27) E-03$ & $(2.51 \pm 1.26) E-03$ & $(4.85 \pm 0.86(E-03$ \\
Pen Branch Discharge at Swamp & $(1.00 \pm 0.13) E-02$ & $(1.38 \pm 0.14) E-02$ & $(4.76 \pm 1.12) E-03$ \\
Steel Creek at Road B & $(1.46 \pm 0.17) E-03$ & $(1.55 \pm 0.70) E-03$ & $(5.09 \pm 1.05) E-03$ \\
Steel Creek Discharge at Swamp & $(2.90 \pm 0.56) E-03$ & $(3.12 \pm 1.05) E-03$ & $2.63 \pm 0.44) E-03$ \\
Steel Creek-Pen Branch Mouth & $(1.25 \pm 0.10) E-02$ & $(1.66 \pm 0.24) E-02$ & $(6.08 \pm 0.30) E-02$ \\
Lower Three Runs Mouth & $(1.05 \pm 0.22) E-03$ & $(2.04 \pm 0.59) E-03$ & $(00.00 \pm 4.13) E-04$ \\
Upper Three Runs Mouth (Control) & $(5.15 \pm 1.88) E-03$ & $(1.70 \pm 0.38) E-03$ & $(3.24 \pm 0.65) E-03$
\end{tabular}




\section{Table 29}

\section{Radioactivity in River and Stream Sediment}

Page 3 of 3

\begin{tabular}{lrrrr}
\hline & & \multicolumn{3}{c}{ Pu-239, PCl/g (dry welght) } \\
\cline { 5 - 5 } Location & River Mlle & 1991 & 1992 & 1993 \\
\hline Savannah River & & & & \\
Below Four Mile Creek & 150.2 & $(0.62 \pm 1.38) E-03$ & $(8.61 \pm 1.15) E-03$ & $(4.29 \pm 3.03) E-04$ \\
Above Little Hell Landing & 136.5 & $(8.71 \pm 1.51) E-04$ & $(1.60 \pm 0.71) E-03$ & $(1.21 \pm 0.32) E-03$ \\
Below Little Hell Landing & 134.0 & $(1.91 \pm 1.71) E-04$ & $(6.64 \pm 9.40) E-04$ & $(7.54 \pm 2.98) E-04$ \\
Above Lower Three Runs & 129.5 & $(2.32 \pm 0.23) E-03$ & $(6.21 \pm 2.54) E-04$ & $(2.48 \pm 5.55) E-04$ \\
Highway 301 & 118.7 & $(1.48 \pm 0.19) E-03$ & $(-0.55 \pm 1.66) E-03$ & $(00.00 \pm 5.64) E-04$ \\
Demier's Landing & 160.5 & $(6.42 \pm 8.30) E-05$ & $(2.76 \pm 0.83) E-03$ & $(9.97 \pm 3.46) E-04$ \\
SRS Streams & & & & \\
Four Mile at Road A-7 & & $(3.16 \pm 0.16) E-02$ & $(1.09 \pm 0.05) E-01$ & $(3.06 \pm 0.25) E-02$ \\
Four Mile A-7A (in a beaver pond) & & $(3.73 \pm 0.06) E-01$ & $(6.03 \pm 0.15) E-01$ & $(1.55 \pm 0.18) E-02$ \\
Four Mile Discharge at Swamp & & $(3.07 \pm 0.33) E-03$ & $(6.01 \pm 1.41) E-03$ & $(3.76 \pm 076) E-03$ \\
Pen Branch Discharge at Swamp & & $(2.78 \pm 0.21) E-02$ & $(2.34 \pm 0.17) E-02$ & $(2.15 \pm 0.22) E-02$ \\
Steel Creek at Road B & & $(4.31 \pm 0.26) E-03$ & $(3.42 \pm 0.83) E-03$ & $(1.24 \pm 0.16) E-02$ \\
Steel Creek Discharge at Swamp & & $(7.14 \pm 0.83) E-03$ & $(1.41 \pm 0.66) E-03$ & $(2.62 \pm 0.45) E-03$ \\
Steel Creek-Pen Branch Mouth & & $(2.56 \pm 0.14) E-02$ & $(1.52 \pm 0.22) E-02$ & $(8.15 \pm 0.36) E-02$ \\
Lower Three Runs Mouth & & $(1.71 \pm 0.23) E-03$ & $(6.29 \pm 3.86) E-04$ & $(1.75 \pm 0.82) E-03$ \\
Upper Three Runs Mouth (Control) & $(6.48 \pm 1.66) E-03$ & $(1.51 \pm 0.38) E-03$ & $(1.52 \pm 0.14) E-02$
\end{tabular}




\section{Table 30}

Radioactivity in Soil

Page 1 of 1

\begin{tabular}{|c|c|c|c|c|}
\hline \multirow[b]{2}{*}{ Location } & \multicolumn{4}{|c|}{$p C l / g \pm \sigma ; d r y$ welght; $0-8 \mathrm{~cm}$ depth } \\
\hline & Sr-90 & Cs-137 & Pu-238 & Pu-239 \\
\hline \multicolumn{5}{|l|}{ F-Area } \\
\hline 2000 Feet East & $(-0.58 \pm 1.83) E-02$ & $(8.71 \pm 0.37) E-01$ & $(6.30 \pm 0.25) E-02$ & $(3.60 \pm 0.09) E-01$ \\
\hline 2000 Feet North & $(-0.15 \pm 1.55) E-02$ & $(2.84 \pm 0.23) E-01$ & $(2.95 \pm 0.23) E-02$ & $(1.25 \pm 0.05) E-01$ \\
\hline 2000 Feet South & $(1.33 \pm 0.27) E-01$ & $(1.26 \pm 0.07) E+00$ & $(7.84 \pm 0.39) E-02$ & $(6.83 \pm 0.36) E-02$ \\
\hline 2000 Feet West & $(0.23 \pm 1.60) E-02$ & $(5.29 \pm 0.29) E-01$ & $(6.51 \pm 0.48) E-02$ & $(8.64 \pm 0.56) E-02$ \\
\hline \multicolumn{5}{|l|}{ H-Area } \\
\hline 2000 Feet East & $(0.98 \pm 1.72) E-02$ & $(7.37 \pm 0.39) E-01$ & $(1.02 \pm 0.09) E-02$ & $(1.78 \pm 0.05) E-01$ \\
\hline 2000 Feet North & $(-0.29 \pm 1.75) E-02$ & $(6.09 \pm 0.32) E-01$ & $(1.61 \pm 0.11) E-02$ & $(1.43 \pm 0.04) E-01$ \\
\hline 2000 Feet South & $(5.18 \pm 1.87) E-02$ & $(1.57 \pm 0.05) E+00$ & $(2.62 \pm 0.15) E-02$ & $(1.51 \pm 0.05) E-01$ \\
\hline 2000 Feet West & $(8.63 \pm 1.86) E-02$ & $(1.03 \pm 0.04) E+00$ & $(2.47 \pm 0.26) E-02$ & $(1.23 \pm 0.06) E-01$ \\
\hline \multicolumn{5}{|l|}{ S-Area } \\
\hline$\# 1$ & $(3.31 \pm 1.76) E-02$ & $(3.53 \pm 0.30) E-01$ & $(7.10 \pm 0.70) E-03$ & $(5.40 \pm 0.22) E-02$ \\
\hline$\# 2$ & $(0.37 \pm 2.60) E-02$ & $(1.09 \pm 0.24) E-01$ & $(3.25 \pm 2.86) E-04$ & $(1.40 \pm 0.52) E-03$ \\
\hline$\# 3$ & $(1.03 \pm 1.56) E-02$ & a & $(3.55 \pm 0.21) E-02$ & $(4.18 \pm 0.23) E-02$ \\
\hline$\# 4$ & $(-0.57 \pm 1.49) E-02$ & & $(0.99 \pm 2.62) E-04$ & $(1.88 \pm 0.46) E-03$ \\
\hline \multicolumn{5}{|l|}{ Z-Area } \\
\hline$\# 1$ & $(-0.02 \pm 2.31) E-02$ & $(7.23 \pm 2.36) E-02$ & $(1.04 \pm 0.29) E-03$ & $(2.62 \pm 0.46) E-03$ \\
\hline$\# 3$ & $(8.25 \pm 2.40) E-02$ & $(2.09 \pm 0.22) E-01$ & $(7.68 \pm 3.08) E-04$ & $(6.31 \pm 0.77) E-03$ \\
\hline$\# 5$ & $(-0.30 \pm 1.56) E-02$ & $(7.15 \pm 0.37) E-01$ & $(6.63 \pm 1.14) E-03$ & $(5.04 \pm 0.31) E-02$ \\
\hline$\# 7$ & $(1.22 \pm 1.66) E-02$ & $(8.20 \pm 0.48) E-01$ & $(5.96 \pm 0.95) E-03$ & $(3.76 \pm 0.24) E-02$ \\
\hline \multicolumn{5}{|l|}{ Burial Ground } \\
\hline $643-26 E-1$ & $(2.64 \pm 1.84) E-02$ & $(2.71 \pm 0.27) E-01$ & Not analyzed & Not analyzed \\
\hline $643-26 E-2$ & $(2.49 \pm 1.72) E-02$ & $(1.32 \pm 0.20) E-01$ & Not analyzed & Not analyzed \\
\hline \multicolumn{5}{|l|}{ Site Perimeter } \\
\hline NE Quadrant & $(-0.37 \pm 1.61) E-02$ & $(4.11 \pm 0.27) E-01$ & $(5.24 \pm 3.87) E-04$ & $(1.09 \pm 0.10) E-02$ \\
\hline NW Quadrant & $(-0.18 \pm 1.62) E-02$ & $(6.52 \pm 0.34) E-01$ & $(1.05 \pm 0.37) E-03$ & $(1.47 \pm 0.11) E-02$ \\
\hline SE Quadrant & $(0.95 \pm 1.63) E-02$ & $(5.14 \pm 0.30) E-01$ & $(7.23 \pm 2.28) E-04$ & $(2.01 \pm 0.10) E-02$ \\
\hline SW Quadrant & $(0.30 \pm 1.64) E-02$ & $(5.56 \pm 0.30) E-01$ & $(1.87 \pm 0.72) E-03$ & $(1.47 \pm 0.24) E-02$ \\
\hline \multicolumn{5}{|l|}{ 100-Mile Radius } \\
\hline Clinton, SC & $(7.72 \pm 1.87) E-02$ & $(3.52 \pm 0.28) E-01$ & $(4.23 \pm 3.63) E-04$ & $(8.35 \pm 1.45) E-03$ \\
\hline Savannah, GA & $(-0.18 \pm 1.48) E-02$ & $(4.41 \pm 1.40) E-02$ & $(1.05 \pm 0.45) E-03$ & $(1.20 \pm 0.47) E-03$ \\
\hline
\end{tabular}

a Blank space indicates activity was below lower limit of detection. 
Table 31

Radioactivity in Vegetation (Quarterly Samples)

Page 1 of 6

\begin{tabular}{|c|c|c|c|c|}
\hline Location & $\begin{array}{c}\text { No. of } \\
\text { Samples }\end{array}$ & Arithmetic Mean $\pm \sigma$ & Maximum $\pm \sigma$ & Minimum $\pm \sigma$ \\
\hline \multicolumn{5}{|l|}{$\mathrm{H}-3, \mathrm{pCl} / \mathrm{g}$ (wet weight) } \\
\hline \multicolumn{5}{|l|}{ On Site } \\
\hline $200-F \# 13$ & 4 & $(1.04 \pm 0.75) E+01$ & $(1.87 \pm 0.01) E+01$ & $(1.29 \pm 0.02) E+00$ \\
\hline $200-F \# 21$ & 4 & $(2.35 \pm 0.95) E+00$ & $(3.68 \pm 0.04) E+00$ & $(1.47 \pm 0.05) E+00$ \\
\hline $200-H \# 10$ & 4 & $(1.72 \pm 2.50) E+00$ & $(5.43 \pm 0.09) E+00$ & $(2.10 \pm 0.19) E-01$ \\
\hline $200-H \# 22$ & 4 & $(3.10 \pm 3.93) E+00$ & $(8.82 \pm 0.16) E+00$ & $(2.29 \pm 0.41) E-01$ \\
\hline $643-26 E-1$ & 4 & $(4.78 \pm 3.11) E+00$ & $(7.56 \pm 0.06) E+00$ & $(9.28 \pm 0.36) E-01$ \\
\hline $643-26 E-2$ & 4 & $(2.67 \pm 1.89) E+00$ & $(4.77 \pm 0.09) E+00$ & $(3.20 \pm 0.19) E-01$ \\
\hline S-Area \#1 & 4 & $(6.41 \pm 3.51) E+00$ & $(9.17 \pm 0.16) E+00$ & $(1.41 \pm 0.04) E+00$ \\
\hline S-Area \#2 & 4 & $(1.83 \pm 0.92) E+00$ & $(3.10 \pm 0.06) E+00$ & $(1.06 \pm 0.04) E+00$ \\
\hline S-Area \#3 & 4 & $(5.45 \pm 8.70) E+00$ & $(1.85 \pm 0.01) E+01$ & $(7.73 \pm 0.27) E-01$ \\
\hline S-Area \#4 & 4 & $(0.73 \pm 1 .(5) E+01$ & $(2.28 \pm 0.04) E+01$ & $(3.25 \pm 0.16) E-01$ \\
\hline Z-Area \#1 & 4 & $(3.98 \pm 4.06) E+00$ & $(9.66 \pm 0.18) E+00$ & $(4.11 \pm 0.32) E-01$ \\
\hline Z-Area \#2 & 4 & $(1.25 \pm 1.03) E+00$ & $(2.62 \pm 0.04) E+00$ & $(2.90 \pm 0.27) E-01$ \\
\hline Z-Area \#3 & 4 & $(1.83 \pm 1.85) E+00$ & $(4.46 \pm 0.08) E+00$ & $(1.26 \pm 0.12) E-01$ \\
\hline Z-Area \#4 & 4 & $(3.30 \pm 2.79) E+00$ & $(6.00 \pm 0.12) E+00$ & $(2.83 \pm 0.47) E-01$ \\
\hline Z-Area \#5 & 4 & $(1.84 \pm 1.22) E+00$ & $(3.15 \pm 0.05) E+00$ & $(1.96 \pm 0.18) E-01$ \\
\hline Z-Area \#6 & 4 & $(0.99 \pm 1.05) E+00$ & $(2.50 \pm 0.04) E+00$ & $(9.33 \pm 1.31) E-02$ \\
\hline Z-Area \#7 & 4 & $(2.42 \pm 2.33) E+00$ & $(5.38 \pm 0.05) E+00$ & $(2.70 \pm 0.32) E-01$ \\
\hline Z-Area \#8 & 4 & $(4.75 \pm 3.89) E+00$ & $(9.19 \pm 0.05) E+00$ & $(1.75 \pm 0.16) E-01$ \\
\hline \multicolumn{5}{|l|}{ Site Perimeter } \\
\hline Allendale Gate & 4 & $(0.54 \pm 1.01) E-01$ & $(2.04 \pm 0.39) E-01$ & $(-1.66 \pm 1.77) E-02$ \\
\hline Barnwell Gate & 4 & $(1.30 \pm 1.23) E-01$ & $(2.98 \pm 0.30) E-01$ & $(6.38 \pm 5.84) E-03$ \\
\hline D-Area & 4 & $(5.30 \pm 4.77) E-01$ & $(1.05 \pm 0.05) E+00$ & $(6.69 \pm 0.73) E-02$ \\
\hline Darkhorse@ Williston Gate & 4 & $(1.13 \pm 0.60) E-01$ & $(1.67 \pm 0.41) E-01$ & $(4.01 \pm 1.18) E-02$ \\
\hline East Talatha & 4 & $(2.03 \pm 1.21) \mathrm{E}-01$ & $(3.27 \pm 0.46) \mathrm{E}-01$ & $(6.18 \pm 2.55) E-02$ \\
\hline Green Pond & 4 & $(2.82 \pm 4.68) \mathrm{E}-01$ & $(9.80 \pm 0.36) E-01$ & $(-0.27 \pm 1.74) E-02$ \\
\hline Highway $125 @$ Road A-14 & 4 & $(2.69 \pm 2.52) E-01$ & $(5.30 \pm 0.30) E-01$ & $(0.95 \pm 2.62) E-02$ \\
\hline Highway $21 / 167$ & 4 & $(1.68 \pm 0.83) E-01$ & $(2.74 \pm 0.26) E-01$ & $(8.85 \pm 2.72) E-02$ \\
\hline Highway39 @ Williston Gate & 4 & $(1.49 \pm 1.61) \mathrm{E}-01$ & $(3.74 \pm 0.62) E-01$ & $(2.30 \pm 0.84) E-02$ \\
\hline Jackson & 4 & $(3.67 \pm 5.65) \mathrm{E}-01$ & $(1.20 \pm 0.05) E+00$ & $(2.51 \pm 2.75) E-02$ \\
\hline Patterson Mill Road & 4 & $(4.88 \pm 7.19) E-02$ & $(1.54 \pm 0.16) \mathrm{E}-01$ & $(-6.31 \pm 8.01) E-03$ \\
\hline
\end{tabular}


Table 31

Radioactivity in Vegetation (Quarterly Samples)

Page 2 of 6

\begin{tabular}{|c|c|c|c|c|}
\hline Location & $\begin{array}{l}\text { No. of } \\
\text { Samples }\end{array}$ & Arithmetlc Mean $\pm \sigma$ & Maximum $\pm \sigma$ & Minimum $\pm \sigma$ \\
\hline Talatha Gate & 3 & $(2.71 \pm 2.30) E-01$ & $(5.15 \pm 0.53) E-01$ & $(5.82 \pm 3.15) E-02$ \\
\hline West Jackson & 4 & $(3.13 \pm 4.16) E-01$ & $(9.06 \pm 0.25) E-01$ & $(2.18 \pm 0.92) E-02$ \\
\hline Windsor Road & 4 & $(1.18 \pm 0.93) E-01$ & $(2.27 \pm 0.28) E-01$ & $(3.12 \pm 0.98) E-02$ \\
\hline \multicolumn{5}{|l|}{ 25-Mile-Radius } \\
\hline Allendale, SC & 4 & $(1.06 \pm 7.10) E-02$ & $(1.10 \pm 0.36) E-01$ & $(-5.93 \pm 5.36) E-02$ \\
\hline Langley, SC & 4 & $(1.58 \pm 2.97) E-01$ & $(5.97 \pm 0.24) E-01$ & $(-5.80 \pm 5.55) E-02$ \\
\hline Springfield, SC & 4 & $(1.33 \pm 1.08) E-01$ & $(2.42 \pm 0.44) E-01$ & $(1.03 \pm 0.79) E-02$ \\
\hline Waynesboro, GA & 4 & $(-0.42 \pm 4.95) E-02$ & $(5.82 \pm 1.35) E-02$ & $(-6.27 \pm 4.81) E-02$ \\
\hline \multicolumn{5}{|l|}{ 100-Mile-Radius } \\
\hline Columbia, SC & 4 & $(6.98 \pm 7.37) E-02$ & $(1.52 \pm 0.13) E-01$ & $(4.31 \pm 7.03) E-03$ \\
\hline Greenville, SC & 4 & $(3.03 \pm 3.94) E-02$ & $(8.86 \pm 1.88) E-02$ & $(0.54 \pm 3.42) E-02$ \\
\hline Macon, GA & 5 & $(0.91 \pm 1.43) E-01$ & $(3.46 \pm 0.63) F-01$ & $(2.25 \pm 6.31) E-03$ \\
\hline Savannah, GA & 4 & $(5.31 \pm 8.80) E-02$ & $(1.51 \pm 0.39) E-01$ & $(-3.41 \pm 6.12) E-02$ \\
\hline \multicolumn{5}{|c|}{ Cs-137a, pCl/g (dry weight) The typical lower limit of detection for Cs-137 in vegetation was $1.00 E-01 \mathrm{pCl/g}$. } \\
\hline \multicolumn{5}{|l|}{ On Site } \\
\hline $643-26 E-1$ & $1 / 4$ & $(2.15 \pm 0.42) E-01$ & & \\
\hline $643-26 E-2$ & $0 / 4$ & & & \\
\hline F-Area & $2 / 4$ & $(6.31 \pm 6.45) E-01$ & $(1.09 \pm 0.08) E \div 00$ & $(1.74 \pm 0.45) E-01$ \\
\hline H-Area & $2 / 4$ & $(1.17 \pm 1.37) E+00$ & $(2.14 \pm 0.07) E+00$ & $(2.02 \pm 0.37) E-01$ \\
\hline S-Area \#1 & $1 / 4$ & $(1.35 \pm 0.39) E-01$ & & \\
\hline S-Area \#2 & $0 / 4$ & & & \\
\hline S-Area \#3 & $2 / 4$ & $(2.73 \pm 2.13) E-01$ & $(4.24 \pm 0.52) E-01$ & $(1.22 \pm 0.28) E-01$ \\
\hline S-Area \#4 & $2 / 4$ & $(1.14 \pm 0.79) E-01$ & $(1.70 \pm 0.48) E-01$ & $(5.80 \pm 1.93) E-02$ \\
\hline Z-Area \#1 & $0 / 4$ & & & \\
\hline Z-Area \#2 & $0 / 4$ & & & \\
\hline Z-Area \#3 & $3 / 4$ & $(1.13 \pm 0.11) E-01$ & $(1.19 \pm 0.32) E-01$ & $(1.00 \pm 0.37) E-01$ \\
\hline Z-Area \#4 & $0 / 4$ & & & \\
\hline Z-Area \#5 & $0 / 4$ & & & \\
\hline Z-Area \#6 & $1 / 4$ & $(1.11 \pm 0.34) E-01$ & & \\
\hline
\end{tabular}

a The gamma analysis package currently used by Environmental Monitoring does not force an activity determination if a threshold setting is not met. For these reported radionuclides, "No. of Samples" is displayed as "number with activities quantified/number of samples counted"; only the number quantified are used in the mean, maximum, and minimum generation. 


\section{Table 31}

\section{Radioactivity in Vegetation (Quarterly Samples)}

Page 3 of 6

\begin{tabular}{|c|c|c|c|c|}
\hline Location & $\begin{array}{l}\text { No. of } \\
\text { Samples }\end{array}$ & Arithmetic Mean $\pm \sigma$ & Maximum $\pm \sigma$ & Minimum $\pm \sigma$ \\
\hline Z-Area \#7 & $2 / 4$ & $(4.25 \pm 2.83) E-01$ & $(6.25 \pm 0.58) E-01$ & $(2.25 \pm 0.60) E-01$ \\
\hline Z-Area \#8 & $1 / 4$ & $(1.69 \pm 0.41) E-01$ & & \\
\hline Site Perimeter & $4 / 4$ & $(2.10 \pm 0.80) E-01$ & $(2.98 \pm 0.40) E-01$ & $(1.11 \pm 0.24) E-01$ \\
\hline 25-Mile-Radius & $2 / 4$ & $(1.29 \pm 0.65) E-01$ & $(1.75 \pm 0.27) E-01$ & $(8.33 \pm 1.83) E-02$ \\
\hline 100-Mile-Radius & $1 / 4$ & $(1.48 \pm 0.42) E-01$ & & \\
\hline \multicolumn{5}{|c|}{ Sr-89,90, pCi/g (dry weight) } \\
\hline \multicolumn{5}{|l|}{ On Site } \\
\hline $643-26 E-1$ & 4 & $(2.86 \pm 2.90) E-01$ & $(5.94 \pm 0.96) E-01$ & $(-0.17 \pm 1.13) E-01$ \\
\hline $643-26 E-2$ & 4 & $(1.45 \pm 2.07) E-01$ & $(4.52 \pm 1.03) E-01$ & $(0.51 \pm 7.25) E-02$ \\
\hline F-Area & 4 & $(8.50 \pm 7.58) E-01$ & $(1.77 \pm 0.12) E+00$ & $(0.88 \pm 1.25) E-01$ \\
\hline H-Area & 4 & $(1.39 \pm 0.99) E+00$ & $(2.84 \pm 0.14) E+00$ & $(6.18 \pm 0.83) E-01$ \\
\hline S-Area \#1 & 4 & $(1.33 \pm 1.61) E-01$ & $(3.14 \pm 0.80) E-C 1$ & $(-0.73 \pm 1.07) E-01$ \\
\hline Z-Area \#1 & 4 & $(2.30 \pm 0.91) E-01$ & $(3.54 \pm 0.83) E-01$ & $(1.39 \pm 0.77) E-01$ \\
\hline Site Perimeter & 4 & $(9.36 \pm 8.10) E-01$ & $(1.92 \pm 0.13) E+C 0$ & $(-0.83 \pm 9.29) E-02$ \\
\hline 25-Mlle-Radius & 4 & $(3.93 \pm 1.88) E-01$ & $(5.75 \pm 0.83) E-01$ & $(1.30 \pm 1.05) E-01$ \\
\hline 100-Mile-Radlus & 4 & $(1.63 \pm 1.14) E-01$ & $(2.56 \pm 0.74) E-01$ & $(0.13 \pm 7.15) E-02$ \\
\hline \multicolumn{5}{|c|}{ Gross Alpha, pCl/g (dry weight) } \\
\hline \multicolumn{5}{|l|}{ On Site } \\
\hline $200-F \# 13$ & 4 & $(0.99 \pm 1.50) E+00$ & $(3.14 \pm 1.16) E+00$ & $(-2.46 \pm 2.17) E-01$ \\
\hline $200-F \# 21$ & 4 & $(0.19 \pm 3.75) E-01$ & $(4.40 \pm 5.25) E-01$ & $(-4.59 \pm 3.73) E-01$ \\
\hline $200-H \# 10$ & 4 & $(0.74 \pm 1.13) E+00$ & $(1.89 \pm 0.75) E+00$ & $(-6.49 \pm 4.11) E-01$ \\
\hline $200-H \# 22$ & 4 & $(0.94 \pm 1.39) E+00$ & $(2.97 \pm 0.98) E+00$ & $(-0.67 \pm 3.63) E-01$ \\
\hline $643-26 E-1$ & 4 & $(0.70 \pm 1.17) E+00$ & $(1.83 \pm 1.04) E+00$ & $(-0.50 \pm 1.20) E+00$ \\
\hline $643-26 E-2$ & 4 & $(5.38 \pm 2.30) E-01$ & $(7.77 \pm 6.33) E-01$ & $(2.24 \pm 5.52) E-01$ \\
\hline S-Area \#1 & 4 & $(3.92 \pm 4.80) E-01$ & $(1.02 \pm 0.65) E+00$ & $(-0.80 \pm 3.85) E-01$ \\
\hline S-Area \#2 & 4 & $(8.34 \pm 9.58) E-01$ & $(2.16 \pm 1.32) E+00$ & $(1.08 \pm 6.47) E-01$ \\
\hline S-Area \#3 & 4 & $(-0.52 \pm 1.34) E+00$ & $(4.58 \pm 4.77) E-01$ & $(-2.49 \pm 1.11) E+00$ \\
\hline S-Area \#4 & 4 & $(1.22 \pm 4.09) E-01$ & $(6.79 \pm 5.34) E-01$ & $(-2.44 \pm 2.10) E-01$ \\
\hline Z-Area \#1 & 4 & $(1.32 \pm 0.90) E+00$ & $(2.50 \pm 1.33) E+00$ & $(3.38 \pm 3.93) E-01$ \\
\hline Z-Area \#2 & 4 & $(1.08 \pm 2.12) E+00$ & $(4.25 \pm 1.48) E+00$ & $(-2.54 \pm 2.20) E-01$ \\
\hline Z-Area \#3 & 4 & $(2.77 \pm 5.08) E-01$ & $(7.03 \pm 4.65) E-01$ & $(-4.52 \pm 3.51) E-01$ \\
\hline Z-Area \#4 & 4 & $(6.69 \pm 3.18) E-01$ & $(9.45 \pm 7.98) E-01$ & $(2.15 \pm 3.96) E-01$ \\
\hline
\end{tabular}




\section{Table 31}

Radioactivity in Vegetation (Quarterly Samples)

Page 4 of 6

\begin{tabular}{|c|c|c|c|c|}
\hline Location & $\begin{array}{l}\text { No. of } \\
\text { Samples }\end{array}$ & Arithmetic Mean $\pm \sigma$ & Maximum \pm 0 & Minimum $\pm \sigma$ \\
\hline Z-Area \#5 & 4 & $(1.37 \pm 1.84) E+00$ & $(4.08 \pm 1.64) E+00$ & $(1.01 \pm 6.75) E-01$ \\
\hline Z-Area \#6 & 4 & $(9.83 \pm 8.32) E-01$ & $(1.75 \pm 0.91) E+00$ & $(-1.97 \pm 1.76) E-01$ \\
\hline Z-Area \#7 & 4 & $(0.06 \pm 3.13) E-01$ & $(4.58 \pm 4.90) E-01$ & $(-2.59 \pm 2.09) E-01$ \\
\hline Z-Area \#8 & 4 & $(1.56 \pm 1.90) E+00$ & $(4.30 \pm 1.36) E+00$ & $(-0.52 \pm 3.05) E-01$ \\
\hline \multicolumn{5}{|l|}{ Site Perimeter } \\
\hline Allendale Gate & 4 & $(3.00 \pm 5.29) E-01$ & $(7.44 \pm 5.87) E-01$ & $(-3.07 \pm 2.65) E-01$ \\
\hline Barnwell Gate & 4 & $(2.19 \pm 3.46) E-01$ & $(6.94 \pm 8.30) E-01$ & $(-0.48 \pm 3.23) E-01$ \\
\hline D-Area & 4 & $(8.22 \pm 2.08) E-01$ & $(1.09 \pm 0.61) E+00$ & $(6.27 \pm 6.50) E-01$ \\
\hline Darkhorse @ Williston Gate & 4 & $(0.70 \pm 1.05) E+00$ & $(1.98 \pm 0.89) E+00$ & $(-2.67 \pm 2.32) E-01$ \\
\hline East Talatha & 4 & $(7.66 \pm 2.99) E-01$ & $(1.21 \pm 0.80) E+00$ & $(5.79 \pm 4.95) E-01$ \\
\hline Green Pond & 4 & $(7.75 \pm 5.45) E-01$ & $(1.37 \pm 0.85) E+00$ & $(2.09 \pm 5.05) E-01$ \\
\hline Highway 125@Road A-14 & 4 & $(0.60 \pm 1.11) E+00$ & $(2.22 \pm 1.07) E+00$ & $(-2.95 \pm 2.53) E-01$ \\
\hline Highway $21 / 167$ & 4 & $(4.63 \pm 4.80) E-01$ & $(1.09 \pm 0.74) E+00$ & $(-0.47 \pm 3.17) E-01$ \\
\hline Highway39 @ Williston Gate & 4 & $(6.93 \pm 9.72) E-01$ & $(1.95 \pm 0.95) E+00$ & $(-2.73 \pm 2.43) E-01$ \\
\hline Jackson & 4 & $(0.75 \pm 1.86) E+00$ & $(3.52 \pm 1.45) E+00$ & $(-4.72 \pm 3.81) E-01$ \\
\hline Patterson Mill Road & 4 & $(4.67 \pm 6.63) E-01$ & $(1.24 \pm 0.81) E+00$ & $(-2.79 \pm 2.42) E-01$ \\
\hline Talatha Gate & 4 & $(2.25 \pm 3.28) E-01$ & $(5.56 \pm 6.61) \mathrm{E}-01$ & $(-0.94 \pm 5.13) E-01$ \\
\hline West Jackson & 4 & $(3.56 \pm 3.01) E-01$ & $(6.94 \pm 5.87) \mathrm{E}-01$ & $(-0.32 \pm 4.26) E-01$ \\
\hline Windsor Road & 4 & $(2.91 \pm 5.70) E-01$ & $(7.98 \pm 6.25) E-01$ & $(-3.48 \pm 3.07) E-01$ \\
\hline \multicolumn{5}{|l|}{ 25-Mile-Radius } \\
\hline Allendale, SC & 4 & $(4.54 \pm 6.42) E-01$ & $(1.34 \pm 0.75) E+00$ & $(-0.40 \pm 2.90) E-01$ \\
\hline Langley, SC & 4 & $(-0.09 \pm 3.21) E-01$ & $(2.96 \pm 5.70) E-01$ & $(-4.18 \pm 3.44) E-01$ \\
\hline Springfield, SC & 4 & $(3.93 \pm 7.20) E-01$ & $(1.15 \pm 0.93) E+00$ & $(-3.53 \pm 2.95) E-01$ \\
\hline Waynesboro, GA & 4 & $(8.08 \pm 2.13) E-01$ & $(1.01 \pm 0.85) E+00$ & $(5.39 \pm 6.35) E-01$ \\
\hline \multicolumn{5}{|l|}{ 100-Mile-Radius } \\
\hline Columbia, SC & 4 & $(5.09 \pm 3.74) E-01$ & $(9.73 \pm 9.67) E-01$ & $(1.54 \pm 1.00) E-01$ \\
\hline Greenville, SC & 4 & $(0.57 \pm 1.21) E+00$ & $(1.95 \pm 1.04) E+00$ & $(-7.34 \pm 5.80) E-01$ \\
\hline Macon, GA & 4 & $(0.28 \pm 2.82) E-01$ & $(2.95 \pm 6.33) E-01$ & $(-0.29 \pm 1.70) E+00$ \\
\hline Savannah, GA & 4 & $(1.26 \pm 4.08) E-01$ & $(6.27 \pm 6.50) E-01$ & $(-3.17 \pm 2.76) E-01$ \\
\hline \multicolumn{5}{|l|}{ Gross Beta, pCl/g (dry weight) } \\
\hline \multicolumn{5}{|l|}{ On Site } \\
\hline $200-F \# 13$ & 4 & $(1.33 \pm 0.86) E+01$ & $(2.16 \pm 0.18) E+01$ & $\left(4.37 \pm 1.08^{\prime} E+00\right.$ \\
\hline
\end{tabular}




\section{Table 31}

\section{Radioactivity in Vegetation (Quarterly Samples)}

Page 5 of 6

\begin{tabular}{|c|c|c|c|c|}
\hline Location & $\begin{array}{c}\text { No. of } \\
\text { Samples }\end{array}$ & Arithmetic Mean $\pm \sigma$ & Maximum $\pm \sigma$ & Minimum $\pm \sigma$ \\
\hline $200-F \# 21$ & 4 & $(6.89 \pm 4.23) E+00$ & $(1.20 \pm 0.13) E+01$ & $(2.02 \pm 0.94) E+00$ \\
\hline $200-H \# 10$ & 4 & $(1.47 \pm 1.23) E+01$ & $(3.25 \pm 0.24) E+01$ & $(3.92 \pm 1.03) E+00$ \\
\hline $200-H \# 22$ & 4 & $(1.20 \pm 0.61) E+01$ & $(1.89 \pm 0.17) E+01$ & $(5.55 \pm 1.14) E+00$ \\
\hline $643-26 E-1$ & 4 & $(2.08 \pm 1.31) E+01$ & $(3.53 \pm 0.22) E+01$ & $(5.03 \pm 1.17) E+00$ \\
\hline $643-26 E-2$ & 4 & $(9.45 \pm 5.74) E+00$ & $(1.74 \pm 0.17) E+01$ & $(5.14 \pm 1.15) E+00$ \\
\hline S-Area \#1 & 4 & $(1.26 \pm 0.67) E+01$ & $(1.97 \pm 0.16) E+01$ & $(3.62 \pm 1.05) E+00$ \\
\hline S-Area \#2 & 4 & $(1.76 \pm 1.03) E+01$ & $(2.97 \pm 0.23) E+01$ & $(7.99 \pm 1.11) E+00$ \\
\hline S-Area \#3 & 4 & $(2.66 \pm 2.98) E+01$ & $(7.03 \pm 0.31) E+01$ & $(6.79 \pm 1.27) E+00$ \\
\hline S-Area \#4 & 4 & $(8.97 \pm 2.93) E+00$ & $(1.08 \pm 0.12) E+01$ & $(4.65 \pm 1.13) E+00$ \\
\hline Z-Area \#1 & 4 & $(1.19 \pm 0.82) E+01$ & $(2.36 \pm 0.21) E+01$ & $(4.81 \pm 1.16) E+00$ \\
\hline Z-Area \#2 & 4 & $(1.49 \pm 0.78) E+01$ & $(2.43 \pm 0.21) E+01$ & $(7.99 \pm 1.11) \mathrm{E}+00$ \\
\hline Z-Area \#3 & 4 & $(8.89 \pm 2.72) E+00$ & $(1.19 \pm 0.15) E+01$ & $(5.30 \pm 1.12) E+00$ \\
\hline Z-Area \#4 & 4 & $(8.62 \pm 3.59) E+00$ & $(1.21 \pm 0.17) E+01$ & $(5.41 \pm 1.16) E+00$ \\
\hline Z-Area \#5 & 4 & $(1.83 \pm 1.46) E+01$ & $(3.17 \pm 0.23) \mathrm{E}+01$ & $(1.41 \pm 0.94) E+00$ \\
\hline Z-Area \#6 & 4 & $(7.76 \pm 2.33) E+00$ & $(1.08 \pm 0.14) E+01$ & $(5.17 \pm 0.96) E+00$ \\
\hline Z-Area \#7 & 4 & $(1.12 \pm 0.42) E+01$ & $(1.48 \pm 0.17) E+01$ & $(5.41 \pm 1.13) E+00$ \\
\hline Z-Area \#8 & 4 & $(7.87 \pm 4.69) E+00$ & $(1.25 \pm 0.15) E+01$ & $(3.03 \pm 1.04) E+00$ \\
\hline \multicolumn{5}{|l|}{ Site Perimeter } \\
\hline Allendale Gate & 4 & $(7.64 \pm 2.86) E+00$ & $(1.16 \pm 0.14) E+01$ & $(4.79 \pm 1.08) E+00$ \\
\hline Barnwell Gate & 4 & $(1.20 \pm 0.37) E+01$ & $(1.54 \pm 0.15) E+01$ & $(6.95 \pm 1.22) E+00$ \\
\hline D-Area & 4 & $(1.34 \pm 0.43) E+01$ & $(1.95 \pm 0.20) E+01$ & $(9.92 \pm 1.21) E+00$ \\
\hline Darkhorse @ Williston Gate & 4 & $(1.32 \pm 0.49) E+01$ & $(1.75 \pm 0.19) E+01$ & $(8.55 \pm 1.26) E+00$ \\
\hline East Talatha & 4 & $(1.64 \pm 0.98) E+01$ & $(3.10 \pm 0.23) E+01$ & $(1.04 \pm 0.12) E+01$ \\
\hline Green Pond & 4 & $(1.46 \pm 0.69) E+01$ & $(2.36 \pm 0.21) E+01$ & $(7.93 \pm 1.16) E+00$ \\
\hline Highway $125 @$ Road A-14 & 4 & $(1.37 \pm 0.82) E+01$ & $(2.56 \pm 0.21) E+01$ & $(6.94 \pm 1.09) E+00$ \\
\hline Highway $21 / 167$ & 4 & $(1.29 \pm 0.67) E+01$ & $(2.22 \pm 0.21) E+01$ & $(6.95 \pm 1.23) E+00$ \\
\hline Highway39@ Williston Gate & 4 & $(1.30 \pm 0.74) E+01$ & $(2.36 \pm 0.21) E+01$ & $(6.45 \pm 1.06) E+00$ \\
\hline Jackson & 4 & $(1.50 \pm 1.48) E+01$ & $(3.64 \pm 0.25) E+01$ & $(4.40 \pm 1.10) E+00$ \\
\hline Patterson Mill Road & 4 & $(1.02 \pm 0.60) E+01$ & $(1.88 \pm 0.16) E+01$ & $(5.73 \pm 1.36) E+00$ \\
\hline Talatha Gate & 4 & $(1.80 \pm 1.62) E+01$ & $(4.18 \pm 0.26) E+01$ & $(5.95 \pm 1.06) E+00$ \\
\hline West Jackson & 4 & $(1.14 \pm 0.24) E+01$ & $(1.35 \pm 0.17) E+01$ & $(8.93 \pm 1.20) E+00$ \\
\hline Windsor Road & 4 & $(1.15 \pm 0.15) E+01$ & $(1.35 \pm 0.17) E+01$ & $(9.92 \pm 1.24) E+00$ \\
\hline
\end{tabular}


Table 31

Radioactivity in Vegetation (Quarterly Samples)

Page 6 of 6

\begin{tabular}{lcccc}
\hline Location & $\begin{array}{c}\text { No. of } \\
\text { Samples }\end{array}$ & Arithmetic Mean $\pm \sigma$ & Maximum $\pm \sigma$ & Minimum $\pm \sigma$ \\
\hline 25-Mille-Radius & 4 & $(1.20 \pm 0.77) E+01$ & $(2.22 \pm 0.20) E+01$ & $(4.46 \pm 1.08) E+00$ \\
$\quad \begin{array}{l}\text { Allendale, SC } \\
\text { Langley, SC }\end{array}$ & 4 & $(2.18 \pm 1.20) E+01$ & $(3.37 \pm 0.24) E+01$ & $(7.44 \pm 1.13) E+00$ \\
Springfield, SC & 4 & $(2.03 \pm 1.25) E+01$ & $(3.64 \pm 0.25) E+01$ & $(8.55 \pm 1.26) E+00$ \\
Waynesboro, GA & 4 & $(2.33 \pm 1.11) E+01$ & $(3.84 \pm 0.25) E+01$ & $(1.34 \pm 0.14) E+01$ \\
$\begin{array}{l}\text { 100-Mile-Radius } \\
\text { Columbia, SC }\end{array}$ & 4 & $(1.41 \pm 0.89) E+01$ & $(2.38 \pm 0.19) E+01$ & $(2.34 \pm 0.19) E+00$ \\
Greenville, SC & 4 & $(2.70 \pm 0.89) E+01$ & $(3.61 \pm 0.24) E+01$ & $(1.75 \pm 0.19) E+01$ \\
Macon, GA & 4 & $(3.13 \pm 0.89) E+01$ & $(3.91 \pm 0.26) E+01$ & $(2.03 \pm 0.16) E+01$ \\
Savannah, GA & 4 & $(1.49 \pm 0.50) E+01$ & $(2.22 \pm 0.21) E+01$ & $(1.09 \pm 0.13) E+01$
\end{tabular}




\section{Table 32}

\section{Radioactivity in Vegetation (Composites from Seepage and Retention Basins)}

Page 1 of 1

\begin{tabular}{lcccc}
\hline & \multicolumn{4}{c}{ pCl/g (Dry Welght) } \\
\cline { 2 - 5 } Location & Cs-137 & Sr-89,90 & Gross Alpha & Gross Beta \\
\hline A-Area Seepage Basina & $(9.08 \pm 0.89) E-01$ & $(1.16 \pm 0.05) E+01$ & $(-4.09 \pm 5.12) E-01$ & $(6.09 \pm 0.24) E+01$ \\
C-Area Seepage Basin ${ }^{b}$ & $(1.75 \pm 0.41) E-01$ & $(5.74 \pm 0.20) E+00$ & $(4.20 \pm 4.84) E-01$ & $(3.11 \pm 0.15) E+01$ \\
F Retention Basinc & $(1.94 \pm 0.54) E-01$ & $(1.42 \pm 0.81) E-01$ & $(9.43 \pm 5.50) E-01$ & $(1.63 \pm 0.12) E+01$ \\
F-Area Seepage Basinb & $d$ & $(4.31 \pm 7.65) E-02$ & $(3.97 \pm 4.63) E-01$ & $(1.75 \pm 0.12) E+01$ \\
H Retention Basinc & $(2.77 \pm 0.12) E+00$ & $(3.64 \pm 0.15) E+00$ & $(1.54 \pm 0.70) E+00$ & $(4.09 \pm 0.17) E+01$ \\
H-Area Seepage Basin ${ }^{b}$ & $(1.30 \pm 0.48) E-01$ & $(1.60 \pm 0.82) E-01$ & $(9.60 \pm 5.15) E-01$ & $(1.50 \pm 0.11) E+01$ \\
K Retention Basina & $(1.61 \pm 0.59) E-01$ & $(2.97 \pm 0.87) E-01$ & $(5.73 \pm 4.63) E-01$ & $(1.39 \pm 0.11) E+01$ \\
K-Area Seepage Basina & $(1.72 \pm 0.37) E-01$ & $(1.25 \pm 0.16) E+00$ & $(-1.88 \pm 3.83) E-01$ & $(1.94 \pm 0.13) E+01$ \\
L Chemical Basin ${ }^{a}$ & $(2.04 \pm 0.44) E-01$ & $(1.69 \pm 0.77) E-01$ & $(5.68 \pm 3.86) E-01$ & $(7.43 \pm 0.80) E+00$ \\
L-Area Seepage Basina & $(2.34 \pm 0.65) E-01$ & $(9.11 \pm 1.08) E-01$ & $(9.43 \pm 4.02) E-01$ & $(9.25 \pm 0.84) E+00$ \\
P-Area Seepage Basina & $(5.01 \pm 0.83) E-01$ & $(3.36 \pm 0.88) E-01$ & $(8.98 \pm 4.06) E-01$ & $(5.92 \pm 0.76) E+00$ \\
R-Area Seepage Basin ${ }^{b}$ & $(2.32 \pm 0.46) E-01$ & $(2.70 \pm 0.15) E+00$ & $(8.31 \pm 4.30) E-01$ & $(1.04 \pm 0.09) E+01$
\end{tabular}

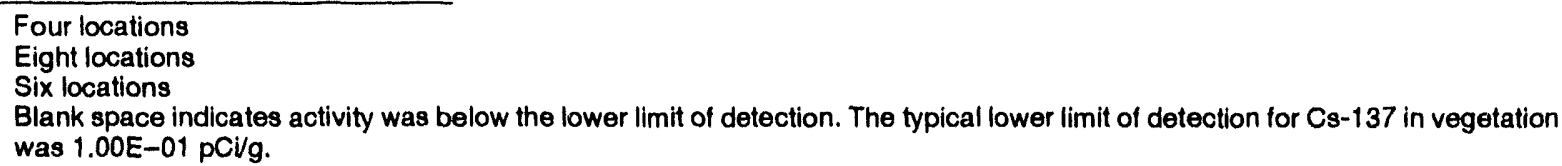


Table 33

Radioactivity in Vegetation (Inside Solid Waste Disposal Facility Fences)

Page 1 of 2

\begin{tabular}{|c|c|c|c|}
\hline \multirow[b]{2}{*}{ Location } & \multicolumn{3}{|c|}{ Dry Welght } \\
\hline & $\mathrm{Cs-137}(\mathrm{pCl} / \mathrm{g})^{b}$ & Gross Alpha (pCl/g) & Gross Beta $(\mathrm{pC} / \mathrm{g})$ \\
\hline IBG-1 Inside Burial Ground & $(3.96 \pm 0.40) E-01$ & $(3.62 \pm 4.15) E-01$ & $(7.05 \pm 1.07) E+00$ \\
\hline IBG-1A Inside Burial Ground & $(4.47 \pm 0.52) E-01$ & $(-2.65 \pm 2.36) E-01$ & $(7.37 \pm 1.13) E+00$ \\
\hline IBG-2 Inside Burial Ground & $\cdot$ & $(-0.59 \pm 3.61) E-01$ & $(9.34 \pm 1.21) E+00$ \\
\hline IBG-3 Inside Burial Ground & $(1.50 \pm 0.42) E-01$ & $(1.57 \pm 3.61) E-01$ & $(7.37 \pm 1.11) E+00$ \\
\hline IBG-3A Inside Burial Ground & $(1.41 \pm 0.32) E-01$ & $(0.23 \pm 4.56) E-01$ & $(1.18 \pm 0.03) E+02$ \\
\hline IBG-4 Inside Burial Ground & $(2.09 \pm 0.48) E-01$ & $(1.72 \pm 4.14) E-01$ & $(1.28 \pm 0.13) E+01$ \\
\hline IBG-4A Inside Burial Ground & $(2.22 \pm 0.10) E+00$ & $(-6.26 \pm 3.01) E-01$ & $(1.48 \pm 0.04) E+02$ \\
\hline IBG-5 Inside Burial Ground & $(2.32 \pm 0.46) E-01$ & $(4.08 \pm 4.86) E-01$ & $(1.67 \pm 0.15) E+01$ \\
\hline IBG-6 Inside Burial Ground & $(5.19 \pm 0.54) E-01$ & $(1.33 \pm 3.33) E-01$ & $(1.52 \pm 0.14) E+01$ \\
\hline IBG-7 Inside Burial Ground & & $(-2.50 \pm 2.28) E-01$ & $(9.67 \pm 1.36) E+00$ \\
\hline IBG-8 Inside Burial Ground & $(1.04 \pm 0.37) E-01$ & $(-0.64 \pm 3.40) E-01$ & $(1.62 \pm 0.14) E+01$ \\
\hline IBG-8A Inside Burial Groul,d & $(2.32 \pm 0.47) E-01$ & $(-4.91 \pm 3.96) E-01$ & $(3.19 \pm 0.20) E+01$ \\
\hline IBG-9 Inside Burial Ground & $(3.39 \pm 0.13) E+00$ & $(-4.23 \pm 2.74) E-01$ & $(7.86 \pm 0.29) E+01$ \\
\hline IBG-9A Inside Burial Ground & $(7.18 \pm 0.90) E-01$ & $(-1.19 \pm 3.73) E-01$ & $(6.26 \pm 0.28) E+01$ \\
\hline IBG-10 Inside Burial Ground & $(7.37 \pm 0.93) E-01$ & $(1.03 \pm 0.62) E+00$ & $(7.86 \pm 0.28) E+01$ \\
\hline IBG-11 Inside Burial Ground & $(1.83 \pm 0.38) E-01$ & $(-2.50 \pm 2.35) E-01$ & $(6.83 \pm 1.25) E+00$ \\
\hline |BG-12 Inside Burial Ground & $(6.80 \pm 0.53) E-01$ & $(-0.63 \pm 4.06) E-01$ & $(2.79 \pm 0.21) E+01$ \\
\hline IBG-13 Inside Burial Ground & $(4.13 \pm 0.66) E-01$ & $(-0.80 \pm 4.73) E-01$ & $(3.02 \pm 0.21) E+01$ \\
\hline IBG-14 Inside Burial Ground & $(1.71 \pm 0.36) E-01$ & $(4.28 \pm 2.00) E-01$ & $(1.01 \pm 1.23) E+01$ \\
\hline IBG-14A Inside Burial Ground & & $(4.44 \pm 4.73) E-01$ & $(1.31 \pm 0.15) E+01$ \\
\hline IBG-15 Inside Burial Ground & & $(-0.42 \pm 3.82) E-01$ & $(1.88 \pm 0.17) E+01$ \\
\hline IBG-16 Inside Burial Ground & $(2.08 \pm 0.08) E+00$ & $(1.31 \pm 4.59) E-01$ & $(6.83 \pm 0.30) E+01$ \\
\hline IBG-17 Inside Burial Ground & $(2.52 \pm 0.41) E-01$ & $(2.67 \pm 5.60) E-01$ & $(1.48 \pm 0.04) E+02$ \\
\hline IBG-18 Inside Burial Ground & & $(6.83 \pm 5.18) E-01$ & $(6.26 \pm 1.22) E+00$ \\
\hline IBG-19 Inside Burial Ground & & $(-4.38 \pm 3.46) E-01$ & $(3.64 \pm 0.23) E+01$ \\
\hline IBG-19A Inside Burial Ground & & $(-0.56 \pm 5.01) E-01$ & $(2.16 \pm 0.20) E+01$ \\
\hline IBG-20 Inside Burial Ground & $(3.66 \pm 0.46) E-01$ & $(2.11 \pm 4.02) E-01$ & $(8.53 \pm 1.32) E+00$ \\
\hline IBG-20A Inside Burial Ground & & $(-3.36 \pm 2.90) E-01$ & $(1.93 \pm 0.18) E+01$ \\
\hline IBG-21 Inside Burial Ground & & $(-3.19 \pm 4.62) E-01$ & $(1.42 \pm 0.04) E+02$ \\
\hline
\end{tabular}

a Blank space indicates activity was below the lower limit of detection. The typical lower limit of detection for Cs-137 in vegetation was $1.00 \mathrm{E}-01 \mathrm{pCi} \mathrm{g}$.

b Value is an average of two samples. 


\section{Table 33}

Radioactivity in Vegetation (Inside Solid Waste Disposal Facility Fences)

Page 2 of 2

\begin{tabular}{|c|c|c|c|}
\hline \multirow[b]{2}{*}{ Location } & \multicolumn{3}{|c|}{ Dry Welght } \\
\hline & Cs-137 (pCl/g) & Gross Alpha (pCl/g) & Gross Beta (pCl/g) \\
\hline |BG-22 Inside Burial Ground & & $(-4.10 \pm 3.37) E-01$ & $(2.84 \pm 0.21) E+01$ \\
\hline IBG-23 Inside Burial Ground & & $(-0.27 \pm 3.90) E-01$ & $(1.02 \pm 0.14) E+01$ \\
\hline IBG-23A Inside Burial Ground & $(5.97 \pm 2.11) E-02$ & $(2.28 \pm 4.33) E-01$ & $(9.10 \pm 1.35) E+00$ \\
\hline IBG-24 Inside Burial Ground & $(1.34 \pm 0.36) E-01$ & $(-2.84 \pm 2.61) E-01$ & $(9.67 \pm 1.38) E+00$ \\
\hline |BG-25 Inside Burial Ground & $(3.48 \pm 0.49) E-01$ & $(-2.62 \pm 2.28) E-01$ & $(1.59 \pm 0.16) E+01$ \\
\hline IBG-26 Inside Burial Ground & & $(4.38 \pm 4.55) E-01$ & $(5.35 \pm 1.18) E+00$ \\
\hline IBG-27 Inside Burial Ground & & $(3.53 \pm 8.63) E-01$ & $(4.15 \pm 0.25) E+01$ \\
\hline IBG-29 Inside Burial Ground & & $(6.83 \pm 5.09) E-01$ & $(5.01 \pm 1.17) E+00$ \\
\hline IBG-30 Inside Burial Ground & & $(5.23 \pm 5.46) E-01$ & $(6.83 \pm 1.28) E+00$ \\
\hline |BG-31 Inside Burial Ground & & $(2.42 \pm 4.50) E-01$ & $(8.28 \pm 1.42) E+00$ \\
\hline IBG-33 Inside Burial Ground & & $(2.42 \pm 4.59) E-01$ & $(1.08 \pm 0.15) E+01$ \\
\hline IBG-34 Inside Burial Ground & & $(7.64 \pm 6.01) E-01$ & $(7.00 \pm 1.38) E+00$ \\
\hline IBG-35 Inside Burial Ground & & $(-2.67 \pm 2.47) E-01$ & $(7.00 \pm 1.37) E+00$ \\
\hline IBG-36 Inside Burial Ground & & $(-0.24 \pm 3.66) E-01$ & $(1.02 \pm 0.15) E+01$ \\
\hline IBG-37 Inside Burial Ground & & $(-2.74 \pm 2.55) E-01$ & $(7.00 \pm 1.37) E+00$ \\
\hline IBG-38 Inside Burial Ground & & $(-0.40 \pm 4.55) E-01$ & $(1.59 \pm 0.18) E+01$ \\
\hline IBG-39 Inside Burial Ground & & $(9.55 \pm 6.07) E-01$ & $(7.00 \pm 1.37) E+00$ \\
\hline IBG-40 Inside Burial Ground & & $(-3.88 \pm 3.49) E-01$ & $(1.40 \pm 0.17) E+01$ \\
\hline IBG-41 Inside Burial Ground & & $(2.36 \pm 4.50) E-01$ & $(9.55 \pm 1.49) E+00$ \\
\hline IBG-42 Inside Burial Ground & & $(-0.26 \pm 4.03) E-01$ & $(1.08 \pm 0.15) E+01$ \\
\hline
\end{tabular}

a Blank space indicates activity was below the lower limit of detection. The typical lower limit of detection for Cs-137 in vegetation was $1.00 \mathrm{E}-01 \mathrm{pCV}$. 


\section{Table 34}

\section{Radioactivity in Vegetation (Outside the Solid Waste Disposal Facility Fences)}

Page 1 of 2

\begin{tabular}{|c|c|c|c|c|}
\hline Location & $\begin{array}{c}\text { No. of } \\
\text { Samples }\end{array}$ & Arithmetic Mean $\pm \sigma$ & Maximum $\pm \sigma$ & Minimum $\pm \sigma$ \\
\hline \multicolumn{5}{|c|}{ Cs-137", pCl/g (dry weight) The typical lower limit of detection of Cs-137 in vegetation was $1.00 E-01 \mathrm{pCl/g}$. } \\
\hline OBG-1 Outside Burial Ground & $1 / 4$ & $(1.19 \pm 0.44) \mathrm{E}-01$ & & \\
\hline OBG-2 Outside Burial Ground & $0 / 4$ & & & \\
\hline OBG-3 Outside Burial Ground & $1 / 4$ & $(1.06 \pm 0.30) E-01$ & & \\
\hline OBG-4 Outside Burial Ground & $0 / 4$ & & & \\
\hline OBG-5 Outside Burial Ground & $0 / 4$ & & & \\
\hline OBG-6 Outside Burial Ground & $1 / 4$ & $(1.18 \pm 0.24) E-01$ & & \\
\hline OBG-7 Outside Burial Ground & $4 / 4$ & $(2.60 \pm 0.95) E-01$ & $(4.00 \pm 0.87) E-01$ & $(1.92 \pm 0.41) E-01$ \\
\hline OBG-8 Outside Burial Ground & $1 / 4$ & $(1.28 \pm 0.35) E-01$ & & \\
\hline OBG-9 Outside Burial Ground & $4 / 4$ & $(3.94 \pm 3.50) E-01$ & $(9.15 \pm 0.70) E-01$ & $(1.66 \pm 0.60) E-01$ \\
\hline OBG-10 Outside Burial Ground & $3 / 4$ & $(2.16 \pm 0.81) E-01$ & $(3.07 \pm 0.75) E-01$ & $(1.50 \pm 0.36) E-01$ \\
\hline OBG-11 Outside Burial Ground & $0 / 4$ & & & \\
\hline OBG-12 Outside Burial Ground & $2 / 4$ & $(2.59 \pm 0.22) E-01$ & $(2.74 \pm 0.40) E-01$ & $(2.44 \pm 0.45) E-01$ \\
\hline OBG-13 Outside Rurial Ground & $0 / 4$ & & & \\
\hline \multicolumn{5}{|l|}{ Gross Alpha, pCi/g (dry welght) } \\
\hline OBG-1 Outside Burial Ground & 4 & $(4.31 \pm 5.22) E-01$ & $(9.43 \pm 7.65) E-01$ & $(-2.82 \pm 6.39) E-01$ \\
\hline OBG-2 Outside Burial Ground & 4 & $(7.02 \pm 9.78) E-01$ & $(1.84 \pm 0.76) E+00$ & $(-3.75 \pm 3.07) E-01$ \\
\hline OBG-3 Outside Burial Ground & 4 & $(2.73 \pm 4.32) E-01$ & $(8.11 \pm 5.41) E-01$ & $(-2.47 \pm 8.35) E-01$ \\
\hline OBG-4 Outside Burial Ground & 4 & $(0.76 \pm 1.35) E+00$ & $(2.66 \pm 1.19) E+00$ & $(-4.59 \pm 3.60) E-01$ \\
\hline OBG-5 Out : de Burial Ground & 4 & $(0.62 \pm 1.28) E+01$ & $(2.54 \pm 0.66) E+01$ & $(-1.13 \pm 0.74) E+00$ \\
\hline OBG-6 Outside Burial Ground & 4 & $(0.03 \pm 1.11) E+00$ & $(9.99 \pm 6.80) E-01$ & $(-1.55 \pm 1.07) E+00$ \\
\hline OBG-7 Outside Burial Ground & 4 & $(1.21 \pm 1.21) E+00$ & $(2.82 \pm 1.51) E+00$ & $(-0.78 \pm 4.33) E-01$ \\
\hline OBG-8 Outside Burial Ground & 4 & $(-0.02 \pm 5.32) E-01$ & $(0.70 \pm 1.05) E+00$ & $(-5.95 \pm 7.83) E-01$ \\
\hline OBG-9 Outside Burial Ground & 4 & $(5.32 \pm 1.24) E-01$ & $(7.06 \pm 6.84) E-01$ & $(4.31 \pm 5.16) E-01$ \\
\hline OBG-10 Outside Burial Ground & 4 & $(2.76 \pm 3.29) E-01$ & $(6.16 \pm 5.07) E-01$ & $(-1.69 \pm 5.69) E-01$ \\
\hline OBG-11 Outside Burial Ground & 4 & $(0.83 \pm 1.05) E+00$ & $(2.33 \pm 1.20) E+00$ & $(-1.40 \pm 6.39) E-01$ \\
\hline OBG-12 Outside Burial Ground & 4 & $(3.74 \pm 5.99) E+00$ & $(1.27 \pm 0.59) E+01$ & $(1.95 \pm 4.70) E-01$ \\
\hline OBG-13 Outside Burial Ground & 4 & $(9.80 \pm 6.81) E-01$ & $(1.76 \pm 1.62) E+00$ & $(1.65 \pm 3.75) E-01$ \\
\hline
\end{tabular}

a The gamma analysis package currently used by Environmental Monitoring does not force an activity determination if a threshold setting is not met. For these reported radionuclides, "No. of Samples" is displayed as "number with activities quantified/number of samples counted"; only the number quantified are used in the mean, maximum, and minimum generation. 
Table 34

Radioactivity in Vegetation (Outside the Solid Waste Disposal Facllity Fences)

Page 2 of 2

\begin{tabular}{llllll}
\hline & $\begin{array}{c}\text { No. of } \\
\text { 8amples }\end{array}$ & Arithmetlo Mean $\pm \sigma$ & Maximum $\pm \sigma$ & Minimum $\pm \sigma$ \\
\hline Location & & & & \\
\hline OBgss Beta, pCl/g (dry weight) & & $(1.56 \pm 0.98) E+01$ & $(2.75 \pm 0.23) E+01$ & $(6.16 \pm 1.17) E+00$ \\
OBG-2 Outside Burial Ground & 4 & $(1.43 \pm 0.85) E+01$ & $(2.41 \pm 0.19) E+01$ & $(5.30 \pm 1.16) E+00$ \\
OBG-3 Outside Burial Ground & 4 & $(1.52 \pm 1.11) E+01$ & $(3.05 \pm 0.21) E+01$ & $(4.16 \pm 1.09) E+00$ \\
OBG-4 Outside Burial Ground & 4 & $(9.16 \pm 2.82) E+00$ & $(1.33 \pm 0.15) E+01$ & $(7.28 \pm 1.23) E+00$ \\
OBG-5 Outside Burial Ground & 4 & $(1.95 \pm 1.09) E+01$ & $(3.46 \pm 0.25) E+01$ & $(8.88 \pm 1.29) E+00$ \\
OBG-6 Outside Burial Ground & 4 & $(1.34 \pm 0.68) E+01$ & $(2.26 \pm 0.22) E+01$ & $(6.49 \pm 1.22) E+00$ \\
OBG-7 Outside Burial Ground & 4 & $(1.91 \pm 0.69) E+01$ & $(2.89 \pm 0.23) E+01$ & $(1.35 \pm 0.15) E+01$ \\
OBG-8 Outside Burial Ground & 4 & $(2.41 \pm 2.12) E+01$ & $(5.24 \pm 0.26) E+01$ & $(7.77 \pm 1.23) E+00$ \\
OBG-9 Outside Burial Ground & 4 & $(1.54 \pm 0.60) E+01$ & $(2.24 \pm 0.18) E+01$ & $(1.03 \pm 0.13) E+01$ \\
OBG-10 Outside Burial Ground & 4 & $(9.52 \pm 3.15) E+00$ & $(1.27 \pm 0.18) E+01$ & $(6.16 \pm 1.16) E+00$ \\
OBG-11 Outside Burial Ground & 4 & $(1.28 \pm 1.0) E+01$ & $(2.63 \pm 0.19) E+01$ & $(5.03 \pm 1.15) E+00$ \\
OBG-12 Outside Burial Ground & 4 & $(1.67 \pm 0.98) E+01$ & $(3.14 \pm 0.21) E+01$ & $(1.03 \pm 0.14) E+01$ \\
OBG-13 Outside Burial Ground & 4 & $(1.16 \pm 0.59) E+01$ & $(1.69 \pm 0.20) E+01$ & $(5.51 \pm 1.03) E+00$
\end{tabular}




\section{Table 35}

\section{Radioactivity in Fooda}

Page 1 of 7

\begin{tabular}{|c|c|c|c|c|c|c|}
\hline Location & $H-3^{b}(p C i / g \pm \sigma)$ & 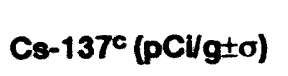 & $\mathrm{Pu}-238^{b}(\mathrm{pCl} / g \pm \sigma)$ & Pu-239b (pCl/g士o) & $\mathrm{Sr}-89,90^{b}(p \mathrm{CV} / \mathrm{g} \pm \sigma)$ & U/Pub $(p C l / g \pm \sigma)$ \\
\hline \multicolumn{7}{|l|}{ Collards } \\
\hline $\begin{array}{l}\text { FS \#1 Below } \\
\text { Barricade \#5 }\end{array}$ & $(3.24 \pm 0.63) E-02$ & & & & $(2.55 \pm 2.51) E-02$ & $(1.29 \pm 1.16) E-02$ \\
\hline $\begin{array}{l}\text { FS \#10 Gracewood } \\
\text { School area }\end{array}$ & $(2.48 \pm 0.60) E-02$ & & $(-7.09 \pm 9.20) E-06$ & $(5.07 \pm 1.43) E-05$ & $(1.03 \pm 0.30) E-01$ & $(5.11 \pm 1.18) E-02$ \\
\hline $\begin{array}{l}\text { FS \#11 Hwy } 19-7 \mathrm{mi} \\
N \text { of Aiken }\end{array}$ & $(0.97 \pm 1.40) E-02$ & & & & $(0.89 \pm 2.74) E-02$ & $(-2.23 \pm 2.29) E-03$ \\
\hline $\begin{array}{l}\text { FS \#12 Springfield } \\
\text { area }\end{array}$ & $(5.20 \pm 1.85) E-02$ & & & & $(0.15 \pm 2.81) E-02$ & $(0.31 \pm 3.45) E-03$ \\
\hline FS \#13 Hilda area & $(3.08 \pm 1.19) E-02$ & & & & $(-0.65 \pm 1.97) E-02$ & $(-2.17 \pm 2.28) E-03$ \\
\hline FS \#14 Uimers area & $(2.36 \pm 0.83) E-02$ & & & & $(2.89 \pm 2.31) E-02$ & $(-2.22 \pm 2.3 i) E-03$ \\
\hline $\begin{array}{l}\text { FS \#15 Control, } \\
\text { Columbia, SC }\end{array}$ & $(2.64 \pm 0.79) E-02$ & & $(2.26 \pm 0.98) E-05$ & $(-0.83 \pm 1.10) E-05$ & $(-1.20 \pm 2.57) E-02$ & $(0.32 \pm 3.45) E-03$ \\
\hline $\begin{array}{l}\text { FS \#2 near Sav. R./E } \\
\text { of Sardis }\end{array}$ & $(1.38 \pm 0.67) E-02$ & & & & $(-1.09 \pm 3.28) E-02$ & $(0.89 \pm 8.00) E-03$ \\
\hline FS \#3 Jackson area & $(2.34 \pm 1.73) E-02$ & & & & $(4.71 \pm 2.83) E-02$ & $(1.26 \pm 1.15) E-02$ \\
\hline FS \#4 Talatha area & $(9.34 \pm 2.30) E-02$ & & $(0.97 \pm 1.19) E-05$ & $(0.00 \pm 1.53) E-05$ & $(1.64 \pm 2.44) E-02$ & $(0.87 \pm 8.06) E-03$ \\
\hline $\begin{array}{l}\text { FS \#5 Darkhorse } \\
\text { area }\end{array}$ & $(7.84 \pm 1.05) E-02$ & $(1.55 \pm 0.55) E-02$ & & & $(-0.15 \pm 2.34) E-02$ & $(-4.99 \pm 5.29) E-03$ \\
\hline FS \#6 Snelling area & $(3.00 \pm 0.75) E-02$ & & $(2.02 \pm 1.01) E-05$ & $(1.51 \pm 1.33) E-05$ & $(4.67 \pm 2.65) E-02$ & $(1.26 \pm 1.16) E-02$ \\
\hline FS \#7 Wade & $(1.91 \pm 0.53) E-02$ & & & & $(3.65 \pm 2.52) E-02$ & $(1.28 \pm 1.17) E-02$ \\
\hline
\end{tabular}

a All results except those for $\mathrm{H}-3$ are expressed in dry weight.

b Blank space indicates that no analyses were performed.

C Blank space indicates that activity was below the lower limit of detection. The typical lower limit of detection for Cs-137 in foodstufts was $7.00 \mathrm{E}-03 \mathrm{pCi} / \mathrm{g}$ 


\section{Table 35 \\ Radioactivity in Food ${ }^{a}$}

Page 2 of 7

\begin{tabular}{|c|c|c|c|c|c|c|}
\hline Location & $H-3^{b}(p C l / g \pm \sigma)$ & Cs-137c (pCilg $\pm \sigma)$ & Pu-238b (pCVg士o) & Pu-239b (pCi/g士o) & Sr-89,90b $(p C i / g \pm \sigma)$ & $U P^{b}$ (pCi/g $\left.\pm \sigma\right)$ \\
\hline FS \#8 Sardis area & $(3.66 \pm 5.14) E-03$ & $(5.06 \pm 0.80) E-02$ & & & $(4.14 \pm 2.37) E-02$ & $(0.71 \pm 7.95) E-03$ \\
\hline $\begin{array}{l}\text { FS \#9 Boyce Dairy } \\
\text { area }\end{array}$ & $(1.45 \pm 0.48) E-02$ & & & & $(0.99 \pm 2.27) E-02$ & $(0.36 \pm 3.45) E-03$ \\
\hline \multicolumn{7}{|l|}{ Greens } \\
\hline $\begin{array}{l}\text { N.E. SC 50-mi. } \\
\text { radius }\end{array}$ & $(1.28 \pm 0.39) E-01$ & $(3.71 \pm 0.80) E-02$ & & & $(4.07 \pm 2.35) E-02$ & $(6.86 \pm 9.99) E-03$ \\
\hline $\begin{array}{l}\text { N.W. SC 50-mi. } \\
\text { radius }\end{array}$ & $(7.80 \pm 3.68) E-02$ & & & & $(4.63 \pm 2.96) E-02$ & $(6.73 \pm 9.92) E-03$ \\
\hline S.E. SC 50-mi. radius & $(0.15 \pm 2.47) E-02$ & & & & $(2.77 \pm 2.70) E-02$ & $(0.37 \pm 3.46) E-03$ \\
\hline \multicolumn{7}{|l|}{ Watermelon } \\
\hline S.E. SC 50-mi. radius & $(3.42 \pm 1.84) E-02$ & & $(1.31 \pm 0.76) E-0.5$ & $(4.34 \pm 4.34) E-06$ & $(2.57 \pm 2.82) E-02$ & $(3.19 \pm 3.57) E-03$ \\
\hline \multicolumn{7}{|l|}{ Soybeans } \\
\hline $\begin{array}{l}\text { N.E. SC 50-mi. } \\
\text { radius }\end{array}$ & $(-9.93 \pm 7.15) E-03$ & & $(2.02 \pm 0.24) E-0.4$ & $(4.40 \pm 8.20) E-06$ & $(-0.60 \pm 2.18) E-02$ & $(1.75 \pm 3.89) E-03$ \\
\hline S.E. SC 50 -mi. radius & $(-1.35 \pm 0.72) E-02$ & & $(6.57 \pm 1.69) E-05$ & $(1.93 \pm 0.86) E-05$ & $(1.74 \pm 2.16) E-02$ & $(1.78 \pm 3.89) E-03$ \\
\hline
\end{tabular}

All results except those for $\mathrm{H}-3$ are expressed in dry weight.

All results except those for $\mathrm{H}-3$ are expressed in dry weight

c Blank space indicates that activity was below the lower limit of detection. The typical lower limit of detection for Cs-137 in foodstults was $7.00 \mathrm{E}-03$ pCi/g. 
Table 35

\section{Radioactivity in Fooda}

Page 3 of 7

\begin{tabular}{|c|c|c|c|c|c|c|}
\hline Location & $H-3^{b}(p C i / g \pm \sigma)$ & $C_{s-137^{c}}(p C V / g \pm \sigma)$ & Pu-238b (pCl/g士o) & $\mathrm{Pu}-239^{b}(\mathrm{PCl} / \mathrm{g} \pm \sigma)$ & $\mathrm{Sr}-89,90^{b}(\mathrm{pCN} / \mathrm{g} \pm)$ & U/Pub $(p C V / g \pm \sigma)$ \\
\hline \multicolumn{7}{|l|}{ Wheat } \\
\hline $\begin{array}{l}\text { N.E. SC 50-mi. } \\
\text { radius }\end{array}$ & & & & & $(-0.38 \pm 2.04) E-02$ & $(-1.07 \pm 8.80) E-03$ \\
\hline $\begin{array}{l}\text { N.W. SC 50-mi. } \\
\text { radius }\end{array}$ & & & & & $(-1.78 \pm 2.02) E-02$ & $(-7.06 \pm 6.44) E-03$ \\
\hline S.E. SC 50-mi. radius & & & & & $(-2.57 \pm 9.86) E-03$ & $(-5.52 \pm 2.16) E-03$ \\
\hline $\begin{array}{l}\text { S.W. GA 50-mi. } \\
\text { radius }\end{array}$ & & & & & $(0.14 \pm 2.20) E-02$ & $(-1.07 \pm 8.80) E-03$ \\
\hline \multicolumn{7}{|l|}{ corn } \\
\hline $\begin{array}{l}\text { FS \#10 Gracewood } \\
\text { School area }\end{array}$ & & $(1.62 \pm 0.51) E-02$ & $(8.29 \pm 5.86) E-06$ & & $(-1.82 \pm 2.01) E-02$ & $(-4.19 \pm 3.82) E-03$ \\
\hline FS \#6 Snelling area & & & $(7.70 \pm 7.32) E-06$ & $(4.32 \pm 6.62) E-06$ & $(-3.72 \pm 2.79) E-02$ & $(6.46 \pm 7.23) E-03$ \\
\hline $\begin{array}{l}\text { N.E. SC 50-mi. } \\
\text { radius }\end{array}$ & & & $(7.71 \pm 9.45) E-06$ & $(-1.54 \pm 1.22) E-05$ & $(-0.20 \pm 3.11) E-02$ & $(-4.11 \pm 3.78) E-03$ \\
\hline S.E. SC 50-mi. radius & & & $(4.01 \pm 8.95) E-06$ & $(3.99 \pm 6.91) E-06$ & $(-0.37 \pm 2.67) E-02$ & $(-4.13 \pm 3.78) E-03$ \\
\hline $\begin{array}{l}\text { FS \#12 Springfiełd } \\
\text { area }\end{array}$ & & & & & $(-1.65 \pm 2.74) E-02$ & $(2.89 \pm 6.25) E-03$ \\
\hline FS \#13 Hilda area & & & & & $(-3.09 \pm 2.64) E-02$ & $(-4.11 \pm 3.78) E-03$ \\
\hline $\begin{array}{l}\text { FS \#2 near Sav. R.J } \\
\text { of Sardis }\end{array}$ & & & & & $(0.20 \pm 2.89) E-02$ & $(6.48 \pm 7.23) E-03$ \\
\hline FS \#3 Jackson area & & & & & $(-0.78 \pm 1.99) E-02$ & $(-0.62 \pm 5.22) E-03$ \\
\hline FS \#7 Wade & & & & & $(-0.88 \pm 2.65) E-02$ & $(2.90 \pm 6.24) E-03$ \\
\hline
\end{tabular}

a All results except those for $\mathrm{H}-3$ are expressed in dry weight.

b Blank space indicates that no analyses were performed.

Blank space indicates that artivity was below the lower limit of detection. The typical lower limit of detection for Cs-137 in foodstuffs was $7.00 \mathrm{E}-03 \mathrm{pCi} / \mathrm{g}$ 


\section{Table 35 \\ Radioactivity in Food ${ }^{a}$}

Page 4 of 7

\begin{tabular}{|c|c|c|c|c|c|c|}
\hline Location & $H-3^{b}(p C l / g \pm \sigma)$ & Cs-137c $(p C l / g \pm \sigma)$ & Pu-238b (pCi/gto) & Pu-239b (pCl/g士o) & Sr-89,90b $(\mathrm{pCi} / \mathrm{g} \pm \sigma)$ & U/Pub $(p C V g \pm \sigma)$ \\
\hline FS \#8 Sardis area & & & & & $(-4.48 \pm 3.04) E-02$ & $(-4.17 \pm 3.82) E-03$ \\
\hline $\begin{array}{l}\text { S.W. GA } 50 \mathrm{mi} \\
\text { radius }\end{array}$ & & & & & $(-0.61 \pm 2.91) E-02$ & $(-4.12 \pm 3.78) E-03$ \\
\hline \multicolumn{7}{|l|}{ Peanuts } \\
\hline $\begin{array}{l}\text { N.E. SC 50-mi. } \\
\text { radius }\end{array}$ & $(5.29 \pm 7.46) E-03$ & & & & & \\
\hline S.E. SC 50-mi. radius & $(-2.27 \pm 0.77) E-02$ & & & & $(-3.83 \pm 3.06) E-02$ & $(3.97 \pm 4.46) E-03$ \\
\hline $\begin{array}{l}\text { S.W. GA 50-mi. } \\
\text { radius }\end{array}$ & $(1.65 \pm 0.83) E-02$ & & & & $(2.76 \pm 2.96) E-02$ & $(3.96 \pm 4.46) E-03$ \\
\hline \multicolumn{7}{|l|}{ Grain } \\
\hline FS \#4 Talatha area & & $(1.50 \pm 0.43) E-02$ & $(7.39 \pm 4.27) E-05$ & $(7.36 \pm 4.25) E-05$ & $(0.39 \pm 2.02) E-02$ & $(-7.04 \pm 6.44) E-03$ \\
\hline $\begin{array}{l}\text { FS \#7 Wade } \\
\text { Plantation vicinity }\end{array}$ & & $(1.77 \pm 0.50) E-02$ & & & $(2.59 \pm 2.26) E-02$ & $(-3.97 \pm 3.64) E-03$ \\
\hline FS \#14 UImers area & & & $(1.28 \pm 0.91) E-05$ & & $(1.93 \pm 2.16) E-02$ & $(-3.98 \pm 3.64) E-03$ \\
\hline FS \#8 Sardis area & & & $(-1.44 \pm 2.50) E-05$ & $(2.87 \pm 2.03) E-05$ & $(0.15 \pm 2.30) E-02$ & $(-3.97 \pm 3.64) E-03$ \\
\hline $\begin{array}{l}\text { FS \#9 Boyce Dairy } \\
\text { area }\end{array}$ & & & $(2.36 \pm 1.18) E-04$ & & $(0.13 \pm 2.14) E-02$ & $(2.81 \pm 6.01) E-03$ \\
\hline $\begin{array}{l}\text { FS \#1 Below } \\
\text { Barricade } \# 5\end{array}$ & & & & & $(2.46 \pm 2.43) E-02$ & $(-7.03 \pm 6.44) E-03$ \\
\hline FS \#10 Gracewood & & & & & $(0.42 \pm 2.08) E-02$ & $(2.79 \pm 6.01) E-03$ \\
\hline
\end{tabular}

A All results except those for $\mathrm{H}-3$ are expressed in dry weight.

b Blank space indicates that no analyses were performed.

c Blank space indicates that activity was below the lower limit of detection. The typical lower limit of detection for Cs-137 in foodstuffs was $7.00 E-03$ pCi/g.

FS \#10 Gracewood

School area 
Table 35

Radioactivity in Food

Page 5 of 7

\begin{tabular}{|c|c|c|c|c|c|c|}
\hline Location & $H-3^{b}(p C i / g \pm \sigma)$ & Cs-137c $(p C l / g \pm \sigma)$ & Pu-238b $(\mathrm{pCl} / \mathrm{g} \pm \sigma)$ & Pu-239b $(p C l / g \pm \sigma)$ & Sr-89,90b $(p C / / g \pm \sigma)$ & $U / P^{b}(p C / / g \pm \sigma)$ \\
\hline $\begin{array}{l}\text { FS \#11 Hwy } 19-7 \mathrm{mi} \\
\mathrm{N} \text { of Aiken }\end{array}$ & & & & & $(1.10 \pm 2.58) E-02$ & $(-5.17 \pm 5.32) E-03$ \\
\hline $\begin{array}{l}\text { FS \#12 Springfield } \\
\text { area }\end{array}$ & & & & & $(-0.68 \pm 2.10) E-02$ & $(-3.97 \pm 3.64) E-03$ \\
\hline FS \#13 Hilda area & & & & & $(-1.55 \pm 2.08) E-02$ & $(-3.97 \pm 3.64) E-03$ \\
\hline $\begin{array}{l}\text { FS \#2 near Sav. R./E } \\
\text { of Sardis }\end{array}$ & & & & & $(0.14 \pm 2.30) E-02$ & $(-7.01 \pm 6.44) E-03$ \\
\hline FS \#3 Jackson area & & & & & $(-2.03 \pm 1.98) E-02$ & $(-1.05 \pm 8.80) E-03$ \\
\hline $\begin{array}{l}\text { FS \#5 Darkhorse } \\
\text { area }\end{array}$ & & & & & $(-0.37 \pm 2.06) E-02$ & $(-1.06 \pm 8.80) E-03$ \\
\hline FS \#6 Snelling area & & & & & $(-0.71 \pm 2.18) E-02$ & $(0.49 \pm 1.06) E-02$ \\
\hline \multicolumn{7}{|r|}{$1030+26015-03$} \\
\hline $\begin{array}{l}\text { FS \#10 Gracewood } \\
\text { School area }\end{array}$ & $(8.25 \pm 1.15) E-02$ & & & $(0.49 \pm 1.09) E-04$ & $(1.57 \pm 2.70) E-02$ & $(-0.30 \pm 2.60) E-03$ \\
\hline $\begin{array}{l}\text { FS \#11 Hwy 19-7mi } \\
N \text { of Aiken }\end{array}$ & $(5.38 \pm 1.01) E-02$ & & & & $(3.03 \pm 2.93) E-02$ & $(-0.31 \pm 2.60) E-03$ \\
\hline $\begin{array}{l}\text { FS \#12 Springfield } \\
\text { area }\end{array}$ & $(-2.08 \pm 4.91) E-03$ & & & & $(2.14 \pm 2.77) E-02$ & $(-0.65 \pm 5.22) E-03$ \\
\hline FS \#13 Hilda area & $(5.23 \pm 2.31) E-02$ & & & & $(-3.58 \pm 1.98) E-02$ & $(-4.14 \pm 3.78) E-03$ \\
\hline FS \#14 Ulmers area & $(2.44 \pm 1.34) E-02$ & & $(1.81 \pm 0.91) E-05$ & & $(1.24 \pm 2.70) E-02$ & $(1.46 \pm 3.14) E-03$ \\
\hline FS \#3 Jackson area & $(8.22 \pm 0.70) E-02$ & & & & $(-3.22 \pm 2.36) E-02$ & $(-4.18 \pm 3.82) E-03$ \\
\hline FS \#4 Talatha area & $(7.84 \pm 1.78) E-02$ & - & & & $(1.00 \pm 2.43) E-02$ & $(2.92 \pm 6.31) E-03$ \\
\hline FS \#6 Snelling area & $(1.49 \pm 0.11) E-01$ & & & & $(-1.79 \pm 2.58) E-02$ & $(1.45 \pm 3.11) E-03$ \\
\hline
\end{tabular}

All results except those for $\mathrm{H}-3$ are expressed in dry weight.

b Blank space indicates that no analyses were performed. 


\section{Table 35 \\ Radioactivity in Food}

Page 6 of 7

\begin{tabular}{|c|c|c|c|c|c|c|}
\hline Location & $H-3^{b}(p c \mid / g \pm \sigma)$ & $C s-137^{c}(p C l / g \pm \sigma)$ & Pu-238b (pCi/g士o) & Pu-239b $(p C l / g \pm \sigma)$ & Sr-89,90b $(p C l / g \pm \sigma)$ & U/Pub $(p C V / g \pm \sigma)$ \\
\hline $\begin{array}{l}\text { FS \#9 Boyce Dairy } \\
\text { area }\end{array}$ & $(1.19 \pm 0.17) E-01$ & & $(0.00 \pm 8.02) E-06$ & $(-5.66 \pm 5.66) E-06$ & $(1.54 \pm 2.77) E-02$ & $(-0.31 \pm 2.60) E-03$ \\
\hline $\begin{array}{l}\text { N.E. SC 50-mi. } \\
\text { radius }\end{array}$ & $(1.24 \pm 1.10) E-02$ & & & & & \\
\hline $\begin{array}{l}\text { N.W. SC 50-mi. } \\
\text { radius }\end{array}$ & $(1.01 \pm 0.21) E-01$ & & & & $(4.12 \pm 3.01) E-02$ & $(-0.31 \pm 2.57) E-03$ \\
\hline S.E. SC 50-mi. radius & $(1.08 \pm 1.18) E-02$ & & $(2.04 \pm 1.52) E-05$ & $(1.35 \pm 0.96) E-05$ & $(-0.06 \pm 2.72) E-02$ & $(-0.30 \pm 2.60) E-03$ \\
\hline \multicolumn{7}{|r|}{$(-0.58 \pm 2.87) E-03$} \\
\hline $\begin{array}{l}\text { N.E. SC 50-mi. } \\
\text { radius }\end{array}$ & $(2.55 \pm 1.06) E-02$ & & & & $(3.72 \pm 1.04) E-02$ & $(-0.58 \pm 2.87) E-03$ \\
\hline $\begin{array}{l}\text { FS \#10 Gracewood } \\
\text { School area }\end{array}$ & & & $(4.83 \pm 2.08) E-05$ & $(-5.33 \pm 5.33) E-06$ & $(2.36 \pm 2.91) E-02$ & \\
\hline $\begin{array}{l}\text { FS \#12 Springfield } \\
\text { area }\end{array}$ & & & $(2.62 \pm 0.95) E-05$ & $(-0.96 \pm 1.17) E-05$ & $(-1.74 \pm 2.62) E-02$ & \\
\hline $\begin{array}{l}\text { FS \#5 Darkhorse } \\
\text { area }\end{array}$ & & & & & $(2.82 \pm 2.78) E-02$ & \\
\hline \multicolumn{7}{|l|}{$\begin{array}{l}\text { area } \\
\text { FS \#7 Wade } \\
\text { Plantation vicinity }\end{array}$} \\
\hline \multicolumn{7}{|r|}{$(-2.19 \pm 2.00) E-03$} \\
\hline $\begin{array}{l}\text { FS \#1 Below } \\
\text { Barricade \#5 }\end{array}$ & $(9.09 \pm 2.38) E-02$ & & $(3.47 \pm 3.48) \bar{\varepsilon}-05$ & $(6.94 \pm 4.91) E-05$ & $(-4.59 \pm 2.70) E-02$ & $(-2.19 \pm 2.00) E-03$ \\
\hline $\begin{array}{l}\text { FS \#10 Gracewood } \\
\text { School area }\end{array}$ & $(6.85 \pm 2.91) E-02$ & & $(1.17 \pm 9.03) E-04$ & $(6.46 \pm 9.32) E-06$ & $(-2.93 \pm 2.66) E-02$ & $(1.48 \pm 3.20) E-03$ \\
\hline $\begin{array}{ll}\text { a } & \text { All results except tho } \\
\text { b Blank space indicate } \\
\text { c Blank space indicate }\end{array}$ & $\begin{array}{l}\text { for } \mathrm{H}-3 \text { are expressec } \\
\text { that no analyses were } \\
\text { that activity was below }\end{array}$ & $\begin{array}{l}\text { n dry weight. } \\
\text { orformed. } \\
\text { e lower limit of detection }\end{array}$ & The typical lower limit of & letection for Cs-137 in for & lstulfs was $7.00 \mathrm{E}-03 \mathrm{pCi} / \mathrm{g}$. & \\
\hline
\end{tabular}




\section{Table 35}

Radioactivity in Food

Page 7 of 7

\begin{tabular}{|c|c|c|c|c|c|c|}
\hline Location & $H-3^{b}(p C l / g \pm \sigma)$ & Cs-137 $(p C i / g \pm \sigma)$ & $\mathrm{Pu}-238^{\mathrm{b}}(\mathrm{pCl} / \mathrm{g} \pm \sigma)$ & Pu-239b $(p C l / g \pm \sigma)$ & $\mathrm{Sr}-89,90^{\mathrm{b}}(\mathrm{pCl} / \mathrm{g} \pm \sigma)$ & U/Pub $(p C / / g \pm \sigma)$ \\
\hline $\begin{array}{l}\text { FS \#11 Hwy } 19-7 \mathrm{mi} \\
\mathrm{N} \text { of Aiken }\end{array}$ & $(5.62 \pm 2.55) E-02$ & $(2.85 \pm 0.71) E-02$ & $\left(3.28 \pm 4.6^{2}\right) E-04$ & $(-2.83 \pm 4.28) E-06$ & $(0.74 \pm 3.10) E-02$ & $(1.53 \pm 3.27) E-03$ \\
\hline FS \#8 Sardis area & $(3.81 \pm 2.59) E-02$ & & $(-1.47 \pm 1.81) E-05$ & $(2.94 \pm 2.08) E-05$ & $(-0.02 \pm 2.93) E-02$ & $(-2.18 \pm 2.00) E-03$ \\
\hline \multicolumn{7}{|l|}{ Beef } \\
\hline FS \#4 Talatha area & & $(5.10 \pm 0.62) E-02$ & & & $(-0.36 \pm 2.36) E-02$ & \\
\hline $\begin{array}{l}\text { FS \#7 Wade } \\
\text { Plantation vicinity }\end{array}$ & & $(2.58 \pm 0.46) E-02$ & $(2.62 \pm 2.31) E-05$ & $(00.00 \pm 1.73) E-05$ & $(1.12 \pm 2.37) E-02$ & \\
\hline FS \#13 Hilda area & & & $(7.53 \pm 6.76) E-06$ & $(-0.40 \pm 1.07) E-05$ & $(0.29 \pm 2.50) E-02$ & \\
\hline $\begin{array}{l}\text { FS \#10 Gracewood } \\
\text { School area }\end{array}$ & & & & & $(2.98 \pm 2.88) E-02$ & \\
\hline NF SC 50-mi. & & & & & $(2.16 \pm 3.06) E-02$ & $(1.78 \pm 3.89) E-03$ \\
\hline
\end{tabular}




\section{Table 36}

\section{Radioactivity in Eggs}

Page 1 of 1

\begin{tabular}{|c|c|c|c|c|}
\hline Location & $\begin{array}{c}\text { No. of } \\
\text { Samples }\end{array}$ & Arithmetic Mean $\pm \sigma$ & Maximum $\pm \sigma$ & Minimum $\pm \sigma$ \\
\hline \multicolumn{5}{|l|}{$\mathrm{H}-3, \mathrm{pCl} / \mathrm{g}$} \\
\hline FS \#1 Below Barricade \#5 & 1 & $(2.47 \pm 0.34) E-01$ & & \\
\hline FS \#10 Gracewood School area & 3 & $(4.58 \pm 4.44) E-02$ & $(9.71 \pm 2.59) E-02$ & $(1.91 \pm 1.25) E-02$ \\
\hline $\begin{array}{l}\text { FS \#2 near Sav. R./E of Sar- } \\
\text { disNO TAG }\end{array}$ & 1 & $(3.87 \pm 1.07) E-02$ & & \\
\hline FS \#4 Talatha area & 2 & $(1.41 \pm 1.41) E-01$ & $(2.41 \pm 0.34) E-01$ & $(4.15 \pm 2.94) E-02$ \\
\hline FS \#5 Darkhorse area NO TAG & 1 & $(2.31 \pm 0.27) E-01$ & & \\
\hline FS \#6 Snelling area & 2 & $(1.32 \pm 0.26) E-01$ & $(1.50 \pm 0.49) E-01$ & $(1.14 \pm 0.16) E-01$ \\
\hline FS \#9 Boyce Dairy area & 2 & $(1.33 \pm 3.64) E-02$ & $(3.91 \pm 2.70) E-02$ & $(-1.24 \pm 5.71) E-02$ \\
\hline \multicolumn{5}{|l|}{ Sr-89,90, pCl/g } \\
\hline $\begin{array}{l}\text { FS \#1 Below Barricade } \\
\# 5^{N O}{ }^{\prime} A G\end{array}$ & 1 & $(0.36 \pm 2.73) E-02$ & & \\
\hline FS \#10 Gracewood School area & 3 & $(-2.80 \pm 1.94) E-02$ & $(-0.90 \pm 2.51) E-02$ & $(-4.78 \pm 4.92) E-02$ \\
\hline $\begin{array}{l}\text { FS \#2 near Sav. R./E of Sar- } \\
\text { dis NO TAG }\end{array}$ & 1 & $(-2.19 \pm 2.35) E-02$ & & \\
\hline FS \#4 Talatha area & 2 & $(-3.33 \pm 6.24) E-02$ & $(1.08 \pm 2.49) E-02$ & $(-7.74 \pm 4.60) E-02$ \\
\hline FS \#5 Darkhorse area NO TAG & 1 & $(1.54 \pm 2.57) E-02$ & & \\
\hline FS \#6 Snelling area & 2 & $(-4.39 \pm 5.04) E-02$ & $(-0.83 \pm 2.00) E-02$ & $(-7.96 \pm 9.00) E-02$ \\
\hline FS \#9 Boyce Dairy area & 2 & $(-4.91 \pm 0.85) E-02$ & $(-4.31 \pm 9.24) E-02$ & $(-5.51 \pm 1.84) E-02$ \\
\hline \multicolumn{5}{|l|}{$\mathrm{U} / \mathrm{Pu}, \mathrm{pCl} / \mathrm{g}$} \\
\hline $\begin{array}{l}\text { FS \#1 Below Barricade } \\
\# 5 \text { NO TAG }\end{array}$ & 1 & $(-2.16 \pm 1.98) E-03$ & & \\
\hline FS \#10 Gracewood School area & 3 & $(-0.21 \pm 1.59) E-03$ & $(1.43 \pm 3.08) E-03$ & $(-0.17 \pm 1.47) E-02$ \\
\hline $\begin{array}{l}\text { FS \#2 near Sav. R./E of Sar- } \\
\text { disNO TAG }\end{array}$ & 1 & $(-2.16 \pm 1.99) E-03$ & & \\
\hline FS \#4 Talatha area & 2 & $(-1.04 \pm 1.04) E-03$ & $(-0.30 \pm 2.57) E-03$ & $(-0.18 \pm 1.47) E-02$ \\
\hline FS \#5 Darkhorse area NO TAG & 1 & $(-0.33 \pm 2.69) E-03$ & & \\
\hline FS \#6 Snelling area & 2 & $(-1.92 \pm 0.27) E-03$ & $(-0.17 \pm 1.47) E-02$ & $(-2.11 \pm 1.93) E-03$ \\
\hline FS \#9 Boyce Dairy area & 2 & $(-1.04 \pm 1.02) E-03$ & $(-0.32 \pm 2.57) E-03$ & $(-0.18 \pm 1.47) E-02$ \\
\hline
\end{tabular}




\section{Table 37}

Radioactivity in Milk

Page 1 of 1

\begin{tabular}{|c|c|c|c|c|}
\hline Location & $\begin{array}{c}\text { No. of } \\
\text { Samples }\end{array}$ & Arithmetic Mean $\pm \sigma$ & Maximum \pm 0 & Minimum $\pm \sigma$ \\
\hline \multicolumn{5}{|l|}{$\mathrm{H}-3, \mu \mathrm{Cl} / \mathrm{mL}$} \\
\hline Dairy (Major Distributor) & 12 & $(0.48 \pm 1.65) E-07$ & $(3.21 \pm 1.20) E-07$ & $(-1.53 \pm 1.40) E-07$ \\
\hline Denmark, SC & 12 & $(1.54 \pm 1.64) E-07$ & $(3.38 \pm 1.23) E-07$ & $(-1.61 \pm 1.40) E-07$ \\
\hline Girard, GA Dairy & 12 & $(3.73 \pm 3.02) E-07$ & $(9.50 \pm 1.60) E-07$ & $(-0.16 \pm 1.43) E-07$ \\
\hline Gracewood, GA Dairy & 11 & $(0.29 \pm 1.39) E-07$ & $(2.31 \pm 1.09) E-07$ & $(-1.37 \pm 1.43) E-07$ \\
\hline Jackson, SC Dairy & 12 & $(7.18 \pm 1.75) E-07$ & $(1.17 \pm 0.13) E-06$ & $(5.79 \pm 1.48) E-07$ \\
\hline Waynesboro, GA Dairy & 12 & $(0.77 \pm 1.67) E-07$ & $(3.21 \pm 1.55) E-07$ & $(-2.30 \pm 1.40) E-07$ \\
\hline N.E. SC 50-Mile Radius & 11 & $(0.89 \pm 1.32) E-07$ & $(3.74 \pm 1.16) E-07$ & $(-0.40 \pm 1.43) E-07$ \\
\hline N.W. SC 50-Mile Radius & 12 & $(0.19 \pm 1.90) E-07$ & $(3.35 \pm 1.48) E-07$ & $(-2.73 \pm 1.41) E-07$ \\
\hline S.E. SC 50-Mile Radius & 12 & $(-0.03 \pm 1.45) E-07$ & $(2.72 \pm 1.19) E-07$ & $(-2.21 \pm 1.41) E-07$ \\
\hline S.W. GA 50-Mile Radius & 12 & $(-0.05 \pm 1.20) E-07$ & $(1.77 \pm 1.19) E-07$ & $(-2.42 \pm 1.42) E-07$ \\
\hline \multicolumn{5}{|l|}{$\mathrm{Sr}-90, \mu \mathrm{Cl} / \mathrm{mL}$} \\
\hline Dairy (Major Distributor) & 2 & $(9.96 \pm 4.59) E-10$ & $(1.32 \pm 1.14) E-09$ & $(0.67 \pm 1.06) E-09$ \\
\hline Denmark, SC & 2 & $(6.41 \pm 3.51) E-10$ & $(0.89 \pm 1.13) E-09$ & $(0.39 \pm 1.41) E-09$ \\
\hline Girard, GA Dairy & 2 & $(2.66 \pm 2.13) E-09$ & $(4.17 \pm 1.37) E-09$ & $(1.16 \pm 0.97) E-09$ \\
\hline Gracewood, GA Dairy & 2 & $(5.34 \pm 0.98) E-10$ & $(0.60 \pm 1.03) E-09$ & $(0.46 \pm 1.06) E-09$ \\
\hline Jackson, SC Dairy & 2 & $(-0.89 \pm 3.80) E-10$ & $(1.80 \pm 9.61) E-10$ & $(-3.58 \pm 9.88) E-10$ \\
\hline Waynesboro, GA Dairy & 2 & $(-0.19 \pm 1.29) E-09$ & $(0.73 \pm 1.45) E-09$ & $(-1.10 \pm 1.19) E-09$ \\
\hline \multicolumn{5}{|c|}{ Cs-137, $\mu \mathrm{Cl} / \mathrm{mL}^{\mathrm{a}}$ (The typical lower limit of detection for Cs-137 in milk was $3.00 \mathrm{E}-09 \mu \mathrm{Cl} / \mathrm{mL}$.) } \\
\hline Dairy (Major Distributor) & $2 / 12$ & $(3.14 \pm 0.30) E-09$ & $(3.35 \pm 1.23) E-09$ & $(2.93 \pm 0.92) E-09$ \\
\hline Denmark, SC & $6 / 12$ & $(4.54 \pm 0.66) E-09$ & $(5.41 \pm 1.30) E-09$ & $(3.80 \pm 1.20) E-09$ \\
\hline Girard, GA Dairy & $7 / 12$ & $(4.05 \pm 0.66) E-09$ & $(5.35 \pm 1.49) E-09$ & $(3.27 \pm 1.18) E-09$ \\
\hline Gracewood, GA Dairy & $2 / 11$ & $(5.62 \pm 0.63) E-09$ & $(6.07 \pm 1.16) E-09$ & $(5.17 \pm 1.44) E-09$ \\
\hline Jackson, SC Dairy & $2 / 12$ & $(4.05 \pm 1.27) \mathrm{E}-09$ & $(4.94 \pm 1.05) E-09$ & $(3.15 \pm 1.04) E-09$ \\
\hline Waynesboro, GA Dairy & $1 / 12$ & $(2.67 \pm 0.87) E-09$ & & \\
\hline N.E. SC 50-Mile Radius & $9 / 12$ & $(5.10 \pm 0.97) E-09$ & $(6.65 \pm 1.52) E-09$ & $(3.58 \pm 0.75) E-09$ \\
\hline N.W. SC 50-Mile Radius & $0 / 12$ & & & \\
\hline S.E. SC 50-Mile Radius & $10 / 12$ & $(4.22 \pm 0.78) E-09$ & $(5.37 \pm 1.55) E-09$ & $(2.78 \pm 0.85) E-09$ \\
\hline S.W. GA 50-Mile Radius & $5 / 12$ & $(3.59 \pm 1.16) E-09$ & $(5.34 \pm 1.38) E-09$ & $(2.53 \pm 0.80) E-09$ \\
\hline
\end{tabular}

a The gamma analysis package currently used by Environmental Monitoring does not force an activity determination if a threshold setting is not met. For these reported radionuclides, "No. of Samples" is displayed as "number with activities quantified/number of samples counted"; only the number quantified are used in the mean, maximum, and minimum generation. 


\section{Table 38}

\section{Radloactivity in Fish}

Page 1 of 14

\begin{tabular}{ll} 
Location & $\begin{array}{c}\text { No. of } \\
\text { Samples }\end{array}$ Arithmetic Mean $\pm \sigma$ \\
\hline H-3, pCl/g & \\
\hline Off Site (Edible)
\end{tabular}

Augusta Lock and Dam

$\begin{array}{lllll}\text { Bass } & 3 & (1.70 \pm 1.19) E-02 & (2.60 \pm 0.77) E-02 & (0.35 \pm 2.43) E-02 \\ \text { Bream } & 3 & (5.27 \pm 5.87) E-02 & (1.20 \pm 0.22) E-01 & (1.17 \pm 2.56) E-02\end{array}$

Beaver Dam Creak River Mouth

Bream

Catfish

1

3

Four Mile Creek River Mouth

Bream

Hwy-301 Bridge Area

Bass
Bream
Catfish

L3R Creek River Mouth

Bass
Bream
Cattish

Steel Creek River Mouth

Bass
Bream
Catfish

U3R Creok River Mouth

Catfish

$$
(4.32 \pm 0.72) E-02
$$

$(1.59 \pm 1.07) E-01$

$(2.44 \pm 0.28) E-01$

$(3.83 \pm 0.72) E-02$

\begin{abstract}
$(2.86 \pm 1.88) E-01$
$(8.38 \pm 3.79) E-01$
\end{abstract}

$(4.14 \pm 0.13) E-01$

$(7.05 \pm 0.94) E-02$

$(1.12 \pm 0.02) E+00$

$(4.07 \pm 0.14) E-01$

$(1.24 \pm 1.57) E-01$

(2.35 \pm 0.23$) E-01$

$(1.33 \pm 1.08) E-02$

$(1.06 \pm 0.54) E-01$

$(1.66 \pm 0.15) E-01$

$(6.47 \pm 1.18) E-02$

$(1.24 \pm 0.67) E-01$

$(1.96 \pm 0.18) E-01$

$(6.36 \pm 0.61) E-02$

1

$(9.92 \pm 1.15) E-02$

$(1.55 \pm 0.54) E-01$

(2.17 \pm 0.22$) E-01$

$(1.18 \pm 0.15) E-01$

3

$(1.01 \pm 0.25) E-01$

$(1.30 \pm 0.18) E-01$

$(8.53 \pm 1.19) E-02$

2

$(1.45 \pm 0.04) E+00$

$(1.47 \pm 0.02) E+00$

$(1.42 \pm 0.02) E+00$

$(1.53 \pm 0.70) E-01$

$(2.01 \pm 0.13) E-01$

$(7.25 \pm 0.85) \mathrm{E}-02$

3

$(1.52 \pm 0.50) E-01$

$(2.08 \pm 0.17) E-01$

$(1.11 \pm 0.08) E-01$

3

$(1.24 \pm 0.37) E-01$

$(1.61 \pm 0.15) E-01$

$(8.75 \pm 0.96) E-02$

West Bank Landing

Bass

Bream

Crappie
$(-3.37 \pm 1.87) E-02$

$(-1.62 \pm 1.38) E-02$

$(-1.57 \pm 0.33) E-02$
$(-1.25 \pm 2.96) E-02$

$(-1.92 \pm 2.00) E-02$ 


\section{Table 38}

Radioactivity in Fish

Page 2 of 14

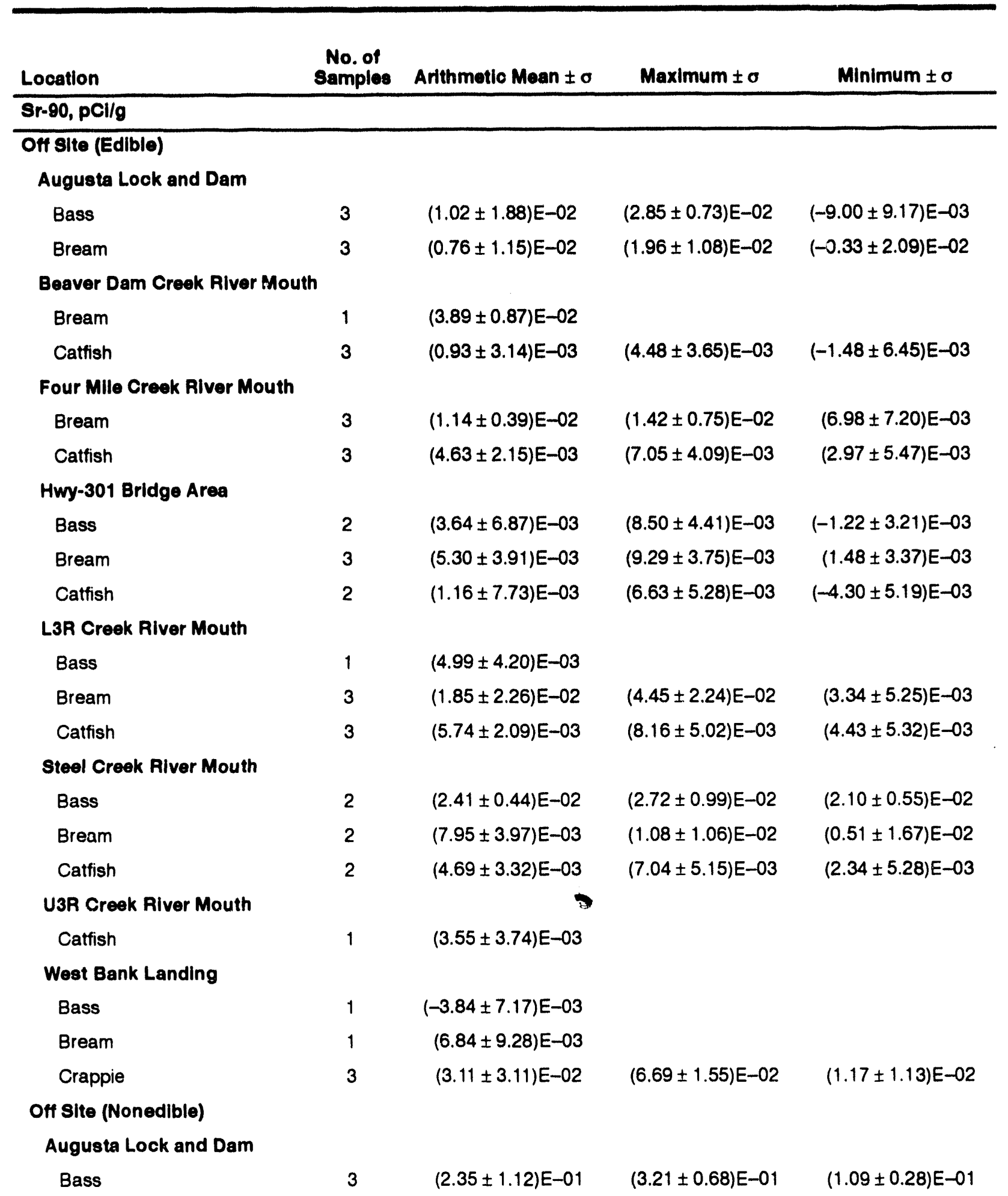




\section{Table 38}

\section{Radioactivity In Fish}

Page 3 of 14

\begin{tabular}{|c|c|c|c|c|}
\hline Location & $\begin{array}{l}\text { No. of } \\
\text { Samples }\end{array}$ & Artthmotlo Moan $\pm \sigma$ & Maximum $\pm \sigma$ & Minimum $\pm \sigma$ \\
\hline Bream & 3 & $(3.40 \pm 3.20) E-01$ & $(6.99 \pm 0.75) E-01$ & $(8.60 \pm 2.76) E-02$ \\
\hline \multicolumn{5}{|c|}{ Beaver Dam Creek River Mouth } \\
\hline Bream & 1 & $(9.77 \pm 0.98) E-01$ & & \\
\hline Catfish & 3 & $(4.79 \pm 3.04) E-01$ & $(6.95 \pm 2.29) E-01$ & $(1.31 \pm 0.49) E-01$ \\
\hline \multicolumn{5}{|c|}{ Four Mile Creek River Mouth } \\
\hline Bream & 3 & $(1.44 \pm 0.38) E+00$ & $(1.82 \pm 0.33) E+00$ & $(1.05 \pm 0.38) E+00$ \\
\hline Catfish & 3 & $(2.06 \pm 1.37) E-01$ & $(3.02 \pm 0.65) E-01$ & $(4.88 \pm 5.81) E-02$ \\
\hline \multicolumn{5}{|c|}{ Hwy-301 Bridge Area } \\
\hline Bass & 2 & $(8.72 \pm 1.10) E-02$ & $(9.50 \pm 2.87) E-02$ & $(7.94 \pm 2.61) E-02$ \\
\hline Bream & 3 & $(1.43 \pm 0.16) E-01$ & $(1.56 \pm 0.35) E-01$ & $(1.25 \pm 0.31) E-01$ \\
\hline Catfish & 3 & $(2.16 \pm 1.04) E-01$ & $(2.96 \pm 0.67) E-01$ & $(9.84 \pm 2.70) E-02$ \\
\hline \multicolumn{5}{|c|}{ L3R Creek River Mouth } \\
\hline Bass & 1 & $(2.13 \pm 0.59) E-01$ & & \\
\hline Bream & 3 & $(2.06 \pm 1.10) E-01$ & $(3.19 \pm 0.58) E-01$ & $(9.87 \pm 2.76) E-02$ \\
\hline Catfish & 2 & $(1.95 \pm 2.17) E-01$ & $(3.48 \pm 0.62) E-01$ & $(4.18 \pm 5.34) E-02$ \\
\hline \multicolumn{5}{|c|}{ Steel Creek River Mouth } \\
\hline Bass & 2 & $(1.34 \pm 0.90) E-01$ & $(1.98 \pm 0.42) E-01$ & $(7.01 \pm 2.66) E-02$ \\
\hline Bream & 3 & $(1.29 \pm 0.55) E-01$ & $(1.91 \pm 0.34) E-01$ & $(8.94 \pm 2.70) E-02$ \\
\hline Catfish & 3 & $(3.81 \pm 5.30) E-01$ & $(9.92 \pm 3.06) E-01$ & $(7.49 \pm 2.45) E-02$ \\
\hline \multicolumn{5}{|c|}{ U3R Creek River Mouth } \\
\hline Cattish & 3 & $(2.04 \pm 1.62) E-01$ & $(3.18 \pm 0.66) E-01$ & $(1.80 \pm 1.99) E-02$ \\
\hline \multicolumn{5}{|c|}{ West Bank Landing } \\
\hline Bass & 1 & $(3.37 \pm 0.64) E-01$ & & \\
\hline Bream & 1 & $(1.97 \pm 0.51) E-01$ & & \\
\hline Crappie & 3 & $(2.90 \pm 0.49) E-01$ & $(3.37 \pm 0.59) E-01$ & $(2.39 \pm 0.62) E-01$ \\
\hline \multicolumn{5}{|c|}{ Cs-137, pCl/ga (The typical lower IImit of detection for Cs-137 in fish was $4.2 E-02 \mathrm{pCl} / \mathrm{g}$.) } \\
\hline \multicolumn{5}{|c|}{ Off Site (Edible) } \\
\hline \multicolumn{5}{|c|}{ Hwy-17A Bridge Area } \\
\hline Bass & $2 / 2$ & $(1.04 \pm 0.40) E-01$ & $(1.33 \pm 0.23) E-01$ & $(7.59 \pm 2.07) E-02$ \\
\hline
\end{tabular}

a The gamma analysis package currently used by Environmental Monitoring does not force an activity determination if a threshold setting is not met. For these reported radionuclides, "No. of Samples" is displayed as "number with activities quantified/number of samples counted"; only the number quantified are used in the mean, maximum, and minimum generation. 
Table 38

Radioactlvity in Fish

Pege 4 of 14

\begin{tabular}{ccccc}
\hline Location & $\begin{array}{c}\text { No. of } \\
\text { Samples }\end{array}$ & Arthmotio Mean \pm 0 & Maximum \pm 0 & MInimum $\pm \sigma$ \\
\hline Mullet & $3 / 3$ & $(2.54 \pm 2.67) E-01$ & $(5.61 \pm 0.31) E-01$ & $(7.73 \pm 2.07) E-02$
\end{tabular}

Augusta Look and Dam

$\begin{array}{lllll}\text { Bass } & 2 / 2 & (2.42 \pm 2.53) E-01 & (4.21 \pm 0.32) E-01 & (6.39 \pm 2.09) E-02 \\ \text { Bream } & 1 / 2 & (1.88 \pm 0.40) E-01 & \end{array}$

Beaver Dam Creak River Mouth

$\begin{array}{lllll}\text { Bream } & 1 / 1 & (7.07 \pm 0.43) E-01 & \\ \text { Cattish } & 1 / 3 & (7.39 \pm 2.21) E-02 & (7.39 \pm 2.21) E-02 & (7.39 \pm 2.21) E-02\end{array}$

Four Mlle Creok RIver Mouth

$\begin{array}{lllll}\text { Bream } & 1 / 3 & (1.52 \pm 0.43) E-01 & & \\ \text { Catfish } & 2 / 3 & (7.22 \pm 1.18) E-02 & (8.05 \pm 1.95) E-02 & (6.39 \pm 1.86) E-02\end{array}$

Hwy-301 Bridge Area

Bass

Bream

Catfish

L3R Creok River Mouth

Bass

Steol Creok River Mouth

$\begin{array}{ll}\text { Bass } & 2 / 2 \\ \text { Bream } & 1 / 2 \\ \text { Catfish } & 3 / 3\end{array}$

Stokes Bluff Landing

Bass
Bream
Catfish

U3R Creek River Mouth

Catfish

$2 / 3$

$(6.95 \pm 1.37) E-02$

$(7.93 \pm 2.39) E-02$

$(5.98 \pm 1.67) E-02$
Bass
$1 / 1$
$(2.54 \pm 0.32) E-01$
Crappie
$1 / 2$
$(4.50 \pm 0.60) E-01$ 
Table 38

Radloactivity in Fish

Page 8 of 14

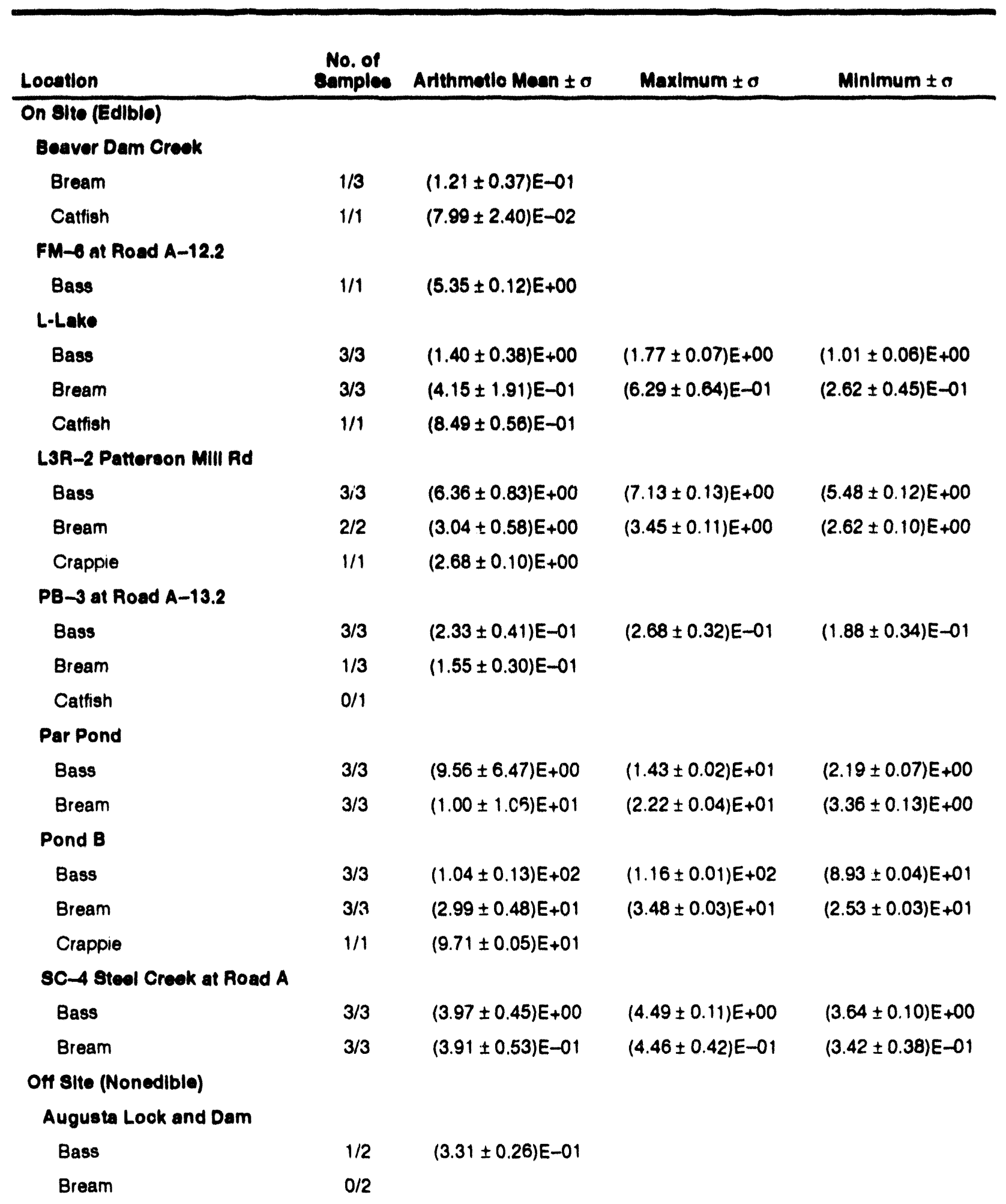


Table 38

Radloactivity in Fish

Page 6 of 14

\begin{tabular}{|c|c|c|c|c|}
\hline Loontion & $\begin{array}{c}\text { No. of } \\
\text { eamples }\end{array}$ & Arthmotio Moen \pm 0 & Maximum \pm 0 & Minimum \pm 0 \\
\hline \multicolumn{5}{|c|}{ Beaver Dam Creok Rlver Mouth } \\
\hline Bream & $1 / 1$ & $(1.43 \pm 0.40) E-01$ & & \\
\hline Catfish & $2 / 3$ & $(4.01 \pm 1.79) E-02$ & $(5.27 \pm 1.61) E-02$ & $(2.74 \pm 0.60) E-02$ \\
\hline \multicolumn{5}{|c|}{ Four Mllo Creck Alver Mouth } \\
\hline Bream & $1 / 3$ & $(1.17 \pm 0.31) E-01$ & & \\
\hline Catfigh & $1 / 3$ & $(8.63 \pm 2.33) E-02$ & & \\
\hline \multicolumn{5}{|c|}{ Huy-301 Bridge Aree } \\
\hline Bass & $1 / 2$ & $(5.67 \pm 1.88) E-02$ & & \\
\hline Bream & $0 / 3$ & & & \\
\hline Cattish & $2 / 3$ & $(6.67 \pm 3.40) E-02$ & $(9.08 \pm 2.42) E-02$ & $(4.27 \pm 1.40) E-02$ \\
\hline \multicolumn{5}{|c|}{ LSR Creok River Mouth } \\
\hline Bass & $1 / 1$ & $(3.50 \pm 0.37) E-01$ & & \\
\hline Bream & $3 / 3$ & $(3.23 \pm 2.26) E-01$ & $(5.46 \pm 0.42) E-01$ & $(9.40 \pm 1.75) E-02$ \\
\hline Catfish & $2 / 2$ & $(1.67 \pm 0.08) E-01$ & $(1.73 \pm 0.24) E-01$ & $(1.61 \pm 0.19) E-01$ \\
\hline \multicolumn{5}{|c|}{ Steol Creek Alver Mouth } \\
\hline Bass & $2 / 2$ & $(7.08 \pm 1.50) E-01$ & $(8.14 \pm 0.27) E-01$ & $(6.02 \pm 0.36) E-01$ \\
\hline Bream & $2 / 2$ & $(8.08 \pm 0.62) E-02$ & $(8.52 \pm 2.46) E-02$ & $(7.64 \pm 2.36) E-02$ \\
\hline Catfish & $2 / 3$ & $(1.10 \pm 0.11) E-01$ & $(1.18 \pm 0.24) E-01$ & $(1.02 \pm 0.20) E-01$ \\
\hline \multicolumn{5}{|c|}{ U3R Creok Rivor Mouth } \\
\hline Calfish & $0 / 3$ & & & \\
\hline \multicolumn{5}{|c|}{ Woat Bank Landing } \\
\hline Bass & $1 / 1$ & $(1.51 \pm 0.26) E-01$ & & \\
\hline Bream & $1 / 1$ & $(9.68 \pm 3.30) E-02$ & & \\
\hline Crappie & $3 / 3$ & $(2.02 \pm 1.55) E-01$ & $(3.61 \pm 0.46) E-01$ & $(5.19 \pm 1.70) E-02$ \\
\hline \multicolumn{5}{|c|}{ Pu-238, pCl/g } \\
\hline \multicolumn{5}{|c|}{ Off slte (Edlble) } \\
\hline \multicolumn{5}{|c|}{ Augusta Look and Dam } \\
\hline Bass & 1 & $(3.27 \pm 7.32) E-06$ & & \\
\hline Bream & 1 & $(0.00 \pm 2.61) E-05$ & & \\
\hline \multicolumn{5}{|c|}{ Beaver Dam Crook Rlver Mouth } \\
\hline Bream & 1 & $(4.05 \pm 2.14) E-05$ & & \\
\hline Catfish & 3 & $(5.81 \pm 5.10) E-06$ & $(9.54 \pm 6.75) E-06$ & $(0.00 \pm 6.87) E-06$ \\
\hline
\end{tabular}




\section{Table 38}

Radloactivity in Fish

Pege 7 of 14

\begin{tabular}{|c|c|c|c|c|}
\hline Looution & $\begin{array}{c}\text { No. of } \\
\text { eamples }\end{array}$ & Artthmotio Moen \pm 0 & Moximum \pm 0 & Minimum $\pm a$ \\
\hline \multicolumn{5}{|c|}{ Four Mille Creck Alver Mouth } \\
\hline Bream & 2 & $(-5.46 \pm 7.72) E-08$ & $(0.00 \pm 1.59) E-05$ & $(-1.09 \pm 1.09) E-05$ \\
\hline Catfish & 3 & $(-0.78 \pm 7.45) E-08$ & $(0.78 \pm 1.35) E-05$ & $(-5.80 \pm 5.80) E-06$ \\
\hline \multicolumn{5}{|c|}{ Hwy-301 Bridge Area } \\
\hline Bass & 2 & $(2.95 \pm 7.47) E-06$ & $(8.23 \pm 4.76) E-06$ & $(-2.33 \pm 5.22) E-06$ \\
\hline Bream & 3 & $(1.02 \pm 5.18) E-06$ & $(4.52 \pm 4.52) E-06$ & $(-4.94 \pm 3.50) E-06$ \\
\hline Catfigh & 3 & $(0.14 \pm 1.49) E-05$ & $(1.42 \pm 0.54) E-0.5$ & $(-1.50 \pm 0.87) E-05$ \\
\hline \multicolumn{5}{|c|}{ LSA Creak Rlver Mouth } \\
\hline Bream & 3 & $(5.44 \pm 8.37) E-05$ & $(1.51 \pm 1.07) E-04$ & $(00.00 \pm 6.00) E-06$ \\
\hline Cattish & 2 & $(3.11 \pm 9.04) E-06$ & $(9.50 \pm 5.49) E-06$ & $(-3.28 \pm 7.33) E-06$ \\
\hline \multicolumn{5}{|c|}{ Steel Creok River Mouth } \\
\hline Bass & 2 & $(5.31 \pm 8.46) E-05$ & $(1.13 \pm 0.51) E-04$ & $(-0.67 \pm 1.51) E-05$ \\
\hline Bream & 2 & $(0.87 \pm 1.24) E-05$ & $(1.75 \pm 0.78) E-05$ & $(00.00 \pm 5.45) E-06$ \\
\hline Cattish & 3 & $(3.97 \pm 7.93) E-06$ & $(1.19 \pm 0.69) E-05$ & $(-3.97 \pm 3.98) E-06$ \\
\hline \multicolumn{5}{|c|}{ USR Creak Alvor Mouth } \\
\hline Cattish & 3 & $(-2.59 \pm 2.53) E-06$ & $(00.00 \pm 6.04) E-06$ & $(-0.51 \pm 1.13) E-05$ \\
\hline \multicolumn{5}{|c|}{ Weat Bank Landing } \\
\hline Bass & 1 & $(0.00 \pm 2.78) E-05$ & & \\
\hline Crappie & 1 & $(2.00 \pm 1.15) E-05$ & & \\
\hline
\end{tabular}

Off site (Ediblo)

Augueta Look and Dam

Bass

$(9.78 \pm 7.30) E-06$

Beaver Dam Creok Rivor Mouth

Bream

Four Mile Creok River Mouth

Bream

Catfish

1

Hwy-301 Bridge Area

$\begin{array}{llrrr}\text { Bass } & 2 & (-2.95 \pm 7.46) E-06 & (2.33 \pm 4.04) E-06 & (-8.22 \pm 6.13) E-06 \\ \text { Bream } & 3 & (3.81 \pm 3.51) E-06 & (6.92 \pm 3.46) E-06 & (00.00 \pm 3.49) E-06\end{array}$


Table 38

Radloactivity in Fish

Page 8 of 14

\begin{tabular}{|c|c|c|c|c|}
\hline Loontion & $\begin{array}{l}\text { No. of } \\
\text { samplese }\end{array}$ & Arlthmotlo Mean \pm 0 & Maximum \pm 0 & Minimum \pm 0 \\
\hline Catfish & 3 & $(-3.32 \pm 5.76) E-06$ & $(0.00 \pm 3.84) E-06$ & $(-9.87 \pm 7.06) E-06$ \\
\hline \multicolumn{5}{|c|}{ L3A Oreak Rlver Mouth } \\
\hline Bream & 3 & $(-2.51 \pm 4.36) E-05$ & $(4.89 \pm 4.89) E-06$ & $(-7.51 \pm 7.51) E-05$ \\
\hline Catfish & 2 & $(-8.74 \pm 5.56) E-10$ & $(00.00 \pm 6.55) E-06$ & $(00.00 \pm 8.94) E-06$ \\
\hline \multicolumn{5}{|c|}{ Steol Creak Alver Mouth } \\
\hline Base & 2 & $(2.92 \pm 2.24) E-05$ & $(4.50 \pm 5.53) E-05$ & $(1.34 \pm 1.64) E-05$ \\
\hline Bream & 2 & $(1.92 \pm 2.72) E-06$ & $(3.85 \pm 6.66) E-06$ & $(00.00 \pm 6.88) E-06$ \\
\hline Cattish & 2 & $(3.97 \pm 0.03) E-06$ & $(3.99 \pm 2.82) E-06$ & $(3.95 \pm 8.85) E-06$ \\
\hline \multicolumn{5}{|c|}{ USA Crcok Alver Mouth } \\
\hline Catfiah & 3 & $(-4.00 \pm 1.18) E-06$ & $(-2.71 \pm 4.70) E-06$ & $(-5.04 \pm 8.73) E-06$ \\
\hline \multicolumn{5}{|c|}{ Wost Bank Landing } \\
\hline Bases & 1 & $(2.95 \pm 2.60) E-05$ & & \\
\hline Crappie & 1 & $(2.66 \pm 1.63) E-05$ & & \\
\hline
\end{tabular}

\begin{tabular}{l} 
Grose Alpha, pOHg \\
\hline Off alte (Edible) \\
Hury-17A Bridge Ares
\end{tabular}

$\begin{array}{llrrr}\text { Bass } & 2 & (1.66 \pm 1.69) E-01 & (-0.46 \pm 3.53) E-01 & (-2.85 \pm 2.62) E-01 \\ \text { Flounde: } & 1 & (-1.85 \pm 1.98) E-01 & & \\ \text { Mullet } & 3 & (0.23 \pm 2.20) E-01 & (2.41 \pm 3.67) E-01 & (-1.99 \pm 2.00) E-01\end{array}$

Augueta Look and Dam

$\begin{array}{lllll}\text { Bass } & 2 & (-3.77 \pm 0.04) E-01 & (-3.74 \pm 3.42) E-01 & (-3.81 \pm 3.42) E-01 \\ \text { Bream } & 2 & (-0.76 \pm 4.13) E-01 & (2.16 \pm 4.71) E-01 & (-3.68 \pm 3.36) E-01\end{array}$

Beaver Dam Creak Aiver Mouth

Bream

$(-2.52 \pm 2.29) E-01$

Catfish

$(-9.05 \pm 8.56) E-02$

$(-0.41 \pm 3.18) E-01$

$(-1.89 \pm 1.60) E-01$

Four Mill Creek River Mouth

Bream

Catfish

3

$(-0.09 \pm 1.79) E-01$

$(1.72 \pm 3.12) E-01$

$(-1.87 \pm 1.80) E-01$

$(-0.71 \pm 2.01) E-01$

$(1.61 \pm 3.03) E-01$

$(-1.92 \pm 1.80) E-01$

Hwy-301 Bridge Area

$\begin{array}{llrll}\text { Bass } & 2 & (-0.85 \pm 1.49) E-01 & (0.21 \pm 2.96) E-01 & (-1.90 \pm 1.86) E-01 \\ \text { Bream } & 3 & (-0.39 \pm 2.52) E-01 & (2.52 \pm 3.80) E-01 & (-1.85 \pm i .86) E-01 \\ \text { Cattish } & 3 & (1.88 \pm 3.59) E+00 & (6.03 \pm 0.74) E+00 & (-2.03 \pm 1.67) E-01\end{array}$




\section{Table 38}

\section{Radioactivity in Fish}

Page 9 of 14

\begin{tabular}{|c|c|c|c|c|}
\hline Location & $\begin{array}{l}\text { No. of } \\
\text { samples }\end{array}$ & Arthmotlo Mean \pm 0 & Maximum \pm 0 & Minimum $\pm \sigma$ \\
\hline \multicolumn{5}{|c|}{ L3R Creok Rlver Mouth } \\
\hline Bass & 1 & $(0.25 \pm 3.02) E-01$ & & \\
\hline Bream & 3 & $(-1.28 \pm 1.04) E-01$ & $(-0.08 \pm 2.29) E-01$ & $(-1.90 \pm 1.86) E-01$ \\
\hline Catfish & 3 & $(-8.96 \pm 9.85) E-02$ & $(-0.32 \pm 2.47) E-01$ & $(-2.03 \pm 1.67) E-01$ \\
\hline \multicolumn{5}{|c|}{ Steel Creok Rlver Mouth } \\
\hline Bass & 2 & $(-0.75 \pm 1.47) E-01$ & $(0.29 \pm 2.74) E-01$ & $(-1.79 \pm 1.86) E-01$ \\
\hline Bream & 2 & $(1.55 \pm 2.34) E-01$ & $(3.20 \pm 3.31) E-01$ & $(-0.11 \pm 2.39) E-01$ \\
\hline Cattish & 3 & $(-1.53 \pm 0.92) E-01$ & $(-0.47 \pm 2.38) E-01$ & $(-2.13 \pm 1.79) E-01$ \\
\hline \multicolumn{5}{|c|}{ Stokes Bluff Landing } \\
\hline Bass & 3 & $(-0.48 \pm 2.54) E-01$ & $(2.45 \pm 3.64) E-01$ & $(-1.97 \pm 1.98) E-01$ \\
\hline Bream & 2 & $(-0.89 \pm 1.62) E-01$ & $(0.26 \pm 2.93) E-01$ & $(-2.03 \pm 1.98) E-01$ \\
\hline Catfish & 3 & $(0.08 \pm 1.71) E-01$ & $(1.06 \pm 2.65) E-01$ & $(-1.89 \pm 1.60) E-01$ \\
\hline \multicolumn{5}{|c|}{ U3R Creok River Mouth } \\
\hline Catfish & 3 & $(0.55 \pm 2.21) E-01$ & $(2.49 \pm 2.96) E-01$ & $(-1.85 \pm 1.86) E-01$ \\
\hline \multicolumn{5}{|c|}{ Wost Bank Landing } \\
\hline Bream & 1 & $(-0.53 \pm 4.14) E-01$ & & \\
\hline Crappie & 2 & $(-0.69 \pm 4.19) E-01$ & $(2.27 \pm 4.91) E-01$ & $(-3.66 \pm 3.35) E-01$ \\
\hline \multicolumn{5}{|c|}{ On site (Edible) } \\
\hline \multicolumn{5}{|c|}{ Beaver Dam Creak } \\
\hline Bream & 3 & $(0.21 \pm 2.10) E-01$ & $(2.35 \pm 3.54) E-01$ & $(-1.85 \pm 1.60) E-01$ \\
\hline Catfish & 1 & $(-3.44 \pm 3.19) E-01$ & & \\
\hline \multicolumn{5}{|c|}{ FM-6 at Road A-12.2 } \\
\hline Bass & 1 & $(0.15 \pm 3.13) E-01$ & & \\
\hline \multicolumn{5}{|l|}{ L-Lake } \\
\hline Bass & 3 & $(-8.07 \pm 9.10) E-02$ & $(-0.17 \pm 3.33) E-01$ & $(-1.85 \pm 1.86) E-01$ \\
\hline Bream & 3 & $(-0.47 \pm 1.20) E-01$ & $(0.24 \pm 2.80) E-01$ & $(-1.86 \pm 1.87) E-01$ \\
\hline \multicolumn{5}{|c|}{ L3R-2 Patterson MIII Rd } \\
\hline Bass & 2 & $(-2.41 \pm 0.34) E-02$ & $(-0.22 \pm 3.26) E-01$ & $(-0.27 \pm 3.40) E-01$ \\
\hline Bream & 2 & $(0.95 \pm 5.00) E-01$ & $(4.49 \pm 4.66) E-01$ & $(-2.58 \pm 2.45) E-01$ \\
\hline Crappie & 1 & $(4.49 \pm 4.71) E-01$ & & \\
\hline \multicolumn{5}{|c|}{ PB-3 at Road A-13.2 } \\
\hline Bass & 3 & $(-6.44 \pm 9.33) E-02$ & $(-0.10 \pm 2.34) E-01$ & $(-1.72 \pm 1.66) E-01$ \\
\hline
\end{tabular}


Table 38

Radioactivity in Fish

Page 10 of 14

\begin{tabular}{|c|c|c|c|c|}
\hline Looation & $\begin{array}{c}\text { No. of } \\
\text { Samplese }\end{array}$ & Arlthmotic Mean \pm 0 & Maximum \pm 0 & Minimum $\pm \sigma$ \\
\hline Bream & 3 & $(-0.90 \pm 2.01) E-01$ & $(0.27 \pm 2.80) E-01$ & $(-3.22 \pm 2.93) E-01$ \\
\hline Catfish & 1 & $(2.24 \pm 3.45) E-01$ & & \\
\hline \multicolumn{5}{|l|}{ Par Pond } \\
\hline Bass & 3 & $(0.69 \pm 1.35) E-01$ & $(2.24 \pm 3.50) E-01$ & $(-0.17 \pm 2.80) E-01$ \\
\hline Bream & 3 & $(2.14 \pm 3.49) E-01$ & $(6.16 \pm 4.65) E-01$ & $(0.09 \pm 2.80) E-01$ \\
\hline \multicolumn{5}{|l|}{ Pond B } \\
\hline Bass & 3 & $(2.19 \pm 5.52) E-01$ & $(8.45 \pm 6.03) E-01$ & $(-1.97 \pm 3.33) E-01$ \\
\hline Bream & 3 & $(-1.37 \pm 0.91) E-01$ & $(-0.75 \pm 3.40) E-01$ & $(-2.41 \pm 2.00) E-01$ \\
\hline Crappie & 1 & $(-4.46 \pm 3.19) E-01$ & & \\
\hline \multicolumn{5}{|c|}{ SC-4 Steel Creek at Road A } \\
\hline Bass & 3 & $(0.44 \pm 1.03) E-01$ & $(1.63 \pm 3.10) E-01$ & $(-0.15 \pm 2.34) E-01$ \\
\hline Bream & 3 & $(-0.95 \pm 2.71) E-01$ & $(2.18 \pm 4.08) E-01$ & $(-2.52 \pm 2.36) E-01$ \\
\hline \multicolumn{5}{|c|}{ Off site (Nonedible) } \\
\hline \multicolumn{5}{|c|}{ Augusta Lock and Dam } \\
\hline Bass & 2 & $(1.96 \pm 3.41) E-01$ & $(4.38 \pm 4.96) E-01$ & $(-0.45 \pm 3.53) E-01$ \\
\hline Bream & 2 & $(1.94 \pm 0.04) E-01$ & $(1.97 \pm 4.30) E-01$ & $(1.90 \pm 4.11) E-01$ \\
\hline \multicolumn{5}{|c|}{ Beaver Dam Creek River Mouth } \\
\hline Bream & 1 & $(4.69 \pm 5.34) E-01$ & & $\cdots$ \\
\hline Catfish & 3 & $(-9.61 \pm 8.50) E-02$ & $(-0.41 \pm 3.34) E-01$ & $(-1.94 \pm 1.67) E-01$ \\
\hline \multicolumn{5}{|c|}{ Four Mlle Creek River Mouth } \\
\hline Bream & 3 & $(-0.28 \pm 4.26) E-01$ & $(2.23 \pm 4.13) E-01$ & $(-5.20 \pm 4.93) E-01$ \\
\hline Catfish & 3 & $(1.09 \pm 2.07) E-01$ & $(3.48 \pm 3.53) E-01$ & $(-0.12 \pm 2.55) E-01$ \\
\hline \multicolumn{5}{|c|}{ Huy-301 Bridge Area } \\
\hline Bass & 2 & $(-1.03 \pm 1.88) E-01$ & $(0.30 \pm 3.95) E-01$ & $(-2.36 \pm 2.38) E-01$ \\
\hline Bream & 3 & $(3.00 \pm 2.70) E-01$ & $(5.76 \pm 5.34) E-01$ & $(0.36 \pm 3.31) E-01$ \\
\hline Catfish & 3 & $(-2.17 \pm 0.23) E-01$ & $(-1.99 \pm 1.73) E-01$ & $(-2.43 \pm 2.10) E-01$ \\
\hline \multicolumn{5}{|c|}{ L3R Creek River Mouth } \\
\hline Bass & 1 & $(5.37 \pm 4.97) E-01$ & & \\
\hline Bream & 3 & $(0.04 \pm 2.85) E-01$ & $(2.77 \pm 4.07) E-01$ & $(-2.93 \pm 2.43) E-01$ \\
\hline Catfish & 2 & $(4.52 \pm 6.72) E+00$ & $(9.27 \pm 1.34) \mathrm{E}+00$ & $(-2.36 \pm 2.01) E-01$ \\
\hline \multicolumn{5}{|c|}{ Steel Creek River Mouth } \\
\hline Bass & 2 & $(2.56 \pm 0.12) E-02$ & $(0.27 \pm 3.82) E-01$ & $(0.25 \pm 2.99) E-01$ \\
\hline
\end{tabular}




\section{Table 38}

\section{Radioactivity in Fish}

Page 11 of 14

\begin{tabular}{lcccc} 
Location & $\begin{array}{c}\text { No. of } \\
\text { Samples }\end{array}$ & Arithmetic Mean $\pm \sigma$ & Maximum $\pm \sigma$ & Minimum $\pm \sigma$ \\
\hline $\begin{array}{l}\text { Bream } \\
\text { Catfish }\end{array}$ & 2 & $(-7.19 \pm 0.35) E-02$ & $(-0.69 \pm 3.33) E-01$ & $(-0.74 \pm 3.54) E-01$ \\
$\begin{array}{l}\text { U3R Creek River Mouth } \\
\text { Catfish }\end{array}$ & 3 & $(-0.62 \pm 1.80) E-01$ & $(1.24 \pm 3.14) E-01$ & $(-2.36 \pm 2.01) E-01$ \\
$\begin{array}{l}\text { West Bank Landing } \\
\text { Bream }\end{array}$ & 3 & $(0.49 \pm 1.62) E-01$ & $(2.36 \pm 3.60) E-01$ & $(-0.45 \pm 2.31) E-01$ \\
Crappie & 1 & $(2.78 \pm 6.21) E-01$ & & \\
\end{tabular}

Gross Beta, pCl/g

Off Site (Edible)

Hwy-17A Bridge Area

$\begin{array}{lllll}\text { Bass } & 2 & (1.78 \pm 1.26) E+00 & (2.66 \pm 1.06) E+00 & (8.88 \pm 9.62) E-01 \\ \text { Flounder } & 1 & (0.00 \pm 7.80) E-01 & & \\ \text { Mullet } & 3 & (1.95 \pm 0.44) E+00 & (2.23 \pm 0.93) E+00 & (1.45 \pm 0.87) E+00\end{array}$

Augusta Lock and Dam

$\begin{array}{lllll}\text { Bass } & 2 & (2.03 \pm 0.36) E+00 & (2.28 \pm 1.10) E+00 & (1.78 \pm 1.06) E+00 \\ \text { Bream } & 2 & (9.71 \pm 7.81) E-01 & (1.52 \pm 1.04) E+00 & (4.19 \pm 9.62) E-01\end{array}$

Beaver Dam Creek River Mouth

Bream

Catfish

Four Mile Creek River Mouth

Bream

Catfish

Hwy-301 Bridge Area

Bass

Bream

Catfish

L3R Creek River Mouth

Bass
Bream
Catfish

Steel Creek River Mouth

Bass
$(6.86 \pm 8.67) E-01$

$(1.35 \pm 0.49) E+00$

$(1.80 \pm 0.81) E+00$

$(8.32 \pm 7.59) E-01$

\section{$(4.28 \pm 0.75) E+00$}

$(5.15 \pm 1.08) E+00$

$(3.79 \pm 1.00) E+00$

$(4.02 \pm 0.74) E+00$

$(4.52 \pm 1.04) E+00$

(3.17 \pm 0.97$) E+00$

$(2.80 \pm 0.08) E+00$

$(2.86 \pm 0.90) E+00$

$(2.74 \pm 0.92) E+00$

$(1.83 \pm 0.63) E+00$

$(2.52 \pm 0.89) E+00$

$(1.29 \pm 0.81) E+00$

$(1.65 \pm 1.37) E+00$

$(2.83 \pm 0.65) E+00$

$(1.48 \pm 7.16) E-01$

1

$(2.17 \pm 0.93) E+00$

$(2.39 \pm 0.43) E+00$

$(2.86 \pm 0.92) E+00$

$(2.02 \pm 0.87) E+00$

3

$(2.80 \pm 0.88) E+00$

$(3.60 \pm 0.90) E+00$

$(1.85 \pm 0.86) E+00$

2

$(8.49 \pm 4.63) E-01$

$(1.18 \pm 0.81) E+00$

$(5.21 \pm 7.52) E-01$ 


\section{Table 38}

\section{Radioactivity in Fish}

Page 12 of 14

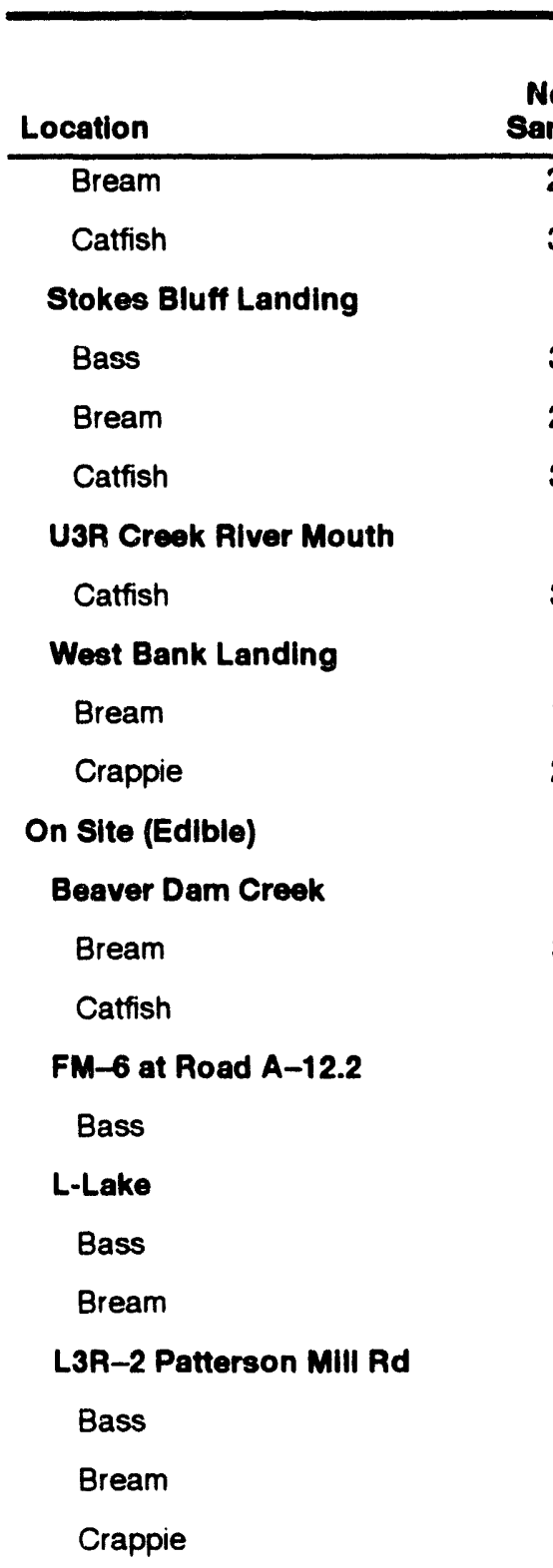

No. of

Samples Arithmetic Mean \pm 0

Maximum $\pm \sigma$

Minimum $\pm \sigma$

$\begin{array}{ll}2 & (4.40 \pm 0.41) E+00 \\ 3 & (1.71 \pm 0.86) E+00\end{array}$

$(4.69 \pm 0.99) E+00$

$(4.11 \pm 0.96) E+00$

$(2.68 \pm 0.91) E+00$

$(1.02 \pm 0.77) E+00$

Stokes Bluff Landing

$3 \quad(1.40 \pm 0.70) E+00$

$(2.15 \pm 0.91) E+00$

$(7.77 \pm 8.24) E-01$

$2 \quad(3.02 \pm 1.90) E+00$

$(4.36 \pm 1.04) E+00$

$(1.67 \pm 0.89) E+00$

$3 \quad(6.91 \pm 4.13) E-01$

$(1.02 \pm 0.77) E+00$

$(2.26 \pm 7.16) E-01$

3

$(1.54 \pm 0.86) E+00$

$(2.49 \pm 0.85) E+00$

$(8.32 \pm 7.59) E-01$

$1 \quad(2.12 \pm 1.18) E+00$

2

$(1.79 \pm 1.29) E+00$

$(2.71 \pm 1.25) E+00$

$(0.88 \pm 1.11) E+00$

On Site (Edible)

Beaver Dam Creek

1

$(2.45 \pm 2.22) E+00$

$(4.82 \pm 1.02) E+00$

$(4.07 \pm 7.30) E-01$

$1 \quad(0.36 \pm 1.08) E+00$

$1 \quad(4.52 \pm 1.06) E+00$

$3 \quad(5.70 \pm 2.85) E+00$

$(7.48 \pm 1.44) E+00$

$(2.41 \pm 0.89) E+00$

3

$(2.41 \pm 0.57) E+00$

$(2.91 \pm 0.92) E+00$

$(1.79 \pm 0.85) E+00$

PB-3 at Road A-13.2

Bass

Catfish

$(1.22 \pm 0.19) E+01$

$(1.36 \pm 0.17) E+01$

$(1.09 \pm 0.16) E+01$

2

$(8.16 \pm 0.96) E+00$

$(8.84 \pm 1.49) E+00$

$(7.48 \pm 1.46) E+00$

1

$(8.16 \pm 1.47) E+00$

Par Pond

$\begin{array}{lllll}\text { Bass } & 3 & (7.67 \pm 3.91) E+00 & (1.18 \pm 0.13) E+01 & (3.98 \pm 0.97) E+00 \\ \text { Bream } & 3 & (7.13 \pm 4.57) E+00 & (1.23 \pm 0.13) E+01 & (3.70 \pm 0.95) E+00\end{array}$




\section{Table 38}

\section{Radioactivity in Fish}

Page 13 of 14

\begin{tabular}{lcccc}
\hline Location & $\begin{array}{c}\text { No. of } \\
\text { Samples }\end{array}$ & Artthmetic Mean \pm 0 & Maximum $\pm \sigma$ & Minimum $\pm \sigma$ \\
\hline $\begin{array}{l}\text { Pond B } \\
\text { Bass }\end{array}$ & 3 & $(9.36 \pm 3.86) E+01$ & $(1.36 \pm 0.04) E+02$ & $(6.03 \pm 0.28) E+01$ \\
$\quad$ Bream & 3 & $(3.25 \pm 1.73) E+01$ & $(4.69 \pm 0.27) E+01$ & $(1.33 \pm 0.14) E+01$ \\
$\quad \begin{array}{l}\text { Crappie } \\
\text { SC-4 Steel Croek at Road A }\end{array}$ & 1 & $(7.31 \pm 0.34) E+01$ & & \\
$\quad$ Bass & 3 & $(7.49 \pm 0.28) E+00$ & $(7.65 \pm 1.11) E+00$ & $(7.18 \pm 1.10) E+00$ \\
Bream & 3 & $(6.30 \pm 2.06) E+00$ & $(8.16 \pm 1.46) E+00$ & $(4.08 \pm 1.27) E+00$
\end{tabular}

Off Slte (Nonedible)

Augusta Lock and Dam

Bass
Bream

$(2.47 \pm 0.90) E+00$

$(3.11 \pm 1.10) E+00$

$(1.84 \pm 1.02) E+00$

Bream

$(1.65 \pm 0.90) E+00$

$(2.28 \pm 1.04) E+00$

$(1.02 \pm 0.96) E+00$

Beaver Dam Creek River Mouth

Bream

$(4.98 \pm 1.13) E+00$

Catfish

$(1.26 \pm 0.80) E+00$

$(2.17 \pm 0.99) E+00$

$(6.86 \pm 8.67) E-01$

Four Mile Creek River Mouth

Bream

$3 \quad(3.60 \pm 0.17) E+00$

$(3.79 \pm 1.04) E+00$

$(3.48 \pm 1.09) E+00$

Catfish

$(3.08 \pm 1.89) E+00$

$(5.20 \pm 1.09) E+00$

$(1.56 \pm 0.89) E+00$

Hwy-301 Bridge Area

Bass
Bream
Catfish

2

3

3

L3R Creek River Mouth

Bass

Bream

Catfish

Steel Creek River Mouth

Bass
Bream
Catfish

U3R Creek River Mouth

Catfish
$(2.54 \pm 0.08) E+00$

$(2.59 \pm 0.96) E+00$

$(1.61 \pm 1.38) E+00$

$(2.82 \pm 0.96) E+00$

$(1.04 \pm 0.84) E+00$

$(2.48 \pm 0.95) E+00$

$(4.80 \pm 6.31) E-01$

$(2.17 \pm 0.95) E+00$

$(1.80 \pm 0.74) E+00$

$(2.63 \pm 0.94) E+00$

$(1.21 \pm 0.85) E+00$

(3.03 \pm 5.91$) E-01$

$(7.21 \pm 8.14) E-01$

$(-1.16 \pm 7.30) E-01$ $(-2.03 \pm 7.01) E-01$
$(2.56 \pm 0.69) E+00$

(3.05 \pm 0.98$) E+00$

$(1.83 \pm 0.00) E+00$

$(1.83 \pm 0.90) E+00$

$(2.43 \pm 0.94) E+00$

$(1.57 \pm 0.78) E+00$

$(1.18 \pm 0.63) E+00$

$(1.84 \pm 0.87) E+00$

$(6.01 \pm 7.59) E-01$

$(1.83 \pm 0.90) E+00$

$(8.93 \pm 8.30) E-01$ 


\section{Table 38}

Radioactivity in Fish

Page 14 of 14

\begin{tabular}{lcccc}
\hline Location & $\begin{array}{c}\text { No. of } \\
\text { Samples }\end{array}$ & Arithmetic Mean $\pm \sigma$ & Maximum $\pm \sigma$ & Minimum $\pm \sigma$ \\
\hline $\begin{array}{l}\text { West Bank Landing } \\
\text { Bream } \\
\text { Crappie }\end{array}$ & 1 & $(4.32 \pm 1.35) E+00$ & & \\
& 3 & $(1.45 \pm 1.52) E+00$ & $(3.20 \pm 1.02) E+00$ & $(0.50 \pm 1.13) E+00$
\end{tabular}




\section{Table 39}

\section{Cesium-137 in Off-Site Deer}

Page 1 of 1

\begin{tabular}{|c|c|c|}
\hline Date & Quadrant & C8-137 (pCl/g) \\
\hline $07-0 C T-93^{a}$ & SE & $0.58 \pm 0.05$ \\
\hline 07-ОСТ-93 & SE & $<L L D^{b}$ \\
\hline $07-0 C T-93$ & SE & $0.46 \pm 0.04$ \\
\hline 07-ОСТ-93 & SE & $0.15 \pm 0.03$ \\
\hline 07-ОСТ-93 & SE & $0.13 \pm 0.03$ \\
\hline 07-ОСТ-93 & SE & $0.16 \pm 0.02$ \\
\hline 07-ОСТ-93 & SE & $0.20 \pm 0.04$ \\
\hline 07-ОСТ-93 & SE & <LLD \\
\hline 07-ОCT-93 & SE & $0.24 \pm 0.04$ \\
\hline 07-ОСТ-93 & SE & $0.44 \pm 0.04$ \\
\hline $07-0 \mathrm{CT}-93$ & SE & $0.24 \pm 0.03$ \\
\hline 07-ОСТ-93 & SE & $0.18 \pm 0.04$ \\
\hline 02-NOV-93 & SE & $0.19 \pm 0.03$ \\
\hline 02-NOV-93 & SE & $0.11 \pm 0.03$ \\
\hline 02-NOV-93 & SE & $<L L D$ \\
\hline 02-NOV-93 & SE & $0.36 \pm 0.05$ \\
\hline 02-NOV-93 & SE & $0.36 \pm 0.04$ \\
\hline 02-NOV-93 & SE & $0.16 \pm 0.03$ \\
\hline 02-NOV-93 & SE & $0.09 \pm 0.03$ \\
\hline 02-NOV-93 & SE & $0.08 \pm 0.03$ \\
\hline 02-NOV-93 & SE & $0.91 \pm 0.06$ \\
\hline 02-NOV-93 & SE & $0.34 \pm 0.04$ \\
\hline 17-DEC-93 & NW & $1.76 \pm 0.07$ \\
\hline 17-DEC-93 & NW & $0.21 \pm 0.03$ \\
\hline 17-DEC-93 & NW & $<L L D$ \\
\hline 17-DEC-93 & NW & $0.06 \pm 0.02$ \\
\hline 17-DEC-93 & NW & $1.33 \pm 0.09$ \\
\hline 17-DEC-93 & NW & $<L L D$ \\
\hline 17-DEC-93 & NW & $0.54 \pm 0.04$ \\
\hline
\end{tabular}

a Date that sample was collected from the hunt club, not necessarily the date that deer was killed

b Less than lower limit of detection $(0.042 \mathrm{pCl} / \mathrm{g})$ 
Table 40

Comparison of Field and Laboratory Cesium-137 Measurements in Deer and Hogs

Page 1 of 7

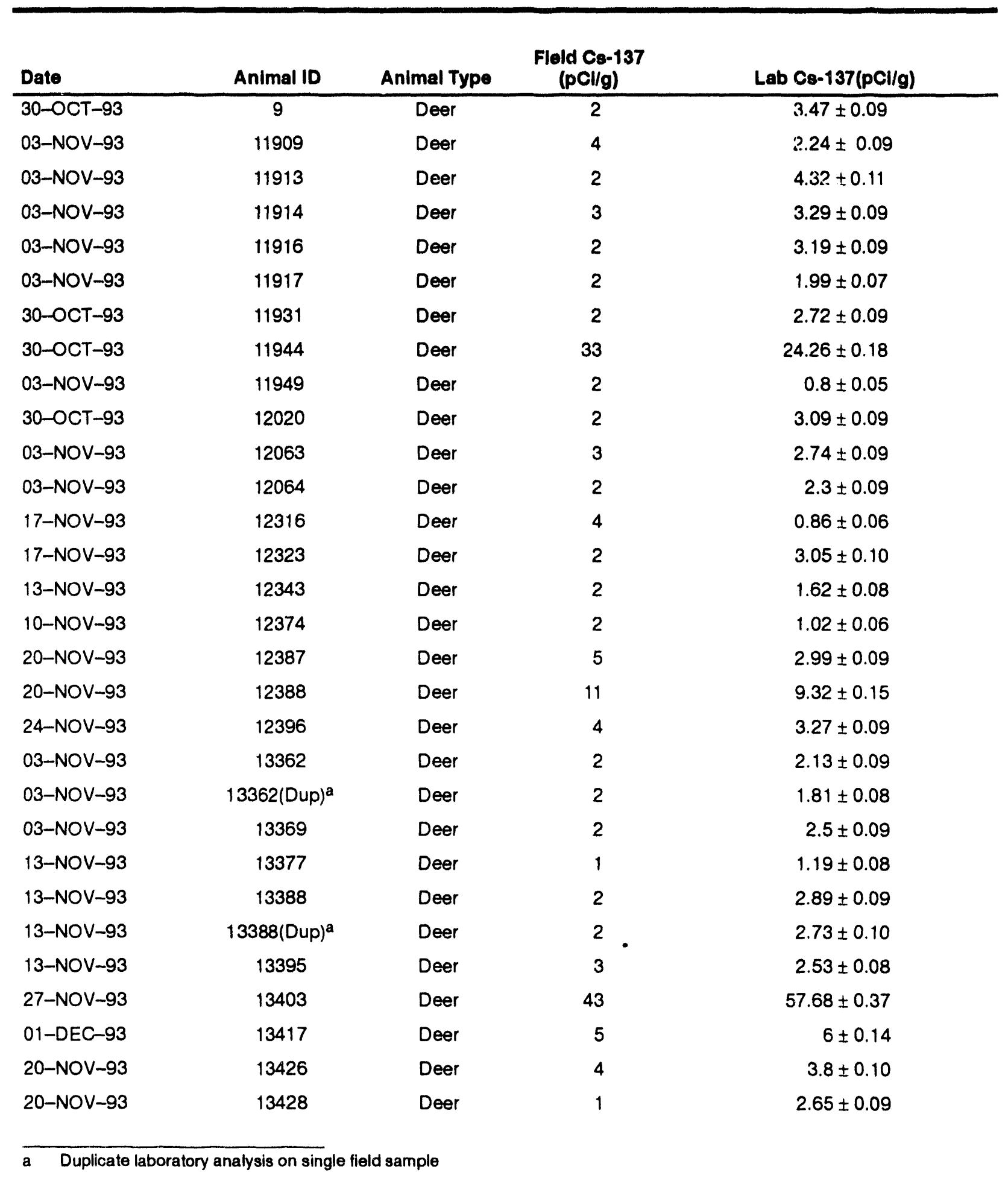




\section{Table 40}

\section{Comparlson of Field and Laboratory Ceslum-137 Measurements} In Deor and Hogs

Pago 2 of 7

\begin{tabular}{|c|c|c|c|c|}
\hline Date & AnImal ID & Anlmal Type & $\begin{array}{l}\text { Fleld Cs-137 } \\
\text { (pC//g) }\end{array}$ & Lab Cs-137(pCl/g) \\
\hline $24-N O V-93$ & 13448 & Deer & 7 & $5.54 \pm 0.12$ \\
\hline 10-NOV-93 & 13464 & Deer & 4 & $2.56 \pm 0.08$ \\
\hline $10-N O V-93$ & $13464(\text { Dup })^{a}$ & Deer & 4 & $2.9 \pm 0.09$ \\
\hline 17-NOV-93 & 13473 & Deer & 2 & $0.22 \pm 0.05$ \\
\hline $10-N O V-93$ & 13494 & Deer & 2 & $0.75 \pm 0.05$ \\
\hline 03-NOV-93 & 13509 & Hog & 26 & $34.05 \pm 0.29$ \\
\hline 03-NOV-93 & 13513 & Deer & 4 & $1.58 \pm 0.07$ \\
\hline 03-NOV-93 & 13518 & Deer & 1 & $1.52 \pm 0.07$ \\
\hline 06-NOV-93 & 13524 & Deer & 6 & $5.83 \pm 0.12$ \\
\hline $30-O C T-93$ & 13601 & Deer & 3 & $2.06 \pm 0.07$ \\
\hline $30-\supset C T-93$ & 13610 & Deer & 3 & $3.27 \pm 0.10$ \\
\hline $30-0 C T-93$ & 13612 & Deer & 4 & $2.37 \pm 0.08$ \\
\hline O6-NOV-93 & 13636 & Deer & 6 & $4.05 \pm 0.10$ \\
\hline 06-NOV-93 & 13638 & Deer & 6 & $5.31 \pm 0.12$ \\
\hline 10-NOV-93 & 13669 & Deer & 8 & $5.41 \pm 0.11$ \\
\hline 24-NOV-93 & 13689 & Deer & 8 & $9.06 \pm 0.15$ \\
\hline 24-NOV-93 & 13695 & Deer & 3 & $2.86 \pm 0.09$ \\
\hline $30-O C T-93$ & 13723 & Deer & 5 & $4.92 \pm 0.11$ \\
\hline $30-O C T-93$ & 13726 & Deer & 4 & $1.47 \pm 0.07$ \\
\hline 03-NOV-93 & 13734 & Deer & 18 & $19.23 \doteq 0.24$ \\
\hline 13-NOV-93 & 13741 & Deer & 1 & $0.78 \pm 0.05$ \\
\hline 13-NOV-93 & 13743 & Deer & 3 & $4.57 \pm 0.12$ \\
\hline 06-NOV-93 & 13747 & Deer & 2 & $1.82 \pm 0.07$ \\
\hline 06-NOV-93 & 13752 & Deer & 3 & $1.99 \pm 0.07$ \\
\hline $10-N O V-93$ & 13780 & Deer & 1 & $1.48 \pm 0.06$ \\
\hline 10-NOV-93 & 13783 & Deer & 4 & $2.2 \pm 0.08$ \\
\hline $10-N O V-93$ & 13784 & Deer & 2 & $2.49 \pm 0.08$ \\
\hline 10-NOV-93 & 13789 & Deer & 4 & $2.92 \pm 0.09$ \\
\hline O6-NOV-93 & 13845 & Deer & 4 & $4.62 \pm 0.11$ \\
\hline 13-NOV-93 & 13878 & Deer & 2 & $1.7 \pm 0.09$ \\
\hline
\end{tabular}


Table 40

Comparison of Fleld and Laboratory Cssium-137 Measurements in Deer and Hogs

Page 3 of 7

\begin{tabular}{|c|c|c|c|c|}
\hline Date & Animal ID & Animal Type & $\begin{array}{l}\text { Fiold } C_{2-137} \\
\text { (pcl/g) }\end{array}$ & Lab Cs-137(pCl/g) \\
\hline $17-$ NOV-93 & 13887 & $\mathrm{Hog}$ & 6 & $11.5 \pm 0.17$ \\
\hline 17-NOV-93 & 13888 & Hog & 5 & $8.09 \pm 0.15$ \\
\hline 17-NOV-93 & 13889 & Hog & 5 & $8.11 \pm 0.14$ \\
\hline 17-NOV-93 & 13905 & $\mathrm{Hog}$ & 13 & $18.07 \pm 0.17$ \\
\hline 17-NOV-93 & 13907 & Deer & 3 & $3.32 \pm 0.10$ \\
\hline 17-NOV-93 & 13907 (Dup) $^{a}$ & Deer & 3 & $3.09 \pm 0.09$ \\
\hline 17-NOV-93 & 13919 & Deer & 1 & $1.08 \pm 0.06$ \\
\hline 17-NOV-93 & 13925 & Deer & 1 & $1.16 \pm 0.07$ \\
\hline 03-NOV-93 & 13933 & Deer & 3 & $1.52 \pm 0.07$ \\
\hline 03-NOV-93 & 13957 & Deer & 3 & $3.72 \pm 0.12$ \\
\hline 27-NOV-93 & 13998 & Deer & 10 & $10.19 \pm 0.15$ \\
\hline 11-DEC-93 & 14011 & Deer & 5 & $3.87 \pm 0.10$ \\
\hline 11-DEC-93 & 14013 & Deer & 4 & $4.13 \pm 0.10$ \\
\hline 11-DEC-93 & 14013 (Dup) $^{a}$ & Deer & 4 & $4.29 \pm 0.10$ \\
\hline 01-DEC-93 & 14301 & Deer & 2 & $1.13 \pm 0.57$ \\
\hline 04-DEC-93 & 14323 & Deer & 7 & $7.21 \pm 0.13$ \\
\hline 04-DEC-93 & 14325 & Deer & 6 & $6.5 \pm 0.13$ \\
\hline 04-DEC-93 & 14325 (Dup) $^{\mathfrak{a}}$ & Deer & 6 & $6.45 \pm 0.13$ \\
\hline 04-DEC-93 & 14339 & Deer & 9 & $9.55 \pm 0.16$ \\
\hline 04-DEC-93 & 14354 & Deer & 1 & $0.52 \pm 0.04$ \\
\hline 04-DEC-93 & 14359 & Deer & 6 & $6.11 \pm 0.13$ \\
\hline 08-DEC-93 & 14374 & Deer & 14 & $13.74 \pm 0.18$ \\
\hline 08-DEC-93 & 14384 & Deer & 11 & $11.21 \pm 0.19$ \\
\hline 08-DEC-93 & 14407 & Deer & 12. & $13.73 \pm 0.18$ \\
\hline 08-DEC-93 & 14412 & Deer & 7 & $7.65 \pm 0.14$ \\
\hline 08-DEC-93 & 14415 & Deer & 10 & $8.58 \pm 0.14$ \\
\hline 08-DEC-93 & 14426 & Deer & 10 & $12.78 \pm 0.17$ \\
\hline 08-DEC-93 & 14428 & Deer & 13 & $14.27 \pm 0.18$ \\
\hline 08-DEC-93 & 14428 (Dup) $^{a}$ & Deer & 13 & $13.19 \pm 0.20$ \\
\hline 08-DEC-93 & 14430 & Deer & 10 & $10.7 \pm 0.16$ \\
\hline
\end{tabular}


Table 40

Comparison of Fleld and Laboratory Ceslum-137 Measurements in Deer and Hogs

Page 4 of 7

\begin{tabular}{|c|c|c|c|c|}
\hline Date & Animal ID & Anlmal Type & $\begin{array}{l}\text { Fiold Ce-137 } \\
\text { (pCl/g) }\end{array}$ & Lab Ca.137(pCV/g) \\
\hline 08-DEC-93 & 14443 & Deer & 7 & $6.15 \pm 0.14$ \\
\hline O8-DEC-93 & 14450 & Deer & 8 & $9.3 \pm 0.15$ \\
\hline 08-DEC-93 & 14454 & Deer & 4 & $3.42 \pm 0.10$ \\
\hline 08-DEC-93 & 14458 & Deer & 5 & $6.09 \pm 0.12$ \\
\hline 08-DEC-93 & $14458(D u p)^{a}$ & Deer & 5 & $6.27 \pm 0.13$ \\
\hline 08-DEC-93 & 14460 & Deer & 5 & $5.59 \pm 0.13$ \\
\hline 08-DEC-93 & 14461 & Deer & 4 & $5.7 \pm 0.14$ \\
\hline 11-DEC-93 & 14473 & Deer & 3 & $2.84 \pm 0.09$ \\
\hline 11-DEC-93 & 14475 & Hog & 17 & $22.29 \pm 0.22$ \\
\hline 11-DEC-93 & 14477 & Deer & 10 & $15.53 \pm 0.19$ \\
\hline 11-DEC-93 & 14485 & Deer & 5 & $8.53 \pm 0.14$ \\
\hline 15-DEC-93 & 14702 & Deer & 8 & $8.45 \pm 0.14$ \\
\hline 15-DEC-93 & 14716 & Deer & 7 & $4.06 \pm 0.07$ \\
\hline 15-DEC-93 & 14716 (Dup) ${ }^{a}$ & Deer & 7 & $7.63 \pm 0.14$ \\
\hline 15-DEC-93 & 14735 & Deer & 21 & $30.54 \pm 0.27$ \\
\hline 15-DEC-93 & 14741 & Deer & 6 & $8.75 \pm 0.15$ \\
\hline 15--ЕЕС-93 & 14750 & Deer & 5 & $7.85 \pm 0.14$ \\
\hline 01-DEC-93 & 14905 & Deer & 7 & $8.78 \pm 0.15$ \\
\hline 01-DEC-93 & 14905 (Dup) $^{a}$ & Deer & 7 & $4.78 \pm 0.08$ \\
\hline 04-DEC-93 & 14915 & Deer & 5 & $6.19 \pm 0.13$ \\
\hline 04-DEC-93 & 14916 & Deer & 8 & $9.71 \pm 0.15$ \\
\hline 04-DEC-93 & 14922 & Deer & 4 & $5.09 \pm 0.12$ \\
\hline 11-DEC-93 & 14929 & Deer & 10 & $15.3 \pm 0.22$ \\
\hline 11-DEC-93 & 14931 & Deer & 10 & $16.6 \pm 0.20$ \\
\hline 08-DEC-93 & 14957 & Deer & 13 & $17.19 \pm 0.21$ \\
\hline 08-DEC-93 & 14961 & Deer & 14 & $16.7 \pm 0.20$ \\
\hline 15-DEC-93 & 14984 & Deer & 7 & $6.56 \pm 0.12$ \\
\hline 13-NOV-93 & 15013 & Deer & 2 & $0.99 \pm 0.06$ \\
\hline 13-NOV-93 & 15021 & Deer & 1 & $1.06 \pm 0.07$ \\
\hline 13-NOV-93 & 15025 & Deer & 1 & $1.86 \pm 0.08$ \\
\hline
\end{tabular}

a Duplicate laboratory analysis on single field sample 
Table 40

Comparison of Fleld and Laboratory Ceslum-137 Measurements in Deer and Hogs

Page 5 of 7

\begin{tabular}{|c|c|c|c|c|}
\hline Date & Animal ID & Anlmal Type & $\begin{array}{l}\text { Flold Co-137 } \\
\text { (pCl/g) }\end{array}$ & Lab Ca-137(po1/g) \\
\hline 10-NOV-93 & 15035 & Deer & 3 & $1.82 \pm 0.07$ \\
\hline 10-NOV-93 & 15037 & Deer & 4 & $3.32 \pm 0.09$ \\
\hline $10-N O V-93$ & 15051 & Deer & 1 & $1.21 \pm 0.06$ \\
\hline 10-NOV-93 & 15054 & Deer & 2 & $1.93 \pm 0.08$ \\
\hline 10-NOV-93 & 15054 (Dup) & Deer & 2 & $1.73 \pm 0.07$ \\
\hline $10-N O V-93$ & 15064 & Deer & 6 & $5.3 \pm 0.11$ \\
\hline 13-NOV-93 & 15071 & Deer & 1 & $0.6 \pm 0.05$ \\
\hline 13-NOV-93 & 15081 & Ceer & 9 & $8.39 \pm 0.17$ \\
\hline $17-\mathrm{NOV}-93$ & 15094 & Deer & 1 & $0.79 \pm 0.04$ \\
\hline 04-DEC-93 & 15112 & Deer & 9 & $12.74 \pm 0.18$ \\
\hline 01-DEC-93 & 15120 & Deer & 7 & $9.38 \pm 0.15$ \\
\hline 17-NOV-93 & 15135 & Deer & 1 & $0.99 \pm 0.07$ \\
\hline 17-NOV-93 & 15141 & Deer & 3 & $1.89 \pm 0.08$ \\
\hline 27-NOV-93 & 15160 & Deer & 1 & $7.09 \pm 0.13$ \\
\hline 04-DEC-93 & 15179 & Deer & 1 & $0.99 \pm 0.05$ \\
\hline 04-DEC-93 & $15179(D u p)^{a}$ & Deer & 1 & $0.96 \pm 0.06$ \\
\hline 08-DEC-93 & 15184 & Deer & 8 & $11.74 \pm 0.20$ \\
\hline 08-DEC-93 & 15186 & Deer & 13 & $13.25 \pm 0.21$ \\
\hline 08-DEC-93 & $15186(\text { Dup })^{a}$ & Deer & 13 & $14.16 \pm 0.18$ \\
\hline 17-NOV-93 & 15201 & Deer & 5 & $0.79 \pm 0.06$ \\
\hline 17-NOV-93 & 15205 & Deer & 3 & $1.13 \pm 0.07$ \\
\hline 17-NOV-93 & 15206 & Deer & 1 & $0.9 \pm 0.06$ \\
\hline 17-NOV-93 & 15208 & Deer & 4 & $1.59 \pm 0.08$ \\
\hline 20-NOV-93 & 15221 & Deer & 5 & $1.79 \pm 0.07$ \\
\hline 13-NOV--93 & 15225 & $\mathrm{Hog}$ & 6 & $12.05 \pm 0.20$ \\
\hline 20-NOV-93 & 15227 & Hog & 17 & $19.68 \pm 0.25$ \\
\hline 20-NOV-93 & 15228 & $\mathrm{Hog}$ & 17 & $19.01 \pm 0.25$ \\
\hline 20-NOV-93 & 15231 & Deer & 5 & $2.66 \pm 0.09$ \\
\hline 20-NOV-93 & 15236 & Deer & 24 & $39.85 \pm 0.24$ \\
\hline 20-NOV-93 & 15238 & Deer & 6 & $5.76 \pm 0.14$ \\
\hline
\end{tabular}


Table 40

Comparison of Fleld and Laboratory Ceslum-137 Measurements In Deer and Hogs

Page 6 of 7

\begin{tabular}{|c|c|c|c|c|}
\hline Dato & Animal I0 & Anlmal Type & $\begin{array}{l}\text { Flold Ce-137 } \\
(p C l / g)\end{array}$ & Lab Ce-137(pCl/g) \\
\hline $20-N O V-93$ & 15241 & Deer & 3 & $4.8 \pm 0.11$ \\
\hline 20-NOV-93 & 15246 & Deer & 5 & $3.82 \pm 0.10$ \\
\hline 24-NOV-93 & 15250 & Deer & 9 & $7.37 \pm 0.14$ \\
\hline 20-NOV-93 & 15258 & Deer & 3 & $0.87 \pm 0.03$ \\
\hline 20-NOV-93 & 15258(Dup)" & Deer & 3 & $2.46 \pm 0.08$ \\
\hline $20-N O V-93$ & 15267 & $\mathrm{Hog}$ & 12 & $17.36 \pm 0.24$ \\
\hline 20-NOV-93 & 15270 & Deer & 8 & $4.71 \pm 0.11$ \\
\hline 20-NOV-93 & 15274 & $\mathrm{Hog}$ & 16 & $20.23 \pm 0.26$ \\
\hline 20-NOV-93 & 15275 & $\mathrm{Hog}$ & 15 & $21.72 \pm 0.26$ \\
\hline 27-NOV-93 & 15290 & Deer & 8 & $7.09 \pm 0.13$ \\
\hline 01-DEC-93 & 15294 & Deer & 8 & $9.32 \pm 0.15$ \\
\hline 27-NOV-93 & 15314 & Deer & 4 & $2.93 \pm 0.09$ \\
\hline 01-DEC-93 & 15333 & Deer & 9 & $9.73 \pm 0.15$ \\
\hline 01-DEC-93 & 15337 & Deer & 3 & $3.02 \pm 0.10$ \\
\hline $01-D E C-93$ & $15337(D u p)^{a}$ & Deer & 3 & $3.07 \pm 0.09$ \\
\hline O4-DEC-93 & 15340 & Deer & 8 & $8.64 \pm 0.15$ \\
\hline 01-DEC-93 & 15357 & Deer & 7 & $8.09 \pm 0.14$ \\
\hline 01-DEC-93 & 15362 & Deer & 2 & $3.08 \pm 0.09$ \\
\hline 01-DEC-93 & 15363 & Deer & 2 & $2.38 \pm 0.08$ \\
\hline 01-DEC-93 & 15383 & $\mathrm{H} \circ \mathrm{g}$ & 2 & $1.23 \pm 0.07$ \\
\hline 01-DEC-93 & 15388 & Deer & 1 & $1.34 \pm 0.07$ \\
\hline 01-DEC-93 & 15392 & $\mathrm{Hog}$ & 14 & $20.83 \pm 0.26$ \\
\hline 24-NOV-93 & 15402 & Deer & 5 & $4.62 \pm 0.10$ \\
\hline 24-NOV-93 & 15408 & Deer & 3 & $2.9 \pm 0.09$ \\
\hline 24-NOV-93 & 15419 & Deer & 7 & $6.78 \pm 0.13$ \\
\hline 24-NOV-93 & 15432 & Deer & 7 & $7.97 \pm 0.14$ \\
\hline 24-NOV-93 & 15432 (Dup) & Deer & 7 & $3.37 \pm 0.05$ \\
\hline 24-NOV-93 & 15440 & Deer & 4 & $5 \pm 0.11$ \\
\hline 24-NOV-93 & 15450 & Deer & 1 & $2.87 \pm 0.09$ \\
\hline 20-NOV-93 & 15464 & Deer & 7 & $4.06 \pm 0.11$ \\
\hline
\end{tabular}

a Duplicate laboratory analysis on single field sample 
Table 40

Comparison of Fleld and Laboratory Ceslum-137 Measurements In Deor and Hogs

Page 7 of 7

\begin{tabular}{|c|c|c|c|c|}
\hline Date & Animal ID & Animal Type & $\begin{array}{l}\text { Flold Ce-137 } \\
\text { (pc//g) }\end{array}$ & Lab Ce-137(pC/g) \\
\hline $20-$ NOV -93 & 15471 & Deer & 4 & $1.98 \pm 0.07$ \\
\hline 27-NOV-93 & 15481 & Deer & 5 & $2.85 \pm 0.09$ \\
\hline $01-D E C-93$ & 15496 & Deer & 5 & $5.16 \pm 0.12$ \\
\hline 01-DEC-93 & 15498 & Deer & 4 & $4.18 \pm 0.10$ \\
\hline 15-DEC-93 & 15659 & Deer & 3 & $2.44 \pm 0.08$ \\
\hline 01-DEC-93 & 15804 & Deer & 1 & $0.81 \pm 0.05$ \\
\hline 04-DEC-93 & 15816 & Deer & 5 & $8.3 \pm 0.15$ \\
\hline 04-DEC-93 & 15826 & Deer & 7 & $7.3 \pm 0.13$ \\
\hline 08-DEC-93 & 15847 & Deer & 4 & $3.25 \pm 0.09$ \\
\hline 11-DEC-93 & 15852 & Deer & 5 & $6.23 \pm 0.12$ \\
\hline 11-DEC-93 & 15854 & Deer & 9 & $10.24 \pm 0.16$ \\
\hline 15-DEC-93 & 15868 & Deer & 18 & $19.61 \pm 0.21$ \\
\hline 11-DEC-93 & 15899 & Deer & 6 & $6.46 \pm 0.13$ \\
\hline 04-DEC-93 & 15901 & Deer & 11 & $9.83 \pm 0.16$ \\
\hline 04-DEC-93 & 15906 & Deer & 8 & $7.37 \pm 0.14$ \\
\hline 08-DEC-93 & 15915 & Deer & 12 & $12.99 \pm 0.18$ \\
\hline 08-DEC-93 & 15931 & Deer & 4 & $9.42 \pm 0.15$ \\
\hline 15-DEC-93 & 15947 & Deer & 9 & $12.41 \pm 0.17$ \\
\hline 15-DEC-93 & 15992 & Deer & 5 & $4.44 \pm 0.11$ \\
\hline
\end{tabular}


Table 41

Summary of Ceslum-137 in Deer

Page 1 of 1

\begin{tabular}{|c|c|c|c|}
\hline Yoer & Number of Deer & Average, pCV/g* & Maximum, $\mathrm{pCl} / \mathrm{g}^{\star}$ \\
\hline 1965 & 198 & 10 & 10 \\
\hline 1966 & 541 & 6 & 24 \\
\hline 1967 & 1,032 & 9 & 104 \\
\hline 1968 & 669 & 11 & 74 \\
\hline $1969 b$ & 889 & 15 & 204 \\
\hline 1970 & 864 & 18 & 77 \\
\hline 1971 & 865 & 11 & 48 \\
\hline 1972 & 808 & 8 & 38 \\
\hline 1973 & 1.158 & 6 & 31 \\
\hline 1974 & 1,551 & 5 & 52 \\
\hline 1975 & 1,391 & 9 & 36 \\
\hline 1976 & 1,357 & 11 & 41 \\
\hline 1977 & 1,271 & 10 & 42 \\
\hline 1978 & 1,287 & 5 & 65 \\
\hline 1979 & 1,079 & 10 & 98 \\
\hline 1980 & 961 & 10 & 98 \\
\hline 1981 & 1,791 & 8 & 47 \\
\hline 1982 & 2,063 & 14 & 73 \\
\hline 1983 & 1,597 & 4 & 16 \\
\hline 1984 & 1,038 & 6 & 23 \\
\hline 1985 & 1,022 & 7 & 30 \\
\hline 1986 & 944 & 7 & 29 \\
\hline 1987 & 606 & 5 & 45 \\
\hline 1988 & 855 & 10 & 60 \\
\hline 1989 & 710 & 7 & 25 \\
\hline 1990 & 1,071 & 6 & 18 \\
\hline 1991 & 1,092 & 1 & 21 \\
\hline 1992 & 1,519 & 6 & 22 \\
\hline 1993 & 1,553 & 5 & 43 \\
\hline
\end{tabular}

a Values are field measurements.

b Approximately $20 \%$ of deer were monitored before 1969; since 1969, each deer has been monitored. 
Table 42

Strontlum in Deer and Hog Muscle

Page 1 of 2

\begin{tabular}{|c|c|c|c|}
\hline Date & Animal ID & Animal Type & $8 \mathrm{r}-90(\mathrm{pCl} / \mathrm{g})$ \\
\hline $30-0$ СТ-93 & 13610 & Deer & $0.008 \pm 0.004$ \\
\hline $30-0 \mathrm{CT}-93$ & 11944 & Deer & $0.002 \pm 0.003$ \\
\hline 03-NOV-93 & 13509 & Hog & $0.009 \pm 0.005$ \\
\hline 03-NOV-93 & 13362 & Deer & $0.002 \pm 0.003$ \\
\hline 03-NOV-93 & 11914 & Deer & $0.014 \pm 0.006$ \\
\hline 03-NOV-93 & 11917 & Deer & $0.010 \pm 0.004$ \\
\hline 10-NOV-93 & 15054 & Deer & $-0.001 \pm 0.004$ \\
\hline 10-NOV-93 & 15054 (Dup) $^{a}$ & Deer & $0.011 \pm 0.004$ \\
\hline 10-NOV-93 & 13783 & Deer & $0.001 \pm 0.003$ \\
\hline $10-N O V-93$ & 13464 & Deer & $0.039 \pm 0.006$ \\
\hline 10-NOV-93 & 13464 (Dup) $^{a}$ & Deer & $0.006 \pm 0.004$ \\
\hline 13-NOV-93 & 13388 & Deer & $-0.004 \pm 0.003$ \\
\hline 13-NOV-93 & 13388 (Dup) & Deer & $0.001 \pm 0.004$ \\
\hline 17-NOV-93 & 13905 & Hog & $0.012 \pm 0.007$ \\
\hline 17-NOV-93 & 13907 & Deer & $0.006 \pm 0.006$ \\
\hline 17-NOV-93 & 13907 (Dup) $^{\mathrm{a}}$ & Deer & $0.001 \pm 0.003$ \\
\hline 20-NOV-93 & 15258 & Deer & b \\
\hline 20-NOV-93 & 15258 (Dup) $^{a}$ & Deer & $0.005 \pm 0.003$ \\
\hline 20-NOV-93 & 15267 & Hog & $0.001 \pm 0.007$ \\
\hline 20-NOV-93 & 15228 & Hog & b \\
\hline 20-NOV-93 & 15274 & Hog & $0.013 \pm 0.007$ \\
\hline 20-NOV-93 & 15275 & Hog & $0.005 \pm 0.006$ \\
\hline 20-NOV-93 & 15227 & Hog & $0.034 \pm 0.011$ \\
\hline 20-NOV-93 & 15241 & Deer & $0.012 \pm 0.005$ \\
\hline 24-NOV-93 & 15432 & Deer & $0.003 \pm 0.002$ \\
\hline 24-NOV-93 & $15432(\text { Dup })^{a}$ & Deer & b \\
\hline 27-NOV-93 & 13403 & Deer & b \\
\hline 01-DEC-93 & 14905 & Deer & $0.005 \pm 0.003$ \\
\hline 01-DEC-93 & 14905 (Dup) $^{\mathrm{a}}$ & Deer & $-0.001 \pm 0.002$ \\
\hline 01-DEC-93 & 15363 & Deer & $-0.004 \pm 0.005$ \\
\hline 01-DEC-93 & 15392 & Hog & $0.076 \pm 0.014$ \\
\hline \begin{tabular}{ll|} 
a & Duplicate \\
b & Less than
\end{tabular} & gle field sample & & \\
\hline
\end{tabular}


Table 42

Strontium in Deer and Hog Muscle

Page 2 of 2

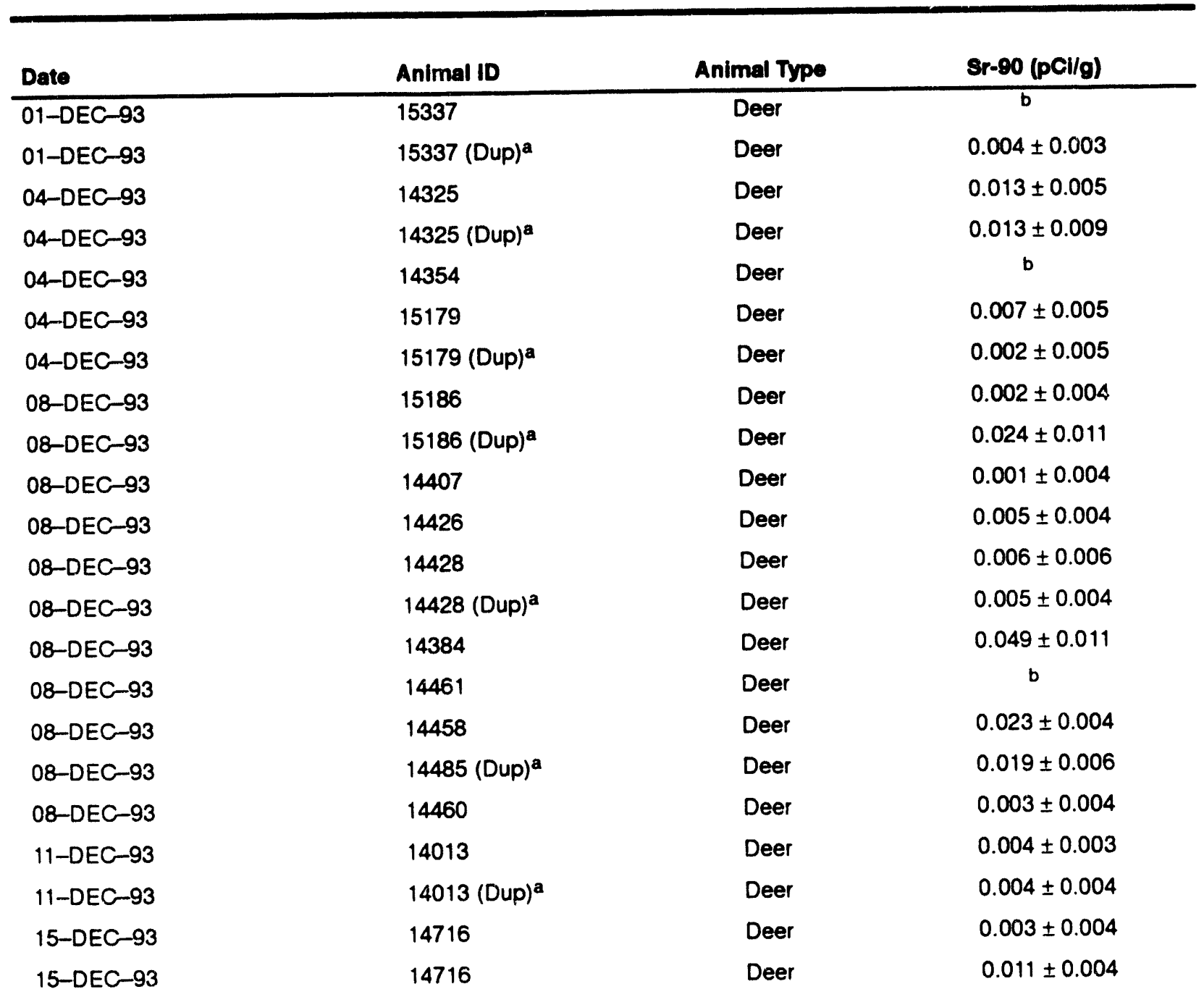


Table 43

Strontium in Deer Bone

Page 1 of 1

\begin{tabular}{|c|c|c|c|}
\hline Date & Animal ID & Animal Type & Sr-90 \\
\hline $30-0 C T-93$ & 13610 & Deer & $9.58 \pm 1.36$ \\
\hline 10-NOV-93 & 13783 & Deer & $9.67 \pm 1.18$ \\
\hline 10-NOV-93 & 13464 & Deer & $8.20 \pm 1.30$ \\
\hline 13-NOV-93 & 13388 & Deer & $6.15 \pm 1.41$ \\
\hline 17-NOV-93 & 13907 & Deer & $6.44 \pm 1.29$ \\
\hline 20-NOV-93 & 15258 & Deer & $9.92 \pm 1.11$ \\
\hline 24-NOV-93 & 15432 & Deer & $10.76 \pm 1.93$ \\
\hline 27-NOV-93 & 13403 & Deer & $5.23 \pm 1.02$ \\
\hline 01-DEC-93 & 14905 & Deer & $7.38 \pm 1.42$ \\
\hline 01-DEC-93 & 15337 & Deer & $4.26 \pm 1.72$ \\
\hline 04-DEC-93 & 14325 & Deer & $9.68 \pm 1.09$ \\
\hline 04-DEC-93 & 15179 & Deer & $4.09 \pm 0.85$ \\
\hline U8-DEC-93 & 15186 & Deer & $13.40 \pm 1.27$ \\
\hline 08-DEC-93 & 14428 & Deer & $7.93 \pm 1.72$ \\
\hline 11-DEC-93 & 14013 & Deer & $5.38 \pm 0.94$ \\
\hline 15-DEC-93 & 14716 & Deer & $7.11 \pm 0.99$ \\
\hline
\end{tabular}




\section{Table 44}

Parameters Used for Adult Consumption Rates and for Atmospheric

Dose Calculations ${ }^{a}$

Page 1 of 1

\begin{tabular}{|c|c|c|c|}
\hline Pathway & $\begin{array}{l}\text { Average } \\
\text { Individual }\end{array}$ & $\begin{array}{l}\text { Maximum } \\
\text { Individual }\end{array}$ & Population \\
\hline Fruits, vegetables, and grains (kg/yr) & 163 & 276 & 163 \\
\hline Leafy vegetables (kg/yr) & 21 & 43 & 21 \\
\hline Milk (L/yr) & 120 & 230 & 120 \\
\hline Meat (beef) (kg/yr) & 43 & 81 & 43 \\
\hline Inhalation ( $\left.\mathrm{m}^{3} / \mathrm{yr}\right)$ & 8,000 & 8,000 & 8,000 \\
\hline $\begin{array}{l}\text { Fraction of dose received after taking structural } \\
\text { shielding into account }\end{array}$ & 0.7 & 0.7 & 0.5 \\
\hline
\end{tabular}

a Values developed by SRTC for Savannah River Site [Hamby, 1991] 
Table 45

Parameters Used for Adult Consumption Rates and for Liquild Dose Calculations ${ }^{a}$

Page 1 of 1

\begin{tabular}{|c|c|c|c|}
\hline Pathway & $\begin{array}{c}\text { Average } \\
\text { Individual }\end{array}$ & $\begin{array}{l}\text { Maximum } \\
\text { Individual }\end{array}$ & Population \\
\hline Water consumption (ப/yr) & 370 & 730 & 370 \\
\hline Fish consumption (kg/yr) & 9 & 19 & 9 \\
\hline Marine invertebrates $(\mathrm{kg} / \mathrm{yr})$ & 2 & 8 & 2 \\
\hline Boating ${ }^{b}$ & $N / A^{c}$ & 21 & $1,100,000$ \\
\hline Swimming ${ }^{b}$ & $N / A^{c}$ & 8.9 & 160,000 \\
\hline Shoreline recreation ${ }^{b}$ & $N / A^{c}$ & 23 & 960,000 \\
\hline \multicolumn{4}{|l|}{ Water Treatment Plants } \\
\hline Water consumption ( $(\mathrm{yr}$ ) & 370 & 730 & 370 \\
\hline
\end{tabular}

a Values developed by SRTC for Savannah River Site [Hamby, 1991]

b Maximum individual values are in units of hours/year; population values are in units of person-hours.

c Not applicable

d Dose is calculated for the average individual, maximum individual, and population through the drinking water pathway from the downriver Beaufort-Jasper Water Treatment Plant (near Beaufort, South Carolina) and Cherokee Hill Water Treatment Plant (Port Wentworth, Georgia) 
Table 46

50-Mile-Radius (80 km) Milk, Meat, and Vegetation Production Around SRS

Page 1 of 2

\begin{tabular}{lccccccc}
\multicolumn{5}{l}{ Site Annual Milk Production (L) } & & & \\
Dir (mileo) & $0-5$ & $5-10$ & $10-20$ & $20-30$ & $30-40$ & $40-50$ & Total \\
\hline N & 0.0 & 0.0 & $4.200 E+04$ & $6.900 E+04$ & $1.000 E+06$ & $5.300 E+06$ & $6.411 E+06$ \\
NNE & 0.0 & 0.0 & $4.200 E+04$ & $6.900 E+04$ & $2.100 E+05$ & $5.000 E+05$ & $8.210 E+05$ \\
NE & 0.0 & 0.0 & $3.200 E+04$ & $1.000 E+06$ & $2.700 E+06$ & $2.000 E+06$ & $5.732 E+06$ \\
ENE & 0.0 & 0.0 & $2.500 E+04$ & $1.200 E+06$ & $4.400 E+06$ & $5.200 E+06$ & $1.083 E+07$ \\
E & 0.0 & 0.0 & $2.500 E+04$ & $1.400 E+06$ & $3.900 E+06$ & $4.900 E+06$ & $1.023 E+07$ \\
ESE & 0.0 & 0.0 & $2.500 E+04$ & $5.600 E+05$ & $3.000 E+04$ & $4.900 E+05$ & $1.105 E+06$ \\
SE & 0.0 & 0.0 & $2.500 E+04$ & $0.000 E+00$ & $0.000 E+00$ & $0.000 E+00$ & $2.500 E+03$ \\
SSE & 0.0 & 0.0 & $4.800 E+05$ & $8.600 E+05$ & $1.200 E+06$ & $1.200 E+06$ & $3.740 E+06$ \\
S & 0.0 & 0.0 & $1.000 E+06$ & $2.100 E+06$ & $3.000 E+06$ & $3.500 E+06$ & $9.600 E+06$ \\
SSW & 0.0 & 0.0 & $9.900 E+05$ & $3.800 E+06$ & $7.400 E+06$ & $7.600 E+06$ & $1.979 E+07$ \\
SW & 0.0 & 0.0 & $9.900 E+05$ & $2.200 E+06$ & $5.800 E+06$ & $4.800 E+06$ & $1.379 E+07$ \\
WSW & 0.0 & 0.0 & $9.900 E+05$ & $1.700 E+06$ & $2.400 E+06$ & $3.500 E+06$ & $8.590 E+06$ \\
W & 0.0 & 0.0 & $6.700 E+05$ & $1.300 E+06$ & $2.200 E+06$ & $3.600 E+06$ & $7.770 E+06$ \\
WNW & 0.0 & 0.0 & $2.300 E+05$ & $1.100 E+06$ & $1.200 E+06$ & $2.000 E+06$ & $4.530 E+06$ \\
NW & 0.0 & 0.0 & $4.200 E+04$ & $3.800 E+05$ & $1.400 E+06$ & $1.000 E+06$ & $2.822 E+06$ \\
NNW & 0.0 & 0.0 & $4.200 E+04$ & $6.900 E+04$ & $1.700 E+06$ & $3.400 E+06$ & $5.211 E+06$ \\
Total & 0.0 & 0.0 & $5.62 E+06$ & $1.781 E+07$ & $3.954 E+07$ & $4.899 E+07$ & $1.110 E+08$
\end{tabular}

Site Annual Meat Production (kg)

\begin{tabular}{lccccccc} 
Dir (miles) & $\mathbf{0 - 5}$ & $\mathbf{5 - 1 0}$ & $\mathbf{1 0 - 2 0}$ & $\mathbf{2 0 - 3 0}$ & $\mathbf{3 0 - 4 0}$ & $\mathbf{4 0 - 5 0}$ & Total \\
\hline N & 0.0 & 0.0 & $5.300 E+04$ & $8.800 E+04$ & $2.500 E+05$ & $9.800 E+05$ & $1.371 E+06$ \\
NNE & 0.0 & 0.0 & $5.300 E+04$ & $8.800 E+04$ & $2.000 E+05$ & $4.100 E+05$ & $7.510 E+05$ \\
NE & 0.0 & 0.0 & $7.100 E+04$ & $1.700 E+05$ & $3.500 E+05$ & $4.500 E+05$ & $1.041 E+06$ \\
ENE & 0.0 & 0.0 & $8.300 E+04$ & $2.000 E+05$ & $4.600 E+05$ & $5.700 E+05$ & $1.313 E+06$ \\
E & 0.0 & 0.0 & $8.300 E+04$ & $1.900 E+05$ & $3.400 E+05$ & $5.100 E+05$ & $1.123 E+06$ \\
ESE & 0.0 & 0.0 & $8.300 E+04$ & $1.900 E+05$ & $2.200 E+05$ & $2.500 E+05$ & $7.430 E+05$ \\
SE & 0.0 & 0.0 & $1.200 E+05$ & $2.100 E+05$ & $2.600 E+05$ & $3.000 E+05$ & $8.900 E+05$ \\
SSE & 0.0 & 0.0 & $1.100 E+05$ & $1.900 E+05$ & $2.600 E+05$ & $2.900 E+05$ & $8.500 E+05$ \\
S & 0.0 & 0.0 & $9.400 E+04$ & $1.500 E+05$ & $2.000 E+05$ & $2.700 E+05$ & $7.140 E+05$ \\
SSW & 0.0 & 0.0 & $9.500 E+04$ & $1.800 E+05$ & $2.900 E+05$ & $3.900 E+05$ & $9.550 E+05$ \\
SW & 0.0 & 0.0 & $9.500 E+04$ & $1.700 E+05$ & $2.700 E+05$ & $3.200 E+05$ & $8.550 E+05$
\end{tabular}


Table 46

50-Mile-Radius (80 km) Milk, Meat, and Vegetation Production Around SRS

Page 2 of 2

Site Annual Meat Production (kg), Cont'd.

\begin{tabular}{lccccccc} 
Dir (miles) & $0-5$ & $5-10$ & $10-20$ & $20-30$ & $30-40$ & $40-50$ & Total \\
\hline WSW & 0.0 & 0.0 & $9.500 E+04$ & $1.600 E+05$ & $2.300 E+05$ & $4.000 E+05$ & $8.850 E+05$ \\
W & 0.0 & 0.0 & $5.800 E+04$ & $1.000 E+05$ & $2.100 E+05$ & $4.100 E+05$ & $7.780 E+05$ \\
WNW & 0.0 & 0.0 & $4.800 E+04$ & $6.200 E+04$ & $1.300 E+05$ & $2.900 E+05$ & $5.300 E+05$ \\
NW & 0.0 & 0.0 & $5.300 E+04$ & $8.000 E+04$ & $2.800 E+05$ & $2.700 E+05$ & $6.830 E+05$ \\
NNW & 0.0 & 0.0 & $5.300 E+04$ & $8.800 E+04$ & $3.300 E+05$ & $6.200 E+05$ & $1.091 E+06$ \\
Total & 0.0 & 0.0 & $1.247 E+06$ & $2.316 E+06$ & $4.280 E+06$ & $6.730 E+06$ & $1.457 E+07$
\end{tabular}

Site Annual Vegetation Production (kg)

\begin{tabular}{lccccccc} 
Dir (miles) & $\mathbf{0 - 5}$ & $\mathbf{5 - 1 0}$ & $\mathbf{1 0 - 2 0}$ & $.20-30$ & $.30-40$ & $.40-50$ &. Total \\
\hline N & 0.0 & 0.0 & $3.600 E+05$ & $6.000 E+05$ & $8.400 E+05$ & $8.700 E+05$ & $2.670 E+06$ \\
NNE & 0.0 & 0.0 & $3.600 E+05$ & $6.000 E+05$ & $5.100 E+05$ & $6.300 E+03$ & $1.476 E+06$ \\
NE & 0.0 & 0.0 & $3.600 E+05$ & $6.900 E+05$ & $1.000 E+06$ & $5.000 E+05$ & $2.550 E+06$ \\
ENE & 0.0 & 0.0 & $3.600 E+05$ & $6.600 E+05$ & $1.200 E+06$ & $1.500 E+06$ & $3.270 E+06$ \\
E & 0.0 & 0.0 & $3.600 E+05$ & $5.900 E+05$ & $8.500 E+05$ & $1.400 E+06$ & $3.200 E+06$ \\
ESE & 0.0 & 0.0 & $3.600 E+05$ & $2.200 E+06$ & $1.900 E+06$ & $1.100 E+06$ & $5.560 E+06$ \\
SE & 0.0 & 0.0 & $2.500 E+06$ & $4.500 E+06$ & $3.000 E+06$ & $1.100 E+06$ & $1.110 E+07$ \\
SSE & 0.0 & 0.0 & $1.700 E+06$ & $2.900 E+06$ & $3.600 E+06$ & $1.100 E+06$ & $9.300 E+06$ \\
S & 0.0 & 0.0 & $7.200 E+04$ & $5.400 E+05$ & $8.400 E+05$ & $9.700 E+05$ & $2.422 E+06$ \\
SSW & 0.0 & 0.0 & $3.500 E+02$ & $1.200 E+05$ & $2.500 E+05$ & $1.100 E+05$ & $4.804 E+05$ \\
SW & 0.0 & 0.0 & $3.500 E+02$ & $7.800 E+02$ & $2.200 E+03$ & $3.100 E+05$ & $3.133 E+05$ \\
WSW & 0.0 & 0.0 & $3.500 E+02$ & $5.800 E+02$ & $9.900 E+02$ & $2.900 E+03$ & $4.820 E+03$ \\
W & 0.0 & 0.0 & $4.500 E+04$ & $2.300 E+04$ & $1.700 E+04$ & $5.200 E+04$ & $1.370 E+05$ \\
WNW & 0.0 & 0.0 & $2.600 E+05$ & $3.800 E+04$ & $4.500 E+05$ & $1.100 E+06$ & $1.848 E+06$ \\
NW & 0.0 & 0.0 & $3.600 E+05$ & $4.300 E+05$ & $8.400 E+05$ & $1.100 E+06$ & $2.730 E+06$ \\
NNW & 0.0 & 0.0 & $3.600 E+05$ & $6.000 E+05$ & $8.400 E+05$ & $1.100 E+06$ & $2.900 E+06$ \\
Total & 0.0 & 0.0 & $7.458 E+06$ & $1.449 E+07$ & $1.614 E+07$ & $1.232 E+07$ & $5.041 E+07$
\end{tabular}




\section{Table 47}

Input Parameters for Atmospheric Transport Model

Page 1 of 1

Release Locations for 1991 Maximum and Average Individualsa

Release Source Area

\begin{tabular}{|c|c|c|c|c|}
\hline$F, H, P, K, C$ & M & D & SRL & $\begin{array}{l}\text { Diffuse } \\
\text { and } \\
\text { Fugitive }\end{array}$ \\
\hline 61 & 0 & 16 & 31 & 0 \\
\hline 58000 & 50041 & 20938 & 51863 & 58000 \\
\hline 62000 & 104828 & 65284 & 106670 & 62000 \\
\hline
\end{tabular}

Release height (m)

Release location (site coord,)

East

North

62000

104828

65284

02000

a Operating period for maximum individual and average individual taken as 74 years, l.e., two times actual operating period; operating period for population dose taken to be 201 years, i.e., 100-year environmental dose commitment 
Table 48

Site-Specific Parameters Used in Liquid Dose Calculations

Page 1 of 1

River flow rate at SRS for 1992 (cubic feet/second)

14,800

River dilution in estuary

Transit time from process areas to river (hr)

Transit time from SRS to water treatment plants (hr)

Water treatment time $(\mathrm{hr})$

Edible aquatic food harvest ( $\mathrm{kg} / \mathrm{yr}$ )

Fish - sport

$35,000^{\mathrm{a}}$

Fish - commercial

$2,700^{a}$

Invertebrates - saltwater

$390,000^{a}$

Irrigation $^{\text {a }}$

None $e^{a}$

Shore width factor

0.2

Fish bioaccumulation factor for cesium

3,000

a Values developed by SRTC for Savannah River Site [Hamby, 1991] 


\section{Table 49}

Meteorologlcal Data for 1987-1991

Page 1 of 7

USNRC Computer Code-XOQDOQ, Vorlon 2.0 Run Dato: 93.071 (BAL 6/29/83 VEABION) 43824 Wind State H-Area 60 minute $62 \mathrm{~W} 87-01$ Stabillty from Slgma A

Joint Frequency Distribution of Wind 8pead and Direotlon:

Atmosphoric stablilty Clase A Extromely Unstable Conditions

\begin{tabular}{lllllllll}
\hline $\begin{array}{c}\text { (M/8) } \\
\text { UMAX }\end{array}$ & N & NNE & NE & ENE & E & EsE & 8E & s8E \\
\hline 2.00 & 0.374 & 0.406 & 0.372 & 0.422 & 0.402 & 0.374 & 0.404 & 0.356 \\
4.00 & 0.872 & 0.735 & 0.876 & 0.995 & 0.942 & 0.942 & 0.648 & 0.618 \\
6.00 & 0.573 & 0.260 & 0.157 & 0.187 & 0.148 & 0.073 & 0.066 & 0.094 \\
8.00 & 0.089 & 0.052 & 0.007 & 0.011 & 0.007 & 0.002 & 0.005 & 0.011 \\
12.00 & 0.005 & 0.000 & 0.002 & 0.002 & 0.000 & 0.000 & 0.000 & 0.000 \\
14.10 & 0.000 & 0.000 & 0.000 & 0.000 & 0.000 & 0.000 & 0.000 & 0.000 \\
TOTAL & 1.91 & 1.45 & 1.41 & 1.62 & 1.50 & 1.39 & 1.12 & 1.08
\end{tabular}

Joint Frequency Distribution of Wind Speed and Direction:

Atmospheric Stabillty Claes A Extremely Unstable Conditions

\begin{tabular}{llllllllll}
\hline $\begin{array}{c}\text { (M/8) } \\
\text { UMAX }\end{array}$ & $\mathbf{s}$ & $\mathbf{s s W}$ & $\mathbf{s W}$ & WSW & $\boldsymbol{W}$ & WNW & NW & NNW & TOTAL \\
\hline 2.00 & 0.358 & 0.347 & 0.450 & 0.392 & 0.454 & 0.434 & 0.370 & 0.413 & 6.328 \\
4.00 & 0.739 & 0.721 & 1.002 & 1.282 & 1.294 & 0.942 & 0.534 & 0.602 & 13.746 \\
6.00 & 0.139 & 0.139 & 0.208 & 0.242 & 0.274 & 0.235 & 0.141 & 0.240 & 3.176 \\
8.00 & 0.021 & 0.018 & 0.016 & 0.037 & 0.032 & 0.021 & 0.014 & 0.055 & 0.397 \\
12.00 & 0.000 & 0.007 & 0.009 & 0.009 & 0.002 & 0.007 & 0.002 & 0.009 & 0.055 \\
14.10 & 0.000 & 0.000 & 0.000 & 0.000 & 0.000 & 0.000 & 0.000 & 0.000 & 0.000 \\
TOTAL & 1.26 & 1.23 & $\mathbf{1 . 6 8}$ & $\mathbf{1 . 9 6}$ & $\mathbf{2 . 0 6}$ & $\mathbf{1 . 6 4}$ & $\mathbf{1 . 0 6}$ & $\mathbf{1 . 3 2}$ & $\mathbf{2 3 . 7 0}$
\end{tabular}


Table 49

Meteorological Data for 1987-1991

Page 2 of 7

UENRC Computer CodeXOQDOQ, Vorsion 2.0 Run Dato: 93.071 (BAL 6/20/83 VEA8ION) 43824 Wind Stats H-Area 60 minuto 62M 87-01 Btablilty from Sloma A

Joint Frequenoy Distribution of Wind 8peed and Direotion:

Atmospherio stablilty Clase B Moderatoly Unatable Conditions

\begin{tabular}{lrlllllll}
\hline $\begin{array}{c}\text { (M/8) } \\
\text { UMAX }\end{array}$ & N & NNE & NE & ENE & E & EsE & 8E & 88E \\
\hline 2.00 & 0.075 & 0.078 & 0.087 & 0.098 & 0.052 & 0.055 & 0.057 & 0.048 \\
4.00 & 0.267 & 0.411 & 0.577 & 0.621 & 0.429 & 0.342 & 0.244 & 0.217 \\
6.00 & 0.139 & 0.388 & 0.377 & 0.313 & 0.164 & 0.105 & 0.071 & 0.084 \\
8.00 & 0.007 & 0.082 & 0.027 & 0.007 & 0.005 & 0.011 & 0.002 & 0.009 \\
12.00 & 0.000 & 0.007 & 0.002 & 0.000 & 0.000 & 0.000 & 0.000 & 0.002 \\
14.10 & 0.000 & 0.000 & 0.000 & 0.000 & 0.000 & 0.000 & 0.000 & 0.000 \\
TOTAL & 0.49 & 0.97 & 1.07 & 1.04 & 0.65 & 0.51 & 0.37 & 0.36
\end{tabular}

Joint Frequency Distribution of Wind Speed and Direction:

Atmospheric Stabllity Class B Mnderately Unstable Conditlons

\begin{tabular}{lrllllllll}
\hline $\begin{array}{c}\text { (M/S) } \\
\text { UMAX }\end{array}$ & $\mathbf{s}$ & ssw & sw & WsW & w & WNW & NW & NNW & TOTAL \\
\hline 2.00 & 0.078 & 0.071 & 0.046 & 0.050 & 0.046 & 0.075 & 0.052 & 0.071 & 1.038 \\
4.00 & 0.324 & 0.329 & 0.477 & 0.671 & 0.559 & 0.372 & 0.251 & 0.210 & 6.300 \\
6.00 & 0.187 & 0.212 & 0.317 & 0.507 & 0.511 & 0.356 & 0.126 & 0.089 & 3.945 \\
8.00 & 0.050 & 0.037 & 0.052 & 0.103 & 0.171 & 0.214 & 0.059 & 0.014 & 0.851 \\
12.00 & 0.002 & 0.002 & 0.007 & 0.007 & 0.064 & 0.064 & 0.007 & 0.002 & 0.167 \\
14.10 & 0.000 & 0.000 & 0.000 & 0.000 & 0.000 & 0.000 & 0.000 & 0.000 & 0.000 \\
TOTAL & $\mathbf{0 . 6 4}$ & $\mathbf{0 . 6 5}$ & $\mathbf{0 . 9 0}$ & $\mathbf{1 . 3 4}$ & $\mathbf{1 . 3 5}$ & $\mathbf{1 . 0 8}$ & $\mathbf{0 . 5 0}$ & $\mathbf{0 . 3 9}$ & $\mathbf{1 2 . 3 0}$
\end{tabular}


Table 49

Meteorologlcal Data for 1987-1991

Page 3 of 7

UENRC Computor Code-XOQDOQ, Verelon 2.0 Aun Dato: 93.071 (8RL 6/29/83 VERBION) 43824 Wind etats H-Area 60 minute 62M 87-01 Btablity from 8loma A

Joint Frequenoy Dlatribution of Wind Epeed and Direotlon:

Atmospherlo stablilty Clase C slightly Unatable Conditlone

\begin{tabular}{lrlllllll}
\hline (M/8) & N & NNE & NE & ENE & E & ESE & SE & SSE \\
\hline 2.00 & 0.032 & 0.057 & 0.091 & 0.071 & 0.059 & 0.046 & 0.055 & 0.048 \\
4.00 & 0.173 & 0.573 & 1.125 & 1.031 & 0.600 & 0.408 & 0.411 & 0.370 \\
6.00 & 0.116 & 0.539 & 1.303 & 0.742 & 0.354 & 0.192 & 0.217 & 0.253 \\
8.00 & 0.014 & 0.098 & 0.196 & 0.082 & 0.018 & 0.032 & 0.032 & 0.059 \\
12.00 & 0.002 & 0.011 & 0.000 & 0.002 & 0.002 & 0.005 & 0.000 & 0.027 \\
14.10 & 0.000 & 0.000 & 0.000 & 0.000 & 0.000 & 0.000 & 0.000 & 0.000 \\
TOTAL & 0.34 & 1.28 & 2.72 & 1.93 & 1.03 & 0.08 & 0.71 & 0.76
\end{tabular}

Joint Frequenoy Distribution of Wind Speed and Direotion:

Atmoepheric stablilty Clase C Silghtly Unstablo Conditions

\begin{tabular}{cccccccccc}
\hline $\begin{array}{c}\text { (M/8) } \\
\text { UMAX }\end{array}$ & $\mathbf{s}$ & s9w & sW & WsW & w & WNW & NW & NNW & TOTAL \\
\hline 2.00 & 0.068 & 0.048 & 0.055 & 0.050 & 0.075 & 0.046 & 0.050 & 0.052 & 0.904 \\
4.00 & 0.479 & 0.516 & 0.589 & 0.794 & 0.527 & 0.445 & 0.301 & 0.237 & 8.580 \\
6.00 & 0.470 & 0.461 & 0.561 & 0.687 & 0.643 & 0.564 & 0.212 & 0.119 & 7.432 \\
8.00 & 0.155 & 0.164 & 0.208 & 0.258 & 0.445 & 0.429 & 0.096 & 0.018 & 2.305 \\
12.00 & 0.043 & 0.041 & 0.046 & 0.059 & 0.155 & 0.167 & 0.018 & 0.007 & 0.586 \\
14.10 & 0.000 & 0.000 & 0.000 & 0.002 & 0.000 & 0.002 & 0.000 & 0.000 & 0.005 \\
TOTAL & 1.22 & 1.23 & 1.46 & 1.85 & 1.85 & 1.65 & 0.68 & 0.43 & 19.81
\end{tabular}


Table 49

Meteorological Data for 1987-1991

Page 4 of 7

UBNRC Computer CodeXOQDOQ, Vorelon 2.0 Run Dato: 93.071 (8RL 6/29/83 VEA8ION) 43824 Wind Stats H-Arca 60 minute $62 M$ 87-01 Btabllity from Elgma A

Jolnt Frequenoy Dlotributlon of Wind 8peed and Direotion:

Atmosphorio stablity Class D Neutral Conditione

\begin{tabular}{lllllllll}
\hline $\begin{array}{c}\text { (M/8) } \\
\text { UMAX }\end{array}$ & N & NNE & NE & ENE & $E$ & E8E & 8E & s8E \\
\hline 2.00 & 0.018 & 0.048 & 0.055 & 0.037 & 0.055 & 0.030 & 0.055 & 0.068 \\
4.00 & 0.103 & 0.436 & 1.072 & 0.888 & 0.552 & 0.504 & 0.705 & 0.691 \\
6.00 & 0.123 & 0.434 & 0.853 & 0.584 & 0.395 & 0.440 & 0.653 & 1.164 \\
8.00 & 0.011 & 0.052 & 0.098 & 0.023 & 0.005 & 0.011 & 0.046 & 0.180 \\
12.00 & 0.000 & 0.023 & 0.023 & 0.000 & 0.002 & 0.000 & 0.000 & 0.007 \\
14.10 & 0.000 & 0.000 & 0.000 & 0.000 & 0.000 & 0.000 & 0.000 & 0.000 \\
TOTAL & 0.28 & 0.99 & 2.10 & 1.53 & 1.01 & 0.09 & 1.46 & 2.11
\end{tabular}

Jolnt Frequenoy Dlatribution of Wind Speed and Directlon:

Atmosphorlo Stability Clese D Noutral Conditions

\begin{tabular}{lccccccccc}
\hline $\begin{array}{c}\text { (M/8) } \\
\text { UMAX }\end{array}$ & $\mathbf{8}$ & ssW & sW & WsW & $W$ & WNW & NW & NNW & TOTAL \\
\hline 2.00 & 0.055 & 0.032 & 0.071 & 0.048 & 0.037 & 0.034 & 0.050 & 0.041 & 0.732 \\
4.00 & 0.924 & 0.910 & 0.803 & 0.808 & 0.723 & 0.570 & 0.427 & 0.272 & 10.389 \\
6.00 & 1.451 & 0.778 & 0.901 & 0.769 & 0.776 & 0.648 & 0.317 & 0.089 & 10.376 \\
8.00 & 0.219 & 0.146 & 0.098 & 0.087 & 0.030 & 0.052 & 0.030 & 0.002 & 1.091 \\
12.00 & 0.016 & 0.037 & 0.002 & 0.002 & 0.007 & 0.002 & 0.000 & 0.000 & 0.121 \\
12.10 & 0.000 & 0.000 & 0.000 & 0.000 & 0.000 & 0.000 & 0.000 & 0.000 & 0.000 \\
TOTAL & 2.67 & 1.90 & 1.88 & 1.71 & 1.57 & 1.31 & 0.82 & 0.40 & 22.71
\end{tabular}




\section{Table 49}

\section{Metoorologlcal Data for 1987-1991}

Page 6 of 7

UENRC Computer CodeXOQDOQ 43824 Wind btats H-Area 60 minute

Verelon 2.0 Aun Dato: 03.071 (BAL 6/29/83 VERBION) 62N 87-01 Etablilty from slgma $A$

Joint Frequency Distribution of Wind Speed and Direotion:

Atmospherto stability Clese E Silghtly stable Conditions

\begin{tabular}{lrlrlrlll}
\hline $\begin{array}{c}\text { (MM/S) } \\
\text { UMAX }\end{array}$ & N & NNE & NE & ENE & E & EsE & sE & SSE \\
\hline 2.00 & 0.011 & 0.021 & 0.039 & 0.007 & 0.009 & 0.025 & 0.030 & 0.030 \\
4.00 & 0.062 & 0.274 & 0.637 & 0.477 & 0.297 & 0.333 & 0.456 & 0.701 \\
6.00 & 0.071 & 0.527 & 0.689 & 0.705 & 0.600 & 0.454 & 0.650 & 1.006 \\
8.00 & 0.000 & 0.050 & 0.032 & 0.037 & 0.011 & 0.011 & 0.002 & 0.027 \\
12.00 & 0.000 & 0.002 & 0.002 & 0.000 & 0.000 & 0.000 & 0.000 & 0.000 \\
14.10 & 0.000 & 0.000 & 0.000 & 0.000 & 0.000 & 0.000 & 0.000 & 0.000 \\
TO-AL & 0.14 & 0.87 & 1.45 & 1.23 & 0.02 & 0.82 & 1.14 & 1.76
\end{tabular}

Joint Frequenoy Distribution of Wind Speed and Direotion:

Atmospherto Stabllity Class E silghtly stable Conditione

\begin{tabular}{lrlllllllll}
\hline $\begin{array}{c}\text { (M/8) } \\
\text { UMAX }\end{array}$ & $\mathbf{8}$ & ssW & sW & WsW & W & WNW & NW & NNW & TOTAL \\
\hline 2.00 & 0.016 & 0.016 & 0.011 & 0.011 & 0.021 & 0.014 & 0.023 & 0.021 & 0.303 \\
4.00 & 0.673 & 0.573 & 0.543 & 0.472 & 0.431 & 0.427 & 0.333 & 0.301 & 7.040 \\
6.00 & 1.184 & 0.940 & 0.910 & 0.894 & 0.484 & 0.402 & 0.187 & 0.141 & 9.846 \\
8.00 & 0.043 & 0.018 & 0.043 & 0.009 & 0.005 & 0.002 & 0.002 & 0.000 & 0.294 \\
12.00 & 0.000 & 0.000 & 0.000 & 0.000 & 0.000 & 0.000 & 0.000 & 0.000 & 0.005 \\
12.10 & 0.000 & 0.000 & 0.000 & 0.000 & 0.000 & 0.000 & 0.000 & 0.000 & 0.000 \\
TOTAL & 1.92 & 1.55 & 1.51 & 1.39 & 0.94 & 0.84 & 0.55 & 0.46 & 17.49
\end{tabular}


Table 49

Meteorologlcal Data for 1987-1991

Page 8 of 7

USNAC Computer Code-XOQDOQ, Vorolon 2.0 RUn Dato: 93.071 (BRL 6/29/83 VEA8ION) 43824 Wind stats H-Area 60 minute 62M 87-01 stablity from 8igma A

Joint Frequency Distribution of Wind spead and Direotlon:

Atmosphorio stability Clase F Moderately Etable Conditione

\begin{tabular}{lllllllll}
\hline $\begin{array}{c}(M / 8) \\
\text { UMAX }\end{array}$ & N & NNE & NE & ENE & $E$ & ESE & SE & s8E \\
\hline 2.00 & 0.000 & 0.005 & 0.007 & 0.011 & 0.007 & 0.005 & 0.002 & 0.005 \\
4.00 & 0.018 & 0.052 & 0.087 & 0.039 & 0.023 & 0.075 & 0.089 & 0.091 \\
6.00 & 0.014 & 0.256 & 0.212 & 0.144 & 0.144 & 0.187 & 0.132 & 0.162 \\
8.00 & 0.000 & 0.027 & 0.021 & 0.021 & 0.000 & 0.011 & 0.000 & 0.007 \\
12.00 & 0.000 & 0.000 & 0.000 & 0.000 & 0.000 & 0.050 & 0.000 & 0.000 \\
14.10 & 0.000 & 0.000 & 0.000 & 0.000 & 0.000 & 0.000 & 0.000 & 0.000 \\
TOTAL & 0.03 & 0.34 & 0.33 & 0.21 & 0.17 & 0.28 & 0.22 & 0.26
\end{tabular}

Joint Frequenoy Distribution of Wind Speed and Directlon:

Atmoephorio stablilty Clase F Moderately Stable Conditions

\begin{tabular}{|c|c|c|c|c|c|c|c|c|c|}
\hline $\begin{array}{c}(\mathrm{M} / \mathrm{B}) \\
\text { UMAX }\end{array}$ & 8 & sew & $s w$ & Wsw & $w$ & WNW & NW & NNW & TOTAL \\
\hline 2.00 & 0.007 & 0.014 & 0.018 & 0.007 & 0.007 & 0.005 & 0.011 & 0.005 & 0.114 \\
\hline 4.00 & 0.107 & 0.080 & 0.119 & 0.091 & 0.030 & 0.050 & 0.050 & 0.073 & 1.075 \\
\hline 6.00 & 0.221 & 0.214 & 0.235 & 0.228 & 0.068 & 0.039 & 0.023 & 0.041 & 2.321 \\
\hline 8.00 & 0.018 & 0.014 & 0.018 & 0.007 & 0.000 & 0.000 & 0.000 & 0.000 & 0.144 \\
\hline 12.00 & 0.000 & 0.000 & 0.000 & 0.000 & 0.000 & 0.000 & 0.000 & 0.000 & 0.000 \\
\hline 12.10 & 0.000 & 0.000 & 0.000 & 0.000 & 0.000 & 0.000 & 0.000 & 0.000 & 0.000 \\
\hline TOTAL & 0.35 & 0.32 & 0.39 & 0.33 & 0.10 & 0.09 & 0.08 & 0.12 & 3.65 \\
\hline
\end{tabular}


Table 49

Meteorological Data for 1987-1991

Page 7 of 7

USNRC Computer CodeXOQDOQ, Version 2.0 Run Date: 93.071 (SRL 6/29/83 VERSION) 43824 Wind Stats H-Area 60 minute $62 M 87-91$ Stability from Sigma A

Joint Frequency Distribution of Wind Speed and Direction:

Atmospheric Stability Class G Extremely Stable Conditions

\begin{tabular}{llllcllll}
\hline $\begin{array}{c}\text { (M/S) } \\
\text { UMAX }\end{array}$ & \multicolumn{1}{c}{ N } & NNE & NE & ENE & E & ESE & SE & SSE \\
\hline 2.00 & 0.000 & 0.000 & 0.002 & 0.000 & 0.000 & 0.002 & 0.000 & 0.005 \\
4.00 & 0.002 & 0.000 & 0.000 & 0.000 & 0.000 & 0.002 & 0.009 & 0.002 \\
6.00 & 0.005 & 0.023 & 0.009 & 0.002 & 0.007 & 0.037 & 0.025 & 0.016 \\
8.00 & 0.000 & 0.002 & 0.000 & 0.000 & 0.000 & 0.002 & 0.002 & 0.000 \\
12.00 & 0.000 & 0.000 & 0.000 & 0.000 & 0.000 & 0.000 & 0.000 & 0.000 \\
14.10 & 0.000 & 0.000 & 0.000 & 0.000 & 0.000 & 0.000 & 0.000 & 0.000 \\
TOTAL & $\mathbf{0 . 0 1}$ & $\mathbf{0 . 0 3}$ & $\mathbf{0 . 0 1}$ & $\mathbf{0 . 0 0}$ & $\mathbf{0 . 0 1}$ & $\mathbf{0 . 0 4}$ & $\mathbf{0 . 0 4}$ & $\mathbf{0 . 0 2}$
\end{tabular}

Joint Frequency Distribution of Wind Speed and Direction:

Atmospheric Stability Class G Extremely Stable Conditions

\begin{tabular}{llllllllll}
\hline $\begin{array}{c}\text { (M/S) } \\
\text { UMAX }\end{array}$ & S & SSW & SW & WSW & W & WNW & NW & NNW & TOTAL \\
\hline 2.00 & 0.007 & 0.009 & 0.007 & 0.002 & 0.002 & 0.000 & 0.000 & 0.002 & 0.039 \\
4.00 & 0.007 & 0.007 & 0.027 & 0.009 & 0.005 & 0.002 & 0.002 & 0.000 & 0.075 \\
6.00 & 0.021 & 0.014 & 0.011 & 0.027 & 0.002 & 0.007 & 0.002 & 0.005 & 0.212 \\
8.00 & 0.000 & 0.002 & 0.000 & 0.000 & 0.000 & 0.000 & 0.000 & 0.000 & 0.009 \\
12.00 & 0.000 & 0.000 & 0.000 & 0.000 & 0.000 & 0.000 & 0.000 & 0.000 & 0.000 \\
12.10 & 0.000 & 0.000 & 0.000 & 0.000 & 0.000 & 0.000 & 0.000 & 0.000 & 0.000 \\
TOTAL & $\mathbf{0 . 0 3}$ & $\mathbf{0 . 0 3}$ & $\mathbf{0 . 0 5}$ & $\mathbf{0 . 0 4}$ & $\mathbf{0 . 0 1}$ & $\mathbf{0 . 0 1}$ & $\mathbf{0 . 0 0}$ & $\mathbf{0 . 0 1}$ & $\mathbf{0 . 3 4}$
\end{tabular}


Table 50

50-Mile-Radius (80 km) Population Distribution Around SRS (1990 Census)

Page 1 of 1

\begin{tabular}{lccccc}
\hline Dir (Miles) & $0.0-1$. & $1 .-2$ & $2 .-3$ & $3 .-4$ & 4. \\
\hline N & $0.000 E+00$ & $0.000 E+00$ & $0.000 E+00$ & $0.000 E+00$ & $0.000 E+00$ \\
NNE & $0.000 E+00$ & $0.000 E+00$ & $0.000 E+00$ & $0.000 E+00$ & $0.000 E+00$ \\
NE & $0.000 E+00$ & $0.000 E+00$ & $0.000 E+00$ & $0.000 E+00$ & $0.000 E+00$ \\
ENE & $0.000 E+00$ & $0.000 E+00$ & $0.000 E+00$ & $0.000 E+00$ & $0.000 E+00$ \\
E & $0.000 E+00$ & $0.000 E+00$ & $0.000 E+00$ & $0.000 E+00$ & $0.000 E+00$ \\
ESE & $0.000 E+00$ & $0.000 E+00$ & $0.000 E+00$ & $0.000 E+00$ & $0.000 E+00$ \\
SE & $0.000 E+00$ & $0.000 E+00$ & $0.000 E+00$ & $0.000 E+00$ & $0.000 E+00$ \\
SSE & $0.000 E+00$ & $0.000 E+00$ & $0.000 E+00$ & $0.000 E+00$ & $0.000 E+00$ \\
S & $0.000 E+00$ & $0.00 C E+00$ & $0.000 E+00$ & $0.000 E+00$ & $0.000 E+00$ \\
SSW & $0.000 E+00$ & $0.000 E+00$ & $0.000 E+00$ & $0.000 E+00$ & $0.000 E+00$ \\
SW & $0.000 E+00$ & $0.000 E+00$ & $0.000 E+00$ & $0.000 E+00$ & $0.000 E+00$ \\
WSW & $0.000 E+00$ & $0.000 E+00$ & $0.000 E+00$ & $0.000 E+00$ & $0.000 E+00$ \\
W & $0.000 E+00$ & $0.000 E+00$ & $0.000 E+00$ & $0.000 E+00$ & $0.000 E+00$ \\
WNW & $0.000 E+00$ & $0.000 E+00$ & $0.000 E+00$ & $0.000 E+00$ & $0.000 E+00$ \\
NW & $0.000 E+00$ & $0.000 E+00$ & $0.000 E+00$ & $0.000 E+00$ & $0.000 E+00$ \\
NNW & $0.000 E+00$ & $0.000 E+00$ & $0.000 E+00$ & $0.000 E+00$ & $0.000 E+00$ \\
Total & $0.000 E+00$ & $0.000 E+00$ & $0.000 E+00$ & $0.000 E+00$ & $0.000 E+00$
\end{tabular}

\begin{tabular}{lcccccc}
\multicolumn{1}{c}{ Dir (Miles) } & $\mathbf{5 . - 1 0 .}$ & $\mathbf{1 0 . - 2 0}$ & $\mathbf{2 0}-\mathbf{- 3 0}$ & $\mathbf{3 0}-\mathbf{- 4 0}$. & $\mathbf{4 0}-\mathbf{- 5 0}$. & Total \\
\hline N & $2.600 E+01$ & $5.321 E+03$ & $1.002 E+04$ & $5.067 E+03$ & $1.221 E+04$ & $3.264 E+04$ \\
NNE & $6.000 E+00$ & $1.320 E+03$ & $2.066 E+03$ & $4.445 E+03$ & $1.437 E+04$ & $2.220 E+04$ \\
NE & $1.000 E+00$ & $2.945 E+03$ & $2.928 E+03$ & $5.269 E+03$ & $1.020 E+04$ & $2.134 E+04$ \\
ENE & $2.700 E+01$ & $3.126 E+03$ & $4.483 E+03$ & $5.337 E+03$ & $4.077 E+04$ & $5.375 E+04$ \\
E & $1.550 E+02$ & $6.743 E+03$ & $5.305 E+03$ & $8.812 E+03$ & $4.334 E+03$ & $2.535 E+04$ \\
ESE & $3.600 E+01$ & $1.556 E+03$ & $1.931 E+03$ & $2.711 E+03$ & $3.253 E+03$ & $9.487 E+03$ \\
SE & $2.600 E+01$ & $5.470 E+02$ & $6.511 E+03$ & $6.685 E+03$ & $8.577 E+03$ & $2.235 E+04$ \\
SSE & $4.000 E+01$ & $3.910 E+02$ & $7.690 E+02$ & $1.356 E+03$ & $2.539 E+03$ & $5.095 E+03$ \\
S & $1.000 E+00$ & $5.580 E+02$ & $1.332 E+03$ & $7.251 E+03$ & $3.335 E+03$ & $1.248 E+04$ \\
SSW & $2.000 E+00$ & $8.970 E+02$ & $2.008 E+03$ & $4.181 E+03$ & $2.944 E+03$ & $1.003 E+04$ \\
SW & $1.700 E+01$ & $9.440 E+02$ & $2.240 E+03$ & $2.606 E+03$ & $2.660 E+03$ & $8.467 E+03$ \\
WSW & $6.000 E+01$ & $1.103 E+03$ & $7.112 E+03$ & $2.285 E+03$ & $5.818 E+03$ & $1.638 E+04$ \\
W & $5.500 E+01$ & $3.314 E+03$ & $7.941 E+03$ & $7.994 E+03$ & $6.780 E+03$ & $2.608 E+04$ \\
WNW & $4.490 E+02$ & $3.342 E+03$ & $1.069 E+05$ & $5.031 E+04$ & $1.155 E+04$ & $1.725 E+05$ \\
NW & $2.710 E+02$ & $5.899 E+03$ & $8.793 E+04$ & $2.657 E+04$ & $3.025 E+03$ & $1.237 E+05$ \\
NNW & $3.63 E+02$ & $1.803 E+04$ & $2.716 E+04$ & $6.665 E+03$ & $6.079 E+03$ & $5.830 E+04$ \\
Total & $1.535 E+03$ & $5.603 E+04$ & $2.766 E+05$ & $1.475 E+05$ & $1.384 E+05$ & $6.201 E+05$
\end{tabular}




\section{Table 51}

Maximum Individual Committed Dose at Site Perimeter from Atmospheric Releases Using Consumption of Cow Milk Pathway

Page 1 of 1

\begin{tabular}{|c|c|c|c|c|}
\hline \multirow[b]{2}{*}{ Pathway } & \multicolumn{2}{|c|}{ Average Consumption } & \multicolumn{2}{|c|}{ Maximum Consumption } \\
\hline & $\begin{array}{l}\text { Max.Individual } \\
\text { Dose (mrem) }\end{array}$ & $\begin{array}{l}\text { Percent of } \\
\text { Total Dose }\end{array}$ & $\begin{array}{c}\text { Max.Individual } \\
\text { Dose (mrem) }\end{array}$ & $\begin{array}{l}\text { Percent of } \\
\text { Total Dose }\end{array}$ \\
\hline Plume & $1.60 E-07$ & 0.0002 & $1.60 E-07$ & 0.0001 \\
\hline Ground & $1.65 E-04$ & 0.2 & $1.65 \mathrm{E}-04$ & 0.2 \\
\hline Inhalation & 5.11E-02 & 61.7 & $5.11 E-02$ & 47.4 \\
\hline Vegetation & $2.18 E-02$ & 26.3 & $3.80 E-02$ & 35.2 \\
\hline Cow Milk & $6.86 E-03$ & 8.3 & $1.31 E-02$ & 12.2 \\
\hline Meat & $2.89 E-03$ & 3.5 & $5.45 E-03$ & 5.1 \\
\hline Total & $8.28 E-02$ & & $1.08 E-01$ & \\
\hline
\end{tabular}

By Radionuclide

Average Consumption

Radionuclide

\section{Max.Individual} Dose (mrem)a

Percent of Total Dose

\section{Gases and Vapors}

$\mathrm{H}-3$

C-14

7.37E-02

89.0

2.99E-06

Ar-41

$\mathrm{Kr}-85$

I-129

$|-13|$

$1-133$

$1.55 E-03$

2.29E-06

$1.45 E-06$

Particulates

Sr-90

1.03E-04

Ru-106

Cs-134,137

U-235,238

Pu-238

Pu-239

Am-241,243

Cm-242,244

Total

$6.22 E-08$

$6.44 E-05$

$1.04 E-03$

2.68E-03

$3.05 E-03$

4.04E-04

$1.75 \mathrm{E}-04$

8.28E-02
0.004

1.9

0.003

0.002

0.1

0.0001

0.1

1.3

3.2

3.7

Maximum Consumption

$\begin{array}{cc}\text { Max.Individual } & \text { Percent of } \\ \text { Dose (mrem) } & \text { Total Dose }\end{array}$

$9.60 \mathrm{E}-02$

89.0

$5.43 E-06$

0.005

2.72E-03

2.5

4.45E-06

0.004

$2.22 E-06$

0.002

$1.68 E-04 \quad 0.2$

1.04E-07

0.0001

7.08E-05

0.1

$1.11 \mathrm{E}-03$

1.0

3.31E-03

3.1

$3.75 E-03$

3.5

0.5

4.99E-04

0.5

0.2

2.11E-04

0.2

1.08E-01

a Effective dose equivalent 
Table 52

Maximum Individual Committed Dose at Site Perimeter from Atmospheric Releases Using Consumption of Goat Milk Pathway

Page 1 of 1

By Pathway

\begin{tabular}{|c|c|c|c|c|}
\hline \multirow[b]{2}{*}{ Pathway } & \multicolumn{2}{|c|}{ Average Consumption } & \multicolumn{2}{|c|}{ Maximum Consumption } \\
\hline & $\begin{array}{l}\text { Max.Individual } \\
\text { Dose (mrem) }\end{array}$ & $\begin{array}{l}\text { Percent of } \\
\text { Total Dose }\end{array}$ & $\begin{array}{c}\text { Max.Individual } \\
\text { Dose (mrem)a }\end{array}$ & $\begin{array}{l}\text { Percent of } \\
\text { Total Dose }\end{array}$ \\
\hline Plume & $1.60 E-07$ & 0.0002 & $1.60 E-07$ & 0.0001 \\
\hline Ground & $1.65 E-04$ & 0.2 & $1.65 E-04$ & 0.1 \\
\hline Inhalation & $5.11 E-02$ & 55.8 & $5.11 E-02$ & 40.9 \\
\hline Vegetation & $2.18 E-02$ & 23.8 & $3.80 E-02$ & 30.4 \\
\hline Cow Milk & $1.57 E-02$ & 17.1 & $3.01 E-02$ & 24.1 \\
\hline Meat & $2.89 E-03$ & 3.2 & $5.45 E-03$ & 4.4 \\
\hline Total & $9.16 E-02$ & & $1.25 E-01$ & \\
\hline
\end{tabular}

By Radionuclide

Average Consumption

Radionuclide

Gases and Vapors

$\mathrm{H}-3$

C-14

Ar -41

$\mathrm{Kr}-85$

1-129

$1-131$

$1-133$

\section{Particulates}

Sr-90

Ru-106

Cs-134, 137

U-235, 238

Pu-238

Pu-239

Am-241,243

$\mathrm{Cm}-242,244$

Total
8.25E-02

3.09E-06

$1.59 E-03$

2.58E-06

$1.51 \mathrm{E}-06$

$1.05 E-04$

$6.22 E-08$

$6.79 \mathrm{E}-05$

$1.04 \mathrm{E}-03$

$2.68 \mathrm{E}-03$

$3.05 \mathrm{E}-03$

4.04E-04

$1.75 \mathrm{E}-04$

$9.16 E-02$
Maximum Consumption

$\begin{array}{cc}\text { Max.Individual } & \text { Percent of } \\ \text { Dose (mrem })^{\mathrm{a}} & \text { Total Dose }\end{array}$

1.13E-01

$5.62 \mathrm{E}-06$

0.003

1.7

0.003

0.002

$$
\begin{aligned}
& 2.80 E-03 \\
& 5.01 E-06 \\
& 2.34 E-06
\end{aligned}
$$

0.1
0.0001
0.1
1.1
2.9
3.3
0.4
0.2

$1.72 E-04$

$1.04 \mathrm{E}-07$

$7.76 \mathrm{E}-05$

$1.11 \mathrm{E}-03$

$3.31 E-03$

3.75E-03

4.99E-04

2.11E-04

1.25E-01
2.2

0.004

0.002

0.1

90.4

0.004

0.0001

0.1

0.9

2.6

3.0

0.4

0.2

a Effective dose equivalent 
Table 53

Average Individual Committed Dose at Site Perimeter from Atmospheric Releases Using Consumption of Cow Milk Pathway

Page 1 of 1

By Pathway

Pathway

Individual Dose (mrem)

Percent of

2.97E-08

Total Dosea

Plume

1.03E-04

0.0001

Ground

3.11E-02

0.2

Inhalation

1.34E-02

61.5

Vegetation

4.22E-03

26.5

Cow Milk

$1.78 E-03$

8.3

Meat

5.06E-02

By Radionuclide

Radionuclide

Individual

Percent of Dose (mrem) Total Dose

\section{Gases and Vapors}

$\mathrm{H}-3$

4.53E-02

89.4

C-14

$1.82 E-06$

0.004

Ar-41

$\mathrm{Kr}-85$

1-129

9.99E-04

2.0

$\mid-131$

$7.48 \mathrm{E}-07$

0.001

$1-133$

$3.60 \mathrm{E}-07$

0.001

\section{Particulates}

Sr-90

5.29E-05

0.1

Ru-106

3.98E-08

0.0001

Cs-134,137

4.08E-05

0.1

U-235,238

5.97E-04

1.2

Pu-238

1.65E-03

3.3

Pu-239

Am-241,243

$1.72 \mathrm{E}-03$

3.4

2.32E-04

0.5

6.33E-05

0.1

Total

5.07E-02

a Effective dose equivalent 
Table 54

50-Mile Collective Dose from Atmospheric Releases

Page 1 of 1

\section{By Pathway}

\begin{tabular}{lcc} 
Pathway & $\begin{array}{c}\text { Population Dose } \\
\text { Person-Rem }\end{array}$ & $\begin{array}{c}\text { Percent of } \\
\text { Total Dose }\end{array}$ \\
\hline Plume & $7.37 E-07$ & 0.00001 \\
Ground & $2.65 E-02$ & 0.3 \\
Inhalation & $5.75 E+00$ & 75.4 \\
Vegetation & $1.12 E+00$ & 14.7 \\
Cow Milk & $5.84 E-01$ & 7.7 \\
Meat & $1.46 E-01$ & 1.9 \\
Total & $7.63 E+00$ &
\end{tabular}

By Radlonuclide

Radionuclide

Gases and Vapors

$\mathrm{H}-3$

C-14

$1-129$

$\mid-131$

$1-133$

Particulates

Sr-90

Ru-106

Cs- 134,137

$U-235,238$

Pu-238

Pu-239

Am-241,243

$\mathrm{Cm}-242,244$

Total

$\begin{array}{ll}6.94 E+00 & 91.0 \\ 1.78 E-04 & 0.002 \\ 9.26 E-02 & 1.2 \\ 5.03 E-05 & 0.001 \\ 5.11 E-06 & 0.0001\end{array}$

$4.22 E-03$

0.1

5.37E-06

0.00005

$6.93 E-03$

0.1

9.64E-02

1.3

2.27E-01

3.0

2.26E-01

3.0

3.08E-02

0.4

$6.27 E-03$

0.1

$7.63 E+00$

a Effective dose equivalent 
Table 55

1993 NESHAP Report Data-CAP88 Compared With MAXIGASP

Page1 of 1

Maximum Individual Dose Commitment at Site Boundary from Atmospheric Releases

\begin{tabular}{|c|c|c|c|}
\hline \multicolumn{2}{|c|}{ CAP88 Code } & \multicolumn{2}{|c|}{ MAXIGASP Code } \\
\hline mrem & $\begin{array}{l}\text { Percent } \\
\text { of Dose }\end{array}$ & mrem & $\begin{array}{l}\text { Percent } \\
\text { of Dose }\end{array}$ \\
\hline
\end{tabular}

By Pathway

\begin{tabular}{|c|c|c|c|c|c|}
\hline Plume & $2.00 E-08$ & & 0.00001 & $1.60 E-07$ & 0.000 \\
\hline Ground & 8.71E-05 & & 0.05 & $1.65 E-04$ & 0.2 \\
\hline Inhalation & $5.34 E-02$ & & 29.3 & $5.11 E-02$ & 47.4 \\
\hline Food ${ }^{b}$ & $1.29 \mathrm{E}-01$ & - & 70.7 & $5.66 E-02$ & 52.5 \\
\hline Total & $1.82 E-01$ & & & $1.08 E-01$ & \\
\hline
\end{tabular}

By Radionuclide

\section{Gases and Vapors}

$\mathrm{H}-3^{\mathrm{c}}$

C-14

Ar-41

$\mathrm{Kr}-85$

$1-129$

$\mid-131$

$1-133$

\section{Particulates}

Sr-90

$\mathrm{Ru}-106$

Cs-134, 137

U-235, 238

$\mathrm{Pu}-238$

Pu-239

Am-241,243

$\mathrm{Cm}-242,244$

Total
1.79E-01

4.59E-06

3.29E-04

1.50E-07

2.99E-08

2.84E-05

$7.25 E-08$

3.08E-05

7.46E-04

1.22E-03

$1.18 \mathrm{E}-03$

2.37E-04

5.04E-05

1.82E-01
98.4

0.003

0.18

0.00008

0.00002

0.02

0.00004

0.02

0.41

0.67

0.65

0.13

0.03

$9.60 E-02$

89.0

$5.43 E-06$

0.005

2.5

0.004

4.45E-06

0.002

2.22E-06

$1.68 \mathrm{E}-04$

0.2

0.0001

0.1

1.0

3.1

3.5

0.5

0.2 


\section{Table 56}

1993 NESHAP Report Data-CAP88 Compared With POPGASP

Page1 of 1

\begin{tabular}{|c|c|c|c|}
\hline \multicolumn{4}{|c|}{ Collective Committed Dose from Atmospheric Releases } \\
\hline CA & & POF & \\
\hline Person-Rema & $\begin{array}{l}\text { Percent } \\
\text { of Dose }\end{array}$ & Person-Rem & $\begin{array}{l}\text { Percent } \\
\text { of Dose }\end{array}$ \\
\hline
\end{tabular}

By Pathway

$\begin{array}{llllr}\text { Plume } & 2.14 E-06 & 0.00001 & 7.37 E-07 & 0.00001 \\ \text { Ground } & 9.63 E-03 & 0.05 & 2.65 E-02 & 0.3 \\ \text { Inhalation } & 6.85 E+00 & 34.8 & 5.75 E+00 & 75.4 \\ \text { Food } & 1.28 E+01 & 65.1 & 1.85 E+00 & 24.3 \\ \text { Total } & 1.97 E+01 & & 7.63 E+00 & \end{array}$

By Radionuclide

\section{Gases and Vapors}

$\mathrm{H}-3^{\mathrm{C}}$

C-14

Ar-41

$\mathrm{Kr}-85$

|-129

$1-131$

1-133

\section{Particulates}

Sr-90

Ru-106

Cs-134,137

U-235,238

Pu-238

Pu-239

Am-241,243

Cm-242,244

Total
$1.93 E+01$

$1.12 E-04$

$1.48 \mathrm{E}-02$

7.03E-06

1.87E-06

2 67E-03

9.80E-06

4.25E-03

8.24E-02

1.32E-01

1.28E-01

2.60E-02

5.49E-03

1.97E+01
98.0

0.001

0.08

0.00004

0.01

0.00005

0.02

0.4

0.7

0.6

0.01

0.03
$6.94 E+00$

$1.78 E-04$

9.26E-02

5.03E-05

5.11E-06

4.22E-03

3.57E-06

6.93E-03

9.64E-02

2.27E-01

2.26E-01

3.08E-02

6.27E-03

7.63E+00
91.0

0.002

1.2

0.001

0.00007

a Effective dose equivalent

b Meat, milk, and vegetables

c Dose from tritium in foods calculated with absolute humidity of $11 \mathrm{~g}$ water/cubic meter of air 
Table 57

Maximum Individual Committed Dose from Liquid Releases

Page 1 of 1

\begin{tabular}{|c|c|c|}
\hline $\begin{array}{l}\text { By Pathway } \\
\text { Pathway }\end{array}$ & Individual Dose, mrem & Peroent of Total Dose \\
\hline Fish & $7.78 E-02$ & 54.1 \\
\hline Water & 6.57E-02 & 45.7 \\
\hline Shoreline & 2.45E-04 & 0.2 \\
\hline Swimming & $1.61 E-07$ & 0.0001 \\
\hline Boating & $1.90 E-07$ & 0.0001 \\
\hline Total & 1.44E-01 & \\
\hline \multicolumn{3}{|c|}{ By Radlonucllde } \\
\hline Radionucllde & Individual Dose, mrema & Percent of Total Dose \\
\hline $\mathrm{H}-3$ & $5.83 E-02$ & 40.5 \\
\hline Sr-90 & $7.86 \mathrm{E}-03$ & 5.5 \\
\hline $1-129$ & $6.10 \mathrm{E}-04$ & 0.4 \\
\hline$C \varepsilon-137$ & $7.35 \mathrm{E}-02$ & 51.0 \\
\hline$P m-147$ & 7.84E-07 & 0.0005 \\
\hline$U-235,238$ & $2.14 \mathrm{E}-07$ & 0.0001 \\
\hline Pu-239 & $3.20 E-03$ & 2.2 \\
\hline Total & $1.44 E-01$ & \\
\hline
\end{tabular}

a Effective dose equivalent 
Table 58

Individual Committed Dose from Public Water Supplies at Beaufort-Jasper

Page 1 of 1

\section{Average Coneumption}

Radionuollde

$\mathrm{H}-3$

Sr-90

1-129

Cs-137

Pm-147

$U-235,238$

Pu-239

Total

\section{Maximum Consumption}

Radionucllde

$\mathrm{H}-3$

Sr-90

$1-129$

Cs-137

Pm-147

U-235,238

Pu-239

Total
Indluidual Dose, mrem

1.66E-02

$1.28 E-03$

$1.28 E-04$

2.69E-04

1.38E-07

5.90E-08

8.54E-04

1.91E-02

Indlvidual Dose, mrem*

3.27E-02

2.53E-03

2.52E-04

5.32E- 04

2.73E-07

1.16E-07

1.69E-03

3.77E-02
Percent of Total Dose

\section{7}

6.7

0.7

1.4

0.0007

0.0003

4.5
Percent of Total Dose

86.7

6.7

0.7

1.4

0.0007

0.6003

4.5 


\section{Table 59}

Individual Committed Dose from Public Water Supplies at Port Wentworth

Page 1 of 1

\section{Avorage Consumption}

Padionuollde

$\mathrm{H}-3$

Sr-90

1-129

C8-137

Pm-147

U-235,238

Pu-239

Total

Maximum Consumption

Radionuolide

\begin{tabular}{l}
\hline H-3 \\
Sr-90 \\
I-129 \\
Cs-137 \\
Pm-147 \\
U-235,238 \\
Pu-239 \\
Total
\end{tabular}

Indlvidual Dose, mrom"

2.25E-02

1.75E-03

1.74E-04

3.67E-04

1.88E-07

8.03E-08

$1.16 E-03$

2.60E-02

Indlvidual Doee, mrem

4.44E-02

3.44E-03

3.42E-04

7.22E-04

3.70E-07

1.58E-07

2.29E-03

5.12E-02

\section{Percent of Total Dose}
86.7
6.7
0.7
1.4
0.0007
0.0003
4.5

Peroent of Total Dose

86.7
6.7
0.7
1.4
0.0007
0.0003
4.5

86.7

6.7

0.7

0.0003

4.5

a Effective dose equivalent 
Table 60

Collectlve Dose from Llquild Releases

Pege 1 of 1

\section{By Pathway}

Pathway

Sport Fish

Commercial Fish

Beaufort-Jasper

Port Wentworth

Salt Water Inversion

Recreation-River

Total

By Radlonuollde

Radionuolide

$\mathrm{H}-3$

Sr-90

$1-129$

Cs-137

Pm-147

U-235,238

Pu-239

Total
Collcotive Doee,

Poreon-Rom

$1.41 E-01$
$1.23 E-03$
$9.56 E-01$
$3.89 E-01$
$1.41 E-04$
$1.02 E-02$
$1.50 E+00$

Peroent of

Total Dose

9.4

0.1

63.8

26.0

0.01

0.7

9.4
0.1
3.8
0.0
0.01
0.7




\section{Table 61}

Potential Doses from Irrigation Pathways

Page 1 of 1

Effeotive Dose Equlvalent

Food Typo"

Maximum Individual (mrem)

Population (Porson-rom)

$\begin{array}{ll}\text { Vegetation } & 4.49 E-02 \\ \text { Leafy vegetable } & 7.00 E-03 \\ \text { Milk } & 2.00 E-02 \\ \text { Meat } & 6.57 E-03 \\ \text { Total } & 7.86 E-02\end{array}$

$2.80 E+00$

$1.81 E-01$

2.72E+00

$3.52 E-01$

$6.05 E+00$ 
Table 62

Calculated Doses to Aquatic Blota from SRS Releases

Page 1 of 1

\begin{tabular}{|c|c|c|c|c|c|}
\hline \multirow[b]{2}{*}{ Stream } & \multirow[b]{2}{*}{ Looation } & \multirow[b]{2}{*}{ Flow, of } & \multicolumn{3}{|c|}{ Dose to Blota, rad/da,b } \\
\hline & & & Fish & Invertobrate & Algae \\
\hline $\begin{array}{l}\text { Upper Three } \\
\text { Runs }\end{array}$ & $\begin{array}{l}\text { Tim's Branch } 5 \\
\text { (Road C) }\end{array}$ & 8 & $1.23 E-04$ & $1.44 E-04$ & $8.82 E-05$ \\
\hline $\begin{array}{l}\text { Beaver Dam } \\
\text { Creek }\end{array}$ & Below 400-D & 71 & 3.45E-06 & $6.60 E-06$ & 2.45E-05 \\
\hline Four Mile Creek & Site No. 3 & 11 & $2.39 E-05$ & 2.77E-03 & $1.26 E-03$ \\
\hline Pen Branch & $K-011$ & 33 & $5.23 E-05$ & $6.16 E-05$ & 5.67E-05 \\
\hline Steel Creek & $\begin{array}{l}\text { Steel Creek 2A } \\
\text { above Road B }\end{array}$ & 11 & 5.01E-05 & $9.70 E-05$ & $3.42 E-04$ \\
\hline $\begin{array}{l}\text { Lower Three } \\
\text { Runs }\end{array}$ & $\begin{array}{l}\text { L3R1A above } \\
\text { Road B }\end{array}$ & 55 & $9.26 E-07$ & $2.28 E-08$ & $1.00 E-05$ \\
\hline
\end{tabular}

\begin{tabular}{|c|c|c|c|c|}
\hline \multirow[b]{2}{*}{ Stream } & \multirow[b]{2}{*}{ Location } & \multirow[b]{2}{*}{ Flow, ofe } & \multicolumn{2}{|c|}{ Dose to Blota, $\mathrm{rad} / \mathrm{da}^{a, b}$} \\
\hline & & & Raccoon & Duok \\
\hline $\begin{array}{l}\text { Upper Three } \\
\text { Runs }\end{array}$ & $\begin{array}{l}\text { Tim's Branch } 5 \\
\text { (Road C) }\end{array}$ & 8 & $5.73 E-05$ & $7.59 E-04$ \\
\hline $\begin{array}{l}\text { Beaver Dam } \\
\text { Creek }\end{array}$ & Below 400-D & 71 & $1.19 E-05$ & $3.48 E-04$ \\
\hline Four Mile Creek & Site No. 3 & 11 & $1.15 E-03$ & 1.65E-02 \\
\hline Pen Branch & K-011 & 33 & 3.37E-05 & $6.82 E-04$ \\
\hline Steel Creek & $\begin{array}{l}\text { Steel Creek } 2 A \\
\text { above Road B }\end{array}$ & 11 & 1.75E-04 & 5.10E-03 \\
\hline $\begin{array}{l}\text { Lower Three } \\
\text { Runs }\end{array}$ & $\begin{array}{l}\text { L3R1A above } \\
\text { Road B }\end{array}$ & 55 & 5.04E-06 & $1.50 \mathrm{E}-04$ \\
\hline
\end{tabular}

a Calculated with CRITR methodology incorporated in the LADTAP computer program

b The DOE limit is $1 \mathrm{rad} / \mathrm{d}$. 
Table 63

Dose from Consumption of Fish from Creek Mouths and Highway 301

Page 1 of 2

\begin{tabular}{|c|c|c|c|c|c|c|}
\hline \multicolumn{7}{|c|}{ Number of Composites" } \\
\hline Location & Specles & $H-3$ & Sr-90 & C8-137 & Pu-238 & Pu-239 \\
\hline \multirow[t]{2}{*}{$\mathrm{BDC}$} & Panfish & 1 & 1 & 1 & 1 & 1 \\
\hline & Catfish & 3 & 3 & 3 & 3 & 3 \\
\hline \multirow[t]{2}{*}{ FMC } & Panfish & 3 & 3 & 3 & 2 & 2 \\
\hline & Catfish & 3 & 3 & 3 & 3 & 2 \\
\hline \multirow[t]{3}{*}{ L3R } & Panfish & 3 & 3 & 3 & 3 & 3 \\
\hline & Cattish & 3 & 3 & 3 & 2 & 2 \\
\hline & Bass & 1 & 1 & 1 & & \\
\hline \multirow[t]{3}{*}{ SC } & Panfish & 3 & 2 & 2 & 2 & 2 \\
\hline & Catfish & 3 & 2 & 3 & 3 & 2 \\
\hline & Bass & 2 & 2 & 2 & 2 & 2 \\
\hline U3R & Catfish & 3 & 1 & 3 & 3 & 3 \\
\hline \multirow[t]{3}{*}{ HWY 301} & Panfish & 3 & 3 & 3 & 3 & 3 \\
\hline & Catfish & 3 & 2 & 3 & 3 & 3 \\
\hline & Bass & 2 & 2 & 2 & 2 & 2 \\
\hline
\end{tabular}

Average Concentration in Composites (pCl/g)

\begin{tabular}{lllllll} 
Location & Species & H-3 & Sr-90 & Cs-137 & Pu-238 & Pu-239 \\
\hline BDC & Panfish & $4.32 E-02$ & $3.89 E-02$ & $7.07 E-01$ & $4.05 E-05$ & $-2.42 E-05$ \\
& Catfish & $1.59 E-01$ & $9.30 E-04$ & $4.90 E-02$ & $5.81 E-06$ & $1.58 E-06$ \\
FMC & Panfish & $2.86 E-01$ & $1.14 E-02$ & $9.31 E-02$ & $-5.45 E-06$ & $-1.65 E-05$ \\
& Catfish & $8.39 E-01$ & $4.63 E-03$ & $6.64 E-02$ & $-7.87 E-07$ & $4.32 E-06$ \\
L3R & Panfish & $1.55 E-01$ & $1.85 E-02$ & $4.31 E-01$ & $5.45 E-05$ & $-2.51 E-05$ \\
& Catfish & $1.01 E-01$ & $5.74 E-03$ & $2.02 E-01$ & $3.11 E-06$ & $-8.76 E-10$ \\
& Bass & $9.92 E-02$ & $4.99 E-03$ & $7.41 E-01$ & NA & NA \\
SC & Panfish & $1.53 E-01$ & $7.38 E-03$ & $1.01 E-01$ & $8.75 E-06$ & $1.92 E-06$ \\
& Catfish & $1.52 E-01$ & $4.69 E-03$ & $1.46 E-01$ & $3.98 E-06$ & $3.97 E-06$ \\
& Bass & $1.45 E+00$ & $2.41 E-02$ & $1.26 E+00$ & $5.31 E-05$ & $2.92 E-05$ \\
U3R & Catfish & $1.24 E-01$ & $3.55 E-03$ & $6.18 E-02$ & $-2.59 E-06$ & $-3.90 E-06$ \\
HWY 301 & Panfish & $1.06 E-01$ & $5.30 E-03$ & $6.36 E-02$ & $1.01 E-06$ & $3.81 E-06$ \\
& Catfish & $1.24 E-01$ & $1.17 E-03$ & $1.03 E-01$ & $1.44 E-06$ & $-3.32 E-06$ \\
& Bass & $1.24 E-01$ & $3.64 E-03$ & $5.48 E-02$ & $2.95 E-06$ & $-2.95 E-06$
\end{tabular}

Most composites were 5 fish, but six were 4 fish and one was 3 fish.

b Not available 
Table 63

Dose from Consumption of Fish from Crsek Mouths and Highway 301

Page 2 of 2

Dose from Consumption of $19 \mathrm{~kg} /$ year (42 lbs), mrem a,b

\begin{tabular}{|c|c|c|c|c|c|c|c|}
\hline Location & Specles & $\mathrm{H}-3$ & Sr-90 & C8-137 & Pu-238 & Pu-239 & Total \\
\hline \multirow[t]{2}{*}{$\mathrm{BDC}$} & Panfish & $5.17 E-05$ & $9.61 E-02$ & $6.72 E-01$ & $2.92 E-03$ & & 7.71E-01 \\
\hline & Catfish & $1.90 \mathrm{E}-04$ & $2.30 E-03$ & 4.66E-02 & 4.19E-04 & $1.29 E-04$ & 4.96E-02 \\
\hline \multirow[t]{2}{*}{ FMC } & Panfish & $3.42 E-04$ & 2.82E-02 & 8.84E-02 & & & $1.17 E-01$ \\
\hline & Catfish & $1.00 E-03$ & $1.14 E-02$ & 6.31E-02 & & $3.53 E-04$ & 7.59E-02 \\
\hline \multirow[t]{3}{*}{ L3R } & Panfish & $1.86 \mathrm{E}-04$ & 4.57E-02 & $4.09 E-01$ & $3.93 E-03$ & & 4.59E-01 \\
\hline & Catfish & $1.21 E-04$ & $1.42 E-02$ & $1.92 E-01$ & $2.25 E-04$ & & $2.06 \mathrm{E}-01$ \\
\hline & Bass & 1.19E-04 & $1.23 E-02$ & $7.04 E-01$ & $N A^{c}$ & $N A^{c}$ & 7.16E-01 \\
\hline \multirow[t]{3}{*}{ SC } & Panfish & $1.83 E-04$ & 1.97E-02 & $9.60 \mathrm{E}-02$ & 6.32E-04 & $1.57 E-04$ & 1.17E-01 \\
\hline & Catfish & $1.82 E-04$ & 1.16E-02 & $1.39 E-01$ & 2.87E-04 & $3.24 E-04$ & $1.51 E-01$ \\
\hline & Bass & $1.74 E-03$ & 5.95E-02 & $1.20 E+00$ & $3.83 E-03$ & $2.39 E-03$ & $1.26 E+00$ \\
\hline U3R & Catfish & $1.48 E-04$ & 8.77E-03 & $5.87 E-02$ & & & $6.76 E-02$ \\
\hline \multirow[t]{3}{*}{ HWY 301} & Panfish & $1.27 E-04$ & $1.31 E-02$ & $6.04 E-02$ & $7.29 E-05$ & $3.11 E-04$ & $1.75 \mathrm{E}-01$ \\
\hline & Catfish & $1.48 E-04$ & $2.98 E-03$ & $9.79 E-02$ & $1.04 E-04$ & & $1.01 E-01$ \\
\hline & Bass & $1.84 E-04$ & 8.99E-03 & 5.21E-02 & $2.13 E-04$ & & $6.14 \mathrm{E}-02$ \\
\hline
\end{tabular}

a Dose is based on maximum consumption of $19 \mathrm{~kg} / \mathrm{year}$. To obtain dose from average consumption of fish ( $9 \mathrm{~kg} / \mathrm{year}$ ), multiply doses in this table by 0.474 .

b Dose based on negative concentration of individual radionuclides in fish was not calculated (shaded) and was not used in the total dose column.

c Not availiable 


\section{Table 64}

\section{Site-Specific Parameters Used with CAP88 Code Used for NESHAPS}

\section{Calculations}

Page 1 of 1

Particle size, AMAD

$\mathrm{H}-3, \mathrm{C}-14, \mathrm{Kr}-85$

All other

Environmental dose commitment integrating period, yrs

Maximum individual

Population

Meteorological data

$1987-1991$

Plume rise

None

Number of stacks

Stack heights, $m$

0 and 61

Height of lid, $m$

Rainfall, cm/yr

121.4

Average air temperature, C

Surface roughness length, m

Height of wind measurements, $m$

Average wind speed, $\mathrm{m} / \mathrm{s}$

Population size

Food supply fractions (fraction from local sources)

Vegetable
Meat
Milk




\section{Table 65}

Release Locations for 1993 Maximum and Average Individual Dosesa

Page 1 of 1

\begin{tabular}{lcrrrr} 
& \multicolumn{5}{c}{ Release Source Area } \\
\cline { 2 - 6 } & F,H,P,K,C & M & D & SRTC & Diffuse \& Fugltive \\
\cline { 2 - 6 } Release Height, $m$ & 61 & 0 & 16 & 31 & 0 \\
Release Location & & & & \\
Site Coordinate & & & & \\
East & 58000 & 50041 & 20938 & 51863 & 58000 \\
North & 62000 & 104828 & 65284 & 106670 & 62000
\end{tabular}

a Operating period for maximum individual and average individual dose taken as 78 years, i.e, 2 times actual operating period. Operating period for population dose taken to be 201 years, i.e., 100-year environmental dose commitment 
Table 66

1993 Total Site Releases and Maximum Individual Effective Dose Equivalent by Radionuclide (CAP88 Dose Calculations for 1993 NESHAP Report to EPA)

Page 1 of 1

\begin{tabular}{|c|c|c|c|}
\hline Radlonuclldea & Releases (curles) & $\begin{array}{l}\text { Maximum Indlvidual EDE } \\
\text { (mrem) }\end{array}$ & Percent of Dose \\
\hline $\mathrm{H}-3$ (oxide) & $1.33 E+05$ & $1.79 E-01$ & 98.4 \\
\hline Pu-238 & $1.21 E-03$ & $1.22 E-03$ & 0.7 \\
\hline Pu-239b & $1.08 \mathrm{E}-03$ & $1.18 \mathrm{E}-03$ & 0.6 \\
\hline$U-235,238$ & $1.92 \mathrm{E}-03$ & $7.46 \mathrm{E}-04$ & 0.4 \\
\hline $1-129$ & 4.96E-03 & $3.29 \mathrm{E}-04$ & 0.2 \\
\hline Am-241,243 & $1.43 E-04$ & 2.37E-04 & 0.1 \\
\hline Sr-89,90 (Y-90) & 2.27E-03 & $2.84 \mathrm{E}-05$ & 0.02 \\
\hline $\mathrm{Cm}-242,244$ & $5.64 E-05$ & $5.04 E-05$ & 0.03 \\
\hline Cs-137 (Ba-137m) & $6.34 E-04$ & $3.08 E-05$ & 0.02 \\
\hline C-14 & $1.69 E-02$ & 4.59E-06 & 0.003 \\
\hline $\mathrm{H}-3$ (elemental) & $5.82 \mathrm{E}-04$ & $3.10 E-06$ & 0.002 \\
\hline S-35 & 2.00E-06 & $4.42 E-11$ & 0.00000002 \\
\hline $\mathrm{Ni}-63$ & $2.00 E-07$ & $7.31 E-12$ & 0.000000004 \\
\hline $\mid-131$ & $1.48 E-04$ & $1.50 E-07$ & 0.00008 \\
\hline Ru-106 (Rh-106) & 4.00E-06 & $7.25 E-08$ & 0.00004 \\
\hline $1-133$ & $1.96 \mathrm{E}-03$ & 2.99E-08 & 0.00002 \\
\hline Co-60 & 5.89E-09 & 2.61E-10 & 0.0000001 \\
\hline $\mathrm{Xe}-135$ & $3.19 \mathrm{E}-02$ & $1.84 E-08$ & 0.00001 \\
\hline Cs-134 & $1.49 \mathrm{E}-06$ & $2.28 \mathrm{E}-08$ & 0.00001 \\
\hline Eu-154 & $3.44 E-13$ & $1.29 E-14$ & 0.000000000007 \\
\hline $\operatorname{Ce}-144(\operatorname{Pr}-144,144 m)$ & $1.13 E-13$ & $5.91 E-16$ & 0.0000000000003 \\
\hline Eu-155 & $1.63 \mathrm{E}-13$ & $2.45 E-16$ & 0.0000000000001 \\
\hline Sb-125 & $7.27 E-15$ & $3.69 \mathrm{E}-17$ & 0.00000000000002 \\
\hline Zr-95 (Nb-95) & $2.39 E-14$ & $3.43 E-17$ & 0.00000000000002 \\
\hline Total & & 1.82E-01 & \\
\hline
\end{tabular}
Radionuclides in parentheses are decay daughters that are included in effective dose equivalent (EDE) calculations.
Includes gross alpha (assigned to $\mathrm{Pu}-239$ )

Includes gross beta-gamma (assigned to Sr-90) 
Table 67

National Pollutant Discharge Elimination System Monitoring Data

Page 1 of 23

\section{Permit SC 0000175}

\begin{tabular}{|c|c|c|c|c|c|c|}
\hline Measurement & Units & $\begin{array}{l}\text { Freql } \\
\text { Year }\end{array}$ & Maximum & Minimum & $\begin{array}{l}\text { \# In } \\
\text { Average }\end{array}$ & Average \\
\hline
\end{tabular}

Outfall A-001

Effluent consists of the following types of wastewater: cooling water, steam condensate, and laboratory drain water.

BOD

FLO

O\&G

$\mathrm{PH}$

TMP

TSS

$\begin{array}{ccc}\mathrm{mg} / \mathrm{L} & 16 & 2.1 \\ \mathrm{MGD} & 15 & 0.648 \\ \mathrm{mg} / \mathrm{L} & 15 & <1 \\ \mathrm{pH} & 15 & 8.6 \\ { }^{\circ} \mathrm{C} & 15 & 27 \\ \mathrm{mg} / \mathrm{L} & 15 & 2\end{array}$

2.1

0.648

8.6

2

$$
<1
$$$$
0.36
$$$$
<1
$$$$
6
$$

19

$<1$

15

5

23

1.4

$\begin{array}{rl}6 & \\ 15 & 0.482\end{array}$

Outfall A-003

Effluent consists of tl,e following types of wastewater: cooling water and steam condensate. CR

FLO

$O \& G$

$\mathrm{PH}$

TMP

TSS

$$
\mathrm{mg} / \mathrm{L} \quad 25
$$

MGD 365

$\mathrm{mg} / \mathrm{L} \quad 15$

$\mathrm{pH} \quad 15$

${ }^{\circ} \mathrm{C} \quad 15$

$\mathrm{mg} / \mathrm{L}$

15

\section{Outfall A-005}

Effluent consists of the following types of wastewater: wastewater from animal holding area, steam condensate, steam cleaning wastewater, and cooling water.

BOD
FEC
FLO
O\&G
PERCL
PH
TMP
TRICL
TSS

$\begin{array}{rcc}\mathrm{mg} / \mathrm{L} & 16 & 4.1 \\ \# / 100 \mathrm{~mL} & 15 & 53 \\ \mathrm{MGD} & 15 & 0.648 \\ \mathrm{mg} / \mathrm{L} & 15 & 1.1 \\ \mathrm{ug} / \mathrm{L} & 15 & <5 \\ \mathrm{pH} & 15 & 7.5 \\ { }^{\circ} \mathrm{C} & 15 & 26 \\ \mathrm{ug} / \mathrm{L} & 15 & 9.8 \\ \mathrm{mg} / \mathrm{L} & 15 & 4\end{array}$

$$
<0.02
$$

$$
1.4865
$$$$
0.0194
$$$$
365
$$$$
<1
$$$$
2
$$$$
7.5
$$$$
6.7
$$

$$
4
$$

1.5

0.482 


\section{Table 67}

National Pollutant Discharge Ellmination System Monitoring Data

Page 2 of 23

\section{Permit SC 0000175}

\begin{tabular}{lcccccc} 
Measurement & Units & $\begin{array}{c}\text { Freq/ } \\
\text { Year }\end{array}$ & Maximum & Minimum & $\begin{array}{c}\text { in } \\
\text { Average }\end{array}$ & Average \\
\hline $\begin{array}{l}\text { Outfall A-011 } \\
\text { Effluent consists of the following types }\end{array}$ & of wastewater: cooling water. & & & \\
BOD & $m \cdot \mathrm{g} / \mathrm{L}$ & 13 & 9.2 & $<1$ & 7 & 3.4 \\
FLO & $\mathrm{MGD}$ & 365 & 1.228 & 0 & 365 & 0.091 \\
O\&G & $\mathrm{mg} / \mathrm{L}$ & 12 & 2.3 & $<1$ & 4 & 1.5 \\
PH & $\mathrm{pH}$ & 12 & 8.1 & 6.8 & & \\
TMP & ${ }^{\circ} \mathrm{C}$ & 12 & 31 & 16 & 12 & 22.4 \\
TSS & $\mathrm{mg} / \mathrm{L}$ & 12 & 4 & $<1$ & 5 & 2.2
\end{tabular}

Outfall A-014

Effluent consists of the following types of wastewater: air stripper wastewater and noncontact cooling water.

$\begin{array}{lcccccc}\text { BOD } & \mathrm{mg} / \mathrm{L} & 13 & 3.4 & <1 & 8 & 2.7 \\ \text { FLO } & \mathrm{MGD} & 39 & 5.2531 & 0.2405 & 39 & 2.2159 \\ \text { O\&G } & \mathrm{mg} / \mathrm{L} & 13 & 7.6 & <1 & 3 & 5.4 \\ \text { PERCL } & \mathrm{ug} / \mathrm{L} & 13 & <2 & <2 & & \\ \mathrm{PH} & \mathrm{pH} & 44 & 7.85 & 6 & & \\ \text { TCE } & \mathrm{ug} / \mathrm{L} & 13 & <2 & <2 & & \\ \text { TMP } & { }^{\circ} \mathrm{C} & 38 & 23.8 & 3.5 & 38 & 20 \\ \text { TRICL } & \mathrm{ug} / \mathrm{L} & 13 & 0.8 & <2 & 3 & 2.72 \\ \text { TSS } & \mathrm{mg} / \mathrm{L} & 33 & 33 & <1 & 16 & 4.5\end{array}$

Outfall A-015

Effluent consists of the following types of wastewater: sanitary wastewater.

$\begin{array}{lrrllll}\text { BOD } & \mathrm{mg} / \mathrm{L} & 13 & 6.8 & <1 & 12 & 3.3 \\ \text { FEC } & \# / 100 \mathrm{~mL} & 14 & 14 & <2 & 2 & 10 \\ \text { FLO } & \mathrm{MGD} & 365 & 0.2376 & 0.0317 & 365 & 0.088 \\ \text { PH } & \mathrm{pH} & 12 & 7.6 & 6.9 & & \\ \text { TSS } & \mathrm{mg} / \mathrm{L} & 13 & 13 & 2 & 13 & 4.5\end{array}$


Table 67

\section{National Pollutant Discharge Elimination System Monitoring Data}

Page 3 of 23

\section{Permit SC 0000175}

\begin{tabular}{|c|c|c|c|c|c|c|}
\hline Measurement & Units & $\begin{array}{l}\text { Freql } \\
\text { Year }\end{array}$ & Marimum & Minimum & $\begin{array}{c}\text { \# In } \\
\text { Average }\end{array}$ & Average \\
\hline \multicolumn{7}{|c|}{ Outfall C-001 } \\
\hline \multicolumn{7}{|c|}{ Effluent consists of the following types of wastewater: cooling water. } \\
\hline FLO & MGD & 365 & 0.181 & 0 & 365 & 0.021 \\
\hline O\&G & $\mathrm{mg} / \mathrm{L}$ & 17 & 1.5 & $<1$ & 4 & 1.3 \\
\hline $\mathrm{PH}$ & $\mathrm{pH}$ & 19 & 7 & 5.7 & & \\
\hline TMP & ${ }^{\circ} \mathrm{C}$ & 19 & 28 & 1 & 19 & 18.2 \\
\hline TSS & $\mathrm{mg} / \mathrm{L}$ & 17 & 2 & $<1$ & 7 & 1.4 \\
\hline \multicolumn{7}{|c|}{ Outfall C-003 } \\
\hline \multicolumn{7}{|c|}{ Effluent consists of the following types of wastewater: cooling water. } \\
\hline FLO & MGD & 365 & 2.8437 & 0.0582 & 365 & 0.3779 \\
\hline O\&G & $\mathrm{mg} / \mathrm{L}$ & 17 & 1.5 & $<1$ & 3 & 1.3 \\
\hline $\mathrm{PH}$ & $\mathrm{pH}$ & 17 & 7.3 & 6.1 & & \\
\hline TMP & ${ }^{\circ} \mathrm{C}$ & 17 & 24 & 14 & 17 & 20.9 \\
\hline TSS & $\mathrm{mg} / \mathrm{L}$ & 17 & 1 & $<1$ & 1 & 1 \\
\hline
\end{tabular}

Outfall C-004

Effluent consists of the following types of wastewater: neutralization water, service wastewater, and C-Reactor cooling water.

FLO

MGD 365

102.1154

1.6158

365

11.3777

O\&G

$\mathrm{mg} / \mathrm{L} \quad 14$

1.7

$<1$

1.4

$\mathrm{PH}$

$\mathrm{pH} \quad 14$

8.1

6.1

TMP

${ }^{\circ} \mathrm{C} \quad 346$

29.8

3.7

346

18.4

TSS

$\mathrm{mg} / \mathrm{L} \quad 14$

7

$<1$

11

3.3

Outfall C-004A

Effluent consists of the following types of wastewater: sanitary wastewater.

$\begin{array}{lrrllll}\text { BOD } & \mathrm{mg} / \mathrm{L} & 13 & 12.4 & 2.1 & 13 & 3.9 \\ \text { FEC } & \# / 100 \mathrm{~mL} & 14 & 3 & <2 & 3 & 2 \\ \text { FLO } & \mathrm{MGD} & 365 & 0.0245 & 0 & 365 & 0.0104 \\ \mathrm{PH} & \mathrm{pH} & 12 & 7.8 & 7.1 & & \\ \text { TSS } & \mathrm{mg} / \mathrm{L} & 13 & 7 & 1 & 13 & 3.4\end{array}$




\section{Table 67}

National Pollutant Discharge Ellmination System Monitoring Data

Page 4 of 23

\section{Permit SC 0000175}

\begin{tabular}{|c|c|c|c|c|c|c|}
\hline Measurement & Units & $\begin{array}{l}\text { Freql } \\
\text { Year }\end{array}$ & Maximum & Minimum & $\begin{array}{c}\text { \# in } \\
\text { Average }\end{array}$ & Average \\
\hline
\end{tabular}

Outfall D-001

Effluent consists of the following types of wastewater: cooling water, neutralization water, and heavy water.

$\begin{array}{lrrrrrc}\text { FLO } & \text { MGD } & 365 & 80.7875 & 31.6687 & 365 & 45.6606 \\ \text { O\&G } & \mathrm{mg} / \mathrm{L} & 17 & 1.2 & <1 & 2 & 1.1 \\ \mathrm{PH} & \mathrm{pH} & 23 & 7.4 & 6 & & \\ \text { TMP } & { }^{\circ} \mathrm{C} & 17 & 30.5 & 14 & 17 & 22.1 \\ \text { TSS } & \mathrm{mg} / \mathrm{L} & 17 & 17 & 5 & 17 & 10.1\end{array}$

Outfall D-001A

Effluent consists of the following types of wastewater: sanitary wastewater.

$\begin{array}{lrrlccl}\text { BOD } & \mathrm{mg} / \mathrm{L} & 13 & 5.9 & 1.5 & 13 & 3.3 \\ \text { FEC } & \# / 100 \mathrm{~mL} & 14 & 4 & <2 & 1 & 4 \\ \text { FLO } & \mathrm{MGD} & 365 & 0.023 & 0 & 365 & 0.008 \\ \text { PH } & \mathrm{pH} & 13 & 8 & 7 & & \\ \text { TSS } & \mathrm{mg} / \mathrm{L} & 13 & 15 & 2 & 13 & 4.4\end{array}$

Outfall D-001B

Effluent consists of the following types of wastewater: coal pile runoff.

$\begin{array}{lcccccc}\text { AL } & \mathrm{mg} / \mathrm{L} & 7 & 44.8 & 37.1 & 7 & 40.2 \\ \mathrm{FE} & \mathrm{mg} / \mathrm{L} & 7 & 223 & 111 & 7 & 174.1 \\ \mathrm{FLO} & \mathrm{MGD} & 365 & 0.216 & 0 & 365 & 0.022 \\ \mathrm{MG} & \mathrm{mg} / \mathrm{L} & 7 & 20.5 & 16 & 7 & 17.7 \\ \mathrm{MN} & \mathrm{mg} / \mathrm{L} & 7 & 2.88 & 1.98 & 7 & 2.38 \\ \mathrm{PH} & \mathrm{pH} & 8 & 7.1 & 6.4 & & \\ \mathrm{SO} 4 & \mathrm{mg} / \mathrm{L} & 7 & 1250.00 & 726 & 7 & 1001 \\ \mathrm{TSS} & \mathrm{mg} / \mathrm{L} & 7 & 3 & <1 & 6 & 1.8 \\ \mathrm{ZN} & \mathrm{mg} / \mathrm{L} & 7 & 1.18 & 0.86 & 7 & 1.04\end{array}$




\section{Table 67}

National Pollutant Discharge Elimination System Monitoring Data

Page 5 of 23

\section{Permit SC 0000175}

\section{Measurement}

Freq/

\# \# In

Averege

Average

Outfall D-001C

Effluent consists of the following types of wastewater: ash basin discharge.

$\begin{array}{lcccccc}\text { FLO } & \text { MGD } & 17 & 5.7298 & 2.6237 & 17 & 4.3003 \\ \text { O\&G } & \mathrm{mg} / \mathrm{L} & 16 & 1.5 & <1 & 4 & 1.3 \\ \mathrm{PH} & \mathrm{pH} & 15 & 7.1 & 5.6 & & \\ \text { TSS } & \mathrm{mg} / \mathrm{L} & 17 & 8 & 1 & 17 & 3.3\end{array}$

Outfall D-003

Effluent consists of the following types of wastewater: powerhouse washdown water and water treatment plant wastewater.

$\begin{array}{lrrlccc}\text { FLO } & \text { MGD } & 365 & 1.0341 & 0.0388 & 365 & 0.2336 \\ \text { O\&G } & \mathrm{mg} / \mathrm{L} & 17 & 1.6 & <1 & 2 & 1.6 \\ \mathrm{PH} & \mathrm{pH} & 17 & 7.5 & 6.2 & & \\ \text { TSS } & \mathrm{mg} / \mathrm{L} & 17 & 4 & <1 & 15 & 2\end{array}$

Outfall D-005

Effluent consists of the following types of wastewater: water filter backwash overflow.

FLO

MGD 13

0

0

Outfall D-006

Effluent consists of the following types of wastewater: cooling water, powerhouse washdown, and heavy water process water.

$\begin{array}{lrrrrrr}\text { FEC } & \# / 100 \mathrm{~mL} & 17 & 52 & 27 & 17 & 169 \\ \text { FLO } & \mathrm{MGD} & 365 & 7.7556 & 0.9695 & 365 & 2.2672 \\ \text { O\&G } & \mathrm{mg} / \mathrm{L} & 17 & 1.3 & <1 & 1 & 1.3 \\ \mathrm{PH} & \mathrm{pH} & 17 & 7.5 & 6 & & \\ \text { TMP } & \circ & 277 & 28.2 & 8 & 277 & 18.9 \\ \text { TSS } & \mathrm{mg} / \mathrm{C} & 18 & 29 & 2 & 18 & 7.5\end{array}$

Outfall DW-001

Effluent consists of the foll-wing types of wastewater: washdown water from concrete transit mixer trucks.

$\begin{array}{lrrllll}\text { FLO } & \text { MGD } & 365 & 0.527 & 0 & 365 & 0.08 \\ \text { PH } & \mathrm{pH} & 5 & 7.7 & 6.4 & & \\ \text { TSS } & \mathrm{mg} / \mathrm{L} & 5 & 6 & 2 & 5 & 4.6\end{array}$




\section{Table 67}

National Pollutant Discharge Elimination System Monitoring Data

Page 6 of 23

\section{Permit SC 0000175}

\begin{tabular}{lllll} 
Measurement & Freql & Ynits & Yoar & * In \\
Average & Average \\
\hline
\end{tabular}

\section{Outfall DW-002}

Effluent consists of the following types of wastewater: steam cleaning wastewater, stormwater runoft, and equipment wash water.

$\begin{array}{lrrrrrr}\text { FLO } & \text { MGD } & 23 & 0.036 & 23 & 0.0185 \\ \text { O\&G } & \mathrm{mg} / \mathrm{L} & 23 & 4.6 & <1 & 7 & 2.2 \\ \mathrm{PH} & \mathrm{pH} & 18 & 9.8 & 6 & \end{array}$

Outfall DW-003

Effluent consists of the following types of wastewater: sanitary wastewater from DWPF (S Area).

$\begin{array}{lrrllcc}\text { BOD } & \mathrm{mg} / \mathrm{L} & 16 & 12.2 & <1 & 15 & 6.5 \\ \text { FEC } & \# / 100 \mathrm{~mL} & 15 & 33 & <2 & 1 & 33 \\ \text { FLO } & \mathrm{MGD} & 365 & 0.0302 & 0 & 365 & 0.0123 \\ \text { PH } & \mathrm{pH} & 13 & 8.6 & 6.7 & & \\ \text { TSS } & \mathrm{mg} / \mathrm{L} & 16 & 53 & 2 & 16 & 9.5\end{array}$

Outfall DW-004

Effluent consists of the following types of wastewater: neutralization, cooling water, and steam condensate.

$\begin{array}{lrlllll}\text { BOD } & \mathrm{mg} / \mathrm{L} & 25 & 3.4 & <1 & 19 & 1.7 \\ \mathrm{CHL} & \mathrm{mg} / \mathrm{L} & 23 & 1.18 & <0.1 & 4 & 0.5 \\ \mathrm{FLO} & \mathrm{MGD} & 55 & 0.1584 & 55 & & 0.0414 \\ \mathrm{O} \& \mathrm{G} & \mathrm{mg} / \mathrm{L} & 24 & 3 & <1 & 5 & 1.7 \\ \mathrm{PH} & \mathrm{pH} & 25 & 8.4 & 6.3 & & \\ \mathrm{TMP} & { }^{\circ} \mathrm{C} & 24 & 3 & 15 & 24 & 21.6 \\ \text { TSS } & \mathrm{mg} / \mathrm{L} & 25 & 26 & <1 & 23 & 4.5\end{array}$

Outfall F-001

Effluent consists of the following types of wastewater: non-process cooling water.

$\begin{array}{lrrcccc}\text { FLO } & \text { MGD } & 15 & 0.288 & 0.108 & 15 & 0.1848 \\ \text { O\&G } & \mathrm{mg} / \mathrm{L} & 15 & 2.2 & <1 & 2 & 2.1 \\ \mathrm{PH} & \mathrm{pH} & 15 & 7.7 & 6.7 & & \\ \text { TMP } & { }^{\circ} \mathrm{C} & 15 & 28 & 21 & 15 & 24.3 \\ \text { TSS } & \mathrm{mg} / \mathrm{L} & 15 & 13 & <1 & 12 & 3.8\end{array}$


Table 67

National Pollutant Discharge Ellmination System Monitoring Data

Page 7 of 23

Permit SC 0000175

Measuroment $\quad$ Units $\quad \begin{gathered}\text { Freq } \\ \text { Year }\end{gathered}$ Maximum Minimum Average Average

Outfall F-002

Effluent consists of the following types of wastewater: non-process cooling water.

$\begin{array}{lrrrccc}\text { FLO } & \text { MGD } & 15 & 0.0864 & 0.0288 & 15 & 0.0523 \\ \text { O\&G } & \mathrm{mg} / \mathrm{L} & 15 & 1.6 & <1 & 3 & 1.3 \\ \mathrm{PH} & \mathrm{pH} & 15 & 7.5 & 6.2 & & \\ \text { TMP } & { }^{\circ} \mathrm{C} & 15 & 28 & 11 & 15 & 18.1 \\ \text { TSS } & \mathrm{mg} / \mathrm{L} & 15 & 4 & <1 & 6 & 1.8\end{array}$

\section{Outtall F-003}

Effluent consists of the following types of wastewater: cooling water and steam condensate.

$\begin{array}{lrrlccc}\text { BOD } & \mathrm{mg} / \mathrm{L} & 16 & 2.4 & <1 & 9 & 1.7 \\ \text { FLO } & \mathrm{MGD} & 15 & 0.144 & 0.0216 & 15 & 0.0638 \\ \text { O\&G } & \mathrm{mg} / \mathrm{L} & 15 & 3.2 & <1 & 5 & 1.8 \\ \mathrm{PH} & \mathrm{pH} & 15 & 8.9 & 6.7 & & \\ \mathrm{TMP} & { }^{\circ} \mathrm{C} & 15 & 32 & 13 & 15 & 22.8 \\ \text { TSS } & \mathrm{mg} / \mathrm{L} & 15 & 19 & <1 & 14 & 5.9\end{array}$

Outfall F-003A

Effluent consists of the following types of wastewater: sanitary wastewater.

$\begin{array}{lrccccc}\text { BOD } & \mathrm{mg} / \mathrm{L} & 14 & 6.5 & 1.6 & 14 & 3.2 \\ \text { FEC } & \# / 100 \mathrm{~mL} & 15 & <2 & <2 & & \\ \text { FLO } & \mathrm{MGD} & 365 & 0.0187 & 0 & 365 & 0.0058 \\ \text { PH } & \mathrm{pH} & 12 & 8.8 & 7.5 & & \\ \text { TSS } & \mathrm{mg} / \mathrm{L} & 14 & 18 & 2 & 14 & 7.6\end{array}$

Outfall F-005

Effluent consists of the following types of wastewater: cooling water and steam condensate.

$\begin{array}{lrrcccc}\text { FLO } & \text { MGD } & 15 & 0.18 & 0.0432 & 15 & 0.1003 \\ \text { O\&G } & \mathrm{mg} / \mathrm{L} & 15 & 1.9 & <1 & 4 & 1.4 \\ \mathrm{PH} & \mathrm{pH} & 15 & 7.8 & 6.4 & & \\ \text { TMP } & { }^{\circ} \mathrm{C} & 15 & 28 & 2 & 15 & 23.6 \\ \text { TSS } & \mathrm{mg} / \mathrm{L} & 16 & 36 & <1 & 11 & 7.4\end{array}$




\section{Table 67}

\section{National Pollutant Discharge Ellmination System Monitoring Data}

Page 8 of 23

\section{Permit sc 000u175}

\section{Measurement}

Unit

\section{Froql} Maximum

Minimum

in Average Average

Outfall F-007

Effluent consists of the following types of wastewater: ash basin discharge.

FLO

MGD $\quad 14 \quad 0$

0

S. utfall F-008

Effluent consists of the following types of wastewater: neutralization, cooling water, steam condensate, process wastewater, and Outfall F-012 and Outtall F-013.

\begin{tabular}{|c|c|c|c|c|c|c|}
\hline$A L$ & $\mathrm{mg} / \mathrm{L}$ & 8 & 0.545 & 0.108 & 8 & 0.263 \\
\hline AN & $\mathrm{mg} / \mathrm{L}$ & 8 & 0.12 & $<0.05$ & 2 & 0.09 \\
\hline CR & $\mathrm{mg} / \mathrm{L}$ & 8 & $<0.03$ & $<0.02$ & & \\
\hline $\mathrm{CU}$ & $\mathrm{mg} / \mathrm{L}$ & 8 & 0.0415 & $<0.01$ & 2 & 0.0258 \\
\hline FLO & MGD & 365 & 9.6945 & 0.3555 & 365 & 1595 \\
\hline$H G$ & $\mathrm{mg} / \mathrm{L}$ & 8 & $<0.0005$ & $<0.0001$ & & \\
\hline$M N$ & $\mathrm{mg} / \mathrm{L}$ & 8 & 0.0139 & $<0.03$ & 7 & 0.0111 \\
\hline N03 & $\mathrm{mg} / \mathrm{L}$ & 8 & 2.42 & 0.05 & 8 & 1.04 \\
\hline $\mathrm{NI}$ & $\mathrm{mg} / \mathrm{L}$ & 8 & $<0.05$ & $<0.03$ & & \\
\hline O\&G & $\mathrm{mg} / \mathrm{L}$ & 15 & 2.7 & $<1$ & 3 & 1.8 \\
\hline PB & $\mathrm{mg} / \mathrm{L}$ & 8 & 0.0071 & $<0.003$ & 1 & 0.0071 \\
\hline $\mathrm{PH}$ & $\mathrm{pH}$ & 15 & 7.6 & 6 & & \\
\hline TMP & ${ }^{\circ} \mathrm{C}$ & 15 & 29.5 & 19 & 15 & 23.9 \\
\hline TSS & $\mathrm{mg} / \mathrm{L}$ & 15 & 9 & $<1$ & 14 & 2.4 \\
\hline $\mathrm{U}_{3} \mathrm{O}_{8}$ & $\mathrm{mg} / \mathrm{L}$ & 8 & $<0.1$ & $<0.02$ & & \\
\hline $\mathrm{ZN}$ & $\mathrm{mg} / \mathrm{L}$ & 8 & 0.0977 & 0.02 & 8 & 0.0396 \\
\hline
\end{tabular}

Outfall F-008A

Effluent consists of the following types of wastewater: sanitary wastewater.

$\begin{array}{lrrcccc}\text { BOD } & \mathrm{mg} / \mathrm{L} & 14 & 7.5 & 1.1 & 14 & 2.9 \\ \text { FEC } & \# / 100 \mathrm{~mL} & 15 & <2 & <2 & & \\ \text { FLO } & \mathrm{MGD} & 364 & 0.1022 & 0.0317 & 364 & 0.0609 \\ \mathrm{PH} & \mathrm{pH} & 12 & 7.6 & 6.9 & & \\ \text { TSS } & \mathrm{mg} / \mathrm{L} & 14 & 13 & 2 & 14 & 5.3\end{array}$


Table 67

National Pollutant Dlecharge Ellmination System Monitoring Data

Page 9 of 23

\section{Permit SC 0000175}

\begin{tabular}{|c|c|c|c|c|c|c|}
\hline Measuremont & Unite & $\begin{array}{l}\text { Freq } \\
\text { Yoar }\end{array}$ & Maximum & MInImum & $\begin{array}{c}\begin{array}{c}\text { In } \\
\text { Average }\end{array}\end{array}$ & Avorage \\
\hline
\end{tabular}

Outtall F-012

Effluent consists of the following types of wastewater: stormwater runoff retention basin effiuent.

AL

AN

CR

CU

FLO

HG

MN

$\mathrm{NO}_{3}$

NI

PB

$\mathrm{PH}$

TSS

$\mathrm{U}_{3} \mathrm{O}_{8}$

ZN

Outfall F-013

Effluent consists of the following types of wastewater: cooling water ret untion basin effluent.

$\mathrm{AL}$

AN

CR

CU

FLO

HG

MN

$\mathrm{NO}_{3}$

$\mathrm{NI}$

PB

$\mathrm{PH}$

TSS

$\begin{array}{cc}\mathrm{mg} / \mathrm{L} & 21 \\ \mathrm{mg} / \mathrm{L} & 21 \\ \mathrm{mg} / \mathrm{L} & 21 \\ \mathrm{mg} / \mathrm{L} & 21 \\ \mathrm{MGD} & 23 \\ \mathrm{mg} / \mathrm{L} & 21 \\ \mathrm{mg} / \mathrm{L} & 21 \\ \mathrm{mg} / \mathrm{L} & 21 \\ \mathrm{mg} / \mathrm{L} & 21 \\ \mathrm{mg} / \mathrm{L} & 21 \\ \mathrm{pH} & 21 \\ \mathrm{mg} / \mathrm{L} & 21 \\ \mathrm{mg} / \mathrm{L} & 21 \\ \mathrm{mg} / \mathrm{L} & 21\end{array}$

0.946

0.26

$<0.03$

0.012

0.72

0.007

0.155

1.4

$<0.05$

0.0107

8

24

0.0002

0.396
0.1

$<0.05$

$<0.007$

$<0.004$

0

$<0.0001$

$<0.03$

$<0.02$

$<0.012$

$<0.003$

5.8

4

$<0.02$

0.0813

$\begin{array}{lllllll}\mathrm{AL} & \mathrm{mg} / \mathrm{L} & 23 & 0.791 & <0.05 & 19 & 0.276 \\ \mathrm{mg} / \mathrm{L} & 23 & 0.36 & <0.05 & 2 & 0.26 \\ \mathrm{mg} / \mathrm{L} & 23 & <0.02 & <0.02 & & 0.014 \\ \mathrm{CU} & \mathrm{mg} / \mathrm{L} & 23 & 0.027 & <0.01 & 6 & 0.4837 \\ \mathrm{MGO} & \mathrm{MGD} & 24 & 0.9216 & 24 & & 0.0138^{\prime} \\ \mathrm{MN} & \mathrm{mg} / \mathrm{L} & 23 & <0.0001 & <0.0001 & & 0.18 \\ \mathrm{NO}_{3} & \mathrm{mg} / \mathrm{L} & 22 & 0.0195 & 0.0054 & 22 & 2 \\ \mathrm{NI} & \mathrm{mg} / \mathrm{L} & 23 & 2.03 & <0.02 & 2 & 0.0049 \\ \mathrm{~PB} & \mathrm{mg} / \mathrm{L} & 23 & <0.05 & <0.05 & & 3 \\ \mathrm{PH} & \mathrm{mg} / \mathrm{L} & 23 & 0.0067 & <0.003 & & 3.7\end{array}$

21

4

0.289

0.15

0.012

0.62

0.004

0.031

0.14

0.0063

8.8

0.0002

0.1404 

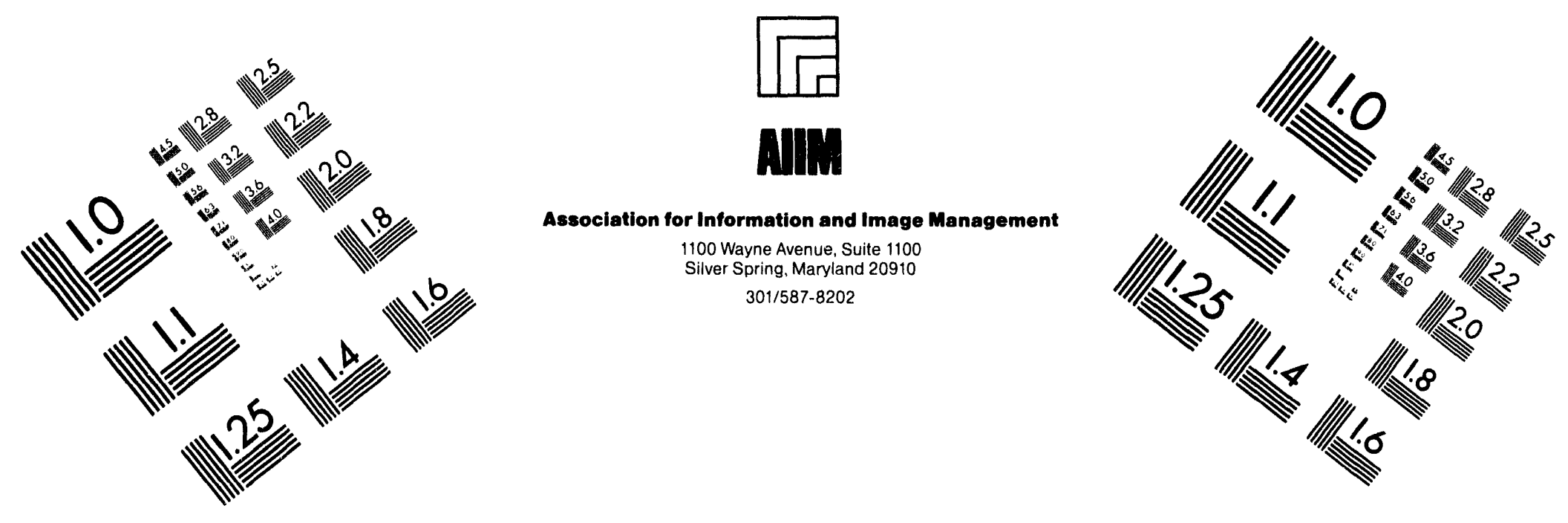

\section{Centimeter}

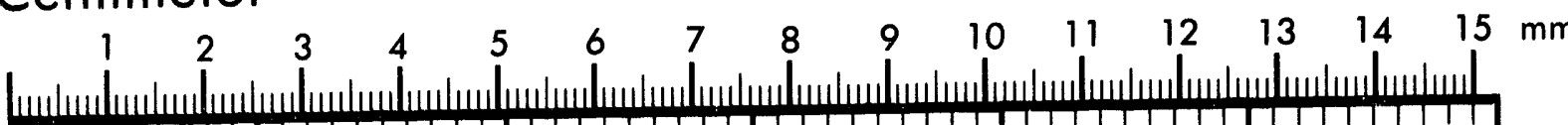

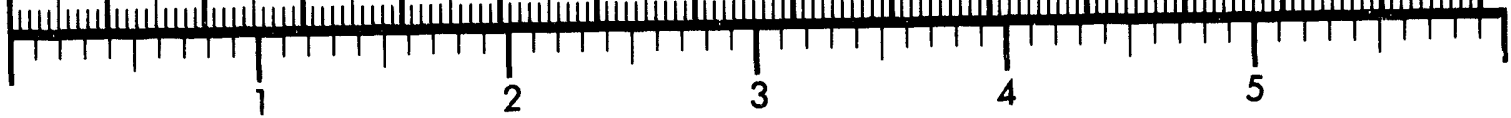
Inches

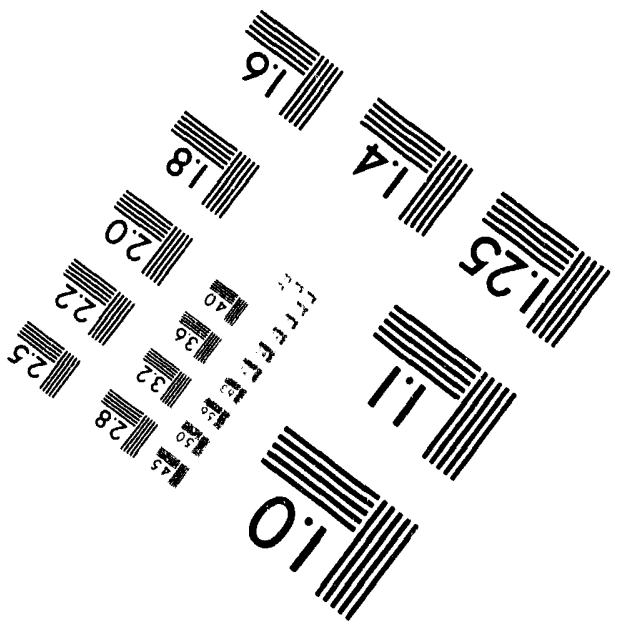

MANUFACTURED TO AIIM STANDARDS

BY APPLIED IMAGE, INC.

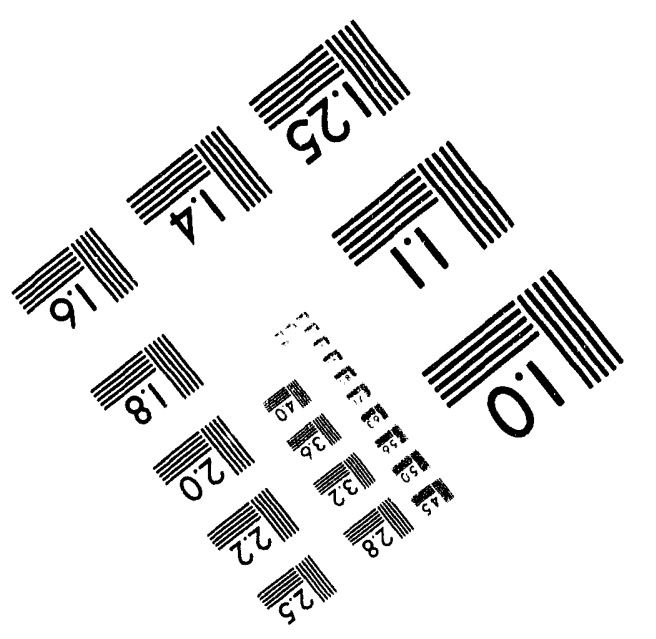



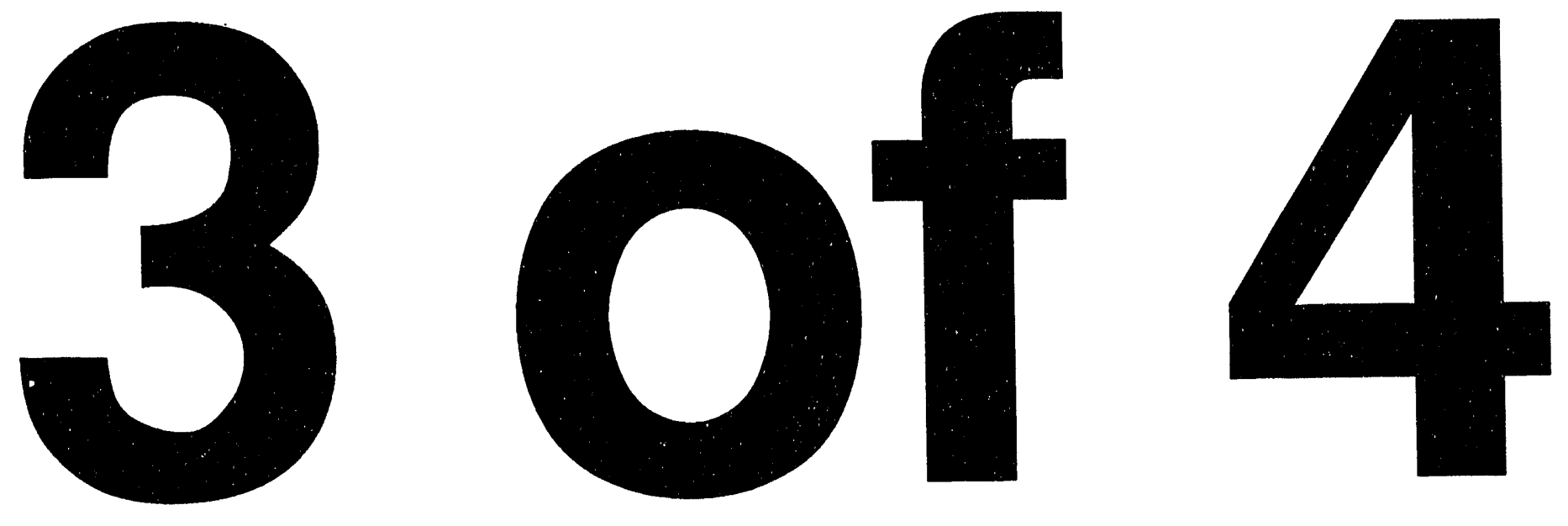
Table 67

National Pollutant Discharge Elimination System Monitoring Data

Page 10 of 23

\section{Permit SC 0000175}

\begin{tabular}{lcccccc} 
Measurement & Units & $\begin{array}{c}\text { Freq/ } \\
\text { Year }\end{array}$ & Maximum & Minimum & $\begin{array}{c}\text { \# in } \\
\text { Average }\end{array}$ & Average \\
\hline Outfall F-013, continued & & & & & & \\
$U_{3} O_{8}$ & $\mathrm{mg} / \mathrm{L}$ & 23 & $<0.02$ & $<0.02$ & & \\
$\mathrm{ZN}$ & $\mathrm{mg} / \mathrm{L}$ & 23 & 0.25 & 0.034 & 23 & 0.098
\end{tabular}

Outfall FS-001

Effluent consists of the following types of wastewater: cooling water and greenhouse wastewater.

FLO MGD $14 \quad 0 \quad 0$

Outfall FS-002

Effluent consists of the following types of wastewater: cooling water and greenhouse wastewater.
FLO
MGD
14
0
0

Outfall $\mathrm{H}-002$

Effluent consists of the following types of wastewater: non-process cooling water and stormwater.

$\begin{array}{lrrcccc}\mathrm{C} & \mathrm{mg} / \mathrm{L} & 6 & 3.77 & <1 & 5 & 1.95 \\ \mathrm{COD} & \mathrm{mg} / \mathrm{L} & 6 & <1 & <10 & & \\ \mathrm{FLO} & \mathrm{MGD} & 16 & 0.216 & 0.0144 & 16 & 0.1427 \\ \mathrm{O} \& \mathrm{G} & \mathrm{mg} / \mathrm{L} & 16 & 5.5 & <1 & 3 & 2.6 \\ \mathrm{PH} & \mathrm{pH} & 16 & 7.8 & 5.9 & & \\ \mathrm{TMP} & { }^{\circ} \mathrm{C} & 16 & 27 & 15 & 16 & 20.3 \\ \text { TSS } & \mathrm{mg} / \mathrm{L} & 16 & 34 & 1 & 16 & 6.1\end{array}$

Outfall $\mathrm{H}-004$

Effluent consists of the following types of wastewater: non-process cooling water.

$\begin{array}{lrrcccc}\text { FLO } & \text { MGD } & 16 & 0.0288 & 0.0014 & 16 & 0.0085 \\ \text { O\&G } & \mathrm{mg} / \mathrm{L} & 16 & 2.4 & <1 & 2 & 2.05 \\ \mathrm{PH} & \mathrm{pH} & 16 & 7.8 & 5.3 & & \\ \text { TMP } & { }^{\circ} \mathrm{C} & 16 & 27 & 7 & 16 & 16.8 \\ \text { TSS } & \mathrm{mg} / \mathrm{L} & 18 & 55 & <1 & 1 & 9.5\end{array}$

Outfall H-006

Effluent consists of the following types of wastewater: stormwater.

C

COD

$\begin{array}{lll}\mathrm{mg} / \mathrm{L} & 1 & <1 \\ \mathrm{mg} / \mathrm{L} & 1 & <1\end{array}$

$<1$
$<1$

$<10$ 
Table 67

National Pollutant Discharge Elimination System Monitoring Data

Page 11 of 23

Permit SC 0000175

\begin{tabular}{lcccccc} 
Measurement & Units & $\begin{array}{c}\text { Freq/ } \\
\text { Year }\end{array}$ & Maximum & Minimum & $\begin{array}{c}\text { \# in } \\
\text { Average }\end{array}$ & Average \\
\hline Outfall H-006, continued & & & & & & \\
FLO & MGD & 3 & 0.0014 & 0 & 3 & 0.0005 \\
O\&G & $\mathrm{mg} / \mathrm{L}$ & 1 & $<1$ & $<1$ & & \\
PH & $\mathrm{pH}$ & 1 & 5.8 & 5.8 &
\end{tabular}

Outfall $\mathrm{H}-007$

Effluent consists of the following types of wastewater: cooling tower blowdown, air compressor cooling water, and freeze protection purge from a water tank filling line.

$\begin{array}{lrllccc}\text { CHL } & \mathrm{mg} / \mathrm{L} & 17 & 0.11 & <0.1 & 3 & 0.06 \\ \text { FLO } & \mathrm{MGD} & 17 & 0.1008 & 0 & 17 & 0.0252 \\ \text { O\&G } & \mathrm{mg} / \mathrm{L} & 15 & 3 & <1 & 4 & 2.0 \\ \mathrm{PH} & \mathrm{pH} & 15 & 6.9 & 5.9 & & \\ \text { TMP } & { }^{\circ} \mathrm{C} & 15 & 28 & 1 & 15 & 16.5 \\ \text { TSS } & \mathrm{mg} / \mathrm{L} & 15 & 32 & <1 & 14 & 8.5\end{array}$

Outfall H-008

Effluent consists of the following types of wastewater: cooling water, steam condensate, neutralization wastewater, and powerhouse wastewater.

$\begin{array}{lrrcccc}\text { FLO } & \text { MGD } & 365 & 5.235 & 0.1163 & 365 & 1.5907 \\ \text { O\&G } & \mathrm{mg} / \mathrm{L} & 16 & 1.9 & <1 & 4 & 1.6 \\ \mathrm{PH} & \mathrm{pH} & 16 & 7.8 & 5.4 & & \\ \text { TMP } & { }^{\circ} \mathrm{C} & 16 & 26 & 12 & 16 & 20.0 \\ \text { TSS } & \mathrm{mg} / \mathrm{L} & 16 & 16 & 2 & 16 & 3.9\end{array}$

Outfall $\mathrm{H}-008 \mathrm{~A}$

Effluent consists of the following types of wastewater: ash basin discharge.

$\begin{array}{lrllccc}\text { FLO } & \text { MGD } & 17 & 1.152 & 0.0014 & 17 & 0.4694 \\ \text { O\&G } & \mathrm{mg} / \mathrm{L} & 16 & 2 & <1 & 3 & 7.9 \\ \mathrm{PH} & \mathrm{pH} & 17 & 7.3 & 5.7 & & \\ \text { TSS } & \mathrm{mg} / \mathrm{L} & 17 & 4 & 1 & 17 & 7\end{array}$


Table 67

National Pollutant Lischarge Elimination System Monitoring Data

Page 12 of 23

\section{Permit SC 0000175}

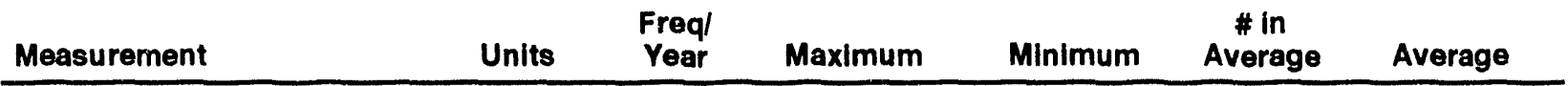

\section{Outfall $\mathrm{H}-012$}

Effluent consists of the following types of wastewater: cooling and steam condensate, Replacement Tritium Facility's decontamination showers, floor drains, Outfall H-017, and Outfall H-018.

\begin{tabular}{|c|c|c|c|c|c|c|}
\hline$A L$ & $\mathrm{mg} / \mathrm{L}$ & 1 & 0.297 & 0.054 & 1 & 0.146 \\
\hline AN & $\mathrm{mg} / \mathrm{L}$ & 11 & 2.2 & $<0.05$ & 3 & 0.8 \\
\hline CR & $\mathrm{mg} / \mathrm{L}$ & 11 & $<0.03$ & $<0.02$ & & \\
\hline $\mathrm{CU}$ & $\mathrm{mg} / \mathrm{L}$ & 11 & 0.021 & $<0.01$ & 6 & 0.016 \\
\hline FLO & MGD & 365 & 2.1328 & 0.0323 & 365 & 0.3604 \\
\hline$H G$ & $\mathrm{mg} / \mathrm{L}$ & 11 & 0.0002 & $<0.0001$ & 3 & 0.0002 \\
\hline MN & $\mathrm{mg} / \mathrm{L}$ & 11 & 0.0394 & $<0.005$ & 1 & 0.0207 \\
\hline $\mathrm{NO}_{3}$ & $\mathrm{mg} / \mathrm{L}$ & 11 & 17.2 & 0.05 & 11 & 3.23 \\
\hline NI & $\mathrm{mg} / \mathrm{L}$ & 11 & 0.032 & $<0.03$ & 1 & 0.032 \\
\hline O\&G & $\mathrm{mg} / \mathrm{L}$ & 16 & 1 & $<1$ & 1 & 1 \\
\hline PB & $\mathrm{mg} / \mathrm{L}$ & 11 & 0.0122 & $<0.003$ & 3 & 0.0089 \\
\hline $\mathrm{PH}$ & $\mathrm{pH}$ & 16 & 7.6 & 6.1 & & \\
\hline $\mathrm{SO}_{4}$ & $\mathrm{mg} / \mathrm{L}$ & 1 & 16.6 & 8.2 & 1 & 11.9 \\
\hline TMP & ${ }^{\circ} \mathrm{C}$ & 16 & 31 & 12.8 & 16 & 21.5 \\
\hline TSS & $\mathrm{mg} / \mathrm{L}$ & 16 & 8 & 1 & 16 & 3.1 \\
\hline $\mathrm{U}_{3} \mathrm{O}_{8}$ & $\mathrm{mg} / \mathrm{L}$ & 11 & $<0.1$ & $<0.02$ & & \\
\hline$Z N$ & $\mathrm{mg} / \mathrm{L}$ & 11 & 0.199 & $<0.05$ & 1 & 0.071 \\
\hline
\end{tabular}

\section{Outfall $\mathrm{H}-013$}

Effluent consists of the following types of wastewater: sanitary wastewater.

$\begin{array}{lrrllll}\text { BOD } & \mathrm{mg} / \mathrm{L} & 14 & 9.6 & 2.1 & 14 & 5.0 \\ \text { FEC } & \# / 100 \mathrm{~mL} & 15 & 2 & <2 & 1 & 2 \\ \text { FLO } & \mathrm{MGD} & 365 & 0.0922 & 0.0085 & 365 & 0.042 \\ \mathrm{PH} & \mathrm{pH} & 12 & 8.2 & 6.9 & & \\ \text { TSS } & \mathrm{mg} / \mathrm{L} & 14 & 1 & <1 & 13 & 5.2\end{array}$

Outfall H-015 (facility not in service but point still on permit)

Effluent consists of the following types of wastewater: non-contact cooling water, steam condensate, and cooling tower blowdown. 
Table 67

National Pollutant Discharge Elimination System Monitoring Data

Page 13 of 23

\section{Permit SC 0000175}

\begin{tabular}{lllll} 
Measurement & Greql \\
Units & Year & Maximum Minimum Average Average & $\begin{array}{c}\text { In } \\
\text { Ave }\end{array}$ \\
\hline
\end{tabular}

Outfall H-016

Effluent consists of the following types of wastewater: process water, cooling water, and stormwater.

\begin{tabular}{|c|c|c|c|c|c|c|}
\hline$A L$ & $\mathrm{mg} / \mathrm{L}$ & 15 & 0.053 & $<0.05$ & 1 & 0.053 \\
\hline$A N$ & $\mathrm{mg} / \mathrm{L}$ & 49 & 0.15 & $<0.01$ & 6 & 0.09 \\
\hline$B O D$ & $\mathrm{mg} / \mathrm{L}$ & 48 & 5 & $<1$ & 31 & 2.1 \\
\hline $\mathrm{CHL}$ & $\mathrm{mg} / \mathrm{L}$ & 13 & 0.37 & $<0.01$ & 3 & 0.17 \\
\hline CR & $\mathrm{mg} / \mathrm{L}$ & 49 & $<0.03$ & $<0.02$ & & \\
\hline $\mathrm{CU}$ & $\mathrm{mg} / \mathrm{L}$ & 49 & 0.013 & $<0.01$ & 2 & 0.012 \\
\hline FLO & MGD & 365 & 0.242 & 0 & 365 & 0.041 \\
\hline$H G$ & $\mathrm{mg} / \mathrm{L}$ & 49 & $<0.0005$ & $<0.0001$ & & \\
\hline$M N$ & $\mathrm{mg} / \mathrm{L}$ & 49 & 0.0343 & $<0.005$ & 3 & 0.0187 \\
\hline $\mathrm{NO}_{3}$ & $\mathrm{mg} / \mathrm{L}$ & 49 & 66 & 1.78 & 49 & 11.16 \\
\hline $\mathrm{NI}$ & $\mathrm{mg} / \mathrm{L}$ & 14 & $<0.05$ & $<0.03$ & & \\
\hline O\&G & $\mathrm{mg} / \mathrm{L}$ & 49 & 10.1 & $<1$ & 7 & 2.8 \\
\hline PB & $\mathrm{mg} / \mathrm{L}$ & 49 & 0.0094 & $<0.0005$ & 9 & 0.0047 \\
\hline $\mathrm{PH}$ & $\mathrm{pH}$ & 24 & 9 & 6.4 & & \\
\hline TMP & ${ }^{\circ} \mathrm{C}$ & 5 & 3 & 14 & 5 & 21.8 \\
\hline TSS & $\mathrm{mg} / \mathrm{L}$ & 49 & 2 & $<1$ & 3 & 1.3 \\
\hline $\mathrm{U}_{3} \mathrm{O}_{8}$ & $\mathrm{mg} / \mathrm{L}$ & 15 & $<0.1$ & $<0.02$ & & \\
\hline $\mathrm{ZN}$ & $\mathrm{mg} / \mathrm{L}$ & 49 & 0.414 & $<0.01$ & 16 & 0.043 \\
\hline
\end{tabular}

Outfall $\mathrm{H}-017$

Effluent consists of the following types of wastewater: stormwater runoff retention basin effluent.

$\begin{array}{lllllll}\mathrm{AL} & \mathrm{mg} / \mathrm{L} & 31 & 0.533 & 0.058 & 31 & 0.220 \\ \mathrm{AN} & \mathrm{mg} / \mathrm{L} & 31 & 0.12 & <0.05 & 4 & 0.09 \\ \mathrm{CR} & \mathrm{mg} / \mathrm{L} & 31 & <0.03 & <0.02 & & \\ \mathrm{CU} & \mathrm{mg} / \mathrm{L} & 31 & 0.013 & <0.01 & 5 & 0.012 \\ \mathrm{FLO} & \mathrm{MGD} & 32 & 1.152 & 0 & 32 & 0.9899 \\ \mathrm{HG} & \mathrm{mg} / \mathrm{L} & 31 & 0.0001 & <0.0001 & 4 & 0.0003 \\ \mathrm{MN} & \mathrm{mg} / \mathrm{L} & 31 & 0.0555 & <0.03 & 3 & 0.02 \\ \mathrm{NO}_{3} & \mathrm{mg} / \mathrm{L} & 31 & 3.36 & <0.02 & 25 & 0.472\end{array}$


Table 67

National Pollutant Discharge Elimination System Monitoring Data

Page 14 of 23

Permit SC 0000175

\begin{tabular}{lcccccc} 
Measurement & Units & $\begin{array}{c}\text { Freq/ } \\
\text { Year }\end{array}$ & Maximum & Minimum & $\begin{array}{c}\text { \# in } \\
\text { Average }\end{array}$ & Average \\
\hline Outfall H-017, continued & & & & & & \\
$\mathrm{NI}$ & $\mathrm{mg} / \mathrm{L}$ & 31 & $<0.05$ & $<0.03$ & & \\
$\mathrm{~PB}$ & $\mathrm{mg} / \mathrm{L}$ & 31 & 0.0166 & $<0.003$ & 8 & 0.0071 \\
$\mathrm{PH}$ & $\mathrm{pH}$ & 31 & 9.3 & 5.9 & & \\
$\mathrm{TSS}$ & $\mathrm{mg} / \mathrm{L}$ & 31 & 15 & 1 & 31 & 7 \\
$\mathrm{U}_{3} \mathrm{O}_{8}$ & $\mathrm{mg} / \mathrm{L}$ & 31 & $<0.1$ & $<0.01$ & & \\
$\mathrm{ZN}$ & $\mathrm{mg} / \mathrm{L}$ & 31 & 0.328 & 0.1 & 31 & 0.189
\end{tabular}

Outfall H-018

Effluent consists of the following types of wastewater: cooling water retention basin effluent.

$\begin{array}{lcccccc}\mathrm{AL} & \mathrm{mg} / \mathrm{L} & 4 & 0.101 & 0.064 & 4 & 0.081 \\ \mathrm{AN} & \mathrm{mg} / \mathrm{L} & 4 & <0.05 & <0.05 & & \\ \mathrm{CR} & \mathrm{mg} / \mathrm{L} & 4 & <0.02 & <0.02 & & \\ \mathrm{CU} & \mathrm{mg} / \mathrm{L} & 4 & 0.016 & <0.01 & 1 & 0.016 \\ \mathrm{FLO} & \mathrm{MGD} & 6 & 0.864 & 6 & & 0.576 \\ \mathrm{HG} & \mathrm{mg} / \mathrm{L} & 4 & <0.0001 & 0.0001 & & \\ \mathrm{MN} & \mathrm{mg} / \mathrm{L} & 4 & 0.0266 & 0.008 & 4 & 0.0173 \\ \mathrm{NO}_{3} & \mathrm{mg} / \mathrm{L} & 4 & 0.84 & 0.67 & 4 & 0.72 \\ \mathrm{NI} & \mathrm{mg} / \mathrm{L} & 4 & <0.05 & <0.05 & & \\ \mathrm{~PB} & \mathrm{mg} / \mathrm{L} & 4 & 0.0056 & <0.003 & 2 & 0.0047 \\ \mathrm{PH} & \mathrm{pH} & 4 & 6.8 & 6.6 & & \\ \mathrm{TSS} & \mathrm{mg} / \mathrm{L} & 4 & 4 & 2 & 4 & 2.75 \\ \mathrm{U}_{3} \mathrm{O}_{8} & \mathrm{mg} / \mathrm{L} & 4 & <0.02 & <0.02 & & \\ \mathrm{ZN} & \mathrm{mg} / \mathrm{L} & 4 & 0.219 & 0.054 & 4 & 0.164\end{array}$

Outfall K-001

Effluent consists of the following types of wastewater: cooling water.

$\begin{array}{lrrlrrr}\text { FLO } & \text { MGD } & 15 & 0.0576 & 0 & 15 & 0.0113 \\ \text { O\&G } & \mathrm{mg} / \mathrm{L} & 7 & 2 & <1 & 1 & 2 \\ \mathrm{PH} & \mathrm{pH} & 7 & 7.3 & 6.1 & & \end{array}$


Table 67

National Pollutant Discharge Elimination System Monitoring Data

Page 15 of 23

\section{Permit SC 0000175}

\begin{tabular}{lcccccc} 
Measurement & Units & $\begin{array}{c}\text { Freq/ } \\
\text { Year }\end{array}$ & Maximum & Minimum & $\begin{array}{c}\text { \# in } \\
\text { Average }\end{array}$ \\
\hline Outfall K-001, continued & & & & & & \\
Average
\end{tabular}

\section{Outfall K-006}

Effluent consists of the following types of wastewater: nonprocess cooling water, ash sluice leakage, and powerhouse wastewater.

$\begin{array}{lrrrrrr}\text { FLO } & \text { MGD } & 15 & 0.216 & 0.0029 & 15 & 0.0318 \\ \text { O\&G } & \mathrm{mg} / \mathrm{L} & 15 & 2.8 & <1 & 5 & 1.9 \\ \mathrm{PH} & \mathrm{pH} & 15 & 7.3 & 6.5 & & \\ \text { TMP } & { }^{\circ} \mathrm{C} & 15 & 28 & 15.5 & 15 & 21.4 \\ \text { TSS } & \mathrm{mg} / \mathrm{L} & 15 & 4 & <1 & 12 & 2.1\end{array}$

\section{Outfall $\mathrm{K}-008$}

Effluent consists of the following types of wastewater: nonprocess cooling water.

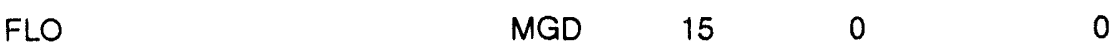

Outfall K-010

Effluent consists of the following types of wastewater: nonprocess cooling water.

$\begin{array}{lrrrccc}\text { FLO } & \text { MGD } & 17 & 0.072 & 0.0014 & 17 & 0.0334 \\ \text { O\&G } & \mathrm{mg} / \mathrm{L} & 16 & 6.2 & <1 & 4 & 3.1 \\ \mathrm{PH} & \mathrm{pH} & 15 & 7.6 & 6.6 & & 15 \\ \mathrm{TMP} & { }^{\circ} \mathrm{C} & 15 & 27 & 13 & 20.9 \\ \text { TSS } & \mathrm{mg} / \mathrm{L} & 17 & 84 & 2 & 17 & 20.1\end{array}$

Outfall K-012

Effluent consists of the following types of wastewater: sanitary wastewater.

$\begin{array}{lrrrrrr}\text { BOD } & \mathrm{mg} / \mathrm{L} & 14 & 7.6 & 1.4 & 14 & 3.8 \\ \text { FEC } & \# / 100 \mathrm{~mL} & 15 & 6 & <2 & 2 & 4 \\ \text { FLO } & \mathrm{MGD} & 365 & 0.0302 & 0.0029 & 365 & 0.0123 \\ \mathrm{PH} & \mathrm{pH} & 12 & 8.1 & 7 & & \\ \text { TSS } & \mathrm{mg} / \mathrm{L} & 14 & 13 & 1 & 14 & 4.4\end{array}$


Table 67

National Pollutant Discharge Elimination System Monitoring Data

Page 16 of 23

Permit SC 0000175

\begin{tabular}{lllll} 
Measurement & Units & $\begin{array}{c}\text { Freq/ } \\
\text { Year }\end{array}$ Maximum & Minimum Average Average \\
\hline
\end{tabular}

Outfall L -007

Effluent consists of the following types of wastewater: treated low activity wastewater, treated sanitary and neutralization tank waste, reactor cooling water, water treatment plant backflush, and reservoir overflow.

$\begin{array}{lrrcccc}\text { FLO } & \text { MGD } & 365 & 111.8099 & 0.9048 & 365 & 62.4061 \\ \text { O\&G } & \mathrm{mg} / \mathrm{L} & 15 & 1.4 & <1 & 2 & 1.4 \\ \mathrm{PH} & \mathrm{pH} & 15 & 7.9 & 6.2 & & \\ \text { TMP } & { }^{\circ} \mathrm{C} & 365 & 27.7 & 6.8 & 365 & 16.5 \\ \text { TSS } & \mathrm{mg} / \mathrm{L} & 16 & 48 & 2 & 16 & 8.3\end{array}$

Outfall L-007A

Effluent consists of the following types of wastewater: sanitary wastewater.

$\begin{array}{lrrllll}\text { BOD } & \mathrm{mg} / \mathrm{L} & 14 & 3.4 & <1 & 11 & 2.4 \\ \text { FEC } & \# / 100 \mathrm{~mL} & 14 & 6 & <2 & 2 & 4 \\ \text { FLO } & \mathrm{MGD} & 365 & 0.0288 & 0 & 365 & 0.0067 \\ \text { PH } & \mathrm{pH} & 12 & 8.7 & 7.01 & & \\ \text { TSS } & \mathrm{mg} / \mathrm{L} & 14 & 6 & 2 & 14 & 4\end{array}$

Outfall L-008

Effluent consists of the following types of wastewater: nonprocess cooling water and water reservoir sump wastewater.

$\begin{array}{lrrcccc}\text { FLO } & \text { MGD } & 15 & 0.576 & 0.0144 & 15 & 0.2424 \\ \text { O\&G } & \mathrm{mg} / \mathrm{L} & 15 & 4.5 & <1 & 4 & 2.5 \\ \mathrm{PH} & \mathrm{pH} & 15 & 7.2 & 6.3 & & \\ \mathrm{TMP} & { }^{\circ} \mathrm{C} & 15 & 29 & 11 & 15 & 20.7 \\ \text { TSS } & \mathrm{mg} / \mathrm{L} & 15 & 15 & <1 & 14 & 4.6\end{array}$

Outfall $L-010$

Effluent consists of the following types of wastewater: stormwater.

FLO MGD $\quad 3 \quad 000$

Outfall M-004

Effluent consists of the following types of wastewater: Liquid Effluent Treatment Facility wastewater.

\begin{tabular}{|c|c|c|c|}
\hline$C D$ & $\mathrm{mg} / \mathrm{L}$ & 13 & $<0.05$ \\
\hline$A G$ & $\mathrm{mg} / \mathrm{L}$ & 13 & $<0.0025$ \\
\hline
\end{tabular}


Table 67

National Pollutant Discharge Elimination System Monitoring Data

Page 17 of 23

\section{Permit SC 0000175}

\begin{tabular}{lcccccc} 
Measurement & Units & $\begin{array}{c}\text { Freql } \\
\text { Year }\end{array}$ & Maximum & Minimum & $\begin{array}{c}\text { \# in } \\
\text { Average }\end{array}$ & Average \\
\hline Outfall M-004, continued & $\mathrm{mg} / \mathrm{L}$ & 46 & 1.3 & $<0.05$ & 18 & 0.273 \\
$\mathrm{AL}$ & $\mathrm{mg} / \mathrm{L}$ & 9 & $<0.005$ & $<0.005$ & & \\
$\mathrm{CN}$ & $\mathrm{mg} / \mathrm{L}$ & 13 & $<0.1$ & $<0.02$ & & \\
$\mathrm{CR}$ & $\mathrm{mg} / \mathrm{L}$ & 46 & 0.03 & $<0.004$ & 1 & 0.03 \\
$\mathrm{CU}$ & $\mathrm{MGD}$ & 56 & 0.0366 & 0 & 56 & 0.0198 \\
$\mathrm{FLO}$ & $\mathrm{mg} / \mathrm{L}$ & 22 & 1700 & 51.1 & 22 & 1089.5 \\
$\mathrm{NO}_{3}$ & $\mathrm{mg} / \mathrm{L}$ & 46 & $<0.3$ & $<0.012$ & & \\
$\mathrm{NI}$ & $\mathrm{mg} / \mathrm{L}$ & 47 & 11.9 & $<1$ & 17 & 2.6 \\
$\mathrm{O} \& \mathrm{GG}$ & $\mathrm{mg} / \mathrm{L}$ & 45 & 0.0225 & $<0.0012$ & 5 & 0.0115 \\
$\mathrm{~PB}$ & $\mathrm{pH}$ & 3 & 7.8 & 6.5 & & \\
$\mathrm{PH}$ & $\mathrm{mg} / \mathrm{L}$ & 22 & 17.3 & 0.238 & 22 & 4.313 \\
$\mathrm{PHOS}$ & $\mathrm{mg} / \mathrm{L}$ & 46 & 14 & 1 & 46 & 4.5 \\
$\mathrm{TSS}$ & $\mathrm{mg} / \mathrm{L}$ & 46 & 0.128 & $<0.02$ & 3 & 0.052 \\
$\mathrm{U}_{3} \mathrm{O}_{8}$ & $\mathrm{mg} / \mathrm{L}$ & 13 & 0.085 & $<0.01$ & 6 & 0.065 \\
$\mathrm{ZN}$ & & & & &
\end{tabular}

Outfall M-005

Effluent consists of the following types of wastewater: air stripper.

\begin{tabular}{|c|c|c|c|c|c|}
\hline FLO & MGD & 54 & 0.8064 & 0 & 0.6993 \\
\hline PERCL & $u g / L$ & 53 & $<5$ & $<2$ & \\
\hline $\mathrm{PH}$ & $\mathrm{pH}$ & 29 & 7.9 & 5.6 & \\
\hline TRICL & $u g / L$ & 53 & $<5$ & $<2$ & \\
\hline
\end{tabular}

Outfall P-005

Effluent consists of the following types of wastewater: ash basin discharge.

FLO MGD $\quad 14 \quad 000$ 
Table 67

National Pollutant Discharge Elimination System Monitoring Data

Page 18 of 23

Permit SC 0000175

\begin{tabular}{|c|c|c|c|c|c|c|}
\hline Measurement & Units & $\begin{array}{l}\text { Freql } \\
\text { Year }\end{array}$ & Maximum & Minimum & $\begin{array}{c}\text { \# In } \\
\text { Average }\end{array}$ & Average \\
\hline
\end{tabular}

Outfall P-007

Effluent consists of the following types of wastewater: Powerhouse wastewater and nonprocess cooling water.

$\begin{array}{lrrlccc}\mathrm{AL} & \mathrm{mg} / \mathrm{L} & 5 & 0.405 & 0.075 & 5 & 0.230 \\ \mathrm{FE} & \mathrm{mg} / \mathrm{L} & 5 & 0.622 & 0.237 & 5 & 0.406 \\ \text { FLO } & \mathrm{MGD} & 33 & 2.3913 & 0 & 33 & 0.1588 \\ \mathrm{O \& G} & \mathrm{mg} / \mathrm{L} & 5 & 1.3 & <1 & 1 & 1.3 \\ \mathrm{PH} & \mathrm{pH} & 5 & 7 & 6.4 & & \\ \text { TMP } & { }^{\circ} \mathrm{C} & 5 & 28 & 18 & 5 & 22.4 \\ \text { TSS } & \mathrm{mg} / \mathrm{L} & 5 & 7 & 1 & 5 & 3.2\end{array}$

Outfall P-013

Effluent consists of the following types of wastewater: neutralization, reactor process, and cooling water.

$\begin{array}{lrrcccc}\text { FLO } & \text { MGD } & 365 & 86.6042 & 0.0452 & 365 & 7.1395 \\ \text { O\&G } & \mathrm{mg} / \mathrm{L} & 13 & 2.6 & <1 & 3 & 2.0 \\ \mathrm{PH} & \mathrm{pH} & 13 & 7.2 & 6.2 & & \\ \text { TMP } & { }^{\circ} \mathrm{C} & 266 & 27.7 & 6.4 & 266 & 17.5 \\ \text { TSS } & \mathrm{mg} / \mathrm{L} & 13 & 8 & <1 & 6 & 4\end{array}$

Outfall P-014

Effluent consists of the following types of wastewater: sanitary wastewater.

$\begin{array}{lrrllll}\text { BOD } & \mathrm{mg} / \mathrm{L} & 15 & 7.4 & <1 & 12 & 4.2 \\ \text { FEC } & \# / 100 \mathrm{~mL} & 15 & 2 & <2 & 1 & 2 \\ \text { FLO } & \mathrm{MGD} & 365 & 0.0288 & 0.0014 & 365 & 0.0088 \\ \text { PH } & \mathrm{pH} & 12 & 8.6 & 7.9 & & \\ \text { TSS } & \mathrm{mg} / \mathrm{L} & 15 & 14 & 2 & 15 & 5\end{array}$

Outfall P-019

Effluent consists of the following types of wastewater: P-Reactor cooling water.

$\begin{array}{lrrccrr}\text { FLO } & \text { MGD } & 365 & 56.8744 & 0 & 365 & 6.4398 \\ \text { O\&G } & \mathrm{mg} / \mathrm{L} & 12 & 2.5 & <1 & 4 & 1.8 \\ \mathrm{PH} & \mathrm{pH} & 12 & 7.2 & 6.1 & & \end{array}$


Table 67

National Pollutant Discharge Elimination System Monitoring Data

Page 19 of 23

Permit SC 0000175

\begin{tabular}{lcccccc} 
Measurement & Units & $\begin{array}{c}\text { Freq/ } \\
\text { Year }\end{array}$ & Maximum & Minimum & $\begin{array}{c}\# \text { in } \\
\text { Average }\end{array}$ & Average \\
\hline Outfall P-019, continued & & & & & & \\
TMP & ${ }^{\circ} \mathrm{C}$ & 354 & 26.2 & 5.7 & 354 & 17.5 \\
TSS & $\mathrm{mg} / \mathrm{L}$ & 12 & 7 & $<1$ & 11 & 3
\end{tabular}

Outfall PP-001

Effluent consists of the following types of wastewater: stormwater.

$\begin{array}{lcllccc}\text { FLO } & \text { MGD } & 8 & 0.72 & 0 & 8 & 0.1971 \\ \text { O\&G } & \mathrm{mg} / \mathrm{L} & 6 & 2 & <1 & 1 & 2 \\ \mathrm{PH} & \mathrm{pH} & 6 & 6.9 & 5.9 & & \end{array}$

Outfall S-002 (CS-002)

Effluent consists of the following types of wastewater: automotive shop and cooling wastewater.

$\begin{array}{lrrcccr}\text { AL } & \mathrm{mg} / \mathrm{L} & 1 & 19.4 & 19.4 & 1 & 19.4 \\ \text { BOD } & \mathrm{mg} / \mathrm{L} & 1 & 12.1 & 12.1 & 1 & 12.1 \\ \text { FLO } & \mathrm{MGD} & 5 & 0.0043 & 0 & 5 & .0001 \\ \text { O\&G } & \mathrm{mg} / \mathrm{L} & 1 & <1 & <1 & & \\ \mathrm{PH} & \mathrm{pH} & 1 & 6.1 & 6.1 & & \\ \text { TMP } & { }^{\circ} \mathrm{C} & 1 & 17 & 17 & 1 & 17 \\ \text { TSS } & \mathrm{mg} / \mathrm{L} & 1 & 451 & 451 & 1 & 451\end{array}$

Outfall S-008

Effluent consists of the following types of wastewater: stormwater runoff and cooling wastewater.

$$
\text { FLO MGD } 13 \quad 0 \quad 0
$$

Outfall S-011

Effluent consists of the following types of wastewater: sanitary wastewater from Central Shops Area wastewater treatment plant.

$\begin{array}{lrrcccc}\text { BOD } & \mathrm{mg} / \mathrm{L} & 14 & 4.9 & <1 & 12 & 2.9 \\ \text { FEC } & \# / 100 \mathrm{~mL} & 14 & <2 & <2 & & \\ \text { FLO } & \mathrm{MGD} & 365 & 0.0533 & 0 & 365 & 0.0215 \\ \mathrm{PH} & \mathrm{pH} & 12 & 8.4 & 7 & & \\ \text { TSS } & \mathrm{mg} / \mathrm{L} & 14 & 4 & 1 & 14 & 2.6\end{array}$


Table 67

National Pollutant Discharge Elimination System Monitoring Data

Page 20 of 23

\section{Permit SC 0000175}

\begin{tabular}{lllll} 
Measurement & Enits & Freql & Year & \# In \\
Mverage & Average \\
\hline
\end{tabular}

\section{Outfall S-014}

Effluent consists of the following types of wastewater: stormwater.

$\begin{array}{lllll}\text { FLO } & \text { MGD } & 3 & 0 & 0\end{array}$

Outfall SC-004

Effluent consists of the following types of wastewater: Steel Creek water monitoring below the dam at Road $A$.

$\begin{array}{lcccccc}\text { AG } & \mathrm{mg} / \mathrm{L} & 12 & 0.187 & <0.0005 & 1 & 0.187 \\ \mathrm{AS} & \mathrm{mg} / \mathrm{L} & 12 & <0.005 & <0.003 & & \\ \mathrm{BA} & \mathrm{mg} / \mathrm{L} & 12 & 0.0216 & <0.03 & 1 & 0.0127 \\ \mathrm{CD} & \mathrm{mg} / \mathrm{L} & 12 & <0.01 & <0.005 & & \\ \mathrm{CR} & \mathrm{mg} / \mathrm{L} & 12 & <0.03 & <0.02 & & \\ \mathrm{DO} & \mathrm{mg} / \mathrm{L} & 365 & 17.4 & 3.7 & 365 & 8.3 \\ \mathrm{FLO} & \mathrm{MGD} & 365 & 293.4202 & 4.0071 & 365 & 91.134 \\ \mathrm{HG} & \mathrm{mg} / \mathrm{L} & 12 & 0.0004 & <0.0001 & 1 & 0.0004 \\ \mathrm{NO}{ }_{3} & \mathrm{mg} / \mathrm{L} & 12 & 0.17 & 0.05 & 12 & 0.1 \\ \mathrm{~PB} & \mathrm{mg} / \mathrm{L} & 12 & 0.0021 & <0.003 & 1 & 0.0021 \\ \mathrm{PH} & \mathrm{pH} & 12 & 7.9 & 5.9 & & \\ \mathrm{PHOS} & \mathrm{mg} / \mathrm{L} & 12 & 0.059 & 0.015 & 12 & 0.030 \\ \mathrm{SE} & \mathrm{mg} / \mathrm{L} & 12 & <0.005 & <.0003 & & \\ \text { TMP } & { }^{\circ} \mathrm{C} & 364 & 31 & 9 & 364 & 18.9\end{array}$

Outfall T-001

Effluent consists of the following types of wastewater: artificial stream water.
FLO
MGD 13
0.0029
0
13
0.0002

Outfall T-005

Effluent consists of the following types of wastewater: cooling water, boiler blowdown, and neutralized acid solutions.

$\begin{array}{lrrcccc}\text { BOD } & \mathrm{mg} / \mathrm{L} & 1 & 4 & <1 & 4 & 2.0 \\ \text { FLO } & \text { MGD } & 14 & 0.216 & 0 & 14 & 0.0207 \\ \text { O\&G } & \mathrm{mg} / \mathrm{L} & 9 & <1 & <1 & & \\ \text { PH } & \mathrm{pH} & 9 & 7.5 & 6.3 & & \end{array}$




\section{Table 67}

National Pollutant Discharge Elimination System Monitoring Data

Page 21 of 23

\section{Permit SC 0000175}

\begin{tabular}{lrrrrrr} 
Measurement & Units & $\begin{array}{c}\text { Freq/ } \\
\text { Year }\end{array}$ & Maximum & Minimum & $\begin{array}{c}\text { \# in } \\
\text { Average }\end{array}$ & Average \\
\hline Outfall T-005, continued & & & & & & \\
TMP & ${ }^{\circ} \mathrm{C}$ & 9 & 27 & 13 & 9 & 21.7 \\
TSS & $\mathrm{mg} / \mathrm{L}$ & 9 & 19 & $<1$ & 8 & 3.5
\end{tabular}

Outfall T-007

Effluent consists of the following types of wastewater: sanitary wastewater from B-Area.

$\begin{array}{lrrlccc}\text { BOD } & \mathrm{mg} / \mathrm{L} & 25 & 7 & 1.5 & 25 & 3.0 \\ \text { CHL } & \mathrm{mg} / \mathrm{L} & 24 & 46 & 0.15 & 24 & 9.3 \\ \text { FEC } & \# / 100 \mathrm{~mL} & 25 & 74 & -2 & 2 & 38 \\ \text { FLO } & \mathrm{MGD} & 365 & 0.0487 & 0 & 365 & 0.0093 \\ \text { PH } & \mathrm{pH} & 23 & 8.4 & 6.8 & & . \\ \text { TSS } & \mathrm{mg} / \mathrm{L} & 25 & 11 & <1 & 24 & 3.1\end{array}$

Outfall $x-004$

Effluent consists of the following types of wastewater: cooling water.

$\begin{array}{lrrcccc}\text { FLO } & \text { MGD } & 365 & 0.4718 & 0.0129 & 365 & 0.0447 \\ \text { O\&G } & \mathrm{mg} / \mathrm{L} & 17 & 2.5 & <1 & 3 & 1.8 \\ \mathrm{PH} & \mathrm{pH} & 17 & 7.2 & 6.2 & & \\ \mathrm{TMP} & { }^{\circ} \mathrm{C} & 17 & 32 & 21 & 17 & 25.1 \\ \text { TSS } & \mathrm{mg} / \mathrm{L} & 17 & 3 & <1 & 13 & 1.5\end{array}$

\section{Outfall $x-008$}

Effluent consists of the following types of wastewater: cooling water, cooling tower blowdown, and domestic water overflow.

$\begin{array}{lrlllll}\text { AL } & \mathrm{mg} / \mathrm{L} & 17 & 0.142 & <0.05 & 5 & 0.090 \\ \mathrm{FE} & \mathrm{mg} / \mathrm{L} & 17 & 1.4 & 0.153 & 17 & 0.992 \\ \text { FL.O } & \mathrm{MGD} & 17 & 0.432 & 0.144 & 17 & 0.274 \\ \text { O\&G } & \mathrm{mg} / \mathrm{L} & 17 & 1.9 & <1 & 4 & 1.4 \\ \mathrm{PH} & \mathrm{pH} & 18 & 6.9 & 5.9 & & \\ \text { TMP } & { }^{\circ} \mathrm{C} & 17 & 3 & 21 & 17 & 24.8 \\ \text { TSS } & \mathrm{mg} / \mathrm{L} & 17 & 8 & <1 & 15 & 2.9\end{array}$


Table 67

National Pollutant Discharge Elimination System Monitoring Data

Page 22 of 23

\section{Permit SC 0000175}

Measurement

Units

Freql

Maximum

Minimum

$\#$ in

Year

Outfall X-011

Effluent consists of the following types of wastewater: cooling water, cooling tower blowdown, and domestic water overflow.

FLO

$$
\begin{array}{llll}
\text { MGD } & 13 \quad 0.0144 & <0.0014 \quad 12
\end{array}
$$

0.0032

\section{Outfall $X-013$}

Effluent consists of the following types of wastewater: sanitary wastewater.

$\begin{array}{lrrrrrr}\text { BOD } & \mathrm{mg} / \mathrm{L} & 14 & 4.9 & 1.1 & 14 & 2.4 \\ \text { FEC } & \# / 100 \mathrm{~mL} & 14 & 2 & <2 & 4 & 2 \\ \text { FLO } & \mathrm{MGD} & 365 & 5.4144 & 0 & 365 & 0.0195 \\ \mathrm{PH} & \mathrm{pH} & 12 & 8.8 & 7 & & \\ \text { TSS } & \mathrm{mg} / \mathrm{L} & 14 & 21 & 2 & 14 & 8.3\end{array}$

Outfall $x-014$

Effluent consists of the following types of wastewater: treated wastewater from TNX-ETP.

$\begin{array}{lcccccc}\text { BEN } & \mathrm{ug} / \mathrm{L} & 5 & <2 & <0.08 & & \\ \text { BOD } & \mathrm{mg} / \mathrm{L} & 48 & 16.1 & <1 & 37 & 4.9 \\ \mathrm{C} & \mathrm{mg} / \mathrm{L} & 5 & 9.57 & 1.21 & 5 & 4.29 \\ \mathrm{FLO} & \mathrm{MGD} & 100 & 0.035 & 0 & 100 & 0.0066 \\ \mathrm{HG} & \mathrm{ug} / \mathrm{L} & 5 & 1.53 & <0.0001 & 34 & 0.2603 \\ \mathrm{PHE} & \mathrm{mg} / \mathrm{L} & 5 & 0.008 & <0.002 & 19 & 0.004 \\ \text { O\&G } & \mathrm{mg} / \mathrm{L} & 49 & 6.4 & <1 & 14 & 2.2 \\ \mathrm{PH} & \mathrm{pH} & 44 & 8.35 & 6.4 & & \\ \text { TSS } & \mathrm{mg} / \mathrm{L} & 5 & 68 & 1 & 5 & 7.8\end{array}$

Outfall $Y-001$

Effluent consists of the following types of wastewater: cooling water and locomotive wash water.
FLO
MGD
13
0
0 
Table 67

National Pollutant Discharge Elimination System Monitoring Data

Page 23 of 23

\section{Permit SC 0044903}

\begin{tabular}{|c|c|c|c|c|c|c|}
\hline Measurement & Units & $\begin{array}{l}\text { Freql } \\
\text { Year }\end{array}$ & Maximum & Minimum & $\begin{array}{c}\# \text { In } \\
\text { Average }\end{array}$ & Average \\
\hline \multicolumn{7}{|l|}{ Outfall A-001A } \\
\hline \multicolumn{7}{|c|}{ Effluent consists of the following types of wastewater: air stripper effluent. } \\
\hline FLO & MGD & 357 & 0.0835 & 0 & 357 & 0.071 \\
\hline PERCL & ug/L & 48 & $<5$ & $<$ & & \\
\hline $\mathrm{PH}$ & $\mathrm{pH}$ & 304 & 8.1 & 4.5 & & \\
\hline TRICL & $u g / L$ & 48 & $<5$ & $<2$ & & \\
\hline
\end{tabular}

Outfall A-028 (facility not yot in service)

Effluent consists of the following types of wastewater: Powerhouse wastewater, water from floor drains, cooling water, and well flush water.

Outfall A-029 (facility not yet in service)

Effluent consists of the following types of wastewater: well flush water and tank overflow.

Outfall K-018 (formeriy Outfall K-011)

Effluent consists of the following types of wastewater: neutralization, $K$-Reactor cooling tower blowdown, and reservoir wastewater.

$\begin{array}{lrrrrrr}\mathrm{AL} & \mathrm{mg} / \mathrm{L} & 24 & 0.801 & 0.074 & 24 & 0.263 \\ \mathrm{BOD} & \mathrm{mg} / \mathrm{L} & 25 & 6.1 & <1 & 23 & 2.1 \\ \mathrm{CHL} & \mathrm{mg} / \mathrm{L} & 104 & 0.18 & <0.1 & 8 & 0.11 \\ \text { FEC } & \# / 100 \mathrm{~mL} & 24 & 308 & 8 & 24 & 55 \\ \text { FLO } & \mathrm{MGD} & 365 & 78 & 365 & & 22.5174 \\ \text { O\&G } & \mathrm{mg} / \mathrm{L} & 24 & 2 & <1 & & 1.5 \\ \mathrm{PH} & \mathrm{pH} & 361 & 7.8 & 5.6 & & \\ \text { TMP } & \circ \mathrm{C} & 365 & 26.8 & 7.5 & 365 & 17.8 \\ \text { TSS } & \mathrm{mg} / \mathrm{L} & 24 & 6 & 1 & 24 & 3.3\end{array}$

Outfall X008A (Facillity not yet in service)

Effluent consists of the following types of wastewater: sanitary wastewater. 
Table 68

National Pollutant Discharge Elimination System Stormwater Monitoring Data

Page 1 of 9

Permit SCR000000

Measurement

Units

Type Sample

Outfall B-008

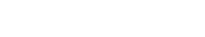

BOD

TSS

COD

TKN

$\mathrm{NO}_{2}, \mathrm{NO}_{3}$

$\mathrm{PO}_{4}-\mathrm{P}$

TOC

$\mathrm{pH}$

AIR TEMP

WATER TEMP

DO

RAIN GAUGE

TOTAL RAIN

LAST 0.1 RAIN

Grab

$12 / 14 / 93$

$\mathrm{mg} / \mathrm{L}$

$\mathrm{mg} / \mathrm{L}$

$\mathrm{mg} / \mathrm{L}$

$\mathrm{mg} / \mathrm{L}$

$\mathrm{mg} / \mathrm{L}$

$\mathrm{mg} / \mathrm{L}$

$\mathrm{mg} / \mathrm{L}$

${ }^{\circ} \mathrm{C}$

${ }^{\circ} \mathrm{C}$

$\mathrm{mg} / \mathrm{L}$

in.

in.

Outfall CS-006

BOD

TSS

$O \& G$

PHENOL

COD

TKN

$\mathrm{NO}_{2}, \mathrm{NO}_{3}$

$\mathrm{PO}_{4}-\mathrm{P}$

TOC

AL
3.9

12.0

11.00

0.40

0.26

0.068

4.40

7.3

11.0

6.9

11.8

Approx. 0.5

Approx. 0.76

$12 / 11 / 93$

6.4

4.0

44.40

0.74

0.68

0.149

17.00

$$
\begin{array}{r}
0.400 \\
9 / 21 / 93
\end{array}
$$

$\begin{array}{cc}\text { Background } & \text { Composite } \\ 5 / 10 / 93 & 5 / 19 / 93 \\ <1.0 & 8.6 \\ 14.0 & 177.0 \\ <1.0 & \\ 0.003 & \\ <10.0 & 19.80 \\ 0.27 & 1.23 \\ 0.02 & 0.50 \\ 0.053 & \\ 3.58 & 8.46 \\ 0.375 & 8.680\end{array}$




\section{Table 68}

National Pollutant Discharge Elimination System Stormwater Monitoring Data

Page 2 of 9

\section{Permit SCR000000 \\ Measurement \\ Outfall CS-006, continued}

Units

Type Sample

AG

CR

$\mathrm{CU}$

FE

MG

NI

PB

ZN

BENZENE

TETRACHLOROETHYLENE

TRICHLOROETHYLENE

1,1,1-/TRICHLOROETHYLENE

$\begin{array}{ccc}\mathrm{mg} / \mathrm{L} & <0.0005 & <0.0005 \\ \mathrm{mg} / \mathrm{L} & 0.020 & <0.02 \\ \mathrm{mg} / \mathrm{L} & <0.01 & 0.010 \\ \mathrm{mg} / \mathrm{L} & 2.700 & 10.700 \\ \mathrm{mg} / \mathrm{L} & 0.506 & 2.950 \\ \mathrm{mg} / \mathrm{L} & <0.05 & <0.05 \\ \mathrm{mg} / \mathrm{L} & <0.003 & 0.016 \\ \mathrm{mg} / \mathrm{L} & 0.017 & 0.157\end{array}$

$\mathrm{mg} / \mathrm{L}$

$<0.8$

$\mu \mathrm{g} / \mathrm{L} \quad<2.0$

$\mu \mathrm{g} / \mathrm{L} \quad<2.0$

$\mathrm{pH}$

$\mu g / L$

$<2.0$

AIR TEMP

WATER TEMP

DO

RAIN GAUGE

LAST 0.1 RAIN

$\begin{array}{rr}{ }^{\circ} \mathrm{C} & 30.0 \\ { }^{\circ} \mathrm{C} & 27.5 \\ \mathrm{mg} / \mathrm{L} & 6.5\end{array}$

in.

0.200

$5 / 13 / 93$

Outfall E-001

300

$\mathrm{mg} / \mathrm{L}$

9/20/93

TSS

$\mathrm{mg} / \mathrm{L}$

2.5

TDS

$\mathrm{mg} / \mathrm{L}$

13.0

58.0

$\mathrm{SO}_{4}$

$\mathrm{mg} / \mathrm{L}$

12.6

BROMIDE

$\mathrm{mg} / \mathrm{L}$

$<0.4$

O\&G

$\mathrm{mg} / \mathrm{L}$

$<1.0$

$\mathrm{CN}$

PHENOL

$\mathrm{mg} / \mathrm{L}$

$\mathrm{mg} / \mathrm{L}$
$<0.02$

$<0.002$

\section{Grab}

10/30/93

1.5

9.0

67.0

5.2

$<0.1$

$<1.0$

$<0.005$

$<0.002$
Grab 12/14/93

1.1

9.0

51.0

7.5

$<0.1$

$<1.0$

$<0.005$

0.003 
Table 68

National Pollutant Discharge Elimination System Stormwater Monitoring Data

Page 3 of 9

\section{Permit SCR000000}

Measurement

Outfall E-001, continued

COD

$\mathrm{NH}_{3}-\mathrm{N}$

TKN

$\mathrm{NO}_{2}, \mathrm{NO}_{3}$

$\mathrm{PO}_{4}-\mathrm{P}$

TOC

AL

AG

AS

B

$B A$

$C D$

$\mathrm{CR}$

CU

FE

HG

MG

MN

NI

PB

SB

SE

SN

U

$\mathrm{ZN}$

MG (DISSOLVED)

TETRACHLOROETHYLENE

TRICHLOROETHYLENE

1,1,1-/TRICHLOROETHYLENE $\mathrm{pH}$
Units

Type Sample

\begin{tabular}{|c|c|c|c|}
\hline $\mathrm{mg} / \mathrm{L}$ & $<10$ & $<10$ & $<10$ \\
\hline $\mathrm{mg} / \mathrm{L}$ & $<0.05$ & $<0.05$ & $<0.05$ \\
\hline $\mathrm{mg} / \mathrm{L}$ & 0.33 & $<0.2$ & $<0.2$ \\
\hline $\mathrm{mg} / \mathrm{L}$ & 0.18 & 0.13 & 0.14 \\
\hline $\mathrm{mg} / \mathrm{L}$ & 0.0760 & 0.0940 & 0.044 \\
\hline $\mathrm{mg} / \mathrm{L}$ & 3.00 & 2.60 & 1.40 \\
\hline $\mathrm{mg} / \mathrm{L}$ & 0.6210 & 3.4700 & 1.62 \\
\hline $\mathrm{mg} / \mathrm{L}$ & $<0.0005$ & $<0.0005$ & $<0.0005$ \\
\hline $\mathrm{mg} / \mathrm{L}$ & $<0.003$ & $<0.003$ & $<0.003$ \\
\hline $\mathrm{mg} / \mathrm{L}$ & $<0.03$ & 0.0520 & $<0.03$ \\
\hline $\mathrm{mg} / \mathrm{L}$ & 0.0138 & 0.0187 & $<0.005$ \\
\hline $\mathrm{mg} / \mathrm{L}$ & $<0.01$ & $<0.01$ & $<0.01$ \\
\hline $\mathrm{mg} / \mathrm{L}$ & $<0.02$ & $<0.02$ & $<0.02$ \\
\hline $\mathrm{mg} / \mathrm{L}$ & $<0.01$ & $<0.01$ & $<0.01$ \\
\hline $\mathrm{mg} / \mathrm{L}$ & 0.7000 & 3.3600 & 1.22 \\
\hline $\mathrm{mg} / \mathrm{L}$ & $<0.0001$ & $<0.0001$ & $<0.0001$ \\
\hline $\mathrm{mg} / \mathrm{L}$ & 1.1900 & 0.9820 & 1.29 \\
\hline $\mathrm{mg} / \mathrm{L}$ & 0.0157 & 0.0363 & 0.0095 \\
\hline $\mathrm{mg} / \mathrm{L}$ & $<0.05$ & $<0.05$ & $<0.05$ \\
\hline $\mathrm{mg} / \mathrm{L}$ & $<0.003$ & 0.0040 & $<0.003$ \\
\hline $\mathrm{mg} / \mathrm{L}$ & $<0.01$ & $<0.01$ & $<0.01$ \\
\hline $\mathrm{mg} / \mathrm{L}$ & $<0.003$ & $<0.015$ & $<0.003$ \\
\hline $\mathrm{mg} / \mathrm{L}$ & $<0.005$ & $<0.005$ & $<0.005$ \\
\hline $\mathrm{mg} / \mathrm{L}$ & $<0.02$ & $<0.02$ & $<0.02$ \\
\hline $\mathrm{mg} / \mathrm{L}$ & $<0.01$ & 0.0180 & $<0.01$ \\
\hline $\mathrm{mg} / \mathrm{L}$ & 1.4100 & 0.9450 & 1.24 \\
\hline$\mu \mathrm{g} / \mathrm{L}$ & $<2.0$ & 2.0 & $<2.0$ \\
\hline$\mu g / L$ & $<2.0$ & 2.0 & $<2.0$ \\
\hline \multirow[t]{2}{*}{$\mu g / L$} & $<2.0$ & $<2.0$ & $<2.0$ \\
\hline & 6.2 & 6.6 & 6.4 \\
\hline
\end{tabular}




\section{Table 68}

National Pollutant Discharge Elimination System Stormwater Monitoring Data

Page 4 of 9

\section{Permit SCR000000}

Measurement

Units

Type Sample

Outfall E-001, continued

AIR TEMP

TOTAL RAIN

LAST 0.1 RAIN

Outfall GS-001

$B O D$

TSS

$\mathrm{SO}_{4}$

O\&G

COD

$\mathrm{NH}_{3}-\mathrm{N}$

TKN

$\mathrm{NO}_{2}, \mathrm{NO}_{3}$

$\mathrm{PO}_{4}-\mathrm{P}$

TOC

CR

CU

HG

NI

PB

SB

SN

$\mathrm{pH}$

AIR TEMP

WATER TEMP
${ }^{\circ} \mathrm{C}$

${ }^{\circ} \mathrm{C}$

$\mathrm{mg} / \mathrm{L}$

in.

in.

23.0

21.0

6.0

0.13

0.50

9/18/93

Grab

$12 / 14 / 93$

$\mathrm{mg} / \mathrm{L}$

$\mathrm{mg} / \mathrm{L}$

1.0

$<1.0$

$\mathrm{mg} / \mathrm{L}$

2.9

$\mathrm{mg} / \mathrm{L} \quad<1.0$

$\mathrm{mg} / \mathrm{L}$

$<10.00$

$\mathrm{mg} / \mathrm{L}$

$<0.05$

$\mathrm{mg} / \mathrm{L}$

$<0.2$

$\mathrm{mg} / \mathrm{L}$

0.26

$\mathrm{mg} / \mathrm{L}$

0.047

$\mathrm{mg} / \mathrm{L}$

1.30

$\mathrm{mg} / \mathrm{L}$

$<0.02$

$\mathrm{mg} / \mathrm{L}$

$<0.01$

$\mathrm{mg} / \mathrm{L}$

$<0.0001$

$\mathrm{mg} / \mathrm{L}$

$<0.05$

$\mathrm{mg} / \mathrm{L}$

0.0785

$\mathrm{mg} / \mathrm{L}$

$<0.01$

$\mathrm{mg} / \mathrm{L}$

$<0.005$

5.4

${ }^{\circ} \mathrm{C}$

11.0

${ }^{\circ} \mathrm{C}$
20.0

10.0

17.5

7.1

8.0

11.1

3.200

Approx. 0.5

Approx. 0.75

$10 / 22 / 93$

$12 / 11 / 93$ 
Table 68

National Pollutant Discharge Elimination System Stormwater Monitoring Data

Page 5 of 9

\section{Permit SCR000000}

Measurement

Units

Type Sample

Outfall GS-001, continued

DO

$\mathrm{mg} / \mathrm{L}$

12.4

RAIN GAUGE

TOTAL RAIN

in. Approx. 0.2

LAST 0.1 RAIN

in. Approx. 0.7

$12 / 11 / 93$

Outfall H-007A

\begin{tabular}{|c|c|c|c|c|}
\hline & & $\begin{array}{c}\text { Grab } \\
12 / 14 / 93\end{array}$ & $\begin{array}{c}\text { Composite } \\
8 / 4 / 93\end{array}$ & $\begin{array}{c}\text { Composite } \\
10 / 17 / 93\end{array}$ \\
\hline BOD & $\mathrm{mg} / \mathrm{L}$ & 1.2 & 7.3 & \\
\hline TSS & $\mathrm{mg} / \mathrm{L}$ & 82.0 & 59.0 & 13.0 \\
\hline $\mathrm{SO}_{4}$ & $\mathrm{mg} / \mathrm{L}$ & 11.1 & 15.8 & 11.4 \\
\hline O\&G & $\mathrm{mg} / \mathrm{L}$ & 1.300 & & \\
\hline COD & $\mathrm{mg} / \mathrm{L}$ & $<10$ & 28.30 & 43.10 \\
\hline TKN & $\mathrm{mg} / \mathrm{L}$ & 0.23 & 0.50 & 0.54 \\
\hline $\mathrm{NO}_{2}, \mathrm{NO}_{3}$ & $\mathrm{mg} / \mathrm{L}$ & 0.35 & 2.25 & 0.69 \\
\hline $\mathrm{PO}_{4}-\mathrm{P}$ & $\mathrm{mg} / \mathrm{L}$ & 0.133 & 0.201 & 0.062 \\
\hline TOC & $\mathrm{mg} / \mathrm{L}$ & 1.90 & 16.40 & 10.00 \\
\hline$A L$ & $\mathrm{mg} / \mathrm{L}$ & 2.740 & 4.660 & 2.400 \\
\hline FE & $\mathrm{mg} / \mathrm{L}$ & 3.850 & 6.480 & 1.100 \\
\hline $\mathrm{pH}$ & & 6.5 & & \\
\hline AIR TEMP & ${ }^{\circ} \mathrm{C}$ & 10.0 & & \\
\hline WATER TEMP & ${ }^{\circ} \mathrm{C}$ & 7.7 & & \\
\hline DO & $\mathrm{mg} / \mathrm{L}$ & 11.8 & & \\
\hline RAIN GAUGE & in. & Approx. 0.5 & & \\
\hline TOTAL RAIN & in. & 0.580 & 0.530 & 0.42 \\
\hline LAST 0.1 RAIN & & $12 / 11 / 93$ & $7 / 24 / 93$ & $9 / 21 / 93$ \\
\hline
\end{tabular}


Table 68

National Pollutant Discharge Elimination System Stormwater Monitoring Data

Page 6 of 9

Permit SCR000000

Measurement

Units

Type Sample

Outfall K-002

BOD
TSS
O\&G
COD
TKN
$\mathrm{NO}_{2}, \mathrm{NO}_{3}$
PO 4 -P
TOC
PH
AIR TEMP
WATER TEMP
DO
RAIN GAUGE
TOTAL RAIN
LAST 0.1 RAIN
OUtFaII K-004

BOD

TSS

O\&G

COD

TKN

$\mathrm{NO}_{2}, \mathrm{NO}_{3}$

$\mathrm{PO}_{4}-\mathrm{P}$

TOC

$\mathrm{pH}$

\section{Grab}

$6 / 25 / 93$

$\mathrm{mg} / \mathrm{L}$

$\mathrm{mg} / \mathrm{L}$

$\mathrm{mg} / \mathrm{L}$

$\mathrm{mg} / \mathrm{L}$

$\mathrm{mg} / \mathrm{L}$

$\mathrm{mg} / \mathrm{L}$

$\mathrm{mg} / \mathrm{L}$

$\mathrm{mg} / \mathrm{L}$

14.4

137.0

$<1.0$

$<10.0$

0.90

0.44

0.293

10.30

7.3

${ }^{\circ} \mathrm{C}$

${ }^{\circ} \mathrm{C}$

25.0

27.0

$\mathrm{mg} / \mathrm{L}$

in.

in.

$13 / 93$

0.460

0.49

0.490

$6 / 13 / 93$
Composite

6/25/93

21.5

63.0

29.50

0.62

0.64

0.173

16.30
Background

$5 / 11 / 93$

$\mathrm{mg} / \mathrm{L}$

$\mathrm{mg} / \mathrm{L}$

$\mathrm{mg} / \mathrm{L}$

$\mathrm{mg} / \mathrm{L}$

$\mathrm{mg} / \mathrm{L}$

$\mathrm{mg} / \mathrm{L}$

$\mathrm{mg} / \mathrm{L}$

$\mathrm{mg} / \mathrm{L}$

$<1.0$

8.0

$<1.0$

$<10.0$

0.22

$<0.05$

0.127

3.62
7.1
Grab

6/25/93

15.8

1560.0

$<1.0$

88.50

1.28

1.80

1.140

15.80

6.0
Composite

$6 / 11 / 93$

13.2

120.0

51.20

0.90

0.89

0.33

11.60 
Table 68

National Pollutant Discharge Elimination System Stormwater Monitoring Data

Page 7 of 9

Permit SCR000000

Measurement

Units

Type Sample

Outfall K-004, continued

AIR TEMP

WATER TEMP

DO

TRC

RAIN GAUGE

TOTAL RAIN

LAST 0.1 RAIN

$\begin{array}{rrr}{ }^{\circ} \mathrm{C} & 23.6 & 27.0 \\ { }^{\circ} \mathrm{C} & 22.0 & 24.7 \\ \mathrm{mg} / \mathrm{L} & 5.9 & 8.0 \\ & <1.0 & \end{array}$

in.

0.460

in.

0.790

$6 / 12 / 93$

$6 / 1 / 93$

Outrail D-001A

ASBESTOS

TOTAL RAIN

t/sq mm

Grab

Composite

$11 / 8 / 93$

$11 / 8 / 93$

$<7.81$

$<7.81$

in.

0.550

0.550

LAST 0.1 RAIN

$7 / 24 / 93$

$7 / 24 / 93$

Outfall D-006A

Outrall D-ODB

ASBESTOS

TOTAL RAIN

LAST 0.1 RAIN

$\begin{array}{rrr} & \text { Grab } & \text { Composite } \\ & 12 / 13 / 93 & 12 / 13 / 93 \\ \text { t/sq mm } & 812.500 & 437.500 \\ \text { in. } & 0.360 & 0.360 \\ & 12 / 4 / 93 & 12 / 4 / 93\end{array}$

Outfall G-020

$\begin{array}{lccc} & & \text { Grab } & \text { Composite } \\ & & 12 / 14 / 93 & 11 / 5 / 93 \\ \text { BOD } & \mathrm{mg} / \mathrm{L} & 4.9 & 4.4 \\ \text { TSS } & \mathrm{mg} / \mathrm{L} & 109.0 & 58.0 \\ \text { O\&G } & \mathrm{mg} / \mathrm{L} & <1.0 & \\ \text { PHENOL } & \mathrm{mg} / \mathrm{L} & 0.002 & \\ \text { COD } & \mathrm{mg} / \mathrm{L} & 37.40 & 35.80 \\ \text { TKN } & \mathrm{mg} / \mathrm{L} & 0.61 & 0.51\end{array}$


Table 68

National Pollutant Discharge Elimination System Stormwater Monitoring Data

Page 8 of 9

\section{Permit SCR000000}

Measurement

Outfall G-020, continued

$\mathrm{NO}_{2}, \mathrm{NO}_{3}$

$\mathrm{PO}_{4}-\mathrm{P}$

TOC

$\mathrm{pH}$

AIR TEMP

WATER TEMP

DO

RAIN GAUGE

LAST 0.1 RAIN

Outfall 8-005

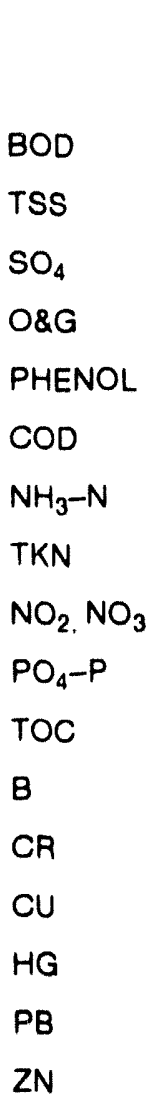

Units

Type Sample

$$
\mathrm{mg} / \mathrm{L}
$$

0.16

0.11

$m g / L$

0.128

0.157

$\mathrm{mg} / \mathrm{L}$

9.10

15.00

6.5

${ }^{\circ} \mathrm{C}$

${ }^{\circ} \mathrm{C}$

$\mathrm{mg} / \mathrm{L}$

in.

10.0

7.7

11.4

Approx. 0.5

12/11/93

Approx. 0.5

10/29/93

Grab

Background

12/14/93

5/10/193

$\mathrm{mg} / \mathrm{L}$

2.3

$<1.0$

10.0

5.0

$\mathrm{mg} / \mathrm{L}$

7.2

15.9

$\mathrm{mg} / \mathrm{L}$

$<1.0$

$<1.0$

$\mathrm{mg} / \mathrm{L}$

$<0.002$

$<0.002$

$\mathrm{mg} / \mathrm{L}$

14.90

11.50

$\mathrm{mg} / \mathrm{L}$

$\mathrm{mg} / \mathrm{L}$

$<0.05$

0.98

0.0500

$\mathrm{mg} / \mathrm{L}$

0.69

$<0.2$

$\mathrm{mg} / \mathrm{L}$

0.3460

5.41

0.6670

$\mathrm{mg} / \mathrm{L}$

6.97

3.90

$\mathrm{mg} / \mathrm{L}$.

$<0.03$

$<0.03$

$\mathrm{mg} / \mathrm{L}$

$<0.02$

$<0.02$

$\mathrm{mg} / \mathrm{L}$

$<0.01$

$<0.01$

$\mathrm{mg} / \mathrm{L}$

$<0.0001$

$<0.0001$

$\mathrm{mg} / \mathrm{L}$

$\mathrm{mg} / \mathrm{L}$

$<0.003$

0.011

0.072

0.133 
Table 68

National Pollutant Discharge Elimination System Stormwater Monitoring Data

Page 9 of 9

Permit SCR000000

Measurement

Unlts

Type Sample

Outfall s-005, continued

BENZENE

$\mathrm{mg} / \mathrm{L}$

$<0.8$

$<0.8$

FECAL COLIFORM

10

6.4

10

$\mathrm{pH}$

${ }^{\circ} \mathrm{C}$

28.0

6.6

AIR TEMP

WATER TEMP

${ }^{\circ} \mathrm{C}$

23.8

11.0

DO

$\mathrm{mg} / \mathrm{L}$

5.9

7.4

TRC

0.02

RAIN GAUGE

in.

Approx. 0.5

TOTAL RAIN

in.

0.580

LAST 0.1 RAIN

$12 / 11 / 93$

Outfall $x-001$

BOD
TSS
$\mathrm{COD}$
$\mathrm{TKN}$
$\mathrm{NO}_{2}, \mathrm{NO}_{3}$
$\mathrm{PO}_{4}-\mathrm{P}$
TOC
$\mathrm{CD}$
$\mathrm{CR}$
CU
HG
TOTAL RAIN
LAST O.1 RAIN

\section{Composite \\ 8/4/193}

$\mathrm{mg} / \mathrm{L}$

$\mathrm{mg} / \mathrm{L}$

$\mathrm{mg} / \mathrm{L}$

$\mathrm{mg} / \mathrm{l}$

$\mathrm{mg} / \mathrm{L}$

$\mathrm{mg} / \mathrm{L}$

$\mathrm{mg} / \mathrm{L}$

$\mathrm{mg} / \mathrm{L}$

$\mathrm{mg} / \mathrm{L}$

$\mathrm{mg} / \mathrm{L}$

$\mathrm{mg} / \mathrm{L}$

in.

10.2

41.0

46.60

0.81

1.27

0.287

16.40

$<0.01$

$<0.03$

0.031

$<0.0001$

0.720

$7 / 24 / 93$

\section{Composite}

11/5/93

2.6

29.0

$<10.00$

0.25

0.68

0.179

3.10

$<0.01$

$<0.02$

$<0.01$

$<0.0001$

0.40

$10 / 29 / 93$ 
Table 69

Savannah River Water Quality

Page 1 of 6

River-2 (Above SRS)

\begin{tabular}{|c|c|c|c|c|c|c|c|}
\hline Paramoter & Units & Jan & Feb & Mar & April & May & June \\
\hline $\begin{array}{l}\text { Water } \\
\text { volume }\end{array}$ & $\mathrm{L}$ & $2.31 E 12$ & 1.57E12 & $1.76 E 12$ & 1.51E12 & $7.99 E 11$ & $6.76 \mathrm{E} 11$ \\
\hline Temperature $^{b}$ & ${ }^{\circ} \mathrm{C}$ & 10.2 & 9.0 & 9.5 & 14.1 & 18.3 & 22.1 \\
\hline $\mathrm{pH}^{\mathrm{b}}$ & $\mathrm{pH}$ & 6.2 & 6.3 & 6.8 & 6.4 & 6.3 & 6.2 \\
\hline $\begin{array}{l}\text { Dissolved } \\
\text { oxygenb }\end{array}$ & $\mathrm{mg} / \mathrm{L}$ & 9.9 & 11.5 & 11.1 & 10.7 & 8.6 & 8.4 \\
\hline Alkalinity & $\mathrm{mg} / \mathrm{L}$ & 13 & 17 & 13 & 14 & 19 & 19 \\
\hline Conductivityb & $\mu \mathrm{mhos} / \mathrm{cm}$ & 54 & 68 & 54 & 55 & 86 & 88 \\
\hline Turbidity & NTU & 24 & 30 & 16 & 17 & 16 & 10 \\
\hline $\begin{array}{l}\text { Suspended } \\
\text { solids }\end{array}$ & $\mathrm{mg} / \mathrm{L}$ & 15 & 18 & 9 & 9 & 17 & 12 \\
\hline Volatile solids & $\mathrm{mg} / \mathrm{L}$ & 1 & 2 & 2 & 3 & 3 & 2 \\
\hline $\begin{array}{l}\text { Total } \\
\text { dissolved } \\
\text { solids }\end{array}$ & $\mathrm{mg} / \mathrm{L}$ & 52 & 61 & 48 & 71 & 71 & 69 \\
\hline Total solids & $\mathrm{mg} / \mathrm{L}$ & 67 & 79 & 57 & 80 & 88 & 81 \\
\hline Fixed residue & $\mathrm{mg} / \mathrm{L}$ & 14 & 17 & 7 & 6 & 15 & 10 \\
\hline $\begin{array}{l}\text { Chemical } \\
\text { oxygen } \\
\text { demand }\end{array}$ & $\mathrm{mg} / \mathrm{L}$ & $N D^{c}$ & ND & ND & ND & ND & ND \\
\hline Chloride & $\mathrm{mg} / \mathrm{L}$ & 4 & 5 & 4 & 4 & 10 & 8 \\
\hline $\begin{array}{l}\text { Nitrogen (as } \\
\mathrm{NO}_{2} / \mathrm{NO}_{3} \text { ) }\end{array}$ & $\mathrm{mg} / \mathrm{L}$ & 0.18 & 0.22 & 0.19 & 0.22 & 0.24 & 0.21 \\
\hline Sulfate & $\mathrm{mg} / \mathrm{L}$ & 5 & 5 & 4 & 4 & 6 & 7 \\
\hline Phosphate P & $\mathrm{mg} / \mathrm{L}$ & ND & ND & ND & ND & ND & ND \\
\hline Aluminum & $\mathrm{mg} / \mathrm{L}$ & & & 0.946 & & & 0.685 \\
\hline $\begin{array}{l}\text { Ammonia } \\
\text { nitrogen }\end{array}$ & $\mathrm{mg} / \mathrm{L}$ & 0.04 & 0.04 & 0.04 & 0.06 & 0.13 & 0.12 \\
\hline Calcium & $\mathrm{mg} / \mathrm{L}$ & & & 3.10 & & & 3.20 \\
\hline Copper & $\mathrm{mg} / \mathrm{L}$ & & & ND & & & ND \\
\hline Cadmium & $\mathrm{mg} / \mathrm{L}$ & & & ND & & & ND \\
\hline Magnesium & $\mathrm{mg} / \mathrm{L}$ & & & 1.30 & & & 1.08 \\
\hline Manganese & $\mathrm{mg} / \mathrm{L}$ & & & 0.088 & & & 0.067 \\
\hline Mercury & $\mu g / L$ & & & ND & & & ND \\
\hline Nickel & $\mathrm{mg} / \mathrm{L}$ & & & ND & & & ND \\
\hline Sodium & $\mathrm{mg} / \mathrm{L}$ & & & 4.87 & & & 7.02 \\
\hline Iron & $\mathrm{mg} / \mathrm{L}$ & & & 1.39 & & & 0.857 \\
\hline Lead & $\mathrm{mg} / \mathrm{L}$ & & & 0.002 & & & ND \\
\hline Chromium & $\mathrm{mg} / \mathrm{L}$ & & & ND & & & ND \\
\hline Zinc & $\mathrm{ma} / \mathrm{h}$ & & & ND & & & ND \\
\hline
\end{tabular}

a Metals are analyzed quarterly from a continuous flow composite.

b Field measurement

c None detected 
Table 69

Savannah River Water Quality

Page 2 of 6

River-2 (Above SRS)

\begin{tabular}{|c|c|c|c|c|c|c|c|}
\hline Parameter" & Units & July & Aug & Sopt & Oot & Nov & Doc \\
\hline $\begin{array}{l}\text { Water } \\
\text { volume }\end{array}$ & $L$ & 6.00E11 & $5.78 E 11$ & $4.73 \mathrm{E} 11$ & 4.67E11 & $4.78 E 11$ & $5.03 E 11$ \\
\hline Temperature $^{b}$ & ${ }^{\circ} \mathrm{C}$ & 24.8 & 23.4 & 23.5 & 20.9 & 15.3 & 14.3 \\
\hline $\mathrm{pH}^{\mathrm{b}}$ & $\mathrm{pH}$ & 6.5 & 6.3 & 6.1 & 6.0 & 6.3 & 6.4 \\
\hline $\begin{array}{l}\text { Dissolved } \\
\text { oxygenb }\end{array}$ & $\mathrm{mg} / \mathrm{L}$ & 8.0 & 8.2 & 6.8 & 8.6 & 8.6 & 8.9 \\
\hline Alkalinity & $\mathrm{mg} / \mathrm{L}$ & 17 & 17 & 19 & 20 & 19 & 20 \\
\hline Conductivityb & $\mu \mathrm{mhos} / \mathrm{cm}$ & 85 & 81 & 107 & 88 & 94 & 91 \\
\hline Turbidity & NTU & 5.8 & 5.3 & 5.9 & 4.0 & 8.1 & 5.1 \\
\hline $\begin{array}{l}\text { Suspended } \\
\text { solids }\end{array}$ & $\mathrm{mg} / \mathrm{L}$ & 15 & 13 & 7 & 10 & 7 & 5 \\
\hline Volatile solids & $\mathrm{mg} / \mathrm{L}$ & 1 & 1 & 1 & 1 & NDC & 3 \\
\hline $\begin{array}{l}\text { Total } \\
\text { dissolved } \\
\text { solids }\end{array}$ & $\mathrm{mg} / \mathrm{L}$ & 67 & 63 & 65 & 67 & 75 & 59 \\
\hline Total solids & $\mathrm{mg} / \mathrm{L}$ & 82 & 76 & 72 & 77 & 82 & 64 \\
\hline Fixed residue & $\mathrm{mg} / \mathrm{L}$ & 14 & 12 & 6 & 9 & 8 & 3 \\
\hline $\begin{array}{l}\text { Chemical } \\
\text { oxygen } \\
\text { demand }\end{array}$ & $\mathrm{mg} / \mathrm{L}$ & ND & ND & ND & ND & ND & ND \\
\hline Chloride & $\mathrm{mg} / \mathrm{L}$ & 8 & 6 & 13 & 10 & 10 & 9 \\
\hline $\begin{array}{l}\text { Nitrogen (as } \\
\mathrm{NO}_{2} / \mathrm{NO}_{3} \text { ) }\end{array}$ & $\mathrm{mg} / \mathrm{L}$ & 0.31 & 0.23 & 0.22 & 0.19 & 0.17 & 0.27 \\
\hline Sulfate & $\mathrm{mg} / \mathrm{L}$ & 7 & 6 & 8 & 6 & 7 & 8 \\
\hline Phosphate P & $\mathrm{mg} / \mathrm{L}$ & ND & ND & ND & ND & ND & ND \\
\hline Aluminum & $\mathrm{mg} / \mathrm{L}$ & & & 0.318 & & & 0.174 \\
\hline $\begin{array}{l}\text { Ammonia } \\
\text { nitrogen }\end{array}$ & $\mathrm{mg} / \mathrm{L}$ & 0.08 & 0.05 & 0.07 & 0.08 & 0.09 & 0.08 \\
\hline Calcium & $\mathrm{mg} / \mathrm{L}$ & & & 3.94 & & & 4.24 \\
\hline Copper & $\mathrm{mg} / \mathrm{L}$ & & & ND & & & ND \\
\hline Cadmium & $\mathrm{mg} / \mathrm{L}$ & & & ND & & & ND \\
\hline Magnesium & $\mathrm{mg} / \mathrm{L}$ & & & 1.26 & & & 1.38 \\
\hline Manganese & $\mathrm{mg} / \mathrm{L}$ & & & 0.081 & & & 0.076 \\
\hline Mercury & $\mu \mathrm{g} / \mathrm{L}$ & & & ND & & & ND \\
\hline Nickel & $\mathrm{mg} / \mathrm{L}$ & & & ND & & & ND \\
\hline Sodium & $\mathrm{mg} / \mathrm{L}$ & & & 11.50 & & & 11.60 \\
\hline Iron & $\mathrm{mg} / \mathrm{L}$ & & & 0.612 & & & 0.410 \\
\hline Lead & $\mathrm{mg} / \mathrm{L}$ & & & ND & & & ND \\
\hline Chromium & $\mathrm{mg} / \mathrm{L}$ & & & ND & & & ND \\
\hline Zinc & mall & & & ND & & & ND \\
\hline
\end{tabular}

Metals are analyzed quarterly from a continuous flow composite.

Field measurement

c None detected 
Table 69

Savannah River Water Quality

Page 3 of 6

\begin{tabular}{|c|c|c|c|c|c|c|c|}
\hline \multicolumn{8}{|c|}{ River-3B (Below Plant Vogtle) } \\
\hline Parameter" & Units & Jan & Feb & Mar & Aprll & May & June \\
\hline $\begin{array}{l}\text { Water } \\
\text { volume }\end{array}$ & $\mathrm{L}$ & $2.54 \mathrm{E} 12$ & 1.73E12 & $1.94 E 12$ & $1.66 E 12$ & $8.79 E 11$ & 7.44E11 \\
\hline Temperatureb & ${ }^{\circ} \mathrm{C}$ & 10.3 & 9.1 & 9.5 & 15.2 & 18.6 & 22.6 \\
\hline $\mathrm{pH}^{\mathrm{b}}$ & $\mathrm{pH}$ & 6.2 & 6.2 & 6.8 & 6.6 & 6.2 & 6.0 \\
\hline $\begin{array}{l}\text { Dissolved } \\
\text { oxygenb }\end{array}$ & $\mathrm{mg} / \mathrm{L}$ & 9.1 & 11.1 & 10.9 & 9.9 & 8.4 & 7.9 \\
\hline Alkalinity & $\mathrm{mg} / \mathrm{L}$ & 14 & 15 & 15 & 13 & 20 & 19 \\
\hline Conductivityb & $\mu \mathrm{mhos} / \mathrm{cm}$ & 57 & 58 & 54 & 55 & 84 & 89 \\
\hline Turbidity & NTU & 27 & 23 & 16 & 14 & 17 & 10 \\
\hline $\begin{array}{l}\text { Suspended } \\
\text { solids }\end{array}$ & $\mathrm{mg} / \mathrm{L}$ & 11 & 19 & 8 & 10 & 12 & 11 \\
\hline Volatile solids & $\mathrm{mg} / \mathrm{L}$ & 2 & 1 & 2 & 6 & 2 & 1 \\
\hline $\begin{array}{l}\text { Total } \\
\text { dissolved } \\
\text { solids }\end{array}$ & $\mathrm{mg} / \mathrm{L}$ & 58 & 53 & 47 & 53 & 60 & 68 \\
\hline Total solids & $\mathrm{mg} / \mathrm{L}$ & 69 & 72 & 55 & 63 & 72 & 79 \\
\hline Fixed residue & $\mathrm{mg} / \mathrm{L}$ & 9 & 19 & 6 & 4 & 10 & 10 \\
\hline $\begin{array}{l}\text { Chemical } \\
\text { oxygen } \\
\text { demand }\end{array}$ & $\mathrm{mg} / \mathrm{L}$ & $N D^{c}$ & ND & ND & ND & ND & ND \\
\hline Chloride & $\mathrm{mg} / \mathrm{L}$ & 4 & 4 & 4 & 4 & 9 & 9 \\
\hline $\begin{array}{l}\text { Nitrogen (as } \\
\mathrm{NO}_{2} / \mathrm{NO}_{3} \text { ) }\end{array}$ & $\mathrm{mg} / \mathrm{L}$ & 0.19 & 0.26 & 0.19 & 0.17 & 0.27 & 0.18 \\
\hline Sulfate & $\mathrm{mg} / \mathrm{L}$ & 5 & 5 & 4 & 4 & 7 & 8 \\
\hline Phosphate P & $\mathrm{mg} / \mathrm{L}$ & ND & ND & ND & ND & ND & ND \\
\hline Aluminum & $\mathrm{mg} / \mathrm{L}$ & & & 0.745 & & & 0.614 \\
\hline $\begin{array}{l}\text { Ammonia } \\
\text { nitrogen }\end{array}$ & $\mathrm{mg} / \mathrm{L}$ & 0.07 & 0.07 & 0.03 & 0.06 & 0.14 & 0.10 \\
\hline Calcium & $\mathrm{mg} / \mathrm{L}$ & & & 3.10 & & & 3.49 \\
\hline Copper & $\mathrm{mg} / \mathrm{h}$ & & & ND & & & ND \\
\hline Cadmium & $\mathrm{mg} / \mathrm{L}$ & & & ND & & & ND \\
\hline Magnesium & $\mathrm{mg} / \mathrm{L}$ & & & 1.23 & & & 1.12 \\
\hline Manganese & $\mathrm{mg} / \mathrm{L}$ & & & 0.069 & & & 0.064 \\
\hline Mercury & $\mu g / L$ & & & ND & & & ND \\
\hline Nickel & $\mathrm{mg} / \mathrm{L}$ & & & ND & & & ND \\
\hline Sodium & $\mathrm{mg} / \mathrm{L}$ & & & 5.04 & & & 7.15 \\
\hline Iron & $\mathrm{mg} / \mathrm{L}$ & & & 1.26 & & & 0.906 \\
\hline Lead & $\mathrm{mg} / \mathrm{L}$ & & & 0.003 & & & ND \\
\hline Chromium & $\mathrm{mg} / \mathrm{L}$ & & & ND & & & ND \\
\hline Zinc & $\mathrm{mg} / \mathrm{L}$ & & & ND & & & ND \\
\hline $\begin{array}{ll}\text { a } & \text { Metals a } \\
\text { b } & \text { Field me } \\
\text { c } & \text { None det }\end{array}$ & $\begin{array}{l}\text { nalyzed } \\
\text { urement } \\
\text { ted }\end{array}$ & & ous flow cor & site. & & & \\
\hline
\end{tabular}


Table 69

Savannah River Water Quality

Page 4 of 6

\begin{tabular}{|c|c|c|c|c|c|c|c|}
\hline \multicolumn{8}{|c|}{ River-3B (Below Plant Vogtle) } \\
\hline Parameter & Units & July & Aug & Sept & Oct & Nov & Dec \\
\hline $\begin{array}{l}\text { Water } \\
\text { volume }\end{array}$ & $\bar{L}$ & $6.60 \mathrm{E} 11$ & 6.36E11 & $5.20 E 11$ & $5.14 \mathrm{E} 11$ & $5.25 E 11$ & 5.53E11 \\
\hline Temperature $^{b}$ & ${ }^{\circ} \mathrm{C}$ & 25.5 & 25.0 & 25.0 & 21.1 & 15.5 & 14.2 \\
\hline $\mathrm{pH}^{\mathrm{b}}$ & $\mathrm{pH}$ & 6.7 & 6.3 & 6.4 & 6.0 & 6.5 & 6.1 \\
\hline $\begin{array}{l}\text { Dissolved } \\
\text { oxygenb }\end{array}$ & $\mathrm{mg} / \mathrm{L}$ & 7.1 & 8.3 & 8.2 & 8.3 & 8.9 & 8.6 \\
\hline Alkalinity & $\mathrm{mg} / \mathrm{L}$ & 24 & 19 & 20 & 20 & 21 & 21 \\
\hline Conductivityb & $\mu \mathrm{mhos} / \mathrm{cm}$ & 108 & 93 & 95 & 82 & 100 & 93 \\
\hline Turbidity & NTU & 6.5 & 5.9 & 5.7 & 3.9 & 8.5 & 5.5 \\
\hline $\begin{array}{l}\text { Suspended } \\
\text { solids }\end{array}$ & $\mathrm{mg} / \mathrm{L}$ & 12 & 12 & 3 & 8 & 13 & 5 \\
\hline Volatile solids & $\mathrm{mg} / \mathrm{L}$ & $N D^{c}$ & 1 & ND & 1 & 2 & 2 \\
\hline $\begin{array}{l}\text { Total } \\
\text { dissolved } \\
\text { solids }\end{array}$ & $\mathrm{mg} / \mathrm{L}$ & 80 & 68 & 68 & 71 & 84 & 63 \\
\hline Total solids & $\mathrm{mg} / \mathrm{L}$ & 92 & 80 & 71 & 79 & 97 & 68 \\
\hline Fixed residue & $\mathrm{mg} / \mathrm{L}$ & 12 & 11 & 3 & 8 & 11 & 4 \\
\hline $\begin{array}{l}\text { Chemical } \\
\text { oxygen } \\
\text { demand }\end{array}$ & $\mathrm{mg} / \mathrm{L}$ & ND & ND & ND & ND & ND & ND \\
\hline Chloride & $\mathrm{mg} / \mathrm{L}$ & 11 & 8 & 11 & 12 & 12 & 8 \\
\hline $\begin{array}{l}\text { Nitrogen (as } \\
\mathrm{NO}_{2} / \mathrm{NO}_{3} \text { ) }\end{array}$ & $\mathrm{mg} / \mathrm{L}$ & 0.32 & 0.25 & 0.23 & 0.22 & 0.18 & 0.35 \\
\hline Sulfate & $\mathrm{mg} / \mathrm{L}$ & 9 & 7 & 8 & 7 & 7 & 8 \\
\hline Phosphate P & $\mathrm{mg} / \mathrm{L}$ & ND & ND & ND & ND & ND & ND \\
\hline Aluminum & $m g / L$ & & & 0.363 & & & 0.190 \\
\hline $\begin{array}{l}\text { Ammonia } \\
\text { nitrogen }\end{array}$ & $\mathrm{mg} / \mathrm{L}$ & 0.06 & 0.06 & 0.08 & 0.09 & 0.09 & 0.12 \\
\hline Calcium & $\mathrm{mg} / \mathrm{L}$ & & & 4.52 & & & 4.45 \\
\hline Copper & $\mathrm{mg} / \mathrm{L}$ & & & ND & & & ND \\
\hline Cadmium & $\mathrm{mg} / \mathrm{L}$ & & & ND & & & ND \\
\hline Magnesium & $\mathrm{mg} / \mathrm{L}$ & & & 1.30 & & & 1.35 \\
\hline Manganese & $\mathrm{mg} / \mathrm{L}$ & & & 0.071 & & & 0.071 \\
\hline Mercury & $\mu g / L$ & & & ND & & & ND \\
\hline Nickel & $\mathrm{mg} / \mathrm{L}$ & & & ND & & & ND \\
\hline Sodium & $\mathrm{mg} / \mathrm{L}$ & & & 12.10 & & & 11.90 \\
\hline Iron & $\mathrm{mg} / \mathrm{L}$ & & & 0.677 & & & 0.453 \\
\hline Lead & $\mathrm{mg} / \mathrm{L}$ & & & ND & & & ND \\
\hline Chromium & $\mathrm{mg} / \mathrm{L}$ & & & ND & & & ND \\
\hline Zinc & $\mathrm{mg} / \mathrm{L}$ & & & 0.017 & & & ND \\
\hline
\end{tabular}

Metals are analyzed quarterly from a continuous flow composite.
b Field measurement

c None detected 
Table 69

Savannah River Water Quality

Page 5 of 6

\begin{tabular}{|c|c|c|c|c|c|c|c|}
\hline \multicolumn{8}{|c|}{ River-10 (Below SRS) } \\
\hline Parametera & Units & Jan & Feb & Mar & April & May & June \\
\hline $\begin{array}{l}\text { Water } \\
\text { volume }\end{array}$ & $\mathrm{L}$ & $2.68 E 12$ & 1.94E12 & $2.08 E 12$ & $1.94 E 12$ & 8.73E11 & $7.29 E 11$ \\
\hline Temperature $^{b}$ & ${ }^{\circ} \mathrm{C}$ & 11.3 & 9.8 & 9.1 & 15.6 & 19.1 & 22.9 \\
\hline $\mathrm{pH}^{\mathrm{b}}$ & $\mathrm{pH}$ & 6.5 & 6.0 & 6.4 & 6.4 & 6.1 & 6.7 \\
\hline $\begin{array}{l}\text { Dissolved } \\
\text { oxygen b }\end{array}$ & $\mathrm{mg} / \mathrm{L}$ & 8.8 & 9.7 & 10.5 & 9.8 & 7.2 & 7.5 \\
\hline Alkalinity & $\mathrm{mg} / \mathrm{L}$ & 13 & 15 & 13 & 14 & 21 & 19 \\
\hline Conductivityb & $\mu \mathrm{mhos} / \mathrm{cm}$ & 58 & 63 & 54 & 61 & 92 & 89 \\
\hline Turbidity & NTU & 28 & 14 & 15 & 12 & 16 & 10 \\
\hline $\begin{array}{l}\text { Suspended } \\
\text { solids }\end{array}$ & $\mathrm{mg} / \mathrm{L}$ & 6 & 5 & 5 & 6 & 16 & 15 \\
\hline Volatile solids & $\mathrm{mg} / \mathrm{L}$ & 2 & 1 & 3 & 3 & 4 & 2 \\
\hline $\begin{array}{l}\text { Total } \\
\text { dissolved } \\
\text { solids }\end{array}$ & $\mathrm{mg} / \mathrm{L}$ & 60 & 51 & 49 & 68 & 67 & 69 \\
\hline Total solids & $\mathrm{mg} / \mathrm{L}$ & 66 & 56 & 54 & 74 & 83 & 84 \\
\hline Fixed residue & $\mathrm{mg} / \mathrm{L}$ & 4 & 4 & 2 & 3 & 13 & 13 \\
\hline $\begin{array}{l}\text { Chemical } \\
\text { oxygen } \\
\text { demand }\end{array}$ & $\mathrm{mg} / \mathrm{L}$ & $N D^{c}$ & ND & ND & ND & ND & ND \\
\hline Chloride & $\mathrm{mg} / \mathrm{L}$ & 5 & 5 & 4 & 4 & 10 & 8 \\
\hline $\begin{array}{l}\text { Nitrogen (as } \\
\mathrm{NO}_{2} / \mathrm{NO}_{3} \text { ) }\end{array}$ & $\mathrm{mg} / \mathrm{L}$ & 0.20 & 0.29 & 0.18 & 0.18 & 0.29 & 0.20 \\
\hline Sulfate & $\mathrm{mg} / \mathrm{L}$ & 5 & 6 & 5 & 4 & 7 & 8 \\
\hline Phosphate P & $\mathrm{mg} / \mathrm{L}$ & ND & ND & ND & ND & ND & ND \\
\hline Aluminum & $\mathrm{mg} / \mathrm{L}$ & & & 0.838 & & & 0.606 \\
\hline $\begin{array}{l}\text { Ammonia } \\
\text { nitrogen }\end{array}$ & $\mathrm{mg} / \mathrm{L}$ & 0.05 & 0.09 & 0.03 & 0.02 & 0.11 & 0.04 \\
\hline Calcium & $\mathrm{mg} / \mathrm{L}$ & & & 3.25 & & & 3.73 \\
\hline Copper & $\mathrm{mg} / \mathrm{L}$ & & & ND & & & ND \\
\hline Cadmium & $\mathrm{mg} / \mathrm{L}$ & & & ND & & & ND \\
\hline Magnesium & $\mathrm{mg} / \mathrm{L}$ & & & 1.21 & & & 1.11 \\
\hline Manganese & $\mathrm{mg} / \mathrm{L}$ & & & 0.04 & & & 0.059 \\
\hline Mercury & $\mu g / L$ & & & ND & & & ND \\
\hline Nickel & $\mathrm{mg} / \mathrm{L}$ & & & ND & & & ND \\
\hline Sodium & $\mathrm{mg} / \mathrm{L}$ & & & 5.28 & & & 7.26 \\
\hline Iron & $\mathrm{mg} / \mathrm{L}$ & & & 1.15 & & & 0.948 \\
\hline Lead & $\mathrm{mg} / \mathrm{L}$ & & & 0.003 & & & ND \\
\hline Chromium & $\mathrm{mg} / \mathrm{L}$ & & & ND & & & ND \\
\hline Zinc & $\mathrm{mg} / \mathrm{L}$ & & & ND & & & ND \\
\hline
\end{tabular}

Metals are analyzed quarterly from a continuous flow composite.

Field measurement

None detected 
Table 69

Savannah River Water Quality

Page 6 of 6

River-10 (Below SRS)

\begin{tabular}{|c|c|c|c|c|c|c|c|}
\hline Parameter & Units & July & Aug & Sopt & Oot & Nov & Dec \\
\hline $\begin{array}{l}\text { Water } \\
\text { volume }\end{array}$ & $L$ & 6.03E11 & $5.23 E 11$ & $4.30 \mathrm{E} 11$ & 4.00E11 & $4.34 \mathrm{E} 11$ & 5.22E11 \\
\hline Temperatureb & ${ }^{\circ} \mathrm{C}$ & 25.7 & 25.1 & 24.5 & 20.7 & 15.0 & 13.6 \\
\hline $\mathrm{pH}^{\mathrm{b}}$ & $\mathrm{pH}$ & 6.4 & 6.4 & 6.0 & 6.3 & 6.3 & 6.3 \\
\hline $\begin{array}{l}\text { Dissolved } \\
\text { oxygen b }^{\mathrm{b}}\end{array}$ & $\mathrm{mg} / \mathrm{L}$ & 6.9 & 6.4 & 6.2 & 8.4 & 8.8 & 8.4 \\
\hline Alkalinity & $\mathrm{mg} / \mathrm{L}$ & 21 & 20 & 17 & 21 & 24 & 22 \\
\hline Conductivityb & $\mu \mathrm{mhos} / \mathrm{cm}$ & 101 & 99 & 96 & 105 & 106 & 100 \\
\hline Turbidity & NTU & 5.7 & 7.1 & 5.2 & 3.6 & 8.0 & 5.5 \\
\hline $\begin{array}{l}\text { Suspended } \\
\text { solids }\end{array}$ & $\mathrm{mg} / \mathrm{L}$ & 12 & 11 & 11 & 8 & 10 & 7 \\
\hline Volatile solids & $\mathrm{mg} / \mathrm{L}$ & 1 & 2 & 1 & 1 & 1 & 3 \\
\hline $\begin{array}{l}\text { Total } \\
\text { dissolved } \\
\text { solids }\end{array}$ & $\mathrm{mg} / \mathrm{L}$ & 69 & 68 & 71 & 77 & 90 & 66 \\
\hline Total solids & $\mathrm{mg} / \mathrm{L}$ & 81 & 79 & 82 & 85 & 100 & 73 \\
\hline Fixed residue & $\mathrm{mg} / \mathrm{L}$ & 11 & 10 & 10 & 8 & 9 & 4 \\
\hline $\begin{array}{l}\text { Chemical } \\
\text { oxygen } \\
\text { demand }\end{array}$ & $\mathrm{mg} / \mathrm{L}$ & $N^{c}$ & ND & ND & ND & ND & ND \\
\hline Chloride & $\mathrm{mg} / \mathrm{L}$ & 10 & 9 & 11 & 12 & 11 & 10 \\
\hline $\begin{array}{l}\text { Nitrogen (as } \\
\mathrm{NO}_{2} / \mathrm{NO}_{3} \text { ) }\end{array}$ & $\mathrm{mg} / \mathrm{L}$ & 0.31 & 0.26 & 0.24 & 0.26 & 0.25 & 0.31 \\
\hline Sulfate & $\mathrm{mg} / \mathrm{L}$ & 9 & 8 & 8 & 8 & 8 & 9 \\
\hline Phosphate P & $\mathrm{mg} / \mathrm{L}$ & ND & ND & ND & ND & ND & ND \\
\hline Aluminum & $\mathrm{mg} / \mathrm{L}$ & & & 0.331 & & & 0.182 \\
\hline $\begin{array}{l}\text { Ammonia } \\
\text { nitrogen }\end{array}$ & $\mathrm{mg} / \mathrm{L}$ & 0.04 & 0.04 & 0.04 & 0.06 & 0.07 & 0.07 \\
\hline Calcium & $\mathrm{mg} / \mathrm{L}$ & & & 4.58 & & & 5.09 \\
\hline Copper & $\mathrm{mg} / \mathrm{L}$ & & & ND & & & ND \\
\hline Cadmium & $\mathrm{mg} / \mathrm{L}$ & & & ND & & & ND \\
\hline Magnesium & $\mathrm{mg} / \mathrm{L}$ & & & 1.24 & & & 1.34 \\
\hline Manganese & $\mathrm{mg} / \mathrm{L}$ & & & 0.063 & & & 0.064 \\
\hline Mercury & $\mu \mathrm{g} / \mathrm{L}$ & & & ND & & & ND \\
\hline Nickel & $\mathrm{mg} / \mathrm{L}$ & & & ND & & & ND \\
\hline Sodium & $\mathrm{mg} / \mathrm{L}$ & & & 11.9 & & & 12.7 \\
\hline Iron & $\mathrm{mg} / \mathrm{L}$ & & & 0.701 & & & 0.516 \\
\hline Lead & $\mathrm{mg} / \mathrm{L}$ & & & ND & & & ND \\
\hline Chromium & $\mathrm{mg} / \mathrm{L}$ & & & ND & & & ND \\
\hline Zinc & $\mathrm{mg} / \mathrm{L}$ & & & 0.012 & & & ND \\
\hline
\end{tabular}

Metals are analyzed quarterly from a continuous flow composite.

Field measurement

c None detected 
Table 70

SRS Stream Water Quallty

Page 1 of 21

\begin{tabular}{|c|c|c|c|c|c|c|c|}
\hline $\begin{array}{l}\text { Tims Branch } 5 \\
\text { Parameter }\end{array}$ & Units & Jan & Fob & Mar & April & May & June \\
\hline Water volume & $L$ & 1.10E09 & $6.35 E 08$ & 9.34E08 & 6.37E08 & $4.33 \mathrm{E} 08$ & $3.96 \mathrm{E08}$ \\
\hline Temperatureb $^{b}$ & ${ }^{\circ} \mathrm{C}$ & 11.6 & 11.5 & 12.5 & 17.6 & 18.1 & 21.7 \\
\hline $\mathrm{pH}^{\mathrm{b}}$ & $\mathrm{pH}$ & 7.4 & 6.6 & 6.4 & 6.7 & 6.3 & 6.9 \\
\hline $\begin{array}{l}\text { Dissolved } \\
\text { oxygenb }\end{array}$ & $\mathrm{mg} / \mathrm{L}$ & 10.6 & 13.0 & 10.5 & 10.0 & 8.9 & 7.8 \\
\hline Alkalinity & $\mathrm{mg} / \mathrm{L}$ & 11 & 10 & 16 & 15 & 14 & 19 \\
\hline Conductivityb & $\mu \mathrm{mhos} / \mathrm{cm}$ & 44 & 69 & 134 & 92 & 104 & 82 \\
\hline $\begin{array}{l}\text { Total organic } \\
\text { carbon }\end{array}$ & & 6.56 & 2.66 & 2.49 & 4.14 & 6.65 & 3.60 \\
\hline Turbidity & NTU & 13 & 7.3 & 20 & 11 & 33 & 12 \\
\hline $\begin{array}{l}\text { Suspended } \\
\text { solids }\end{array}$ & $\mathrm{mg} / \mathrm{L}$ & 8 & 9 & 31 & 7 & 31 & 12 \\
\hline Volatile solids & $\mathrm{mg} / \mathrm{L}$ & $N D^{c}$ & 3 & 9 & 4 & 10 & 4 \\
\hline $\begin{array}{l}\text { Total dissolved } \\
\text { solids }\end{array}$ & $\mathrm{mg} / \mathrm{L}$ & 37 & 52 & 95 & 103 & 76 & 64 \\
\hline Total solids & $\mathrm{mg} / \mathrm{L}$ & 45 & 61 & 126 & 110 & 107 & 76 \\
\hline Fixed residue & $\mathrm{mg} / \mathrm{L}$ & 8 & 7 & 22 & 3 & 21 & 8 \\
\hline $\begin{array}{l}\text { Chemical } \\
\text { oxygen demand }\end{array}$ & $\mathrm{mg} / \mathrm{L}$ & ND & ND & ND & ND & 24 & ND \\
\hline $\begin{array}{l}\text { Total Kjeldahl } \\
\text { Nitrogen }\end{array}$ & $\mathrm{mg} / \mathrm{L}$ & 0.27 & 0.25 & 0.56 & 0.37 & 0.95 & 0.22 \\
\hline Chloride & $\mathrm{mg} / \mathrm{L}$ & 3 & 3 & 4 & 3 & 4 & 3 \\
\hline $\begin{array}{l}\text { Nitrogen (as } \\
\mathrm{NO}_{2} / \mathrm{NO}_{3} \text { ) }\end{array}$ & $\mathrm{mg} / \mathrm{L}$ & 0.46 & 2.26 & 7.31 & 2.61 & 3.33 & 1.31 \\
\hline Sulfate & $\mathrm{mg} / \mathrm{L}$ & 3 & 6 & 15 & 10 & 12 & 5 \\
\hline Phosphate P & $\mathrm{mg} / \mathrm{L}$ & ND & ND & ND & ND & ND & ND \\
\hline Aluminum & $\mathrm{mg} / \mathrm{L}$ & & & 0.535 & & & 0.727 \\
\hline $\begin{array}{l}\text { Ammonia } \\
\text { nitrogen }\end{array}$ & $\mathrm{mg} / \mathrm{L}$ & 0.02 & 0.03 & 0.03 & 0.03 & 0.05 & 0.01 \\
\hline Calcium & $\mathrm{mg} / \mathrm{L}$ & & & 1.25 & & & 1.25 \\
\hline Copper & $\mathrm{mg} / \mathrm{L}$ & & & ND & & & ND \\
\hline Cadmium & $\mathrm{mg} / \mathrm{L}$ & & & ND & & & ND \\
\hline Magnesium & $\mathrm{mg} / \mathrm{L}$ & & & 0.484 & & & 0.442 \\
\hline Manganese & $\mathrm{mg} / \mathrm{L}$ & & & 0.068 & & & 0.075 \\
\hline Mercury & $\mu g / L$ & & & ND & & & ND \\
\hline Nickel & $\mathrm{mg} / \mathrm{L}$ & & & ND & & & ND \\
\hline Sodium & $\mathrm{mg} / \mathrm{L}$ & & & 12.9 & & & 14.9 \\
\hline Iron & $\mathrm{mg} / \mathrm{L}$ & & & 1.55 & & & 2.22 \\
\hline Lead & $\mathrm{mg} / \mathrm{L}$ & & & 0.004 & & & ND \\
\hline Chromium & $\mathrm{mg} / \mathrm{L}$ & & & ND & & & ND \\
\hline Zinc & $\mathrm{mg} / \mathrm{L}$ & & & ND & & & ND \\
\hline $\begin{array}{ll}\text { a } & \text { Metals are ane } \\
\text { b } & \text { Field measure } \\
\text { c None detectec }\end{array}$ & $\begin{array}{l}\text { lyzed quarterly } \\
\text { ments }\end{array}$ & m a monthl & rab composite & & & & \\
\hline
\end{tabular}


Table 70

SRS Stream Water Quality

Page 2 of 21

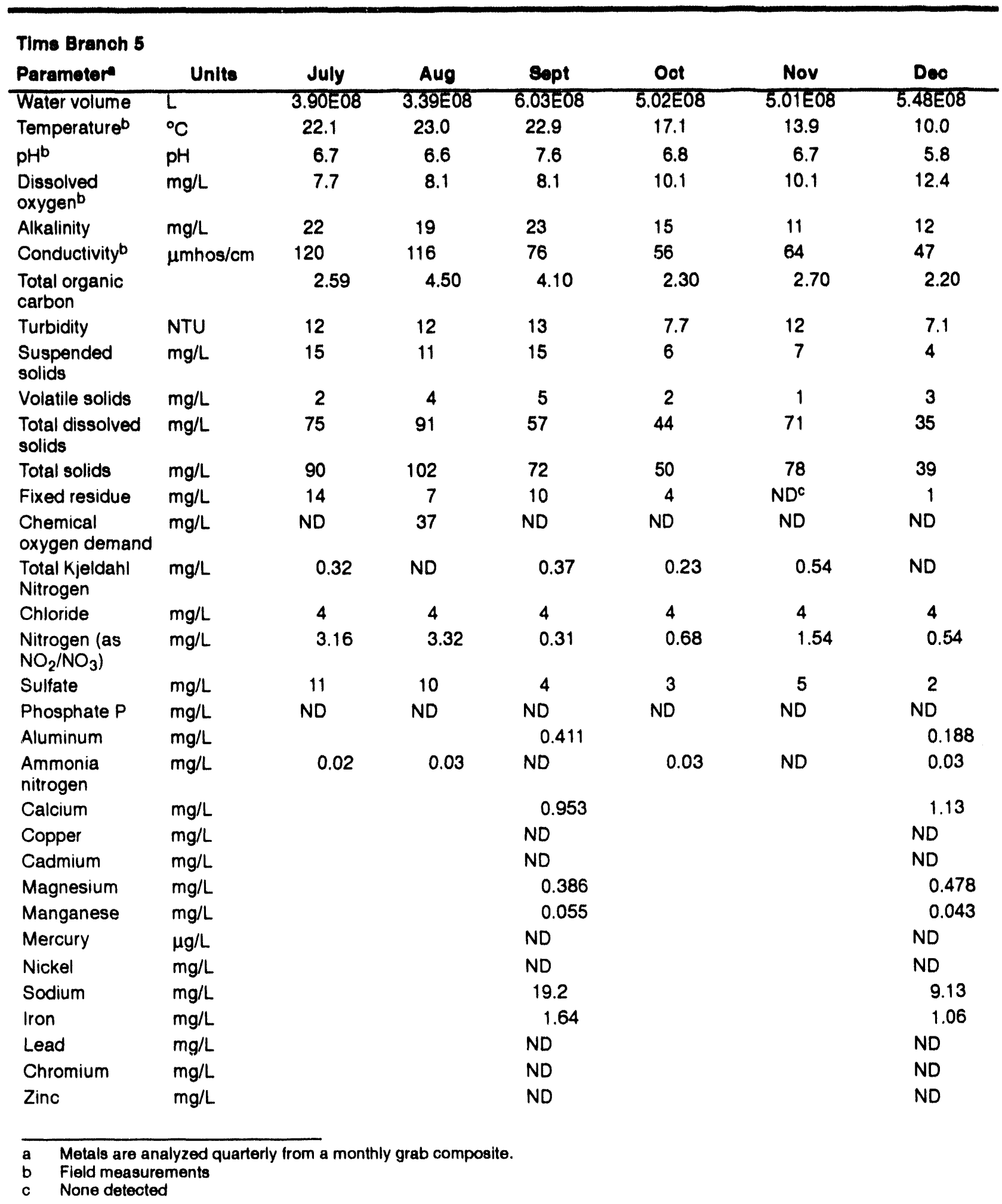


Table 70

SRS Stream Water Quality

Page 3 of 21

\begin{tabular}{|c|c|c|c|c|c|c|c|}
\hline \multicolumn{8}{|c|}{ Steal Creok 4 at Road A } \\
\hline Parameten & Units & Jan & Fob & Mar & April & May & June \\
\hline \multirow{2}{*}{$\begin{array}{l}\text { Water volume } \\
\text { Temperatureb }\end{array}$} & $\mathrm{L}$ & $1.10 E 10$ & $1.32 E=10$ & 2.51E10 & 1.54E10 & 1.05E10 & $9.78 \mathrm{E} 09$ \\
\hline & ${ }^{\circ} \mathrm{C}$ & 11.8 & 10.2 & 11.6 & 19.3 & 19.5 & 25.8 \\
\hline $\mathrm{pH}^{\mathrm{b}}$ & $\mathrm{pH}$ & 6.9 & 6.5 & 6.3 & 6.8 & 6.4 & 6.7 \\
\hline $\begin{array}{l}\text { Dissolved } \\
\text { oxygen b }\end{array}$ & $\mathrm{mg} / \mathrm{L}$ & 9.8 & 10.6 & 11.4 & 9.9 & 7.9 & 6.7 \\
\hline Alkalinity & $\mathrm{mg} / \mathrm{L}$ & 11 & 11 & 12 & 12 & 11 & 12 \\
\hline Conductivityb & $\mu \mathrm{mhos} / \mathrm{cm}$ & 58 & 55 & 55. & 62 & 57 & 64 \\
\hline $\begin{array}{l}\text { Total organic } \\
\text { carbon }\end{array}$ & & 6.43 & 3.10 & 3.29 & 3.53 & 4.06 & 3.48 \\
\hline Turbidity & NTU & 2.9 & 2.4 & 4.1 & 3.3 & 3.0 & 3.1 \\
\hline $\begin{array}{l}\text { Suspended } \\
\text { solids }\end{array}$ & $\mathrm{mg} / \mathrm{L}$ & 5 & 3 & 3 & $N^{c}$ & 3 & 5 \\
\hline Volatile solids & $\mathrm{mg} / \mathrm{L}$ & 2 & 2 & 1 & ND & 3 & 1 \\
\hline $\begin{array}{l}\text { Total dissolved } \\
\text { solids }\end{array}$ & $\mathrm{mg} / \mathrm{L}$ & 44 & 40 & 39 & 67 & 42 & 45 \\
\hline Total solids & $\mathrm{mg} / \mathrm{L}$ & 49 & 43 & 42 & 67 & 45 & 50 \\
\hline Fixed residue & $\mathrm{mg} / \mathrm{L}$ & 3 & 2 & 2 & ND & 1 & 4 \\
\hline $\begin{array}{l}\text { Chemical } \\
\text { oxygen demand }\end{array}$ & $\mathrm{mg} / \mathrm{L}$ & ND & ND & ND & ND & ND & ND \\
\hline $\begin{array}{l}\text { Total Kjeldahl } \\
\text { Nitrogen }\end{array}$ & $\mathrm{mg} / \mathrm{L}$ & 0.20 & 0.23 & ND & 0.25 & 0.28 & 0.18 \\
\hline Chloride & $\mathrm{mg} / \mathrm{L}$ & 6 & 6 & 5 & 4 & 4 & 5 \\
\hline $\begin{array}{l}\text { Nitrogen (as } \\
\mathrm{NO}_{2} / \mathrm{NO}_{3} \text { ) }\end{array}$ & $\mathrm{mg} / \mathrm{L}$ & 0.10 & 0.14 & 0.13 & 0.15 & 0.10 & 0.12 \\
\hline Sulfate & $\mathrm{mg} / \mathrm{L}$ & 5 & 5 & 5 & 5 & 4 & 6 \\
\hline Phosphate P & $\mathrm{mg} / \mathrm{L}$ & ND & ND & ND & ND & ND & ND \\
\hline Aluminum & $\mathrm{mg} / \mathrm{L}$ & & & 0.071 & & & 0.138 \\
\hline $\begin{array}{l}\text { Ammonia } \\
\text { nitrogen }\end{array}$ & $\mathrm{mg} / \mathrm{L}$ & 0.04 & 0.03 & 0.04 & 0.03 & 0.04 & 0.04 \\
\hline Calcium & $\mathrm{mg} / \mathrm{L}$ & & & 2.28 & & & 2.25 \\
\hline Copper & $\mathrm{mg} / \mathrm{L}$ & & & ND & & & ND \\
\hline Cadmium & $\mathrm{mg} / \mathrm{L}$ & & & ND & & & ND \\
\hline Magnesium & $\mathrm{mg} / \mathrm{L}$ & & & 0.981 & & & 0.947 \\
\hline Manganese & $\mathrm{mg} / \mathrm{L}$ & & & 0.024 & & & ND \\
\hline Mercury & $\mu \mathrm{g} / \mathrm{L}$ & & & ND & & & ND \\
\hline Nickel & $\mathrm{mg} / \mathrm{L}$ & & & ND & & & ND \\
\hline Sodium & $\mathrm{mg} / \mathrm{L}$ & & & 5.72 & & & 5.44 \\
\hline Iron & $\mathrm{mg} / \mathrm{L}$ & & & 0.224 & & & 0.213 \\
\hline Lead & $\mathrm{mg} / \mathrm{L}$ & & & 0.004 & & & ND \\
\hline Chromium & $\mathrm{mg} / \mathrm{L}$ & & & ND & & & ND \\
\hline Zinc & $\mathrm{mg} / \mathrm{L}$ & & & 0.014 & & & ND \\
\hline $\begin{array}{ll}\text { a } & \text { Metals are ane } \\
\text { b } & \text { Field measure } \\
\text { c } & \text { None detected }\end{array}$ & $\begin{array}{l}\text { alyzed quarte } \\
\text { ments } \\
\text { d }\end{array}$ & ma month & grab comp & & & & \\
\hline
\end{tabular}


Table 70

SRS Stream Water Quality

Page 4 of 21

\begin{tabular}{|c|c|c|c|c|c|c|c|}
\hline Paramotor & Unite & July & Aug & Sopt & Oot & Nov & Dec \\
\hline Water volume & $L$ & 7.75E09 & 6.61E09 & $8.531=09$ & $4.52=09$ & 6.27E09 & 7.37E09 \\
\hline Temperature $^{b}$ & ${ }^{\circ} \mathrm{C}$ & 29.6 & 28.0 & 27.2 & 22.4 & 16.7 & 14.0 \\
\hline $\mathrm{pH}^{\mathrm{b}}$ & $\mathrm{pH}$ & 7.0 & 6.8 & 7.9 & 7.2 & 6.7 & 5.9 \\
\hline $\begin{array}{l}\text { Dissolved } \\
\text { oxygenb }\end{array}$ & $\mathrm{mg} / \mathrm{l}$ & 7.0 & 6.5 & 6.4 & 7.2 & 10.8 & 8.9 \\
\hline Alkalini†y & $\mathrm{mg} / \mathrm{L}$ & 12 & 12 & 13 & 13 & 12 & 13 \\
\hline Conductivityb & $\mu \mathrm{mhos} / \mathrm{cm}$ & 70 & 76 & 66 & 68 & 70 & 70 \\
\hline $\begin{array}{l}\text { Total organic } \\
\text { carbon }\end{array}$ & & 3.35 & 3.80 & 3.00 & 2.80 & 3.10 & 2.70 \\
\hline Turbidity & NTU & 2.8 & 3.0 & 3.0 & 2.3 & 1.9 & 2.1 \\
\hline $\begin{array}{l}\text { Suspended } \\
\text { solids }\end{array}$ & $\mathrm{mg} / \mathrm{L}$ & 3 & 3 & 3 & 3 & 3 & 2 \\
\hline Volatile solids & $\mathrm{mg} / \mathrm{L}$ & 1 & 1 & 1 & 1 & $N D^{c}$ & 1 \\
\hline $\begin{array}{l}\text { Total dissolved } \\
\text { solids }\end{array}$ & $\mathrm{mg} / \mathrm{L}$ & 39 & 50 & 46 & 45 & 63 & 46 \\
\hline Total solids & $\mathrm{mg} / \mathrm{L}$ & 42 & 53 & 49 & 48 & 66 & 48 \\
\hline Fixed residue & $\mathrm{mg} / \mathrm{L}$ & 2 & 2 & 2 & 3 & 3 & 1 \\
\hline $\begin{array}{l}\text { Chemical } \\
\text { oxygen demand }\end{array}$ & $\mathrm{mg} / \mathrm{L}$ & ND & ND & ND & ND & ND & ND \\
\hline $\begin{array}{l}\text { Total Kjeldahl } \\
\text { Nitrogen }\end{array}$ & $\mathrm{mg} / \mathrm{L}$ & 0.29 & ND & 0.23 & 0.28 & ND & ND \\
\hline Chloride & $\mathrm{mg} / \mathrm{L}$ & 6 & 7 & 8 & 9 & 8 & 7 \\
\hline $\begin{array}{l}\text { Nitrogen (as } \\
\mathrm{NO}_{2} / \mathrm{NO}_{3} \text { ) }\end{array}$ & $\mathrm{mg} / \mathrm{L}$ & 0.10 & 0.08 & 0.06 & 0.06 & ND & 0.17 \\
\hline Sulfate & $\mathrm{mg} / \mathrm{L}$ & 6 & 6 & 6 & 6 & 6 & 6 \\
\hline Phosphate P & $\mathrm{mg} / \mathrm{L}$ & ND & ND & ND & ND & ND & ND \\
\hline Aluminum & $\mathrm{mg} / \mathrm{L}$ & & & ND & & & 0.060 \\
\hline $\begin{array}{l}\text { Ammonia } \\
\text { nitrogen }\end{array}$ & $\mathrm{mg} / \mathrm{L}$ & 0.04 & 0.05 & 0.02 & 0.04 & ND & 0.05 \\
\hline Calcium & $\mathrm{mg} / \mathrm{L}$ & & & 1.92 & & & 2.17 \\
\hline Copper & $\mathrm{mg} / \mathrm{L}$ & & & ND & & & ND \\
\hline Cadmium & $\mathrm{mg} / \mathrm{L}$ & & & ND & & & ND \\
\hline Magnesium & $\mathrm{mg} / \mathrm{L}$ & & & 1.04 & & & 1.16 \\
\hline Manganese & $\mathrm{mg} / \mathrm{L}$ & & & 0.008 & & & 0.019 \\
\hline Mercury & $\mu g / L$ & & & ND & & & ND \\
\hline Nickel & $\mathrm{mg} / \mathrm{L}$ & & & ND & & & ND \\
\hline Sodium & $\mathrm{mg} / \mathrm{L}$ & & & 7.86 & & & 8.53 \\
\hline Iron & $\mathrm{mg} / \mathrm{L}$ & & & 0.053 & & & 0.093 \\
\hline Lead & $\mathrm{mg} / \mathrm{L}$ & & & ND & & & ND \\
\hline Chromium & $\mathrm{mg} / \mathrm{L}$ & & & ND & & & ND \\
\hline Zinc & $\mathrm{mg} / \mathrm{L}$ & & & 0.01 & & & ND \\
\hline
\end{tabular}


Table 70 SRS Stream Water Quality

Pege 5 of 21

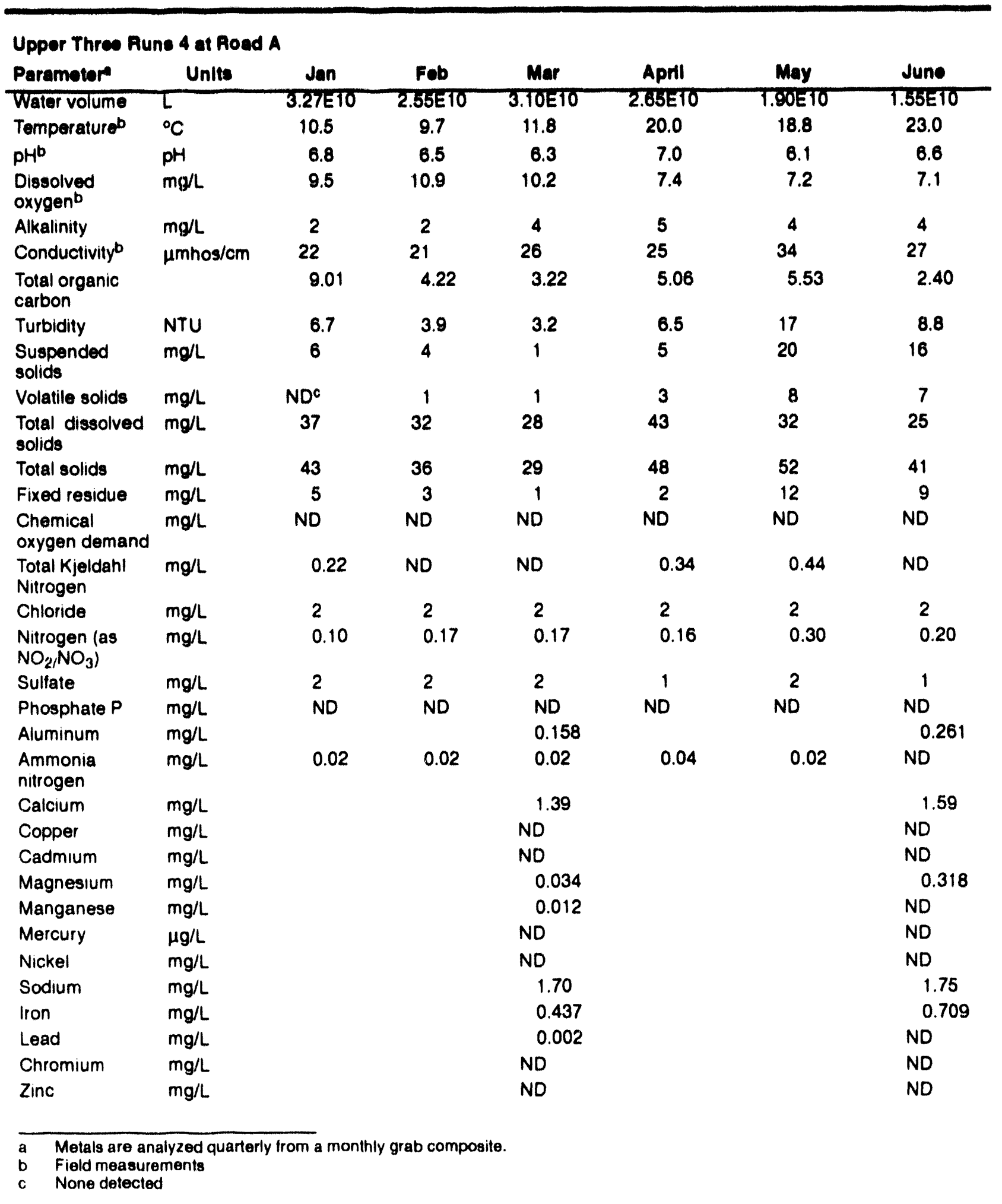


Table 70

SRS Stream Water Quallty

Page 6 of 21

\begin{tabular}{|c|c|c|c|c|c|c|c|}
\hline Paramutore & Unite & July & Aug & Eopt & Oot & Nov & Deo \\
\hline Water volume & $L$ & T.44E10 & T.34E 10 & T.68ETO & T.55ETO & T.87E10 & 1.89E10 \\
\hline Temperatureb & ${ }^{\circ} \mathrm{C}$ & 24.4 & 24.0 & 23.1 & 17.4 & 13.2 & 10.1 \\
\hline $\mathrm{pH}^{\mathrm{b}}$ & pH & 6.0 & 6.3 & 8.0 & 7.2 & 6.4 & 5.2 \\
\hline $\begin{array}{l}\text { Dissolved } \\
\text { oxygent }\end{array}$ & $\mathrm{mg} / \mathrm{L}$ & 7.6 & 7.2 & 5.0 & 9.1 & 12.5 & 8.5 \\
\hline Alkalinity & $m g / L$ & 3 & 4 & 4 & 4 & 3 & 3 \\
\hline Conductivityb & $\mu \mathrm{mhos} / \mathrm{cm}$ & 40 & 28 & 24 & 22 & 24 & 23 \\
\hline $\begin{array}{l}\text { Total organic } \\
\text { carbon }\end{array}$ & & 4.62 & 3.50 & 2.30 & 2.00 & 3.30 & 2.80 \\
\hline Turbidity & NTU & 4.9 & 16 & 13 & 4.7 & 3.8 & 2.6 \\
\hline $\begin{array}{l}\text { Suspended } \\
\text { solids }\end{array}$ & $\mathrm{mg} / \mathrm{L}$ & 10 & 13 & 8 & 6 & 4 & 2 \\
\hline Volatile solids & $\mathrm{mg} / \mathrm{L}$ & 3 & 5 & 4 & 2 & NDC & 2 \\
\hline $\begin{array}{l}\text { Total dissolved } \\
\text { solids }\end{array}$ & $\mathrm{mg} / \mathrm{L}$ & 27 & 29 & 20 & 26 & 47 & 19 \\
\hline Total solids & $\mathrm{mg} / \mathrm{L}$ & 37 & 42 & 29 & 32 & 51 & 21 \\
\hline Fixed residue & $\mathrm{mg} / \mathrm{L}$ & 8 & 8 & 5 & 4 & 4 & ND \\
\hline $\begin{array}{l}\text { Chemical } \\
\text { oxygen demand }\end{array}$ & $\mathrm{mg} / \mathrm{L}$ & ND & ND & ND & ND & ND & ND \\
\hline $\begin{array}{l}\text { Total Kjeldahl } \\
\text { Nitrogen }\end{array}$ & $\mathrm{mg} / \mathrm{L}$ & 0.38 & ND & ND & ND & ND & ND \\
\hline Chloride & $\mathrm{mg} / \mathrm{L}$ & 3 & 2 & 3 & 2 & 3 & 3 \\
\hline $\begin{array}{l}\text { Nitrogen (as } \\
\mathrm{NO}_{2}, \mathrm{NO}_{3} \text { ) }\end{array}$ & $\mathrm{mg} / \mathrm{L}$ & 0.16 & 0.19 & 0.12 & 0.13 & 0.12 & 0.10 \\
\hline Sulfate & $\mathrm{mg} / \mathrm{L}$ & 3 & 2 & 1 & 1 & 2 & 2 \\
\hline Phosphate P & $\mathrm{mg} / \mathrm{L}$ & ND & ND & ND & ND & ND & ND \\
\hline Aluminum & $\mathrm{mg} / \mathrm{L}$ & & & 0.220 & & & 0.018 \\
\hline $\begin{array}{l}\text { Ammonia } \\
\text { nitrogen }\end{array}$ & $\mathrm{mg} / \mathrm{L}$ & 0.02 & 0.03 & ND & 0.02 & ND & 0.02 \\
\hline Calcium & $\mathrm{mg} / \mathrm{L}$ & & & 1.71 & & & 1.47 \\
\hline Copper & $\mathrm{mg} / \mathrm{L}$ & & & ND & & & ND \\
\hline Cadmium & $\mathrm{mg} / \mathrm{h}$ & & & ND & & & ND \\
\hline Magnesium & $\mathrm{mg} / \mathrm{L}$ & & & 0.356 & & & 0.336 \\
\hline Manganese & $\mathrm{mg} / \mathrm{L}$ & & & 0.034 & & & 0.018 \\
\hline Mercury & $\mu g / L$ & & & ND & & & ND \\
\hline Nickel & $\mathrm{mg} / \mathrm{L}$ & & & ND & & & ND \\
\hline Sodium & $\mathrm{mg} / \mathrm{L}$ & & & 2.01 & & & 1.44 \\
\hline Iron & $\mathrm{mg} / \mathrm{L}$ & & & 0.624 & & & 0.363 \\
\hline Lead & $\mathrm{mg} / \mathrm{L}$ & & & ND & & & ND \\
\hline Chromium & $\mathrm{mg} / \mathrm{L}$ & & & ND & & & ND \\
\hline Zinc & $\mathrm{mg} / \mathrm{L}$ & & & ND & & & ND \\
\hline
\end{tabular}


Table 70

SRS Stream Water Quality

Pege 7 of 21

\begin{tabular}{|c|c|c|c|c|c|c|c|}
\hline \multicolumn{8}{|c|}{ Four Mllo Oreak at Road AT } \\
\hline Paramotore & Units & Jan & Fob & Mar & April & May & June \\
\hline \multirow{2}{*}{$\begin{array}{l}\text { Warer volume } \\
\text { Temperatureb }\end{array}$} & $\tau$ & 2.41E0' & T.50E00 & $3.221=09$ & $2.04 E 09$ & $9.49 \mathrm{E} 08$ & T.19E09 \\
\hline & ${ }^{\circ} \mathrm{C}$ & 10.1 & 11.1 & 11.8 & 19.9 & 19.8 & 24.8 \\
\hline $\mathrm{pH}^{\mathrm{b}}$ & pH & 6.8 & 6.6 & 6.3 & 6.9 & 6.1 & 6.6 \\
\hline $\begin{array}{l}\text { Dissolved } \\
\text { oxygent }\end{array}$ & $\mathrm{mg} / \mathrm{h}$ & 9.6 & 11.3 & 10.0 & 9.0 & 6.4 & 6.4 \\
\hline Alkalinity & $\mathrm{mg} / \mathrm{l}$ & 6 & 5 & 5 & 9 & 8 & 13 \\
\hline Conductivityb & umhoo/cm & 57 & 54 & 63 & 55 & 61 & 72 \\
\hline $\begin{array}{l}\text { Total organic } \\
\text { carbon }\end{array}$ & & 4.50 & 2.30 & 2.36 & 3.84 & 4.99 & 2.99 \\
\hline Turbidity & NTU & 7.3 & 3.9 & 6.3 & 4.4 & 21 & 4.6 \\
\hline $\begin{array}{l}\text { Suspended } \\
\text { solids }\end{array}$ & $\mathrm{mg} / \mathrm{L}$ & 6 & 7 & 7 & 2 & 9 & 4 \\
\hline Volatile solids & $\mathrm{mg} / \mathrm{L}$ & 1 & 2 & $N D^{\circ}$ & 1 & 3 & 1 \\
\hline $\begin{array}{l}\text { Total dissolved } \\
\text { solids }\end{array}$ & $\mathrm{mg} / \mathrm{L}$ & 42 & 40 & 40 & 67 & 50 & 62 \\
\hline Total solids & $\mathrm{mg} / \mathrm{L}$ & 48 & 47 & 47 & 69 & 59 & 66 \\
\hline Fixed residue & $\mathrm{mg} / \mathrm{L}$ & 5 & 6 & 7 & 1 & 6 & 3 \\
\hline $\begin{array}{l}\text { Chemical } \\
\text { oxygen demand }\end{array}$ & $\mathrm{mgl}$ & ND & ND & ND & ND & ND & ND \\
\hline $\begin{array}{l}\text { Total Kjeldahl } \\
\text { Nitrogen }\end{array}$ & $\mathrm{mgl}$ & 0.21 & ND & ND & 0.48 & 0.37 & ND \\
\hline Chloride & $\mathrm{mg} / \mathrm{L}$ & 3 & 3 & 3 & 3 & 3 & 3 \\
\hline $\begin{array}{l}\text { Nitrogen (as } \\
\mathrm{NO}_{2} / \mathrm{NO}_{3} \text { ) }\end{array}$ & $\mathrm{mg} / \mathrm{L}$ & 1.45 & 1.81 & 2.28 & 1.42 & 1.89 & 1.88 \\
\hline Sulfate & $\mathrm{mg} / \mathrm{L}$ & 9 & 7 & 6 & 4 & 5 & 5 \\
\hline Phosphate P & $\mathrm{mg} / \mathrm{h}$ & ND & ND & ND & ND & ND & ND \\
\hline Aluminum & $\mathrm{mg} / \mathrm{L}$ & & & 0.340 & & & 0.325 \\
\hline $\begin{array}{l}\text { Ammonia } \\
\text { nitrogen }\end{array}$ & $\mathrm{mg} / \mathrm{L}$ & 0.04 & 0.04 & 0.04 & 0.03 & 0.03 & 0.01 \\
\hline Calcium & $\mathrm{mg} / \mathrm{L}$ & & & 2.24 & & & 2.86 \\
\hline Copper & $\mathrm{mg} / \mathrm{L}$ & & & ND & & & ND \\
\hline Cadmium & $\mathrm{mg} / \mathrm{L}$ & & & ND & & & ND \\
\hline Magnesium & $\mathrm{mg} / \mathrm{L}$ & & & 0.565 & & & 0.605 \\
\hline Manganese & $\mathrm{mg} / \mathrm{L}$ & & & 0.104 & & & 0.093 \\
\hline Mercury & $\mu g / L$ & & & ND & & & ND \\
\hline Nickel & $\mathrm{mg} / \mathrm{L}$ & & & ND & & & ND \\
\hline Sodium & $\mathrm{mg} / \mathrm{L}$ & & & 6.29 & & & 7.53 \\
\hline Iron & $\mathrm{mg} / \mathrm{L}$ & & & 0.727 & & & 1.14 \\
\hline Lead & $\mathrm{mg} / \mathrm{L}$ & & & 0.003 & & & ND \\
\hline Chromium & $\mathrm{mg} / \mathrm{L}$ & & & ND & & & ND \\
\hline Zinc & $\mathrm{mg} / \mathrm{L}$ & & & 0.011 & & & ND \\
\hline $\begin{array}{ll}\text { a } & \text { Metals are ana } \\
\text { b } & \text { Field measure } \\
\text { c } & \text { None delected }\end{array}$ & $\begin{array}{l}\text { lyzed quarterl } \\
\text { ments }\end{array}$ & na monthly & grab composite & & & & \\
\hline
\end{tabular}


Table 70

\section{SRS Stream Wator Quality}

Pege 8 of 21

\begin{tabular}{|c|c|c|c|c|c|c|c|}
\hline \multicolumn{8}{|c|}{ Four Mllo Creok of Road A7 } \\
\hline Paramotor & Unite & July & Aug & Eept & Oot & Nov & Doo \\
\hline Water volume & $\mathrm{L}$ & $7.12=08$ & $7.21=08$ & T.26E09 & T.171E09 & T.06E09 & T.04E09 \\
\hline Temperatureb & ${ }^{\circ} \mathrm{C}$ & 25.5 & 23.0 & 24.2 & 18.0 & 13.1 & 10.0 \\
\hline $\mathrm{pH}^{\mathrm{b}}$ & $\mathrm{pH}$ & 6.7 & 6.6 & 7.7 & 6.8 & 6.4 & 5.7 \\
\hline $\begin{array}{l}\text { Dissolved } \\
\text { oxygent }\end{array}$ & $\mathrm{mg} / \mathrm{L}$ & 6.5 & 8.1 & 6.8 & 8.0 & 11.1 & 9.6 \\
\hline Alkalinity & $m g / L$ & 18 & 9 & 16 & 10 & 6 & 8 \\
\hline Conductivityb & $\mu \mathrm{mhos} / \mathrm{cm}$ & 90 & 116 & 76 & 75 & 74 & 79 \\
\hline $\begin{array}{l}\text { Total organic } \\
\text { carbon }\end{array}$ & & 2.67 & 3.50 & 2.00 & 1.40 & 1.80 & 1.50 \\
\hline Turbidity & NTU & 7.1 & 6.6 & 4.2 & 2.8 & 1.2 & 2.0 \\
\hline $\begin{array}{l}\text { Suspended } \\
\text { solids }\end{array}$ & $\mathrm{mg} / \mathrm{l}$ & 4 & 3 & 3 & 3 & 5 & 2 \\
\hline Volatile solids & $\mathrm{mg} / \mathrm{L}$ & 1 & 2 & 1 & 2 & 1 & 2 \\
\hline $\begin{array}{l}\text { Total dissolved } \\
\text { solids }\end{array}$ & $\mathrm{mg} / \mathrm{L}$ & 52 & 58 & 58 & 54 & 78 & 64 \\
\hline Total solids & $\mathrm{mg} / \mathrm{L}$ & 56 & 61 & 61 & 57 & 83 & 66 \\
\hline Fixed residue & $\mathrm{mg} / \mathrm{l}$ & 4 & 2 & 3 & . 2 & 4 & $N^{c}$ \\
\hline $\begin{array}{l}\text { Chemical } \\
\text { oxygen demand }\end{array}$ & $\mathrm{mg} / \mathrm{l}$ & ND & ND & ND & ND & ND & ND \\
\hline $\begin{array}{l}\text { Total Kjeldahl } \\
\text { Nitrogen }\end{array}$ & $\mathrm{mg} / \mathrm{L}$ & ND & ND & ND & ND & ND & ND \\
\hline Chloride & $\mathrm{mg} / \mathrm{L}$ & 4 & 2 & 4 & 4 & 5 & 4 \\
\hline $\begin{array}{l}\text { Nitrogen (as } \\
\mathrm{NO}_{2} / \mathrm{NO}_{3} \text { ) }\end{array}$ & $\mathrm{mg} / \mathrm{L}$ & 1.50 & 273 & 1.58 & 2.04 & 2.72 & 2.85 \\
\hline Sulfate & $\mathrm{mg} / \mathrm{L}$ & 4 & 6 & 6 & 8 & 9 & 6 \\
\hline Phosphate P & $\mathrm{mg} / \mathrm{L}$ & ND & ND & ND & ND & ND & ND \\
\hline Aluminum & $\mathrm{mg} / \mathrm{L}$ & & & 0.152 & & & 0.080 \\
\hline $\begin{array}{l}\text { Ammoni } \\
\text { nitrogen }\end{array}$ & $\mathrm{mg} / \mathrm{L}$ & 0.03 & 0.03 & ND & 0.02 & ND & 0.04 \\
\hline Calcium & $\mathrm{mg} / \mathrm{L}$ & & & 3.35 & & & 3.10 \\
\hline Copper & $\mathrm{mg} / \mathrm{L}$ & & & ND & & & ND \\
\hline Cadmium & $\mathrm{mg} / \mathrm{L}$ & & & ND & & & ND \\
\hline Magnesium & $\mathrm{mg} / \mathrm{L}$ & & & 0.596 & & & 0.636 \\
\hline Manganese & $\mathrm{mg} / \mathrm{L}$ & & & 0.089 & & & 0.079 \\
\hline Mercury & $\mu g / L$ & & & ND & & & ND \\
\hline Nickel & $\mathrm{mg} / \mathrm{L}$ & & & ND & & & ND \\
\hline Sodium & $\mathrm{mg} / \mathrm{l}$ & & & 10.6 & & & 9.37 \\
\hline Iron & $\mathrm{mg} / \mathrm{L}$ & & & 0.944 & & & 0.364 \\
\hline Lead & $\mathrm{mg} / \mathrm{L}$ & & & ND & & & ND \\
\hline Chromium & $\mathrm{mg} / \mathrm{L}$ & & & ND & & & ND \\
\hline Zinc & $\mathrm{mg} / \mathrm{L}$ & & & ND & & & ND \\
\hline
\end{tabular}




\section{Table 70}

SRS Stroam Water Quality

Page 9 of 21

\begin{tabular}{|c|c|c|c|c|c|c|c|}
\hline \multicolumn{8}{|l|}{ Crouch Branch } \\
\hline Paramotor & Unite & Jan & Fab & Mar & April & May & June \\
\hline Water volume & $\tau$ & $4.86 E 07$ & $2.26=07$ & 4.48E07 & $2.06 \mathrm{E} 07$ & 1.67E06 & $3.90 \mathrm{E} 06$ \\
\hline Temperatureb & ${ }^{\circ} \mathrm{C}$ & 11.2 & 10.2 & 11.3 & 17.8 & 19.3 & 21.0 \\
\hline $\mathrm{pH}^{\mathrm{b}}$ & $\mathrm{pH}$ & 6.2 & 5.8 & 6.2 & 6.2 & 6.3 & 6.0 \\
\hline $\begin{array}{l}\text { Dissolved } \\
\text { oxygenb }\end{array}$ & $\mathrm{mg} / \mathrm{L}$ & 6.7 & 10.5 & 7.5 & 6.8 & 5.4 & 3.9 \\
\hline Conductivityb & $\mu \mathrm{mhos} / \mathrm{cm}$ & 69 & 86 & 56 & 61 & 108 & 60 \\
\hline Turbidity & NTU & 28 & 103 & 18 & 16 & 71 & 26 \\
\hline $\begin{array}{l}\text { Suspended } \\
\text { solids }\end{array}$ & $\mathrm{mg} / \mathrm{L}$ & 7 & 26 & 5 & 5 & 32 & 12 \\
\hline
\end{tabular}

a Metals are analyzed quarterly from a monthly grab composite.

b Field measurements 


\section{Table 70}

\section{SRS Stream Water Quality}

Page 10 of 21

\begin{tabular}{|c|c|c|c|c|c|c|c|}
\hline $\begin{array}{l}\text { Crouch Branch } \\
\text { Paramotore }\end{array}$ & Unite & July & Aug & Sept & Oot & Nov & Deo \\
\hline Water volume & $I$ & 3.27EO6 & 3.65E06 & 1.69E07 & $1.91 E 06$ & 4.41E06 & 6.61E06 \\
\hline Temperatureb & ${ }^{\circ} \mathrm{C}$ & 24.0 & 26.6 & 23.7 & 17.8 & 13.0 & 9.5 \\
\hline $\mathrm{pH}^{\mathrm{b}}$ & $\mathrm{pH}$ & 6.2 & 7.9 & 6.6 & 6.0 & 5.9 & 7.1 \\
\hline $\begin{array}{l}\text { Dissolved } \\
\text { oxygen }^{b}\end{array}$ & $\mathrm{mg} / \mathrm{L}$ & 3.0 & 5.6 & 3.9 & 3.9 & 6.6 & 7.5 \\
\hline Conductivityb & $\mu \mathrm{mhos} / \mathrm{cm}$ & 100 & 133 & 125 & 73 & 83 & 93 \\
\hline Turbidity & NTU & 16 & 45 & 19 & 15 & 54 & 18 \\
\hline $\begin{array}{l}\text { Suspended } \\
\text { solids }\end{array}$ & $\mathrm{mg} / \mathrm{L}$ & 12 & 18 & 10 & 8 & 34 & 6 \\
\hline
\end{tabular}

a Metals are analyzed quarterly from a monthly grab composite.

b Field measurements 


\section{Table 70}

\section{SRS Stream Water Quality}

Page 11 of 21

\begin{tabular}{|c|c|c|c|c|c|c|c|}
\hline \multicolumn{8}{|c|}{ Lower Three Runs-2 } \\
\hline Parameter & Units & Jan & Feb & Mar & April & May & June \\
\hline Water volume & $\mathrm{L}$ & 1.19E10 & $6.93 \mathrm{E} 09$ & 9.27E09 & 9.19E09 & 5.16 EO9 & $4.67 E 09$ \\
\hline Temperature $^{b}$ & ${ }^{\circ} \mathrm{C}$ & 10.3 & 10.7 & 11.5 & 17.7 & 20.0 & 24.0 \\
\hline $\mathrm{pH}^{\mathrm{b}}$ & $\mathrm{pH}$ & 6.2 & 5.9 & 6.6 & 6.3 & 6.5 & 6.3 \\
\hline $\begin{array}{l}\text { Dissolved } \\
\text { oxygen b }\end{array}$ & $\mathrm{mg} / \mathrm{L}$ & 9.6 & 10.1 & 10.2 & 7.8 & 7.2 & 6.7 \\
\hline Alkalinity & $\mathrm{mg} / \mathrm{L}$ & 10 & 12 & 17 & 16 & 17 & 21 \\
\hline Conductivityb & $\mu \mathrm{mhos} / \mathrm{cm}$ & 44 & 56 & 60 & 61 & 57 & 60 \\
\hline Turbidity & NTU & 2.9 & 2.3 & 3.1 & 2.2 & 4.2 & 5.0 \\
\hline $\begin{array}{l}\text { Suspended } \\
\text { solids }\end{array}$ & $\mathrm{mg} / \mathrm{L}$ & 4 & 4 & 5 & $N D^{c}$ & 5 & 10 \\
\hline Volatile solids & $\mathrm{mg} / \mathrm{L}$ & 2 & 2 & 1 & ND & 4 & 2 \\
\hline $\begin{array}{l}\text { Total dissolved } \\
\text { solids }\end{array}$ & $\mathrm{mg} / \mathrm{L}$ & 33 & 40 & 41 & 69 & 51 & 46 \\
\hline Tutal solids & $\mathrm{mg} / \mathrm{L}$ & 37 & 44 & 46 & 69 & 56 & 56 \\
\hline Fixed residue & $\mathrm{mg} / \mathrm{L}$ & 2 & 2 & 4 & ND & 1 & 8 \\
\hline $\begin{array}{l}\text { Chemical } \\
\text { oxygen demand }\end{array}$ & $\mathrm{mg} / \mathrm{L}$ & ND & ND & ND & ND & ND & ND \\
\hline Chloride & $\mathrm{mg} / \mathrm{L}$ & 3 & 3 & 3 & 3 & 3 & 3 \\
\hline $\begin{array}{l}\text { Nitrogen (as } \\
\mathrm{NO}_{2} / \mathrm{NO}_{3} \text { ) }\end{array}$ & $\mathrm{mg} / \mathrm{L}$ & 0.11 & 0.14 & 0.12 & 0.12 & 0.08 & 0.18 \\
\hline Sulfate & $\mathrm{mg} / \mathrm{L}$ & 4 & 4 & 3 & 3 & 2 & 3 \\
\hline Phosphate P & $\mathrm{mg} / \mathrm{L}$ & ND & ND & ND & ND & ND & ND \\
\hline Aluminum & $\mathrm{mg} / \mathrm{L}$ & & & 0.078 & & & 0.092 \\
\hline $\begin{array}{l}\text { Ammonia } \\
\text { nitrogen }\end{array}$ & $\mathrm{mg} / \mathrm{L}$ & 0.02 & 0.02 & 0.04 & 0.04 & 0.06 & 0.04 \\
\hline Calcium & $\mathrm{mg} / \mathrm{L}$ & & & 5.63 & & & 7.68 \\
\hline Copper & $\mathrm{mg} / \mathrm{L}$ & & & ND & & & ND \\
\hline Cadmium & $\mathrm{mg} / \mathrm{L}$ & & & ND & & & ND \\
\hline Magnesium & $\mathrm{mg} / \mathrm{L}$ & & & 0.564 & & & 0.553 \\
\hline Manganese & $\mathrm{mg} / \mathrm{L}$ & & & 0.017 & & & ND \\
\hline Mercury & $\mu g / L$ & & & ND & & & ND \\
\hline Nickel & $\mathrm{mg} / \mathrm{L}$ & & & ND & & & ND \\
\hline Sodium & $\mathrm{mg} / \mathrm{L}$ & & & 2.28 & & & 1.97 \\
\hline Iron & $\mathrm{mg} / \mathrm{L}$ & & & 0.275 & & & 0.266 \\
\hline Lead & $\mathrm{mg} / \mathrm{L}$ & & & 0.002 & & & ND \\
\hline Chromium & $\mathrm{mg} / \mathrm{L}$ & & & ND & & & ND \\
\hline Zinc & $\mathrm{mg} / \mathrm{L}$ & & & ND & & & 0.031 \\
\hline
\end{tabular}

a Metals are analyzed quarterly from a monthly grab composite.

c None detected 
Table 70

SRS Stroam Water Quality

Page 12 of 21

\begin{tabular}{|c|c|c|c|c|c|c|c|}
\hline \multicolumn{8}{|c|}{ Lower Three Runs-2 } \\
\hline Parameter & Units & July & Aug & Sept & Oct & Nov & Dec \\
\hline Water volume & $\mathrm{L}$ & 4.52E09 & 4.15E09 & 4.84E09 & $4.40 \mathrm{EO9}$ & 5.37E09 & 5.34E09 \\
\hline Temperature & ${ }^{\circ} \mathrm{C}$ & 25.0 & 24.9 & 26.0 & 19.8 & 15.0 & 10.8 \\
\hline $\mathrm{pH}^{\mathrm{b}}$ & $\mathrm{pH}$ & 6.6 & 6.2 & 7.0 & 7.5 & 6.7 & 6.8 \\
\hline $\begin{array}{l}\text { Dissolved } \\
\text { oxygen b }^{\text {b }}\end{array}$ & $\mathrm{mg} / \mathrm{L}$ & 6.7 & 6.8 & 7.1 & 8.6 & 9.0 & 7.9 \\
\hline Alkalinity & $\mathrm{mg} / \mathrm{L}$ & 23 & 23 & 24 & 22 & 20 & 21 \\
\hline Conductivityb & $\mu \mathrm{mhos} / \mathrm{cm}$ & 67 & 86 & 65 & 81 & 82 & 63 \\
\hline Turbidity & NTU & 2.0 & 5.0 & 2.1 & 2.6 & 2.2 & 1.1 \\
\hline $\begin{array}{l}\text { Suspended } \\
\text { solids }\end{array}$ & $\mathrm{mg} / \mathrm{L}$ & 9 & 9 & 5 & 4 & 3 & 2 \\
\hline Volatile solids & $\mathrm{mg} / \mathrm{L}$ & 3 & 4 & 2 & 2 & $N D^{c}$ & 3 \\
\hline $\begin{array}{l}\text { Total dissolved } \\
\text { solids }\end{array}$ & $\mathrm{mg} / \mathrm{L}$ & 37 & 47 & 47 & 54 & 63 & 46 \\
\hline Total solids & $\mathrm{mg} / \mathrm{L}$ & 46 & 56 & 52 & 58 & 66 & 48 \\
\hline Fixed residue & $\mathrm{mg} / \mathrm{L}$ & 7 & 5 & 3 & 2 & 3 & ND \\
\hline $\begin{array}{l}\text { Chemical } \\
\text { oxygen demand }\end{array}$ & $\mathrm{mg} / \mathrm{L}$ & ND & 27 & ND & ND & ND & ND \\
\hline Chloride & $\mathrm{mg} / \mathrm{h}$ & 3 & 3 & 4 & 4 & 5 & 4 \\
\hline $\begin{array}{l}\text { Nitrogen (as } \\
\mathrm{NO}_{2} / \mathrm{NO}_{3} \text { ) }\end{array}$ & $\mathrm{mg} / \mathrm{L}$ & 0.14 & 0.13 & 0.10 & 0.10 & ND & 0.10 \\
\hline Sulfate & $\mathrm{mg} / \mathrm{L}$ & 2 & 2 & 3 & 3 & 3 & 4 \\
\hline Phosphate P & $\mathrm{mg} / \mathrm{L}$ & ND & ND & ND & ND & ND & ND \\
\hline Aluminum & $\mathrm{mg} / \mathrm{L}$ & & & ND & & & ND \\
\hline $\begin{array}{l}\text { Ammonia } \\
\text { nitrogen }\end{array}$ & $\mathrm{mg} / \mathrm{L}$ & 0.04 & 0.04 & ND & 0.02 & ND & 0.02 \\
\hline Calcium & $\mathrm{mg} / \mathrm{L}$ & & & 8.94 & & & 12.8 \\
\hline Copper & $\mathrm{mg} / \mathrm{L}$ & & & ND & & & ND \\
\hline Cadmium & $\mathrm{mg} / \mathrm{L}$ & & & ND & & & ND \\
\hline Magnesium & $\mathrm{mg} / \mathrm{L}$ & & & 0.741 & & & 0.79 \\
\hline Manganese & $\mathrm{mg} / \mathrm{L}$ & & & 0.024 & & & 0.013 \\
\hline Mercury & $\mu \mathrm{g} / \mathrm{L}$ & & & ND & & & ND \\
\hline Nickel & $\mathrm{mg} / \mathrm{L}$ & & & ND & & & ND \\
\hline Sodium & $\mathrm{mg} / \mathrm{L}$ & & & 2.98 & & & 2.88 \\
\hline Iron & $\mathrm{mg} / \mathrm{L}$ & & & 0.156 & & & 0.138 \\
\hline Lead & $\mathrm{mg} / \mathrm{L}$ & & & ND & & & ND \\
\hline Chromium & $\mathrm{mg} / \mathrm{L}$ & & & ND & & & ND \\
\hline Zinc & $\mathrm{mg} / \mathrm{L}$ & & & ND & & & ND \\
\hline
\end{tabular}

a Metals are analyzed quarterly from a monthly grab composite.

Field measurements

None detected 
Table 70

SRS Stream Water Quality

Page 13 of 21

\begin{tabular}{|c|c|c|c|c|c|c|c|}
\hline \multicolumn{8}{|c|}{ McQueens Branch } \\
\hline Parameter & Units & Jan & Feb & Mar & April & May & June \\
\hline Water volume & $\mathrm{L}$ & $2.63 E 08$ & 1.34E08 & $2.05 E 08$ & 1.22E08 & 9.34E07 & $5.51 E 07$ \\
\hline Temperatureb & ${ }^{\circ} \mathrm{C}$ & 11.4 & 12.1 & 12.0 & 17.7 & 18.3 & 23.0 \\
\hline $\mathrm{pH}^{\mathrm{b}}$ & $\mathrm{pH}$ & 5.6 & 6.0 & 6.9 & 6.2 & 6.4 & 6.5 \\
\hline $\begin{array}{l}\text { Dissolved } \\
\text { oxygen }\end{array}$ & $\mathrm{mg} / \mathrm{L}$ & 9.5 & 9.1 & 9.4 & 8.7 & 7.6 & 5.8 \\
\hline Conductivityb & $\mu \mathrm{mhos} / \mathrm{cm}$ & 37 & 5 & 45 & 47 & 46 & 40 \\
\hline Turbidity & NTU & 9.1 & 43 & 47 & 5.9 & 21 & 17 \\
\hline $\begin{array}{l}\text { Suspended sol- } \\
\text { ids }\end{array}$ & $\mathrm{mg} / \mathrm{L}$ & 7 & 10 & 22 & 2 & 11 & 82 \\
\hline
\end{tabular}

a Metals are analyzed quarterly from a monthly grab composite.

b Field measurements 
Table 70

SRS Stream Water Quality

Page 14 of 21

\begin{tabular}{|c|c|c|c|c|c|c|c|}
\hline \multicolumn{8}{|c|}{ McQueens Branch } \\
\hline Parameter & Units & July & Aug & Sept & Oot & Nov & Dec \\
\hline Water volume & $\mathrm{L}$ & 7.06E07 & 5.09E07 & 7.79E07 & 1.08E08 & 1.07E08 & $9.72 E 07$ \\
\hline Temperatureb & ${ }^{\circ} \mathrm{C}$ & 24.8 & 23.8 & 23.6 & 17.5 & 13.5 & 10.1 \\
\hline $\mathrm{pH}^{\mathrm{b}}$ & $\mathrm{pH}$ & 6.7 & 6.9 & 6.8 & 6.8 & 6.1 & 7.2 \\
\hline $\begin{array}{l}\text { Dissolved } \\
\text { oxygen }^{\mathrm{b}}\end{array}$ & mg/L & 6.4 & 7.7 & 6.8 & 8.1 & 9.4 & 9.8 \\
\hline Conductivity ${ }^{b}$ & $\mu \mathrm{mhos} / \mathrm{cm}$ & 68 & 55 & 45 & 48 & 50 & 45 \\
\hline Turbidity & NTU & 6.4 & 21 & 5.6 & 3.3 & 3.3 & 3.7 \\
\hline $\begin{array}{l}\text { Suspended sol- } \\
\text { ids }\end{array}$ & $\mathrm{mg} / \mathrm{L}$ & 12 & 7 & 2 & 1 & 2 & 1 \\
\hline
\end{tabular}

a Metals are analyzed quarterly from a monthly grab composite.

b Field measurements 
Table 70

SRS Stream Water Quality

Page 15 of 21

\begin{tabular}{|c|c|c|c|c|c|c|c|}
\hline $\begin{array}{l}\text { Pen Branch-3 } \\
\text { Parameter }\end{array}$ & Units & Jan & Feb & Mar & Aprll & May & June \\
\hline Water volume & $\mathrm{L}$ & $3.66 \mathrm{E} 09$ & $3.34 E 09$ & 5.42E09 & 3.67E09 & $3.40 E 09$ & 2.73E09 \\
\hline Temperature $^{b}$ & ${ }^{\circ} \mathrm{C}$ & 10.3 & 11.9 & 11.1 & 16.9 & 24.6 & 25.4 \\
\hline $\mathrm{pH}^{\mathrm{b}}$ & $\mathrm{pH}$ & 6.0 & 5.9 & 6.8 & 6.2 & 6.7 & 6.3 \\
\hline $\begin{array}{l}\text { Dissolved } \\
\text { oxygen b }\end{array}$ & $\mathrm{mg} / \mathrm{L}$ & 9.2 & 9.5 & 10.6 & 9.7 & 6.3 & 7.4 \\
\hline Alkalinity & $\mathrm{mg} / \mathrm{L}$ & 10 & 11 & 13 & 15 & 15 & 18 \\
\hline Conductivityb & $\mu \mathrm{mhos} / \mathrm{cm}$ & 47 & 52 & 69 & 69.4 & 63 & 70 \\
\hline Turbidity & NTU & 8.3 & 6.4 & 12 & 10 & 12 & 7.0 \\
\hline $\begin{array}{l}\text { Suspended } \\
\text { solids }\end{array}$ & $\mathrm{mg} / \mathrm{L}$ & 9 & 12 & 9 & 4 & 8 & 6 \\
\hline Volatile solids & $\mathrm{mg} / \mathrm{L}$ & 1 & 2 & 2 & 3 & 2 & 2 \\
\hline $\begin{array}{l}\text { Total dissolved } \\
\text { solids }\end{array}$ & $\mathrm{mg} / \mathrm{L}$ & 51 & 47 & 42 & 79 & 55 & 59 \\
\hline Total solids & $\mathrm{mg} / \mathrm{L}$ & 60 & 59 & 51 & 83 & 63 & 65 \\
\hline Fixed residue & $\mathrm{mg} / \mathrm{L}$ & 8 & 8 & 7 & 2 & 6 & 4 \\
\hline $\begin{array}{l}\text { Chemical } \\
\text { oxygen demand }\end{array}$ & $\mathrm{mg} / \mathrm{L}$ & $N D^{c}$ & ND & ND & ND & ND & ND \\
\hline Chloride & $\mathrm{mg} / \mathrm{L}$ & 4 & 3 & 4 & 4 & 6 & 5 \\
\hline $\begin{array}{l}\text { Nitrogen (as } \\
\mathrm{NO}_{2} / \mathrm{NO}_{3} \text { ) }\end{array}$ & $\mathrm{mg} / \mathrm{L}$ & 0.16 & 0.13 & 0.15 & 0.17 & 0.25 & 0.26 \\
\hline Sulfate & $\mathrm{mg} / \mathrm{L}$ & 5 & 4 & 4 & 4 & 4 & 5 \\
\hline Phosphate P & $\mathrm{mg} / \mathrm{L}$ & 0.04 & 0.04 & 0.03 & ND & ND & ND \\
\hline Aluminum & $\mathrm{mg} / \mathrm{L}$ & & & 0.298 & & & 0.231 \\
\hline $\begin{array}{l}\text { Ammonia } \\
\text { nitrogen }\end{array}$ & $\mathrm{mg} / \mathrm{L}$ & 0.03 & 0.09 & 0.02 & 0.03 & 0.04 & 0.02 \\
\hline Calcium & $\mathrm{mg} / \mathrm{L}$ & & & 0.976 & & & 4.15 \\
\hline Copper & $\mathrm{mg} / \mathrm{L}$ & & & 0.05 & & & 0.054 \\
\hline Cadmium & $\mathrm{mg} / \mathrm{L}$ & & & ND & & & ND \\
\hline Magnesium & $\mathrm{mg} / \mathrm{L}$ & & & 0.710 & & & 0.823 \\
\hline Manganese & $\mathrm{mg} / \mathrm{L}$ & & & 0.038 & & & 0.041 \\
\hline Mercury & $\mu g / L$ & & & ND & & & ND \\
\hline Nickel & $\mathrm{mg} / \mathrm{L}$ & & & ND & & & ND \\
\hline Sodium & $\mathrm{mg} / \mathrm{L}$ & & & 3.49 & & & 5.05 \\
\hline Iron & $\mathrm{mg} / \mathrm{L}$ & & & 0.705 & & & 0.704 \\
\hline Lead & $\mathrm{mg} / \mathrm{L}$ & & & 0.002 & & & ND \\
\hline Chromium & $\mathrm{mg} / \mathrm{L}$ & & & ND & & & ND \\
\hline Zinc & $\mathrm{mg} / \mathrm{L}$ & & & ND & & & ND \\
\hline
\end{tabular}

a Metals are analyzed quarterly from a monthly grab composite.
b Field measurements

c None detected 
Table 70

SRS Stream Water Quality

Page 16 of 21

\begin{tabular}{|c|c|c|c|c|c|c|c|}
\hline $\begin{array}{l}\text { Pen Branch-3 } \\
\text { Parametor }\end{array}$ & Units & July & Aug & Sopt & Oot & Nov & Deo \\
\hline Water volume & $L$ & $2.44 E 09$ & $2.56 E 09$ & $3.09 \mathrm{E} 09$ & 3.41 EO & 3.73E09 & 3.71EOS \\
\hline Temperature & ${ }^{\circ} \mathrm{C}$ & 25.5 & 26.5 & 26.2 & 19.1 & 14.5 & 12.2 \\
\hline $\mathrm{pH}^{\mathrm{b}}$ & $\mathrm{pH}$ & 6.6 & 6.5 & 7.8 & 7.4 & 6.7 & 7.4 \\
\hline $\begin{array}{l}\text { Dissolved } \\
\text { oxygenb }\end{array}$ & $\mathrm{mg} / \mathrm{L}$ & 8.1 & 8.7 & 7.9 & 9.4 & 10.2 & 10.5 \\
\hline Alkalinity & $\mathrm{mg} / \mathrm{L}$ & 18 & 19 & 17 & 17 & 15 & 15 \\
\hline Conductivityb & $\mu \mathrm{mhos} / \mathrm{cm}$ & 81 & 88 & 85 & 85 & 89 & 79 \\
\hline Turbidity & NTU & 5.4 & 4.3 & 4.1 & 4.0 & 6.4 & 3.7 \\
\hline $\begin{array}{l}\text { Suspended } \\
\text { solids }\end{array}$ & $\mathrm{mg} / \mathrm{L}$ & 6 & 2 & 3 & 4 & 5 & 4 \\
\hline Volatile solids & $\mathrm{mg} / \mathrm{L}$ & 1 & 1 & 2 & 1 & $N D^{c}$ & 3 \\
\hline $\begin{array}{l}\text { Total dissolved } \\
\text { solids }\end{array}$ & $\mathrm{mg} / \mathrm{L}$ & 45 & 63 & 59 & 56 & 72 & 52 \\
\hline Total solids & $\mathrm{mg} / \mathrm{L}$ & 51 & 65 & 62 & 60 & 77 & 56 \\
\hline Fixed residue & $\mathrm{mg} / \mathrm{L}$ & 5 & 2 & 2 & 3 & 5 & 1 \\
\hline $\begin{array}{l}\text { Chemical } \\
\text { oxygen demand }\end{array}$ & $\mathrm{mg} / \mathrm{L}$ & ND & ND & ND & ND & ND & ND \\
\hline Chloride & $\mathrm{mg} / \mathrm{L}$ & 6 & 7 & 9 & 10 & 8 & 7 \\
\hline $\begin{array}{l}\text { Nitrogen (as } \\
\mathrm{NO}_{2} / \mathrm{NO}_{3} \text { ) }\end{array}$ & $\mathrm{mg} / \mathrm{L}$ & 0.23 & 0.22 & 0.20 & 0.24 & 0.23 & 0.33 \\
\hline Sulfate & $\mathrm{mg} / \mathrm{L}$ & 5 & 6 & 6 & 6 & 7 & 7 \\
\hline Phosphate P & $\mathrm{mg} / \mathrm{L}$ & ND & ND & ND & ND & ND & ND \\
\hline Aluminum & $\mathrm{mg} / \mathrm{L}$ & & & 0.398 & & & 0.096 \\
\hline $\begin{array}{l}\text { Ammoni } \\
\text { nitrogen }\end{array}$ & $\mathrm{mg} / \mathrm{L}$ & 0.02 & 0.03 & ND & ND & 0.02 & 0.03 \\
\hline Calcium & $\mathrm{mg} / \mathrm{L}$ & & & 4.79 & & & 5.03 \\
\hline Copper & $\mathrm{mg} / \mathrm{L}$ & & & 0.041 & & & 0.098 \\
\hline Cadmium & $\mathrm{mg} / \mathrm{L}$ & & & ND & & & ND \\
\hline Magnesium & $\mathrm{mg} / \mathrm{L}$ & & & 0.996 & & & 1.08 \\
\hline Manganese & $\mathrm{mg} / \mathrm{L}$ & & & 0.096 & & & 0.040 \\
\hline Mercury & $\mu \mathrm{g} / \mathrm{L}$ & & & ND & & & ND \\
\hline Nickel & $\mathrm{mg} / \mathrm{L}$ & & & ND & & & ND \\
\hline Sodium & $\mathrm{mg} / \mathrm{L}$ & & & 8.96 & & & 9.35 \\
\hline Iron & $\mathrm{mg} / \mathrm{L}$ & & & 0.786 & & & 0.361 \\
\hline Lead & $\mathrm{mg} / \mathrm{L}$ & & & ND & & & ND \\
\hline Chromium & $\mathrm{mg} / \mathrm{L}$ & & & ND & & & ND \\
\hline Zinc & $\mathrm{mg} / \mathrm{L}$ & & & 0.012 & & & ND \\
\hline
\end{tabular}

a Metals are analyzed quarterly from a monthly grab composite.

b Field measurements

c None detected 
Table 70

SRS Stream Water Quality

Page 17 of 21

\begin{tabular}{|c|c|c|c|c|c|c|c|}
\hline \multicolumn{8}{|c|}{ Four Mile Creek-6 } \\
\hline Parameter & Unlte & Jan & Feb & Mar & April & May & June \\
\hline Water volume & $L$ & 5.45E09 & 6.63E09 & T.26E10 & $3.28=09$ & 2.07E09 & 3.57E09 \\
\hline Temperatureb & ${ }^{\circ} \mathrm{C}$ & 10.4 & 14.0 & 10.8 & 18.6 & 22.5 & 26.0 \\
\hline $\mathrm{pH}^{\mathrm{b}}$ & $\mathrm{pH}$ & 5.7 & 5.9 & 6.5 & 5.8 & 6.6 & 6.4 \\
\hline $\begin{array}{l}\text { Dissolved } \\
\text { oxygen }\end{array}$ & $\mathrm{mg} / \mathrm{L}$ & 9.4 & 9.0 & 10.1 & 9.7 & 8.3 & 7.2 \\
\hline Alkalinity & $\mathrm{mg} / \mathrm{L}$ & 7 & 9 & 9 & 10 & 9 & 13 \\
\hline Conductivity ${ }^{b}$ & $\mu \mathrm{mhos} / \mathrm{cm}$ & 50 & 60 & 30 & 50 & 54 & 60 \\
\hline Turbidity & NTU & 7.2 & 5.8 & 10 & 5.0 & 8.9 & 4.9 \\
\hline $\begin{array}{l}\text { Suspended } \\
\text { solids }\end{array}$ & $\mathrm{mg} / \mathrm{L}$ & 5 & 7 & 6 & 1 & 12 & 4 \\
\hline Volatile solids & $\mathrm{mg} / \mathrm{L}$ & 2 & 2 & 2 & $N D^{c}$ & 3 & 1 \\
\hline $\begin{array}{l}\text { Total dissolved } \\
\text { solids }\end{array}$ & $\mathrm{mg} / \mathrm{L}$ & 46 & 43 & 39 & 66 & 40 & 49 \\
\hline Total solids & $\mathrm{mg} / \mathrm{L}$ & 51 & 50 & 45 & 67 & 52 & 53 \\
\hline Fixed residue & $\mathrm{mg} / \mathrm{L}$ & 4 & 5 & 4 & 1 & 9 & 3 \\
\hline $\begin{array}{l}\text { Chemical } \\
\text { oxygen demand }\end{array}$ & $\mathrm{mg} / \mathrm{L}$ & ND & ND & ND & ND & ND & ND \\
\hline Chloride & $\mathrm{mg} / \mathrm{L}$ & 3 & 3 & 3 & 3 & 3 & 3 \\
\hline $\begin{array}{l}\text { Nitrogen (as } \\
\mathrm{NO}_{2} / \mathrm{NO}_{3} \text { ) }\end{array}$ & $\mathrm{mg} / \mathrm{L}$ & 0.89 & 0.79 & 0.38 & 0.69 & 1.00 & 0.84 \\
\hline Sulfate & $\mathrm{mg} / \mathrm{L}$ & 6 & 6 & 4 & 4 & 4 & 3 \\
\hline Phosphate P & $\mathrm{mg} / \mathrm{L}$ & ND & ND & ND & ND & ND & ND \\
\hline Aluminum & $\mathrm{mg} / \mathrm{L}$ & & & 0.253 & & & 0.143 \\
\hline $\begin{array}{l}\text { Ammonia } \\
\text { nitrogen }\end{array}$ & $\mathrm{mg} / \mathrm{L}$ & 0.04 & 0.04 & 0.02 & 0.04 & 0.04 & 0.04 \\
\hline Calcium & $\mathrm{mg} / \mathrm{L}$ & & & 2.74 & & & 2.82 \\
\hline Copper & $\mathrm{mg} / \mathrm{L}$ & & & ND & & & ND \\
\hline Cadmium & $\mathrm{mg} / \mathrm{L}$ & & & ND & & & ND \\
\hline Magnesium & $\mathrm{mg} / \mathrm{L}$ & & & 0.752 & & & 0.617 \\
\hline Manganese & $\mathrm{mg} / \mathrm{L}$ & & & 0.036 & & & ND \\
\hline Mercury & $\mu \mathrm{g} / \mathrm{L}$ & & & ND & & & ND \\
\hline Nickel & $\mathrm{mg} / \mathrm{L}$ & & & ND & & & ND \\
\hline Sodium & $\mathrm{mg} / \mathrm{L}$ & & & 4.86 & & & 5.01 \\
\hline Iron & $\mathrm{mg} / \mathrm{L}$ & & & 0.696 & & & 0.566 \\
\hline Lead & $\mathrm{mg} / \mathrm{L}$ & & & 0.002 & & & ND \\
\hline Chromium & $\mathrm{mg} / \mathrm{L}$ & & & ND & & & ND \\
\hline Zinc & $\mathrm{mg} / \mathrm{L}$ & & & 0.011 & & & ND \\
\hline
\end{tabular}

a Metals are analyzed quarterly from a monthly grab composite.

Field measurements

None detected 
Table 70

SRS Stream Water Quality

Page 18 of 21

\begin{tabular}{|c|c|c|c|c|c|c|c|}
\hline \multicolumn{8}{|c|}{ Four Mile Creak-0 } \\
\hline Paramotor & Unite & July & Aug & Sopt & Oot & Nov & Dec \\
\hline Water volume & $\tau$ & $2.64 E 09$ & 2.00E09 & $3.09=09$ & $2.39 \mathrm{EOg}$ & 2.47EO9 & $2.54 E 09$ \\
\hline Temperatureb & ${ }^{\circ} \mathrm{C}$ & 25.0 & 24.5 & 26.6 & 17.6 & 13.5 & 10.8 \\
\hline $\mathrm{pH}^{\mathrm{b}}$ & $\mathrm{pH}$ & 6.5 & 6.6 & 6.9 & 7.0 & 6.4 & 7.3 \\
\hline $\begin{array}{l}\text { Dissolved } \\
\text { oxygen }^{b}\end{array}$ & $\mathrm{mg} / \mathrm{L}$ & 7.0 & 7.9 & 7.8 & 10.4 & 9.8 & 10.8 \\
\hline Alkalinity & $\mathrm{mg} / \mathrm{L}$ & 16 & 15 & 13 & 9 & 11 & 12 \\
\hline Conductivityb & $\mu \mathrm{mhos} / \mathrm{cm}$ & 65 & 67 & 65 & 60 & 68 & 73 \\
\hline Turbidity & NTU & 3.0 & 3.6 & 2.0 & 1.8 & 1.8 & 2.3 \\
\hline $\begin{array}{l}\text { Suspended } \\
\text { solids }\end{array}$ & $\mathrm{mg} / \mathrm{L}$ & 2 & 2 & 1 & $N^{c}$ & 2 & 2 \\
\hline Volatile solids & $\mathrm{mg} / \mathrm{L}$ & 1 & ND & 2 & ND & ND & 2 \\
\hline $\begin{array}{l}\text { Total dissolved } \\
\text { solids }\end{array}$ & $\mathrm{mg} / \mathrm{L}$ & 37 & 55 & 48 & 41 & 65 & 50 \\
\hline Total solids & $\mathrm{mg} / \mathrm{L}$ & 39 & 57 & 49 & 41 & 67 & 52 \\
\hline Fixed residue & $\mathrm{mg} / \mathrm{L}$ & 2 & 1 & ND & ND & 2 & ND \\
\hline $\begin{array}{l}\text { Chemical } \\
\text { oxygen demand }\end{array}$ & $\mathrm{mg} / \mathrm{L}$ & ND & ND & ND & ND & ND & ND \\
\hline Chloride & $\mathrm{mg} / \mathrm{L}$ & 4 & 4 & 6 & 4 & 6 & 5 \\
\hline $\begin{array}{l}\text { Nitrogen (as } \\
\mathrm{NO}_{2} / \mathrm{NO}_{3} \text { ) }\end{array}$ & $\mathrm{mg} / \mathrm{L}$ & 0.49 & 0.69 & 0.54 & 0.97 & 1.09 & 1.31 \\
\hline Sulfate & $\mathrm{mg} / \mathrm{L}$ & 4 & 5 & 5 & 5 & 6 & 7 \\
\hline Phosphate P & $\mathrm{mg} / \mathrm{L}$ & ND & ND & ND & ND & ND & ND \\
\hline Aluminum & $\mathrm{mg} / \mathrm{L}$ & & & ND & & & ND \\
\hline $\begin{array}{l}\text { Ammoni } \\
\text { nitrogen }\end{array}$ & $\mathrm{mg} / \mathrm{L}$ & 0.03 & 0.04 & ND & ND & 0.02 & 0.02 \\
\hline Calcium & $\mathrm{mg} / \mathrm{L}$ & & & 3.31 & & & 3.56 \\
\hline Copper & $\mathrm{mg} / \mathrm{L}$ & & & ND & & & ND \\
\hline Cadmium & $\mathrm{mg} / \mathrm{L}$ & & & ND & & & ND \\
\hline Magnesium & $\mathrm{mg} / \mathrm{L}$ & & & 0.634 & & & 0.724 \\
\hline Manganese & $\mathrm{mg} / \mathrm{L}$ & & & 0.024 & & & 0.018 \\
\hline Mercury & $\mu g / L$ & & & ND & & & ND \\
\hline Nickel & $\mathrm{mg} / \mathrm{L}$ & & & ND & & & ND \\
\hline Sodium & $\mathrm{mg} / \mathrm{L}$ & & & 7.52 & & & 7.62 \\
\hline Iron & $\mathrm{mg} / \mathrm{L}$ & & & 0.289 & & & 0.314 \\
\hline Lead & $\mathrm{mg} / \mathrm{L}$ & & & ND & & & ND \\
\hline Chromium & $\mathrm{mg} / \mathrm{L}$ & & & ND & & & ND \\
\hline Zinc & $\mathrm{mg} / \mathrm{L}$ & & & ND & & & ND \\
\hline
\end{tabular}

Metals are analyzed quarterly from a monthly grab composite.

Field measurements

None delected 
Table 70

SR8 Stream Water Quality

Page 19 of 21

\begin{tabular}{|c|c|c|c|c|c|c|c|}
\hline \multicolumn{8}{|c|}{ Upper Three Rune at 1-A } \\
\hline Paramoton & Unlte & Jan & Fob & Mar & Aprll & May & June \\
\hline Water volume & $\tau$ & T.01E10 & $8.16 E 09$ & $9.95 \mathrm{E} 09$ & 9.11EOS & $8.28 \mathrm{EO}$ & 7.42EOg \\
\hline Temperatureb & ${ }^{\circ} \mathrm{C}$ & 12.0 & 13.3 & 13.4 & 18.1 & 18.5 & 20.8 \\
\hline $\mathrm{pH}^{\mathrm{b}}$ & $\mathrm{pH}$ & 6.2 & 5.7 & 6.6 & 6.5 & 5.3 & 6.3 \\
\hline $\begin{array}{l}\text { Dissolved } \\
\text { oxygen b }\end{array}$ & $\mathrm{mg} / \mathrm{L}$ & 9.3 & 9.6 & 8.4 & 9.3 & 7.3 & 7.3 \\
\hline Alkalinity & $\mathrm{mg} / \mathrm{L}$ & 1 & 2 & 1 & 2 & 1 & 2 \\
\hline Conductivityb & $\mu \mathrm{mhos} / \mathrm{cm}$ & 17 & 23 & 17 & 24 & 21 & 30 \\
\hline Turbidity & NTU & 5.6 & 2.7 & 2.6 & 2.6 & 7.4 & 4.8 \\
\hline $\begin{array}{l}\text { Suspended } \\
\text { solids }\end{array}$ & $\mathrm{mg} / \mathrm{L}$ & 5 & 6 & 3 & 1 & 11 & 8 \\
\hline Volatile solids & $\mathrm{mg} / \mathrm{L}$ & 2 & 3 & 1 & 1 & 5 & 2 \\
\hline $\begin{array}{l}\text { Total dissolved } \\
\text { solids }\end{array}$ & $\mathrm{mg} / \mathrm{L}$ & 23 & 26 & 20 & 46 & 26 & 20 \\
\hline Total solids & $\mathrm{mg} / \mathrm{L}$ & 28 & 32 & 23 & 47 & 37 & 28 \\
\hline Fixed residue & $\mathrm{mg} / \mathrm{L}$ & 4 & 3 & 2 & $N^{c}$ & 7 & 6 \\
\hline $\begin{array}{l}\text { Chemical } \\
\text { oxygen dema.1d }\end{array}$ & $\mathrm{mg} / \mathrm{L}$ & ND & ND & ND & ND & ND & ND \\
\hline Chloride & $\mathrm{mg} / \mathrm{L}$ & 2 & 2 & 2 & 2 & 2 & 2 \\
\hline $\begin{array}{l}\text { Nitrogen (as } \\
\mathrm{NO}_{2} / \mathrm{NO}_{3} \text { ) }\end{array}$ & $\mathrm{mg} / \mathrm{L}$ & 0.19 & 0.23 & 0.20 & 0.20 & 0.15 & 0.21 \\
\hline Sulfate & $\mathrm{mg} / \mathrm{L}$ & 1 & 1 & 1 & 1 & 1 & 1 \\
\hline Phosphate P & $\mathrm{mg} / \mathrm{L}$ & ND & ND & ND & ND & ND & ND \\
\hline Aluminum & $\mathrm{mg} / \mathrm{L}$ & & & 0.137 & & & 0.121 \\
\hline $\begin{array}{l}\text { Ammoni } \\
\text { nitrogen }\end{array}$ & $\mathrm{mg} / \mathrm{L}$ & 0.04 & 0.03 & 0.02 & 0.02 & 0.02 & 0.01 \\
\hline Calcium & $\mathrm{mg} / \mathrm{L}$ & & & 0.905 & & & 0.416 \\
\hline Copper & $\mathrm{mg} / \mathrm{L}$ & & & ND & & & ND \\
\hline Cadmium & $\mathrm{mg} / \mathrm{L}$ & & & ND & & & ND \\
\hline Magnesium & $\mathrm{mg} / \mathrm{L}$ & & & 0.349 & & & 0.278 \\
\hline Manganese & $\mathrm{mg} / \mathrm{L}$ & & & 0.123 & & & ND \\
\hline Mercury & $\mu \mathrm{g} / \mathrm{L}$ & & & ND & & & ND \\
\hline Nickel & $\mathrm{mg} / \mathrm{L}$ & & & ND & & & ND \\
\hline Sodium & $\mathrm{mg} / \mathrm{L}$ & & & 1.51 & & & 1.34 \\
\hline Iron & $\mathrm{mg} / \mathrm{L}$ & & & 0.328 & & & 0.336 \\
\hline Lead & $\mathrm{mg} / \mathrm{L}$ & & & 0.003 & & & ND \\
\hline Chromium & $\mathrm{mg} / \mathrm{L}$ & & & ND & & & ND \\
\hline Zinc & $\mathrm{mg} / \mathrm{L}$ & & & ND & & & ND \\
\hline
\end{tabular}


Table 70

SRS Stream Water Quality

Page 20 of 21

\begin{tabular}{|c|c|c|c|c|c|c|c|}
\hline \multicolumn{8}{|c|}{ Upper Three Rune at 1-A } \\
\hline Parametor & Unite & July & Aug & Eopt & Oot & Nov & Dec \\
\hline Water volume & $\mathrm{L}$ & 7.38E09 & 7.15E09 & $7.72=09$ & 7.75E09 & 7.866408 & 8.13E09 \\
\hline Temperatureb & ${ }^{\circ} \mathrm{C}$ & 24.0 & 21.0 & 21.5 & 17.7 & 16.8 & 11.8 \\
\hline $\mathrm{pH}^{\mathrm{b}}$ & pH & 6.8 & 7.6 & 6.8 & 7.4 & 6.5 & 7.4 \\
\hline $\begin{array}{l}\text { Dissolve } \\
\text { oxygen }\end{array}$ & $\mathrm{mg} / \mathrm{L}$ & 8.1 & 7.8 & 7.8 & 9.4 & 9.8 & 9.5 \\
\hline Alkalinity & $\mathrm{mg} / \mathrm{L}$ & 2 & 1 & 2 & 1 & 2 & 2 \\
\hline Conductivity ${ }^{b}$ & $\mu \mathrm{mhos} / \mathrm{cm}$ & 17 & 22 & 17 & 17 & 20 & 20 \\
\hline Turbidity & NTU & 5.3 & 2.5 & 1.8 & 1.8 & 1.8 & 1.8 \\
\hline $\begin{array}{l}\text { Suspended } \\
\text { solids }\end{array}$ & $\mathrm{mg} / \mathrm{L}$ & 3 & 5 & 4 & 2 & 3 & 2 \\
\hline Volatile solids & $\mathrm{mg} / \mathrm{L}$ & 1 & 3 & 2 & 1 & $N D^{c}$ & 2 \\
\hline $\begin{array}{l}\text { Total dissolved } \\
\text { solids }\end{array}$ & $\mathrm{mg} / \mathrm{L}$ & 13 & 23 & 16 & 15 & 33 & 15 \\
\hline Total solids & $\mathrm{mg} / \mathrm{L}$ & 16 & 28 & 20 & 17 & 36 & 17 \\
\hline Fixed residue & $\mathrm{mg} / \mathrm{L}$ & 2 & 2 & 2 & 1 & 3 & ND \\
\hline $\begin{array}{l}\text { Chemical } \\
\text { oxygen demand }\end{array}$ & $\mathrm{mg} / \mathrm{L}$ & ND & ND & ND & ND & ND & ND \\
\hline Chloride & $\mathrm{mg} / \mathrm{L}$ & 3 & 2 & 2 & 2 & 2 & 2 \\
\hline $\begin{array}{l}\text { Nitrogen (a: } \\
\mathrm{NO}_{2} / \mathrm{NO}_{3} \text { ) }\end{array}$ & $. n g / L$ & 0.22 & 0.17 & 0.17 & 0.21 & 0.19 & 0.29 \\
\hline Sulfate & $\mathrm{mg} / \mathrm{L}$ & 1 & 1 & 1 & 1 & 1 & 1 \\
\hline Phosphate P & $\mathrm{mg} / \mathrm{L}$ & ND & ND & ND & ND & ND & ND \\
\hline Aluminum & $\mathrm{mg} / \mathrm{L}$ & & & 0.091 & & & 0.110 \\
\hline $\begin{array}{l}\text { Ammoni } \\
\text { nitrogen }\end{array}$ & $\mathrm{mg} / \mathrm{L}$ & 0.02 & 0.02 & ND & ND & ND & 0.02 \\
\hline Calcium & $\mathrm{mg} / \mathrm{L}$ & & & 0.412 & & & 0.440 \\
\hline Copper & $\mathrm{mg} / \mathrm{L}$ & & & ND & & & ND \\
\hline Cadmium & $\mathrm{mg} / \mathrm{L}$ & & & ND & & & ND \\
\hline Magnesium & $\mathrm{mg} / \mathrm{L}$ & & & 0.301 & & & 0.331 \\
\hline Manganese & $\mathrm{mg} / \mathrm{L}$ & & & 0.006 & & & 0.005 \\
\hline Mercury & $\mu g / L$ & & & ND & & & ND \\
\hline Nickel & $\mathrm{mg} / \mathrm{L}$ & & & ND & & & ND \\
\hline Sodium & $\mathrm{mg} / \mathrm{L}$ & & & 1.41 & & & 1.34 \\
\hline Iron & $\mathrm{mg} / \mathrm{L}$ & & & 0.258 & & & 0.197 \\
\hline Lead & $\mathrm{mg} / \mathrm{L}$ & & & ND & & & ND \\
\hline Chromium & $\mathrm{mg} / \mathrm{L}$ & & & ND & & & ND \\
\hline Zinc & $\mathrm{mg} / \mathrm{h}$ & & & 0.017 & & & ND \\
\hline
\end{tabular}

a Metals are analyzed quarterly from a monthly grab composite.

b Field measurements

c None detected 
Table 70

SRS Stream Water Quallty

Pege 21 of 21

\section{SR8 Stream Water Quality: Herbicides and Volatile Organics}

EMs eamples from the following SCDHEC locatione were analyzed monthly for the conetituente lieted below. Herbloldee and volatile organloe wore not doteoted in any of the analyees.

\author{
Looatlone \\ Tims Branch-5 \\ Four Mile Creek-A7 \\ Steel Creek-4 \\ Upper Three Runs Creek-4
}

\author{
Horbloldee \\ Aldrin \\ Alpha BHC \\ Beta BHC \\ Lindane \\ alpha-Chlordane \\ gamma-Chlordane \\ tech-Chlordane \\ 4,4'-DDD \\ 4,4'-DDE \\ 4,4'-DDT \\ Dieldrin \\ Endosulfan I \\ Endosulfan II \\ Endosulfan Sulfate \\ Endrin \\ Endrin Aldehyde \\ Heptachlor \\ Heptachlor Epoxide \\ Methoxychlor \\ Toxaphene \\ 2, 4-D \\ 2, 4, 5-TP (Silvex) \\ Volatile Organice \\ 111-Trichloroethane \\ Trichloroethene \\ Tetrachloroethene
}




\section{Table 71}

Pesticides and Herblcides in Stream and Rlver Water and Sediment

\section{Page 1 of 1}

amples from the following locutione were analyzed annually for the conetituente lleted below. All analyees (resulte reported by auboontraoted off-elte laboratory, shealy Environmental serviose, Ino., wore below the doteotlon limite.

\begin{tabular}{|c|c|c|c|}
\hline Looatlons & Peotloldes & $\begin{array}{l}\text { Wator } \\
\text { Lower Limit of Dotection } \\
(L L D)-\mu Q / L\end{array}$ & 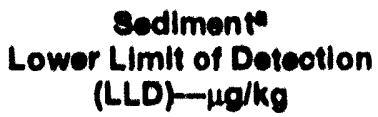 \\
\hline Steel Creek-4 & Aldrin & $<0.05$ & 6.00 \\
\hline Par Pond Pumphouse & Alpha-BHC & $<0.05$ & 6.00 \\
\hline Lower Three Runs-3 & Beta-BHC & $<0.05$ & 4.00 \\
\hline Upper Three Runs at Road A & Delta-BHC & $<0.05$ & 4.00 \\
\hline Four Mile Creak at Road A & Lindane & $<0.05$ & 4.00 \\
\hline Pen Branch at Road A & Chlordane & 1.0 & 33.0 \\
\hline Upper Three Runs at Road F & 4,4-DDD & $<0.05$ & 8.4 \\
\hline River 2 & 4,4-DDE & $<0.05$ & 12.7 \\
\hline \multirow[t]{15}{*}{ River 10} & 4,4-DDr & $<0.05$ & 8.4 \\
\hline & Dieldrif & $<0.05$ & 4.0 \\
\hline & Endosulfan I & $<0.05$ & 6.0 \\
\hline & Endosulfan II & $<0.05$ & 8.4 \\
\hline & Endosulfan sulfate & $<0.05$ & 6.0 \\
\hline & Endrin & $<0.05$ & 8.4 \\
\hline & Endrin aldehyde & $<0.05$ & 6.0 \\
\hline & Endrin ketone & $<0.05$ & 14.7 \\
\hline & Heptachlor & $<0.05$ & 4.0 \\
\hline & Heptachlor epoxide & $<0.05$ & 4.0 \\
\hline & Methoxychlor & $<0.20$ & 27.4 \\
\hline & Toxaphene & $<5.0$ & 167 \\
\hline & \multicolumn{3}{|l|}{ Herbioldes } \\
\hline & $2,4-D$ & $<0.10$ & 21 \\
\hline & 2,4,5-TP (Silvex) & $<0.10$ & 10 \\
\hline
\end{tabular}

a Samples were taken August 6, 1993. 
Table 72

Fecal Collírm Bacterla in SRS Streams and Savannah Rlver

Page 1 of 6

\begin{tabular}{|c|c|c|c|c|c|c|}
\hline Looation & & Colonlea/ $100 \mathrm{~mL}$ & & * of Semples & $\begin{array}{l}\text { Number of } \\
\text { Exceedanoese }\end{array}$ & $\begin{array}{l}\text { Period } \\
\text { of Time }\end{array}$ \\
\hline & JAN MIN & JAN MAX & GEO MEAN & & & \\
\hline RIVER-2 & 28 & 127 & 47 & 4 & 0 & $1 / 4-1 / 26$ \\
\hline RIVER-3B & 28 & 78 & 43 & 4 & 0 & $1 / 4-1 / 26$ \\
\hline RIVER-10 & 41 & 175 & 75 & 4 & 0 & $1 / 4-1 / 26$ \\
\hline U3R-1A & $<2$ & 280 & 48 & 4 & 0 & $1 / 4-1 / 26$ \\
\hline U3R-4 & 74 & 174 & 103 & 4 & 0 & $1 / 4-1 / 26$ \\
\hline FMC-6 & 56 & 230 & 102 & 4 & 0 & $1 / 4-1 / 26$ \\
\hline PB-3 & 42 & 148 & 73 & 4 & 0 & $1 / 4-1 / 26$ \\
\hline BDC & 2 & 125 & 16 & 4 & 0 & $1 / 4-1 / 26$ \\
\hline L3R-2 & 108 & 400 & 179 & 4 & 0 & $1 / 4-1 / 26$ \\
\hline L3R-TCR ${ }^{b}$ & 94 & 320 & 191 & 3 & 0 & $1 / 4-1 / 26$ \\
\hline $5 C-4^{\circ}$ & 10 & 10 & 10 & 1 & 0 & $1 / 11$ \\
\hline TB- $5^{\mathrm{C}}$ & 110 & 110 & 110 & 1 & 0 & $1 / 11$ \\
\hline$F M-A 7^{C}$ & 42 & 42 & 42 & 1 & 0 & $1 / 11$ \\
\hline U $3 R-4^{c}$ & 220 & 220 & 220 & 1 & 1 & $1 / 11$ \\
\hline Location & \multicolumn{3}{|c|}{ Coloniea/100mL } & \# of Samples & $\begin{array}{l}\text { Number of } \\
\text { Excoedances }\end{array}$ & $\begin{array}{l}\text { Period } \\
\text { of Time }\end{array}$ \\
\hline & FEB MIN & FEB MAX & GEO MEAN" & & & \\
\hline RIVER-2 & 26 & 270 & 87 & 4 & 0 & $2 / 1-2 / 23$ \\
\hline RIVER-3B & 26 & 118 & 53 & 4 & 0 & $2 / 1-2 / 23$ \\
\hline RIVER-10 & 38 & 68 & 49 & 4 & 2 & $2 / 1-2 / 23$ \\
\hline U3R-1A & 106 & 691 & 288 & 4 & 0 & $2 / 1-2 / 23$ \\
\hline U3R-4 & 118 & 280 & 186 & 4 & 0 & $2 / 1-2 / 23$ \\
\hline FMC-6 & 50 & 94 & 66 & 4 & 0 & $2 / 1-2 / 23$ \\
\hline PB-3 & 50 & 86 & 67 & 4 & 0 & $2 / 1-2 / 23$ \\
\hline BDC & 7 & 62 & 21 & 4 & 0 & $2 / 1-2 / 23$ \\
\hline L3R-2 & 104 & 330 & 167 & 4 & 0 & $2 / 1-2 / 23$ \\
\hline $5 C-4^{c}$ & 42 & 42 & 42 & 1 & 0 & $2 / 10$ \\
\hline TB-5C & 84 & 84 & 84 & 1 & 0 & $2 / 10$ \\
\hline$F M-A 7^{C}$ & 39 & 39 & 39 & 1 & 0 & $2 / 10$ \\
\hline U3R-4c & 100 & 100 & 100 & 1 & 0 & $2 / 10$ \\
\hline
\end{tabular}

a The standard for South Carolina states that the fecal coliform count should not exceed a geometric mean of $200 / 100 \mathrm{~mL}$, based on five consecutive samples during any 30 -day period, nor shall more than 10 percent of the total samples during any 30 -day period exceed $400 / 100 \mathrm{mg} / \mathrm{L}$.

b L3R-TCR sample location discontinued beginning February 1993

c SCDHEC sample location-one sample collected monthly 
Table 72

Fecal Collform Bacterla In SRS Streams and Savannah River

Page 2 of 6

\begin{tabular}{|c|c|c|c|c|c|c|}
\hline Location & & Colonlea/100mL & & * of Samples & $\begin{array}{l}\text { Number of } \\
\text { Exocedanoses }\end{array}$ & $\begin{array}{l}\text { Perlod } \\
\text { of Time }\end{array}$ \\
\hline & MAR MIN & MAR MAX & GEO MEAN" & & & \\
\hline RIVER-2 & 13 & 320 & 118 & 5 & 0 & $3 / 1-3 / 30$ \\
\hline RIVER-3B & 25 & 144 & 57 & 5 & 0 & $3 / 1-3 / 30$ \\
\hline RIVER-10 & 40 & 290 & 72 & 5 & 0 & $3 / 1-3 / 30$ \\
\hline U3R-1A & 64 & 220 & 117 & 5 & 0 & $3 / 1-3 / 30$ \\
\hline U3R-4 & 56 & 210 & 133 & 5 & 0 & $3 / 1-3 / 30$ \\
\hline FMC-6 & 54 & 430 & 105 & 5 & 1 & $3 / 1-3 / 30$ \\
\hline PB-3 & 20 & 320 & 77 & 5 & 0 & $3 / 1-3 / 30$ \\
\hline$B D C$ & 3 & 121 & 31 & 5 & 0 & $3 / 1-3 / 30$ \\
\hline L3R-2 & 123 & 290 & 225 & 5 & 1 & $3 / 1-3 / 30$ \\
\hline $5 c-4^{b}$ & 2 & 2 & 2 & 1 & 0 & $3 / 3$ \\
\hline TB- $5^{\mathrm{b}}$ & 200 & 200 & 200 & 1 & 0 & $3 / 3$ \\
\hline$F M-A 7^{b}$ & 13 & 13 & 13 & 1 & 0 & $3 / 3$ \\
\hline U3R-4 & 119 & 119 & 119 & 1 & 0 & $3 / 3$ \\
\hline Location & \multicolumn{3}{|c|}{ Colonise/100mL } & \# of Samples & $\begin{array}{l}\text { Number of } \\
\text { Excoedances }\end{array}$ & $\begin{array}{l}\text { Period } \\
\text { of Time }\end{array}$ \\
\hline & APR MIN & APR MAX & GEOMEAN" & & & \\
\hline RIVER-2 & 80 & 200 & 116 & 4 & 0 & $4 / 5-4 / 27$ \\
\hline RIVER-3B & 34 & 124 & 63 & 4 & 0 & $4 / 5-4 / 27$ \\
\hline RIVER-10 & 40 & 210 & 75 & 4 & 0 & $4 / 5-4 / 27$ \\
\hline U3R-1A & 123 & 370 & 200 & 4 & 0 & $4 / 5-4 / 27$ \\
\hline U3R-4 & 100 & 330 & 181 & 4 & 0 & $4 / 5-4 / 27$ \\
\hline FMC-6 & 50 & 118 & 77 & 4 & 0 & $4 / 5-4 / 27$ \\
\hline PB-3 & 42 & 310 & 104 & 4 & 0 & $4 / 5-4 / 27$ \\
\hline $\mathrm{BDC}$ & 16 & 62 & 32 & 4 & 0 & $4 / 5-4 / 27$ \\
\hline L3R-2 & 152 & 580 & 239 & 4 & 2 & $4 / 5-4 / 27$ \\
\hline$S C-4^{b}$ & 15 & 15 & 15 & 1 & 0 & $4 / 15$ \\
\hline TB-5 ${ }^{b}$ & 260 & 260 & 260 & 1 & 0 & $4 / 15$ \\
\hline$F M-A 7^{b}$ & 42 & 42 & 42 & 1 & 0 & $4 / 15$ \\
\hline U $3 R-4^{b}$ & 112 & 112 & 112 & 1 & 0 & $4 / 15$ \\
\hline
\end{tabular}

a The standard for South Carolina states that the fecal coliform count should not exceed a geometric mean of $200 / 100 \mathrm{~mL}$, based on five consecutive samples during any 30 -day period, nor shall more than 10 percent of the total samples during any 30 -day period exceed $400 / 100 \mathrm{mg} / \mathrm{L}$.

b SCDHEC sample location-one sample collected monthly 
Table 72

Fecal Coliform Bacteria in SRS Streams and Savannah River

Page 3 of 6

\begin{tabular}{|c|c|c|c|c|c|c|}
\hline Location & & Colonies/100mL & & \# of Samples & $\begin{array}{l}\text { Number of } \\
\text { Exceedances }\end{array}$ & $\begin{array}{l}\text { Period } \\
\text { of Time }\end{array}$ \\
\hline & MAY MIN & MAY MAX & GEO MEAN- & & & \\
\hline RIVER-2 & 40 & 450 & 101 & 4 & 1 & $5 / 3-5 / 25$ \\
\hline RIVER-3B & 40 & 320 & 139 & 4 & 0 & $5 / 3-5 / 25$ \\
\hline RIVER-10 & 26 & 124 & 47 & 4 & 0 & $5 / 3-5 / 25$ \\
\hline U3R-1A & 191 & 280 & 240 & 4 & 1 & $5 / 3-5 / 25$ \\
\hline U3R-4 & 139 & 230 & 179 & 4 & 0 & $5 / 3-5 / 25$ \\
\hline FMC-6 & 52 & 102 & 77 & 4 & 0 & $5 / 3-5 / 25$ \\
\hline PB-3 & 48 & 260 & 91 & 4 & 0 & $5 / 3-5 / 25$ \\
\hline$B D C$ & 66 & 510 & 130 & 4 & 1 & $5 / 3-5 / 25$ \\
\hline L3R-2 & 104 & 154 & 134 & 4 & 0 & $5 / 3-5 / 25$ \\
\hline$S C-4^{b}$ & 142 & 142 & 142 & 1 & 0 & $5 / 5$ \\
\hline TB-5 & 3,300 & 3,300 & 3,300 & 1 & 2 & $5 / 5$ \\
\hline$F M-A 7^{b}$ & 430 & 430 & 430 & 1 & 2 & $5 / 5$ \\
\hline U $3 R-4^{b}$ & 1,681 & 1,681 & 1,681 & 1 & 2 & $5 / 5$ \\
\hline Location & \multicolumn{3}{|c|}{ Colonies/100mL } & \# of Samples & $\begin{array}{l}\text { Number of } \\
\text { Exceedances }\end{array}$ & $\begin{array}{l}\text { Period } \\
\text { of Time }\end{array}$ \\
\hline & JUNE MIN & JUNE MAX & GEO MEAN" & & & \\
\hline RIVER-2 & 26 & 98 & 56 & 5 & 0 & $6 / 1-6 / 29$ \\
\hline RIVER-3B & 29 & 22.300 & 298 & 5 & 3 & $6 / 1-6 / 29$ \\
\hline RIVER-10 & 5 & 854 & 54 & 5 & 2 & $6 / 1-6 / 29$ \\
\hline U3R-1A & 220 & 370 & 248 & 5 & 1 & $6 / 1-6 / 29$ \\
\hline U3R-4 & 56 & 1,309 & 330 & 5 & 2 & $6 / 1-6 / 29$ \\
\hline FMC -6 & 50 & 92 & 75 & 5 & 0 & $6 / 1-6 / 29$ \\
\hline PB-3 & 78 & 280 & 97 & 5 & 0 & $6 / 1-6 / 29$ \\
\hline $\mathrm{BDC}$ & 29 & 98 & 64 & 5 & 0 & $6 / 1-6 / 29$ \\
\hline L3R-2 & 112 & 3,263 & 256 & 5 & 2 & $6 / 1-6 / 29$ \\
\hline$S C-4^{b}$ & 8 & 8 & 8 & 1 & 0 & $6 / 9$ \\
\hline TB-5b & 390 & 390 & 390 & 1 & 1 & $6 / 9$ \\
\hline$F M-A 7^{b}$ & 88 & 88 & 88 & 1 & 0 & $6 / 9$ \\
\hline$U 3 R-4^{b}$ & 250 & 250 & 250 & 1 & 1 & $6 / 9$ \\
\hline
\end{tabular}

a The standard for South Carolina states that the fecal coliform count should not exceed a geometric mean of $200 / 100 \mathrm{~mL}$, based on five consecutive samples during any 30 -day period, nor shall more than 10 percent of the total samples during any 30-day period exceed 400/100 mg/L.

b SCDHEC sample location-one sample collected monthly 
Table 72

Fecal Coliform Bacteria in SRS Streams and Savannah River

Page 4 of 6

\begin{tabular}{|c|c|c|c|c|c|c|}
\hline \multirow[t]{2}{*}{ Location } & \multicolumn{3}{|c|}{ Colonies/100mL } & \multirow[t]{2}{*}{ \# of Samples } & \multirow[t]{2}{*}{$\begin{array}{l}\text { Number of } \\
\text { Exceedances }\end{array}$} & \multirow[t]{2}{*}{$\begin{array}{l}\text { Period } \\
\text { of Time }\end{array}$} \\
\hline & JUL MIN & JUL MAX & GEO MEAN" & & & \\
\hline RIVER-2 & 56 & 440 & 136 & 4 & 1 & $7 / 6-7 / 27$ \\
\hline RIVER-3B & 40 & 16,900 & 354 & 4 & 2 & $7 / 6-7 / 27$ \\
\hline RIVER-10 & 6 & 40 & 22 & 4 & 0 & $7 / 6-7 / 27$ \\
\hline U3R-1A & 86 & 280 & 155 & 4 & 0 & $7 / 6-7 / 27$ \\
\hline U3R-4 & 120 & 370 & 238 & 4 & 1 & $7 / 6-7 / 27$ \\
\hline FMC-6 & 46 & 400 & 114 & 4 & 0 & $7 / 6-7 / 27$ \\
\hline PB-3 & 37 & 250 & 90 & 4 & 0 & $7 / 6-7 / 27$ \\
\hline $\mathrm{BDC}$ & 52 & 250 & 108 & 4 & 0 & $7 / 6-7 / 27$ \\
\hline L3R-2 & 100 & 750 & 223 & 4 & 2 & $7 / 6-7 / 27$ \\
\hline$s c-4^{b}$ & 8 & 8 & 8 & 1 & 0 & $7 / 22$ \\
\hline TB-5 $5^{b}$ & 390 & 390 & 390 & 1 & 1 & $7 / 22$ \\
\hline$F M-A 7^{b}$ & 88 & 88 & 88 & 1 & 0 & $7 / 22$ \\
\hline$U 3 R-4^{b}$ & 250 & 250 & 250 & 1 & 2 & $7 / 22$ \\
\hline \multirow[t]{2}{*}{ Location } & \multicolumn{3}{|c|}{ Colonies $/ 100 \mathrm{~mL}$} & \# of Samples & $\begin{array}{l}\text { Number of } \\
\text { Excoedances }\end{array}$ & $\begin{array}{l}\text { Period } \\
\text { of Time }\end{array}$ \\
\hline & AUG MIN & AUG MAX & GEO MEAN & & & \\
\hline RIVER-2 & 31 & 88 & 53 & 5 & 0 & $8 / 2-8 / 31$ \\
\hline RIVER-3B & 24 & 570 & 89 & 5 & 1 & $8 / 2-8 / 31$ \\
\hline RIVER-10 & 18 & 240 & 47 & 5 & 0 & $8 / 2-8 / 31$ \\
\hline U3R-1A & 136 & 360 & 169 & 5 & 0 & $8 / 2-8 / 31$ \\
\hline U3R-4 & 52 & 240 & 135 & 5 & 0 & $8 / 2-8 / 31$ \\
\hline FMC-6 & 39 & 134 & 63 & 5 & 0 & $8 / 2-8 / 31$ \\
\hline PB-3 & 56 & 112 & 77 & 5 & 0 & $8 / 2-8 / 31$ \\
\hline $\mathrm{BDC}$ & 21 & 230 & 76 & 5 & 0 & $8 / 2-8 / 31$ \\
\hline L3R-2 & 84 & 112 & 95 & 5 & 0 & $8 / 2-8 / 31$ \\
\hline$s c-4^{b}$ & 29 & 29 & 29 & 1 & 0 & $8 / 4$ \\
\hline TB-5b & 1,263 & 1,263 & 1,263 & 1 & 1 & $8 / 4$ \\
\hline$F M-A 7^{b}$ & 460 & 460 & 460 & 1 & 2 & $8 / 4$ \\
\hline$U 3 R-4^{b}$ & 311 & 311 & 311 & 1 & 1 & $8 / 4$ \\
\hline
\end{tabular}

a The standard for South Carolina states that the fecal coliform count should not exceed a geometric mean of $200 / 100 \mathrm{~mL}$, based on five consecutive samples during any 30 -day period, nor shall more than 10 percent of the total samples during any 30 -day period exceed $400 / 100 \mathrm{mg} / \mathrm{L}$.

b SCDHEC sample location-one sample collected monthly 
Table 72

Fecal Coliform Bacteria in SRS Streams and Savannah River

Page 5 of 6

\begin{tabular}{|c|c|c|c|c|c|c|}
\hline \multirow[t]{2}{*}{ Location } & \multicolumn{3}{|c|}{ Colonies/100mL } & \multirow[t]{2}{*}{ \# of Samples } & \multirow[t]{2}{*}{$\begin{array}{l}\text { Number of } \\
\text { Exceedances }\end{array}$} & \multirow[t]{2}{*}{$\begin{array}{l}\text { Period } \\
\text { of Time }\end{array}$} \\
\hline & SEP MIN & SEP MAX & GEO MEAN & & & \\
\hline RIVER-2 & 40 & 1,960 & 138 & 4 & 1 & $9 / 7-9 / 28$ \\
\hline RIVER-3B & 18 & 94 & 35 & 4 & 0 & $9 / 7-9 / 28$ \\
\hline RIVER-10 & 16 & 90 & 41 & 4 & 0 & $9 / 7-9 / 28$ \\
\hline U3R-1A & 250 & 500 & 331 & 4 & 2 & $9 / 7-9 / 28$ \\
\hline U3R-4 & 211 & 1,495 & 440 & 4 & 2 & $9 / 7-9 / 28$ \\
\hline FMC-6 & 78 & 200 & 117 & 4 & 0 & $9 / 7-9 / 28$ \\
\hline PB-3 & 50 & 260 & 96 & 4 & 0 & $9 / 7-9 / 28$ \\
\hline $\mathrm{BDC}$ & 24 & 76 & 50 & 4 & 0 & $9 / 7-9 / 28$ \\
\hline L3R-2 & 104 & 12,200 & 519 & 4 & 2 & $9 / 7-928$ \\
\hline$s c-4^{b}$ & 46 & 46 & 46 & 1 & 0 & $9 / 10$ \\
\hline TB-5 & 410 & 410 & 410 & 1 & 2 & $9 / 10$ \\
\hline$F M-A 7^{b}$ & 151 & 151 & 151 & 1 & 0 & $9 / 10$ \\
\hline U $3 R-4^{b}$ & 240 & 240 & 240 & 1 & 1 & $9 / 40$ \\
\hline \multirow[t]{2}{*}{ Location } & \multicolumn{3}{|c|}{ Colonies/100mL } & \# of Samples & $\begin{array}{l}\text { Number of } \\
\text { Exceedances }\end{array}$ & $\begin{array}{l}\text { Period } \\
\text { of Time }\end{array}$ \\
\hline & OCTMIN & OCT MAX & GEO MEAN & & & \\
\hline RIVER-2 & 64 & 1,500 & 209 & 4 & 2 & $10 / 4-10 / 26$ \\
\hline RIVER-3B & 26 & 310 & 88 & 4 & 0 & $10 / 4-10 / 26$ \\
\hline RIVER-10 & 29 & 106 & 54 & 4 & 0 & $10 / 4-10 / 26$ \\
\hline U3R-1A & 160 & 480 & 253 & 4 & 2 & $10 / 4-10 / 26$ \\
\hline U3R-4 & 118 & 240 & 184 & $\Delta$ & 0 & $10 / 4-10 / 26$ \\
\hline FMC-6 & 70 & 440 & 191 & 4 & 1 & $10 / 4-10 / 26$ \\
\hline PB-3 & 46 & 173 & 69 & 4 & 0 & $10 / 4-10 / 26$ \\
\hline BDC & 24 & 147 & 63 & 4 & 0 & $10 / 4-10 / 26$ \\
\hline L3R-2 & 150 & 854 & 239 & 4 & 2 & $10 / 4-10 / 26$ \\
\hline$s c-4^{b}$ & 14 & 14 & 14 & 1 & 0 & $10 / 7$ \\
\hline TB-5 & 460 & 460 & 460 & 1 & 2 & $10 / 7$ \\
\hline$F M-A 7^{b}$ & 250 & 250 & 250 & 1 & 1 & $10 / 7$ \\
\hline U3R-4b & 220 & 220 & 220 & 1 & 1 & $10 / 7$ \\
\hline
\end{tabular}

\footnotetext{
a The standard for South Carolina states that the fecal coliform coun should not exceed a geometric mean of 200/100 $\mathrm{mL}$, based on five consecutive samples during any 30 -day period, nor shall more than 10 percent of the total samples during any 30-day period exceed $400 / 100 \mathrm{mg} / \mathrm{L}$.

b SCDHEC sample location-one sample collected monthly
} 
Table 72

Fecal Collform Bacteria in SRS Streams and Savannah River

Page 6 of 6

\begin{tabular}{|c|c|c|c|c|c|c|}
\hline Location & & Colonies/100mL & & \# of Samples & $\begin{array}{l}\text { Number of } \\
\text { Exceedances }\end{array}$ & $\begin{array}{l}\text { Perlod } \\
\text { of Time }\end{array}$ \\
\hline & NOV MIN & NOV MAX & GEO MEANa & & & \\
\hline RIVER-2 & 66 & 550 & 197 & 5 & 1 & $11 / 1-11 / 30$ \\
\hline RIVER-3B & 61 & 490 & 143 & 5 & 2 & $11 / 1-11 / 30$ \\
\hline RIVER-10 & 48 & 836 & 167 & 5 & 2 & $11 / 1-11 / 30$ \\
\hline U3R-1A & 14 & 410 & 128 & 5 & 1 & $11 / 1-11 / 30$ \\
\hline U3R-4 & 100 & 310 & 184 & 5 & 0 & $11 / 1-11 / 30$ \\
\hline FMC-6 & 56 & 170 & 87 & 5 & 0 & $11 / 1-11 / 30$ \\
\hline PB-3 & 46 & 94 & 58 & 5 & 0 & $11 / 1-11 / 30$ \\
\hline BDC & 24 & 1,800 & 103 & 5 & 1 & $11 / 1-11 / 30$ \\
\hline L3R-2 & 72 & 410 & 159 & 5 & 1 & $11 / 1-11 / 30$ \\
\hline$s c-4^{b}$ & 19 & 19 & 19 & 1 & 0 & $11 / 4$ \\
\hline TB-5 & 260 & 260 & 260 & 1 & 1 & $11 / 4$ \\
\hline$F M-A 7^{b}$ & 54 & 54 & 54 & 1 & 0 & $11 / 4$ \\
\hline U $3 R-4^{b}$ & 200 & 200 & 200 & 1 & 0 & $11 / 4$ \\
\hline Location & \multicolumn{3}{|c|}{ Colonies/100mL } & \# of Samples & $\begin{array}{l}\text { Number of } \\
\text { Exceedances }\end{array}$ & $\begin{array}{l}\text { Period } \\
\text { of Time }\end{array}$ \\
\hline & DEC MIN & DEC MAX & GEO MEAN & & & \\
\hline RIVER-2 & 28 & 34 & 31 & 4 & 0 & $12 / 6-12 / 28$ \\
\hline RIVER-3B & 2 & 66 & 18 & 4 & 0 & $12 / 6-12 / 28$ \\
\hline RIVER-10 & 11 & 52 & 22 & 4 & 0 & $12 / 6-12 / 28$ \\
\hline U3R-1A & 34 & 126 & 70 & 4 & 0 & $12 / 6-12 / 28$ \\
\hline U3R-4 & 114 & 210 & 139 & 4 & 0 & $12 / 6-12 / 28$ \\
\hline$F M C-6$ & 23 & 250 & 87 & 4 & 0 & $12 / 6-12 / 28$ \\
\hline PB-3 & 18 & 144 & 53 & 4 & 0 & $12 / 6-12 / 28$ \\
\hline$B D C$ & 8 & 66 & 28 & 4 & 0 & $12 / 6-12 / 28$ \\
\hline L3R-2 & 40 & 370 & 63 & 4 & 0 & $12 / 6-12 / 28$ \\
\hline$s c-4^{b}$ & 18 & 18 & 18 & 1 & 0 & $12 / 2$ \\
\hline TB-5b & 198 & 198 & 198 & 1 & 0 & $12 / 2$ \\
\hline$F M-A 7^{b}$ & 31 & 31 & 31 & 1 & 0 & $12 / 2$ \\
\hline U $3 R-4^{b}$ & 112 & 112 & 112 & 1 & 0 & $12 / 2$ \\
\hline
\end{tabular}

a The standard for South Carolina states that the fecal coliform count should not exceed a geometric mean of $200 / 100 \mathrm{~mL}$, based on five consecutive samples during any 30-day period, nor shall more than 10 percent of the total samples during any 30 -day period exceed $400 / 100 \mathrm{mg} / \mathrm{L}$.

b SCDHEC sample location-one sample collected monthly 
Table 73

Mercury in Fish Flesh

Page 1 of 3

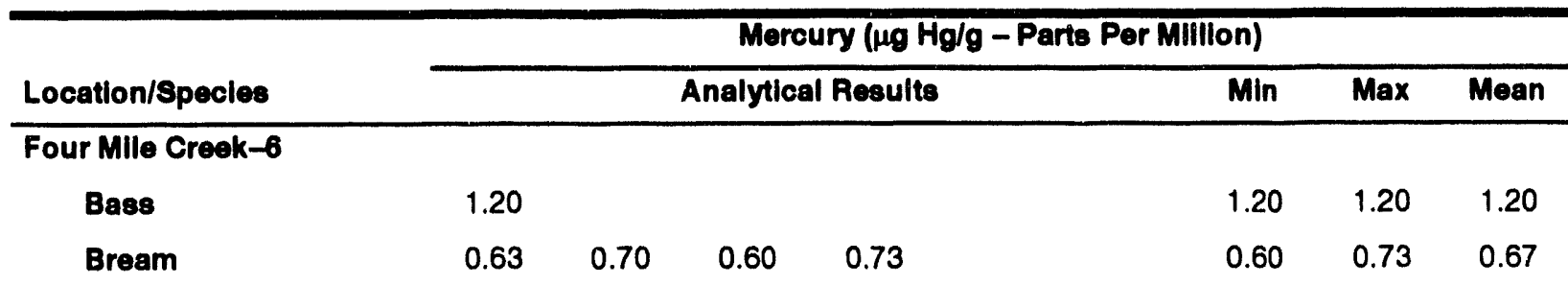

Four Mile Creek

@ Creek Mouth

$\begin{array}{lllllllll}\text { Bream } & 0.17 & 0.10 & 0.10 & 0.03 & 0.23 & 0.03 & 0.17 & 0.12 \\ \text { Cattish } & 1.47 & 0.07 & 0.03 & 0 & 0.20 & 0 & 1.47 & 0.46\end{array}$

Augusta L \& D

Bass

Bream

Beaver Dam Creek

Bass
Bream
Catfish
Creek Mouth

Bream

Catfish

Hwy 17-A Bridge

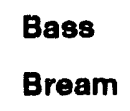

Catfish

Mullet

\section{L-Lake}

Bass

Beaver Dam Creek @ Creok Mouth

\section{$0.77 \quad 0.73$}

$0.33 \quad 0.37$

0.13

0.17

0.53

0.27

0.13

0.03

0.10

0.90

0.73

$0.77 \quad 0.75$

$\begin{array}{lll}0.13 & 0.53 & 0.33\end{array}$

0.03

$0.17 \quad 0.22$

$\begin{array}{llllllll}0 & 0.10 & 0.10 & 0.50 & 0.10 & 0 & 0.50 & 0.19 \\ 0 & 0.10 & & & & 0 & 0.10 & 0.05\end{array}$

$\begin{array}{ccccc}0.60 & 0.60 & 0.30 & 0.17 & 0.07 \\ 0.33 & 0.03 & 0 & 0.03 & 0 \\ 0.23 & 0.20 & & & \\ 0 & 0 & 0 & 0 & 0\end{array}$

$\begin{array}{ccc}0.07 & 0.60 & 0.34 \\ 0 & 0.33 & 0.10 \\ 0.20 & 0.23 & 0.22 \\ 0 & 0 & 0\end{array}$




\section{Table 73}

\section{Mercury in Fish Flesh}

Page 2 of 3

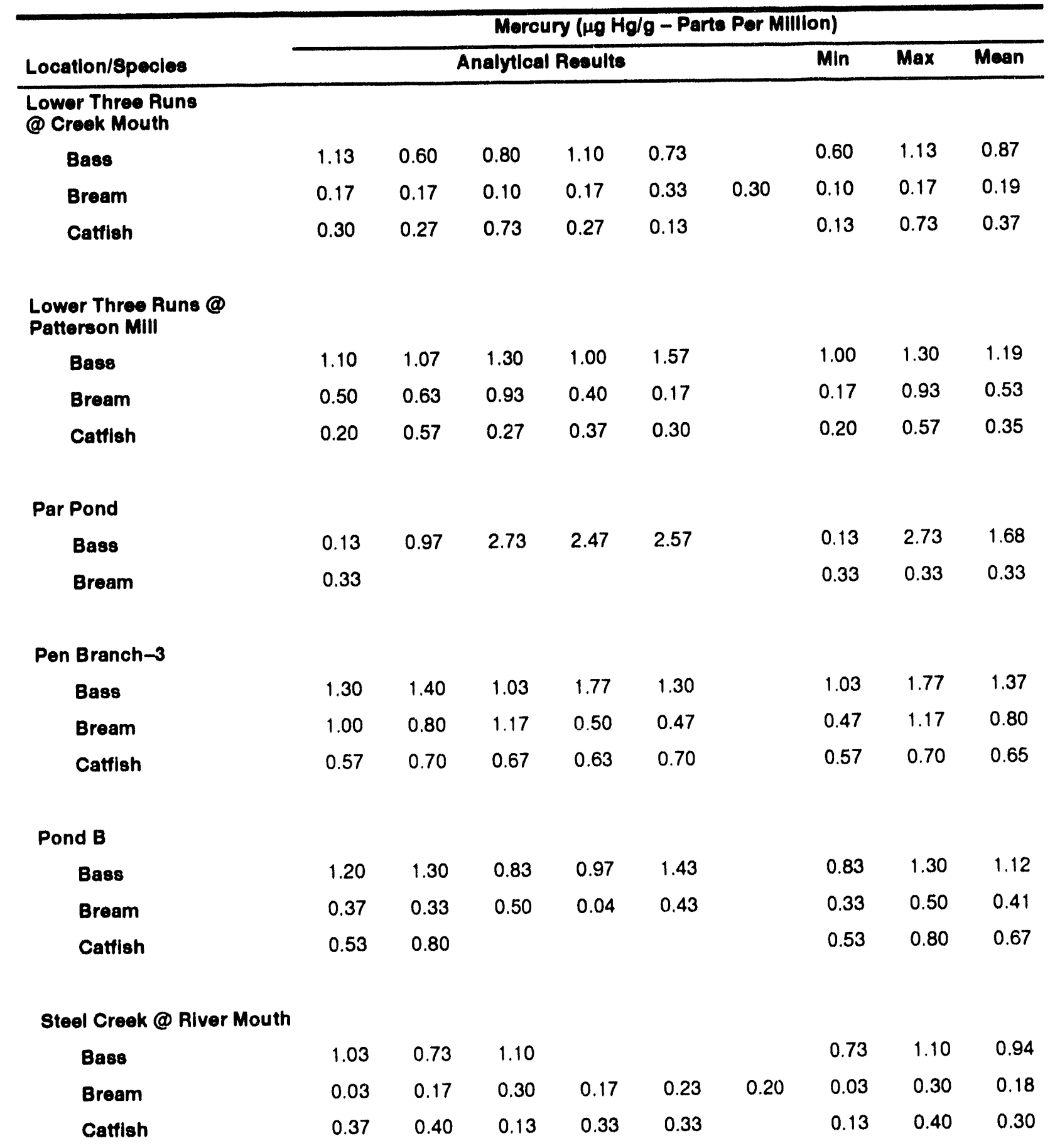


Table 73

Mercury in Fish Flesh

Page 3 of 3

\begin{tabular}{|c|c|c|c|c|c|c|c|c|}
\hline \multirow[b]{2}{*}{ Location/Specles } & \multicolumn{8}{|c|}{ Mercury ( $\mu \mathrm{g} \mathrm{Hg} / \mathrm{g}$ - Parts Per Million) } \\
\hline & \multicolumn{5}{|c|}{ Analytical Results } & Min & $\operatorname{Max}$ & Mean \\
\hline \multicolumn{4}{|l|}{ Steol Creek-4 } & & & & & \\
\hline Bass & 1.03 & 1.17 & 1.40 & 1.63 & 1.57 & 1.03 & 1.63 & 1.35 \\
\hline Bream & 0.53 & 0.33 & 0.33 & 0.27 & & 0.27 & 0.53 & 0.38 \\
\hline Cattish & 1.03 & 0.80 & 0.50 & 0.97 & 0.43 & 0.43 & 1.03 & 0.74 \\
\hline Crapple & 1.27 & & & & & 1.27 & 1.27 & 1.27 \\
\hline \multicolumn{9}{|c|}{$\begin{array}{l}\text { Savannah Rlver } \\
\text { @ U.S. Highway } 301\end{array}$} \\
\hline Bass & 0.67 & 0.57 & 0.43 & 0.70 & 1.00 & 0.43 & 0.70 & 0.64 \\
\hline Bream & 0.30 & 0.13 & 0.17 & 0.50 & 0.13 & 0.13 & 0.50 & 0.27 \\
\hline Catfish & 0.30 & 0.17 & 1.33 & 0.13 & 0.17 & 0.13 & 1.33 & 0.51 \\
\hline \multicolumn{9}{|c|}{ Stokes Bluff Landing } \\
\hline Bass & 0.60 & 0.83 & 0.37 & 0.77 & 0.50 & 0.37 & 0.83 & 0.61 \\
\hline Bream & 0.37 & 0.43 & 0.33 & 0.43 & 0.43 & 0.33 & 0.43 & 0.39 \\
\hline Cattish & 0.23 & 0.63 & 0.37 & 0.23 & 0.53 & 0.23 & 0.63 & 0.41 \\
\hline
\end{tabular}

Upper Three Runs @ Creek Mouth

$\begin{array}{lcccccccc}\text { Bass } & 1.43 & 0.53 & & & & 0.53 & 1.43 & 0.98 \\ \text { Bream } & 0.17 & 0 & 0.20 & 0.10 & 0.07 & 0 & 0.20 & 0.11 \\ \text { Catfish } & 0.27 & 0.33 & 0.13 & 0.10 & 0.20 & 0.10 & 0.33 & 0.21\end{array}$

West Bank Landing

Crapple

$\begin{array}{lllll}0.13 & 0.13 & 0.30 & 0.13 & 0.10\end{array}$

$0.10 \quad 0.30$

0.17 


\section{Table 74}

Chlorocarbon Monitoring Results from A-Area Wells

Page 1 of 4

\begin{tabular}{|c|c|c|c|c|c|c|c|}
\hline \multirow[b]{3}{*}{ Location } & \multirow[b]{3}{*}{ Date } & \multirow{2}{*}{\multicolumn{2}{|c|}{111 Triohloroothane }} & \multirow{2}{*}{\multicolumn{2}{|c|}{$\frac{(\mu / g L)}{\text { Trichloroethylene }}$}} & & \\
\hline & & & & & & \multicolumn{2}{|c|}{ Tetrachloroothylene } \\
\hline & & SR8 & TMA* & 8RS & TMA" & SA8 & TMA: \\
\hline \multicolumn{8}{|c|}{ 3/700 Area Domestlo $\mathrm{H}_{2} \mathrm{O}$, First Quarter } \\
\hline $905-82 A$ & $\operatorname{Jan}-93$ & Not Pulled & & & & & \\
\hline $905-82 A$ & Feb-93 & Not Pulled & & & & & \\
\hline $905-82 A$ & Mar-93 & Not Pulled & & & & & \\
\hline $905-112 G$ & Feb-93 & $N D^{b}$ & ND & ND & ND & ND & ND \\
\hline $905-113 G$ & Feb-93 & $\begin{array}{l}\text { Out of } \\
\text { Service }\end{array}$ & & & & & \\
\hline \multicolumn{8}{|c|}{ 3/700 Area Drinking $\mathrm{H}_{2} \mathrm{O}$, First Quarter } \\
\hline $735-A$ & Jan-93 & ND & ND & ND & ND & ND & ND \\
\hline 735-A & Feb-93 & ND & ND & ND & ND & ND & ND \\
\hline 735-A & Mar-93 & ND & ND & ND & ND & ND & ND \\
\hline 784-A & Feb-93 & ND & ND & ND & ND & ND & ND \\
\hline \multicolumn{8}{|c|}{ TSC Backup Domestlc $\mathrm{H}_{2} \mathrm{O}$, First Quarter } \\
\hline $905-68 \mathrm{~A}$ & Jan-93 & ND & ND & ND & ND & ND & ND \\
\hline $905-68 \mathrm{~A}$ & Feb-93 & ND & ND & ND & ND & ND & ND \\
\hline $905-68 A$ & Mar-93 & ND & ND & ND & ND & ND & ND \\
\hline \multicolumn{8}{|c|}{ 3/700 Area Process $\mathrm{H}_{2} \mathrm{O}$ Well, First Quarter } \\
\hline $905-20 A$ & Jan-93 & ND & 6 & 25 & 56 & ND & 2 \\
\hline $905-20 A$ & Feb-93 & ND & ND & 58 & 57 & ND & ND \\
\hline $905-20 A$ & Mar-93 & ND & ND & 62 & 32 & ND & ND \\
\hline Service $\mathrm{H}_{2} \mathrm{O}$ & Jan-93 & ND & ND & 5 & 12 & ND & ND \\
\hline Service $\mathrm{H}_{2} \mathrm{O}$ & Feb-93 & ND & ND & 6 & 5 & ND & ND \\
\hline Service $\mathrm{H}_{2} \mathrm{O}$ & Mar-93 & ND & ND & 3 & 4 & ND & ND \\
\hline $905-53 A$ & Jan-93 & ND & ND & 52 & 114 & 5 & 13 \\
\hline $905-53 A$ & Feb-93 & ND & ND & 139 & 143 & 12 & 15 \\
\hline $905-53 A$ & Mar-93 & ND & ND & 144 & 128 & 11 & 14 \\
\hline \multicolumn{8}{|c|}{ Other SRS Domestlc $\mathrm{H}_{2} \mathrm{O}$ Supplies, Flrst Quarter } \\
\hline $400-D$ & Feb-93 & ND & ND & ND & ND & ND & ND \\
\hline
\end{tabular}

a Analyses performed by TMA/Eberline Laboratory, Inc.

b None detected 


\section{Table 74}

Chlorocarbon Monitoring Results from A-Area Wells

Page 2 of 4

\begin{tabular}{|c|c|c|c|c|c|c|c|}
\hline \multirow[b]{3}{*}{ Looation } & \multirow[b]{3}{*}{ Date } & \multirow{2}{*}{\multicolumn{2}{|c|}{111 Trlohloroethane }} & \multicolumn{2}{|c|}{$(\mu / g L)$} & & \\
\hline & & & & Trlohlor & Thylene & \multicolumn{2}{|c|}{ Totrachloroothylene } \\
\hline & & 8R8 & TMA & 8R8 & TMAA & SA8 & TMA \\
\hline \multicolumn{8}{|c|}{$3 / 700$ Area Domestlo $\mathrm{H}_{2} \mathrm{O}$, Second Quarter } \\
\hline $905-82 A$ & Apr-93 & $N D^{b}$ & ND & ND & ND & ND & ND \\
\hline $905-82 A$ & May-93 & ND & ND & ND & ND & ND & ND \\
\hline $905-82 A$ & Jun-93 & ND & ND & ND & ND & ND & ND \\
\hline $905-112 G$ & May-93 & ND & ND & ND & ND & ND & ND \\
\hline $905-113 G$ & May-93 & $\begin{array}{l}\text { Out of } \\
\text { Service }\end{array}$ & & & & & \\
\hline \multicolumn{8}{|c|}{ 3/700 Area Drinking $\mathrm{H}_{2} \mathrm{O}$, Second Quarter } \\
\hline 735-A & Apr-93 & ND & ND & ND & ND & ND & ND \\
\hline $735-A$ & May-93 & ND & ND & ND & ND & ND & ND \\
\hline 735-A & Jun-93 & ND & ND & ND & ND & ND & ND \\
\hline $784-A$ & May-93 & ND & ND & ND & ND & ND & ND \\
\hline \multicolumn{8}{|c|}{ TSC Backup Domestlc $\mathrm{H}_{2} \mathrm{O}$, Second Quarter } \\
\hline $905-68 \mathrm{~A}$ & Apr-93 & ND & ND & ND & ND & ND & ND \\
\hline $905-68 \mathrm{~A}$ & May-93 & ND & ND & 1 & ND & 2 & ND \\
\hline $905-68 A$ & Jun-93 & ND & ND & ND & ND & ND & ND \\
\hline \multicolumn{8}{|c|}{ 3/700 Area Process $\mathrm{H}_{2} \mathrm{O}$ Wells, Second Quarter } \\
\hline $905-20 A$ & Apr-93 & ND & ND & 66 & 68 & ND & 3 \\
\hline $905-20 A$ & May-93 & ND & ND & 107 & 112 & 4 & 6 \\
\hline $905-20 A$ & Jun-93 & ND & ND & 113 & 84 & ND & 4 \\
\hline Service $\mathrm{H}_{2} \mathrm{O}$ & Apr-93 & ND & ND & 10 & 3 & ND & ND \\
\hline Service $\mathrm{H}_{2} \mathrm{O}$ & May-93 & ND & ND & 12 & 11 & ND & ND \\
\hline Service $\mathrm{H}_{2} \mathrm{O}$ & Jun-93 & ND & ND & 13 & 12 & ND & ND \\
\hline $905-53 A$ & Apr-93 & ND & ND & 145 & 100 & ND & 10 \\
\hline $905-53 A$ & May-93 & ND & ND & 146 & 190 & ND & 25 \\
\hline $905-53 A$ & Jun-93 & ND & ND & 127 & 158 & 10 & 19 \\
\hline \multicolumn{8}{|c|}{ Other SRS Domestic $\mathrm{H}_{2} \mathrm{O}$ Supplles, Second Quarter } \\
\hline $400-D$ & May-93 & ND & ND & ND & ND & ND & ND \\
\hline
\end{tabular}

a Analyses performed by TMA/Eberline Laboratory, Inc. None detected 
Table 74

Chlorocarbon Monitoring Results from A-Area Wells

Page 3 of 4

\begin{tabular}{|c|c|c|c|c|c|c|c|}
\hline \multirow[b]{3}{*}{ Location } & \multirow[b]{3}{*}{ Date } & \multirow{2}{*}{\multicolumn{2}{|c|}{111 Trlohloroothane }} & \multirow{2}{*}{\multicolumn{2}{|c|}{$\frac{(\mu / g L)}{\text { Trlohloroothylono }}$}} & & \\
\hline & & & & & & \multicolumn{2}{|c|}{ Totraohloroothylene } \\
\hline & & SA8 & TMA* & 8Rs & TMA* & sas & TMA* \\
\hline \multicolumn{8}{|c|}{$3 / 700$ Area Domeatlo $\mathrm{H}_{2} \mathrm{O}$, Third Quarter } \\
\hline $905-82 A$ & Jul-93 & $N D^{b}$ & ND & ND & ND & ND & ND \\
\hline $905-82 A$ & Aug-93 & ND & ND & ND & ND & ND & ND \\
\hline $905-82 A$ & Sep-93 & Not Pulled & & & & & \\
\hline $905-112 G$ & Aug-93 & ND & ND & ND & ND & ND & ND \\
\hline $905-113 G$ & Aug-93 & $\begin{array}{l}\text { Out of } \\
\text { Service }\end{array}$ & & & & & \\
\hline \multicolumn{8}{|c|}{$3 / 700$ Area Drinking $\mathrm{H}_{2} \mathrm{O}$, Third Quartor } \\
\hline 735-A & Jul-93 & ND & ND & ND & ND & ND & ND \\
\hline 735-A & Aug-93 & ND & ND & ND & ND & ND & ND \\
\hline $735-A$ & Sep-93 & ND & ND & ND & ND & ND & ND \\
\hline 784-A & Aug-93 & ND & ND & ND & ND & ND & ND \\
\hline \multicolumn{8}{|c|}{ TSC Backup Domeatic $\mathrm{H}_{2} \mathrm{O}$, Third Quarter } \\
\hline $905-68 A$ & Jul-93 & ND & ND & ND & ND & ND & ND \\
\hline $905-68 A$ & Aug-93 & ND & ND & ND & ND & ND & ND \\
\hline $905-68 A$ & Sep-93 & ND & ND & ND & ND & ND & ND \\
\hline \multicolumn{8}{|c|}{ 3/700 Area Process $\mathrm{H}_{2} \mathrm{O}$ Wolls, Third Quarter } \\
\hline $905-20 A$ & Jul-93 & ND & ND & 74 & 54 & ND & 2 \\
\hline $905-20 A$ & Aug-93 & ND & ND & 134 & 198 & 6 & ND \\
\hline $905-20 A$ & Sep-93 & ND & ND & 81 & 100 & ND & 3 \\
\hline Service $\mathrm{H}_{2} \mathrm{O}$ & Jul-93 & ND & ND & 27 & 20 & ND & ND \\
\hline Service $\mathrm{H}_{2} \mathrm{O}$ & Aug-93 & ND & ND & 58 & 81 & 6 & ND \\
\hline Service $\mathrm{H}_{2} \mathrm{O}$ & Sep-93 & ND & 2 & 5 & 5 & ND & ND \\
\hline $905-53 A$ & Jul-93 & ND & ND & 202 & 133 & 16 & 16 \\
\hline $905-53 A$ & Aug-93 & ND & ND & 129 & 178 & 114 & 20 \\
\hline $905-53 A$ & Sep-93 & ND & ND & 110 & 95 & 18 & 8 \\
\hline \multicolumn{8}{|c|}{$\begin{array}{l}\text { Other SRS Domestic } \mathrm{H}_{2} \mathrm{O} \text { Supplies, Third Quarter } \\
400-D\end{array}$} \\
\hline $400-D$ & Aug-93 & ND & ND & ND & ND & ND & ND \\
\hline
\end{tabular}

a Analyses pertormed by TMA/Ebertine Laboratory, Inc.

b None detected 
Table 74

Chlorocarbon Monitoring Results from A-Area Wells

Page 4 of 4

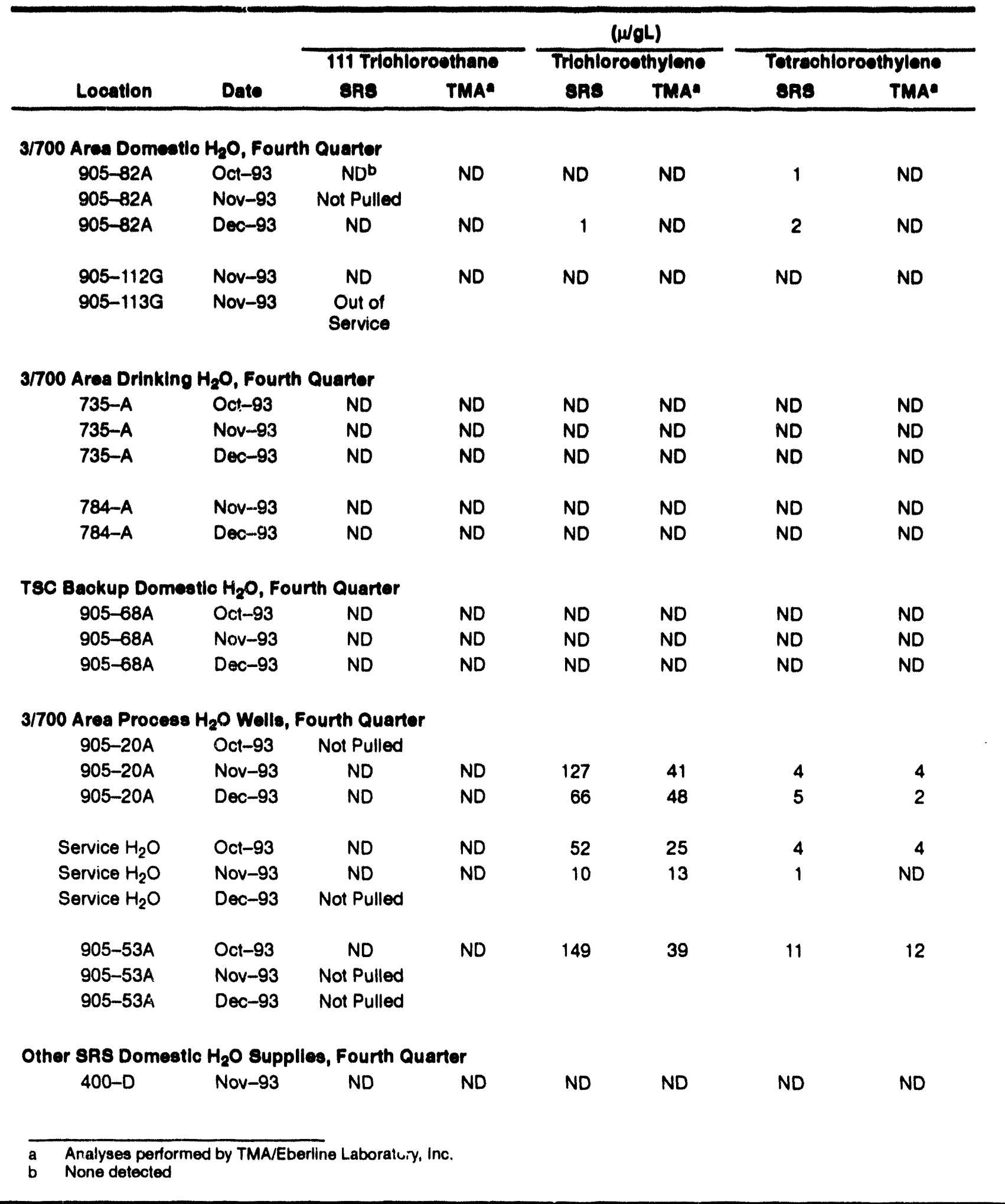


Table 75

Inorganics in sediment

Page 1 of 2

\begin{tabular}{|c|c|c|c|c|c|c|}
\hline $\begin{array}{l}\text { Inorganlos } \\
\text { Conatltuent }\end{array}$ & $\begin{array}{c}\text { Uppor Three } \\
\text { Rune } \\
\text { Road 8-1 }\end{array}$ & $\begin{array}{l}\text { Tinker Creok } \\
\text { Konnedy } \\
\text { Pond Roed }\end{array}$ & $\begin{array}{l}\text { Steal Creak } \\
\text { Road A }\end{array}$ & $\begin{array}{c}\text { Lower Three } \\
\text { Auns } \\
\text { Pattereon } \\
\text { MIII }\end{array}$ & $\begin{array}{l}\text { Pon Branch } \\
\text { Road A }\end{array}$ & $\begin{array}{c}\text { Four Milo } \\
\text { Creok } \\
\text { Road A }\end{array}$ \\
\hline Cyanido & $<0.20$ & $<0.02$ & $<0.02$ & $<0.005$ & $<0.020$ & $<0.020$ \\
\hline Aluminum & $<0.100$ & 0.450 & 1.430 & 0.119 & 0.850 & 1.130 \\
\hline Arsonic & $<0.060$ & $<0.060$ & $<0.030$ & $<0.5$ & $<0.060$ & $<0.060$ \\
\hline Barium & 2.700 & 0.122 & 0.144 & 0.234 & 0.378 & 0.186 \\
\hline Cadmium & 0.116 & 0.010 & 0.010 & $<0.02$ & $<0.010$ & $<0.010$ \\
\hline Calcium & 6.500 & 11.650 & 14.100 & 50.5 & $<12.300$ & 11.100 \\
\hline Chrome & 0.110 & $<0.020$ & $<0.020$ & 0.02 & $<0.020$ & $<0.020$ \\
\hline Copper & 0.020 & $<0.020$ & 0.020 & $<0.01$ & 0.020 & $<0.020$ \\
\hline Iron & $<0.060$ & 0.060 & 0.549 & 0.072 & 0.060 & 0.855 \\
\hline Lead & 0.160 & 0.076 & $<0.060$ & $<0.5$ & 0.708 & $<0.060$ \\
\hline Magnesium & $<0.200$ & $<0.200$ & 0.318 & 1.930 & 0.026 & $<0.200$ \\
\hline Mercury & $<0.001$ & $<0.003$ & $<0.001$ & $<0.01$ & $<0.001$ & $<0.001$ \\
\hline Sodium & & & & 1290 & & \\
\hline Nickel & 0.062 & $<0.020$ & 0.044 & $<0.05$ & 0.026 & 0.064 \\
\hline Selenium & $<0.040$ & $<0.040$ & $<0.040$ & $<0.5$ & $<0.040$ & 0.040 \\
\hline Silver & 0.254 & 0.020 & 0.022 & 0.05 & 0.016 & 0.020 \\
\hline $\begin{array}{l}\text { Uranium } \\
(8 / 6 / 93)\end{array}$ & 30.70 & 29.40 & 25.90 & & 29.30 & 30.60 \\
\hline $\begin{array}{l}\text { Uranium } \\
(12 / 16 / 93)\end{array}$ & $<0.2$ & $<0.2$ & $<0.2$ & $<0.2$ & $<0.2$ & $<0.2$ \\
\hline Zinc & 0.167 & 0.385 & 0.129 & 0.018 & 0.171 & 0.213 \\
\hline
\end{tabular}


Table 75

Inorganics in Sediment

Page 2 of 2

\begin{tabular}{|c|c|c|c|c|c|}
\hline $\begin{array}{l}\text { Inorganios } \\
\text { Constituont }\end{array}$ & $\begin{array}{l}\text { Beaver Dam } \\
\text { Creok } \\
400 \text { Area }\end{array}$ & $\begin{array}{l}\text { Uppor Three } \\
\text { Runs } \\
\text { Road A }\end{array}$ & $\begin{array}{c}\text { Alver } 2 \\
\text { Alver Mill } \\
160\end{array}$ & $\begin{array}{c}\text { Alver } 38 \\
\text { Plant Vogtlo }\end{array}$ & $\begin{array}{l}\text { Rlver } 10 \\
\text { Alver Mile } 120\end{array}$ \\
\hline Cyanide & 0.020 & $<0.020$ & $<0.020$ & $<0.020$ & $<0.020$ \\
\hline Aluminum & 0.470 & 1.690 & 1.010 & 1.030 & 0.904 \\
\hline Arsonic & $<0.060$ & $<0.060$ & $<0.060$ & $<0.050$ & $<0.060$ \\
\hline Barium & 0.296 & 0.220 & 0.746 & 0.420 & 0.222 \\
\hline Cadmium & 0.010 & $<0.010$ & $<0.010$ & $<0.010$ & $<0.010$ \\
\hline Calcium & 18.600 & 8.780 & 8.540 & 5.740 & 2.460 \\
\hline Chrome & $<0.020$ & $<0.020$ & $<0.020$ & $<0.02$ & $<0.020$ \\
\hline Copper & $<0.020$ & 0.046 & 0.024 & $<0.020$ & 0.168 \\
\hline Iron & 0.547 & $<0.060$ & 1.300 & 0.403 & 0.041 \\
\hline Lead & $<0.060$ & $<0.060$ & 0.060 & $<0.060$ & $<0.060$ \\
\hline Magnesium & 5.400 & $<0.200$ & 1.040 & 1.382 & $<0.200$ \\
\hline Mercury & $<0.001$ & $<0.001$ & $<0.001$ & $<0.003$ & $<0.001$ \\
\hline \multicolumn{6}{|l|}{ Sodium } \\
\hline Nickel & 0.042 & 0.108 & 0.048 & 0.036 & 0.048 \\
\hline Selenium & $<0.040$ & $<0.040$ & $<0.04$ & $<0.040$ & $<0.040$ \\
\hline Silver & 0.024 & 0.032 & 0.050 & 0.014 & 0.038 \\
\hline $\begin{array}{l}\text { Uranium } \\
(8 / 6 / 93)\end{array}$ & 29.80 & 32.10 & 31.00 & 29.50 & 28.00 \\
\hline $\begin{array}{l}\text { Uranium } \\
\text { (12/16/93) }\end{array}$ & $<0.2$ & $<0.2$ & $<0.2$ & $<0.2$ & $<0.2$ \\
\hline Zinc & 0.091 & 1.220 & 0.233 & 0.135 & 0.165 \\
\hline
\end{tabular}


Table 76

Blind Sample Results for Conductivity Fleld Measurements

Page 1 of 1

\begin{tabular}{|c|c|c|c|}
\hline \multirow[b]{2}{*}{ Sample Identifloation } & \multicolumn{2}{|c|}{ (umhos/om) } & \multirow[b]{2}{*}{ Difforenoe } \\
\hline & Measured Value & Aotual Value & \\
\hline$B C-01-1$ & 144 & 148 & $1.37 \%$ \\
\hline$B C-01-2$ & 132 & 137 & $3.65 \%$ \\
\hline$B C-01-3$ & 100 & 109 & $8.26 \%$ \\
\hline$B C-02-1$ & 147 & 150 & $2.00 \%$ \\
\hline$B C-02-2$ & 134 & 138 & $2.89 \%$ \\
\hline$B C-02-4$ & 124 & 131 & $5.34 \%$ \\
\hline$B C-03-1$ & 118 & 125 & $5.60 \%$ \\
\hline$B C-03-2$ & 150 & 155 & $3.22 \%$ \\
\hline$B C-03-3$ & 117 & 137 & $14.60 \%$ \\
\hline$B C-04-1$ & 117 & 123 & $4.88 \%$ \\
\hline$B C-04-2$ & 105 & 117 & $10.26 \%$ \\
\hline$B C-04-3$ & 168 & 172 & $2.33 \%$ \\
\hline$B C-05-1$ & 117 & 120 & $2.50 \%$ \\
\hline$B C-05-2$ & 110 & 112 & $1.79 \%$ \\
\hline$B C-05-3$ & 166 & 171 & $2.92 \%$ \\
\hline$B C-06-1$ & 150 & 131 & $14.50 \%$ \\
\hline$B C-06-2$ & 110 & 107 & $2.80 \%$ \\
\hline$B C-06-3$ & 167 & 170 & $1.76 \%$ \\
\hline$B C-07-1$ & 146 & 152 & $3.95 \%$ \\
\hline$B C-07-2$ & 106 & 112 & $5.36 \%$ \\
\hline$B C-07-3$ & 160 & 174 & $8.04 \%$ \\
\hline$B C-08-1$ & 146 & 149 & $2.01 \%$ \\
\hline$B C-08-2$ & 105 & 110 & $4.55 \%$ \\
\hline$B C-08-3$ & 171 & 175 & $2.29 \%$ \\
\hline$B C-09-1$ & 71 & 72 & $1.39 \%$ \\
\hline$B C-09-2$ & 100 & 107 & $6.54 \%$ \\
\hline$B C-09-3$ & 134 & 133 & $0.75 \%$ \\
\hline$B C-10-1$ & 74 & 73 & $1.37 \%$ \\
\hline$B C-10-2$ & 106 & 107 & $0.93 \%$ \\
\hline$B C-10-3$ & 131 & 131 & $0.00 \%$ \\
\hline$B C-11-1$ & 47 & 50 & $6.00 \%$ \\
\hline$B C-11-2$ & 77 & 79 & $2.53 \%$ \\
\hline$B C-11-3$ & 149 & 150 & $0.67 \%$ \\
\hline$B C-12-1$ & 47 & 48 & $2.08 \%$ \\
\hline$B C-12-2$ & 78 & 81 & $3.70 \%$ \\
\hline$B C-12-3$ & 149 & 147 & $1.36 \%$ \\
\hline
\end{tabular}


Table 77

Blind Sample Results for pH Field Measurements

Page 1 of 1

pH Units

\begin{tabular}{|c|c|c|c|}
\hline \multirow[b]{2}{*}{ Sample Identification } & & \multirow[b]{2}{*}{ Difference } \\
\hline & Measured Value & Actual Value & \\
\hline $\mathrm{BpH}-01-1$ & 8.31 & 8.21 & 0.10 units \\
\hline $\mathrm{BpH}-01-2$ & 8.54 & 9.20 & 0.66 units \\
\hline $\mathrm{BpH}-01-3$ & 8.54 & 8.58 & 0.04 units \\
\hline $\mathrm{BpH}-02-1$ & 8.21 & 8.23 & 0.02 units \\
\hline $\mathrm{BpH}-02-2$ & 8.93 & 9.01 & 0.08 units \\
\hline $\mathrm{BpH}-02-3$ & 8.73 & 8.62 & 0.11 units \\
\hline $\mathrm{BpH}-03-1$ & 9.07 & 8.96 & 0.12 units \\
\hline $\mathrm{BpH}-03-2$ & 7.23 & 7.20 & 0.03 units \\
\hline $\mathrm{BpH}-03-3$ & 4.86 & 4.87 & 0.01 units \\
\hline $\mathrm{BpH}-03-4$ & 8.70 & 8.59 & 0.11 units \\
\hline $\mathrm{BpH}-04-1$ & 8.30 & 8.19 & 0.11 units \\
\hline $\mathrm{BpH}-04-2$ & 9.00 & 9.03 & 0.03 units \\
\hline $\mathrm{BpH}-04-3$ & 8.60 & 8.47 & 0.13 units \\
\hline $\mathrm{BpH}-04-4$ & 7.10 & 7.22 & 0.12 units \\
\hline $\mathrm{BpH}-04-5$ & 4.95 & 4.90 & 0.05 units \\
\hline $\mathrm{BpH}-05-1$ & 8.14 & 8.17 & 0.03 units \\
\hline $\mathrm{BpH}-05-2$ & 9.00 & 9.02 & 0.02 units \\
\hline $\mathrm{BpH}-05-3$ & 8.52 & 8.53 & 0.01 units \\
\hline $\mathrm{BpH}-05-4$ & 7.15 & 7.22 & 0.07 units \\
\hline $\mathrm{BpH}-06-1$ & 3.89 & 4.09 & 0.20 units \\
\hline $\mathrm{BpH}-06-2$ & 10.48 & 10.15 & 0.33 units \\
\hline $\mathrm{BpH}-06-3$ & 9.12 & 9.08 & 0.04 units \\
\hline $\mathrm{BpH}-06-4$ & 7.35 & 7.45 & 0.10 units \\
\hline BpH-07-1 & 3.98 & 4.05 & 0.07 units \\
\hline $\mathrm{BpH}-07-2$ & 10.25 & 9.96 & 0.29 units \\
\hline $\mathrm{BpH}-07-3$ & 8.87 & 9.00 & 0.03 units \\
\hline BpH-08-1 & 4.06 & 4.30 & 0.24 units \\
\hline BpH-08-2 & 10.05 & 10.16 & 0.11 units \\
\hline $\mathrm{BpH}-08-3$ & 9.10 & 9.28 & 0.18 units \\
\hline $\mathrm{BpH}-09-1$ & 6.86 & 6.86 & 0.00 units \\
\hline $\mathrm{BpH}-09-2$ & 8.70 & 9.04 & 0.34 units \\
\hline $\mathrm{BpH}-09-3$ & 4.00 & 4.03 & 0.03 units \\
\hline $\mathrm{BpH}-09-4$ & 9.40 & 10.10 & 0.70 units \\
\hline $\mathrm{BpH}-10-1$ & 6.86 & 6.81 & 0.05 units \\
\hline $\mathrm{BpH}-10-2$ & 9.00 & 8.97 & 0.03 units \\
\hline $\mathrm{BpH}-10-3$ & 4.00 & 4.02 & 0.02 units \\
\hline $\mathrm{BpH}-11-1$ & 3.94 & 4.03 & 0.09 units \\
\hline $\mathrm{BpH}-11-2$ & 10.60 & 10.21 & 0.39 units \\
\hline $\mathrm{BpH}-11-3$ & 6.48 & 6.87 & 0.39 units \\
\hline $\mathrm{BpH}-12-1$ & 4.21 & 4.02 & 0.19 units \\
\hline $\mathrm{BpH}-12-2$ & 10.40 & 10.07 & 0.33 units \\
\hline $\mathrm{BpH}-12-3$ & 6.69 & 6.82 & 0.13 units \\
\hline
\end{tabular}




\section{Table 78}

EMS Blind Sample Results for Tritium and Strontium

Page 1 of 1

$\mathrm{pCl} / \mathrm{mL}$

\begin{tabular}{lrrr}
\cline { 2 - 3 } Sample Number & Measured Value & Actual Value & Ratio \\
\hline Tritium & & & \\
105 & $9.15 \pm 0.92$ & $10.10 \pm 0.8$ & $0.91 \pm 0.12$ \\
106 & $9.36 \pm 0.55$ & $9.88 \pm 0.8$ & $0.95 \pm 0.09$ \\
108 & $4.86 \pm 0.82$ & $6.26 \pm 0.8$ & $0.78 \pm 0.16$ \\
110 & $3.38 \pm 0.46$ & $4.25 \pm 0.8$ & $0.80 \pm 0.18$ \\
111 & $3.18 \pm 0.45$ & $2.60 \pm 0.8$ & $1.22 \pm 0.41$ \\
112 & $4.93 \pm 0.50$ & $5.16 \pm 0.8$ & $0.96 \pm 0.18$ \\
113 & $3.25 \pm 0.80$ & $3.71 \pm 0.8$ & $0.88 \pm 0.29$ \\
116 & $0.84 \pm 0.40$ & $1.98 \pm 0.8$ & $0.42 \pm 0.26$ \\
117 & $10.7 \pm 0.57$ & $11.50 \pm 0.8$ & $0.93 \pm 0.08$ \\
119 & $1.25 \pm 0.40$ & $1 . i 6 \pm 0.8$ & $0.71 \pm 0.39$ \\
121 & $4.01 \pm 0.46$ & $3.85 \pm 0.8$ & $1.04 \pm 0.25$ \\
Strontium & & & \\
109 & $8.1 \pm 1.1$ & $9.7 \pm 2.2$ & $0.84 \pm 0.22$
\end{tabular}




\section{Table 79}

EMS Blind Sample Results for Gross Alpha and Beta and

Gamma-Emitting Radionuclides

Page 1 of 1

\begin{tabular}{|c|c|c|c|c|}
\hline \multirow[b]{2}{*}{ Number } & \multirow[b]{2}{*}{ Nuclide } & \multicolumn{2}{|c|}{ pCI/L } & \multirow[b]{2}{*}{ Ratio } \\
\hline & & Measured Value & Actual Value & \\
\hline
\end{tabular}

Gross Alpha and Beta

120

$$
\begin{aligned}
& \text { Gross Alpha } \\
& \text { Gross Beta }
\end{aligned}
$$

$24 \pm 12$

$19.6 \pm 8.9$

$20 \pm 5$

$1.20 \pm 0.67$

$15 \pm 5$

$1.30 \pm 0.74$

Gamma-Emitting Radionuclides

114

$\begin{array}{lc}\text { Co-60 } & 23 \pm 8 \\ \text { Zn-65 } & 94 \pm 34 \\ \text { Ru-106 } & 115 \pm 82 \\ \text { Ba-133 } & 96 \pm 4\end{array}$

$15 \pm 2$

$1.53 \pm 0.57$

$107 \pm 8$

$0.88 \pm 0.32$

$104 \pm 13$

$1.11 \pm 0.80$

$97 \pm 7$

$0.99 \pm 0.08$ 
Table 80

QAD Interlaboratory Comparison of Analytical Results

Page 1 of 2

\begin{tabular}{|c|c|c|c|c|c|c|c|}
\hline Nucllde & Sample & SAS Value & QAD Valuea & $\begin{array}{c}\text { SRS/QAD } \\
\text { Ratio }\end{array}$ & $\begin{array}{l}\text { No. of } \\
\text { Labs }\end{array}$ & $\begin{array}{c}\text { Mean Value } \\
\text { of Labs }\end{array}$ & $\begin{array}{c}\% \text { Labs } \\
\text { within 3o of } \\
\text { QAD Value }\end{array}$ \\
\hline \multicolumn{8}{|c|}{ Water Samples (pCi/L) } \\
\hline \multirow[t]{5}{*}{ Alpha } & $1-29-93$ & $16 \pm 7$ & $34 \pm 9$ & 0.5 & 187 & $17.09 \pm 7.54$ & 34 \\
\hline & $4-20-93$ & $99 \pm 15$ & $95 \pm 24$ & 1.0 & 167 & $97 \pm 20$ & 90 \\
\hline & $7-23-93$ & $7.67 \pm 4$ & $15 \pm 5$ & 0.5 & 176 & $12.06 \pm 4.28$ & 71 \\
\hline & $10-19-93$ & $38 \pm 7$ & $40 \pm 10$ & 1.0 & 153 & $41 \pm 8$ & 90 \\
\hline & $10-29-93$ & $16 \pm 8$ & $20 \pm 5$ & 0.8 & 182 & $14 \pm 4.6$ & 74 \\
\hline \multirow[t]{2}{*}{$\mathrm{Ba}-133$} & $6-11-93$ & $101 \pm 12$ & $99 \pm 10$ & 1.0 & 174 & $97 \pm 6.9$ & 90 \\
\hline & $11-12-93$ & $77 \pm 10$ & $79 \pm 8$ & 1.0 & 172 & $76.4 \pm 6.3$ & 86 \\
\hline \multirow[t]{5}{*}{ Beta } & $1-29-93$ & $36 \pm 9$ & $44.0 \pm 5.0$ & 0.8 & 194 & $41.99 \pm 7.41$ & 72 \\
\hline & $4-20-93$ & $108 \pm 18$ & $177 \pm 27$ & 0.6 & $17 i$ & $156 \pm 18$ & 86 \\
\hline & $7-23-93$ & $31 \pm 7.5$ & $43 \pm 6.9$ & 0.7 & 203 & $37.65 \pm 8.49$ & 80 \\
\hline & $10-19-93$ & $46 \pm 3$ & $58 \pm 10$ & 0.8 & 156 & $53 \pm 6$ & 93 \\
\hline & $10-29-93$ & $14 \pm 8$ & $15 \pm 5$ & 0.9 & 186 & $17 \pm 5$ & 87 \\
\hline \multirow[t]{4}{*}{ Co-60 } & $4-20-93$ & $42 \pm 8$ & $39 \pm 5$ & 1.1 & 137 & $39.4 \pm 2.9$ & 89 \\
\hline & $6-11-93$ & $17 \pm 3$ & $15 \pm 5$ & 1.1 & 178 & $14.9 \pm 2.1$ & 95 \\
\hline & $10-19-93$ & $15 \pm 5$ & $10 \pm 5$ & 1.5 & 137 & $10.4 \pm 1.6$ & 98 \\
\hline & $11-12-93$ & $29 \pm 7$ & $30 \pm 5$ & 1.0 & 178 & $29.7 \pm 2.3$ & 92 \\
\hline \multirow[t]{3}{*}{ Cs-134 } & $4-20-93$ & $26 \pm 6$ & $27 \pm 5$ & 1.0 & 136 & $25.4 \pm 2.14$ & 90 \\
\hline & $10-19-93$ & $12 \pm 5$ & $12 \pm 5$ & 1.0 & 138 & $9.8 \pm 1.9$ & 99 \\
\hline & $11-12-93$ & $52 \pm 6$ & $59 \pm 5$ & 0.9 & 175 & $54.4 \pm 4.5$ & 74 \\
\hline \multirow[t]{4}{*}{ Cs-137 } & $4-20-93$ & $31 \pm 9$ & $32 \pm 5$ & 1.0 & 137 & $32.6 \pm 2.81$ & 92 \\
\hline & $6-11-93$ & $8 \pm 6$ & $5 \pm 5$ & 1.6 & 175 & $5.8 \pm 1.5$ & 93 \\
\hline & $10-19-93$ & $12 \pm 6$ & $10 \pm 5$ & 1.2 & 138 & $10.9 \pm 1.7$ & 99 \\
\hline & $11-12-93$ & $45 \pm 8$ & $40 \pm 5$ & 1.1 & 174 & $42.1 \pm 3.0$ & 87 \\
\hline \multirow[t]{2}{*}{$\mathrm{H}-3$} & $6-04-93$ & $8903 \pm 240$ & $9844 \pm 984$ & 0.9 & 169 & $9592 \pm 689$ & 95 \\
\hline & $11-05-93$ & $6553 \pm 200$ & $7398 \pm 740$ & 0.9 & 166 & $7216 \pm 575$ & \\
\hline Pu-239 & $1-22-93$ & $16.8 \pm 1.2$ & $20.0 \pm 2.0$ & 0.8 & 62 & $18.53 \pm 1.80$ & 53.4 \\
\hline \multirow[t]{2}{*}{$R u-106$} & $6-11-93$ & $104 \pm 33$ & $119 \pm 12$ & 0.9 & 173 & $104 \pm 13$ & 65 \\
\hline & $11-12-93$ & $198 \pm 60$ & $201 \pm 20$ & 1.0 & 173 & $175 \pm 18.3$ & 64 \\
\hline \multirow[t]{4}{*}{ Sr-89 } & $1-15-93$ & $10.7 \pm 1.1$ & $15 \pm 5$ & 0.7 & 95 & $14.53 \pm 3.12$ & 95 \\
\hline & $4-20-93$ & $40 \pm 4.7$ & $41 \pm 5$ & 1.0 & 94 & $37.6 \pm 8.3$ & 67 \\
\hline & $7-16-93$ & $37 \pm 6.3$ & $34 \pm 5$ & 1.1 & 100 & $34.2 \pm 6.67$ & 78 \\
\hline & $10-19-93$ & $16 \pm 3.5$ & $15 \pm 5$ & 1.1 & 91 & $14.0 \pm 3.0$ & 95 \\
\hline
\end{tabular}

a Environmental Protection Agency Quality Assurance Division (QAD)

b Percentage of participating laboratories that had results falling within $3 \sigma$ of $Q A D$ value 
Table 80

QAD Interlaboratory Comparison of Analytical Results

Page 2 of 2

\begin{tabular}{|c|c|c|c|c|c|c|c|}
\hline Nuclide & $\begin{array}{c}\text { Sample } \\
\text { Date }\end{array}$ & SRS Value & QAD Value日 & $\begin{array}{c}\text { SRS/QAD } \\
\text { Ratlo }\end{array}$ & $\begin{array}{l}\text { No. of } \\
\text { Labs }\end{array}$ & $\begin{array}{c}\text { Mean Value } \\
\text { of Labs }\end{array}$ & $\begin{array}{l}\% \text { Labs } \\
\text { within } 30 \text { of } \\
\text { QAD Valueb }\end{array}$ \\
\hline \multirow[t]{4}{*}{ Sr-90 } & $1-15-93$ & $7.0 \pm 1.2$ & $10.0 \pm 5$ & 0.7 & 101 & $9.66 \pm 2.20$ & 98 \\
\hline & $4-20-93$ & $23.7 \pm 4.5$ & $29 \pm 5$ & 0.8 & 101 & $27.8 \pm 3.4$ & 88 \\
\hline & $7-16-93$ & $23 \pm 4.1$ & $25 \pm 5$ & 0.9 & 108 & $24.0 \pm 3.1$ & 92 \\
\hline & $10-19-93$ & $7 \pm 3$ & $10 \pm 5$ & 0.7 & 102 & $10.3 \pm 2.0$ & 97 \\
\hline \multirow[t]{4}{*}{$U$} & $2-12-93$ & $6.67 \pm 15$ & $7.6 \pm 3$ & 0.9 & 121 & $7.16 \pm 1.04$ & 97.5 \\
\hline & $4-20-93$ & $31.6 \pm 0.6$ & $28.9 \pm 3$ & 1.1 & 122 & $27.6 \pm 5.0$ & 75.4 \\
\hline & $8-13-93$ & $23.6 \pm 0.5$ & $25.3 \pm 3$ & 0.9 & 138 & $24.9 \pm 2.8$ & 83 \\
\hline & $10-19-93$ & $24.1 \pm 0.6$ & $15.1 \pm 3$ & 1.6 & 118 & $14.4 \pm 2.63$ & 87 \\
\hline \multirow[t]{2}{*}{$Z n-65$} & $6-11-93$ & $105 \pm 20$ & $103 \pm 10$ & 1.0 & 176 & $107.5 \pm 7.8$ & 86 \\
\hline & $11-12-93$ & $163 \pm 25$ & $150 \pm 15$ & 1.1 & 168 & $156 \pm 9.2$ & 88 \\
\hline
\end{tabular}

Air Filter Samples (pci/filter)

\begin{tabular}{lllllllll} 
Alpha & $8-27-93$ & $23.67 \pm 3$ & $19 \pm 5$ & 1.2 & 155 & $20 \pm 3.27$ & 91 \\
\hline Beta & $8-27-93$ & $50.33 \pm 3.2$ & $47 \pm 5$ & 1.1 & 151 & $49.32 \pm 4.62$ & 70 \\
\hline Cs-137 & $8-27-93$ & $10.33 \pm 5$ & $9 \pm 5$ & 1.1 & 141 & $10 \pm 1.62$ & 97
\end{tabular}

Milk Samples (pCl/L)

\begin{tabular}{ccccccccc} 
Cs-137 & $9-24-93$ & $55 \pm 6$ & $49 \pm 5$ & 1.1 & 90 & $50 \pm 3$ & 96 \\
\hline I-131 & $9-24-93$ & $116 \pm 10$ & $120 \pm 12$ & 1.0 & 84 & $120 \pm 8$ & 92 \\
\hline K-40 & $9-24-93$ & $1754 \pm 188$ & $1679 \pm 84$ & 1.0 & 77 & $1674 \pm 95$ & 80 \\
\hline Sr-90 & $9-24-93$ & $11 \pm 3$ & $25 \pm 5$ & 0.4 & 49 & $20 \pm 5.2$ & 76
\end{tabular}

a Environmental Protection Agency Quality Assurance Division (QAD)

b Percentage of participating laboratories that had results falling within $3 \sigma$ of $Q A D$ value 


\section{Table 81}

QAP Interlaboratory Comparison of Analytical Results

Page 1 of 2

\begin{tabular}{|c|c|c|c|c|c|c|}
\hline Nuclide & SRS Valuea & QAP Valueb & $\begin{array}{l}\text { SRBI } \\
\text { QAP } \\
\text { Ratlo }\end{array}$ & $\begin{array}{c}\text { No. of } \\
\text { Labs }\end{array}$ & $\begin{array}{l}\text { Mean } \\
\text { Value } \\
\text { of Labs }\end{array}$ & $\begin{array}{c}\text { Labs 20\% } \\
\text { QAP } \\
\text { Valueb }\end{array}$ \\
\hline \multicolumn{7}{|c|}{ March-June 1993} \\
\hline \multicolumn{7}{|l|}{ Alr } \\
\hline $\mathrm{Be}-7$ & $27 . \pm 3$ & 27.4 & 0.99 & 57 & 26.9 & $84 \%$ \\
\hline Ce-144 & 16. \pm 0.5 & 19.3 & 0.83 & 52 & 16.4 & $61 \%$ \\
\hline Co-57 & $2.4 \pm 0.2$ & 2.71 & 0.89 & 54 & 2.39 & $76 \%$ \\
\hline Co-60 & $1.7 \pm 0.3$ & 1.7 & 1.00 & 55 & 1.66 & $85 \%$ \\
\hline Cs-134 & 2. \pm 0.3 & 1.96 & 1.02 & 55 & 1.99 & $92 \%$ \\
\hline Cs-137 & 3. \pm 0.1 & 3.07 & 0.98 & 57 & 3.08 & $84 \%$ \\
\hline$M n-54$ & 11. \pm 0.5 & 11.7 & 0.94 & 55 & 11.6 & $91 \%$ \\
\hline Pu-238 & $0.0285 \pm 0.005$ & 0.0363 & 0.79 & 29 & 0.0335 & $69 \%$ \\
\hline Pu-239 & $0.0186 \pm 0.005$ & 0.0234 & 0.79 & 32 & 0.0231 & $85 \%$ \\
\hline$U$ & $0.95 \pm 0.04$ & 1.8 & 0.53 & 12 & 1.87 & $71 \%$ \\
\hline \multicolumn{7}{|l|}{ Soll } \\
\hline Cs- 137 & 1336. \pm 57 & 923. & 1.45 & 61 & 996. & $72 \%$ \\
\hline$K-40$ & 430. \pm 23 & 321. & 1.34 & 55 & 327. & $75 \%$ \\
\hline Pu-239 & $10.58 \pm 0.851$ & 11.6 & 0.91 & 50 & 10.9 & $73 \%$ \\
\hline Sr-Q0 & $17.7 \pm 4.4$ & 41.7 & 0.42 & 37 & 37.4 & $64 \%$ \\
\hline \multicolumn{7}{|l|}{ Vegetation } \\
\hline Cs-137 & $30 . \pm 1.2$ & 24.6 & 1.22 & 50 & 26.6 & $58 \%$ \\
\hline$K-40$ & $440 . \pm 60$ & 383. & 1.15 & 44 & 399. & $65 \%$ \\
\hline Pu-238 & $1.33 \pm .123$ & 1.14 & 1.17 & 24 & 1.22 & $68 \%$ \\
\hline Pu-239 & $.444 \pm .089$ & .323 & 1.37 & 25 & .347 & $45 \%$ \\
\hline Sr-90 & $189.1 \pm 18.9$ & 237. & 0.80 & 25 & 215 & $51 \%$ \\
\hline \multicolumn{7}{|l|}{ Water } \\
\hline Ce-144 & $88 . \pm 8$ & 83.6 & 1.05 & 59 & 87. & $90 \%$ \\
\hline Co-60 & $47 . \pm 2$ & 45.3 & 1.04 & 62 & 47.5 & $90 \%$ \\
\hline Cs-134 & 44. \pm 1 & 42.4 & 1.04 & 61 & 46.1 & $90 \%$ \\
\hline Cs-137 & 55. \pm 2 & 50.8 & 1.08 & 63 & 54.9 & $87 \%$ \\
\hline $\mathrm{H}-3$ & 75. \pm 9.7 & 97. & 0.77 & 43 & 95.6 & $62 \%$ \\
\hline$M n-54$ & 109. \pm 3 & 105. & 1.04 & 61 & 109. & $93 \%$ \\
\hline Pu-238 & $0.481 \pm 0.037$ & 0.494 & 0.97 & 48 & 0.502 & $84 \%$ \\
\hline Pu-239 & $0.814 \pm 0.074$ & 0.828 & 0.98 & 47 & 0.844 & $86 \%$ \\
\hline Sr-90 & $0.99 \pm 0.85$ & 1.03 & 0.96 & 34 & 1.17 & $35 \%$ \\
\hline$U$ & $0.0111 \pm 0.0003$ & 0.0117 & 0.95 & 23 & 0.012 & $84 \%$ \\
\hline
\end{tabular}

\footnotetext{
Values are $B q / L, B q / K g, B q / f i l t e r$, except $U$, whose values are $u g / g$ and $u g / f i l t e r$.

Quality Assessment Program (QAP) conducted by the DOE Environmental Measurements Laboratory (EML)

Percentage of participating laboratories that had results falling between the $\pm 20 \%$ acceptance range
} 
Table 81

QAP Interlaboratory Comparison of Analytical Results

Page 2 of 2

\begin{tabular}{|c|c|c|c|c|c|c|}
\hline Nuclide & SRS Valuea & QAP Valueb & $\begin{array}{l}\text { SRSY } \\
\text { QAP } \\
\text { Ratlo }\end{array}$ & $\begin{array}{l}\text { No. of } \\
\text { Labs }\end{array}$ & $\begin{array}{l}\text { Mean } \\
\text { Value } \\
\text { of Labs }\end{array}$ & $\begin{array}{c}\text { Labs 20\% } \\
\text { QAP } \\
\text { Valueb }\end{array}$ \\
\hline \multicolumn{7}{|c|}{ September-December 1993} \\
\hline \multicolumn{7}{|c|}{ Alr } \\
\hline $\mathrm{Ce}-144$ & $34.5 \pm 1.4$ & 40.3 & 0.86 & 54 & 34.6 & $48 \%$ \\
\hline Co-57 & $15.4 \pm .6$ & 17.3 & 0.89 & 54 & 15.1 & $52 \%$ \\
\hline Co-60 & $20.2 \pm .9$ & 20.5 & 0.99 & 58 & 19.4 & $85 \%$ \\
\hline Cs-134 & $12.2 \pm 1.8$ & 12.2 & 1.00 & 57 & 12.1 & $80 \%$ \\
\hline Cs-137 & $18.5 \pm .8$ & 18.8 & 0.98 & 60 & 18.5 & $78 \%$ \\
\hline$M n-54$ & $15.2 \pm 1.1$ & 15.4 & 0.99 & 56 & 14.9 & $85 \%$ \\
\hline Pu-238 & $.121 \pm .01$ & .129 & 0.94 & 42 & .125 & $90 \%$ \\
\hline Pu-239 & $.073 \pm .006$ & .08 & 0.91 & 39 & .0814 & $75 \%$ \\
\hline $\mathrm{Sb}-125$ & $17.8 \pm 1.1$ & 17.4 & 1.02 & 44 & 17.8 & $78 \%$ \\
\hline Sr-90 & $.9 \pm .1$ & .762 & 1.18 & 33 & .805 & $62 \%$ \\
\hline U & $2.05 \pm .05$ & 5.41 & 0.38 & 22 & 5.3 & $79 \%$ \\
\hline \multicolumn{7}{|l|}{ Soll } \\
\hline Cs-137 & $11 . \pm 1$ & 11.4 & 0.96 & 61 & 12.5 & $72 \%$ \\
\hline$K-40$ & $28 . \pm 4$ & 28.6 & 0.98 & 40 & 29.9 & $44 \%$ \\
\hline Pu-239 & $2.048 \pm .183$ & 1.52 & 1.35 & 34 & 1.63 & $26 \%$ \\
\hline \multicolumn{7}{|l|}{ Vegetation } \\
\hline Co-60 & $7.6 \pm 2.4$ & 6.45 & 1.18 & 45 & 7.13 & $63 \%$ \\
\hline Cs-137 & $94 . \pm 4$ & 89.2 & 1.05 & 56 & 97.2 & $78 \%$ \\
\hline $\mathrm{K}-40$ & $955 . \pm 47$ & 842. & 1.13 & 53 & 946. & $75 \%$ \\
\hline Pu-238 & $.405 \pm .045$ & .463 & 0.87 & 28 & .432 & $68 \%$ \\
\hline Pu-239 & $.807 \pm .087$ & .965 & 0.84 & 29 & .912 & $72 \%$ \\
\hline Sr-90 & 179. \pm 16 & 221. & 0.81 & 30 & 207. & $70 \%$ \\
\hline $\mathrm{Ce}-144$ & $185 . \pm 24$ & 173. & 1.07 & 66 & 175. & $89 \%$ \\
\hline \multicolumn{7}{|l|}{ Water } \\
\hline Co-60 & $105 . \pm 7$ & 99.6 & 1.05 & 71 & 103. & $90 \%$ \\
\hline Cs-134 & 59. \pm 7 & 56.1 & 1.05 & 71 & 58.6 & $84 \%$ \\
\hline Cs-137 & 79. \pm 8 & 75.5 & 1.05 & 73 & 79.1 & $87 \%$ \\
\hline $\mathrm{H}-3$ & $221 . \pm 12$ & 270 & 0.82 & 42 & 255. & $69 \%$ \\
\hline$M n-54$ & $114 . \pm 11$ & 109. & 1.05 & 70 & 115 & $90 \%$ \\
\hline Pu-239 & $.312 \pm .038$ & .338 & 0.92 & 43 & .336 & $71 \%$ \\
\hline Sr-90 & $2.7 \pm 1.3$ & 2.52 & 1.07 & 39 & 2.61 & $70 \%$ \\
\hline U & $.068 \pm .002$ & .0842 & 0.81 & 24 & .0836 & $85 \%$ \\
\hline $\begin{array}{ll}\text { a } & \text { Values a } \\
\text { b } & \text { Quality } \\
\text { c } & \text { Percent }\end{array}$ & $\begin{array}{l}\text { Bq/filter, excep } \\
\text { ogram (QAP) co } \\
\text { ting laboratories }\end{array}$ & $\begin{array}{l}\text { alues are } u g / g \text { and } \\
\text { le DOE Environme } \\
\text { ults falling betweer }\end{array}$ & $\begin{array}{l}\text { /ilter. } \\
\text { l Measur } \\
\pm 20 \% \text { a }\end{array}$ & $\begin{array}{l}\text { ents Labc } \\
\text { eptance } r\end{array}$ & $\begin{array}{l}\text { atory (EML) } \\
\text { ige }\end{array}$ & \\
\hline
\end{tabular}


Table 82

NPDES Blind Sample Results

Page 1 of 1

First Quarter

\begin{tabular}{llcccc} 
NPDES 8ite & Parameter Sampled & Unlts & NPDEs Result & $\begin{array}{c}\text { Blind } \\
\text { Sample } \\
\text { Result }\end{array}$ & Difference \\
\hline $\mathrm{A}-003$ & Chromium & $\mathrm{mg} / \mathrm{L}$ & $<0.02$ & $<0.02$ & $<0.02$ \\
$\mathrm{D}-003$ & Total suspended solids & $\mathrm{mg} / \mathrm{L}$ & 2 & 2 & 0 \\
$\mathrm{D}-003$ & Oil and grease & $\mathrm{mg} / \mathrm{L}$ & $<1$ & $<1$ & $<1$ \\
$\mathrm{~F}-003$ & Total suspended solids & $\mathrm{mg} / \mathrm{L}$ & $<1$ & 2 & $<2$ \\
$\mathrm{~F}-003$ & Oil and grease & $\mathrm{mg} / \mathrm{L}$ & $<1$ & $<1$ & $<1$
\end{tabular}

Third Quarter

\begin{tabular}{lllccc} 
NPDES Site & Parameter Sampled & Units & NPDES Result & $\begin{array}{c}\text { Blind } \\
\text { Sample } \\
\text { Result }\end{array}$ & Difference \\
\hline $\mathrm{A}-001$ & Total suspended solids & $\mathrm{mg} / \mathrm{L}$ & 1 & $<1$ & $<1$ \\
$\mathrm{~A}-001$ & Oil and grease & $\mathrm{mg} / \mathrm{L}$ & $<1$ & $<1$ & $<1$ \\
$\mathrm{~A}-011$ & Oil and grease & $\mathrm{mg} / \mathrm{L}$ & $<1$ & $<1$ & $<1$ \\
$\mathrm{~A}-011$ & Total suspended solids & $\mathrm{mg} / \mathrm{L}$ & $<1$ & 1 & $<1$ \\
$\mathrm{M}-004$ & Nitrates & $\mathrm{mg} / \mathrm{L}$ & 1360 & 1530 & 170 \\
$\mathrm{M}-004$ & Nickel & $\mathrm{mg} / \mathrm{L}$ & $<0.03$ & $<0.03$ & $<0.03$ \\
X-008 & Iron & $\mathrm{mg} / \mathrm{L}$ & 1.02 & 1.04 & 0.02 \\
X-008 & Aluminum & $\mathrm{mg} / \mathrm{L}$ & $<0.05$ & $<0.05$ & $<0.05$
\end{tabular}


Table 83

NPDES Duplicate Sample Results

Page 1 of 6

\begin{tabular}{|c|c|c|c|c|}
\hline \multicolumn{5}{|c|}{ First Quarter } \\
\hline Sample & Analyte & Result 1 & Result 2 & Difference \\
\hline$A-001$ & Biochemical oxygen demand, $\mathrm{mg} / \mathrm{L}$ & $<1.3$ & $<1$ & $<1.3$ \\
\hline A-001 & Oil and grease, $\mathrm{mg} / \mathrm{L}$ & $<1$ & 1 & $<1$ \\
\hline A-003 & Total suspended solids, mg/L & $<1$ & $<1$ & $<1$ \\
\hline A-003 & Oil and grease, $\mathrm{mg} / \mathrm{L}$ & $<1.2$ & $<1$ & $<1.2$ \\
\hline A-003 & Chromium, mg/L & 0.065 & 0.068 & 0.003 \\
\hline$A-003$ & Chromium, mg/L & $<.02$ & 0.095 & $>.075$ \\
\hline$A-003$ & Chromium, mg/L & $<.02$ & $<.02$ & $<.02$ \\
\hline A-005 & Oil and grease, $\mathrm{mg} / \mathrm{L}$ & $<1$ & $<1$ & $<1$ \\
\hline A-005 & Total suspended solids, $\mathrm{mg} / \mathrm{L}$ & $<2$ & $<2$ & $<1$ \\
\hline$A-011$ & Total suspended solids, mg/L & $<1$ & $<1$ & $<1$ \\
\hline$A-011$ & Oil and grease, $\mathrm{mg} / \mathrm{L}$ & 1.3 & 1.2 & 1.1 \\
\hline A-014 & Oil and grease, $\mathrm{mg} / \mathrm{L}$ & $<1$ & $<1$ & $<1$ \\
\hline$A-014$ & Total suspended solids, mg/L & $<1$ & $<1$ & $<1$ \\
\hline C-001 & Oil and grease, $\mathrm{mg} / \mathrm{L}$ & $<1$ & $<1$ & $<1$ \\
\hline$c-001$ & Total suspended solids, mg/L & 1 & 1 & $<1$ \\
\hline$D-001$ & Oil and grease, $\mathrm{mg} / \mathrm{L}$ & $<1$ & 2.9 & $<2.9$ \\
\hline D-001 & Total suspended solids, mg/L & 17 & 18 & 1 \\
\hline C-003 & Total suspended solids, mg/L & $<1$ & $<1$ & $<1$ \\
\hline $\mathrm{C}-003$ & Oil and grease, $\mathrm{mg} / \mathrm{L}$ & 1.5 & $<1$ & $<1.5$ \\
\hline $\mathrm{D}-001 \mathrm{C}$ & Total suspended solids, mg/L & 3 & 3 & 0 \\
\hline D-003 & Total suspended solids, mg/L & 2 & 2 & 0 \\
\hline$D-003$ & Oil and grease, $\mathrm{mg} / \mathrm{L}$ & $<1$ & $<1$ & $<1$ \\
\hline$D-006$ & Total suspended solids, mg/L & 27 & 29 & 2 \\
\hline$D-006$ & Oil and grease, $\mathrm{mg} / \mathrm{L}$ & $<1$ & $<1$ & $<1$ \\
\hline $\mathrm{F}-001$ & Total suspended solids, mg/L & 7 & 1 & 6 \\
\hline$F-001$ & Oil and grease, $\mathrm{mg} / \mathrm{L}$ & $<1$ & 1.1 & $<1.1$ \\
\hline$F-002$ & Total suspended solids, mg/L & 1 & $<1$ & $<1$ \\
\hline $\mathrm{F}-002$ & Oil and grease, $\mathrm{mg} / \mathrm{L}$ & $<1$ & $<1$ & $<1$ \\
\hline
\end{tabular}




\section{Table 83}

\section{NPDES Duplicate Sample Results}

Page 2 of 6

\begin{tabular}{llccc}
\hline $\begin{array}{l}\text { Flrat Quarter } \\
\text { Sample }\end{array}$ & Analyte & Reault 1 & Result 2 & Difference \\
\hline F-003 & Oil and grease, $\mathrm{mg} / \mathrm{L}$ & $<1$ & $<1$ & $<1$ \\
$\mathrm{~F}-003$ & Oil and grease, $\mathrm{mg} / \mathrm{L}$ & $<1$ & $<1$ & $<1$ \\
$\mathrm{~F}-003$ & Biochemical oxygen demand, $\mathrm{mg} / \mathrm{L}$ & $<1$ & $<1$ & $<1$ \\
$\mathrm{~F}-003$ & Total suspended solids, $\mathrm{mg} / \mathrm{L}$ & $<1$ & $<1$ & $<1$ \\
$\mathrm{H}-016$ & Ammonia, $\mathrm{mgN} / \mathrm{L}$ & $<0.05$ & $<0.05$ & $<.05$ \\
$\mathrm{M}-004$ & Nitrate, $\mathrm{mg} / \mathrm{L}$ & 51.1 & 50.1 & 1 \\
$\mathrm{M}-004$ & Uranium, $\mathrm{mg} / \mathrm{L}$ & 0.128 & 0.115 & 0.013 \\
$\mathrm{SC}-004$ & Lead, $\mathrm{mg} / \mathrm{L}$ & $<.003$ & $<.003$ & $<.003$ \\
$\mathrm{SC}-004$ & Phosphate, $\mathrm{mgP} / \mathrm{L}$ & 0.023 & 0.023 & .0 \\
$\mathrm{SC}-004$ & Phosphate, $\mathrm{mgP} / \mathrm{L}$ & 0.025 & 0.02 & 0.005
\end{tabular}


Table 83

\section{NPDES Duplicate Sample Results}

Page 3 of 6

\begin{tabular}{|c|c|c|c|c|}
\hline \multicolumn{5}{|c|}{ Beoond Quartor } \\
\hline Sample & Analyte & Result 1 & Reoult 2 & Difforenoe \\
\hline DW-002 & Oil and grease, $\mathrm{mg} / \mathrm{L}$ & $<1$ & $<1$ & $<1$ \\
\hline$F-005$ & Oil and grease, $\mathrm{mg} / \mathrm{L}$ & $<1$ & $<1$ & $<1$ \\
\hline$F-005$ & Total suspended solids, mg/L & 1 & 2 & 1 \\
\hline$F-008$ & Total suspended solids, mg/L & 2 & 3 & 1 \\
\hline$F-008$ & Total suspended solids, mg/L & 2 & 2 & 0 \\
\hline$F-008$ & Oil and grease, $\mathrm{mg} / \mathrm{L}$ & 2.7 & 2.1 & 0.6 \\
\hline $\mathrm{H}-002$ & Oil and grease, $\mathrm{mg} / \mathrm{L}$ & $<1$ & $<1$ & $<1$ \\
\hline $\mathrm{H}-002$ & Total suspended solids, mg/L & 2 & 2 & 0 \\
\hline $\mathrm{H} 004$ & Oll and grease, $\mathrm{mg} / \mathrm{L}$ & 2.4 & 3.1 & 0.7 \\
\hline$H-008$ & Total suspended solids, mg/L & 3 & 2 & 1 \\
\hline$H-008$ & Oil and grease, mg/L & $<1$ & $<1$ & $<1$ \\
\hline$H-016$ & Oil and grease, mg/L & $<1$ & $<1$ & $<1$ \\
\hline$H-016$ & Total suspended solids, mg/L & $<1$ & $<1$ & $<1$ \\
\hline$H-016$ & Zinc, mg/L & 0.016 & $<.01$ & $<.016$ \\
\hline$K-006$ & Oil and grease, $\mathrm{mg} / \mathrm{L}$ & $<1$ & $<1$ & $<1$ \\
\hline$k-018$ & Aluminum, $\mathrm{mg} / \mathrm{L}$ & $<1$ & $<1$ & $<1$ \\
\hline$K-018$ & Total suspended solids, $\mathrm{mg} / \mathrm{L}$ & 5 & 4 & 1 \\
\hline$K-018$ & Oil and grease, $\mathrm{mg} / \mathrm{L}$ & $<1$ & $<1$ & $<1$ \\
\hline$L-008$ & Total suspended solids, mg/L & $<1$ & $<1$ & $<1$ \\
\hline$L-008$ & Oil and grease, $\mathrm{mg} / \mathrm{L}$ & $<1$ & 1.3 & $<1.3$ \\
\hline$M-004$ & Mercury, mg/L & $<.0001$ & $<.0001$ & $<.0001$ \\
\hline$M-004$ & Phosphate, mgP/L & 4.68 & 6.03 & 1.35 \\
\hline$M-004$ & Nitrate, $\mathrm{mg} / \mathrm{L}$ & 1090 & 1330 & 240 \\
\hline$M-004$ & Total suspended solids, mg/L & 4 & 4 & 0 \\
\hline$M-004$ & Aluminum, $\mathrm{mg} / \mathrm{L}$ & 0.3 & 0.127 & .0 .173 \\
\hline sc-004 & Cadmium, mg/L & $<.01$ & $<.01$ & $<.01$ \\
\hline SC-004 & Phosphate, mgP/L & $<.003$ & $<.003$ & $<.003$ \\
\hline
\end{tabular}


Table 83

NPDES Duplicate Sample Resulte

Page 4 of 8

\begin{tabular}{|c|c|c|c|c|}
\hline \multicolumn{5}{|c|}{ Third Quartor } \\
\hline Sample & Analyte & Rosult 1 & Result 2 & Difference \\
\hline$A-001$ & Total suspended sollds, mg/L & $<1$ & $<1$ & $<1$ \\
\hline$A-001$ & Oll and grease, $\mathrm{mg} / \mathrm{L}$ & $<1$ & $<1$ & $<1$ \\
\hline$A-001$ & Total suspended solids, $\mathrm{mg} / \mathrm{L}$ & 1 & $<1$ & $<1$ \\
\hline$D-001$ & Total suspended solids, mg/L & 10 & 10 & 0 \\
\hline D-001 & Oll and grease, $\mathrm{mg} / \mathrm{L}$ & $<1$ & $<1$ & $<1$ \\
\hline$A-003$ & Total suspended solids, $\mathrm{mg} / \mathrm{L}$ & $<1$ & $<1$ & $<1$ \\
\hline$A-003$ & Oll and grease, $\mathrm{mg} / \mathrm{L}$ & $<1$ & $<1$ & $<1$ \\
\hline$A-011$ & Total suspended solids, $\mathrm{mg} / \mathrm{L}$ & $<1$ & $<1$ & $<1$ \\
\hline$A-011$ & Oil and grease, $\mathrm{mg} / \mathrm{L}$ & $<1$ & $<1$ & $<1$ \\
\hline C-004 & Oil and grease, $\mathrm{mg} / \mathrm{L}$ & $<1$ & $<1$ & $<1$ \\
\hline$C-004$ & Total suspended solids, $\mathrm{mg} / \mathrm{L}$ & 2 & 2 & 0 \\
\hline$D-006$ & Total suspended solids, mg/L & 6 & 6 & 0 \\
\hline$D-006$ & Oil and grease, $\mathrm{mg} / \mathrm{L}$ & $<1$ & $<1$ & $<1$ \\
\hline$F-001$ & Total suspended solids, mg/L & $<1$ & $<1$ & $<1$ \\
\hline$F-001$ & Oil and grease, $\mathrm{mg} / \mathrm{L}$ & $<1$ & $<1$ & $<1$ \\
\hline $\mathrm{H}-012$ & Oil and grease, $\mathrm{mg} / \mathrm{L}$ & $<1$ & $<1$ & $<1$ \\
\hline$H-012$ & Sulfate, $m g / L$ & 16.6 & 16.2 & 0.4 \\
\hline$H-016$ & Biochemical Oxygen demand, $\mathrm{mg} / \mathrm{L}$ & 4.1 & 3.4 & $<.05$ \\
\hline $\mathrm{H}-016$ & Nickel, mg/L & $<.05$ & $<.05$ & $<.05$ \\
\hline$M-004$ & Oil and grease, $\mathrm{mg} / \mathrm{L}$ & 1.5 & $<1$ & $<1.5$ \\
\hline$M-004$ & Nickel, mg/L & $<0.03$ & $<0.0138$ & $<.0138$ \\
\hline$M-004$ & Total suspended solids, mg/L & 4 & 2 & 2 \\
\hline$M-004$ & Aluminum, Mg/L & 0.255 & 0.388 & 0.133 \\
\hline$M-004$ & Nickel, mg/L & $<.25$ & $<.25$ & $<.25$ \\
\hline$M-004$ & Nitrate, mgN/L & 1360 & 1280 & 80 \\
\hline$P-007$ & Aluminum, $\mathrm{mg} / \mathrm{L}$ & 0.075 & 0.06 & 0.015 \\
\hline P-007 & Iron, $\mathrm{mg} / \mathrm{L}$ & 0.237 & 0.222 & 0.015 \\
\hline P-013 & Total suspended solids, $\mathrm{mg} / \mathrm{L}$ & 8 & 7 & 1 \\
\hline$P-013$ & Oil and grease, $\mathrm{mg} / \mathrm{L}$ & $<1$ & $<1$ & $<1$ \\
\hline$P-013$ & Total suspended solids, $\mathrm{mg} / \mathrm{L}$ & $<1$ & $<1$ & $<1$ \\
\hline P-013 & Oil and grease, $\mathrm{mg} / \mathrm{L}$ & 1.6 & $<1$ & $<1.6$ \\
\hline$P-019$ & Oil and grease, mg/L & $<1$ & $<1$ & $<1$ \\
\hline P-019 & Total suspended solids, $\mathrm{mg} / \mathrm{L}$ & 6 & 6 & 0 \\
\hline
\end{tabular}




\section{Table 83}

NPDES Dupllcate Sample Resulte

Page 5 of 0

\begin{tabular}{|c|c|c|c|c|}
\hline \multicolumn{5}{|c|}{ Thled Quarter } \\
\hline Samplo & Analyte & Recult 1 & Result 2 & Difference \\
\hline SC-0C4 & Silver, mg/L & $=.03$ & $<.03$ & $<.03$ \\
\hline sc-004 & Phosphate, mgP/L & 0.037 & 0.034 & 0.003 \\
\hline$x-004$ & Total suspended solids, $\mathrm{mg} / \mathrm{L}$ & 3 & 3 & 0 \\
\hline$x-004$ & Oil and grease, $\mathrm{mg} / \mathrm{L}$ & $<1$ & 1.1 & $<1.1$ \\
\hline$x-008$ & Oil and grease, $\mathrm{mg} / \mathrm{L}$ & 1.9 & 1.5 & 0.4 \\
\hline$x-008$ & Total suspended solids, $\mathrm{mg} / \mathrm{L}$ & 6 & 7 & 1 \\
\hline$x-008$ & Aluminum, mg/L & $<.05$ & $<.05$ & $<.05$ \\
\hline$x-008$ & Aluminum, $\mathrm{mg} / \mathrm{L}$ & $<.05$ & $<.05$ & $<.05$ \\
\hline$x-008$ & Iron, $\mathrm{mg} / \mathrm{L}$ & 1.02 & 0.986 & 0.034 \\
\hline$x-008$ & Iron, mg/L & 1.05 & 1.05 & 0 \\
\hline$x-014$ & Oil and grease, mg/L & $<1$ & $<1$ & $<1$ \\
\hline$x-014$ & Total organic carbon, mg/L & 3.29 & 2.83 & 1.66 \\
\hline$x-014$ & Biochemical oxygen demand, $\mathrm{mg} / \mathrm{L}$ & 1 & $<1$ & $<1$ \\
\hline$x-014$ & Total suspended solids, mg/L & 3 & 3 & 0 \\
\hline
\end{tabular}




\section{Table 83}

\section{NPDES Duplicate Sample Results}

Page 6 of 0

\begin{tabular}{llccc}
\hline $\begin{array}{l}\text { Fourth Quarter } \\
\text { sample }\end{array}$ & Analyte & Reault 1 & Result 2 & Difterenos \\
\hline F-001 & Total suspended solids, $\mathrm{mg} / \mathrm{L}$ & 9 & 8 & 1 \\
F-001 & Oil and grease, $\mathrm{mg} / \mathrm{L}$ & $<1$ & $<1$ & $<1$ \\
$\mathrm{~F}-002$ & Total suspended solids, $\mathrm{mg} / \mathrm{L}$ & $<1$ & $<1$ & $<1$ \\
$\mathrm{~F}-002$ & Oil and grease, $\mathrm{mg} / \mathrm{L}$ & 1.4 & $<1$ & $<1.4$ \\
$\mathrm{~F}-003$ & Total suspended solids, $\mathrm{mg} / \mathrm{L}$ & 9 & 9 & 0 \\
$\mathrm{~F}-005$ & Oil and grease, $\mathrm{mg} / \mathrm{L}$ & $<1$ & 1.2 & $<1.2$ \\
$\mathrm{~F}-005$ & Total suspended solids, $\mathrm{mg} / \mathrm{L}$ & 10 & 11 & 1 \\
$\mathrm{~F}-008$ & Oil and grease, $\mathrm{mg} / \mathrm{L}$ & $<1$ & $<1$ & $<1$ \\
$\mathrm{H}-004$ & Total suspended solids, $\mathrm{mg} / \mathrm{L}$ & 1 & $<1$ & $<1$ \\
$\mathrm{H}-004$ & Oil and grease, $\mathrm{mg} / \mathrm{L}$ & $<1$ & $<1$ & $<1$ \\
$\mathrm{H}-008$ & Oil and grease, $\mathrm{mg} / \mathrm{L}$ & $<1$ & $<1$ & 1 \\
$\mathrm{H}-008$ & Total suspended solids, $\mathrm{mg} / \mathrm{L}$ & 2 & 1 & $<.003$ \\
SC-004 & Selenium, $\mathrm{mg} / \mathrm{L}$ & $<.003$ & $<.003$ &
\end{tabular}




\section{Table 84}

\section{Release and Historical Data from the 195-Foot F-Area Stack}

Page 1 of 1

\begin{tabular}{|c|c|c|c|c|}
\hline Radionuclide & Period & $\begin{array}{l}\text { Release } \\
\text { (mCl) }\end{array}$ & $\begin{array}{l}\text { Concentration } \\
\left(\mathrm{pCl} / \mathrm{m}^{3}\right)\end{array}$ & Type \\
\hline \multirow[t]{3}{*}{ Pu-238 } & December 27-28, 1993 & 166 & 14.5 & a \\
\hline & December 26-30, 1993 & 188 & 3.9 & b \\
\hline & 1993 Weekly Average ${ }^{c}$ & 2 & .03 & b \\
\hline \multirow[t]{3}{*}{ Pu-239 } & December 27-28, 1993 & 18 & 1.5 & a \\
\hline & December 26-30, 1993 & 24 & .5 & b \\
\hline & 1993 Weekly Average $\mathrm{c}$ & 14 & .18 & b \\
\hline
\end{tabular}

a Sample collected from the monitoring system on 195-foot stack

c 1993 weekly average includes samples collected through the middle of November 1993. 


\section{Table 85}

Comparison of Alpha Concentrations Beifore and During the Period of the Nonroutine Release in December 1993

Page 1 of 1

\begin{tabular}{|c|c|c|}
\hline $\begin{array}{l}\text { Environmental Surveillance } \\
\text { Station }\end{array}$ & Perloda & $\begin{array}{l}\text { Alpha Concentration } \\
\left(\mathrm{pCl} / \mathrm{m}^{3}\right)\end{array}$ \\
\hline \multirow[t]{3}{*}{ Darkhorse } & December 22-28, 1993 & .003 \\
\hline & 1993 Maximum & .004 \\
\hline & 1993 Average & .002 \\
\hline \multirow[t]{3}{*}{ Highway 39} & December 22-28, 1993 & .003 \\
\hline & 1993 Maximum & .005 \\
\hline & 1993 Average & .002 \\
\hline \multirow[t]{3}{*}{ Aiken Airport } & December 22-29, 1993 & .001 \\
\hline & 1993 Maximum & .004 \\
\hline & 1993 Average & .002 \\
\hline \multirow[t]{3}{*}{ Aiken State Park } & December 22-29, 1993 & .001 \\
\hline & 1993 Maximum & .004 \\
\hline & 1993 Average & .002 \\
\hline \multirow[t]{3}{*}{ Springfield } & December 22-29, 1993 & .001 \\
\hline & 1993 Maximum & .004 \\
\hline & 1993 Average & .002 \\
\hline \multirow[t]{3}{*}{ Lees } & December 22-29, 1993 & .00038 \\
\hline & 1993 Maximum & .004 \\
\hline & 1993 Average & .002 \\
\hline
\end{tabular}

a 1993 maximums and averages were calculated using 1993 data collected through the first week in December. 
Table 86

Savannah River Swamp TLD Exposure Measurements-1993

Page 1 of 2

\begin{tabular}{|c|c|c|}
\hline Trall & Distance from River (Feet) & $\begin{array}{c}\text { Exposure Rate } \\
(m R / D a y)^{a, b}\end{array}$ \\
\hline 1 & 0 & 0.26 \\
\hline 1 & 585 & 0.38 \\
\hline 1 & 1175 & 0.44 \\
\hline 1 & 1805 & 0.72 \\
\hline 1 & 2150 & 0.75 \\
\hline 1 & 2640 & 0.35 \\
\hline 2 & 0 & 0.33 \\
\hline 2 & 680 & 0.32 \\
\hline 2 & 1330 & 0.34 \\
\hline 2 & 1960 & 0.35 \\
\hline 2 & 2620 & 0.37 \\
\hline 2 & 3100 & 0.45 \\
\hline 2 & 3200 & 0.29 \\
\hline 3 & 0 & 0.34 \\
\hline 3 & 920 & 0.35 \\
\hline 3 & 2055 & 0.28 \\
\hline 4 & 0 & 0.31 \\
\hline 4 & 960 & 0.35 \\
\hline 4 & 1245 & 0.42 \\
\hline 4 & 1690 & 0.34 \\
\hline 4 & 1900 & 0.55 \\
\hline 4 & 2390 & 0.30 \\
\hline 5 & 0 & 0.34 \\
\hline 5 & 1750 & 0.36 \\
\hline 5 & 1880 & 0.43 \\
\hline 5 & 2100 & 0.70 \\
\hline 5 & 2535 & 0.33 \\
\hline
\end{tabular}

a $\quad \mathrm{rnR}=$ milliRoentgen, exposure unit for gamma radiation. One $\mathrm{mR}$ is approximately equal to $1 \mathrm{mrem}$.

b An $18 \%$ uncertainty is associated with each result. 
Table 86

Savannah River Swamp TLD Exposure Measurements—1993

Page 2 of 2

\begin{tabular}{|c|c|c|}
\hline Trall & Distance from River (Feet) & $\begin{array}{c}\text { Exposure Rate } \\
(\mathrm{mR} / \mathrm{Day})^{\mathrm{a}, \mathrm{b}}\end{array}$ \\
\hline 6 & 0 & 0.33 \\
\hline 6 & 1800 & 0.35 \\
\hline 6 & 2300 & 0.41 \\
\hline 6 & 2530 & 0.54 \\
\hline 6 & 2680 & 0.33 \\
\hline 7 & 0 & c \\
\hline 7 & 1900 & 0.33 \\
\hline 7 & 2600 & 0.43 \\
\hline 7 & 2700 & 0.37 \\
\hline 7 & 3100 & 0.40 \\
\hline 7 & 3200 & 0.32 \\
\hline 8 & 0 & 0.30 \\
\hline 8 & 550 & 0.33 \\
\hline 8 & 915 & 0.32 \\
\hline 8 & 1460 & 0.32 \\
\hline 8 & 2005 & 0.34 \\
\hline 8 & 2670 & 0.32 \\
\hline 8 & 2900 & 0.50 \\
\hline 8 & 3000 & 0.41 \\
\hline 9 & 0 & 0.41 \\
\hline 9 & 1680 & 0.43 \\
\hline 9 & 2035 & 0.47 \\
\hline 9 & 2200 & 0.59 \\
\hline 9 & 2525 & 0.36 \\
\hline 10 & 0 & 0.40 \\
\hline 10 & 100 & 0.39 \\
\hline 10 & 240 & 0.31 \\
\hline
\end{tabular}

a $\mathrm{mR}=$ milliRoentgen, exposure unit for gamma radiation. One $\mathrm{mR}$ is approximately equal to $1 \mathrm{mrem}$.

b An $18 \%$ uncertainty is associated with each result.

c Blank space indicates missing TLD results. 
Table 87

Creek Plantation Survey, Swamp Soll Results

Page 1 of 1

\begin{tabular}{rrccc}
\hline & $\begin{array}{c}\text { Distance (Feet } \\
\text { Tromsavannah } \\
\text { River) }\end{array}$ & C8-137 (pCl/g) & Co-60 (pCl/g) & sr 89,90 (pCl/g) \\
\hline 1 & 2150 & $1.05 E+02 \pm 4.90 E-01$ & $3.40 E-01 \pm 4.00 E-02$ & $1.49 E-02 \pm 1.79 E-02$ \\
2 & 3100 & $5.79 E+01 \pm 4.20 E-01$ & $<L L D a$ & $4.43 E-03 \pm 1.53 E-02$ \\
3 & 920 & $1.59 E+00 \pm 9.00 E-02$ & $<L L D$ & $1.25 E-02 \pm 1.64 E-02$ \\
4 & 1900 & $4.77 E+01 \pm 4.40 E-01$ & $1.60 E-01 \pm 3.00 E-02$ & $1.10 E-01 \pm 2.10 E-02$ \\
5 & 2100 & $3.21 E+01 \pm 3.30 E-01$ & $<L L D$ & $4.85 E-02 \pm 2.12 E-02$ \\
6 & 2530 & $7.02 E+01 \pm 6.00 E-01$ & $<L L D$ & $1.60 E-01 \pm 2.54 E-02$ \\
7 & 2700 & $3.11 E+01 \pm 4.20 E-01$ & $<L L D$ & $1.33 E-01 \pm 2.44 E-02$ \\
8 & 2900 & $3.77 E+01 \pm 3.80 E-01$ & $1.00 E-01 \pm 4.00 E-02$ & $1.29 E-01 \pm 2.43 E-02$ \\
9 & 2205 & $3.79 E-01 \pm 3.60 E-01$ & $<L L D$ & $1.52 E-01 \pm 2.51 E-02$ \\
10 & 100 & $2.34 E+01 \pm 2.40 E-01$ & $<L L$ & $3.93 E-02 \pm 1.84 E-02$
\end{tabular}

a Less than the lower limit of detection 


\section{Table 88}

Creek Plantation Survey, Swamp Vegetation Results

Page 1 of 1

\begin{tabular}{|c|c|c|c|}
\hline Trall & $\begin{array}{c}\text { Distance (Foet } \\
\text { From Savannah River) }\end{array}$ & C8-137 (pCl/g) & Sr $89,90(p C / / g)$ \\
\hline 1 & 2150 & $2.75 E+01 \pm 3.90 E-01$ & $3.48 E-02 \pm 7.51 E-02$ \\
\hline 2 & 3100 & $5.00 E-01 \pm 8.00 E-02$ & $1.22 E-01 \pm 8.29 E-02$ \\
\hline 3 & 920 & $<L L D^{a}$ & $2.00 E-02 \pm 7.66 E-02$ \\
\hline 4 & 1900 & $1.34 E+01 \pm 2.70 E-01$ & $4.81 E-01 \pm 9.50 E-02$ \\
\hline 5 & 2100 & $6.67 E+00 \pm 1.90 E-01$ & $2.45 E-01 \pm 8.62 E-02$ \\
\hline 6 & 2530 & $3.87 E+01 \pm 5.50 E-01$ & $9.92 E-01 \pm 1.06 E-01$ \\
\hline 7 & 2700 & $2.00 E+00 \pm 1.30 E-01$ & $5.33 E-01 \pm 1.05 E-01$ \\
\hline 8 & 2900 & $5.60 E+00 \pm 1.90 E-01$ & $2.57 E-01 \pm 8.79 E-02$ \\
\hline 9 & 2205 & $1.08 E+00 \pm 1.10 E-01$ & $7.22 E-01 \pm 9.17 E-02$ \\
\hline 10 & 100 & $1.59 E+00 \pm 9.00 E-02$ & $1.36 E-01 \pm 8.76 E-02$ \\
\hline
\end{tabular}




\section{Table 89}

Creek Plantation Survey, Swamp Results 1990, 1991, 1993

Page 1 of 3

\begin{tabular}{|c|c|c|c|c|c|c|c|}
\hline Trail & $\begin{array}{l}\text { Distance } \\
\text { (Feet from } \\
\text { Savannah } \\
\text { River) }\end{array}$ & $\begin{array}{c}\text { Soil, Cs-137 } \\
\text { (pCi/g) }\end{array}$ & $\begin{array}{c}\text { Soil, Sr-89,90 } \\
\text { (pCl/g) }\end{array}$ & $\begin{array}{c}\text { Soil, Co-60 } \\
\text { (pClig) }\end{array}$ & $\begin{array}{c}\text { Veg, Cs-137 } \\
\text { (pCi/g) }\end{array}$ & $\begin{array}{c}\text { Veg, Sr-89,90 } \\
\text { (pCl/g) }\end{array}$ & $\begin{array}{c}\text { Veg, Co-60 } \\
\text { (pCV/g) }\end{array}$ \\
\hline \multirow[t]{4}{*}{1} & 2150 & & & & & & \\
\hline & 1990 & $<L_{L} D^{a}$ & $2.10 E-01$ & $\angle L L D$ & $1.51 E+01$ & $1.60 \mathrm{E}-01$ & $1.50 E-01$ \\
\hline & 1991 & $1.38 E+01$ & $2.00 E-02$ & $\angle L L D$ & $4.00 \mathrm{E}-01$ & $5.20 E-01$ & CLLD \\
\hline & 1993 & $1.05 E+02$ & $1.00 \mathrm{E}-02$ & $3.40 E-01$ & $2.75+01$ & $3.48 E-02$ & DLD \\
\hline \multirow[t]{4}{*}{2} & 3100 & & & & & & \\
\hline & 1990 & <LLD & $9.00 E-02$ & $\angle L L D$ & $<L D$ & $6.50 E-01$ & DLI \\
\hline & 1991 & $1.10 E+00$ & $2.00 \mathrm{E}-02$ & $<L L D$ & $<L D$ & $2.00 E-02$ & DLA \\
\hline & 1993 & $5.79 E+01$ & $1.00 \mathrm{E}-02$ & $\angle L L D$ & $5.00 \mathrm{E}-01$ & $1.22 E-01$ & CLD \\
\hline \multirow[t]{4}{*}{3} & 920 & & & & & & \\
\hline & 1990 & 0.8 & $<L L D$ & $4.00 \mathrm{E}-01$ & $<L L D$ & $2.50 \mathrm{E}-01$ & $\angle L D$ \\
\hline & 1991 & $2.60 E+00$ & $3.00 E-02$ & <LLD & $<L D$ & $2.40 \mathrm{E}-01$ & $\angle L D$ \\
\hline & 1993 & $1.60 E+00$ & $1.00 \mathrm{E}-02$ & $<L L D$ & <LLD & $2.00 E-02$ & CLD \\
\hline
\end{tabular}

a Less than the lower limit of detection 


\section{Table 89}

Creek Plantation Survey, Swamp Results 1990, 1991, 1993

Page 2 of 3

\begin{tabular}{|c|c|c|c|c|c|c|c|}
\hline Trall & $\begin{array}{c}\text { Distance } \\
\text { (Feet from } \\
\text { Savannah } \\
\text { River) } \\
\end{array}$ & $\begin{array}{c}\text { Soll, Cs-137 } \\
\text { (pCl/g) }\end{array}$ & $\begin{array}{c}\text { Soil, Sr-89,90 } \\
\text { (pCl/g) }\end{array}$ & $\begin{array}{c}\text { Soll, Co-60 } \\
\text { (pCi/g) }\end{array}$ & $\begin{array}{l}\text { Veg, Cs-137 } \\
\text { (pcl/g) }\end{array}$ & $\begin{array}{l}\text { Veg, Sr-89,90 } \\
\text { (pCl/g) }\end{array}$ & $\begin{array}{c}\text { Veg, Co-60 } \\
\text { (pCl/g) }\end{array}$ \\
\hline \multirow[t]{4}{*}{4} & 1900 & & & & & & \\
\hline & 1990 & 92.2 & $1.40 \mathrm{E}-01$ & $5.00 E-01$ & $1.11 E+01$ & $3.20 E-01$ & $<U D^{a}$ \\
\hline & 1991 & $7.60 E+01$ & $3.00 E-02$ & $3.00 \mathrm{E}-01$ & $5.70 E+00$ & $3.40 E-01$ & $\angle L D$ \\
\hline & 1993 & $4.77 E+01$ & $1.10 E-01$ & $2.00 E-01$ & $1.34 E+01$ & $4.81 E-01$ & CLD \\
\hline \multirow[t]{4}{*}{5} & 2105 & & & & & & \\
\hline & 1990 & 58.2 & $1.90 E-01$ & $4.00 E-01$ & $1.40 E+00$ & $4.60 E-01$ & CLLD \\
\hline & 1991 & $<L L D$ & <LLD & $\angle L L D$ & $<\amalg D$ & $\angle L L D$ & ALD \\
\hline & 1993 & $3.21 E+01$ & $5.00 E-02$ & $\angle L L D$ & $6.67 E+00$ & $2.45 E-01$ & LLD \\
\hline \multirow[t]{4}{*}{6} & 2530 & & & & & & \\
\hline & 1990 & 41.4 & $1.00 \mathrm{E}-01$ & $2.00 E-01$ & $4.50 E+00$ & $7.00 E-01$ & CLD \\
\hline & 1991 & $6.88 E+01$ & $1.00 \mathrm{E}-02$ & $1.00 E-01$ & $1.76 E+01$ & $9.70 E 01$ & LLD \\
\hline & 1993 & $7.02 E+01$ & $1.60 E-01$ & $<L L D$ & $3.87 E+01$ & $9.92 E-01$ & CLD \\
\hline \multirow[t]{4}{*}{7} & 2700 & & & & & & \\
\hline & 1990 & 29.9 & $3.00 E-02$ & $<L L D$ & $<L L D$ & $<L D$ & \&LD \\
\hline & 1991 & $2.77 E+02$ & $3.00 \mathrm{E}-02$ & $1.00 E+00$ & $<L L D$ & $2.50 E-01$ & $\triangle 1 L D$ \\
\hline & 1993 & $3.11 E+01$ & $1.30 \mathrm{E}-01$ & $<L L D$ & $2.00 E+00$ & $5.33 E-01$ & $\angle L D$ \\
\hline
\end{tabular}


Table 89

Creek Plantation Survey, Swamp Results 1990, 1991, 1993

Page 3 of 3

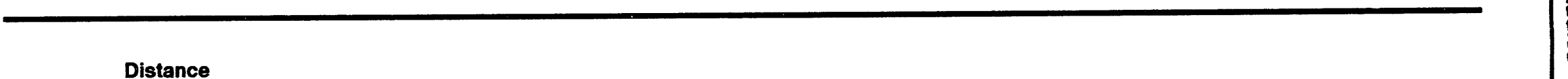

\begin{tabular}{|c|c|c|c|c|c|c|c|}
\hline Trail & $\begin{array}{c}\text { (Feet from } \\
\text { Savannah } \\
\text { River) }\end{array}$ & $\begin{array}{l}\text { Soil, Cs-137 } \\
\text { (pCi/g) }\end{array}$ & $\begin{array}{c}\text { Soil, Sr-89,90 } \\
\text { (pCl/g) }\end{array}$ & $\begin{array}{c}\text { Soil, Co-60 } \\
\text { (pCl/g) }\end{array}$ & $\begin{array}{c}\text { Veg, Cs-137 } \\
\text { (pCi/g) }\end{array}$ & $\begin{array}{c}\text { Veg, Sr-89,90 } \\
\text { (pCV/g) }\end{array}$ & $\begin{array}{c}\text { Veg, Co-60 } \\
\text { (pcl/g) }\end{array}$ \\
\hline \multirow[t]{4}{*}{8} & 2900 & & & & & & \\
\hline & 1990 & 66.4 & $8.00 \mathrm{E}-02$ & $<L^{\prime} D^{a}$ & $5.40 E+00$ & $2.80 E-01$ & CLLD \\
\hline & 1991 & $6.80 E+01$ & $2.00 E-02$ & $1.00 E-01$ & $2.30 E+00$ & $1.80 \mathrm{E}-01$ & LLD \\
\hline & 1993 & $3.77 E+01$ & $1.30 \mathrm{E}-01$ & $1.00 E-01$ & $5.60 \mathrm{E}+00$ & $2.57 E-01$ & CLD \\
\hline
\end{tabular}

$9 \quad 2205$

$\begin{array}{lcccccr}1990 & 61.5 & 1.70 E-01 & 2.00 E-01 & 1.34 E+01 & 5.20 E-01 & 4 L D \\ 1991 & 3.66 E+01 & 6.00 E-02 & 2.00 E-01 & 7.20 E+00 & 2.40 E-01 & 4.72 E-01 \\ 1993 & 3.79 E+01 & 1.50 E-01 & L L L D & 1.08 E+00 & 4 L D\end{array}$

$10 \quad 100$

$\begin{array}{lcccccr}1990 & 19.1 & 6.00 E-02 & 1.00 E-01 & 5.00 E-01 & 2.60 E-01 & 4 L D \\ 1991 & 2.24 E+01 & 2.00 E-02 & L L L D & 1.07 E+01 & 2.40 E-01 & 1.36 E-01 \\ 1993 & 2.34 E+01 & 4.00 E-02 & L L L D & 1.59 E+00 & 4 L D\end{array}$

$\overline{\text { a Lese }}$ ! $n$ the lower limit of detection 


\section{Table 90}

Pen Branch Core Samples

Page 1 of 4

\begin{tabular}{|c|c|c|c|}
\hline Location & $\begin{array}{c}\text { Depth } \\
\text { (Inches) }\end{array}$ & $C_{8-137}(p C / / g)$ & $\mathrm{Co-60}(\mathrm{pCl} / \mathrm{g})$ \\
\hline 1 NWa Bank & $0-3$ & $\angle L L D^{b}$ & $<L L D$ \\
\hline 1 NW Bank & $3-6$ & $<L L D$ & $<L L D$ \\
\hline 1 NW Bank & $6-9$ & $<L L D$ & $<L L D$ \\
\hline 1 NW High Ground & $0-3$ & $5.40 E-01 \pm 6.00 E-02$ & $<L L D$ \\
\hline 1 NW High Ground & $3-6$ & $3.30 E-01 \pm 4.00 E-02$ & $<L L D$ \\
\hline 1 NW High Ground & $6-9$ & $3.80 E-01 \pm 5.00 E-02$ & $<L L D$ \\
\hline 1 NW High Ground & $9-12$ & $<L L D$ & $<L L D$ \\
\hline $1 \mathrm{SE}^{\mathrm{c}}$ Bank & $0-3$ & $5.00 E-02 \pm 3.00 E-02$ & <LLD \\
\hline 1 SE Bank & $3-6$ & $<L L D$ & $<L L D$ \\
\hline 1 SE Bank & $6-9$ & $<L L D$ & $<L L D$ \\
\hline 1 SE Bank & $9-12$ & $<L L D$ & $<L L D$ \\
\hline 1 SE Flood Plain & $0-3$ & $<L L D$ & $<L L D$ \\
\hline 1 SE Flood Plain & $3-6$ & <LLD & $<L L D$ \\
\hline 1 SE Flood Plain & $6-9$ & $3.60 E-01 \pm 4.00 E-02$ & $<L L D$ \\
\hline 1 SE High Ground & $0-3$ & $5.60 \mathrm{E}-01 \pm 7.00 \mathrm{E}-02$ & $<L L D$ \\
\hline 1 SE High Ground & $3-6$ & $2.20 E-01 \pm 4.00 E-02$ & <LLD \\
\hline 2 NW Bank & $0-3$ & $1.09 E+00 \pm 6.00 E-02$ & $1.60 E+00 \pm 7.00 E-02$ \\
\hline 2 NW Bank & $3-6$ & $5.60 E-01 \pm 6.00 E-02$ & $8.90 E-01 \pm 5.00 E-02$ \\
\hline 2 NW Bank & $6-9$ & $<L L D$ & $<L L D$ \\
\hline 2 NW Flood Plain & $0-3$ & $7.40 E-01 \pm 6.00 E-02$ & $<L L D$ \\
\hline 2 NW Flood Plain & $3-6$ & $3.40 \mathrm{E}-01 \pm 4.00 \mathrm{E}-02$ & $<L L D$ \\
\hline 2 NW Flood Plain & $6-9$ & $2.50 E-01 \pm 6.00 E-02$ & $1.10 E-01 \pm 2.00 E-02$ \\
\hline 2 NW Flood Plain & $9-12$ & $<L L D$ & $<L L D$ \\
\hline 2 NW High Ground & $0-3$ & $1.60 E-01 \pm 4.00 E-02$ & $<L L . D$ \\
\hline 2 NW High Ground & $3-6$ & $2.50 E-01 \pm 4.00 E-02$ & $<L L D$ \\
\hline 2 NW High Ground & $6-9$ & $1.30 E-01 \pm 4.00 E-02$ & $<L L D$ \\
\hline 2 NW High Ground & $9-12$ & $<L L D$ & <LLD \\
\hline 2 SE Bank & $0-3$ & $6.80 E-01 \pm 6.00 E-02$ & $2.30 E-01 \pm 3.00 E-02$ \\
\hline 2 SE Bank & $3-6$ & <LLD & $<L L D$ \\
\hline
\end{tabular}


Table 90

Pen Branch Core Samples

Page 2 of 4

\begin{tabular}{|c|c|c|c|}
\hline Location & $\begin{array}{c}\text { Depth } \\
\text { (Inchee) }\end{array}$ & C8-137 (pCl/g) & Co-60 (pCl/g) \\
\hline 2 SE $^{\mathrm{a}}$ Bank & $6-9$ & $\angle L L D^{b}$ & $<L L D$ \\
\hline 2 SE Bank & $9-12$ & $<L L D$ & $<L L D$ \\
\hline 2 SE Flood Plain & $0-3$ & $6.50 E-01 \pm 6.00 E-02$ & $3.20 E-01 \pm 3.00 E-02$ \\
\hline 2 SE Flood Plain & $3-6$ & $<L L D$ & $<L L D$ \\
\hline 2 SE High Ground & $0-3$ & $2.20 E-01 \pm 4.00 E-02$ & $<L L D$ \\
\hline 2 SE High Ground & $3-6$ & $1.30 E-01 \pm 2.00 E-02$ & $<L L D$ \\
\hline 2 SE High Ground & $6-9$ & $<L L D$ & $<L L D$ \\
\hline 2 SE High Ground & $9-12$ & $<L L D$ & $<L L D$ \\
\hline $3 N^{C}$ Bank & $0-3$ & $8.50 E-01 \pm 7.00 E-02$ & $<L L D$ \\
\hline 3 NW Bank & $3-6$ & $4.40 E-01 \pm 4.00 E-02$ & $<L L D$ \\
\hline 3 NW Flood Plain & $0-3$ & $4.40 E-01 \pm 5.00 E-02$ & $9.00 \mathrm{E}-02 \pm 2.00 \mathrm{E}-02$ \\
\hline 3 NW Flood Plain & $3-6$ & $1.70 \mathrm{E}-01 \pm 3.00 \mathrm{E}-\Omega 2$ & $<L L D$ \\
\hline 3 NW High Ground & $0-3$ & $3.90 \mathrm{E}-01 \pm 4.00 \mathrm{E}-02$ & $<L L D$ \\
\hline 3 NW High Ground & $3-6$ & $1.70 E-01 \pm 5.00 E-02$ & $<L L D$ \\
\hline 3 NW High Ground & $6-9$ & $<L L D$ & $<$ LLL.D \\
\hline 3 NW High Ground & $9-12$ & $<L L D$ & $<L L D$ \\
\hline 3 SE Bank & $0-3$ & $1.82 E+00 \pm 9.00 E-02$ & $<L L D$ \\
\hline 3 SE Bank & $3-6$ & $3.20 E-01 \pm 5.00 E-02$ & $<L L D$ \\
\hline 3 SE Flood Plain & $0-3$ & $2.20 \mathrm{E}-01 \pm 7.00 \mathrm{E}-02$ & $<L L D$ \\
\hline 3 SE Flood Plain & $3-6$ & $1.30 \mathrm{E}-01 \pm 3.00 \mathrm{E}-02$ & $<L L D$ \\
\hline 3 SE Flood Plain & $6-9$ & $8.60 E-01 \pm 7.00 E-02$ & $<L L D$ \\
\hline 3 SE High Ground & $0-3$ & $2.40 E-01 \pm 5.00 E-02$ & $<L L D$ \\
\hline 3 SE High Ground & $3-6$ & $1.40 \mathrm{E}-01 \pm 4.00 \mathrm{E}-02$ & $<L L D$ \\
\hline 3 SE High Ground & $6-9$ & $<L L D$ & $<L L D$ \\
\hline 3 SE High Ground & $9-12$ & $<L L D$ & $<L L D$ \\
\hline 4 NW Bank & $0-3$ & $<L L D$ & $<L L D$ \\
\hline 4 NW Bank & $3-6$ & $<L L D$ & $<L L D$ \\
\hline 4 NW Bank & $6-9$ & $<L L D$ & $<L L D$ \\
\hline 4 NW Flood Plain & $0-3$ & $3.40 \mathrm{E}-01 \pm 5.00 \mathrm{E}-02$ & $<L L D$ \\
\hline
\end{tabular}




\section{Table 90}

\section{Pen Branch Core Samples}

Page 3 of 4

\begin{tabular}{|c|c|c|c|}
\hline Location & $\begin{array}{c}\text { Depth } \\
\text { (Inohes) }\end{array}$ & $c_{8-137}(p c 1 / q)$ & $C 0.60(p 01 / g)$ \\
\hline 4 NWa Flood Plain $^{\circ}$ & $3-6$ & $1.60 E-01 \pm 4.00 E-02$ & $\angle L L D^{b}$ \\
\hline 4 NW Flood Plain & $6-9$ & $<L L D$ & $<L L D$ \\
\hline 4 NW High Ground & $0-3$ & $1.00 E-01 \pm 4.00 E-02$ & $<L L D$ \\
\hline 4 NW High Ground & $3-6$ & $<L L D$ & $<L L D$ \\
\hline 4 NW High Ground & $6-9$ & $<L L D$ & $<L L D$ \\
\hline 4 NW High Ground & $9-12$ & $<L L D$ & $<L L D$ \\
\hline 4 SEC Bank $^{c}$ & $0-3$ & $1.77 E+00 \pm 1.00 E-01$ & $2.23 E+00 \pm 7.00 E-02$ \\
\hline 4 SE Bank & $3-6$ & $1.63 E+00 \pm 7.00 E-02$ & $1.28 E+00 \pm 6.00 E-02$ \\
\hline 4 SE Bank & $6-9$ & $1.55 E+00 \pm 7.00 E-02$ & $1.66 E+00 \pm 6.00 E-02$ \\
\hline 4 SE Flood Plain & $0-3$ & $9.93 E+00 \pm 1.40 E-01$ & $1.96 \mathrm{E}+00 \pm 7.00 \mathrm{E}-02$ \\
\hline 4 SE Flood Plain & $3-6$ & $1.51 E+00 \pm 8.00 E-02$ & $2.55 E+00 \pm 7.00 E-02$ \\
\hline 4 SE High Ground & $0-3$ & $2.04 E+00 \pm 8.00 E-02$ & $1.46 E+00 \pm 6.00 E-02$ \\
\hline 4 SE High Ground & $3-6$ & $8.70 E-01 \pm 6.00 E-02$ & $3.39 E+00 \pm 8.00 E-02$ \\
\hline 5 NW Bank & $0-3$ & $2.00 \mathrm{E}-01 \pm 6.00 \mathrm{E}-02$ & $8.00 E-02 \pm 2.00 E-02$ \\
\hline 5 NW Bank & $3-6$ & $1.09 E+00 \pm 7.00 E-02$ & $4.80 \mathrm{E}-01 \pm 4.00 \mathrm{E}-02$ \\
\hline 5 NW Bank & $6-9$ & $1.47 E+00 \pm 7.00 E-02$ & $1.79 E+00 \pm 6.00 E-02$ \\
\hline 5 NW Flood Plain & $0-3$ & $2.50 E-01 \pm 5.00 E-0 ?$ & $<L L D$ \\
\hline 5 NW Flood Plain & $3-6$ & $5.20 E-01 \pm 7.00 E-02$ & $<L L D$ \\
\hline 5 NW Flood Plain & $6-9$ & $1.00 E-01 \pm 4.00 E-02$ & $<L L D$ \\
\hline 5 NW Flood Plain & $9-12$ & $<L L D$ & $<L L D$ \\
\hline 5 NW High Ground & $0-3$ & $2.60 E-01 \pm 5.00 E-02$ & $<L L D$ \\
\hline 5 NW High Ground & $3-6$ & $<L L D$ & $<L L D$ \\
\hline 5 SE Bank & $0-3$ & $<L L D$ & $1.20 E-01 \pm 3.00 E-02$ \\
\hline 5 SE Bank & $3-6$ & $3.80 E-01 \pm 5.00 E-02$ & $<L L D$ \\
\hline 5 SE Bank & $6-9$ & $6.40 E-01 \pm 5.00 E-02$ & $8.00 E-01 \pm 5.00 E-02$ \\
\hline 5 SE Bank & $9-12$ & $2.40 E-01 \pm 4.00 E-02$ & $3.30 E-01 \pm 3.00 E-02$ \\
\hline 5 SE Flood Plain & $0-3$ & $6.60 \mathrm{E}-01 \pm 6.00 \mathrm{E}-02$ & $<L L D$ \\
\hline 5 SE Flood Plain & $3-6$ & $3.70 E-01 \pm 5.00 E-02$ & $<L L D$ \\
\hline 5 SE Flood Plain & $6-9$ & $<L L D$ & <LLD \\
\hline
\end{tabular}


Table 90

Pen Branch Core Samples

Page 4 of 4

\begin{tabular}{|c|c|c|c|}
\hline Looation & $\begin{array}{c}\text { Depth } \\
\text { (Inoheo) }\end{array}$ & Co-137 (pC//g) & Co-60 (pcl/g) \\
\hline 5 SEa High Ground & $0-3$ & $7.30 \mathrm{E}-01 \pm 8.00 \mathrm{E}-02$ & $\angle L L D^{b}$ \\
\hline 5 SE High Ground & $3-6$ & $<L L D$ & $<L L D$ \\
\hline 5 SE High Ground & $6-9$ & <LLD & $<L L D$ \\
\hline 5 SE High Ground & $9-12$ & <LLD & $<L L D$ \\
\hline 6 NWC Bank $^{\circ}$ & $0-3$ & $2.03 E+00 \pm 9.00 E-02$ & $9.50 E-01 \pm 5.00 E-02$ \\
\hline 6 NW Bank & $3-6$ & $5.20 E+00 \pm 1.20 E-01$ & $1.76 E+00 \pm 6.00 E-02$ \\
\hline 6 NW Bank & $6-9$ & $2.21 \theta+01 \pm 2.10 E-01$ & $1.77 E+00 \pm 7.00 E-02$ \\
\hline 6 NW Flood Plain & $0-3$ & $1.70 E-01 \pm 3.00 E-02$ & $<L L D$ \\
\hline 6 NW Flood Plain & $3-6$ & <LLD & <LLD \\
\hline 6 NW Flood Plain & $6-9$ & <LLD & $<L L D$ \\
\hline 6 NW High Ground & $0-3$ & <LLD & $<L L D$ \\
\hline 6 NW High Ground & $3-6$ & $<L L D$ & $<I L D$ \\
\hline 6 NW High Ground & $6-9$ & <LLD & <LLD \\
\hline 6 SE Bank & $0-3$ & $7.70 E-01 \pm 6.00 E-02$ & $1.23 E+00 \pm 6.00 E-02$ \\
\hline 6 SE Bank & $3-6$ & $2.55 E+00 \pm 1.00 E-01$ & $1.40 E+00 \pm 5.00 E-02$ \\
\hline 6 SE Bank & $6-9$ & $9.10 \mathrm{E}-01 \pm 6.00 \mathrm{E}-02$ & $1.26 E+00 \pm 6.00 E-02$ \\
\hline 6 SE Flood Plain & $0-3$ & $7.20 \mathrm{E}-01 \pm 6.00 \mathrm{E}-02$ & $4.60 E-01 \pm 4.00 E-02$ \\
\hline 6 SE Flood Plain & $3-6$ & $6.00 \mathrm{E}-01 \pm 5.00 \mathrm{E}-02$ & $1.31 E+00 \pm 6.00 E-02$ \\
\hline 6 SE Flood Plain & $6-9$ & $4.60 E-01 \pm 5.00 E-02$ & $<L L D$ \\
\hline 6 SE High Ground & $0-3$ & $2.80 E-01 \pm 6.00 E-02$ & $<L L D$ \\
\hline 6 SE High Ground & $3-6$ & $<L L D$ & $<L L D$ \\
\hline
\end{tabular}

\footnotetext{
South or aide of the stream 
Table 91

Pen Branch Survey-Soil Results

Page 1 of 2

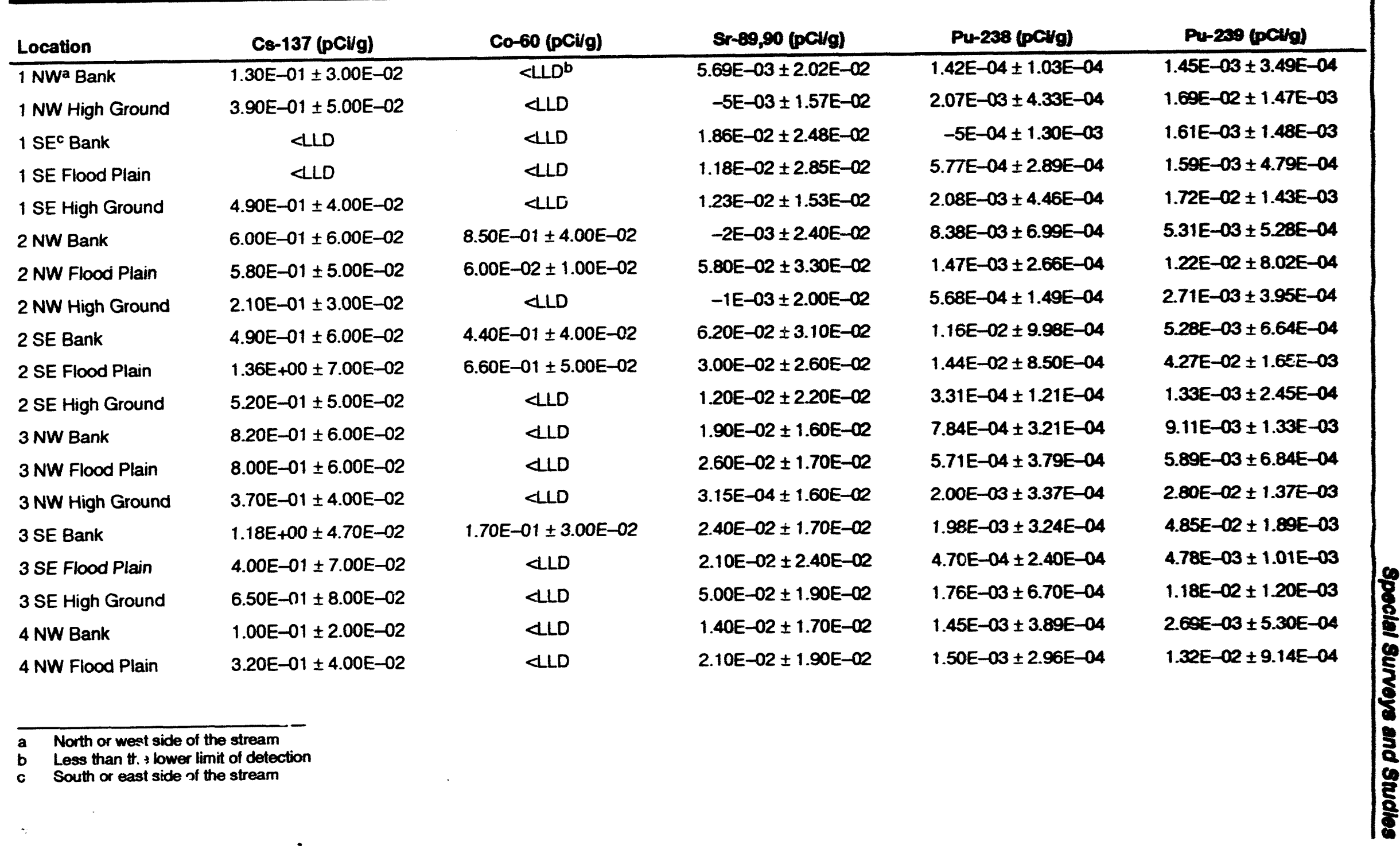



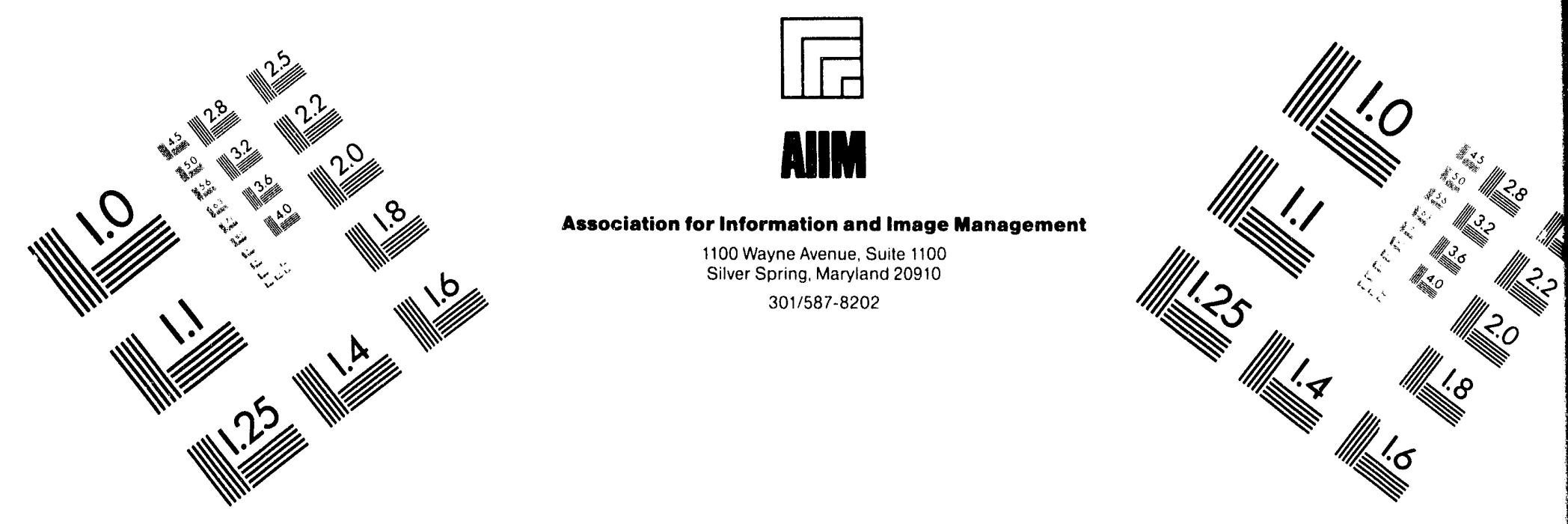

\section{Centimeter}

$\left.\right|_{1}$

Inches
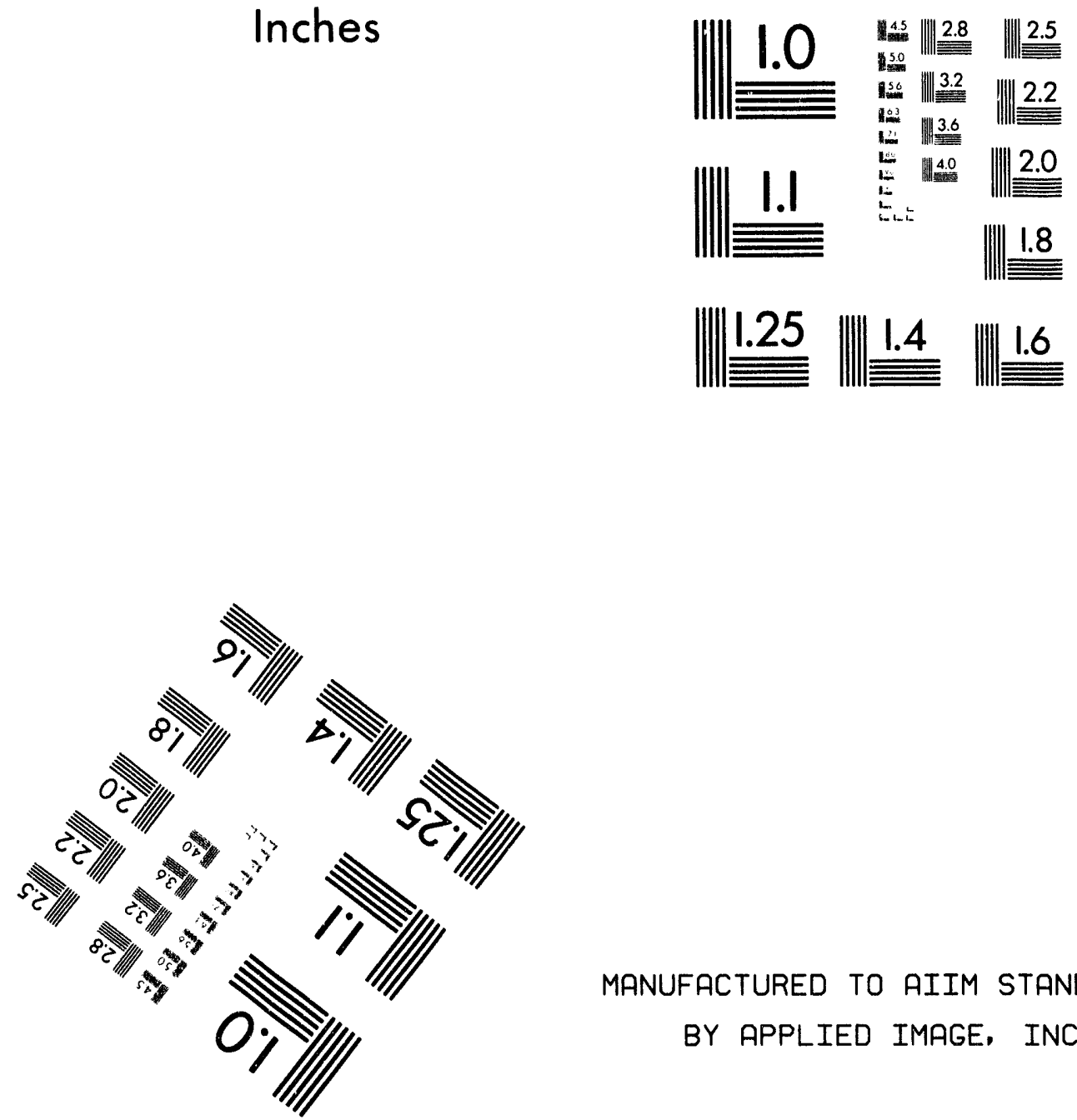

MANUFACTURED TO AIIM STANDARDS

$$
\text { BY APPLIED IMAGE, INC. }
$$

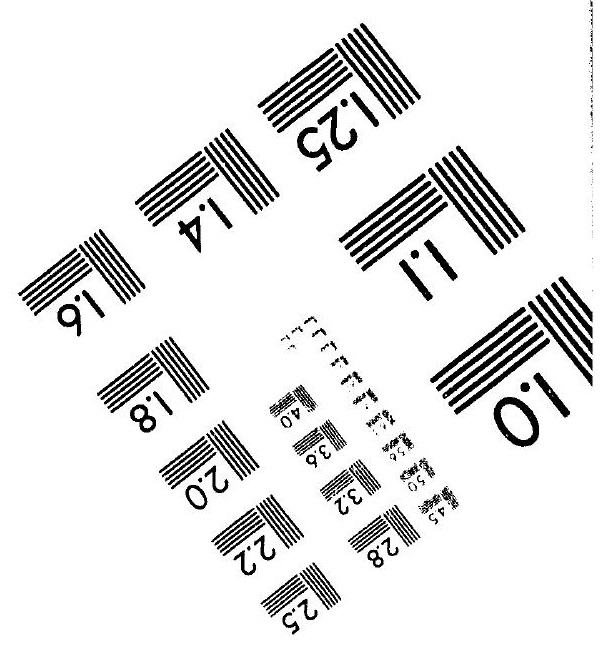



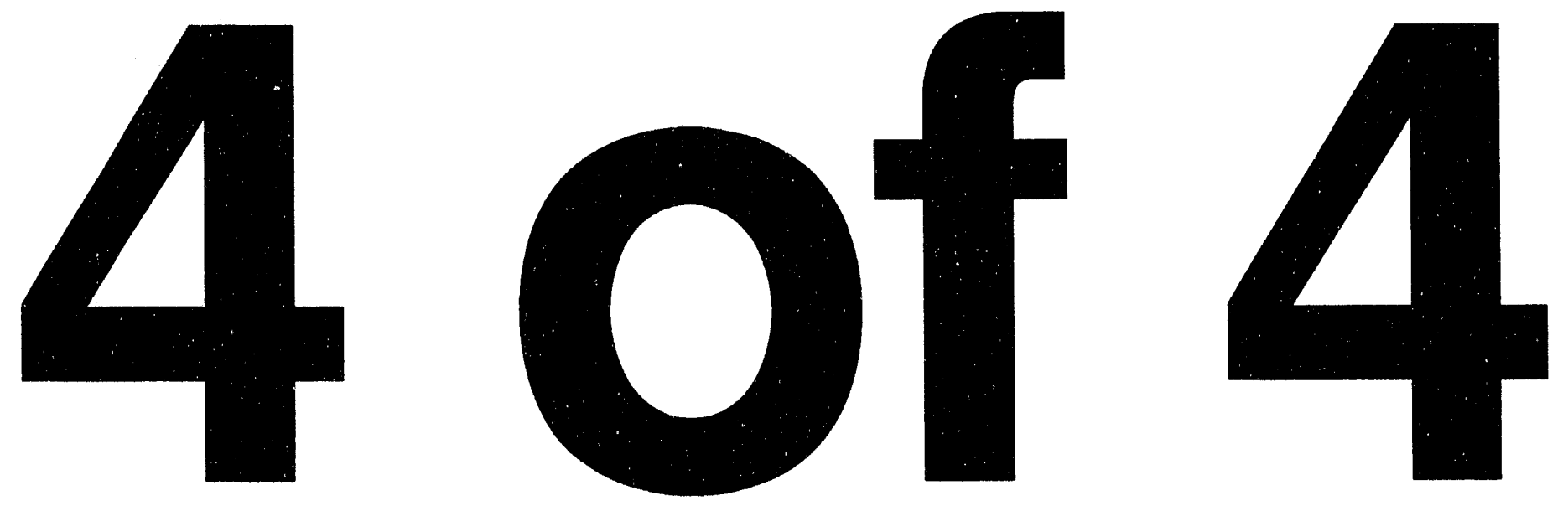
Table 91

Pen Branch Survey-Soil Results

Page 2 of 2

\begin{tabular}{|c|c|c|c|c|c|}
\hline Location & Cs-137 (pCi/g) & $\mathrm{Co}-60(\mathrm{pCl} / \mathrm{g})$ & Sr-89,90 (pCi/g) & $\mathrm{Pu}-238$ (pCl/g) & Pu-239 (pCl/g) \\
\hline 4 NWa High Ground & $2.20 E-01 \pm 3.00 E-02$ & $<$ LLD $^{\mathrm{b}}$ & $4.90 \mathrm{E}-02 \pm 1.80 \mathrm{E}-02$ & $2.97 E-03 \pm 4.29 E-04$ & $1.74 E-03 \pm 4.74 E-04$ \\
\hline 4 SEC Bank $^{c}$ & $2.07 E+00 \pm 8.00 E-02$ & $2.50 E+00 \pm 7.00 E-02$ & $9.40 E-02 \pm 2.90 E-02$ & $7.73 E-03 \pm 4.65 E-04$ & $7.80 E-02 \pm 2.66 E-03$ \\
\hline 4 SE Flood Plain & $5.82 E+00 \pm 1.30 E-01$ & $3.70 E+00 \pm 9.00 E-02$ & $1.60 E-02 \pm 1.80 E-02$ & $5.38 E-02 \pm 6.07 E-04$ & $1.78 E-01 \pm 4.91 E-03$ \\
\hline 4 SE High Ground & $4.02 E+00 \pm 1.00 E-01$ & $3.08 E+00 \pm 8.00 E-02$ & $5.92 E-02 \pm 1.93 E-02$ & $6.56 E-03 \pm 9.55 E-04$ & $1.31 E-01 \pm 4.68 E-03$ \\
\hline 5 NW Bank & $9.00 E-02 \pm 3.00 E-02$ & $<L L D$ & $5.67 E-03 \pm 1.72 E-02$ & $2.18 \mathrm{E}-04 \pm 9.77 \mathrm{E}-05$ & $-2 E-04 \pm 3.37 E-04$ \\
\hline 5 NW Flood Plain & $4.40 E-01 \pm 5.00 E-02$ & <LLD & $-4 E-04 \pm 1.84 E-02$ & $3.76 E-04 \pm 1.68 E-04$ & $8.93 E-03 \pm 8.39 E-04$ \\
\hline 5 NW High Ground & $4.60 E-01 \pm 4.00 E-02$ & $<L L D$ & $2.80 \mathrm{E}-02 \pm 2.00 \mathrm{E}-02$ & $3.86 E-04 \pm 2.97 E-04$ & $1.53 E-02 \pm 9.77 E-04$ \\
\hline 5 SE Bank & <LLD & $<L L D$ & $1.10 E-02 \pm 1.80 E-02$ & $7.70 E-04 \pm 2.57 E-04$ & $1.02 E-02 \pm 2.97 E-04$ \\
\hline 5 SE Flood Plain & $6.40 \mathrm{E}-01 \pm 4.00 \mathrm{E}-02$ & <LLD & $8.80 E-02 \pm 3.20 E=02$ & $1.78 \mathrm{E}-03 \pm 4.10 \mathrm{E}-04$ & $1.55 \mathrm{E}-02 \pm 1.25 \mathrm{E}-03$ \\
\hline 5 SE High Ground & $1.90 E-01 \pm 5.00 E-02$ & $<L L D$ & 4.27E-03 $\pm 1.90 E-02$ & $1.08 E-03 \pm 2.63 E-04$ & $1.29 E-02 \pm 9.46 E-04$ \\
\hline 6 NW Bank & $9.30 \mathrm{E}-01 \pm 5.00 \mathrm{E}-02$ & $3.80 E-01 \pm 3.00 E-02$ & $6.39-03 \pm 1.50 E-02$ & $3.62 E-03 \pm 4.51 E-04$ & $9.01 E-03 \pm 7.89 E-04$ \\
\hline 6 NW Flood Plain & $6.00 \mathrm{E}-02 \pm 2.00-\mathrm{E} 02$ & $<L L D$ & $-1 E-02 \pm 1.50 E-02$ & $-2 E-04 \pm 1.43 E-04$ & $1.31 E-03 \pm 4.42 E-04$ \\
\hline 6 NW High Ground & $1.20 E-01 \pm 3.00 E-02$ & $<L L D$ & $-3 E-02 \pm 1.50 E-02$ & $4.68 E-04 \pm 3.41 E-04$ & $4.08 E-03 \pm 5.61 E-04$ \\
\hline 6 SE Bank & $6.70 E-01 \pm 5.00 E-02$ & $6.40 \mathrm{E}-01 \pm 4.00 \mathrm{E}-02$ & $-8 E-04 \pm 1.50 E-02$ & $3.23 E-03 \pm 4.61 E-04$ & $6.45 E-03 \pm 6.48 E-04$ \\
\hline 6 SE Flood Plain & $1.43 E+00 \pm 9.00 E-02$ & $8.60 \mathrm{E}-01 \pm 6.00 \mathrm{E}-02$ & $6.00 E-02 \pm 2.80 E-02$ & $9.11 E-03 \pm 8.90 E-04$ & $1.78 E-02 \pm 1.27 E-03$ \\
\hline 6 SE High Ground & $2.70 \mathrm{E}-01 \pm 3.00 \mathrm{E}-02$ & $<L L D$ & $-3 E-02 \pm 2.10 E-02$ & $3.94 E-04 \pm 1.97 E-04$ & $6.60 E-03 \pm 8.17 E-04$ \\
\hline $\begin{array}{ll}\text { a North or west side } \\
\text { b Less than the lower } \\
\text { c South or east side c }\end{array}$ & $\begin{array}{l}\text { stream } \\
\text { t of detection } \\
\text { stream }\end{array}$ & & & & \\
\hline
\end{tabular}




\section{Table 92 \\ Pen Branch Survey, Vegetation Results}

Page 1 of 2

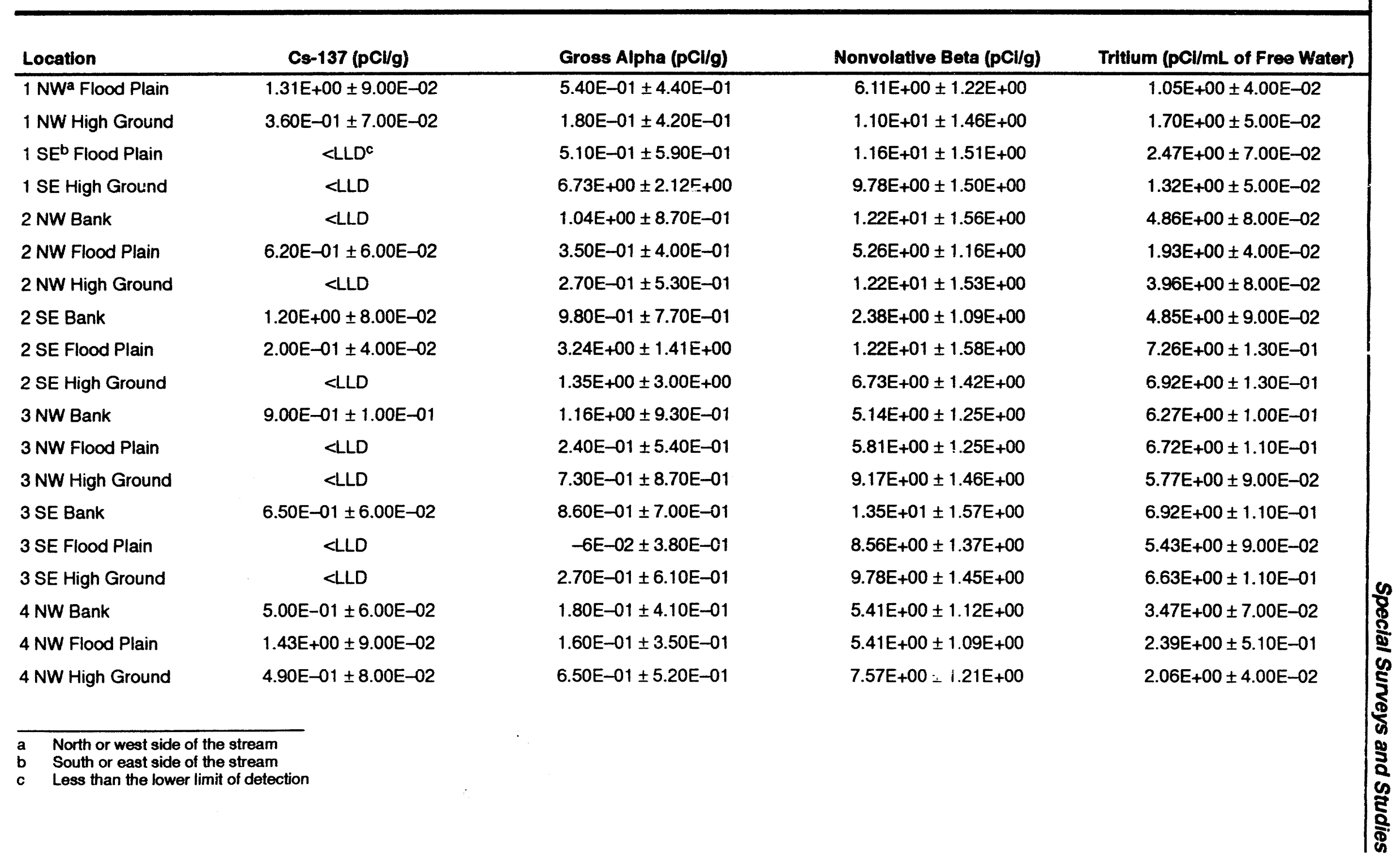


Table 92

Pen Branch Survey, Vegetation Results

Page 2 of 2

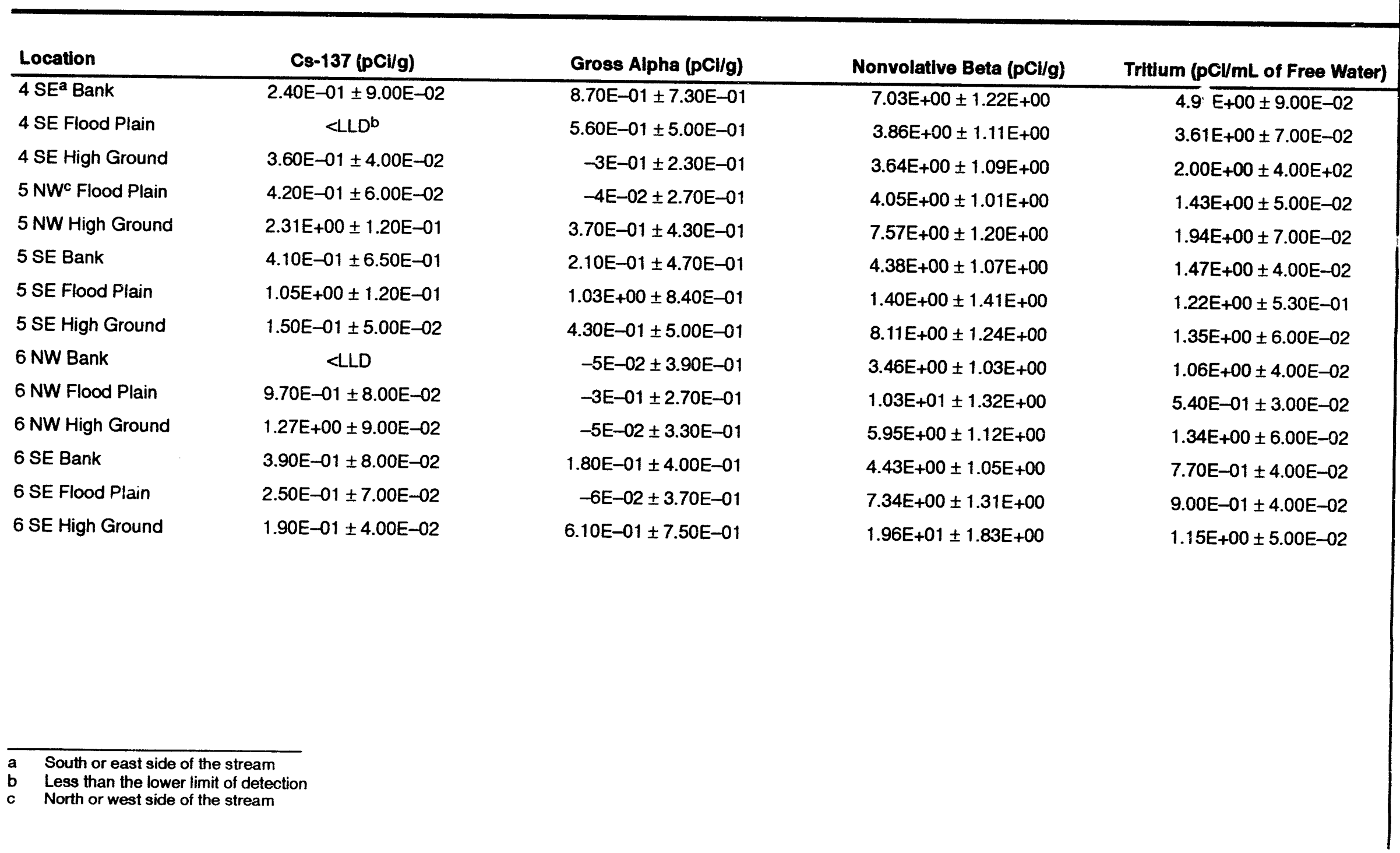



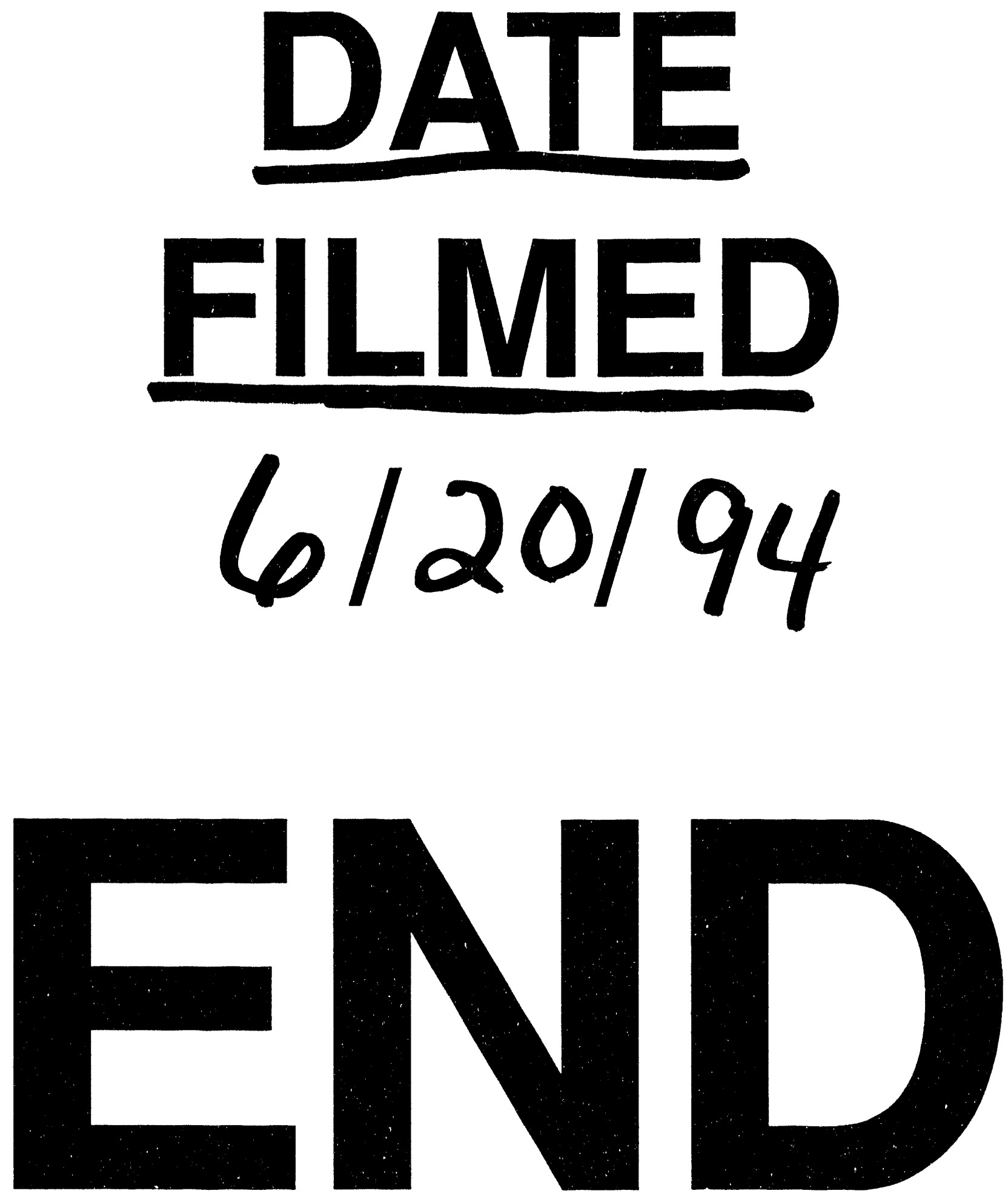
